Deutsche Gesellschaft für

Geschichte und Theorie der Biologie

\title{
Annals of the History and Philosophy of Biology \\ Volume 18 (2013)
}

formerly Jahrbuch für

Geschichte und Theorie der Biologie

Karl Porges

Evolutionsbiologie im Biologieunterricht der SBZ/DDR

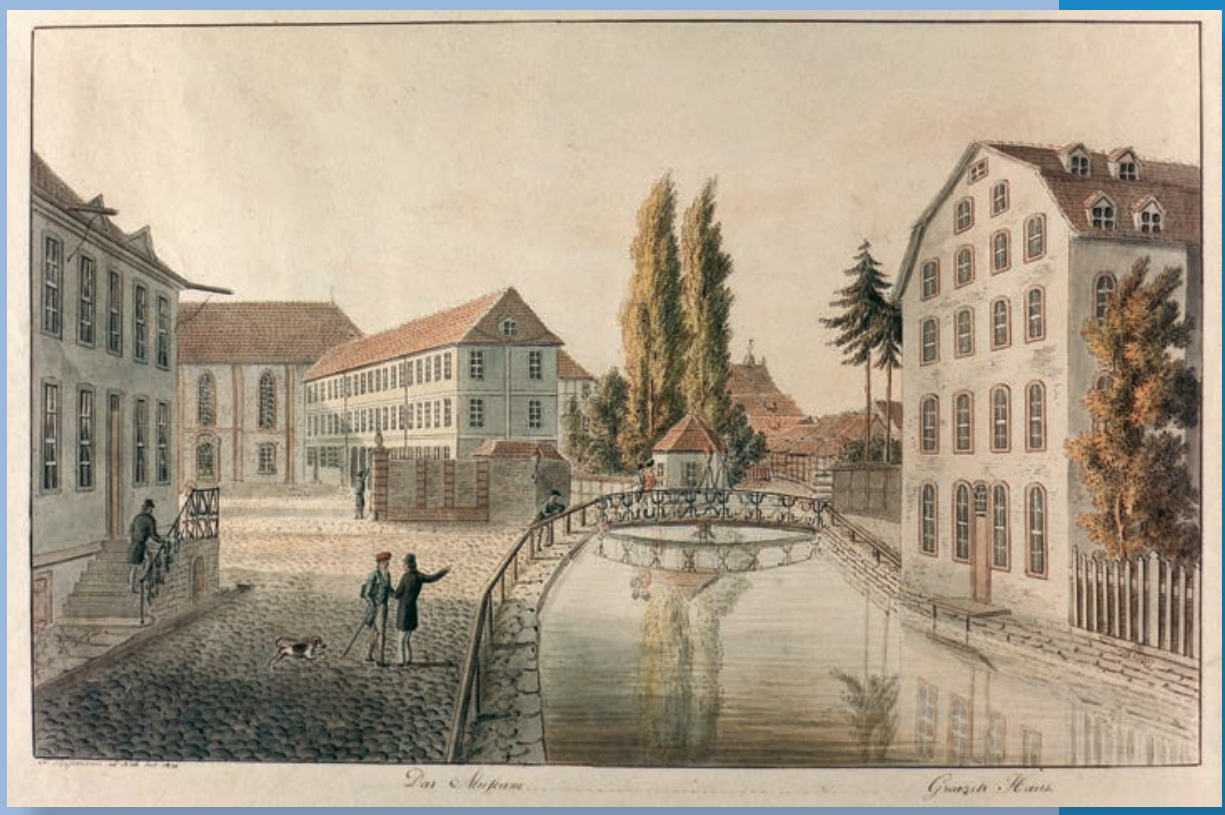


Manuscripts should be submitted to the managing editor. Submissions will be peer reviewed. The preferred language is English. Articles in German should be accompanied by a short (max. 1000 words) summary in English.

Managing Editor

Dr. Christian Reiß

Professur für Wissenschaftsgeschichte

Universität Regensburg

93040 Regensburg

Germany

Email: Christian.Reiss@psk.uni-regensburg.de

Editors

Uwe Hoßfeld, Jena, Germany

Lennart Olsson, Jena, Germany

Christian Reiß, Regensburg, Germany

Editorial Board

Ingo Brigandt, Edmonton, Canada

Ariane Dröscher, Bologna, Italy

Eve-Marie Engels, Tübingen, Germany

Gabriel W. Finkelstein, Denver, USA

Nick Hopwood, Cambridge, UK

Thomas Junker, Frankfurt/Main, Germany

Ulrich Kutschera, Kassel, Germany

Georgy S. Levit, Kassel, Germany

Amos Morris-Reich, Haifa, Israel

Staffan Müller-Wille, Exeter, UK

Kärin Nickelsen, Munich, Germany

Hans-Jörg Rheinberger, Berlin, Germany

Robert Richards, Chicago, USA

Marsha L. Richmond, Detroit, USA

Nicolaas A. Rupke, Lexington, USA

Hans-Konrad Schmutz, Zürich/Winterthur, Switzerland

Michal Simunek, Prague, Czech Republic

Georg Töpfer, Berlin, Germany

David M. Williams, London, UK

Volker Wissemann, Gießen, Germany 

Deutsche Gesellschaft für Geschichte und Theorie der Biologie (Ed.)

Annals of the History and Philosophy of Biology Vol. 18 (2013)

This work is licensed under a Creative Commons Attribution-ShareAlike 4.0 International License.

(c) (i) (2) 
Annals of the History and Philosophy of Biology; Volume 18 (2013)

Universitätsverlag Göttingen 2018 
Deutsche Gesellschaft für

Geschichte und Theorie der

Biologie (Ed.)

Annals of the History and

Philosophy of Biology

Vol. 18 (2013)

Karl Porges

Evolutionsbiologie im Biologieunterricht der SBZ/DDR

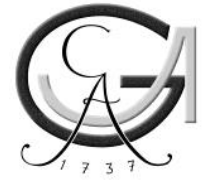

Universitätsverlag Göttingen 2018 
Bibliographic information published by the Deutsche Nationalbibliothek The Deutsche Nationalbibliothek lists this publication in the Deutsche Nationalbibliografie; detailed bibliographic data are available on the Internet at http://dnb.dnb.de.

\title{
Address of the author
}

Karl Porges

Research Group for Biology Education

Institute of Zoology and Evolutionary Research

Faculty of Biological Sciences

Friedrich Schiller University Jena

Am Steiger 3, Bienenhaus

07743 Jena

Germany

Email: karl.porges@uni-jena.de

This is a revised version of Porges' doctoral dissertation that was submitted to the Faculty of Biological Sciences at the Friedrich Schiller University Jena in 2015 to fulfill the requirements for the degree of doctor rerum naturalium (Dr. rer. nat.).

\author{
Managing Editor of the Annals of the History and Philosophy of Biology \\ Dr. Christian Reiß \\ Professur für Wissenschaftsgeschichte \\ Universität Regensburg \\ 93040 Regensburg \\ Germany \\ Email: Christian.Reiss@psk.uni-regensburg.de
}

Cover Picture: Friedrich Besemann: Leinekanal mit akademischem Museum und Graetzelhaus. Aquarellierte Federzeichung 1860. Graphische Sammlung des

Städtischen Museums Göttingen

Layout: Karl Porges

Cover Design: Kilian Klapp, Maren Büttner

(C) 2018 Universitätsverlag Göttingen

https://univerlag.uni-goettingen.de

ISBN: 978-3-86395-333-1

DOI: https://doi.org/10.17875/gup2018-1056

eISSN: 2512-5923 


\section{Danksagung}

Viele Kolleginnen und Kollegen sowie Freunde haben zur Entstehung dieses Projekts beigetragen und meine Arbeit intellektuell und persönlich unterstützt.

An erster Stelle ist es mir ein Anliegen, Uwe Hoßfeld (Jena) zu danken. Er eröffnete mir die Möglichkeit berufsbegleitender Forschungsarbeit, die mein Leben mit neuen Einsichten und Erfahrungen bereicherte. Sein entgegengebrachtes Vertrauen, die nützlichen Literaturhinweise, die fachlich-freundschaftlichen Ratschläge und die intensive Betreuung, trotz der teilweise zu bewältigenden Entfernung, ermöglichten mir den Schaffensprozess. Den wissenschaftlichen Mitarbeitern Georg S. Levit, Michael Markert und Kirsten Gesang der AG Biologiedidaktik in Jena, die mir mit Rat und Tat bei pragmatischen, menschlichen und fachlichsachlichen Aspekten weiter geholfen haben, gilt ebenso mein Dank.

Den Mitgliedern der Internationalen Gesellschaft für historische und systematische Schulbuch- und Bildungsmedienforschung e. V. (IGSBi) danke ich für die freundliche und konstruktive Kritik, die ich auf den Jahrestagungen der Gesellschaft kennen lernen durfte. Die Vorträge, die ich zu verschiedenen Anlässen hören und halten konnte, haben mein Wissen über den interdisziplinären Ansatz von Schulbuchforschung nachhaltig beeinflusst. Allen voran gilt mein Dank hier Frau Eva Matthes (Augsburg) und Frau Sylvia Schütze (Hannover).

Frau Gertrud Kummer (Berlin) danke ich insbesondere für ihr Interesse an meiner Arbeit und die gemeinsamen Gesprächen sowie vielfältigen und kritischen Anregungen. Ihre Lebenserinnerungen bieten einen reichen Fundus, wenn es um die Beschäftigung mit Schule im Allgemeinen und Biologieschulbüchern der DDR im Speziellen geht. Auch dem Biologiedidaktiker Erwin Zabel (Güstrow) bin ich für den aufschlussreichen und anregenden Briefwechsel und die Hinweise zur Geschichte der Sektion Schulbiologie zu Dank verpflichtet.

Meinen Eltern und Geschwistern, meiner Frau und meinen Kindern danke ich herzlich für Liebe, Verständnis, Beistand und Geduld. Ihnen widme ich diese Arbeit. 



\section{Inhalt}

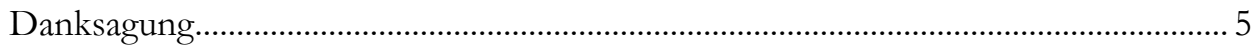

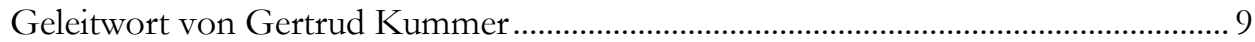

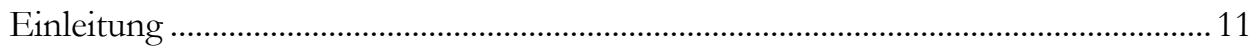

Zum Stellenwert der Schulbuchforschung............................................................... 19

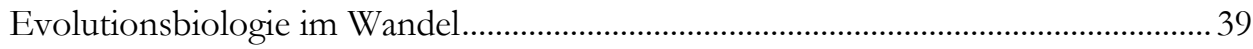

Das Bildungssystem der DDR und das Schulfach Biologie......................................61

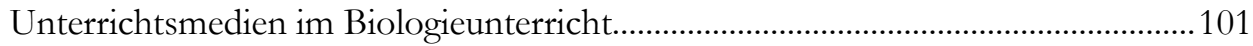

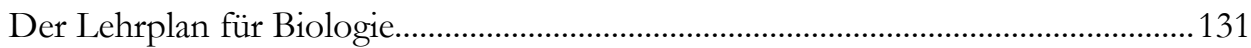

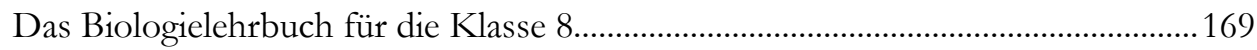

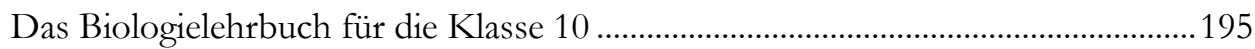

Das Biologielehrbuch für die Klasse 12 ..............................................................227

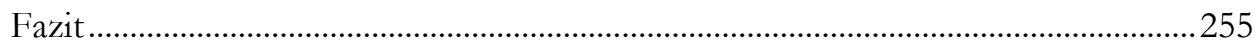

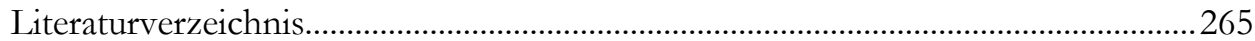

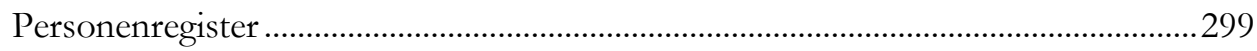

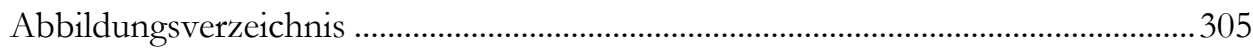

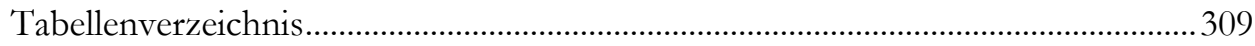





\section{Geleitwort von Gertrud Kummer}

Das hier vorliegende Buch vermittelt einen interessanten Einblick in das Bildungssystem der SBZ/DDR. Der Verfasser konzentriert sich dabei auf den Anteil der Biologie im Allgemeinen und der Evolutionstheorie im Speziellen im Gesamtprogramm. Er gibt einen Überblick über Umfang und inhaltliche Schwerpunkte des Bereichs Evolutionstheorie - in den Lehrplänen meist als Entwicklungsgeschichte und Abstammungslehre bezeichnet - und die unterschiedliche Schwerpunktsetzung in dieser Entwicklung. Er belegt seine Untersuchungsergebnisse durch zahlreiche Beispiele, Tabellen und Vergleiche und führt meines Erachtens zu wesentlichen Erkenntnissen, die auch und gerade für Gegenwart und Zukunft wichtige Hinweise zu Inhalt und Gestaltung der biologischen Bildung an allen Schulen bzw. Schulsystemen geben und große Bedeutung haben.

Ich selbst habe die hier aufgezeichneten Entwicklungen hautnah miterlebt, war ich doch von 1946 bis 1989 in der Volksbildung tätig, unterrichtete zuerst in der Unterstufe, ab 1950 dann als Fachlehrerin für Biologie in den Klassen 5 bis 12. Im September 1960 begann dann meine Tätigkeit beim Verlag Volk und Wissen Berlin. Dort war ich zunächst als Redakteurin tätig. 1962 wurde ich zur Leiterin der Abteilung Biologie berufen. Diese Abteilung war verantwortlich für die Entwicklung und Herausgabe von Lehrbüchern Biologie für alle Klassenstufen sowie für lehrplanbezogene Literatur (fachwissenschaftliche und fachbezogene didaktischmethodische) für Biologielehrerinnen und -lehrer. Seitdem war ich maßgeblich in 
die Entwicklung der Biologie-Lehrpläne einbezogen und wesentlich an deren Umsetzung beteiligt. Wie bekannt, begann der Biologieunterricht in allen Schulen und für alle Schülerinnen und Schüler der DDR in Klasse 5 und endete nach Klasse 10 in der Polytechnischen Oberschule (POS) bzw. 12 in der Erweiterten Oberschule (EOS). Jede Schülerin und jeder Schüler absolvierte also während seiner Schulzeit insgesamt 440 Stunden (POS) bzw. 622 Stunden (EOS) Biologieunterricht. Die letzten sechs Wochen am Ende der Klasse 12 standen für die Abiturvorbereitung zur Verfügung. Die Stoffauswahl und vor allem die Stoffanordnung folgte von Anfang an dem biologischen System: Klasse 5 Wirbeltiere, Klasse 6 Wirbellose, Klasse 7 Mikroorganismen sowie in jeder Klassenstufe Vertreter bestimmter Pflanzenfamilien. In Klasse 8 stand Bau und Funktion des menschlichen Körpers (und gesunde Lebensweise), in der Klasse 9 angewandte Biologie (Ökologie und Umweltschutz, Pflanzenanbau, Tierhaltung), in der 10. Kasse Abstammungslehre und Genetik im Lehrplan. In der 11. Klasse waren Anatomie und Physiologie des Menschen (unter Betonung der Vorgänge im Körper), in der Klasse 12 Evolutionstheorie und Genetik Unterrichtsgegenstand. Dabei wurde von Anfang an Wert auf exakte, dem Alter angepasste wissenschaftlich fundierte Kenntnisvermittlung gelegt. So wurden in den Klassen 5 bis 9 (bzw. 11) die Grundlagen geschaffen, um die Entstehung des Lebens und die Entwicklung der Organismen und die Sicherung ihres Fortbestandes im Unterricht erfolgreich zu behandeln. Das war besonders wichtig, weil dieses Stoffgebiet schwer zu veranschaulichen ist - durch Originale fast nur in Museen, sonst vor allem durch Abbildungen. Trotzdem fanden gerade diese Themen bei vielen Schülerinnen und Schülern besonderes Interesse, konnten sie doch aufgrund bereits erworbener Kenntnisse selbst Vergleiche anstellen, Ursachen und Wirkungen erkennen, Zusammenhänge finden. Ihnen wurde bewusst, dass die Welt erkennbar ist. Damit war ein wesentliches Bildungs- und Erziehungsziel erreicht - die Herausbildung eines wissenschaftlichen Weltbildes als Grundlage einer wissenschaftlichen Weltanschauung. Ehemalige Schülerinnen und Schüler, die noch heute Kontakt zu mir halten, betonen immer wieder, wie wichtig diese wissenschaftliche, an Fakten orientierte Vermittlung von Erkenntnissen und Einsichten gerade auch im Biologieunterricht für ihre weitere Entwicklung war. Das zeigt aber zugleich, wie groß die Verantwortung der Gesellschaft für ihr Bildungs- und Erziehungssystem, für die Entwicklung ihrer Nachkommen ist. Die Kinder sind das Wertvollste, was eine Gesellschaft hat, denn sie sichern den Fortbestand, also die Zukunft.

Vielleicht regt dieses Buch dazu an, in den verschiedenen Bundesländern (die ja jeweils eigene Lehrprogramme und Unterrichtsgestaltungen haben) ähnliche Untersuchungen durchzuführen. Vergleiche zwischen Programmen und Zielen könnten zu deren Weiterentwicklung führen oder wünschenswerte, vielleicht sogar dringend erforderliche Änderungen bewirken und so zum Fortbestand unserer Gesellschaft beitragen.

Berlin im Juli 2017, Gertrud Kummer 


\section{Einleitung}

„Da alles Bestehende eine Geschichte hat, erfordert die Erkenntnis der Dinge auch die Erkenntnis ihrer Entwicklung." (Bach et al. 1987: 91)

Die Institution Schule ist ein Grundpfeiler unserer modernen Gesellschaft, denn im Unterricht werden „Qualifikations- und Sozialisationsleistungen im Hinblick auf die Erhaltung und Sicherung der Gesellschaft" erbracht (Fees 2006: 82). Doch sie ist ebenso „eine historisch entstandene und damit auch stets zu hinterfragende Lösung, dem Anspruch des Menschen auf Bildung zu entsprechen“ (ebd.). Beides führt dazu, dass Schule ,in ihrer Existenz abhängig von historischen und sozialen Kontexten“ ist und „einem ständigen Reformdruck“ unterliegt (ebd.: 86). Der Historiker Sellin (2008: 154) betont: „Wo immer der Mensch handelt, sind Mentalitäten, wo immer er sich über sein Verhalten äußert und Rechenschaft ablegt, sind Ideologien im Spiel.“ Dies wirkt sich letztlich auch auf schulische Inhalte aus, die in Lehr- und Lernmaterialien festgeschrieben sind. Lässig (2010: 199) resümiert folgerichtig, dass „Wissen [...] bis heute abhängig von dem [ist], was gesellschaftlich vorstrukturiert und medial vermittelt wird“. Für Lehrwerke bedeutet dies, dass sie „immer auch ein Spiegel ihrer Zeit sind“ (Jürgens 2006: 406) und jegliche Ver- 
änderungen an ihnen auch auf gesellschaftliche Veränderungen hinweisen. Nach Neuner (1989: 15) lassen sich gar ,aus der Analyse von Lehrplänen und anderen Unterrichtsmaterialien [...] die direktesten Rückschlüsse auf verfolgte Absichten und Ziele, auf Ideen und Werte, auf vertretene Theorien ziehen“.

Aus diesen Überlegungen ergeben sich zwei allgemeine Hypothesen. Erstens verändern sich die Darstellung und der Stellenwert schulischer Vorgaben in den Lehr- und Lernmaterialien. Zweitens stehen diese Modifikationen in den Materialien der allgemeinbildenden Schulen im Zusammenhang mit der soziokulturellen Entwicklung eines Staates und der Genese des Bildungssystems. Das Modell des didaktischen Dreiecks ergänzt dieses Gedankengebäude. Der mittels Lehrplänen administrativ vorgegeben Lehrstoff, der die Grundlage der Interaktion zwischen Lehrkraft und Schüler bildet, beeinflusst den Unterricht. Grundlegend bilden also historische Lehrpläne und Lehrbücher einen Zugang zu vergangenen Unterrichtsvorstellungen. Lehr- und Lernmaterialien, die einer staatlichen Kontrolle, dem Erkenntniszugewinn der jeweiligen Fachwissenschaft im Speziellen, der Pädagogik und Didaktik im Allgemeinen sowie dem technischen Fortschritt unterliegen, sind somit Zeugen gesellschaftlicher und (fach-)wissenschaftlicher Entwicklungen.

Eine Analyse von Lehr- und Lernmaterialien, die das Ziel verfolgt, ein Abbild der inhaltlichen, strukturellen und visuellen Realitäten der Quellen zu erstellen, ermöglicht folglich einen Einblick in das gesellschaftlich gewünschte Schulgeschehen. Einleitend befasst sich das erste Kapitel dieser Studie mit der Schulbuchforschung und der aus ihr hervorgehenden Lehrplan- und Lehrbuchtheorie sowie methodologischen Überlegungen. Trotz bestehender Fachdidaktiken resümiert Pöggeler (2003), dass die Geschichte des Schulbuches nicht in allen schulpädagogischen Teildisziplinen erforscht ist. Mittlerweile existiert jedoch eine Vielzahl von Beiträgen, die diesen Überlegungen folgt und sich mit historischen Analysen einzelner Schulfächer beschäftigt (vgl. u. a. Fischer 2004; Stürmer 2014; Wagner 2016). Befassen sich diese Arbeiten dabei mit dem Bildungssystem der Deutschen Demokratischen Republik (DDR), so wird deutlich, dass die Lehrpläne eine ,verbindliche Grundlage für den Unterricht" darstellten und „mit gewissen Einschränkungen den Unterricht selbst“" widerspiegelten (Tille 1992a: 321). In dieser Linie verortet sich auch die hier vorliegende Studie. ${ }^{1}$ Dennoch sollte der Fehler vermieden werden, die Ergebnisse mit der Unterrichtsrealität gleichzusetzen. Vielmehr ermöglichen Schulbuchanalysen, die sich von technologischen und prognostischen Forschungen abgrenzen, retrospektiv Ergebnisse und Entwicklungen zu beschreiben und „für eine bereits geschehene und vorliegende Wirkung (B) nachträglich Ursachen (A) zu postulieren“" (Krüger und Vogt 2007: 5). Die Auseinandersetzung mit der Schulgeschichte der DDR als Teil der Zeitgeschichte

1 Die Arbeit wurde 2015 als Dissertation an der Friedrich-Schiller-Universität in Jena verteidigt. Sie versteht sich als überarbeite Version und integriert Elemente einzelner Veröffentlichungen des Autors im Sinn einer zusammenfassenden Darstellung (vgl. u. a. Porges 2014; Porges \& Hoßfeld 2017; Porges im Druck). 
ist auch insofern relevant, da die Auswirkungen der Bildungs- und Erziehungsinhalte der DDR nachwirken. Zeitzeugen und mit ihnen ihre Schulbildung, die stets auch den Blick auf die Welt prägt, sind Teil unserer Gesellschaft. ${ }^{2}$

Bisher weitgehend unbeachtet blieb eine wissenschaftshistorische Beschäftigung mit der Geschichte des Biologieunterrichts in der DDR. Zwar betonen die Biologiedidaktiker Berck und Graf (2010: 279), „dass es ,den“ Biologieunterricht nicht gibt“. Jedoch erkennen sie, dass sich „,aus dem Studium der Geschichte des naturwissenschaftlichen, speziell des biologischen Unterrichts [...] Einsichten ergeben [können], was an historischen Erfahrungen heute effektiv zu übernehmen oder abzulehnen ist" (ebd.: 22). Vor diesem Hintergrund kann eine Auseinandersetzung mit der Geschichte des Biologieunterrichtes auf dem Gebiet der ehemaligen Sowjetischen Besatzungszone (SBZ) sowie der DDR zumindest Denkanstöße für Entwicklungen und notwendige Reformen liefern, um die vielfältigen Herausforderungen der Gegenwart zu meistern.

Innerhalb des Fächerkanons liegt eine zentrale Bedeutung des Schulfaches Biologie in seiner Funktion als „Brückenfach zwischen Natur-, Sozial- und Geisteswissenschaften auf der einen Seite sowie Naturwissenschaften und ihre technischen Anwendungen auf der anderen Seite“"3. Die Mannigfaltigkeit der Inhalte des Faches charakterisiert Meisert (2008) durch die Begriffe Vielfalt und Komplexität biologischer Phänomene sowie Funktionalität. Innerhalb der Biowissenschaften wiederum stellt die Fachdisziplin Evolutionsbiologie ${ }^{4}$ ein verbindendes, da historisch-erklärendes, Element dar (Gropengießer \& Kattmann 2006). Evolutionstheorien sind folglich „für ein grundlegendes Verständnis von Biologie unabdingbar“ und sollten ,einen zentralen Platz im Biologieunterricht einnehmen“ (Wallin 2011: 122). Das zweite Kapitel der vorliegenden Studie zeichnet die historische Entwicklung des Konstrukts Evolutionsbiologie nach, definiert wesentliche Begriffe und diskutiert Befunde schulbiologisch relevanter evolutionsbiologischer Forschungsfelder. Exemplarisch werden Auseinandersetzungen um die Evolutionsbiologie als eine Folge gesellschaftlicher Diskurse vorgestellt. Der Fokus liegt hier auf Entwicklungen im Rahmen der sozialistisch-kommunistischen Ideologie des

2 Gertrud Kummer (*1929) erinnert sich, dass der Biologieunterricht in der DDR zum Kennenlernen der Lebensformen, der Natur und ihrer Bedeutung und ihres Schutzes sowie zur Kenntnis des eigenen Körpers und auch zur Anwendung des Gelernten führen sollte. Sie arbeitete seit 1960 beim Verlag Volk und Wissen und war dort von 1962 bis 1989 als Abteilungsleiterin für die Buchredaktion in der Sektion Biologie verantwortlich (Interview vom 28.12.2016).

3 Sekretariat der Ständigen Konferenz der Kultusminister der Länder in der Bundesrepublik Deutschland 2004, S. 5.

4 Die Begriffe Abstammungslehre, Darwinismus, Deszendenzlehre, Entwicklungslehre, Evolution und Evolutionstheorie(n), standen zu verschiedenen Zeiten im Vordergrund. Im Verlauf der Arbeit wird allgemein von evolutionsbiologischen Inhalten gesprochen, ohne jedoch die zeittypischen Begriffe auszusparen. Entsprechende Definitionen, die die Beziehungen zentraler (historischer) Begriffe untereinander verdeutlichen und sie gleichzeitig voneinander abgrenzen, erfolgen im Kapitel Evolutionsbiologie im Wandel. 
19. und 20. Jahrhunderts sowie in aktueller Perspektive auf dem Darwin-Jahr 2009, (populär)wissenschaftlichen und kreationistischen Bestrebungen.

Trotz oder gerade wegen ihres Bildungsbeitrags stehen evolutionsbiologische Inhalte nicht nur im Zentrum wissenschaftlicher, sondern auch gesellschaftlicher und somit schulpolitischer Diskurse. Die Erklärung der Evolutionsprozesse war und ist, mehr als andere wissenschaftliche Erkenntnisse, von gesellschaftlichen Reaktionen begleitet, da sie oftmals im Widerspruch zu tradierten Vorstellungen steht und, wenn auch von Charles Darwin (1809-1882) nicht gewollt, Ausgangspunkt für sozialwissenschaftliche Interpretationen, politische Ansichten und Deutungen bot (Hoßfeld \& Brömer 2001; Engels 2009). Bereits 1877 forderte Ernst Haeckel (1834-1919) die Einbindung evolutionsbiologischer Inhalte in den Schulunterricht. Er stieß jedoch seinerzeit auf heftigen Widerstand. Der Fall Hermann Müller (1829-1883) führte in Preußen gar zur Abschaffung des Biologieunterrichtes in den oberen Klassen der höheren Lehranstalten (Hoßfeld 2010). Zwar ist die unterrichtliche Behandlung der Evolutionsbiologie heute Standard, dennoch zeigen aktuelle Diskussionen um den Kreationismus, dass eine Anerkennung wissenschaftlicher Erkenntnisse stets neu erstritten werden muss (Kutschera 2008; Junker 2011; Waschke \& Lammers 2011; Porges 2016a).

Auch aktuelle Reformen im Thüringer Bildungswesen, wie die Abschaffung des Biologieunterrichtes in der Orientierungsstufe, verdeutlichen, dass für die Evolutionsbiologie als grundlegend erachtete Erkenntnisse keinen sicheren Stand haben (Beese 2012). Graf (2009: 4) resümiert, dass „der zentralen Bedeutung der Evolutionsbiologie in der Schule [...] nur unzureichend Rechnung getragen [wird, da die Behandlung] erst in den Abschlussklassen [...] und damit viel zu spät [erfolgt]". Einen Grund sieht Krull (2011: 6) darin, dass vor dem Hintergrund der Schrecken des Nationalsozialismus „diese Disziplin bis heute in den Lehrplänen deutscher Schulen und Hochschulen ein Schattendasein führt" (vgl. auch Griffhorn \& Langlet 2006). Es verwundert daher nicht, dass sich erst im Jahr 2002 ein Arbeitskreis Evolutionsbiologie im Verband deutscher Biologen und biowissenschaftlicher Fachgesellschaften (vdbiol) gründete. Hier greift schlicht die Tatsache, dass „das Lehrgebiet Evolutionsbiologie an deutschen Universitäten unterrepräsentiert war" (Kutschera \& Hoßfeld 2012: 20). Andererseits ist das Bemühen erkennbar, die Fachdisziplin Evolutionsbiologie bereits in der Grundschule zu verankern (MarquardtMau \& Rojek 2011; Rojek et al. 2012; Graf et al. 2015). Denn sie liefert selbst vor dem Hintergrund eines inklusiven Bildungssettings einen Beitrag zum Verständnis der Welt (Porges \& Porges 2017). Auch die Nationale Akademie der Wissenschaften Deutschlands (2017: 35) betont, dass „Maßnahmen zur Stärkung der Evolutionsbiologie im Schulunterricht von herausragender Bedeutung" sind und schlägt daher „Verbesserungen im Curriculum des Biologie- bzw. naturwissenschaftlichen Unterrichts und in den Unterrichtsmaterialien" vor. 
Debatten um die Evolutionsbiologie und ihre Behandlung im Unterricht sind letztlich als Folge gesellschaftlicher Entwicklungen in politischen Systemen zu verstehen. Eine wissenschaftshistorische Auseinandersetzung mit dieser Fachdisziplin ermöglicht somit einen Einblick in das komplexe Wechselspiel zwischen Wissenschaft und Gesellschaft. Vor diesem spannungsreichen Hintergrund soll die vorliegende Studie klären, wie evolutionsbiologische Inhalte auf dem Gebiet der SBZ/DDR Eingang in den Unterricht fanden, welche Bedeutung ihr im Rahmen von Schule und Bildung beigemessen wurde und welche inhaltlichen Präferenzen in relevanten Lehr- und Lernmaterialien verortet waren. Ziel der vorliegenden Analyse ist es, anhand der Genese von Stellenwert und Darstellung evolutionsbiologischer Inhalte im Lehrplan und Schulbuch für den Biologieunterricht auf dem Gebiet der SBZ/DDR exemplarisch die enge Verknüpfung zu politischgesellschaftlichen und wissenschaftlichen Entwicklungen und Diskursen aufzuzeigen. Dabei begreift sie die Auseinandersetzungen um die Evolutionsbiologie und ihre Behandlung im Unterricht als eine Folge gesellschaftlicher und wissenschaftlicher Diskurse, Entwicklungen und politischer Systeme.

Die Arbeit verfolgt dabei zwei wesentliche Ziele: Erstens kategorisiert sie die in den Dokumenten abgebildeten Inhalte der Evolutionsbiologie und verdeutlicht so die Genese von Stellenwert und Darstellung. Zweitens stellt sie Präferenzen und deren Veränderungen in den Kontext schulpolitischer, gesellschaftlicher und wissenschaftlicher Diskurse. Die entsprechenden Rahmenbedingungen schulischer Lehr- und Lernmaterialien werden im dritten Kapitel vorgestellt. Konkret betrifft das die strukturelle Entwicklung des allgemeinbildenden Schulsystems in der SBZ/DDR, die Ausbildung der Lehrkräfte und die Phasen der Lehrplanentwicklung. Anschließend wird das Schulfach Biologie auf dem ehemaligen Gebiet der SBZ/DDR skizziert und die Entwicklung und die Aufgabenfelder der Sektion Schulbiologie der Biologischen Gesellschaft in der DDR nachgezeichnet.

Zwar sind Studien zum Biologieunterricht auf dem Gebiet der DDR unter verschiedenen Schwerpunktsetzungen vorhanden ${ }^{5}$, ein Desiderat bleibt jedoch eine leitfadenorientierte Auseinandersetzung mit den Schulmaterialien zum inhaltlich zentralen Aspekt Evolutionsbiologie in der SBZ/DDR. ${ }^{6}$ Nicht unerwähnt bleiben soll der richtungsweisende Beitrag Evolutionsbiologie und Schule von Rommel (2006). ${ }^{7}$ Die vorliegende Arbeit, die sich als Beitrag zur Geschichte des Biologie-

5 Porges et al. (2016) dokumentieren in einem Aufsatz die Entwicklung der (Mendel-)Genetik in den Lehr- und Lernmaterialien der SBZ/DDR.

6 Für die Zeit nach 1989 geht Scholl (2014) der Frage nach, inwieweit Biologielehrbücher der Oberstufe in Deutschland im Bereich Evolutionsbiologie hilfreiche Unterrichtsmittel sind.

7 Rommel (2006) befasste sich im Rahmen ihrer Examensarbeit an der AG Biologiedidaktik in Jena mit einer Analyse der Lehrpläne und Schulbücher der DDR. Hier erfolgt bereits eine „Gegenüberstellung modifizierter Lehrplan- und Schulbuchauflagen [...] nach qualitativen und quantitativen Aspekten“ (ebd., S. 22). Deskriptive und vergleichende Betrachtungen von Lehrplänen und Lehrbüchern der DDR der Klassen 8 und 10 bilden das Fundament dieser Arbeit, wobei ein Schwerpunkt auf der Darstellung evolutionsbiologischer Inhalte am Beispiel 
unterrichtes in Deutschland versteht, liefert im vierten Kapitel eine Bestandsaufnahme der Lehrpläne und Schullehrbücher des Biologieunterrichts sowie weiterer Unterrichtsmedien und verortet diese im Rahmen einer historischen Quellenforschung zusammengestellten Dokumente in ihrem historischen Kontext. Sie bezieht Lehrpläne und Lehrbücher der 8., 10. und 12. Klassenstufe sowie Direktiven, Korrekturen und Anweisungen zum Lehrplan mit ein. Dabei wird die Entwicklung der Schulbiologie am Beispiel der Evolutionsbiologie chronologisch rekonstruiert.

Die Biologielehrpläne als administrative Vorgaben für die Unterrichtsplanung sowie das Biologieschulbuch, das als biologiedidaktisches Dokument eine zentrale Rolle im Unterrichtsprozess einnimmt und die Geschichte des Biologieunterrichtes widerspiegelt (Berck \& Graf 2010), stehen im Zentrum der Analyse. Diese nimmt ideologische Vorgaben, das Layout, die Fachinhalte und die didaktischmethodischen Besonderheiten fakten- und leitfadenorientiert in den Blick und bietet einen Pool quantifizierbarer Daten. Inhaltliche Entwicklungen, Veränderungen sowie Anpassungen werden herausgestellt und Interpretationsansätze angeboten, die neben der publizistischen die gesellschaftliche Ebene beleuchten. Exemplarisch wird die Rolle der Lehr- und Lernmaterialien bei gesellschaftlichen Transformationsprozessen betont. Dabei ermöglicht der historische Abstand, „die zeitbedingten politischen und ideologischen Färbungen aus einer distanzierten Perspektive zu betrachten“ (Wagner 2016: 7).

Die Kapitel fünf bis acht dienen der Ergebnisdarstellung der Lehrplan- und Lehrbuchanalyse, nach dem in Kapitel eins vorgestellten methodischen Leitfaden. Abschließend greift das neunte Kapitel auf die Fragestellungen zurück, um letztlich die Wirkungen des gesellschaftlichen Wandels auf die gewählten Bildungsmedien aufzuzeigen. Ein Erkenntniszugewinn entsteht, da erstmals die Evolutionsbiologie als Lehr- und Lerneinheit im Gesellschaftsbereich Schule im genannten Zeitraum empirisch erfasst, interpretiert und kontextualisiert wird. Die Analyse der Lehrwerke und Lehrpläne der SBZ/DDR ermöglicht einen Einblick in den Schulalltag, da in allen Klassen einer Jahrgangsstufe innerhalb einer Schulart auf dem Staatsgebiet der DDR die gleichen Lehrpläne und Unterrichtsmedien genutzt wurden. Somit ist eine Vergleichbarkeit der Medien für alle Schülerinnen und Schüler eines Jahrgangs möglich. Dies ist ein entscheidender Unterschied zum Schulwesen der Bundesrepublik Deutschland (BRD). Da hier die Zuständigkeit über das Schul- und Hochschulwesen bei den Bundesländern liegt, können Teile des Bildungssystems sowie Lehr- und Lernmaterialien unterschiedlich sein. Die

der Homologen Organe liegt. Die Einteilung in Epochen orientiert sich dabei an der Einführung neuer Lehrpläne. Trotz der umfangreichen Auseinandersetzung mit dem Thema erschwert die Vorgehensweise einen direkten Vergleich der Materialien. Zwar wurde erkannt, dass sich zur Analyse die Erarbeitung eines Leitfadens anbietet, jedoch wurde diese Idee aufgrund „der (inhaltlichen und thematischen) Größe des Stoffgebietes und der zum Teil beachtlichen Modifikationen [...] nicht umgesetzt" (ebd.), wodurch ein Mangel an quantitativen Daten entstand. Ebenso verweist Rommel noch auf Lücken in der Quellensammlung. 
Arbeit will als Teil der historischen Schulbuch- und Lehrplanforschung und somit der historischen Bildungsforschung auf diesem Weg letztlich einen Beitrag zur Geschichte des Biologieunterrichtes im deutschsprachigen Raum leisten. Anhand dieser Gesamtschau soll, dem Selbstverständnis der Schulbuchforschung entsprechend, ein Beitrag ,zum besseren Verständnis der Schule als Wissensvermittlerin in der Gesellschaft" entstehen (Depaepe \& Simon 2003: 66). 



\section{Zum Stellenwert der Schulbuchforschung}

„Das Schulbuch ist ein sehr komplexer Gegenstand; die (wenigen) vorliegenden Schulbuchtheorien machen das überzeugend deutlich.“ (Matthes \& Schütze 2014: 9)

\section{Das Schulbuch im Fokus}

Sandfuchs (2010) verweist darauf, dass der Schulbuchfrage historisch betrachtet, ein hoher Stellenwert zukommt, was die Beachtung aller Schulfächer und ihrer Lehrbücher erfordert. Er legt dar, dass, „wann immer in der Schulgeschichte eine Steigerung der Qualität von Schule und Unterricht angestrebt wird, [...] neue Lehrpläne entworfen, neue Methoden ,erfunden' und propagiert und zu beiden passend neue Schulbücher verfasst" werden (ebd., S. 11). Dies wird insbesondere nach einschneidenden politischen Veränderungen, wie beispielsweise nach 1945 oder 1989 aktuell oder wenn es das Ansehen eines Verlages betrifft (Pöggeler 2003).

Das Nachdenken über das Schulbuch begann mit der Erfindung des Buchdrucks (Wiater 2003), wobei Comenius bereits im 15. Jahrhundert eine Schulbuchtheorie begründete. Die international vergleichende Schulbuchforschung setzte im 
19. Jahrhundert, nach dem ersten Weltkrieg, mit Untersuchungen durch den Völkerbund ein. Der Fokus lag dabei auf politischen Inhalten und der Suche nach notwendigen Korrekturen (Pöggeler 2003). In der BRD erlebte die Schulbuchforschung in den 1970er Jahren einen deutlichen Aufschwung. Fritsche (1992, S. 9) verwies jedoch bereits vor über zwei Jahrzehnten auf „ein Unbehagen über das vermeintlich unklare Selbstverständnis und die mangelnde Wissenschaftlichkeit der Disziplin“. Der Augsburger Schulpädagoge Werner Wiater (2003, S. 12) stellt fest, dass die Schulbuchforschung in Deutschland „,nicht die Bedeutung [besitzt], die ihr aus schultheoretischer und schulpraktischer Sicht zukommen sollte". Tröhler und Oelkers (2005, S. 97) fordern daher, dass Forschungssettings „größere Zeitspannen, mehrere Regionen oder Länder und vor allem auch diverse Lernfächer umfassen" müssen. Dennoch ist Deutschland neben den Ländern Frankreich, Spanien und Kanada Vorreiter in der Schulbuchforschung (Depaepe \& Simon 2003). Neuere Untersuchungen belegen das Bemühen um eine historische und systematische Auseinandersetzung mit Schulmaterialien verschiedener Fächer der SBZ/DDR (Stürmer 2014; Wagner 2016).

In Deutschland sind es vor allem das Georg-Eckert-Institut für internationale Schulbuchforschung in Braunschweig (GEI), die Internationale Gesellschaft für historische und systematische Schulbuch- und Bildungsmedienforschung e. V. (IGSBi) mit Sitz in Bayern und die Bibliothek für Bildungsgeschichtliche Forschung (BBF) des Deutschen Institutes für Internationale Pädagogische Forschung (DIPF), die in diesem Bereich Sammlungstätigkeit und Forschungsarbeit leisten. Auch das Leibniz-Institut für die Pädagogik der Naturwissenschaften und Mathematik in Kiel sowie die Deutsche Nationalbibliothek an den Standorten Frankfurt am Main und Leipzig besitzen im Bereich Schulbuch beachtliche Bestände. Der Fokus der Sammlung des GEI, das internationale und binationale Projekte zu historischen und politischen Themen organisiert, liegt dabei auf Schulbüchern der gesellschaftswissenschaftlichen Fächer, nicht aber der Naturwissenschaften. Die IGSBi, die sich im Jahr 1997 gründete, organisiert ebenfalls Veranstaltungen und publiziert Forschungsergebnisse zur Förderung der Schulbuchforschung. Die Beiträge der Jahresversammlungen erscheinen in der Reihe Beiträge zur historischen und systematischen Scbulbuchforscbung im Julius Klinkhardt Verlag (u. a. Knecht et al. 2014; Matthes \& Schütze 2016a, 2016b).

Depaepe und Simon (2003) verweisen darauf, dass in der Schulbuchforschung bisher vor allem die gesellschaftswissenschaftlichen Fächer berücksichtigt wurden. Dennoch bestehen auch für das Biologieschulbuch Analysen unter verschiedenen Fragestellungen. Eine gute Übersicht bieten Unterbrunner (2006) sowie Berck und Graf (2010). Eine weitere „wertvolle Quelle zur Geschichte des Biologieunterrichtes" (Hoßfeld o. J.) weist in Deutschland die Lehrsammlung der Arbeitsgruppe Biologiedidaktik der Friedrich-Schiller-Universität Jena auf, deren Bestand bis in die 1950er Jahre zurückreicht (ebd.). Hier finden sich für das Fachgebiet Evolutionsbiologie unter anderem Rollbilder, Dias und Modelle für den Unterricht. 
Von einer Inventarisierung des Materials ausgehend, sind die zentralen Schwerpunkte der Schulbuchforschung Untersuchungen ideologisch belasteter Themenfelder sowie das Aufzeigen der Entwicklung bestimmter Fachinhalte (Depaepe \& Simon 2003). Wiater (2003) gliedert Forschungsschwerpunkte in kulturhistorische Forschung, Medienforschung, Forschung unter fachwissenschaftlichen und fachdidaktischen Aspekten, Textanalyse-Forschung sowie historische Quellenforschung. Gleichfalls spezifiziert er für die Schulbuchforschung drei Vorgehensweisen: historisches, systematisches, und vergleichendes Forschen. Für das historische Forschen, das in dieser Arbeit zum Tragen kommt, schlägt Wiater (ebd.) ein grundlegendes Ablaufmodell vor: (1) Festlegung des Forschungsinteresses, (2) Sichtung aller absichtlich und unabsichtlich überlieferten Quellen und (3) Auswertung des Datenmaterials als historische Tatsache mit Hilfe hermeneutischer und sozialwissenschaftlicher Methoden.

Für Kiper (2001) wird durch die Reproduzierbarkeit von Unterrichtsmedien objektivierter Unterricht wiederholbar. Die Terminologie Unterrichtsmedien steht seit den 1970er Jahren für die Begriffe Unterrichts-, Lehr-, Lern- sowie Arbeitsmittel, deren gemeinsame Merkmale Verfügbarkeit, Reproduzierbarkeit, Multiplizierbarkeit, Ökonomie, Evaluierungsmöglichkeit sowie Zweck-MittelRationalität sind (vgl. ebd.). Ihnen liegen Bildungsvorgaben in Form von Lehrplänen zu Grunde. Berck und Graf (2010) argumentieren, dass die Beurteilung eines Biologielehrplanes ohne eine Lehrplantheorie nicht möglich ist. Auch für die Einschätzung von Schulbüchern ist eine Schulbuchtheorie nützlich. Im Folgenden geht es daher insbesondere um Charakteristika von Lehrplänen und Schullehrbüchern, aus denen sich Beurteilungskriterien ableiten lassen.

Wiater (2006) zeigt auf, dass Lehrpläne staatlichen Vorgaben unterliegen. In diesem Zusammenhang wird durch Lehrpläne deutlich, welches Wissen (Fakten, Zusammenhänge, Kompetenzen) als zentral oder peripher eingestuft wird (Lässig 2010). Darüber hinaus werden Lehrpläne durch Lehrkräfte wahrgenommen und stellen für Unterricht und Erziehung ein Regulativ sowie eine Hilfe für das Lernen dar (Wiater 2006). Hinsichtlich Umfang und Richtung inhaltlicher Vorgaben unterscheiden sich Bildungspläne (Beitrag zur Menschenbildung durch Schule), Rahmenpläne (Leitbestimmung, größere Freiräume bei der Umsetzung möglich), Kerncurricula (Angabe der zu erreichenden Standards) und Curricula (Unterricht wird festgelegt: Ziele, Inhalte, Methoden, Medien). ${ }^{8}$ Allgemein versteht Wiater (2006: 169) unter dem Begriff Lehrplan eine:

„Zusammenstellung von ausgewählten Lehrstoffen, die zur Erreichung eines vorgegebenen Lehrziels in einer bestimmten Reihenfolge angeordnet wurden; als Plan unterstützt er didaktische Überlegungen zur Realisierung des entsprechenden Unterrichts und ermöglicht die Überprüfung des intendierten Lehr-Lern-Erfolgs. Erfolgt die Erstellung und Ausgabe des

8 Vgl. Gropengießer 2006; Wiater 2006. 
Lehrplans auf die Initiative und unter Kontrolle des Staates, so kann gesagt werden: Lehrpläne kodifizieren und norminieren den Schulunterricht, zumindest sind sie ein diesbezügliches direktes Lenkungsmittel des Staates.“

Auch der Biologiedidaktiker Gropengießer (2006: 191) definiert Lehrpläne als „amtliche oder schulische Dokumente zur Regulierung des Unterrichts, in denen mindestens inhaltliche Vorgaben gemacht werden, und zwar für bestimmte Schularten, Schulstufen, Fächer oder Lernbereiche in bestimmten Jahrgangsstufen“".

Dagegen gilt als Schulbuch jeder gedruckte und gebundene Text, der in der Schule als Lehr- und Lernmittel eingesetzt werden kann (Unterbrunner 2006). Auch Pöggeler (2013) versteht unter Schulbuch jedes in der Schule eingesetzte Buch. Eine Abgrenzung zu anderer Literatur, ob wissenschaftlich oder populärwissenschaftlich, besteht in trivialer Weise darin, dass das Studium von Schulbüchern meist unfreiwillig erfolgt (Detlev 1993: 7). Ferner durchlaufen Schulbücher in Deutschland grundsätzlich ein staatliches Prüfungsverfahren (Schulbuchapprobation), wobei Zulassungspraxis jedoch heterogen ist (Fey 2016). Wiater (2003: 13) verweist daher zu Recht darauf, dass Schulbücher „ein indirektes Mittel der staatlichen Beeinflussung“" sind. Das bildungspolitische Steuerungsmodell ist entsprechend eng mit dem Schulbuch verknüpft. Lehrbücher im modernen Schulsystem besitzen als „privilegierte Medien“ (Lässig 2010: 199) somit zwei Eigenschaften. Sie werden inhaltlich einerseits vom Staat beeinflusst, geprägt sowie zertifiziert. Andererseits sind sie statische Medien, über die langfristig wirkende Wahrnehmungsmuster und Deutungen kommuniziert werden (ebd.). Lehrpläne und Schulbücher geben daher einen Einblick in das Unterrichtsgeschehen und in gesellschaftlich anerkannte Vorstellungen bzw. gewünschte Inhalte. Gleichzeitig hinken sie aber zwangsläufig dem aktuellen Forschungsstand hinterher, was sich u. a. aus Abläufen der Produktion sowie den staatlichen Zulassungsverfahren ergibt (Unterbrunner 2006; Lässig 2010).

Allgemein werden Schulbücher als „pädagogische Hilfsmittel“ (Stein 1994: 752) verstanden, deren Bedeutung für den Unterricht sich aus einer Mehrwertigkeit hinsichtlich Lehrplan sowie Lehr- und Lernbuch ergibt. Sie gelten als ,zum Leben erweckte Lehrpläne“ (Heinze 2005: 9), da sie auf Lehrplänen basierend selektives autorisiertes Wissen transportieren (Lässig 2010). Inhalte und Strukturen der Schulbücher repräsentieren zumindest in Teilen Inhalte und Strukturen der Lehrpläne. Da sich Lehrkräfte häufig direkt auf das Lehrbuch beziehen (Stein 1994), nutzen sie somit die vorgegebene Inhaltsstruktur. Dies wirkt auf die Schüler zurück, da die inhaltliche Struktur den Lernerfolg beeinflusst (Meyer 2004). Zwar erschweren Schullehrbücher durch ihre festgelegte Struktur einen situationsund erfahrungsbezogenen Unterricht, doch der entscheidende Vorteil liegt in ihrer Vergleichbarkeit innerhalb einer Schule und der Möglichkeit, es durch weitere Unterrichtsmedien zu ergänzen (Jürgens 2006). Dadurch nimmt das Schulbuch im Kanon der Unterrichtsmedien eine zentrale Funktion im Unterricht ein. Entsprechend kommt ihnen als „Leitmedium“ (Heinze 2005: 13) des Unterrichtes, wenn- 
gleich schulfachspezifisch differierend, ebenso eine ,zentrale Steuerungsfunktion im Schulsystem“ (ebd.: 9) zu.

Rein formal gliedert Choppin (1992) die Struktur des Schullehrbuches in die Bereiche Text und Paratext. Hierbei sind Schullehrbücher durch einen hohen Anteil an Abbildungen charakterisiert (Unterbrunner 2006). Auch Sujew (1986) hat die Struktur von Schullehrbüchern ausführlich erörtert. Den Lehrbuchtext definiert er als:

„das grundlegende verbale System des allgemeinen Lehrbuchmodells, das in der Strukturhierarchie der Spitze am nächsten steht; er bildet das ,Grundskelett' des Lehrbuches, gibt dessen Inhalt wieder und sichert eine folgerichtige und umfassende Darstellung und Erörterung des Stoffes entsprechend dem Lehrplan; der Text ist Träger der grundlegenden Information, bestimmt Wesen und Umfang des zur Aneignung vorgesehenen Bildungsinhalts“ (ebd.: 97, Hervorhebung im Original).

Tabelle 1: Funktionen von Schulbüchern (verändert nach Jürgens 2006)

Funktionen von Schulbüchern (Auswahl)

- Information: wissenschaftlich korrekt und zielgruppengerecht

- Struktur: Abbildung von Lehrplanvorgaben

- Repräsentation: Repräsentation des Lehrplans

- Steuerung: Auswertung des Materials durch Aufgabenstellungen

- Motivation: Aufforderung zur thematischen Auseinandersetzung

- Anregung

- Differenzierung: z. B. durch parallele Angebote

- Übung: Lernpsychologische Berücksichtigung von Lerntypen

- Kontrolle: Aufgabenstellungen in verschiedenen Anforderungsbereichen

- Wiederholung: Nachschlagen (Merksätze, Glossar u. ä.)

- Sicherung: Zusammenfassung

Schulbücher weisen ferner vielfältige didaktisch-methodische Funktionen in unterschiedlicher Ausprägung auf (Tab. 1) und können davon einige, zum Beispiel durch Arbeitshefte oder elektronische Medien, auslagern. Die Verbindung von Schulbüchern mit begleitenden und ergänzenden Materialien für eine Zielgruppe, die durch Lehrpläne vorgegebene Lerninhalte abbilden und für den schulischen Lehr- und Lernprozess als Arbeitsmittel zur Anwendung gelangen, werden unter dem Begriff Lehrwerk geführt (Jürgens 2006). Ergänzende Materialien für Schüler sind neben Arbeitsheften auch digitale Medien. Eine besondere, der modernen Medienwelt gerechte Form, ist das Projekt Digitale Schulbücher der Bildungsmedien Verlage. Es ermöglicht die Einrichtung einer digitalen Bibliothek, in der Schulbücher nach der Freischaltung online oder offline genutzt werden können. Neuere Analysen der Bildungsmedienforschung berücksichtigen den Trend, dass die Unterrichtspraxis zunehmend von kostenlosen Lehrmitteln aus dem Internet geprägt wird (Neumann 2015; Fey 2015). 
Für die Lehrenden sind Lehrerbände, Kopiervorlagen sowie Lernkontrollen Teile des Lehrwerkes. Lehrwerke konkretisieren somit Lehrpläne und unterliegen als Gesamtkonzept ebenfalls einer staatlichen Prüfung, bevor sie auf verschiedenen Wegen vorgestellt und eingeführt werden. Die Praxis stellt hierbei an die Lehrwerke verschiedenste Anforderungen je nach Verwendungszweck und Rahmenbedingungen (Tab. 2). Im Unterschied zu anderer Schulliteratur sind Biologielehrbücher dabei selten Lesebücher, sondern meist Lehr-, Lern- und Arbeitsbücher sowie Materialsammlungen (Unterbrunner 2006).

Tabelle 2: Anforderungen aus der Praxis an ein Lehrbuch - eine Auswahl (verändert nach Jürgens 2006)

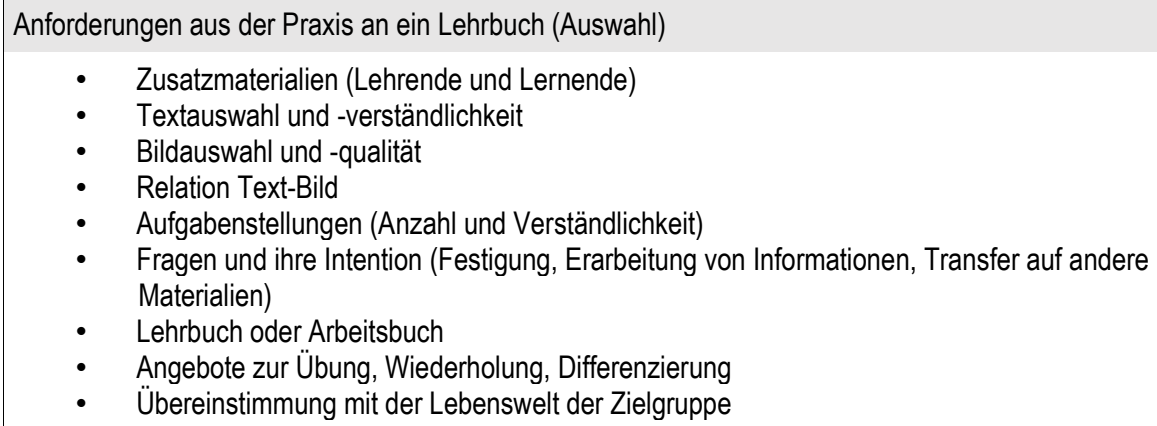

Letztlich ist das Schullehrbuch im Rahmen einer Schulbuchtheorie „erstens [...] Produkt und Faktor gesellschaftlicher Prozesse, zweitens [...] Arbeitsmittel, Lernhilfe und Gegenstand des schulischen Lernprozesses und drittens [...] Element in einer multimedialen Lernumgebung" (Wiater 2003: 12). Zusammenfassend erklärt sich das Schulbuch daher als ein Produkt didaktisch-methodischer, pädagogischer und politischer Überlegungen. Stein (1994) betrachtet das Schulbuch in diesem Kontext als Informatorium, Paedagogikum und Politikum.

\section{Methodische Überlegungen}

Einen zeitlichen und räumlichen Rahmen erfährt die vorliegende Analyse durch das historische Bestehen der SBZ/DDR von 1945 bis 1989. Durch den Rückgriff auf historische Schriftzeugnisse und die Konzentration auf das Fachgebiet Evolutionsbiologie wird der Untersuchungsgegenstand abgesteckt. Da Aussagen über Schulbücher und Lehrpläne getroffen werden, entspricht die Richtung der Analyse, dem inhaltsanalytischen Kommunikationsmodell von Mayring (2010) folgend, einer Dokumentenanalyse. Vor dem soziokulturellen und gesellschaftspolitischen Hintergrund der SBZ/DDR erfolgt die Reflexion der Ergebnisse (Abb. 1). Das folgende Kapitel erörtert das Untersuchungsdesign und stellt die Untersuchungsverfahren vor, die der Dokumentenanalyse zu Grunde liegen. 


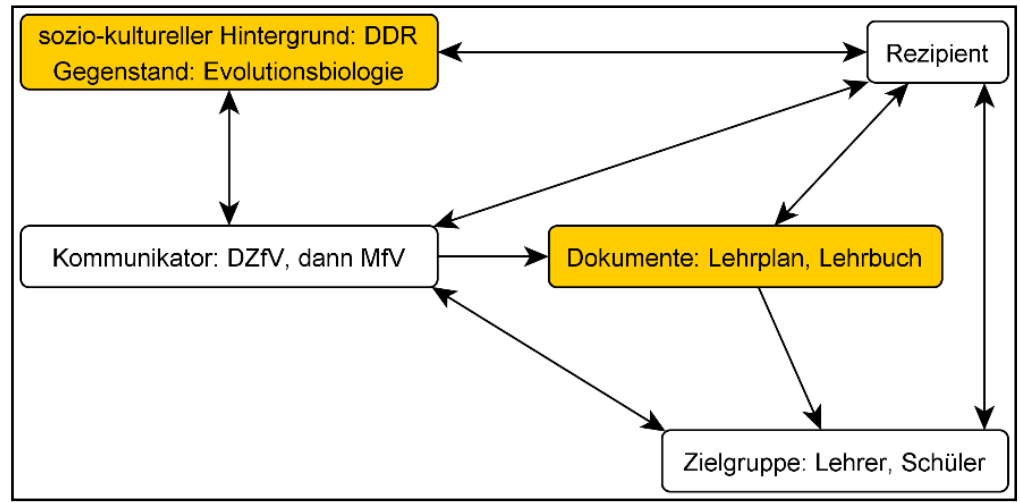

Abbildung 1: Inhaltsanalytisches Kommunikationsmodell (verändert nach Mayring 2010)

Im Rahmen einer historischen Quellenforschung mit dem Forschungsschwerpunkt Schulbuchforschung und um weitere Analysen zu ermöglichen, erfolgte im ersten Schritt die bibliographische Erfassung relevanter Materialien. Als relevant wurden hierbei Lehrbücher und Lehrpläne betrachtet, in denen evolutionsbiologische Kapitel vorhanden waren. Da alle Quellen vollständig erschlossen werden konnten, bestand für die Analyse kein limitierender Faktor. Somit basieren die Ergebnisse nicht auf einer Einzelfallorientierung oder repräsentativen Stichprobe, sondern auf der Gesamterhebung eines zeitlich und räumlich begrenzten Raumes. Die zeitliche Datierung der Quellen orientierte sich dabei ,,an der Chronologie der Quellen selbst“ (Jordan 2005: 113).

Hinsichtlich der Lehrpläne leistete das Bestandsverzeichnis der Bibliothek für Bildungsgeschichtliche Forschung des Deutschen Institutes für Internationale Pädagogische Forschung mit dem Titel Lehrpläne der Sowjetischen Besatzungszone (SBZ) und der Deutschen Demokratischen Republik (DDR) eine erste Orientierung (Dornhof 1994: 92-97). Weitere Recherchen füllten noch vorhandene Lücken und führten zur Erstellung eines Bestandsverzeichnisses für relevante Lehrbücher. Das Ziel der Bibliographie, epochal bedeutsame Dokumente bzw. Quellen zu erfassen, konnte durch das Auffinden von Erstauflagen und veränderten Folgeauflagen erreicht werden. In Fällen, in denen Erstauflagen nicht zugänglich waren, ermöglichten unveränderten Neuauflagen notwendige Rückschlüsse. Auch wurden, wie Wiater (2003) fordert, mit Blick auf die Real- und Sozialgeschichte weitere themenbezogene Quellen einbezogen, um das Schulbuch in seiner Zeit angemessen zu erschließen. Der Fokus lag hierbei auf Unterrichtsmedien, Fachbüchern und 
Zeitschriften (insbesondere die Zeitschrift Biologie in der Schule) ${ }^{9}$ sowie Quellen zu Institutionen sowie den (schul-)rechtlichen und gesellschaftlichen Zuständen.

Recherche, Quellenarbeit und Datenerhebung erfolgte, insofern nicht durch private Bestände abgesichert, an der Deutschen Nationalbibliothek in Leipzig, den Universitätsbibliotheken in Erfurt und Potsdam sowie der AG für Biologiedidaktik in Jena. Ausgehend von der Tatsache, dass die Sammlung der Deutschen Nationalbibliothek alle in Deutschland seit 1913 veröffentlichten Medienwerke umfasst (Jockel 2014), wurde ein Großteil der Recherchearbeit vor Ort geleistet. Da jedoch nicht alle Werke zu jeder Zeit verfügbar waren, u. a. durch notwendige Restaurationen, dienten die genannten Einrichtungen der Ergänzung. Zusätzlich entstand durch eigene Sammlungstätigkeit ein breit gefächerter Materialfundus als Grundlage für diese und weiterführende Arbeiten. ${ }^{10}$

Zur Erstellung eines möglichst umfassenden Bildes der gewählten Zeitdokumente sowie zur Beantwortung der Forschungsfragen liegt der Quellenauswertung ein Analyseraster zu Grunde, das nach Weinbrenner (1992) einem multidimensionalen Forschungsansatz folgt. Es fokussiert auf Aspekte aus den Bezugssystemen Wissenschaftstheorie, Design, Fachdidaktik Biologie und Fachwissenschaft Biologie (Tab. 3). Die Dimension Wissenschaftstheorie dient einer Rekonstruktion des erkenntnistheoretischen Ansatzes in den Dokumenten (Weinbrenner 1992). Berck und Graf (2010) verweisen darauf, dass Biologieschulbücher Ideologien darstellen und es für die Beurteilung eines Schulbuches bedeutsam ist, zu prüfen, wie dies geschieht. Der Schwerpunkt der Analyse liegt hierbei auf der Bildung von Ideologien in den Lehrplänen, verbunden mit der Frage nach Paradigmen in den Erziehungs- und Orientierungsaussagen. Die Aufnahme der Dimension Design begründet sich bereits aus der formalen Struktur der Lehrbücher und ihren zu Grunde liegenden pädagogischen sowie psychologischen Überlegungen (Sujew 1986; Choppin 1992). Weinbrenner (1992: 45) betonte bereits vor über 20 Jahren:

„Da das Schulbuch von diesen Gestaltungsfaktoren ganz wesentlich bestimmt wird, kann eine zukünftige Schulbuchanalyse, die sich auf die Beurteilung von Schulbüchern als Ganzes richtet, nicht mehr ohne die Berücksichtigung der Dimension ,Design' durchgeführt werden.“

Dies gilt umso mehr, wenn durch lange Untersuchungszeiträume, wie in der vorliegenden Arbeit, signifikante Veränderungen zu erwarten sind. Im Interesse eines Gesamteindrucks erfasst die Analyse sowohl äußere (Einband, Format, Umfang) als auch innere Gesichtspunkte (typografische Besonderheiten, farbliche Gestaltung, Abbildungen).

9 Die Zeitschrift für Lehrkräfte Biologie in der Schule erschien erstmals im Jahr 1952 im Verlag Volk und Wissen. Sie war in dieser Form die erste Zeitschrift für den naturwissenschaftlichen Unterricht in der DDR. Ihre Entwicklung bis 1989 hat Engel (2008) ausführlich dargelegt.

10 Entsprechende Ergebnisse dieser Quellensammlung finden sich auch bei Porges 2014, 2016b; Porges et al. 2016; Porges \& Hoßfeld 2017. 
Tabelle 3: Bezugssysteme und Aspekte des Forschungsansatzes (verändert nach Weinbrenner 1992)

\begin{tabular}{|c|c|}
\hline Bezugssystem & Aspekt \\
\hline Wissenschaftstheorie & - Ideologien \\
\hline Design & $\begin{array}{ll}\text { - } & \text { Äußeres Design } \\
\text { - } & \text { Inneres Design }\end{array}$ \\
\hline Fachdidaktik & $\begin{array}{ll}\text { - } & \text { Strukturierung und Sequenzierung } \\
\text { - } & \text { Richtlinienbezug }\end{array}$ \\
\hline Fachwissenschaft & $\begin{array}{ll}\text { - } & \text { Wissenschaftlicher Sachstand } \\
\text { - } & \text { Paradigmenwechsel }\end{array}$ \\
\hline
\end{tabular}

Um einer Orientierung auf das Fach Biologie als Unterrichtsfach gerecht zu werden, sind ferner Aspekte aus dem Bezugssystem Fachdidaktik Teil des multidimensionalen Forschungsansatzes. Der Fokus liegt hierbei auf der Inhaltsstruktur der Dokumente, den daraus abzuleitenden Präferenzen sowie der Aufdeckung von Begründungszusammenhängen. Letztlich befähigt eine Analyse der Inhaltsstruktur, die Übereinstimmung der Schulbücher mit den Vorgaben aus den Lehrplänen zu überprüfen und so die Lehrplan-Lehrbuch-Verschränkung aufzuzeigen. Da die Dokumente inhaltliche Veränderungen erkennen lassen, dient das Referenzsystem Fachwissenschaft Biologie dem Ziel, den Informationsgehalt der Inhalte herauszustellen und an ausgewählten Inhalten zu prüfen, welches Wissen zu welcher Zeit in den Materialien präsent war.

Da alle vier Bezugssysteme in der Hauptsache den Aspekt Evolutionsbiologie beleuchten sollen, ist dieser Forschungsansatz als Partialanalyse zu verstehen. Die Problematik einer Totalanalyse sieht Weinbrenner darin:

„daß Schulbuchforschung wegen ihrer nicht einholbaren Komplexität nur als Partialanalyse möglich ist oder umgekehrt, daß innerhalb eines Forschungsprojektes ein Schulbuch bzw. ein Set von Schulbüchern immer nur im Hinblick auf bestimmte Merkmale, Inhalte oder Funktionen untersucht werden kann, aber nicht als ,Ganzes“" (Weinbrenner 1992: 50, Hervorhebung im Original).

Die Quellenauswertung folgt den von Meyers (1976) vorgeschlagenen Analysearten für historisch-politische Schulbücher und verbindet diese in drei aufeinander aufbauenden Stufen. Die Datenerhebung ist der erste Schritt. Sie beinhaltet die Auswertung der Quellen in Einzelanalysen. Die Reihenfolge der Bearbeitung orientiert sich dabei, dem Prinzip der Komplexitätsreduzierung folgend, an der Chronologie der Quellen. Anschließend erfolgt in einem zweiten Schritt die Zusammenstellung der gewonnenen Daten nach Jahrgangsstufen (Gruppenanalysen). In einem dritten Schritt wird ein Schulbuchvergleich vollzogen, der dadurch charakterisiert ist, dass „Bücher [...] aus verschiedenen Epochen [...] miteinander 
verglichen werden“ (Meyers 1976: 50). Hierbei werden Entwicklungsprozesse in ihrer Gesamtheit aufgezeigt und im Kontext der Schulentwicklung der DDR verortet. Die Quellenlage bringt es mit sich, dass Auswertungen ausschließlich vertikal (Längsschnittanalysen bzw. diachrone Betrachtungen) erfolgen können. Da es in den einzelnen Epochen weder konkurrierende Verlage noch Lehrbücher gab und die jeweils aktuell gültigen Lehrpläne für das gesamte Gebiet der DDR galten, bleiben horizontale Analysen (Querschnittanalysen bzw. synchrone Betrachtungen) auf Einzelanalysen beschränkt. Die Art der Quellenauswertung erfolgt produktorientiert. Hierbei ,interessiert das Schulbuch als Unterrichtsmedium und als Mittel der visuellen Kommunikation" (Weinbrenner 1992: 35). Eine wirkungsorientierte Analyse, d. h. Untersuchungen zu Auswirkungen der Dokumente auf die Zielgruppe, ist insofern problematisch, da der zurückliegende Zeitraum subjektive Verzerrungen der Zeitzeugen erwarten lässt. Ferner ist eine prozessorientierte Analyse denkbar, die den „Lebensay)klus des Scbulbuchs“ (ebd.: 34, Hervorhebung im Original) untersucht. Wenngleich wirkungs- und prozessorientierte Schulbuchanalysen lohnenswerte Forschungsfelder sind, ist letztlich eine produktorientierte Analyse dem Forschungsinteresse nach zielführend.

Pöggeler (2003: 34, Hervorhebung im Original) verweist darauf, dass bisher nur wenig versucht wurde, „die methodologischen Besonderheiten zu präzisieren, die beim Thema Schulbuch entstehen“. Er fordert in diesem Zusammenhang ein interdisziplinäres Vorgehen und verweist auf Methoden, „die so formal sind, dass sie von allen Wissenschaften verwandt werden können. Das gilt z. B. für die (zunächst von der empirischen Soziologie entwickelte) Inhaltsanalyse“ (ebd.: 34f.). In diesem Kontext legt Pöggeler (ebd.) dar, dass sich methodologische Besonderheiten der Schulbuchforschung vor allem aus ihrer Interdisziplinarität ergeben. Der in den Quellen vorliegende Datentyp (Texte, Abbildungen) ist qualitativer Art. Hierbei wird einer anwendungsbezogenen Definition von Kuckartz (2012: 14) gefolgt: „Als quantitative Daten werden numerische Daten, also Zahlen, bezeichnet. Qualitative Daten sind demgegenüber vielfältiger, es kann sich um Texte, aber auch um Bilder [...] und anderes mehr handeln." Für die Analyse der in Schulbüchern enthaltenen Daten fordert Meyers (1976) eine Verschränkung hermeneutischer und quantifizierender Verfahren. Dieser Forderung folgend, werden unter dem Primat der Interdisziplinarität die qualitativen Daten qualitativ und quantitativ ausgewertet. Die qualitative Auswertung erfolgt mittels interpretativer Textauswertung bzw. qualitativer Inhaltsanalyse, wie sie von Mayring (2010) und Kuckartz (2012) vertreten wird. Kuckartz (2012: 6, Hervorhebung im Original) spricht dabei von „kategoriebasierte[n] Methoden zur systematischen Analyse qualitativer Daten" und Mayring (2010: 13, Hervorhebung im Original) von ,kategoriegeleitete [r] Textanalyse". Mayring (2010: 13) versteht unter Inhaltsanalyse als einer sozialwissenschaftlichen Methode letztlich die Möglichkeit in Quellen fixierte Kommunikation systematisch, regel- und theoriegeleitet zu analysieren. Er formuliert drei Grundformen des Interpretierens: Zusammenfassung, Strukturierung und Expli- 
kation. In der strukturierenden Inhaltsanalyse sieht er wiederum die ,zentralste inhaltsanalytische Technik" (Mayring 2010: 92) und unterteilt diese u. a. in formale, inhaltliche und typisierende Strukturierung (Abb. 2).

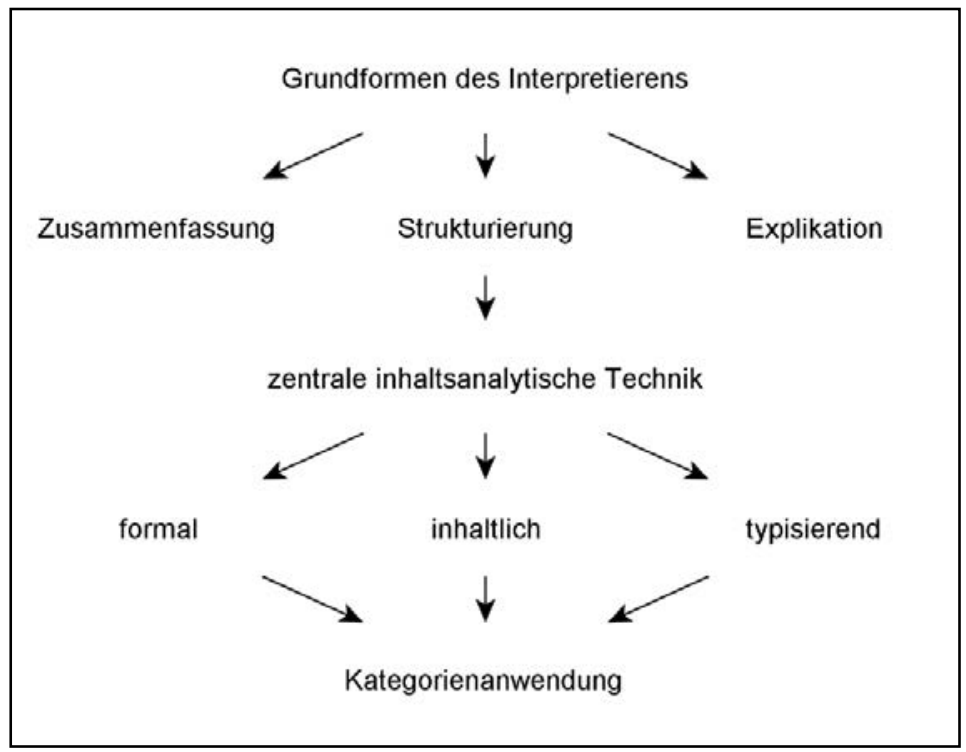

Abbildung 2: Grundformen des Interpretierens (verändert nach Mayring 2010)

Alle drei Grundformen kommen in dieser Arbeit zur Anwendung, mit dem Ziel (a) Strukturen des Materials herauszuarbeiten, (b) ausgewählte Aspekte zusammenzufassen und (c) markante Ausprägungen zu beschreiben. Diese Techniken folgen innerhalb der Analyse folgendem Ablaufmodell (ebd.):

(a) Ablaufmodell der formalen Strukturierung

1. Bestimmung des formalen Kriteriums

2. Materialdurchlauf und Kodierung mittels Kategoriensystem

3. Zusammenstellung der Feinstruktur

4. Konstruktion der Grobstruktur

(b) Ablaufmodell der inhaltlichen Strukturierung

1. Festlegung der inhaltlichen Haupt- und Subkategorien

2. Materialdurchlauf und Kodierung mittels Kategoriensystem

3. Paraphrasierung des Materials

4. Zusammenfassung pro Sub- und Hauptkategorien 
(c) Ablaufmodell der typisierenden Strukturierung

1. Bestimmung der Typisierungsdimension

2. Materialdurchlauf

3. Bestimmung der typischen Ausprägung

4. Bestimmung und Beschreibung der Prototypen

Neben den genannten qualitativen Ansätzen kommen Raum- und Frequenzanalysen als quantitative Verfahren zur Beantwortung der Forschungsfragen zur Anwendung. Diese sind für Schulbuchanalysen besonders geeignet, da sie „ein relativ hohes Maß an Intersubjektivität“ (Uhe 1976: 92) erlauben. Bei Raumanalysen wird davon ausgegangen, dass der Umfang eines Themenkomplexes etwas darüber aussagt, welche Bedeutung ihm die Autorin bzw. der Autor beigemessen hat (ebd.). Bei Frequenzanalysen wird, ähnlich der Raumanalyse, davon ausgegangen, dass von der Häufigkeit der Nennung von Begriffen und Personen im Darstellungstext sowie der Anzahl der Abbildungen auf die Bedeutung, die ihr die Autorin bzw. der Autor beimisst, geschlossen werden kann. In Verbindung mit Raumanalysen dienen so gewonnen Daten einer Zunahme ihrer Kausalität. Durch den Einsatz von Software für qualitative Datenanalyse (engl. „Qualitative Data Analysis“, QDA) werden in diesem Kontext Worthäufigkeitslisten und daraus resultierende Wortwolken erstellt. Dieses Visualisierungstool signalisiert anschaulich über die Schriftgröße die Häufigkeit der Begriffe. Des Weiteren werden die Vorworte der Lehrpläne sowie exemplarisch Lehrbuchtexte deskriptiv-analytisch erschlossen. Auf eine vollständige Darlegung der Lehrbuchtexte wird jedoch verzichtet, da bereits durch Rommel (2006) eine ausführliche deskriptive Arbeit vorliegt.

Das gewählte Untersuchungsdesign sowie die methodische Vorgehensweise (Inhaltsanalyse) erfordert die Bildung von thematischen Kategorien als methodische Hilfsmittel zur Erfassung von Inhalten. Dieser Schritt ist nach Meyers (1976: 49) „besonders wichtig, da er über das Ergebnis der Gesamtuntersuchung mitentscheidet“". Aus diesem Grund erfolgten im Prozess der Kategorienbildung unter Hinzunahme von Referenzliteratur (Tab. 4) eine mehrfache Durchsicht aller Materialien sowie die Durchführung von Pretests. Aus einem deduktiv-induktiven Zugang und im Meinungsaustausch mit den wissenschaftlichen Mitarbeitern der AG Biologiedidaktik der Friedrich-Schiller-Universität in Jena resultierten sieben sich ausschließende Kategorien, die den gesamten zu untersuchenden Inhalt (Text und Abbildungen) umfassen: Theorien, Biogenese, Neontologie, Paläontologie, Systematik, Hominisation, Didaktisches (Abb. 3). Die hier vorgestellte Reihenfolge der Kategorien orientiert sich an den Thüringer Lehrplänen von 2012 für den Erwerb des Hauptschul- und Realschulabschlusses sowie für den Erwerb der allgemeinen Hochschulreife, die drei Themenfelder aufweisen: Evolutionstheorien, Belege für die Evolution sowie Entwicklung des Menschen aus tierischen Vorfahren. Um die Reliabilität der Daten zu gewährleisten, werden im Folgenden die Kategorien vorgestellt und eingegrenzt. 
Tabelle 4: Primärquellen und Referenzliteratur (Auswahl)

Primärquellen (SBZ/DDR): 26 Lehrpläne, Direktiven und methodische Hilfen sowie 12 Lehrbücher

Referenzliteratur (BRD):

Studienliteratur:

Campbell, N. A. (1997): Biologie. Heidelberg etc.: Spektrum.

Kutschera, U. (2008): Evolutionsbiologie. 3. Aufl. Stuttgart: Eugen Ulmer KG.

Mayr, E. (2005): Das ist Evolution. 3. Aufl. München: Goldmann.

Storch, V., Welsch, U. \& Wink, M. (2007): Evolutionsbiologie. 2. Aufl. Berlin etc.: Springer.

Wehner, R. \& Gehring, W. (1995). Zoologie. 23. Aufl. Stuttgart etc.: Georg Thieme.

Schulbücher:

Bayrhuber, H. \& Kull, U. (1998): Linder Biologie. Lehrbuch für die Oberstufe. 21. Aufl. Hannover: Schroedel.

Grümme, T., Krull, H.-P., Küttner, R. \& Nolte, M. (2010): Markl Biologie Oberstufe. Arbeitsbuch. Stuttgart: Ernst Klett.

Högermann, C. \& Meißner, K. (Hrsg.) (2001): Biologie plus. Gymnasium Klassen 9/10. Thüringen. Berlin: Cornelsen.

Miram, W. \& Scharf, K.-H. (1988): Biologie heute S II. Hannover: Schroedel.

Miram, W. \& Scharf, K.-H. (1997): Biologie heute S II. Ein Lehr- und Arbeitsbuch. Hannover:

Schroedel.

Paul, A. (Hrsg.) (2004): Biologie heute entdecken SII. Ein Lehr- und Arbeitsbuch. Braun-

schweig: Schroedel.

Lehrpläne:

TMBWK (Thüringer Ministerium für Bildung, Wissenschaft und Kultur) (Hrsg.) (2012): Lehr-

plan für den Erwerb des Hauptschul- und Realschulabschlusses. Biologie.

TMBWK (Thüringer Ministerium für Bildung, Wissenschaft und Kultur) (Hrsg.) (2012): Lehrplan für den Erwerb der allgemeinen Hochschulreife. Biologie.

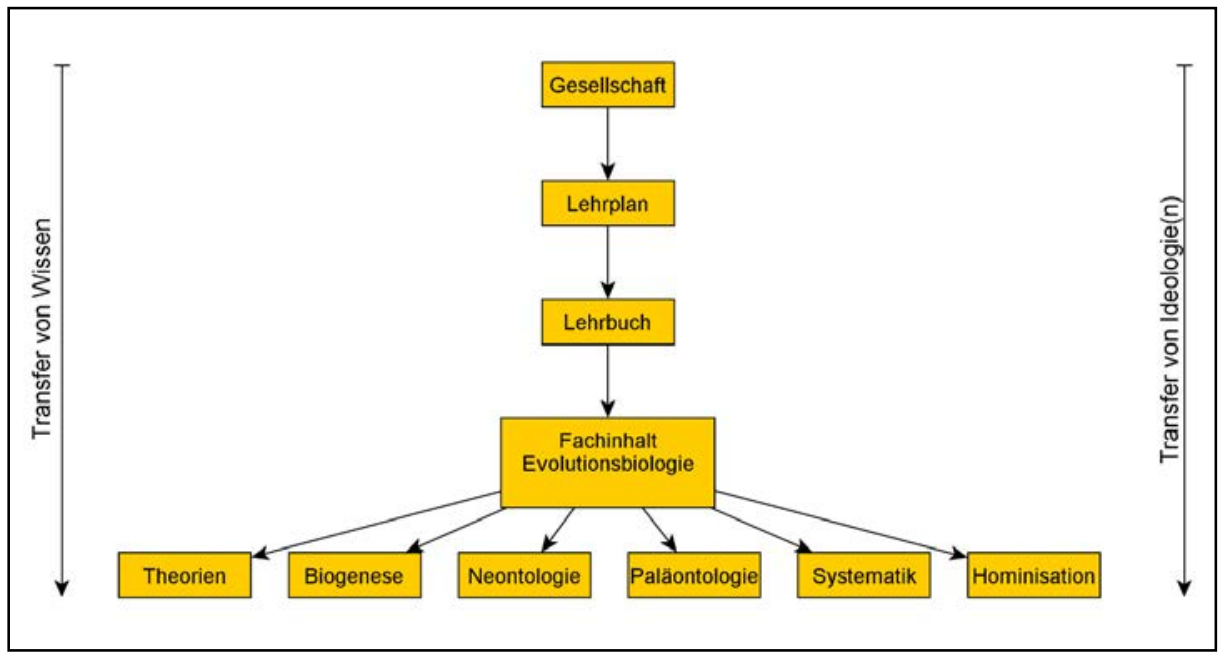

Abbildung 3: Kategoriensystem 
Kategoriensystem

Die Kategorie Theorien umfasst alle Aussagen über Evolutionstheorien, außer Theorien, die die Entstehung des Lebendigen erklären (s. dazu Kategorie Biogenese). Ferner sind alle Aussagen über das Leben und Werk historischer Persönlichkeiten, die im Zusammenhang mit der Entwicklung von Evolutionstheorien Erwähnung finden, Teil dieser Kategorie. Dies bedeutet, dass neben historischen und biografischen Darstellungen auch Aussagen über jeweils aktuelle Mechanismen sowie Ursachen der Evolution (Evolutionsfaktoren) dieser Kategorie zugeordnet werden. Inhalte aus den Fachgebieten Genetik und Züchtung (auch Lyssenkoismus bzw. Schöpferischer Darwinismus), innerhalb evolutionsbiologischer Kapitel behandelt, sind ebenso integrativer Bestandteil. Ausführliche Informationen dazu finden sich in der Fachliteratur (u. a. Mayr 2002; Junker \& Hoßfeld 2009).

Die Kategorie Biogenese beinhaltet alle Aussagen über die chemische Evolution. Dazu gehören beispielsweise Angaben zu Theorien (u. a. Koazervatentheorie, Ursuppe), die die Entstehung des Lebendigen erklären. Auch Angaben zu Ursprungstheorien bzw. der Kritik an diesen (Schöpfungsglaube) sowie Persönlichkeiten, die im Kontext mit der Erforschung der Biogenese vorgestellt werden (u. a. Oparin, Miller), sind Teil dieser Kategorie. Eine Zuordnung erfahren ferner Aussagen über die Merkmale des Lebendigen. Ein Überblick über die Forschungslage finden sich u. a. bei Storch et al. (2007) und Kutschera (2008).

Die Kategorie Neontologie umfasst alle Aussagen aus neontologischer Forschung. Dies beinhaltet Resultate und Untersuchungen aus den Bereichen der Biogeografie, der vergleichenden Anatomie und Morphologie (Homologie, Analogie) sowie der Entwicklungsbiologie (Rudimente, Atavismen, Biogenetisches Grundgesetz). Belege für den Ablauf der Evolution aus dem Bereich der Neontologie werden in der Fachliteratur (u. a. Kutschera 2008) näher erläutert.

Die Kategorie Paläontologie setzt sich aus allen Aussagen der allgemeinen (Mechanismen der Fossilisation) und speziellen Paläontologie (Beschreibung und Klassifizierung von Fossilien) zusammen. Ferner sind paläobiologische Inhalte (Paläobotanik und -zoologie) sowie Aussagen zu geologischen Ären, Unter-Ären und Alterswerten in diese Kategorie integriert. Auch fossile Übergangsformen und Aussagen zu lebenden Fossilien sind hier eingeordnet. In der Literatur zur Evolutionsbiologie wird diese Kategorie ausführlich beschrieben (u. a. Storch et al. 2007; Kutschera 2008).

Die Kategorie Systematik beinhaltet alle Aussagen zur natürlichen Systematik, einem Teilgebiet der beschreibenden Biologie, insofern sie innerhalb evolutionsbiologischer Kapitel behandelt wird. Dies betrifft Angaben zur Vielfalt der Organismen und ihrer Verwandtschaftsverhältnisse. Erkenntnisse aus der molekularen Phylogenetik, die die klassische Systematik erweitern, werden u. a. von Kutschera (2008) dargelegt. 
Die Kategorie Hominisation umfasst alle Aussagen zur Evolution des Menschen. Dies betrifft die Bereiche der Biogenese physischer Merkmale, der Tradigenese (Werkzeuggebrauch, Verwendung des Feuers u. a.) sowie Fossilien der Hominisation und Verwandtschaftsverhältnisse. Die Abstammung des Menschen ist ausführlich in der Fachliteratur beschrieben (u. a. Henke \& Rothe 2003; Storch et al. 2007).

Die Kategorie Didaktisches beinhaltet Kapitel zur Wiederholung und Systematisierung sowie Einleitungen, insofern diese explizit vom Lerninhalt abgegrenzt sind. Hierbei ergeben sich zwangsläufig Unschärfen zu allen bisher genannten Kategorien. Weiterführende Kodierungen erfolgen hierbei nicht, da ausschließlich das didaktische Element von Interesse ist. Frage- bzw. Aufgabenstellungen, die direkt im Lerninhalt integriert sind, werden im Kodierprozess den jeweiligen Kategorien (1 bis 6) zugeordnet.

Grundlegend wurden für alle Untersuchungsverfahren der vorliegenden Studie folgende Analyseeinheiten definiert: Kodiereinheit (Minimum) ein Satz, Kontexteinheit (Maximum) das gesamte Material der jeweiligen Quelle, Auswertungseinheit entsprechend dem Kategoriensystem. Allerdings offenbarte die Durchsicht der Quellen in vielen Fällen eine Verflechtung der Kategorien. Diese Beobachtung bestätigten auch der Analyse vorgeschaltete Pretests. Um eine eindeutige Zuordnung zu gewährleisten, wurden die jeweiligen Aussagen im Kontext betrachtet. Generell gilt, im Sinn einer Kodierregel, der thematische Zusammenhang, da Unschärfen in der Darstellung immanenter Bestandteil evolutionsbiologischer Inhalte und Verflechtungen somit fachspezifisch sind. Diese inhärente Charakteristik soll im Folgenden an zwei Ankerbeispielen verdeutlicht werden:

„Über die Entstehung der verschiedenen Arten haben die Menschen im Verlauf der Jahrtausende verschiedene Auffassungen entwickelt." (Kummer et al. 1988: 73)

Dieser Satz isoliert betrachtet, entspricht dem Kodiersystem der Kategorie Theorien. Kontextuell ist das erste Ankerbeispiel Teil eines einleitenden Abschnittes im Lehrbuch zu Beginn des evolutionsbiologischen Themenfeldes. Demnach erfolgt die Zuordnung zur Kategorie Didaktisches.

„Zahlreiche Fossilfunde sowie eingehende Vergleiche zwischen dem Menschen und den heute noch lebenden Tieren lassen eindeutig erkennen, daß der Mensch nicht plötzlich durch einen Schöpfungsakt auf die Erde gekommen sein kann." (Bach et al. 1968: 64)

Dieses Zitat für sich genommen, enthält dem Kodiersystem entsprechend Elemente bzw. Begriffe aus den Kategorien Paläontologie, Biogenese, Neontologie und Hominisation. Da dieser Satz jedoch integraler Bestandteil eines Abschnittes über die Evolution des Menschen ist, erfolgt die Zuordnung zur Kategorie Hominisation. 


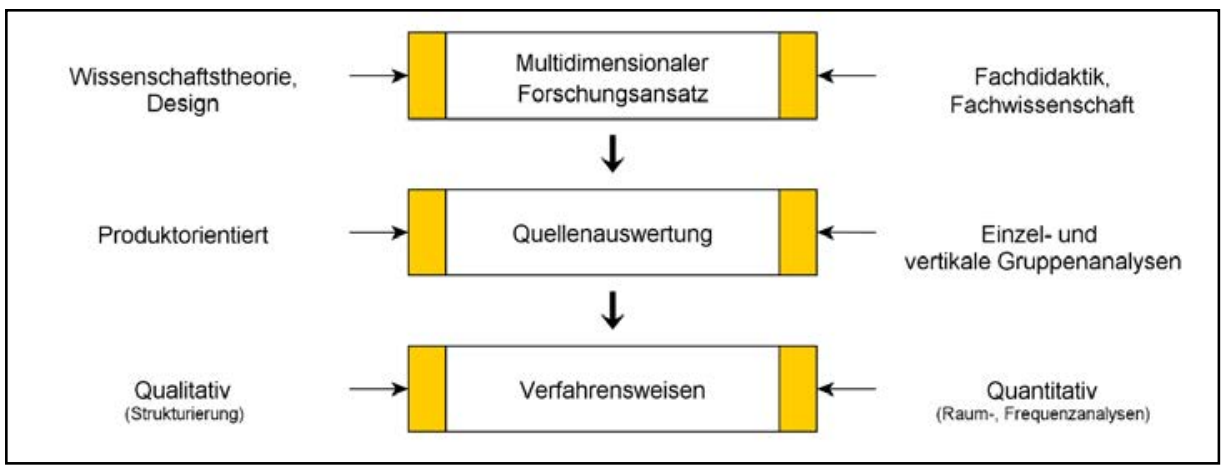

Abbildung 4: Forschungsdesign

Dem Forschungsinteresse folgend, ergab sich aus den vorgestellten methodologischen Überlegungen ein Kriterienkatalog, der der Beurteilung der Lehrpläne und Lehrbücher zu Grunde liegt. Basierend auf dem bereits vorgestellten multidimensionalen Forschungsansatz (Design, Fachdidaktik, Fachwissenschaft), bildet dieser Katalog gleichsam die weitere Gliederung der vorliegenden Arbeit. Für die Aufarbeitung des Quellenmaterials wurden aus den allgemeinen Forschungsfragen weitere Leitfragen spezifiziert und ihnen adäquate Methoden zugeordnet (Abb. 4). Im Rahmen der historischen Quellenforschung erfolgte darüber hinaus die Erfassung formaler Angaben (Erscheinungsjahr, Titel, Autorin bzw. Autor). Hier interessierte die Frage, ob ein Wechsel der Autorinnen und Autoren mit inhaltlichen Veränderungen in den Materialien verbunden war.

Beurteilungskriterien

\section{Beurteilung der Lehrpläne}

Vorwort (wissenschaftstheoretischer Zugang): Welche Hinweise auf evolutionsbiologische Inhalte integrierten die Autorinnen und Autoren in den Vorworten der Lehrpläne und welche Einstellungen sowie Verhaltensweisen (Erziehungs- und Orientierungsaussagen) wurden durch diese Aussagen gefordert? Diese Fragen zu klären, dient die Analyse der Lehrplanvorworte. Der Zugang erfolgt deskriptiv, fokussiert auf diejenigen Textstellen, die einen Bezug zur Evolutionsbiologie aufweisen und mündet in einer Zusammenstellung sowie Interpretation relevanter Passagen. Eine typisierend strukturierende qualitative Inhaltsanalyse ordnet ferner die Orientierungsaussagen in vier typische, merkmalshomogene Denkfiguren. Typ 1 der Lehrpläne beinhalten im Vorwort eine Verflechtung ideologischer Orientierungen und evolutionsbiologischer Inhalte. Lehrpläne vom Typ 2 weisen im Vorwort lediglich sachbezogene Aussagen auf, ohne diese in Beziehung zu ideologischen Orientierungen zu setzen. Lehrpläne vom Typ 3 beinhalten im Vorwort ideologische Orientierungen, setzen diese jedoch nicht in Beziehung zu evoluti- 
onsbiologischen Fachinhalten. Lehrplanvorworte vom Typ 4 enthalten weder sachbezogene Aussagen zum Thema Evolutionsbiologie noch ideologische Orientierungen. Die Ergebnisdarstellung erfolgt verbal-interpretativ und visuell in Tabellenform (Tab. 5).

Tabelle 5: Typologie von Sach- und Ideologiebezug zum Aspekt Evolutionsbiologie

\begin{tabular}{|llll|}
\hline \multicolumn{1}{|c|}{ ja } & \multicolumn{1}{c|}{ Ideologiebezug } \\
Sachbezug & ja & $\begin{array}{l}\text { Typ 1: sachbezogene, ideologische } \\
\text { Orientierung }\end{array}$ & Typ 2: sachbezogene Orientierung \\
& nein & $\begin{array}{l}\text { Typ 3: ideologische Orientierung } \\
\text { Typ 4: Orientierung ohne Sach- und } \\
\text { Ideologiebezug }\end{array}$ \\
\hline
\end{tabular}

Struktur (fachdidaktischer Zugang): Die Strukturierung und Sequenzierung evolutionsbiologischer Lehrplaninhalte zu erschließen und Präferenzen aufzuzeigen, ist das Ziel der Analyse der Stoffpläne. Ermittelt werden der Umfang der Jahresstunden sowie der absolute und relative Stundenumfang für evolutionsbiologische Inhalte. Ferner erfolgt eine Zusammenstellung der Themen des Jahrgangs (Grobstruktur) sowie der Gliederung der evolutionsbiologischen Inhalte (Feinstruktur). Mittels formal strukturierender, qualitativer Inhaltsanalyse entlang der Kategorien, basierend auf einer Kodierung der Stoffpläne, wird die inhaltliche Gliederung der Feinstruktur aufgezeigt. Eine Raumanalyse verdeutlicht die kategoriale Struktur und dient einer vertiefenden Darstellung thematischer Schwerpunkte und Präferenzen. Als Einheiten liegen der Raumanalyse Stundenangaben zu Grunde. Daraus resultierende Prozentwerte werden im Interesse der Lesbarkeit gerundet. Die Ergebnisdarstellung erfolgt verbal-interpretativ und visuell in Form von Tabellen, Balken- und gestapelten Säulendiagrammen.

\section{Beurteilung der Lehrbücher}

Design: Welche Besonderheiten im Layout waren für die relevanten Lehrbücher charakteristisch? Die Analyse des Designs hilft, diese Frage zu klären. Dazu wird im ersten Schritt das äußere Design hinsichtlich Gestaltung, Handlichkeit und Information der Einbände beschrieben. Eine kategorienbasierte Auswertung der Abbildungen auf dem Cover verdeutlicht Präferenzen und stellt Beziehungen zu Fachinhalten im Lehrbuchtext heraus. Raumanalysen zeigen ferner den Umfang bzw. die Genese des Umfangs der Lehrbücher auf. Der Fokus liegt auf dem Gesamt- sowie dem Textumfang des Lehrbuches, dem absoluten und relativen Anteil evolutionsbiologischer Themen am Text, dem absoluten Anteil aller übrigen Themen sowie dem absoluten Anteil des Beiwerkes. Als Einheiten dienen den Raumanalysen Seitenzahlen, wobei jede angefangene Seite zählt. 
Der zweite Schritt, die Analyse des inneren Designs der Lehrbücher, beinhaltet die Erfassung von Hilfsmitteln zur Wissenserschließung (Beiwerk), wie Inhaltsund Sachverzeichnis, Glossar sowie Literaturangaben. Auch Besonderheiten im Text, denen eine didaktisch-methodische Funktion zu Grunde liegt, wie auffällige Formatierungen (Unterschiede in der Schriftgröße, Fettdruck, Kursivschrift) sowie als Merksätze und Aufgabenstellungen abgegrenzte Textbestandteile werden aufgenommen. Ferner sind die Innenseiten der Einbände, Farbtafeln und Kunstdrucktafeln und deren Beziehungen zu evolutionsbiologischen Themen Untersuchungsgegenstand. Erfasst wird weiterhin die Anzahl der Abbildungen des gesamten Lehrbuches und der evolutionsbiologischen Themen, die Abbildungen pro Seite sowie deren Genese. Eine kategorienbasierte Analyse der Abbildungen zeigt letztlich inhaltliche Präferenzen in der Visualisierung auf. Die Ergebnisdarstellung des äußeren und inneren Designs erfolgt verbal-interpretativ und visuell in Form von Tabellen und (gestapelten) Säulendiagrammen.

Struktur (fachdidaktischer Zugang): Ziel der Strukturanalyse der Lehrbücher ist es, die Strukturierung und Sequenzierung der Inhalte zu erschließen und Präferenzen aufzuzeigen. Der Zugang erfolgt deskriptiv, fokussiert auf die Gliederung evolutionsbiologischer Kapitel. Raumanalysen stellen den absoluten und relativen Umfang der Kapitel dar, betonen Besonderheiten in Umfang sowie Gliederung und lassen Rückschlüsse auf den Stellenwert einzelner Kapitel zu. Den Raumanalysen liegen dabei Seitenzahlen als Einheiten zu Grunde, wobei jede angefangene Seite gezählt wird. Ferner zeigt eine Kodierung der Lehrbuchtexte, welche Kategorien in den relevanten Kapiteln vorhanden sind. Dem Kodierprozess liegen hier Zeilen als Einheiten zu Grunde, mit der Vorgabe, dass angefangene Zeilen als ganze Zeilen, Zeilen neben Bildern als halbe Zeilen und Überschriften, Bildunterschriften sowie Fußnoten nicht zählen. Bei Zeilenschnitten erfährt jede Kategorie eine Zeilenzuweisung. Dadurch auftretende Unregelmäßigkeiten verändern das Gesamtbild nicht wesentlich und sind zu vernachlässigen. Worthäufigkeitslisten der Kapitelüberschriften, erstellt und als Wortwolke visualisiert, zeigen die biologischen Begriffe der Überschriften auf, die ihrerseits die in den Lehrbuchtexten enthaltenen Inhalte spiegeln. Abschließend erfolgt die Analyse der Textbestandteile nach dem Ablaufmodell der formal strukturierenden, qualitativen Inhaltsanalyse in Verbindung mit einer Raumanalyse zur Bestimmung von Abfolge und Umfang der Kategorien im Textfluss. Dieser Schritt basiert ebenfalls auf einer Kodierung der Lehrbuchtexte. Die Darstellung der Ergebnisse erfolgt verbal-interpretativ sowie visuell in Form von Wortwolken, Säulendiagrammen und Tabellen.

Fachinhalte (fachwissenschaftlicher Zugang): Die Frage zu klären, welches Wissen zu welcher Zeit in den Lehrbüchern angeboten wurde, ergibt sich durch die Analyse der evolutionsbiologischen Fachinhalte. Der Zugang erfolgt mittels inhaltlich strukturierender qualitativer Inhaltsanalyse exemplarisch anhand der Kategorien Theorien, Neontologie und Paläontologie. Innerhalb der Kategorie Theorien wird eine kategorienbasierte Auswertung entlang der Subkategorien 
Darwinismus, Neodarwinismus, Schöpferische Biologie und Synthetische Theorie vorgenommen. Frequenzanalysen informieren in diesem Kontext über Häufigkeiten von, für die jeweiligen Epochen charakteristischen, Personennamen. Übersichten zu Darwins Leben und Werk, Ankerbeispiele für die Darwin'schen Theorien und gelistete Bildbelege ermöglichen innerhalb der Subkategorie Darwinismus eine vergleichende Analyse der Lehrbücher. In der Kategorie Neontologie wird eine kategorienbasierte Auswertung entlang der Subkategorien Homologien, Rudimente, Atavismen, Analogien, Biogeografie und Embryologie durchgeführt. Der Fokus liegt hier auf den Text- und Bildbelegen. Die Auswertung der Kategorie Paläontologie basiert auf den Subkategorien Systemtabellen, Entwicklungsreihen, Zwischenformen und Fossilien. Übersichten sowie Text- und Bildbelege bilden die Schwerpunkte der vergleichenden Darstellung. Die Präsentation der Ergebnisse erfolgt verbal-interpretativ sowie visuell in Form von Säulendiagrammen und Übersichtstabellen zu den Themen Darwins Leben und Werk, Ankerbeispiele der Darwin'schen Theorien, Entwicklungsreihen sowie Fossilien. 



\title{
Evolutionsbiologie im Wandel
}

\begin{abstract}
„Wissenschaftliche Revolutionen hat es seit Kopernikus immer wieder gegeben, aber keine hat einen solchen Umbruch im abendländischen Denken bewirkt wie Darwins Beobachtungen und Theorien." (Mayr 2008: 9)
\end{abstract}

\section{Vom Evolutionsgedanken zur Wissenschaftsdisziplin}

Das folgende Kapitel reflektiert über die Entwicklung des Fachgebietes Evolutionsbiologie von der Vergangenheit bis zur Gegenwart. Es werden relevante Phasen in der Entwicklung der Wissenschaftsdisziplin Evolutionsbiologie aufgezeigt und deren Begrifflichkeiten bestimmt. Anschließend wird eine Auswahl an Indizien für Evolutionsprozesse vorgestellt. Diese Darlegungen bilden den theoretischen Hintergrund für die im Rahmen der Dokumentenanalyse (Schulbuch) aufgegriffenen Kategorien Theorien, Neontologie und Paläontologie. 
Evolutionsvorstellungen - ein historischer Abriss

Wenngleich echte Evolutionsvorstellungen von der Antike bis ins 18. Jahrhundert weitgehend fehlten, so finden sich bereits bei den Vorsokratikern Anaximandros (ca. 611-546 v. Chr.) und Empedokles (ca. 495-435 v. Chr.) einige Ansätze in Form von Ursprungstheorien (Junker \& Hoßfeld 2009). Empedokles hat aus heutiger Sicht „eine allerdings etwas phantastische Theorie von der Fortentwicklung und vom Überleben des Tüchtigsten aufgestellt“ (Russell 2009: 76). Plato (428/7349/8 v. Chr.) und Aristoteles (384-322 v. Chr.), die von allen Philosophen von der Antike bis zur Neuzeit auf die darauf folgenden Epochen den größten Einfluss ausübten (Russell 2009), vertraten hingegen keine evolutionistische Einstellung. Plato postulierte ewige Prinzipien und eine schöpferische Kraft und Aristoteles vertrat das statische Konzept einer Stufenleiter (Junker \& Hoßfeld 2009). „Gedanken, die später für die moderne Evolutionstheorie entscheidend wurden“ (ebd.: 27), finden sich dagegen bei den Epikureern ${ }^{11}$. Ein Schüler Epikurs (342/1$271 / 0$ v. Chr.) war der römische Dichter Lukrez (99-55 v. Chr.). Von Empedokles beeinflusst (Russel 2009), vertrat er in seinem Werk De rerum natura die Ansicht, „daß kein Ding aus nichts entsteht auf göttliche Weise“ (Lukrez 2008: 19). Er setzte sich mit dem Entwicklungsgedanken auseinander und beschrieb ,als erster das Prinzip der Selektion“ (Schäfers 2008: 41).

Darwin (2004: 11) sprach ,von bloßen Andeutungen bei den klassischen Schriftstellern" und verwies stattdessen auf die wissenschaftlichen Leistungen des 18. und 19. Jahrhunderts. Im Jahrhundert der Aufklärung wurde der Versuch unternommen, mit Hilfe neuer Erkenntnisse an die Gedankengebäude der Antike anzuknüpfen (Junker \& Hoßfeld 2009). Insbesondere durch das Wirken von Carl von Linné (1707-1778) entstand im 18. Jahrhundert die Biologie als Wissenschaft vom Lebendigen (Jahn \& Schmitt 2001). Aufgrund seiner Auffassungen gelang es, „den Schritt zu einer biologisch arbeitenden und denkenden Anthropologie/Menschenkunde zu vollziehen“ (Hoßfeld 2001: 87). Zwar ist nicht ersichtlich, dass Linné an der Konstanz der Arten und am Schöpfungsbericht zweifelte, doch verzichtete er in der letzten Auflage seines Werkes Systema naturae auf die Bemerkung, dass keine neuen Arten entstehen (Jahn \& Schmitt 2001; Storch et al. 2007).

Immanuel Kant (1724-1804), „der Begründer des deutschen Idealismus“ (Russell 2009: 712), wird „des ,gewagten Abenteuers der Vernunft' halber [...] zum ,Vorläufer Darwins` ernannt“" (Löther 2004: 117). Er äußerte in seinen Werken Vermutungen über die verwandtschaftlichen Beziehungen der Lebewesen und deren Entwicklung, lehnte jedoch die Evolutionstheorie ab (Kutschera 2008; Junker \& Hoßfeld 2009). Einer der bekanntesten Naturforscher dieser Zeit war Georges-Louis Leclerc Buffon (1707-1788), der in seinem Werk Histoire Naturelle ohne theologische Einflüsse auskam und die Naturgeschichte entscheidend prägte (Rieppel 2001; Junker \& Hoßfeld 2009). Zwar konnte er die Diskontinuität der

${ }^{11}$ Schule der hellenistischen Epoche. 
Arten, wie auch Charles Bonnet (1720-1788), Étienne Geoffroy Saint-Hilaire (1772-1844), Jean Baptiste de Lamarck (1744-1829) oder Darwin nicht befriedigend aufklären, doch erkannte er das Problem ihrer Transformation sowie deren Auslegung und schuf „ein intellektuelles Gebäude, das den meisten Naturforschern bis hin zu Charles Darwin zur Orientierung diente“ (Rieppel 2001: 31).

Als „Begründer (Urvater) der Evolutionsforschung“ (Kutschera 2008: 26) gilt heute Lamarck, wenngleich sein Hauptwerk viel eher in der Begründung der Zoologie der Wirbellosen zu verorten ist (Lefèvre 2001). Er wurde nicht nur mit dem jungen Darwin bekannt, er erkannte auch die Existenz der Evolution. Lamarck vertrat, im Gegensatz zur Katastrophentheorie des französischen Naturforschers Georges Cuvier (1769-1832), die Ansicht, dass sich das Leben kontinuierlich entwickelt und die Veränderungen der lebenden Materie nicht auf geologische Katastrophen zurückzuführen ist (Storch et al. 2001). Cuvier, der die vergleichend funktionelle Anatomie begründete, vertrat die Unveränderlichkeit der Arten. Saint-Hilaire war ein weiterer bekannter französischer Evolutionist seiner Zeit. Er postulierte die Einheit des Bauplans (unité de composition) für das gesamte Tierreich und versuchte, die evolutionäre Abwandlung dieses Bauplans zu zeigen (Junker \& Hoßfeld 2009). Eine Auseinandersetzung zwischen ihm und Cuvier ging unter der Anteilnahme von Johann Wolfgang von Goethe (1749-1832) als Pariser Akademiestreit in die Geschichte ein. Im Ergebnis „trat eine völlige Erschöpfung der Naturphilosophie ein, und es folgte [...] eine lange Periode, innerhalb deren alle Kräfte sich auf die Spezialforschung warfen" (Weismann 1904: 20, Hervorhebung im Original).

Ein entscheidender Schritt hin zu einer wissenschaftlichen Erklärung der Entstehung und Veränderung der Arten gelang Charles Robert Darwin und Alfred Russell Wallace (1823-1913). Zuvor finden sich schon ,einige schwache Versuche“ (ebd.: 23) bei Robert Chambers (1802-1871), Charles Victor Naudin (18151899) und Hermann Schaaffhausen (1816-1893). Einen entscheidenden Einfluss auf das Leben und das Werk von Darwin übte seine fünfjährige Forschungsreise aus, die er als Begleiter des Kapitäns Robert Fritz-Roy (1805-1865) auf dem Segelschiff H.M.S. Beagle antrat (Barlow 2008; Darwin 2008; Junker 2009). Darwin (2008: 19) selbst äußerte tiefste Dankbarkeit, dass ihm die „Gelegenheit zum Studium der Naturgeschichte der verschiedenen Länder" vergönnt war. Auch Wallace, der die evolutionäre Biogeographie mit begründete, fand auf seinen Reisen eine Erklärung für den Mechanismus der Evolution (Glaubrecht 2013). Darwins einflussreiches Werk On the Origins of Species by means of Natural Selections, or the Preservation of Favoured Races in the Struggel for Life (1859) revolutionierte alle bis dato gültigen Vorstellungen der Artentstehung. Für ihn war klar, wenn diese oder ähnliche „Ansichten über die Entstehung der Arten allgemein zugegeben werden, [...] eine große Umwälzung der Naturwissenschaften die Folge sein" muss (Darwin 2004: 672). Dem durch sein Werk ausgelösten gesellschaftlich-wissenschaftlichen Diskurs enthielt er sich jedoch und verbrachte die meiste Zeit auf seinem Landsitz 
in Down. Eine ähnliche wohl überlegte Vorgehensweise wählte er bei der Veröffentlichung seiner Ansichten über die Abstammung des Menschen, als er schrieb:

„Viele Jahre hindurch habe ich Notizen über den Ursprung oder die Abstammung des Menschen gesammelt, ohne die Absicht, etwas darüber zu veröffentlichen; ich war im Gegenteil entschlossen, nichts davon in die Öffentlichkeit zu bringen, weil ich fürchtete, damit nur die Vorurteile gegen meine Ansichten zu vermehren." (Darwin 2009: 9)

Trotz dieser Zurückhaltung, so belegen bekannte Karikaturen, hat die Frage nach der Herkunft des Menschen die damalige anthropozentrische „Gesellschaft besonders betroffen“ (Leinfelder 2007: 286). Als "die prominentesten Vertreter“ (Hoßfeld 2012a: 81) der biologisch-anthropologischen Forschung dieser Zeit gelten Ernst Haeckel und Thomas Henry Huxley (1825-1895). Der Engländer Huxley war einer der ersten Anhänger Darwins. Er setzte sich ,in zahlreichen wissenschaftlichen und populären Schriften konsequent für die Darwin'sche Abstammungslehre ein und bezeichnete sich selbst als ,Generalagenten Darwins"“ (Schulz 2001: 130). In Deutschland gehörte in der Anfangszeit des Darwinismus der Jenaer Universitätsprofessor Ernst Haeckel zu den engagiertesten Vertretern (Haeckel 1909; Hoßfeld 2010). Für ihn war „die Abstammungslehre (Phylogenetik) nicht nur der Kern der Evolutionstheorie, sondern synonym damit. Hierin zeigte sich ein großer Unterschied zu Darwin, dessen Hauptinteresse den Ursachen und Mechanismen der Evolution galt" (Fischer et al. 2008: 6). Dass Evolutionstheoretiker nach 1859 nicht alle fünf Theorien von Charles Darwin anerkannten, sah Ernst Mayr (1904-2005) in der Beweislage und in den herrschenden Ideologien begründet (Mayr 1995; Mayr 2002). Obwohl Haeckel, im Gegensatz zu Darwin, den Zufall als Evolutionsfaktor ablehnte, wird er als Deutscher Darwin gewürdigt (Fischer et al. 2008: 7; Hoßfeld 2010: 9). In seinen Ansichten gefestigt, polarisierte er aktiv die Diskurse seiner Zeit und ließ sich 1904 in Rom zum Gegenpapst ausrufen (Hoßfeld 2007b: 45).

Ein Meilenstein im Wirken von Ernst Haeckel war die Eröffnung des Phyletischen Museums in Jena, dessen Grundsteinlegung zu Goethes Geburtstag am 28. August 1907 erfolgte. Die Fresken am Gebäude sowie die Werke Kunstformen der Natur und Wanderbilder sind Dokumente seiner Reisen und zeigen neben seiner wissenschaftlichen Arbeit sein künstlerisches Schaffen (Fischer et al. 2008; Haeckel 2009; Hoßfeld 2010). Er sah darin für „die moderne bildende Kunst und das moderne, mächtig emporgeblühte Kunstgewerbe [...] eine reiche Fülle neuer und schöner Motive“" (Haeckel 2009a: 4). Wie schon Huxley in England so nutzte auch Haeckel die Möglichkeit, den Evolutionsgedanken über populärwissenschaftliche Werke zu verbreiten (Haeckel 2009b). Nicht zuletzt durch Huxleys und Haeckels Wirken, war bereits einige Jahrzehnte nach Darwins Hauptwerk „die Evolution der Organismen [...] von Fachleuten als Faktum anerkannt" (Kutschera 2009: 138, Hervorhebung im Original). 
Wissenschaftsdisziplin Evolutionsbiologie

In der Retrospektive prägten Erkenntnisse aus den Bereichen der Evolutionsbiologie wissenschaftliche und öffentliche Debatten der letzten 200 Jahre. Sie ist als Teilgebiet der Biologie ,aus der Verknüpfung zahlreicher biologischer Disziplinen, ursprünglich speziell der Populationsbiologie (Demökologie), mit der Darwinschen Evolutionstheorie (Darwinismus) hervorgegangen“ (Freudig \& Sauermost 2000a: 282). Mit Blick auf aktuelle Studienliteratur steht heute insbesondere ihr interdisziplinärer Charakter im Vordergrund, denn „das Gedankengebäude der Evolutionsbiologie ist komplex und verbindet Wissenschaften wie Geologie, Paläontologie, Astro- und Geophysik, Klimatologie, Ökologie, Biochemie, Molekularund Zellbiologie, Botanik, Mikrobiologie sowie Zoologie" (Storch et al. 2007: VI). Aus antiken Vorstellungen über klassische Evolutionstheorien bis hin zu ihrer heutigen Form entwickelte sich die moderne Evolutionstheorie zu einem „System zahlreicher Unter-Theorien“ (Kutschera 2009: 305). Sie wird als Erweiterte Synthetische Theorie der biologischen Evolution, als synthetischer Darwinismus bzw. als Wissenschaftsdisziplin Evolutionsbiologie bezeichnet (Kutschera 2008; Junker \& Hoßfeld 2009).

Darwin, Wallace und Haeckel gebrauchten in ihren Werken das Wort Evolution nur selten bzw. gar nicht (Kutschera 2008). Der Begriff Evolution, der sich aus dem Lateinischen evolvere (abwickeln) bzw. evolutio (ausrollen) ableitet, „legt das Auswickeln einer bereits existenten Struktur nahe“ und bedeutet im Allgemeinen Veränderung in allumfassender Hinsicht (Junker \& Hoßfeld 2009: 16). Im 18. Jahrhundert in der Präformationslehre (Auswickeln präformierter Strukturen) verwandt, führte in den 1860er Jahren der Philosoph Herbert Spencer (18201903) das Wort Evolution als Synonym für die Veränderung der Arten ein (ebd.). $\mathrm{Da}$ zu dieser Zeit bekannte fossile Belege eine Komplexitätszunahme zeigten, wurde der Begriff Evolution ,irrtümlicherweise mit dem Schlagwort ,Höherentwicklung" (bzw. ,Perfektionierung) gleichgesetzt" (Kutschera 2009b: 137). Eine gerichtete Entwicklung im Verlauf der Evolution bzw. die Ansicht der Mensch sei das am höchsten entwickelte Lebewesen steht jedoch im Widerspruch zu den heutigen Erkenntnissen der Evolutionsbiologie (Gould 1999; Kutschera 2009b; Kattmann 2008; Paul 2009). In den 1970er Jahren definierte der Freiburger Zoologe Günther Osche (1926-2009) den Begriff Evolution wie folgt:

„Ausgehend von gemeinsamen Ahnen, muss es im Verlauf der Stammesgeschichte (Phylogenese) der Organismen zu einer Transformation von deren Gestalt, Funktion und Lebensweise, und das heißt zur Bildung neuer Arten und Organisationstypen, gekommen sein. Diesen Prozess, der im Hinblick auf die Eigenschaften dazu führt, dass im Laufe der Generationenfolge die Nachfahren einer Tierart ,andersartig' werden im Vergleich zu ihren Vorfahren, nennen wir Evolution." (Osche 1979: 9f., Hervorhebung im Original) 
Mayr (2005a: 14) formulierte prägnant, dass Evolution heute „der wichtigste Begriff in der gesamten Biologie“ ist. Damit festigte er die viel zitierte Aussage des russisch-amerikanischen Evolutionsbiologen Theodosius Dobzhansky (19001975) „Nothing in Biology makes sense, except in the light of evolution“ (1973: 125). Der Begriff organische Evolution fasst heute zwei Vorgängen, die voneinander unabhängig sind. Dies sind Veränderungen in der Zeit und im Raum (Transformation und Diversifikation). Kutschera (2008: 37) betont, dass ,die biologische Evolution [...] ein zeitlich-räumlicher Entwicklungsprozess [ist], der stattgefunden hat, noch heute andauert und letztendlich die Vielfalt der Lebensformen auf der Erde (Biodiversität) hervorgebracht hat". Ebenso legen Junker und Hoßfeld (2009: 16) dar, dass die moderne Sichtweise der biologischen Evolution Vorgänge in Zeit und Raum einschließt:

„Nach der modernen Auffassung umfasst die biologische Evolution zudem zwei voneinander weitgehend unabhängige Vorgänge: Die Weiterentwicklung von Arten in der Zeit (Transformation) und ihre Aufspaltung im Raum (Speziation). Es handelt sich dabei um Veränderungen in den Eigenschaften von Populationen von Organismen, die die Lebenszeit eines einzelnen Individuums überschreiten.“

Während der Begriff Evolution zunächst Vorgänge bzw. Prozesse verdeutlicht, liefern Evolutionstheorien Erklärungsansätze. In diesem Kontext war die Definition der Deszendenztheorie von August Weismann (1834-1914) richtungsweisend:

„Die Deszendenztheorie ist eine Entwicklungstheorie, sie begnügt sich nicht damit, wie die frühere Wissenschaft, die vorhandenen Lebensformen als gegebene hinzunehmen und zu beschreiben, sondern sie faßt sie als gewordene, und zwar durch einen Entwicklungsprozeß gewordene auf, sucht die Stufen dieser Entwicklung zu erforschen und die treibenden Kräfte zu entdecken, welche ihr zugrunde liegen. Sie ist, kurz gesagt, der Versuch einer wissenschaftlichen Erklärung der Entstehung und Mannigfaltigkeit der Lebewelt.“ (Weismann 1904: 1, Hervorhebung im Original)

Die Abstammungslehre oder auch Deszendenzlehre wird heute verstanden als „die wissenschaftliche Lehre, daß die heutigen, zum Teil hochorganisierten Lebewesen von einfacheren Vorfahren in früheren Erdzeitaltern abstammen und mehr oder weniger nah miteinander verwandt sind" (Freudig \& Sauermost 1999: 28). Für Diamond (2005: 7) stellen die Evolutionstheorien dabei „das tiefgreifendste, machtvollste Gedankengebäude" der letzten 200 Jahre dar. Heute verschaffen sie als „Meilenstein der Biowissenschaften [...] dem biologischen Wissen ein vereinigendes Gerüst" (Wallin 2011: 122) und werden verstanden als die „Theorie von der Entwicklung der Mannigfaltigkeit, der gemeinsamen Abstammung der Lebe- 
wesen [...] und den Ursachen des evolutionären Wandels der belebten Welt" (Freudig \& Sauermost 2000a: 284). Junker und Hoßfeld (2009: 16) sehen ihren grundlegenden Gedanken darin, ,dass die Welt weder unveränderlich noch vor kurzer Zeit entstanden ist. [...] Es wird weiter angenommen, dass die Veränderungen kumulativ sind und zu einer völlig neuen Situation führen“.

Die historisch gewachsene Vielzahl der Theorien zum Ursprung der Arten ordnet Kutschera (2015) in drei Bereiche: die übernatürliche Erschaffung des Lebendigen, die Panspermie-Hypothese von Svante Arrhenius (1859-1927) und die Deszendenztheorien u. a. von Lamarck 1809 (Lamarckismus) sowie von Darwin und Wallace 1858/59 (Darwinismus). Mit Blick auf die Artentstehung schlagen Junker und Hoßfeld (2009) eine Unterteilung in vier Positionen vor, ohne eine klare Abgrenzung zu postulieren: Ursprungstheorien, die Konstanz der Arten, Evolutionstheorien und die Theorie der gemeinsamen Abstammung.

Die letzten drei Phasen in der Entwicklung der Evolutionstheorien werden von den meisten Autorinnen und Autoren als Darwinismus, Neodarwinismus und Synthetische Theorie bezeichnet (Reif et al. 2000). Von der Erklärung durch Selektion ausgehend, spezifizieren Junker und Hoßfeld (2009) die wissenschaftliche Weiterentwicklung des Darwinismus in vier Etappen:

1. klassischer Darwinismus (nach 1859)

2. Neodarwinismus (nach 1883)

3. genetischer und populationsgenetischer Darwinismus (1915 bis 1932)

4. synthetischer Darwinismus (1930 bis 1950)

Für die letzte Phase schlagen sie (ebd.: 174) den Begriff synthetischer Darwinismus vor, „da die neue Evolutionstheorie historisch und inhaltlich eindeutig auf Darwins Theorien zurückgeht“. Die Entwicklung der Synthetischen Theorie bzw. des synthetischen Darwinismus vollzog sich nach Reif et al. (2000) in vier Stufen:

1. „Roots“ (bis und um 1920)

2. „Preparation“ (die zweite Hälfte der 1920er Jahre und die erste Hälfte der 1930er Jahre)

3. „Formation“ (1930 bis 1950)

4. „Reception“ (1950 bis heute)

In der Zeit nach 1950 bis in die Gegenwart entwickelte und etablierte sich die Erweiterte Synthetische Theorie (Expanded Synthesis) bzw. Evolutionsbiologie (vgl. Kutschera 2015). Günter Osche, der die Biogenetische Grundregel von Ernst Haeckel „in seine Version der modernen Evolutionstheorie integriert[e], ist [...] einer der Wegbereiter" (Kutschera 2010a: 260). Kutschera (2007a: 24) skizziert die Evolutionsbiologie mit den Worten: „Ausgehend vom dokumentierten Faktum Evolution befasst sich diese Generaldisziplin der Biowissenschaften mit der Rekonstruktion der Phylogenese der Organismen und deren Antriebskräften." Ergänzend sei hier noch auf das von Kutschera (2009) vorgelegte Synade-Modell der 
Makroevolution hingewiesen. Er betont, dass die Symbiogenese, die natürliche Selektion und die dynamische Erde die entscheidenden Faktoren für Artenwandel und Biodiversifitätszunahme sind.

Für das wissenschaftlich fundierte Gedankengebäude zur Entstehung der Arten, das von Darwin und Wallace entwickelt wurde, führte Wallace den Begriff Darwinismus ein (Freudig \& Sauermost 2000b). Ernst Mayr veröffentlichte 1982 in The Growth of Biological Thought und auch in späteren Werken, dass sich der Darwinismus aus fünf ineinandergreifenden Theorien zusammensetzt (vgl. auch Mayr 2002; 2005b; 2010). Dies sind die Evolution als solche, die Theorie der gemeinsamen Abstammung der Organismen, der Gradualismus, die Theorie der Vervielfältigung der Arten sowie die Theorie der natürlichen Auslese (Tab. 6). Haeckel betonte den zentralen Stellenwert der Selektionstheorie indem er darlegte, dass „die Selektionstheorie von Darwin [...] die kausale Begründung der von Goethe und Lamarck aufgestellten Deszendenztheorie“ ist (Hoßfeld 2010: 98). Der Darwinismus, als Konstruktion der Rezeption verstanden, enthält Theoreme, die es ermöglichten, das biologische Wissen neu zu ordnen und zu interpretieren (Engels 2009). Von verschiedenen Disziplinen mit unterschiedlichen Forschungsfragen untersucht, bleibt die Unterscheidung der Evolutionstheorien von Charles Darwin auch für die moderne Evolutionsbiologie von Bedeutung (Sommer 2011).

Tabelle 6: Weiterentwicklung der Postulate des klassischen Darwinismus zum synthetischen Darwinismus (vgl. u. a. Junker \& Hoßfeld 2009; Mayr 2010)

\begin{tabular}{|c|c|c|c|}
\hline $\begin{array}{l}\text { Klassischer } \\
\text { Darwinismus }\end{array}$ & Neodarwinismus & $\begin{array}{l}\text { Populationsgeneti- } \\
\text { scher Darwinismus }\end{array}$ & $\begin{array}{l}\text { Synthetischer } \\
\text { Darwinismus }\end{array}$ \\
\hline $\begin{array}{l}\text { Evolution } \\
\text { als solche }\end{array}$ & \multirow{5}{*}{$\begin{array}{l}\text { Überwindung des } \\
\text { Lamarck'schen Prin- } \\
\text { zips }\end{array}$} & \multirow{5}{*}{$\begin{array}{l}\text { Überwindung des } \\
\text { Mutationismus }\end{array}$} & \multirow{2}{*}{$\begin{array}{l}\text { Speziation durch } \\
\text { geographische } \\
\text { Isolation }\end{array}$} \\
\hline $\begin{array}{l}\text { Gemeinsame } \\
\text { Abstammung }\end{array}$ & & & \\
\hline $\begin{array}{c}\text { Vervielfältigung } \\
\text { der Arten }\end{array}$ & & & \multirow{3}{*}{$\begin{array}{c}\text { Genetische Variation } \\
\text { durch Mutation und } \\
\text { Rekombination }\end{array}$} \\
\hline Gradualismus & & & \\
\hline $\begin{array}{l}\text { Natürliche } \\
\text { Selektion }\end{array}$ & & & \\
\hline
\end{tabular}

Der Begriff Neodarwinismus, durch George John Romanes (1848-1894) geprägt, bezeichnet die Weiterentwicklung des Darwinismus durch August Weismann. Dieser war einer der bedeutendsten Evolutionsbiologen des 20. Jahrhunderts in Deutschland. Weismann wandte sich in seinen Überlegungen den Faktoren der Evolution zu, betonte die natürliche Selektion als Mechanismus der Evolution und nahm Erkenntnisse der Zytologie und der Genetik in seine Arbeiten auf. Während Darwin und Haeckel die Vererbung erworbener Eigenschaften in ihre Betrachtungen integrierten, kritisierte Weismann das Lamarck'sche Prinzip sowie die Pangenesistheorie und trug somit entschieden zu deren Überwindung bei (Reifet al. 2000). Unterstützt wurde er in dieser Sicht durch Wallace. Anstatt in der 
Vererbung erworbener Eigenschaften erkannte Weismann in der Rekombination die Ursache erblicher Variabilität der Organismen (Weismann 1904; Zirnstein 2001; Junker \& Hoßfeld 2009; Kutschera 2015). Seine Theorie der Kontinuität des Keimplasmas, in der er eine Trennung von Keim- und Somazellen postulierte, ließ den Schluss zu, dass „Veränderungen des Organismus keinen Einfluss auf die Keimzellen und ihr Kernplasma haben können“ (Junker \& Hoßfeld 2009: 157). Kutschera (2014: 369) resümiert heute, dass „diese Weismann'sche These vom ,unsterblichen Leben' [...] sich bewahrheitet [hat] und zu den bleibenden Erkenntnissen der klassischen Biologie" zählt.

Anfang des 20. Jahrhunderts entstand mit der Wiederentdeckung der Arbeiten Gregor Johann Mendels (1822-1884) die Genetik als Wissenschaft, deren Erkenntnisse letztlich die Selektionstheorie stützten. Anfangs dominierte jedoch die Mutationstheorie von Hugo de Vries (1884-1935), in der die Selektionstheorie nur eine untergeordnete Rolle spielte (Sohn 2001; Junker \& Hoßfeld 2009). Als einer der ersten Biologen verband Julian Huxley (1887-1975) die Mendel'sche Genetik mit Darwins Evolutionstheorie (Schulz 2001). Die moderne Synthese wurde u. a. in den Werken von Dobzhansky Genetics and the Origin of Species (1937), Huxley Evolution: The Modern Synthesis (1942) und Mayr Systematics and the Origin of Species (1942) begründet. Weitere Impulse lieferten George Gaylord Simpson (19021984), George Ledyard Stebbins (1906-2000) und Bernhard Rensch (1900-1990). Simpson prägte als erstes den Begriff ,Synthetic Theory' (Kutschera 2008; Junker $\&$ Hoßfeld 2009). Biologen wie Mayr und Simpson, die über die Synthese schrieben, verbreiteten über die Synthetische Theorie drei Vorstellungen (Reif et al. 2000):

1. Die Synthetische Theorie stellt einen Paradigmenwechsel in der Theorie der Evolution dar.

2. Sie ist ein einmaliger Prozess in der Biologiegeschichte.

3. Sie wird durch Verbindung verschiedener Methoden (theoretischmathematischer und experimenteller sowie Feldforschung) ermöglicht.

Reif et al. (ebd.: 43f.) betonen: „In contrast to neo-Darwinism the Synthetic Theory includes modern genetics, population genetics, systematics and theories of speciation and macroevolution." Der Beitrag der Synthetischen Theorie, die vordergründig „eine Theorie der Evolutionsmechanismen“ ist (Junker \& Hoßfeld 2009: 175), bestand in der empirischen Überprüfung der Wirkung der Evolutionsfaktoren Mutation, Rekombination, Selektion, Isolation und Gendrift (Reif et al. 2000). Mayr (2005a) verwies darauf, dass die Synthese der Evolutionsforschung ab den 1930er Jahren zu einer allgemeinen Einigkeit führte und die Entwicklungen auf dem Gebiet der Molekularbiologie die Darwin'sche Lehre weiter festigten. 
Belege aus paläontologischer und neontologischer Forschung

Belege für den Ablauf der biologischen Evolution steuern viele Wissenschaftsdisziplinen bei und bilden das Fundament der modernen Evolutionstheorie. Nach Simpson (1984: 157) muss ,eine akzeptable allgemeine Evolutionstheorie [...] Befunde und Schlußfolgerungen ganz unterschiedlicher Forschungsgebiete berücksichtigen, die sich mit Organismen, lebendig oder tot, beschäftigen“. Mayr (2010: 236) argumentiert, dass ,,in den fast anderthalb Jahrhunderten seit Darwin [...] diese Beweise so überwältigend [wurden], daß die Biologen von der Evolution nicht mehr als Theorie sprechen, sondern sie als Tatsache betrachten". Darwin selbst ging nach seiner Reise mit dem Forschungsschiff Beagle „streng nach den Prinzipien Bacons vor und trug ohne jede Theorie möglichst umfassend Tatsachen zusammen“ (Darwin 2008: 129). Ein Querschnitt klassischer und neuer Indizien für die biologische Evolution aus Bereichen der Neontologie und Paläontologie soll, mit Blick auf die Schwerpunkte der Lehrbuchanalyse im Folgenden dargelegt werden.

Wenngleich das Interesse an Fossilien schon dem Neandertaler nachgesagt wird (Ivanov et al. o. J.), wurde die Paläontologie als Wissenschaft erst im 19. Jahrhundert durch Georges Cuvier begründet (Simpson 1984). Sie untersucht und interpretiert fossile Zeugnisse der Erdgeschichte (Kutschera 2008). Junker (2001: 381) betont, dass „die Paläontologie [...] für die Evolutionstheorie von besonderer Bedeutung [ist], da ihre empirischen Befunde, die Fossilien, direkte Beweise für die Geschichte der Organismen darstellen“. Die Fossilien als „Zeugen vergangenen Lebens" (Gensel et al. 1999: 5) ermöglichen einen Einblick in vergangene Erdzeitalter. Der Begriff Fossil bedeutet Versteinerung und wurde durch Georg Bauer, genannt Agricola (1494-1555) geprägt (Kutschera 2009b). Die Paläontologie befasst sich mit den Mechanismen der Fossilisation (allgemeine Paläontologie) und der Beschreibung sowie Klassifizierung von Fossilien (spezielle Paläontologie). Die spezielle Paläontologie, die auch als Paläobiologie bezeichnet wird, gliedert sich in Paläobotanik, Paläozoologie und Mikropaläobiologie (Kutschera 2008). Othenio Abel (1875-1946) lieferte in seinem Werk Grundzüge der Paläobiologie der Wirbeltiere (1911) eine Definition des Begriffs Paläobiologie und gilt nach Kutschera (2009b) als ihr Begründer.

Der Prozess der Fossilisation bzw. die Entstehung von Fossilien ist zumeist an Sedimentgestein gebunden und entscheidet über Qualität und Art der Erhaltung. Häufig vorkommende Erhaltungsformen sind Steinkern, Versteinerung, Inkohlung, Abdruck und Einschluss. Seltener treten Mumifizierungen auf. Rode (2007) betont, dass von den Organismen zumeist Hartteile, seltener Weichteile als Fossilien erhalten bleiben. Das nur ein geringer Teil der Organismen als Fossilien erhalten bleibt, erklärt sich durch die Tätigkeit aasfressender Organismen sowie die Prozesse der Fäulnis, Auflösung und mechanischen Beanspruchung (ebd.). Auf die Lückenhaftigkeit fossiler Belege wies bereits Darwin (2004: 231) hin, als er 
formulierte: „Die Erdrinde ist ein großes Museum; aber ihre naturgeschichtliche Sammlung ist unvollständig und sagt über bedeutende Zeitabschnitte nichts aus.“

Durch Leitfossilien, deren Vorhandensein in bestimmten geologischen Schichten typisch und auf diese begrenzt ist, lassen sich Rückschlüsse auf das Alter von Gesteinsschichten ziehen (Rohde o. J.). Neben dieser relativen ist zudem eine absolute Altersbestimmung durch radioaktive Elemente (radiometrische Datierung) möglich (Storch et al. 2007; Kutschera 2009, 2015). Die so gewonnen Daten erlauben eine zeitliche Gliederung der Erdgeschichte in einer stratigrafischen Tabelle. Als Äonen definiert, bilden Archaikum, Proterozoikum und Phanerozoikum die größten Zeiteinheiten. Das Zeitalter der erkennbaren Lebensspuren (Phanerozoikum) gliedert die moderne Geologie in die Ären Paläozoikum, Mesozoikum und Känozoikum (Kutschera 2015; Tab. 7).

Tabelle 7: Geologische Zeitskala bzw. stratigrafische Tabelle (verändert nach Kutschera 2015)

\begin{tabular}{|l|l|r|}
\hline Äon/Ära & Periode & $\begin{array}{l}\text { Beginn vor Mio. } \\
\text { Jahren }\end{array}$ \\
\hline Känozoikum & Quartär & 2,6 \\
& Tertiär & 66,0 \\
\hline Mesozoikum & Kreide & 145,0 \\
& Jura & 201,0 \\
& Trias & 252,0 \\
\hline Paläozoikum & Perm & 299,0 \\
& Karbon & 359,0 \\
& Devon & 419,0 \\
& Silur & 444,0 \\
& Ordovizium & 485,0 \\
& Kambrium & 541,0 \\
\hline Proterozoikum & & 2500,0 \\
\hline Archaikum & & 4600,0 \\
\hline
\end{tabular}

Stetig anwachsende Fossilfunde liefern auch heute noch neue Erkenntnisse, wie das Vorhandensein differenzierter Organismen im Präkambrium sowie die Einordnung der Gattung Pliohippus (statt Hipparion) als Vorfahren rezenter Pferde (Storch et al. 2001). Die Vielzahl der Fossilien ermöglicht das Erstellen von Entwicklungsreihen. So ist die Evolution der Pferde heute gut belegt und ein „klassische[s] Beispiel für Makroevolution“ (Kutschera 2008: 121; vgl. auch Mayr 2005a). Auch die Abstammung der Walartigen konnte seit 1990 rekonstruiert werden (Kutschera 2015). Ein besonderer Stellenwert kommt den fossilen Zwischenformen (conneting links) zu, die den graduellen Wandel im Bauplan der Organismen (Postulat des Synthetischen Darwinismus) belegen (ebd.). Eine klassische und bekannte Übergangsform zwischen Vogel und Reptil ist der Urvogel Archaeopteryx. Ein Fund aus neuerer Zeit stellt dagegen die Zwischenform Tiktaalik dar, die den 
Übergang von den Fischen zu den Amphibien vervollständigt und dokumentiert (Shubin 2008).

Anders als die Paläontologie untersucht neontologische Forschung rezente Organismen mit dem Ziel, „Rückschlüsse auf historische Vorgänge“ zu ziehen (Kutschera 2015: 43). Dazu gehören u. a. die Tier- und Pflanzenzucht, die Biogeographie, die vergleichende Anatomie sowie die Entwicklungsbiologie. Mayr verweist darauf, dass:

„die Manifestationen der gemeinsamen Abstammung, wie sie die vergleichende Anatomie, vergleichende Embryologie, Systematik und Biogeografie zum Vorschein brachten, [...] so überzeugend [waren], daß schon zehn Jahre nach Veröffentlichung von Die Entstehung der Arten die meisten Biologen von der Evolution durch gemeinsame Abstammung überzeugt waren“ (Mayr 2010: 239, Hervorhebung im Original).

Darwin war selbst ein leidenschaftlicher Taubenzüchter. Der Tierzucht entnahm er die Tatsache, dass Individuen voneinander abweichen und einen Großteil ihrer individuellen Verschiedenartigkeit vererben (Darwin 2004). Er „merkte bald, daß die Selektion [Zuchtwahl] der Schlüssel zum Erfolg des Menschen beim Züchten erfolgreicher Tier- und Pflanzenrassen ist" (Darwin 2008: 129). Darwin stützte sich auch auf Erkenntnisse aus der Biogeographie. Gleich zwei Kapitel widmete er in Die Entstehung der Arten der geografischen Verbreitung von Tieren und Pflanzen, die durch die Evolutionstheorie eine Erklärung fand. Die Erlebnisse während seiner Reise (Galapagos, Feuerland u. a.) mit dem Forschungsschiff Beagle ließen in ihm bereits vorher den Gedanken an eine Evolution reifen, wie Andeutungen in seinem Reisebericht nahelegen (Darwin 2008; Voss 2008).

Eine zentrale Rolle für die Evolutionstheorie nehmen die aus Homologien gewonnen Erkenntnisse der vergleichenden Anatomie ein. Unter dem Begriff Homologie, der auf Richard Owen (1804-1892) zurückgeht (Rupke 2001), wird heute die „Ähnlichkeit von Merkmalen zwischen Organismen, die auf gemeinsame Vorfahren zurückführbar ist" (Kutschera 2015: 356), verstanden. Im Gegensatz dazu beruhen Analogien auf konvergenter oder paralleler Evolution im Sinne einer Funktions- und Anpassungsähnlichkeit. Zur Ermittlung von Homologien wird das Kriterium der Lage, der speziellen Qualität der Strukturen sowie der Verknüpfung durch Zwischenformen herangezogen (Storch et. al. 2007; Kutschera 2015).

Auch die Entwicklungsbiologie liefert Belege für den Ablauf der Evolution anhand von Untersuchungen an rudimentären Strukturen und Atavismen. Rudimente, die nach Darwin (2004: 627) „den Stempel der Nutzlosigkeit tragen“, stellen rückgebildete Organe der Vorfahren dar, sind jedoch, wie Storch et al. (2007) darlegen, nicht zwingend funktionslos (z. B. muskuläre Ansatzstellen für Penis und After an rudimentären Beckenknochen der Wale). Atavismen hingegen (beispielsweise bei Pferde mit einer zweiten Zehe) treten nur bei einzelnen Individuen 
auf und werden als „Rückschläge in Ahnenstadien“ interpretiert (Kutschera 2015: 51).

Das von Ernst Haeckel aufgestellte Biogenetische Grundgesetz, das heute als biogenetische Regel bezeichnet wird, ist trotz aller Kritik in seiner Grundaussage gültig (Hoßfeld et al. 2011; Kutschera 2015). Bestimmte Merkmale werden in der Embryonalentwicklung rekapituliert und weisen auf Abstammung hin (Mayr 2005a). Haeckel schuf u. a. die Begriffe Ontogenie (Individualentwicklung) sowie Phylogenie (Stammesentwicklung) und betonte ihre Verbindung wie folgt: „Die Ontogenesis ist die kurze und schnelle Rekapitulation der Phylogenesis“ (Haeckel, zit. n. Hoßfeld 2010: 112). Fortschritte in der Entwicklungsbiologie brachten zudem eine neue Fachdisziplin, die Evolutionäre Entwicklungsbiologie (Evo-Devo) hervor. Kernelemente sind hier Vergleiche von Entwicklungsgenen verschiedener Spezies, die die Formbildung steuern und aus denen entsprechende Schlussfolgerungen gezogen werden. Eine grundlegende Erkenntnis ist, dass alle Tiere (Animalia) mit gemeinsamen Entwicklungsgenen ausgestattet sind (Carroll 2008a, 2008b; Hoßfeld et al. 2011).

\section{Evolutionsbiologie und Gesellschaft}

Das folgende Kapitel zeigt den Stellenwert evolutionsbiologischer Erkenntnisse für sozialistisch-kommunistische Ideologien sowie die Darstellung der Evolutionsbiologie in der Gesellschaft des 21. Jahrhunderts auf. In Kurzform wird die Weltanschauung auf dem Staatsgebiet der DDR und ihre Rückwirkung auf das Handlungsfeld Schule charakterisiert. Ebenso wird die Beziehung ausgewählter Protagonisten sozialistisch-kommunistischer Ideologien zu den Erkenntnissen der Darwin'schen Evolutionstheorie aufgezeigt. Weiterhin werden Trends und Kontroversen, wie die Auseinandersetzung mit dem Kreationismus, sowie das DarwinJahr 2009 und die Bemühungen der science community um Aufklärung beleuchtet. Hierbei wird neben wissenschaftlicher Literatur auch Literatur aus dem populärwissenschaftlichen Bereich vorgestellt, da die Übergänge meist fließend und für Lehrkräfte gleichermaßen von Interesse sind. Ohne einen Anspruch auf Vollständigkeit zu erheben, ist es Ziel, einen Überblick und somit Zugänge zum Thema Evolutionsbiologie und Gesellschaft anzubieten.

Evolutionsbiologie im Kontext sozialistisch-kommunistischer Ideologien

Ideologie wird gemeinhin verstanden als ein System von Weltanschauungen sowie (politischen) Grundeinstellungen und Wertungen (Wermke et al. 2006). Zur Zeit der DDR wurde betont, dass diese Ideen insbesondere durch Produktionsverhältnisse bedingt bestimmte Klasseninteressen zum Ausdruck bringen (Böhme et al. 1973). Dem politisch-gesellschaftlichen Leben in der DDR, als sozialistischem Staat, lagen die Prinzipien des Marxismus-Leninismus zu Grunde, die als wissen- 
schaftliche Ideologie verstanden wurden (Engels 1966a; vgl. Neuner 1996). Der Artikel 1 der Verfassung der DDR (Anonymus 1976: 9) verdeutlichte diese politische Grundhaltung: „Die Deutsche Demokratische Republik ist ein sozialistischer Staat der Arbeiter und Bauern. Sie ist die politische Organisation der Werktätigen in Stadt und Land unter Führung der Arbeiterklasse und ihrer marxistischleninistischen Partei.“

Detlev (1993: 74) resümiert, dass „die Schulbiologie [...] neben naturwissenschaftlichen Inhalten auch Bio-Politische Elemente [umfasst]. Diese finden, sobald im Rahmen der Säkularisierung der Mensch Objekt der Schulbiologie ist, Eingang in den Unterricht". Im Handlungsfeld Schule interessierte daher neben Fachinhalten auch die „ideologisch-erzieherische Wirkung des Fachunterrichts“ (Neuner 1996: 209). Ein Ziel des Unterrichtes war es, ,zusammen mit der Stoffvermittlung vor allem die Herausbildung politisch-ideologischer Grundüberzeugungen zu unterstützen" (Graef et al. 1985: 9). An die Biologielehrerinnen und lehrer stellten sich folgende Anforderungen:

„Der Lehrer muß seine Begeisterung für die Sache des Sozialismus und für sein Fach seinen Schülern weitergeben. Die sozialistische Erziehung ist ein Grundanliegen seiner Arbeit und erfolgt dementsprechend in Einheit mit dem biologischen Stoff.“ (Graef et al. 1985: 11)

Dietrich $^{12}$ (1972: 16) argumentiert, dass „Aussagen über einzelne Ziel- und Inhaltsaspekte der Allgemeinbildung [...] nur auf dem Boden des MarxismusLeninismus stehen [können], wenn sie abgeleitet werden vom Beziehungsgefüge der gesellschaftlichen Entwicklung“. Die weltanschauliche Bedeutung der Evolutionsbiologie beeinflusste den Biologieunterricht auf dem Gebiet der DDR (Lengert 1959), da insbesondere an evolutionsbiologischen Themen der „Kampf zwischen philosophischem Materialismus und philosophischem Idealismus“ (Löther \& Schellhorn 1964: 7) ausgetragen wurde.

Die Verbindung evolutionsbiologischer Inhalte mit politischen Überzeugungen erklärt sich aus ihrem historischen Kontext. Karl Marx (1818-1883) selbst verstand den Sozialismus als wissenschaftlichen Begriff (Russell 2009). Russell (ebd.: 732) betont, dass Marx in seinen Ansichten durch die Naturwissenschaften inspiriert wurde. Insbesondere Darwins Erkenntnisse beeinflussten und bestätigten sein materialistisches Weltbild. Das Marx den Wunsch hegte, die Erstausgabe von Das Kapital Darwin zu widmen, spiegelt seine Bewunderung für den englischen Forscher wider (Fischer et al. 2008). Harald Wessel äußerte im Jahr 1977 in der Tageszeitung Neues Deutschland dagegen die Auffassung, dass vielmehr der englische Biologe und Sozialist Edward Aveling (1849-1898) eine Widmung wünschte. Als Abteilungsleiter für Propaganda und Wissenschaft in der Redaktion

12 Gerhard Dietrich (1927-1986) war zu dieser Zeit Direktor der Sektion Pädagogik/Psychologie der Karl-Marx-Universität in Leipzig und ordentliches Mitglied der Akademie der pädagogischen Wissenschaften der DDR. 
der Tageszeitung (Baumgartner \& Hebig 1997) verwies er im Artikel auf einen Brief Avelings an Darwin vom 12. Oktober 1880.

„Erstens hatte Darwin in einem Handschreiben vom 1. Oktober 1873 an Marx Worte hoher Anerkennung für den 1867 erschienenen ersten Band des ,Kapitals‘ gefunden. Zweitens gab es im Herbst 1880 praktisch keine Ausgabe des ,Kapitals‘, die Marx hätte Darwin öffentlich widmen können.“ (Wessel 1977: 15)

Auch Friedrich Engels (1820-1895), der die marxistische Weltanschauung mitbegründete (Böhme et al. 1973), würdigte in seinen Schriften Darwins Leistungen.

„Es war bezeichnend, daß fast gleichzeitig mit Kants Angriff auf die Ewigkeit des Sonnensystems C. F. Wolff 1759 den ersten Angriff auf die Beständigkeit der Arten erließ und die Abstammungslehre proklamierte. Aber was bei ihm nur noch geniale Antizipation, das nahm bei Oken, Lamarck, Baer feste Gestalt an und wurde genau 100 Jahre später, 1859, von Darwin sieghaft durchgeführt." (Engels 1966b: 59)

Darüber hinaus integrierte Engels in seinen Werken den Entwicklungsgedanken Darwins. Er argumentierte, dass sich der Mensch aus dem Tierreich aufgrund der Fähigkeit entwickelte, seine Umwelt bewusst gestalten und in seinem Sinn verändern zu können, um sich über die Willkür der Natur zu erheben (Engels 1966b). Vor allem in der Produktion sah er dies bestätigt. Gleichzeitig erkannte Engels aber auch eine Diskrepanz zwischen den Möglichkeiten und den tatsächlichen Erfolgen und band in seine Argumentation zur gesellschaftlichen Situation Englands Darwins Kampf ums Dasein ein.

„Darwin wusste nicht, welch bittre Satire er auf die Menschen und besonders auf seine Landsleute schrieb, als er nachwies, daß die freie Konkurrenz, der Kampf ums Dasein, den die Ökonomen als höchste geschichtliche Errungenschaft feiern, der Normalzustand des Tierreichs ist." (Engels 1966b: 63, Hervorhebung im Original)

In seiner Schrift Anteil der Arbeit an der Menschwerdung des Affen bezog sich Engels (1966c: 68) ebenfalls auf Darwin: „Darwin hat uns eine annähernde Beschreibung dieser unsrer Vorfahren gegeben. Sie waren über und über behaart, hatten Bärte und spitze Ohren und lebten in Rudeln auf Bäumen.“ Das Engels in seiner Rede zum Begräbnis von Marx dessen Leistungen mit denen Darwins verglich, hebt den Einfluss, den Darwin auf Marx und Engels ausübte, besonders hervor: „Wie Darwin das Gesetz der Entwicklung der organischen Natur, so entdeckt Marx das Entwicklungsgesetz der menschlichen Geschichte“ (Engels 1966d: 152).

In Russland nahm der Darwinismus bereits vor der Oktoberrevolution von 1917 eine zentrale Stellung ein und übte einen bedeutenden Einfluss auf die Gesellschaft und die Naturwissenschaften aus. Nach der Revolution galt er als 
„Grundstein der marxistischen Philosophie“ (Kolchinsky 2001: 157). Nikolai Iwanowitsch Bucharin (1888-1938), ein russischer Politiker und Philosoph, schrieb, dass „,nur die marxistische Synthese [...] dem großen Werk Darwins eine fortschrittliche funktionale Rolle“ verleiht (Bucharin 2001: 153, Hervorhebung im Original). Der Darwinismus wurde letztlich als Hilfe für den Aufbau des Sozialismus wahrgenommen. Bucharin betonte:

„Wenn der in die gemeinsame Kette der marxistischen Weltanschauung eingegliederte Darwinismus früher die Rolle eines Rammbocks gegen die Theologie gespielt hat, erhält er heute - hauptsächlich durch die Genetik - für die Arbeiterklasse eine praktische Bedeutung auch unter dem Aspekt der Produktion.“ (ebd.: 151, Hervorhebung im Original)

Trofim Denissowitsch Lyssenko (1898-1976) nutzte schließlich die „Anschlussfähigkeit des Darwinismus an die Staatsideologie“ (Kolchinsky 2001: 164) und entwickelte den sogenannten Schöpferischen Darwinismus, der auch als Lyssenkoismus oder Mitschurin-Biologie bekannt wurde. Er legte die Vererbung erworbener Eigenschaften sowie die gerichtete Veränderlichkeit erworbener Eigenschaften durch Umweltbedingungen seinem Gedankenkonstrukt zu Grunde. Obwohl sein Ideengebäude im Gegensatz zu Befunden der genetischen Forschung stand, entwickelte sich der Lyssenkoismus ab den 1930er Jahren durch die Unterstützung Josef Wissarionowitsch Stalins (1878-1953) zu „eine[r] unentbehrliche[n] Komponente des stalinschen Marxismus" (Kolchinsky 2001: 164). In diesem Umfeld propagierte Lyssenko verschiedene Versprechungen, wie beispielsweise die Veränderung von Sommer- und Winterweizen. Anfang der 1930er Jahre wurde dadurch das Verfahren der Jarowisation (Kältebehandlung angekeimter Samen) bekannt. Wenngleich Anhänger des schöpferischen Darwinismus bzw. des Lyssenkoismus über viele Jahre zur Elite des Landes gehörten, konnte dieser bis zum Ende der 1960er Jahre überwunden werden.

Auch in der DDR verbreiteten sich bis Mitte der 1960 die Theorien Lyssenkos. Dies geschah unter Mitwirkung des Jenaer Zoologen Georg Schneider (1909-1970), der im Jahr 1947 in Jena den Urania Verlag wieder gründete (UraniaVerlag 1974; Links 2010). Zur Propagierung des schöpferischen Darwinismus nutzte er seine Stellung als Direktor des Ernst-Haeckel-Hauses und als Professor für Theoretische Biologie in Jena (Hoßfeld \& Breidbach 2007). Der Lyssenkoismus, der sich aufgrund seiner politisch gewollten Anerkennung ausschließlich auf den Gebieten sozialistischer Staaten ausbreitete, fand letztlich Eingang auch in die Schul- und Hochschullehrbücher sowie Lehrpläne der DDR (Hoßfeld \& Brömer 2001). Sein Einfluss auf die Hochschulausbildung in Jena war jedoch nicht nachhaltig (Knorre et al. 2007; Hoßfeld 2012b). Bis in die erste Hälfte der 1960er Jahre verhinderte der Einfluss des Lyssenkoismus dennoch die Integration der klassischen Genetik in die Lehr- und Lernmaterialien der allgemeinbildenden Schule der DDR. Löther (2010: 84) berichtet in seinen Erinnerungen, 
dass „der Lyssenkoismus [...] in den Lehrplänen und Lehrbüchern [...] breit berücksichtigt [wurde], während die Behandlung der Mendelschen Regeln im Biologieunterricht offiziell als Kündigungsgrund galt.“ Dennoch zeigt Laitko (2010: 129), dass ein „schlichtes Totalitarismusmodell“ nicht geeignet ist, „das Verhältnis von Politik und Wissenschaft in der DDR angemessen zu reflektieren.“

Unabhängig vom Aufkommen und dem Niedergang der lamarckistisch orientierten Lehren Lyssenkos wurden Darwins Evolutionstheorien von Vertretern einer materialistischen Weltanschauung, auf deren Ansichten das gesellschaftlichpolitische Leben der DDR basierte, geschätzt. Ferner erfuhr auch das Wirken von Ernst Haeckel beispielsweise durch Wladimir Ilitsch Lenin (1870-1924), Walter Ulbricht (1893-1973) sowie Mao Tse-Tung (1893-1976) Zuspruch und Anerkennung (Fischer et al. 2008; Hoßfeld \& Breidbach 2008). Die Tageszeitung Neues Deutschland, das Organ des Zentralkomitees der Sozialistischen Einheitspartei Deutschlands (ZK der SED), veröffentlichte Artikel wie 100 Jahre Darwinismus (Kirschke 1959) und Übrigens ist der Darwin gan₹ famos (Wessel 1974).

Trends und Kontroversen im Darwin Jahr 2009

Anlässlich des Darwin-Jubiläums vor über 50 Jahren betonte der Nobelpreisträger Hermann Joseph Muller (1890-1967): „Hundert Jahre ohne Darwin sind genug! Sorgen wir dafür, daß das mit dem Jahre 1959 begonnene Jahrhundert ein Jahrhundert mit Darwin wird“" (zit. n. Heberer 2004: 686). Erneut ein halbes Jahrhundert später jährte sich Charles Darwins Geburtstag bereits zum 200. und das Erscheinen seines Buches On the Origin of Species zum 150. Mal. Beide Jubiläen fanden, im Gegensatz zu anderen Jubiläen, wie dem 150. Todestag von Alexander von Humboldt (1769-1859), dem 175. Geburtstag von Ernst Haeckel, dem Erscheinen von Lamarcks Philosophie zoologique vor 200 Jahren sowie dem 275. Geburtstag von Caspar Friedrich Wolff (1734-1794), im Darwin-Jahr vielfältige Beachtung. Engels (2009) resümiert, dass Darwins Popularität und Präsenz in Literatur und Medien im Vergleich zu anderen großen Denkern, wie Kopernikus, Galilei und Newton bereits vor 2009 ungleich größer war.

Im ersten Jubiläumsjahr 1909 lag der Fokus neben Darwin auch auf dessen Vorläufer Lamarck. Ernst Haeckel (1909: 6, Hervorhebung im Original) betonte in einer Festrede zur Hundertjährigen Geburtstagsfeier von Charles Darwin im Volkshaus zu Jena: „Kurz, in zwei Worte zusammengefasst, ist das Hauptverdienst der Lamarck-Darwinschen Theorie die endgültige Lösung der großen ,Schöpfungsfrage‘ [...]."Während jedoch eine Festkultur eher die Ausnahme bildete, änderte sich dies im zweiten Jubiläumsjahr 1959 aufgrund größerer Veranstaltungen sowie der Veröffentlichung von Sammlungs- und Tagungsbänden auf internationalem Niveau (Hoßfeld 2009). Das dritte Jubiläumsjahr 2009 war neben wissenschaftlichen Veranstaltungen insbesondere durch populärwissenschaftliche Akzente charakterisiert. Mit diesem Jahr wurden zu und über Darwin, ähnlich einem Popstar, Merchandising Artikel, wie CDs, Plakate, Fanartikel, Kleidungs- 
stücke und anderes mehr zum Kauf angeboten. Wenn auch heute nicht mehr von der Lamarck-Darwin'schen Theorie, sondern von der Darwin-Wallace'schen Theorie gesprochen wird (Hoßfeld 2009), so stand die Person Wallace weit weniger im Fokus der Feierlichkeiten, Veranstaltungen und Veröffentlichungen als Darwin. Auch die Tagespresse widmete sich vermehrt Charles Darwin und der Evolutionsbiologie. Zeitschriften wie das Wissenschaftsmagazin der Max-PlanckGesellschaft zur Förderung der Wissenschaften e. V. (MPG) sowie das Organ des Deutschen Vereins zur Förderung des mathematischen und naturwissenschaftlichen Unterrichts (MNU) brachten Themenhefte bzw. Sonderausgaben heraus. Ein weiteres Charakteristikum war die Eröffnung zahlreicher themenbezogener Webseiten, wie die Virtuelle Fachbibliothek der Universitätsbibliothek Johann Christian Senckenberg in Frankfurt am Main, die seitdem ausführliche Informationen über Darwin anbieten. Eine Übersicht relevanter Webseiten befindet sich auch auf der Internetpräsenz des Arbeitskreises (AK) Evolutionsbiologie. Ferner wurden Sonder- und Dauerausstellungen nicht nur in Museen eingerichtet, wie Evolution schafft Vielfalt im Expo-Park Hannover (Kattmann et al.: 2009), der Evolutionssaal im Phyletischen Museum in Jena sowie die Gastausstellung Wege zum Menschen: Hominiden Evolution im Museum für Naturkunde in Berlin. Darüber hinaus, teilweise aus dem Englischen übersetzt, erschien allein auf dem deutschen Markt eine Vielzahl an (populär)wissenschaftlicher Lektüre, ,so dass man seit vielen Jahren von der ,Darwin-Buchindustrie“ spricht“" (Kutschera 2009b: 18). Mit Blick auf Darwin wurden nach Hoßfeld (2009a; 2009b) vorwiegend sieben Themenbereiche publiziert bzw. diskutiert: Biografik, Beagle-Reise, Kinderbuchliteratur, Philosophie (Geisteswissenschaften), (Evolutions)biologie, allgemeine (europäische) Biologie und Wiederveröffentlichungen (z. T. als kommentierte Reprints).

Der Trend, wissenschaftliche Erkenntnisse einer breiteren Öffentlichkeit zugänglich zu machen, war und ist im Themenfeld Evolutionsbiologie deutlich spürbar. Der Verband Biologie, Biowissenschaften und Biomedizin in Deutschland (VBIO), der im Jahr 2007 aus der Fusion des vdbiol und dem Verbund biowissenschaftlicher und biomedirinischer Gesellschaften (vbbm) hervorging, unterstützt entsprechende Bemühungen. Diese werden als notwendig erachtet, da auch noch im 21. Jahrhundert eine Diskrepanz zwischen wissenschaftlichen Erkenntnissen und öffentlich-gesellschaftlichen Denkmustern besteht. Der Geschäftsführer der GiordanoBruno-Stiftung (gbs) Schmidt-Salomon (2006: 7) spricht in diesem Kontext von einer „Zeit der Ungleichzeitigkeit“, in der hoch entwickelte Technologien alten Weltbildern gegenüberstehen. Alternativ vertritt die Stiftung eine naturalistische Weltsicht, die von einer „Leitkultur von Humanismus und Aufklärung" getragen wird (ebd.: 8, Hervorhebung im Original). Auch der Arbeitskreis Evolutionsbiologie, der mit Unterstützung von Ernst Mayr am 27. Oktober 2002 in Potsdam gegründet wurde und bis 2017 ein Kooperationspartner des VBIO war, vertritt eine natura- 
listische Weltsicht (Kutschera o. J.). ${ }^{13}$ Primäres Ziel war es vorerst, wie vom damaligen vdbiol-Präsident Hans-Jörg Jacobsen formuliert, „die Evolutionsbiologie öffentlichkeitswirksam nach außen darzustellen und ideologisch begründete Gegenströmungen, d. h. den biblischen Kreationismus einzudämmen" (Kutschera \& Hoßfeld 2012: 20).

Richard Dawkins, streitbarer Evolutionsbiologe, Religionskritiker und bis 2008 Inhaber des Charles-Simonyi-Lehrstuhls Public Understanding of Science an der Universität Oxford, publizierte in diesem Sinn eine Reihe (populär)wissenschaftlicher Bücher. Er verfolgte u. a. die Ziele, „die wichtigsten Verständnisschwierigkeiten zu benennen und zu beseitigen“ (2010a: 7), die Geschichte des Lebendigen nachzuzeichnen sowie Belege für die Evolution als Tatsache zu liefern (Dawkins 2009; Dawkins 2010a). Seine Denkweise lässt eine klare Linie und Positionierung erkennen und seine Werke polarisieren nicht nur zwischen Wissenschaft und Religion, sondern auch innerhalb der Biowissenschaften. Mit den Werken Das Egoistische Gen (2007) und Der erweiterte Phänotyp (2010b), so urteilt Dawkins (2010a: 7) selbst, bietet er „einen ungewohnten Blick auf die altbekannte Theorie der natürlichen Selektion“. In diesem Zusammenhang warnt Rose (2000: 8) mit Blick auf die Soziobiologie und die Theorie der egoistischen Gene vor einem biologischen Determinismus, den er als „Ultra-Darwinismus“ bezeichnet. Neffe (2008: 478) argumentiert gar, dass „Soziobiologen und ihre jüngsten Ableger, die Evolutionspsychologen, [...] uns auf Steinzeitniveau zurück[werfen]“. Die Anthropologen Henke und Rothe (2003: 5) sehen hingegen in der Theorie der egoistischen Gene die Überwindung des Darwin'schen Paradoxon:

„Die ganze Herausforderung der Evolutionstheorie, eben nicht nur die physische Evolution der Organismen, sondern auch die Verhaltensevolution von Tier und Mensch auf biologischer Grundlage zu erklären, erfolgte erst mit der Begründung der Soziobiologie unter anderem durch William D. Hamilton, Edward O. Wilson und Richard Dawkins.“

Insbesondere die Auseinandersetzung, die Dawkins als Religionskritiker führte (vgl. dazu auch Dawkins 2010a), brachte ihm letztlich im Jahr 2007 den erstmals durch die gbs dotierten Deschner-Preis ein (Schmidt-Salomon \& Held 2011). Der Autor Karlheinz Deschner (1924-2014), der in Bamberg geboren wurde, verfasste u. a. die Kriminalgeschichte des Christentums und sah die Motive für seine Arbeit in der Aufarbeitung von Unrecht (Deschner 2013).

Auch Ernst Mayr publizierte mit dem Ziel, eine gebildete Öffentlichkeit zu erreichen. So formulierte der 1937 in Boston geboren Physiologe und Evolutionsbiologe Jared Mason Diamond treffend:

13 Der AK Evolutionsbiologie agiert seit 2017 eigenständig und kooperiert mit dem National Center for Science Education (NCSE) in Oakland, CA, USA, der Richard Dawkins Foundation für Vernunft \& Wissenschaft und der Huffington Post - EditionDE. 
„Aber so hervorragend die zur Verfügung stehende Literatur auch ist, so lässt sie doch eine Lücke: Es fehlt die Darstellung der Evolution auf mittlerer Ebene, die nicht nur für Fachleute geschrieben ist, sondern auch für die gebildete Öffentlichkeit, mit besonderem Schwergewicht auf der Erläuterung von Phänomenen und Abläufen der Evolution.“ (Diamond 2005: 11, Hervorhebung im Original)

Weitere Veröffentlichungen leisteten auch Thomas Junker und Sabine Paul, die beide bis Juni 2014 als Mitglieder des wissenschaftlichen Beirates der gbs auftraten, u. a. mit ihrem Buch Der Darwin Code (2010). Diese keinesfalls vollständige Liste ausgewählter Autorinnen und Autoren zeigt das Angebot von Literaturbeiträgen zu wissenschaftshistorischen und modernen Aspekten der Evolutionsbiologie für das gebildete Laienpublikum auf. Gleichzeitig wird auch das gestiegene Interesse der Leserschaft an evolutionsbiologischen Themen und Informationen deutlich.

Trotz dieser Bemühungen im Bereich der Öffentlichkeitsarbeit und Bildung sowie umfangreicher Erkenntnisse aus den Geo- und Biowissenschaften, die belegen, dass die Evolution eine Tatsache ist, bleiben Zweifel in großen Teilen der Bevölkerung. Da Debatten häufig emotional geführt werden, fragen Junker und Paul (2009: 8) nach Gründen für die „Angst vor der Evolutionstheorie“. Dass diese Frage berechtigt ist, belegen aktuelle Diskussionen um den Kreationismus (Graf 2011), der verstanden wird als „das Dogma, wonach der gesamte Kosmos inklusive aller Lebewesen zumindest im Wesentlichen in ihrer heutigen Form von einem Schöpfergott erschaffen wurde“ (Graf \& Lammers 2011: 9). Der Kreationismus, definiert als „wörtlich verstandener biblischer Schöpfungsglaube“, stellt hierbei ein grenzübergreifendes Phänomen dar (Kutschera 2008: 236, Hervorhebung im Original).

Die verschieden stark betriebenen Bemühungen Bibeltexte als Tatsachenberichte auszulegen, hat zahlreiche Varianten des Kreationismus nicht nur in den Vereinigten Staaten hervorgebracht (Brasseur 2011). Versionen des Schöpfungsglaubens sind dem Arbeitskreis Evolutionsbiologie zufolge Flache-ErdeKreationismus, Junge-Erde-Kreationismus, Alte-Erde-Intelligent DesignKreationismus und Theistische Evolution (Kutschera 2010b). Glaubensgemeinschaften, wie die evangelikale Studiengemeinschaft Wort und Wissen haben in ihren Reihen zwar keine ausgewiesene Evolutionsforscher, dennoch betonen sie ihren wissenschaftlichen Anspruch in diesem Fachgebiet (Kutschera 2012; Porges 2016a). Anhänger des Intelligent-Designs (ID), eine Variante des Kreationismus, „,vermeiden zur Rechtfertigung ihres Standpunktes in aller Regel religiöses Argumentieren. Als Letztursache für Entstehen und Veränderung von Lebewesen wird nicht Gott verantwortlich gemacht, sondern meist ein nicht näher spezifizierter intelligenter Designer“ (Graf \& Lammers 2011: 13). Grundlegend widersprechen diese Ansichten den Prinzipien des methodischen Naturalismus und sind daher aus wissenschaftlicher Sicht inakzeptabel. 
Neben Veröffentlichungen wie Streitpunkt Evolution (Kutschera 2004) und Kreationismus in Deutschland (Kutschera 2007) wirkt der Arbeitskreis Evolutionsbiologie auch über digitale Medien (You-Tube Videos) seit 2010 aufklärerisch. Die im Arbeitskreis tätigen renommierten Evolutionsbiologen zeigen in ihren Bestrebungen unzweideutig die Fehler in der Argumentationskette von Vertretern kreationistischer Vorstellungen auf. In neueren Untersuchungen wird der Glaube selbst zum Gegenstand wissenschaftlicher Forschung. Im Buch Gott, Gene und Gehirn (Vaas \& Blume 2009) steht beispielsweise die Frage nach den evolutionsund neurobiologische Grundlagen der Religiosität im Fokus der Betrachtungen. Dennoch ist eine abschließende gesellschaftliche Anerkennung der biologischen Evolution trotz Erkenntniszugewinn und intensiver Öffentlichkeitsarbeit nicht erreicht.

Storch et al. (2001: VI) reflektieren, dass nicht alle Menschen die Evolution als Tatsache akzeptieren, sondern in einem ,seit Jahrtausenden gepflegt[en]“ Schöpfungsglauben verharren. Der Umgang damit trägt im öffentlichen Raum viele Facetten und differenziert auch seitens der Wissenschaften. Mayr (2005a) betonte bereits, dass jene, die sich nicht überzeugen lassen wollen, selbst durch eine detaillierte Darstellung der Belege nicht zu überzeugen sind. Beispielhaft seien hier die Bemühungen von Hans-Joachim Zillmer angeführt, dessen Werke in Buchhandlungen unter der Rubrik Wissenschaft zu finden sind. Neben der vorgestellten populärwissenschaftlichen Literatur findet der Leser Titel, wie Darwins Irrtum, Irrtümer der Erdgeschichte sowie Die Evolutionslïge. Demnach wird im Sinne einer Glaubensfreiheit dem Leser die Entscheidung überlassen, welche Werke er liest. Die Zuordnung zur Rubrik Wissenschaft in Buchhandlungen suggeriert jedoch, dass diese und ähnliche Darlegungen wissenschaftlich seriös sind (Kutschera 2007a; Leinfelder 2007).

Kutschera (2009a: 18) formuliert folgerichtig: „Man könnte diese laienhaften, mit Fehlern durchsetzten Schreibereien ignorieren, würden sie nicht von einem großen, Fach-unkundigen Publikum gekauft, gelesen und für ernsthafte Wissenschaft gehalten werden.“ Darüber hinaus sind Tendenzen feststellbar, den Kreationismus an Schulen und Universitäten zu etablieren. Sei es über die Forderung, die Schöpfungslehre im Biologieunterricht mit einzubeziehen (Brasseur 2011; Kutschera \& Hoßfeld 2012), über Bildungsmedien (Kutschera 2004, 2007b; Junker 2011), über Weiterbildungen (Kutschera 2004), die Ablehnung von Abituraufgaben im Fach Biologie (Beese 2014) oder mit dem Ziel der Missionierung verbundene Hochschultage (Steinhaus 2014). Die Gefahren sind offensichtlich und belegen, trotz aller Erfolge, dass der zu Beginn zitierte Ausspruch Mullers auch heute noch einer Hervorhebung bedarf. 



\title{
Das Bildungssystem der DDR und das Schulfach Biologie
}

\begin{abstract}
„Die Schrecken der Vergangenheit, die Not der Gegenwart, aber auch das Bewußtsein von einer unvermeidbar kommenden besseren Zukunft gibt uns die Kraft, das Schwerste zu meistern und unser demokratisches Aufbauwerk zu Ende zu führen.“ (Wandel o. J.: 50f.)
\end{abstract}

\section{Das allgemeinbildende Schulsystem}

Strukturwandel

Institutionell war die Bildungsgeschichte der DDR geprägt durch einen Strukturwandel des allgemeinbildenden Schulsystems, der sich in drei Etappen vollzog (Köhler 2008; Abb. 5, Tab. 8). Das Schulsystem der DDR war somit kein statisches Produkt, sondern gleichermaßen ein Bestandteil und ein Spiegel gesellschaftlicher Veränderungen. 


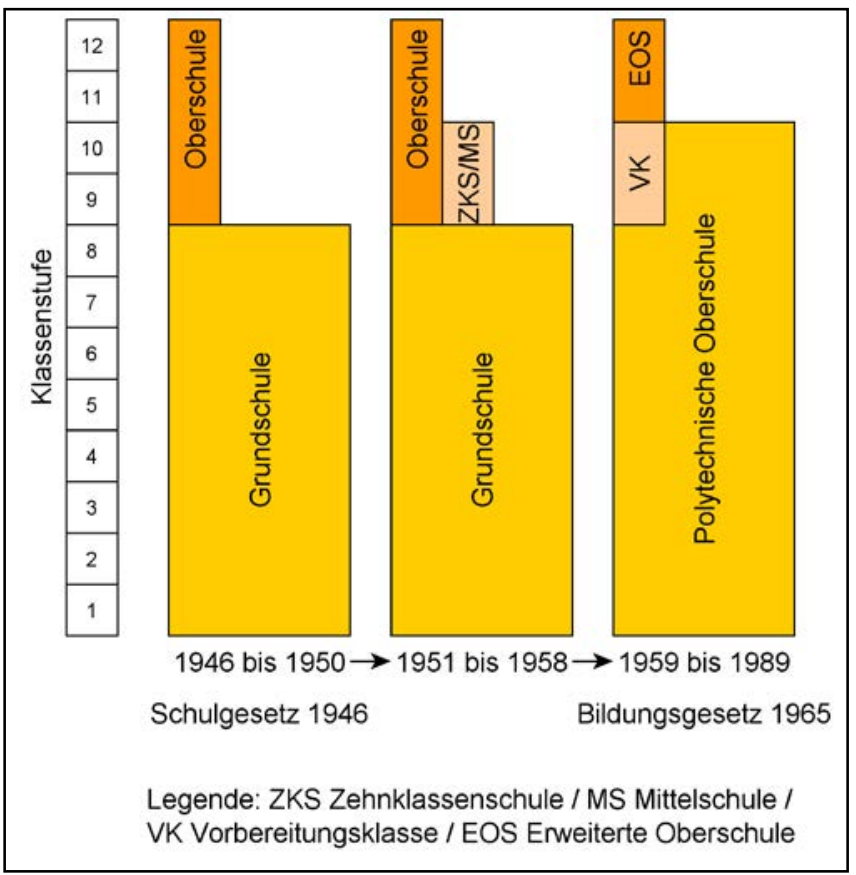

Abbildung 5: Strukturwandel des allgemeinbildenden Schulsystems der SBZ/DDR (verändert nach Köhler 2008)

Tabelle 8: Geschichte der Schule der DDR - eine Auswahl wesentlicher Ereignisse

\begin{tabular}{|c|c|c|}
\hline & Datum & Wesentliche Ereignisse \\
\hline & 27. Juli 1945 & Bildung der Deutschen Zentralverwaltung für Volksbildung \\
\hline$\stackrel{\Phi}{c}$ & 25. August 1945 & Befehl Nr. 40 der SMAD (Vorbereitung der Schulen zum Schulbetrieb) \\
\hline$\sum^{\pi}$ & 1. Oktober 1945 & Wiedereröffnung des Schulbetriebs \\
\hline 美 & 12. Oktober 1945 & Gründung des Verlags Volk und Wissen \\
\hline 忌 & 7. März 1946 & Gründung der FDJ \\
\hline & 1. September 1946 & Gesetz zur Demokratisierung der deutschen Schule \\
\hline & 12. Juni 1946 & Befehl Nr. 205 der SMAD (Einrichtung Pädagogischer Fakultäten) \\
\hline & 1. Juli 1946 & Einführung von Lehrplänen für Grund- und Oberschulen \\
\hline & 15. bis 17. August 1946 & 1. Pädagogischer Kongress \\
\hline & 10. September 1947 & 2. Pädagogischer Kongress (Grundsätze der Erziehung) \\
\hline & 21. Mai 1941 & $\begin{array}{l}\text { Umwandlung von Vorstudienanstalten in Arbeiter und Bauern } \\
\text { Fakultäten (ABF) }\end{array}$ \\
\hline & 23. bis 25 . August 1949 & $\begin{array}{l}\text { 4. Pädagogischer Kongress (Ablehnung reformpädagogischer } \\
\text { Bestrebungen) }\end{array}$ \\
\hline & 24. August 1949 & Schulpolitische Richtlinien für die neue demokratische Schule \\
\hline & 1. September 1949 & $\begin{array}{l}\text { (Beschluss des Parteivorstands der SED) } \\
\text { Gründung des Deutschen Pädagogischen Zentralinstituts (DPZI) }\end{array}$ \\
\hline & 7. Oktober 1949 & Gründung der DDR \\
\hline & 15. Dezember 1950 & Schulpflichtaesetz \\
\hline
\end{tabular}




\begin{tabular}{|c|c|c|}
\hline 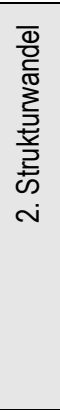 & $\begin{array}{l}\text { 22. Februar } 1951 \\
\text { 1. September } 1951 \\
\text { 23. Juli } 1952 \\
\text { 15. Mai } 1953 \\
\text { 1. Januar } 1955 \\
\text { 15. bis } 18 \text {. Mai } 1956 \\
\text { 1. September } 1958\end{array}$ & $\begin{array}{l}\text { 2. Hochschulreform (Verordnung über die Neuorganisation des } \\
\text { Hochschulwesens) } \\
\text { Einführung neuer Lehrpläne (Grund- und Oberschulen) und Eröffnung } \\
\text { von Zehnjahrschulen } \\
\text { Auflösung der Länder der SBZ in } 14 \text { Bezirke } \\
\text { Verordnung über die Neureglung der Ausbildung der Lehrer an den } \\
\text { allgemeinbildenden Schulen [...] } \\
\text { Gründung des Zentralinstituts für Lehrerweiterbildung } \\
\text { 5. Pädagogischer Kongress } \\
\text { Aufnahme polytechnischen Unterrichts an allgemeinbildenden } \\
\text { Schulen }\end{array}$ \\
\hline 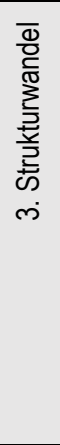 & $\begin{array}{l}\text { 1. September } 1959 \\
\text { 1. Dezember } 1959 \\
\text { 1. September } 1960 \\
\text { 3. bis } 5 \text {. Juni } 1961 \\
\text { 25. Februar } 1965 \\
\text { 1968/69 } \\
\text { 3. April } 1969\end{array}$ & $\begin{array}{l}\text { Einführung des Lehrplanwerkes für die zehnklassige } \\
\text { allgemeinbildende polytechnische Oberschule } \\
\text { Gesetz über die sozialistische Entwicklung des Schulwesens } \\
\text { Einführung eines neuen Systems in der Lehrerausbildung } \\
\text { 6. Pädagogischer Kongress } \\
\text { Gesetz über das einheitliche sozialistische Bildungssystem } \\
\text { 3. Hochschulreform (eingeleitet durch die 4. Hochschulkonferenz von } \\
\text { 1967) } \\
\text { Beschluss des Staatsrates der DDR regelte die Weiterführung der } 3 \text {. } \\
\text { Hochschulreform und die Entwicklung des Hochschulwesens bis } \\
\text { 1975 } \\
\text { 9. Pädagogischer Kongress }\end{array}$ \\
\hline
\end{tabular}

Einen entscheidenden Einfluss auf den Wiederaufbau der Gesellschaft in der SBZ übte die Sowjetische Militäradministration in Deutschland (SMAD) aus (Weber 2006). Mit dem Befehl Nr. 17 vom 27. Juli 1945 Errichtung von Zentralverwaltungen gründete sich auch die Deutsche Zentralverwaltung für Volksbildung (DZfV), die später in Deutsche Verwaltung für Volksbildung (DVfV) umbenannt wurde ${ }^{14}$ (SMAD-Befehl Nr. 17/45). Paul Wandel (1905-1995), der zu dieser Zeit als Chefredakteur bei der Zeitung Deutsche Volkszeitung arbeitete, übernahm die Führung der Verwaltung (Baumgartner \& Hebig 1997). Zwar besaß die DZfV „keine gesetzgeberische Befugnis“15, doch kam ihr die Aufgabe zu, „die Tätigkeit der Schulverwaltungen in den Ländern und Provinzen zusammenzufassen, zu koordinieren, anzuleiten und zu kontrollieren“ (Günther \& Uhlig 1969: 26). Mit dem Befehl Nr. 40 vom 25. August 1945 legte die SMAD fest, den Unterricht an allen Schulen der SBZ am 1. Oktober 1945 wieder aufzunehmen (SMAD-Befehl Nr. 40/45). ${ }^{16}$ Vertreter verschiedener Parteien äußerten bereits 1945 den Wunsch

14 Die Namensgebung DZfV hatte sich hinsichtlich der Westzonen und der föderalen Länder der SBZ seit etwa Mitte 1946 „als unangemessen erwiesen“ (Geißler 2000: 68).

15 Geißler (2000: 68) resümiert, dass „die Zentralverwaltungen [...] von sowjetischer Seite zunächst als Grundstock eventuell möglicher, dem Alliierten Kontrollrat unterstellter gesamtdeutscher Verwaltungen gedacht" waren.

16 Am 1. Oktober 1945 nahmen 10.822 allgemeinbildende Schulen die Arbeit auf (Günther \& Uhlig 1969). 
nach einer Schulreform mit Bildungschancen für alle (Weber 2006). ${ }^{17}$ Der sich daraus entwickelnde Neubeginn im Schulwesen wurde als antifaschistischdemokratische Schulreform bezeichnet (Neuner 2009).18 Zentrales Rechtsdokument war das Gesetz, zur Demokratisierung der Deutschen Schule, das am 12. Juni 1946 in Thüringen verkündet wurde und am 1. September 1946 in Kraft trat. ${ }^{19}$ Es regelte die Ziele der Schulreform, die Aufgaben der Schulverwaltung und legte die Struktur einer demokratischen Einheitsschule fest (Reuter 1998). Die Grundgedanken des Gesetzes waren der einheitliche Aufbau des Erziehungs- und Bildungswesens, das Recht auf Bildung an allen Bildungseinrichtungen sowie die Übertragung schulischer Angelegenheiten an den Staat (Lang 1946).

Eine achtiährige Grundschule als demokratische Einheitsschule konzipiert, ersetzte in der Folge die bisherige Dreigliedrigkeit des Schulwesens (Geißler et al. 1996). ${ }^{20}$ Privatschulen wurden aufgelöst, eingegliedert oder umgewandelt, um ,jede Abseits-Erziehung und Absonderung in der schulischen Jugenderziehung" (Lang 1946: 11) zu unterbinden. Nach Abschluss der Grundschule konnte eine dreijährige Berufs- oder vierjährige Oberschule besucht werden. Der Unterricht in der Grundschule sah wissenschaftsbezogenen Fachunterricht und Fremdsprache vor, mit dem Ziel, „die traditionelle volkstümliche Bildung zu überwinden und alle Kinder in den Bereich jener Bildungsmöglichkeiten zu führen, die aus proletarischer Sicht [...] als Privileg sozialer Eliten galten“ (Geißler et al. 1996: 19). Für die Klassen 7 und 8 der Grundschule „war vorgesehen, neben dem obligatorischem Kernunterricht wahlfreie Kurse anzubieten“ (Geißler 2000: 92). ${ }^{21}$ Dieser Kursunterricht gliederte sich in einen neusprachlichen, altsprachlichen und mathematischnaturwissenschaftlichen Zweig und bereitete im Grunde auf das Kurssystem der Oberschule vor (Köhler 2008; Abb. 6). ${ }^{22}$

17 Das Zentralkomitee der KPD und der Zentralausschuss der SPD veröffentlichten am 18. Oktober einen gemeinsamen Aufruf zur demokratischen Schulreform (Günther \& Uhlig 1969; Geißler 2000). Eine am 4. November durchgeführte gemeinsame Veranstaltung im Berliner Admiralpalast „trug das Reformanliegen - in der Presse eher nachrangig behandelt - in die Öffentlichkeit" (Geißler 2000: 56).

18 Als ,erste[s] Dokument der beginnenden antifaschistisch-demokratischen Schulreform“ gilt der Beschluss des Berliner Senats Vorläufige Richtlinien für die Wiedereröffnung des Schulwesens vom 11. Juni 1945 (Günther \& Uhlig 1969).

19 In Erinnerung an diesen Tag erklärte die Regierung der DDR den 12. Juni am 4. Mai 1951 zum Tag des Lehrers (Günther \& Uhlig 1969). Die Arbeit und die gesellschaftliche Funktion der Lehrerschaft sollten an diesem Tag im Fokus der Öffentlichkeit stehen. Auch die Verleihung des, bereits seit dem 12. August 1949 gestifteten, Ehrentitels Verdienter Lebrer des Volkes erfolgte an diesem Tag. Dieser Ehrentitel war, zusammen mit dem Ehrentitel Verdienter Arat des Volkes, der am längsten verliehene in der DDR (Hubrich 2013).

20 Eine zentrale Aufgabe der Schulreform war „die Hebung des Bildungsniveaus der Volkschule auf das Niveau der Mitteschule“ (Geißler 2000: 92).

21 Diese Differenzierung galt in Thüringen bereits ab Klasse 5 (Geißler 2000).

22 Der Kursunterricht an den Oberschulen wurde im Juni 1951 eingestellt (Geißler 2000). 


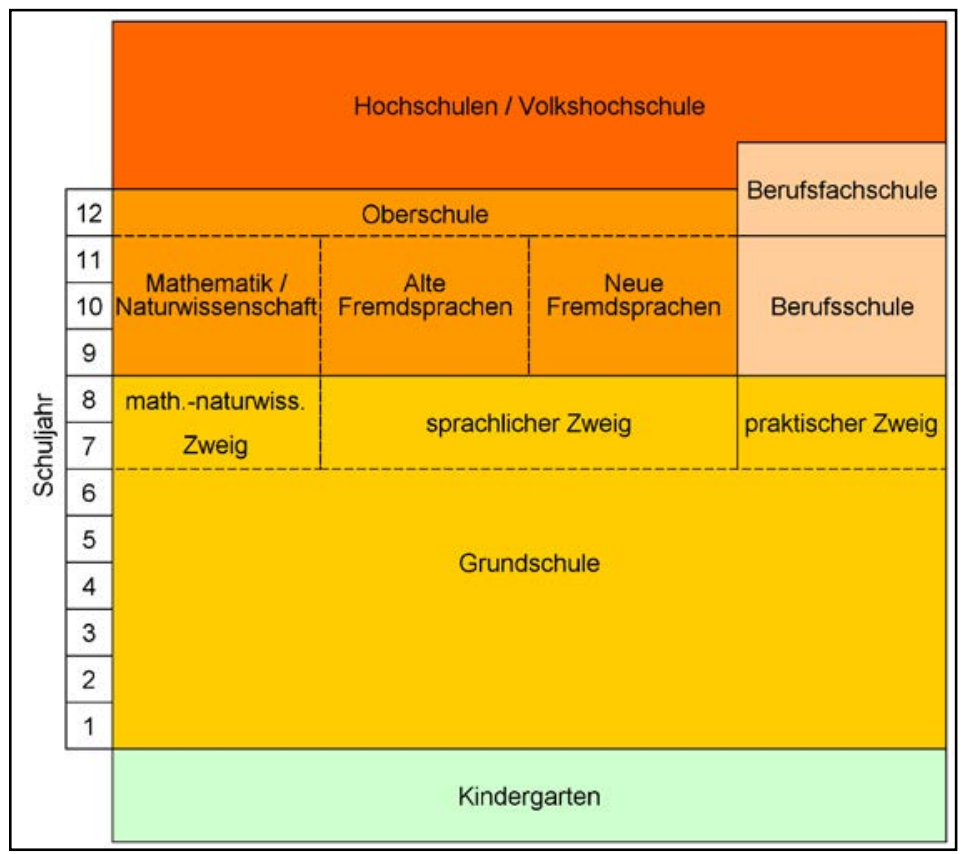

Abbildung 6: Struktur des allgemeinbildenden Schulwesens der SBZ/DDR bis 1950 (verändert nach Lang 1946)

Bereits im Herbst 1947 wurden eine Reduzierung der ehemals einklassigen Schulen und ein Anstieg der ausgebauten achtklassigen Schulen festgestellt (Weber 2006). Auf dem 2. Pädagogischen Kongress ${ }^{23}$ von 1947 fasste Paul Wandel die bis dahin erreichten Veränderungen zusammen.

„Das Ziel unserer Erziehung wurde weiter geklärt, der organisatorische Umbau unseres Schulwesens, der Einbau der verschiedenen Schularten in das System unserer gegliederten Einheitsschule im ganzen abgeschlossen; erfolgreich wurde der Ausbau unserer Grundschule durchgeführt, vor allem in Bezug auf die Entwicklung der bisher einstufigen Landschule zu einer mehrstufigen und des Aufbaus des fremdsprachlichen und des Kursunterrichts in der Mittelstufe“" (Wandel o. J.: 22f.).

Die Ziele der antifaschistisch-demokratischen Schulreform „,waren 1948/49 weitgehend verwirklicht“ (Günther \& Uhlig 1969: 67). Gleichfalls aber wurde deutlich, dass „an den Schulen der Einflußgewinn der SED gering geblieben war“ (Geißler 2000: 245). Die Sozialistischen Einheitspartei Deutschlands (SED) entwickelte daher ihr schulpolitisches Programm weiter und verabschiedete am 24. August 1949 Schulpo-

23 Zwischen dem Ende des Krieges und der Gründung der DDR am 7. Oktober 1949 fanden begleitend vier Pädagogische Kongresse statt (Reuter 1998). 
litische Richtlinien für die deutsche demokratische Schule. Diese bestimmten „die vollausgebaute achtklassige Schule zum Normaltyp der Grundschule“ (Günther \& Uhlig 1969: 67) und „bedeuteten den bis dahin deutlichsten Versuch der SED, [...] auch im Bildungswesen ihren Herrschaftsanspruch durchzusetzen" (Geißler 2000: 248).

Mit Gründung der DDR am 7. Oktober 1949 entstanden neue politische Rahmenbedingungen, die zu einer neuen Dynamik in der Schulpolitik führten (Geißler 2000). Aus den bestehenden institutionellen Strukturen der DVfV konstituierte sich das Ministerium für Volksbildung der DDR (MfV). ${ }^{24}$ Es war zuständig „für die Gestaltung der pädagogischen und administrativen Verhältnisse in den staatlichen Kindergärten und Heimen, den allgemeinbildenden Schulen und in den nichtuniversitären Lehrerbildungseinrichtungen" (ebd.: 254). Die Verfassung der DDR sicherte in den Artikeln 34 bis 40 die Grundgedanken des Gesetzes zur Demokratisierung der Deutschen Schule (Günther \& Uhlig 1969). Doch wurde bereits im „Frühjahr $1950[\ldots]$ einen umfassende, Reorganisation“ des Schulwesens geplant" (Geißler 2000: 263). Dies führte zu zentralen schulrechtlichen Vorgaben, wie das Gesetz. über die Schulpflicht in der Deutschen Demokratischen Republik vom 15. Dezember 1950.

Auf dem 3. Parteitag der SED wurde die Einführung von Zehnklassenschulen festgelegt, um ,ihr altes Ziel, eine Oberschulbildung für alle Kinder des Volkes, zu verwirklichen" (Günther \& Uhlig 1969: 72). Ab 1951 eröffneten neben den Grund- und Oberschulen zusätzlich die ersten Zehnklassenschulen ${ }^{25}$, in denen die Lehrkräfte nach den Lehrplänen des mathematisch-naturwissenschaftlichen Zweiges der Oberschulen unterrichteten (Köhler 2008). ${ }^{26}$ Ziel war es, die Oberschulbildung auf zwei Jahre zu verkürzen, einen direkten Zugang zu Fachschulen zu ermöglichen und „Kenntnisse und Einsichten zu vermitteln sowie Fähigkeiten und Fertigkeiten zu entwickeln, die für den Besuch von Fachschulen zur Heranbildung mittlerer Kader notwendig sind" (MfV 1951c: S. 3). Jedoch entstand kein „eigenständiger, abgerundeter und differenzierter Bildungsgang“ (Geißler 2000). Bereits im Jahr 1953 erfuhr dieser Versuch daher seine Einstellung. Statt dem Abschluss der Zehnklassenschule wurde mit dem Schuljahr 1953/54 in Klasse 10 der Oberschulen die Prüfung der Mittleren Reife eingeführt. Dies ermöglichte vorzeitigen Abgängern der Oberschule ein Fachschulstudium. Nach dem 4. Par-

24 Das MfV besaß zunächst „eine nachrangige Stellung“ und ,war erst seit dem Jahre 1952 weitgehend stabil“" (Geißler 2000: 253f.).

25 Vornehmlich wurde sie ,in ländlichen Regionen und Industriegebieten eingerichtet“. Sie war einerseits „Ersatz für die mit den Schulgesetzen von 1946 aufgehobene, in der Bevölkerung aber als Bildungsgang tradierte Mittelschule“. Andererseits wirkte sie politisch dahin, „der noch immer weit überwiegend von Kindern nichtproletarischer sozialer Herkunft besuchten Oberschule das Monopol auf Vermittlung weiterführender Allgemeinbildung zu schmälern“ (Geißler 2000: 266).

26 Die Lehrpläne für die Zehnklassenschulen waren ,wenig mehr als die Kurzfassung des jeweiligen Gesamtlehrgangs der Oberschule, oder es war der Oberschulstoff der Klassen 9 bis 10 komplett übernommen wurden" (Geißler 2000: 267). 
teitag der SED im Jahr 1955 wurde erneut mit dem Aufbau der Zehnklassenschule begonnen und diese in einer Anordnung des MfV vom 11. Mai 1955 „erstmalig als die künftige obligatorische Schule für alle Kinder bezeichnet" (Günther \& Uhlig 1969: 84). Im Jahr 1955 entstand schließlich unter der Bezeichnung Mittelschule eine neue Form der Zehnklassenschule (Köhler 2008). ${ }^{27}$ In Folge der 3. Parteikonferenz der SED und dem Ministerratsbeschluss vom 15. März 195628 sollte sie bis „1960 etwa 40 Prozent der Schüler erfassen und bis zum Jahre 1965 zur obligatorischen allgemeinbildenden Schule entwickelt werden“ (Günther \& Uhlig 1969: 94). Zwar war unklar, ,welche Aufgabe der Oberschule mit dem Aufbau der Mittelschule zufallen würde“, doch „blieb das Thema Oberschule im MfV auch aufgrund von Anfragen und der Diskussion in der Lehrerschaft aktuell“ (Geißler 2000: 447).

Das MfV leitete im Schuljahr 1958/59 „eine neue Etappe im Aufbau der Mittelschule ein“ (Günther \& Uhlig 1969: 110). ${ }^{29}$ Die Abteilungen Volksbildung der Bezirke und Kreise erarbeiteten „Perspektivpläne für die Entwicklung ihrer allgemeinbildenden Schulen zu Mittelschulen“, um die Einführung der zehnjährigen Allgemeinbildung vorzubereiten (ebd.). Im Januar 1959 formulierte das Zentralkomitee (ZK) der SED auf seiner 4. Tagung Thesen über die sozialistische Entwicklung des Schulwesens (Akademie für Staats- und Rechtswissenschaft der DDR 1984). Auch hier wurde der „Vorschlag unterbreitet, eine Oberschulbildung für alle Kinder aufzubauen“ (Günther \& Uhlig 1969: 120). Mit der Bezeichnung Oberschule sollte sich „der neue, einheitliche Schultyp“ mit qualitativ höhere Bildung auch begrifflich gegenüber der früheren Schule abgrenzen (Geißler 2000: 512). Der Einsicht folgend, „daß die qualitativen Veränderungen im Schulwesen [...] einer gesetzlichen Absicherung bedurften“, verabschiedete die Volkskammer am 2. Dezember 1959 nach einer breit angelegten Diskussion ${ }^{30}$ das Gesetz über die sozialistische Entwicklung des Schulwesens in der Deutschen Demokratischen Republik (ebd.). ${ }^{31}$ Das geforderte Abschlussniveau wurde dann schrittweise von acht auf zehn Jahre angehoben, da die zehnjährige allgemeinbildende polytechnische Oberschule (POS)

27 Im September 1955 setzte sich die Bildungslandschaft aus 9566 Grundschulen, 406 Zehnklasseschulen (Mittelschulen) und 419 Oberschulen zusammen (Günther \& Uhlig 1969).

28 Beschluss über die Aufgaben und den Aufbau der Mittelschulen in der Deutschen Demokratischen Republik.

29 Ein weiteres Charakteristikum der Epoche ab 1959 war die Verbindung von Unterricht und produktiver Arbeit. Im Schuljahr 1958/59 wurde der polytechnische Unterricht durch verbindliche Lehrpläne eingeführt und manifestierte sich in den Schulgesetzen von 1959 und 1965 (ebd.; Reischock 2009).

30 Über „den Sinn und Zweck“ der Thesen und die damit verbunden Konsequenzen fand eine öffentliche Diskussion in mehr als 70000 Versammlungen statt (Günther \& Uhlig 1969: 121). Geißler (2000: 513) spricht hier von einer „Inszenierung einer breiten, gelenkten ,Volksdiskussion““, die durch Beiträge in den Medien flankiert wurde, um „die Öffentlichkeit auf die bevorstehende ,sozialistische Entwicklung des Schulwesens" einzustimmen“.

31 Im Vergleich mit den schulpolitischen Veränderungen 1945/46 und den 1950er Jahren, „ging der Einführung des neuen Schulgesetzes eine relativ lange öffentliche Vorbereitungsphase voraus" (Geißler 2000: 512). 
zur Grundlage des Schulsystems wurde (Akademie für Staats- und Rechtswissenschaft der DDR 1984).

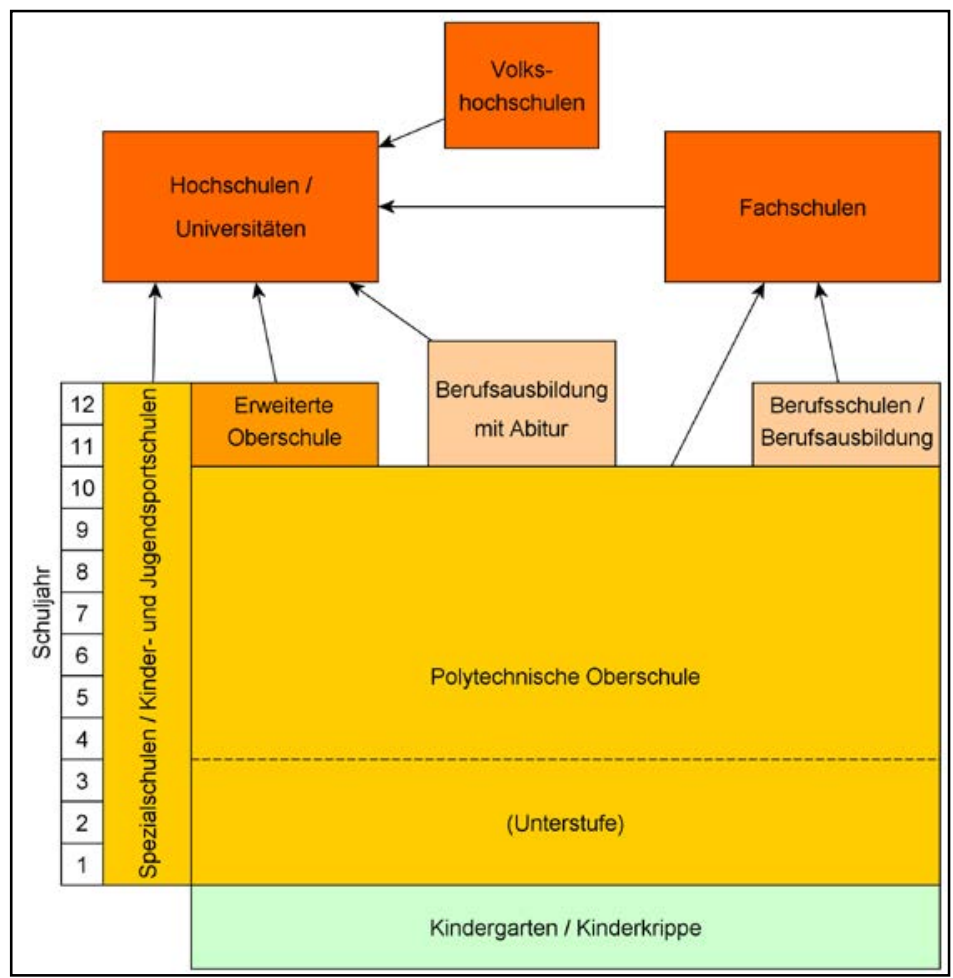

Abbildung 7: Struktur des allgemeinbildenden Schulwesens der DDR seit dem Ende 1980er Jahre (verändert nach Köhler 2008)

Zwar manifestierten sich mit dem Schulgesetz „bedeutende Veränderungen im Bildungswesen“, doch waren aufgrund neuer Entwicklungen „manche seiner Bestimmungen [...] nur temporär von Bedeutung“ (Geißler 2000: 515). Da sich auch an die pädagogischen Wissenschaften die Frage stellte, wie ihre „Wirksamkeit [...] bei der weiteren Entwicklung der sozialistischen Schule wesentlich erhöht werden" kann, erarbeitete das Deutsche Pädagogische Zentralinstitut (DPZI) eine Konzeption für ein Forschungsprogramm (Günther \& Uhlig 1969: 136). Diese wurde 1962 vom MfV und vom Staatssekretariat für das Hoch- und Fachschulstudium bestätigt. ${ }^{32}$ Auf Vorschlag des 6. Parteitages der SED arbeitete eine

32 Borneleit (2003: 136) resümiert, dass ,nach herrschender Theorie [...] die Pädagogik in gesetzmäßige Entwicklungszusammenhänge der Gesellschaft eingefügt" war. Ebenso betont er, dass „zwischen Kernproblemen der Gesellschaft und Schulproblemen [...] ein Zusammenhang gesehen" wurde. 
Kommission des Ministerrates ${ }^{33}$ Grundsätze für ein neues Bildungsgesetz aus. Ein erster Entwurf, der auf wissenschaftlichen Vorarbeiten des DPZI beruhte, erschien Anfang des Jahres 1964 (Günther \& Uhlig 1969). Nach einer monatelangen öffentlichen Diskussion wurde das Gesetz über das einheitliche sozialistische Bildungssystem ausgearbeitet und am 25. Februar 1965 durch die Volkskammer beschlossen. Als zentrales schulisches Rechtsdokument regelte das Gesetz die zehnjährige Oberschulpflicht (vgl. Rockstuhl 2006; Weber 2006).

„Es gewährleistet den einheitlichen Bildungs- und Erziehungsprozeß [...]. Sein Kernstück ist die allgemeinbildende zehnklassige polytechnische Oberschule, die damit zur Schule des ganzen Volkes entwickelt wird. Inhalt und Ziel des einheitlichen sozialistischen Bildungswesens sind, sozialistische Persönlichkeiten heranzubilden, die eine allseitige hohe Bildung mit einem festen Klassenstandpunkt vereinen, den Anforderungen der modernen Produktion und der anderen gesellschaftlichen Bereiche auch künftig gewachsen sind und unerschüttlich die Sache des Sozialismus in der Klassenauseinandersetzung mit dem Imperialismus vertreten." (Diehl et al. 1978: 461)

Im Zentrum der Bildungslandschaft stand jetzt als Pflichtschule die POS mit Unterstufe von Klasse 1 bis 4 und Oberstufe von Klasse 5 bis 10 (Abb. 7). Das Gesetz über das einheitliche sozialistische Bildungssystem ergab aber wesentliche Veränderungen für die erweiterten Oberschulen (EOS), die ,künftig auf der Oberschule aufbauen und die Schüler in 2 Jahren zum Abitur führen" sollte (Günther \& Uhlig 1969: 178). Um den direkten Anschluss an die POS zu gestatten, musste folglich das Niveau der 9. und 10. Klasse erhöht werden. Zwischen den Schuljahren 1967/68 und 1980/81 erfolgte die Vorbereitung auf die EOS daher in Vorbereitungsklassen, in denen geeignete Schülerinnen und Schüler aus den Jahrgänge 9 und 10 zusammengefasst wurden. Sie blieben im Sinn des Bildungsgesetzes jedoch Schülerinnen und Schüler der POS und legten am Ende der 10. Klasse eine Prüfung ab (Wernecke 1967a; Günther \& Uhlig 1969). Grundlegend ergaben sich die Anpassungen im Bildungswesen aus der parteilichen Sichtweise, dass „die sozialistischen Produktionsverhältnisse gesiegt hatten und die wesentlichen Aufgaben der Übergangsperiode vom Kapitalismus zum Sozialismus gelöst worden waren“, wodurch der Schwerpunkt der gesellschaftlichen Entwicklung neu ausgerichtet werden konnte (Diehl et al. 1978: 456). Infolge des Gesetzes über das einheitliche sozialistische Bildungssystem und dem neuen Lehrplanwerk wurde die POS innerhalb des Bildungssystems als ein Teilsystem verstanden, „das mit allen anderen Elementen des Bildungssystems [...] mannigfach verbunden ist" (Günther 1969: 19f.). Als Pflichtschule kam ihr die Aufgabe zu,

33 Staatliche Kommission zur Gestaltung des einheitlichen sozialistischen Bildungssystems. 
Das Bildungssystem der DDR und das Schulfach Biologie

„die Grundlagen für die allseitige Entwicklung der sozialistischen Persönlichkeit zu legen, alle Schülerinnen und Schüler auf schöpferische produktive Arbeit, auf aktive Mitwirkung an den Angelegenheiten der sozialistischen Gesellschaft vorzubereiten, sie zu bewußten sozialistischen Staatsbürgern zu erziehen und die Voraussetzungen für alle weiterführenden Bildungswege und die ständige Weiterbildung zu legen "Günther 1969: 20, Hervorhebung im Original)

Ausbildung der Lehrkräfte

Der Neubeginn im Schulwesen war mit einer umfassenden Entnazifizierung verbunden ${ }^{34}$, die „,bei steigenden Schülerzahlen zu einer dramatischen Unterbesetzung der Schulen mit Lehrkräften“ führte (Geißler 2000: 104) und eine personelle Neubesetzung erforderte. ${ }^{35}$ Die freien Stellen übernahmen ,Zehntausende neue Menschen, mit gutem Willen, ehrlicher Absicht, aber sehr oft ohne pädagogische Vorbildung“" (Wandel o. J.: 20). ${ }^{36}$ Doch die Art der Ausbildungswege und Einstellungsmodalitäten sowie das Auftreten der Schulverwaltungen eröffneten im schulischen Bereich berufliche Perspektiven (Kirchhöfer 2009). Etwa 15000 Neulehrerinnen und -lehrer erhielten nach einer Aufnahmeprüfung bereits im Schuljahr 1945/46 eine Anstellung im Schulbetrieb. Diese konnten sich entsprechend der Möglichkeiten in kurzen Lehrgängen weiterbilden. Ab Januar 1946 fanden dann für Neulehrerinnen und -lehrer achtmonatigen Lehrgängen statt. ${ }^{37}$ Zum Schuljahr 1946/47 wurden etwa 25000 weitere Einstellungen vorgenommen, so dass die alte Lehrerschaft nur noch etwa ein Drittel ausmachte (Günther \& Uhlig 1969; Herbst et al. 1994). So ersetzten sukzessiv kurzfristig ausgebildete Neulehrerinnen und lehrer die abgesetzten Lehrkräfte (Weber 2006). Mit dieser „neue Lehrerschaft“ sollte „ein neuer demokratischer Geist" in der Schule einziehen (Wandel o. J.: 22f.). Sie stellten letztlich das „Schlüsselsymbol der DDR-Gesellschaft in der Phase der antifaschistisch-demokratischen Schulreform von 1945 bis 1949“ dar (Kirchhöfer 2009: 140). Mit ihrer Hilfe gelang es auch, bis 1959 das Landschulwe-

34 Nach Günther und Uhlig (1969: 29) gibt es ,,in der deutschen Schulgeschichte wenige Maßnahmen, die auf einen so wütenden Widerstand [...] stießen, wie diese Säuberungen der Lehrerschaft“. Geißler (2000: 104) betont, dass ,nach der Entlassungswelle [...] im Frühjahr 1946 wieder einzelne Zulassungen zum Schuldienst“ erfolgten. „Eine ,scharfe Nachprüfung“ aller Entlassungsfälle" wurde durch Ausführungsbestimmungen der DZfV geregelt.

35 Die Entnazifizierung im Schuldienst führt zu einem ,plötzlichen Ausfall von mehr als 20000 Lehrern [...], so daß vorübergehend eine kritische Situation entstand“" (Günther \& Uhlig 1969: 29).

36 Gertrud Kummer (2016a: 120) erinnert sich, dass ,in den Tageszeitungen, auf Flugblättern und Plakaten [...] interessierte junge Menschen aufgefordert [wurden], sich in einer Kurzausbildung auf einen wichtigen, zukunftsträchtigen Beruf, den des Lehrers, vorzubereiten“. Die „Neuen unterrichteten wie die Altlehrer 32 Stunden pro Woche, verkauften die Schulhefte, verteilten die wenigen Schulbücher" (ebd.: 121).

37 Ab 1946 diente die Schriftenreihe Lernen und Lebren und ab 1947 die Pädagogische Bibliothek als pädagogisch-methodische Unterstützung für den Unterricht (Günther \& Uhlig 1969). Aus den achtmonatigen Kursen entwickelten sich 1950 die Institute für Lehrerbildung (IfL). 
$\operatorname{sen}^{38}$, die Einklassenschulen sowie der Mehrklassenunterricht abzuschaffen (Koch 2012).

Auf der Grundlage der SMAD-Befehle Nr. 205 vom 12. Juli und Nr. 237 vom 2. August 1946 richteten die Universitäten und die Technische Hochschule Dresden Pädagogische Fakultäten ein ${ }^{39}$, um ,eine alte Forderung fortschrittlicher bürgerlicher Pädagogen zu erfüllen [...]: die Hochschulausbildung für alle Lehrer“ (Günther \& Uhlig 1969: 48). Hier nahmen im Wintersemester 1946/47 die ersten Studentinnen und Studenten eine dreijährige Ausbildung zu Mittelstufenlehrerinnen und -lehrern auf (Lang 1946; Günther \& Uhlig 1969).40 Die sechs Universitäten auf dem Gebiet der SBZ eröffneten gemäß dem Befehl Nr. 50 der SMAD vom 04. September 1945 nach dem 2. Weltkrieg den Lehrbetrieb am:

15. Oktober 1945, Friedrich-Schiller-Universität Jena (FSU),

29. Januar 1946, Humboldt-Universität zu Berlin (HU Berlin, HUB),

1. Februar 1946, Martin-Luther-Universität Halle-Wittenberg (MLU),

5. Februar 1946, Karl-Marx-Universität Leipzig (KMU),

15. Februar 1946, Ernst-Moritz-Arndt-Universität Greifswald (EMAU),

25. Februar 1946, Wilhelm-Pieck-Universität Rostock (WPU).

Der besondere Stellenwert der Lehrerausbildung zeigte sich in der finanziellen Unterstützung bzw. Entlastung durch Schulgeldfreiheit, Stipendien und darin, dass „den Lehrern als erster Kategorie der Berufstätigen die Gehälter erhöht und eine bevorzugte materielle Versorgung ausgesprochen" wurde (Wandel o. J.: 4). ${ }^{41}$ Zwar studierten 1947 etwa 2000 Lehramtsanwärter an den Pädagogischen Fakultäten, dennoch blieb die Situation prekär, da weiterhin 40000 Menschen ohne pädagogische Ausbildung im Lehrberuf tätig waren (ebd.). Daher sollten die Pläne und Methoden der Pädagogischen Fakultäten so gestaltet werden, „daß sie den allgemeinen Rahmen der ganzen Lehrerausbildung, einschließlich der Fortbildung der 40000 Lehrer, bis zu ihrer ersten und zweiten Lehrerprüfung erfassen“ (ebd.). Ziel

38 Die Landschulreform wurde bis 1955/56, ,im wesentlichen abgeschlossen“. Es entstanden 1745 Zentralschulen, so dass „Stadt- und Landschulen [...] eine Bildung von gleichem Umfang und gleicher Höhe" vermittelten (Günther \& Uhlig 1969: 84).

39 Die Pädagogische Fakultät Dresden wurde 1950 auf die Ausbildung von Berufsschullehrerinnen und -lehrern umgestellt (Günther \& Uhlig 1969: 48).

40 Im Schuljahr 1949/50 gingen die ersten Absolventinnen und Absolventen in die Praxis. In diesem Jahr wurde auch „der Unterricht in den Oberklassen der Grundschule nach dem Fachlehrerprinzip erteilt“" sowie erstmals ,an allen Grundschulen Versetzungs- und Abschlußprüfungen durchgeführt" (Günther \& Uhlig 1969: 71).

41 Einen Grund für diese Unterstützungen sehen Günther und Uhlig (1969: 47) darin, dass „Neulehrer den Beruf leichtfertig wechselten oder aber von den Schwierigkeiten erdrückt wurden. Deshalb organisierten die SED, die demokratischen Verwaltungen und auch die sowjetischen Besatzungsorgane eine Reihe von Maßnahmen, um die Arbeitsbedingungen der Lehrer zu verbessern." Dennoch befanden sich insbesondere die Lehrkräfte an den Grundschulen zu Beginn der 1950er Jahre „nach wie vor in einem Zustand hoher Instabilität und großer beruflicher Beanspruchung“ (Geißler 2000: 336). 
war es, die Ausbildung an den pädagogischen Fakultäten im „Interesse des einheitlichen inneren und äußeren Aufbaues [...] [des] gesamten Schulwesens" zu vereinheitlichen (Lang 1946: 13). Unter der Prämisse, diese zu verbessern und die (schul-)politischen Ziele zu verwirklichen, entstanden Pädagogische Hochschulen (PH). Die ersten eröffneten 1947 in Berlin und 1948 in Potsdam.42

Die Veränderungen im Schulwesen, die infolge der Schulpolitischen Richtlinien der SED und der Gründung der DDR vorangetrieben wurden, bezogen sich nicht nur auf die Schulstruktur, sondern auch auf die Ausbildung der Lehrkräfte. Diese „zielte[n] in einem ersten Schritt darauf ab, die noch immer erheblichen Divergenzen in der Gestaltung der einzelnen Kurse abzubauen" (Geißler 2000: 269) und „eine große Anzahl von Lehrern auszubilden“ (Günther \& Uhlig 1969: 74). Ab September 1950 eröffneten Institute für Lehrerbildung (IfL) ${ }^{43}$, die anfangs eine dreijährige Ausbildung für Lehrerinnen und Lehrer der Klassen 1 bis 8 ermöglichten (Geißler 2000). Damit auch die Neulehrerinnen und -lehrer eine Fachausbildung erwerben und mit „den Anforderungen des wissenschaftlichen Fachunterrichts in der Grundschule Schritt [...] halten" konnten, richtete das DPZI im Jahr 1951 ein Fernstudium für Mittelstufenlehrerinnen und -lehrer ein (Günther \& Uhlig 1969: 74). Ein Fernstudium für Oberstufenlehrerinnen und -lehrer an der PH Potsdam ergänzte in der Folgezeit dieses Angebot (ebd.). ${ }^{44}$

Auf Beschluss des Politbüros des ZK der SED ${ }^{45}$ vom 29. Juli 1952 sollte, „die politisch-ideologische Arbeit unter den Lehrern und die Lehrerausbildung" verbessert werden (ebd.: 78). Zukünftig sollten ,nur noch voll ausgebildete Lehrer an den Schulen der DDR unterrichten“, was ein „festes System der Lehrerbildung“ erforderte (ebd.: 85). In Folge des 3. Parteitages der SED beschloss der Ministerrat in einer Verordnung ${ }^{46}$, die Ausbildung der Unterstufenlehrerinnen und -lehrer (Klassen 1 bis 4) ${ }^{47}$ auf die IfL zu übertragen. Die Pädagogischen Institute bildeten Abiturientinnen und Abiturienten zu Mittelstufenlehrerinnen und -lehrern (Klasse

42 Seit 1948 existierte in Potsdam die Brandenburgische Landeshochschule. Die PH in Potsdam wurde ab 20. Oktober 1948 aufgebaut, da in der Mark Brandenburg zu dieser Zeit keine Hochschule mit Pädagogischer Fakultät existierte (Günther \& Uhlig 1969). Im Jahr 1951 erhielt die PH den Namen „Karl Liebknecht“.

43 Auf Beschluss der Ministerkonferenz vom 29. Juli 1950 (Geißler 2000).

44 Eine Forderung auf dem 3. Parteitag der SED von 1950 lautete, ,,die Bildungs- und Erziehungsinhalte an Schulen und Hochschulen künftig auf der Grundlage des dialektischen Materialismus zu entwickeln“ (Herbst et al. 1994: 770). Daraus resultierte im Jahr 1951 die Verordnung über die Neuorganisation des Hochschulwesens - Gesellschaftswissenschaftliches Grundstudium an den Universitäten und Hochschulen. Ein entsprechendes Grundlagenstudium war nun für alle Studenten obligatorisch (ebd.). Auch die obligatorische Weiterbildung der Lehrkräfte fand ab 1951 „nach einheitlichen Themenplänen statt, die für Veröffentlichungen sowjetischer Wissenschaftler [einen] breiten Raum vorsahen" (Günther \& Uhlig 1969: 89).

45 Zur Erböbung des wissenschaftlichen Niveaus des Unterrichts und zur Verbesserung der Parteiarbeit an den allgemeinbildenden Schulen.

46 Verordnung über die Neuregelung der Ausbildung der Lehrer an den allgemeinbildenden Schulen, der Pionierleiter, der Kindergärtnerinnen und der Erzieher in Heimen und Horten vom 15. Mai 1953.

47 Die Ausbildung für Lehrerinnen und Lehrern der Klassen 1 bis 4 dauerte zwei Jahre. 
5 bis 8) aus ${ }^{48}$, während die Ausbildung der Oberschullehrerinnen und -lehrer an den Universitäten und der PH Potsdam verblieb (ebd.; Geißler 2000).

„Die Verordnung vom 15. Mai 1953 beendete die Zeit der revolutionären Übergangslösungen, mit deren Hilfe auf der Grundlage einer klaren schulpolitischen Konzeption ein neuer Lehrkörper geschaffen war. Es entstand ein System der Lehrerbildung, das in der Folgezeit ergänzt und weiterentwickelt wurde, aber in seiner Grundstruktur unverändert blieb.“ (Günther \& Uhlig 1969: 85)

Aufgrund einer vom Ministerrat erlassenen Verordnung49 vom 4. August 1955 verlängerte sich die Dauer der Ausbildung für Lehrerinnen und Lehrer der Klassen 5 bis 8 von zwei auf drei Jahre. Auch für Fachlehrerinnen und -lehrer mit zwei Fächern (Klassen 5 bis 10) veränderten sich mit dem Wintersemester 1958/59 die Studieninhalte und die Prüfungsbedingungen, wodurch sich die Studiendauer auf vier Jahre verlängerte (Herbst et al. 1994).50

Die Einführung des polytechnischen Unterrichtes und die Anhebung der Schulpflicht auf zehn Jahre, stellten neue Anforderungen an den Lehrerberuf. Eine Hauptaufgabe im Schuljahr 1959/60 lautete, ,alle Lehrer auf der Grundlage des neuen Lehrplanwerkes" methodisch und fachlich vorzubereiten (Günther \& Uhlig 1969: 124). Dies führte ab 1959 zu Reformen in der Ausbildung. Ab 1960 sah das MfV und das Staatssekretariat für Hoch- und Fachschulwesen „eine Ausbildung für 2 Unterrichtsfächer der Klassen 5-10, eine polytechnische Ausbildung aller Studierenden, eine gründliche Ausbildung im Fach Marxismus-Leninismus und in den Erziehungswissenschaften sowie einen schulpraktischen Tag in der Woche" vor (Herbst et al. 1994: 770). ${ }^{51}$ Zum Teil begannen Absolventinnen und Absolventen diese Ausbildung bereits 1960 (Günther \& Uhlig 1969).52 Im Zusammenhang mit dem Aufbau der zehnklassigen Oberschule stieg die benötigte Anzahl ausgebildeter Lehrkräfte an. ${ }^{53} \mathrm{Da}$ die Kapazitäten an den Einrichtungen nicht ausreichten, wurden bis Mitte der 1960er Jahre Sonderwege eröffnet. Dazu

48 Diese führte auch zur Neugründung von PI mit Hochschulcharakter, die teilweise aus den IfL hervorgingen. An ihnen erfolgte in einem viersemestrigen Studium die Ausbildung von Mittelstufenlehrerinnen und -lehrern für die Klassen 5 bis 8 (Herbst et al. 1994).

49 Verordnung über die Verbesserung der Ausbildung der Mittelstufenlebrer für die allgemeinbildende Schule und die Qualifizierung der wissenschaftlichen Kader für die Lehrerbildung.

50 Ein Bericht der Hauptschulinspektion aus dem Jahr 1956 nannte als „Hauptursache“ für das Problem der Überlastung bei Schülerinnen und Schülern, „die methodische Arbeit der Lehrer und deren Mangel an psychologischen Kenntnissen" (Geißler 2000: 442).

51 Plan zur sozialistischen Entwicklung der Lehrerausbildung vom 14. April 1960.

52 Zukünftige Oberstufenlehrerinnen und -lehrer absolvierten ein vierjähriges Studium an Universtäten, Hochschulen und PI. Unterstufenlehrerinnen und -lehrer absolvierten ein dreijähriges Studium an PI (Günther \& Uhlig 1969).

53 Schätzungen zufolge sollten die Schülerzahlen „bis zum Schuljahr 1964/65 um etwa 800000“ ansteigen. Entsprechend waren „Aufwendungen für die Ausbildung vieler Lehrer“ nötig (Günther \& Uhlig 1969: 121). 
gehörte auch die Auslagerung von Ausbildungsinhalten in ein Fernstudium (Wilms 2009).

Im Programm der SED, das auf dem 6. Parteitag im Januar 1963 beschlossen wurde, trat die Einbettung der Lehrerkräfte in den gesellschaftlichen Kontext sowie die Forderung nach politischer, wissenschaftlicher und pädagogischer Bildung deutlich hervor. In Folge des Parteitages und dem daraus resultierenden Gesetz. über das einheitliche sozialistische Bildungssystem vom 25. Februar 1965 entstand „ein in sich geschlossenes System der Ausbildung von Lehrern und Erziehern“ (Richter 1967: 9). ${ }^{54}$ Diese Neukonzipierung und Weiterentwicklung der Ausbildung der Fachlehrerinnen und -lehrer führte dazu, dass ,alle Fachlehrerstudenten einheitlich an allen Universitäten, lehrerbildenden Hochschulen sowie an den Pädagogischen Instituten nach neuen, auf diesen Grundlinien aufbauenden Studienprogrammen ausgebildet" wurden (ebd.: 16). Das Studium an Universitäten und Hochschulen umfasste vier Jahre und konnte mit dem Diplom abgeschlossen werden (Richter 1972). Neben dem Hauptfach, dem Nebenfach und den dazugehörigen Methodiken waren Marxismus-Leninismus, Pädagogik und Psychologie obligatorische Bestandteile des Studiums.

Die Gründung weiterer Pädagogischer Hochschulen vollzog sich ab Ende der 1960er Jahre aus bereits bestehenden Instituten für Lehrerfortbildung (IfL) bzw. aus Pädagogischen Instituten (PI):

- PH „Karl Friedrich Wilhelm Wander“ Dresden seit 1967 (PI seit 1952)

- PH „Dr. Theodor Neubauer“ Erfurt/Mühlhausen seit 1969

(Mühlhausen: IfL seit 1949, PI seit 1953; Erfurt: PI seit 1953)

- PH „Liselotte Herrmann“ Güstrow seit 1972 (PI seit 1952)

- PH „Clara Zetkin“ Leipzig seit 1972 (PI seit 1953)

- PH „Erich Weinert“ Magdeburg seit 1972 (IfL seit 1951, PI seit 1962)

- PH „Ernst Schneller“ Zwickau seit 1973 (PI seit 1965)

- $\quad \mathrm{PH}$, „N. K. Krupskaja“ Halle-Köthen seit 1988 (Halle: IfL seit 1952, PH seit 1972; Köthen: PH seit 1974)

- $\quad$ PH „Edwin Hoernle“ Neubrandenburg seit 1989 (1988 Verlegung des PI aus Templin)

In den 1980er Jahren standen 17 Pädagogische Schulen und 30 IfL der Ausbildung von Unterstufenlehrerinnen und -lehrern, von Kindergärtnerinnen und Kindergärtnern sowie Erzieherinnen und Erziehern zur Verfügung. Zulassungsbedingung war der Abschluss der 10. Klasse. Die Ausbildung von Oberstufen- bzw. Diplomfachlehrerinnen und -lehrern (Klasse 5 bis 10) erfolgte auf Hochschulebene in einem Zeitraum von fünf Jahren an $\mathrm{PH}$, Technischen Hochschulen und den

54 Das Gesetz über das einheitliche sozialistische Bildungssystem der DDR regelte im 8. Abschnitt in den Paragrafen 26 bis 29 die Aus- und Weiterbildung der Lehrer und Erzieher (Rockstuhl 2006). 
Pädagogischen Fakultäten der Universitäten. Studienmöglichkeiten bestanden im Direkt- und im Fernstudium (Butzmann 1988; Herbst et al. 1994).

Für den Unterricht in den 11. und 12. Klassen der EOS war kein gesondertes Studium erforderlich. Die dort tätigen Lehrkräfte wurden nach mehrjähriger Berufserfahrung an einer POS in der Regel aufgrund ihrer politischen Haltung und fachlichen Leistung durch die Schulverwaltung für den höheren Schuldienst ausgewählt (Herbst et al. 1994). Die Mehrzahl der Diplomlehrerinnen und -lehrer erwarb an den PH die Lehrbefähigung für zwei Fächer. Die Lehrerausbildung an den IfL und den PH fiel in den Zuständigkeitsbereich des MfV, ebenso wie das Zentralinstitut für Weiterbildung der Lehrer und Erzieher in Ludwigsfelde ${ }^{55}$, das die Bezirkskabinette für die Weiterbildung der Lehrkräfte sowie Erzieherinnen und Erzieher leitete. Alle anderen Einrichtungen für die Ausbildung von Lehrkräften, wie Universitäten, Technische Hochschulen (Berufsschullehrerinnen- und lehrer), Musik- und Sporthochschulen sowie Fachschulen fielen in den Zuständigkeitsbereich des Ministeriums für Hoch- und Fachschulen und anderen Fachministerien (Reuter 1998).

\section{Lehrplanentwicklung}

In der Gesamtschau vollzog sich die Entwicklung der Lehrpläne im Zeitraum von 1945 bis 1989 in fünf Phasen (Neuner 2009) und war eingebettet in „das marxistisch-leninistische bildungstheoretische Denken [...] [, das] wissenschaftliche Bildung mit wissenschaftlicher Ideologie" verband (Neuner 1989: 85, Hervorhebung im Original). Bereits am 20. September 1945 erließ die DZfV Richtlinien für die Aufstellung von vorläufigen Lehrplänen für die Volksschulen ${ }^{56}$, denn ,ausgearbeitete Lehrpläne existierten nicht" (Günther \& Uhlig 1969: 32, Hervorhebung im Original). Die ersten Lehrpläne, die sich an Richtlinien und Befehlen der sowjetischen Besatzungsmacht orientierten, traten schließlich im Herbst 1946 in Kraft. Sie enthielten konkrete Stoffpläne und fokussierten auf einen wissenschaftlichen Fachunterricht, der mit dem Neuaufbau im Schulwesen eingerichtet wurde. Aufgrund der schwierigen Situation formulierten die Lehrpläne vorerst Zielvorstellungen für vollgegliederte Schulen, die über ausreichend Lehrkräfte verfügten (Geißler 2000). Die Pläne knüpften an reformpädagogische Ideen der Weimarer Zeit, insbesondere der Arbeitsschule, an (Pietsch 1959; Neuner 2009). Dabei war der Versuch kennzeichnend, „eine gewisse Synthese zwischen reformerischen und eher traditionellen Unterrichtsauffassungen herzustellen“ (Geißler 2000: 93). Da die Lehrpläne jedoch in „kurzer Zeit von einem begrenzten Personenkreis“ ausgearbeitet wurden und sich „die Verfasser [...] kaum auf Unterrichtserfahrungen in der neuen Schule

55 Das Zentralinstitut für Weiterbildung der Lehrer und Erzieher wurde 1962 gegründet (Tille 1993b).

56 Ausführungsbestimmungen zum Befehl Nr. 40 der SMAD vom 25. August 1945. 
Das Bildungssystem der DDR und das Schulfach Biologie

stützen“ konnten, wurden die Pläne bereits 1947 präzisiert (Günther \& Uhlig 1969: 43).

Mit Gründung der DDR gründete sich im Jahr 1949 auch das DPZI in Berlin. Übergeordnetes Ministerium war das MfV. Die Aufgabe des DPZI bestand darin, Lehrpläne für die allgemeinbildenden Schulen und Lehrerbildungsinstitute auszuarbeiten (Wendt 1957; Zabel 2009). ${ }^{57}$ Beeinflusst durch den 4. Pädagogischen Kongress 1949 in Leipzig wurde reformpädagogischen Bestrebungen der ersten Rahmenlehrpläne eine Absage erteilt und der Weg für eine neue Schulpolitik bereitet. Diese sah für den Kampf gegen den Imperialismus eine „Lern- und Leistungsschule sowjetrussischen Typs“ vor (Neuner 2009: 129), um „durch ,Leistungssteigerung' im Unterricht akuten volkswirtschaftlichen Zielstellungen zuzuarbeiten“" (Geißler 2000: 280). ${ }^{58}$ Dies zeigte Auswirkungen auf die Lehrpläne, die im Schuljahr 1951/52 in Kraft traten. ${ }^{59}$ Ihnen lagen „,vor allem sowjetische Vorbilder zugrunde“ und sie setzten ,übergangslos hohe Anforderungen für die gesamte Grundschularbeit“ (ebd.: 287). Die Herausgabe der Lehrpläne wurde „als ein bedeutender Schritt vorwärts auf dem Wege des ständigen Kampfes um die Erhöhung der Leistungen“ (DPZI 1952: 145) angesehen. Sie „fußten konsequent und parteilich auf der Wissenschaft des Marxismus-Leninismus" und gingen dabei „von einer einheitlichen inhaltlichen Konzeption aus“ (Günther \& Uhlig 1969: 70). Gegenüber den Lehrplänen von 1946 gaben die neuen Lehrpläne „,nun nicht mehr ein unter Umständen erreichbares Stoffmaximum, sondern den überall verbindlich zu vermittelnden Unterrichtsstoff an" (Geißler 2000: 287). Gleichfalls verwies jedoch das DPZI auf einen Mangel an notwendigen Praxisüberprüfungen und forderte in diesem Zusammenhang die Lehrerschaft auf, Vorschläge zur Ver-

57 Später zeichnete die im Jahr 1970 gegründete Akademie der Pädagogischen Wissenschaften für die Lehrpläne verantwortlich. Beauftragte Fachkräfte aus der Didaktik und der Fachwissenschaft reichten Vorschläge ein, die von Gutachtern gelesen wurden. Zu den Gutachtern gehörten Lehrkräfte, Didaktikerinnen und Didaktiker, Fachwissenschaftlerinnen und -wissenschaftler, Mitarbeiterinnen und Mitarbeiter der Akademie der Wissenschaften und des Verlags Volk und Wissen. Nach Beratungen der Autoren und Gutachter wurde ein Vorschlag beim übergeordneten $\mathrm{MfV}$ eingereicht und in einer gemeinsamen Sitzung aller Beteiligten unter Vorsitz der Bildungsministerin Margot Honecker (1927-2016) beschlossen. Kummer berichtete (Interview vom 28.12.2016), dass Margot Honecker mit Fragen zur Sache und schriftlichen Anmerkungen interessiert und konstruktiv die Entwicklung der Biologielehrpläne vorantrieb.

58 Die Auseinandersetzungen um reformpädagogische Strömungen begannen bereits 1946 und waren methodisch sowie politisch-ideologisch geprägt. Mit dem beginnenden Ost-WestKonflikt leitete die SED 1948 eine ideologische Offensive ein, die auch vom 3. Pädagogischen Kongress 1948 mitgetragen wurde. Eine verstärkte politisch-ideologische Erziehungsarbeit und die Erhöhung des Leistungsniveaus im Unterricht, um „der Volkswirtschaft qualifizierte Kader zuzuführen“, standen bereits hier im Zentrum der Schulpolitik (Günther \& Uhlig 1969: 53).

59 Die Herausgabe der Lehrpläne war mit einer veränderten Stundentafel verbunden. Sie enthielten „erste Hinweise für die polytechnische Bildung“ und erweiterten den naturwissenschaftlichen Unterricht (ebd.: 70). 
besserung einzureichen (DPZI 1952). ${ }^{60}$ Da die Pläne jedoch letztlich als „Gegenstück zur 46er Reform“ wahrgenommen wurden (Neuner 2009: 129), kam es in den folgenden Jahren ,zu zahlreichen [...] Veränderungen, ohne jedoch das Grundmuster der Lehrpläne aufzugeben“ (Geißler 2000: 289). ${ }^{61}$ Infolge der hohen Belastung der Schülerinnen und Schüler setzte eine anhaltende Schulkritik ein, die im Schuljahr 1955/56 zur „Entlastung“ und „Lockerung des formal-verbindlichen Charakters“ der Pläne sowie zur „Beschränkung auf Rahmenlehrpläne in den [...] Fächern Heimatkunde und Werken“ führte (Geißler 2000: 442). Die Leitung des MfV kam infolge eines Berichts der Hauptschulinspektion aus dem Jahr 1956 überein, „Umfang und Inhalt einer zeitgemäßen Allgemeinbildung“ zu bestimmen sowie „die Stunden- und Lehrpläne zu verändern“ (ebd.).

Im Februar 1959 leitete schließlich das MfV in einem Artikel der Deutschen Lehrerzeitung eine Diskussion über ein neues Lehrplanwerk ein. Eine erste Grundkonzeption erarbeitete das DPZI in Kooperation mit Wissenschaftlerinnen und Wissenschaftlern sowie Lehrkräften anderer Einrichtungen. Dieser Entwurf einer Grundkonzeption für das Lehrplanwerk der zehnklassigen allgemeinbildenden polytechnischen Oberschule wurde der „Öffentlichkeit zur Prüfung und Kritik [...] übergeben“ und am 1. September 1959 obligatorisch eingeführt (Günther \& Uhlig 1969: 139). ${ }^{62}$ Die schrittweise Erweiterung von acht auf zehn Schuljahre und die damit einhergehenden inhaltlichen Neuausrichtungen wurden durch Übergangslehrpläne begleitet. Ebenso erschienen ,vielfältige Materialien für die Hand des Lehrers zur Umsetzung des Lehrplanwerkes in die Praxis“ (ebd.).

„Erstmals in der deutschen Schulgeschichte wurde eine in sich geschlossene, relativ einheitliche, wenn auch noch nicht in allen Details hinreichend koordinierte Auffassung von Wesen, Struktur und Inhalten einer zehnjährigen Allgemeinbildung unter Einschluß der polytechnischen Bildung für alle Kinder des Volkes entwickelt.“ (Günther \& Uhlig 1969: 125)

Infolge des Gesetzes des einheitlichen sozialistischen Bildungssystems von 1965 traten erneut neue Lehrpläne in Kraft. Zwar hatten sich die Vorgaben von 1959 „,in der Schulpraxis vielfach bewährt“, doch wurden sie „ausgehend vom Stand und von den Entwicklungstendenzen der gesamten gesellschaftlichen Entwicklung“ präzisiert und diese jahrgangsweise mit dem Schuljahr 1965/66 eingeführt (Günther \& Uhlig 1969: 172f.). ${ }^{63}$ Im neuen Lehrplanwerk wurden ,alle Unterrichtsfächer zu

60 Kritik übten die Lehrkräfte vor allem an der Stofffülle, infolgedessen bereits 1952 „die Pläne erstmals korrigiert und die Stundentafel verändert" wurden (Geißler 2000: 289).

61 Mit dem Politbürobeschluss der SED vom 29. Juli 1952 „nahm die SED-Führung die Schule direkt in die, Verteidigungsbereitschaft'. Entsprechend wurden deutliche Neuakzentuierungen in Lehrplan und Unterricht vorgenommen" (Geißler 2000: 360).

62 Das MfV erhielt ,5000 schriftliche Stellungnahmen [...] zu dieser Grundkonzeption“ und für die „endgültige Fassung des Lehrplanwerkes wurden 350 Wissenschaftler [...] konsultiert“ (Günther \& Uhlig 1969: 126).

63 Bereits 1964 trat ein neuer Lehrplan für das Fach Staatsbürgerkunde in Kraft. 
einem Ganzen zusammengefügt“ und „auf die Verwirklichung des komplexen Ziels der allseitig entwickelten sozialistischen Persönlichkeit gerichtet" (Neuner 1969: 67). Stärker als in den früheren Lehrplänen führte der Aufbau der Lehrgänge durch Linienführung zu einem „hohen Grad an Koordinierung“ (Drefenstedt 1969: 84). Das Gesetz über das einheitliche sozialistische Bildungssystem regelte im $\ 23$ (1), dass der Unterricht nach staatlichen Lehrplänen zu erteilen ist, „die die Wissenschaftlichkeit und Systematik des Unterrichts gewährleisten" (Rockstuhl 2006: 39). Auch sollte „der grundlegende Bildungsinhalt für einen längeren Zeitraum“ festgelegt und ,in Gemeinschaftsarbeit von pädagogischen Wissenschaftlern, Lehrern und Wissenschaftlern anderer Bereiche erarbeitet werden" (ebd.).

Die letzte Phase der Lehrplanentwicklung in der DDR begann 1982 und dauerte bis zur Wiedervereinigung beider deutscher Staaten an. Sie folgte anders als die bisherigen Phasen einem Modernisierungsdruck, da die letzte Neubearbeitung bereits fast zwei Jahrzehnte zurück lag. Vordergründiges Charakteristikum war das Bestreben nach „Rücknahme von überzogener Verwissenschaftlichung“ (Neuner 2009: 136).

\section{Verlag Volk und Wissen}

Mit dem SMAD-Befehl Nr. 70 vom 25. September 1945 gründete die DZfV die Volk und Wissen Verlags-GmbH, die bereits am 12. Oktober 1945 in Berlin und Leipzig ihre Arbeit aufnahm und 1951 volkseigen wurde (SMAD-Befehl Nr. 70/45; Links 2010). Die Entwicklung des Verlages war ,aufs Engste mit der Entwicklung der Schule verbunden“, denn ,letztlich dient[e] die gesamte Arbeit des Verlages der Entwicklung der sozialistischen Schule und damit der Arbeit des Lehrers" (Anonymus 1985: 387). Die ersten Lehrbücher konnte der Verlag bereits im Herbst 1945 herausgeben ${ }^{64}$, da schon „im Sommer 1945 [...] Vorarbeiten für die Schulbuchproduktion aufgenommen" wurden (Günther \& Uhlig 1969: 32). Die Manuskripte für die neuen Werke entstanden ,in aller Eile durch die Bearbeitung einiger Lehrbücher der Weimarer Republik“" (ebd.). ${ }^{65}$ So wurden in der Anfangszeit der DZfV nicht nur „Lehrpläne für alle Schularten und Wahlfächer [...] geschaffen oder neubearbeitet“, sondern über den Verlag auch „,neue Lehrbücher für alle Unterrichtsfächer gestaltet" (Wandel o. J.: 22f.). Bis 1948 verbesserte sich die Situation schließlich soweit, dass „,die bearbeiteten Lehrbücher aus der Zeit der Weimarer Republik [...] allmählich durch neue Lehrbücher ersetzt" werden konnten (Günther \& Uhlig 1969: 44). Auch die Versorgung mit Lehrbüchern und Lehrmitteln konnte deutlich gesteigert werden. Lieferte der Verlag Volk und Wissen 1945 noch 4,1 Millionen Schulbücher aus, so betrug die Jahresproduktion

64 Bis Ende des Jahres 1945 stellte der Verlag 4116000 Schulbücher fertig (Günther \& Uhlig 1969: 32).

65 Im Schuljahr 1945/46 wurde „noch kein Geschichtsunterricht erteilt“, da er „,von Grund auf umgestaltet werden“ musste und ,dazu längere Vorarbeiten nötig [waren] als in den anderen Fächern“ (Günther \& Uhlig 1969: 43). 
1946 bereits 9,3 Millionen. Im Jahr 1947 steigerte der Verlag seine Produktion auf 11 Millionen, 1948 auf 13,6 Millionen und 1949 schließlich auf 14,6 Millionen Exemplare (ebd.).

Für die Hände der Lehrkräfte gab Gertrud Rosenow ab 1946 im Verlag Volk und Wissen die Reihe Lernen und Lehren. Methodische Schriften für lernende Lehrer heraus und unterstützte auf diesem Weg die Arbeit der Neulehrerinnen und -lehrer. Die Aufgabe des Verlages, den Lehrerinnen und -lehrern „direkte Hilfen für die stoffliche und methodische Gestaltung des Unterrichts zu geben“, zeigte sich auch an der Entwicklung von Zeitschriften (Anonymus 1985: 387). Neben den Zeitschriften die neue schule ${ }^{66}$ und Pädagogik, die ab 1946 erschienen, entwickelte der Verlag ab 1948 ,systematisch Fachzeitschriften für die einzelnen Unterrichtsfächer“ (ebd.). ${ }^{67}$ Durch die Herausgabe der Sammelbücherei sowie von 1946 bis 1950 $\operatorname{der} A B C$-Zeitung und der Schulpost erhielten auch die Kinder einen „Lese- und Wissensstoff in einer Zeit, [...] [in der] es noch kaum Kinderliteratur gab“ (ebd.). Die Versorgung mit Zeitschriften durch den Verlag Volk und Wissen konnte zwischen 1946 und 1949 verdoppelt werden. Lieferte der Verlag 1946 noch 5,8 Millionen Zeitschriften aus, so betrug die Jahresproduktion 1949 bereits 11,7 Millionen Exemplare (Günther \& Uhlig 1969: 44).

Infolge des Gesetzes zur Demokratisierung der deutschen Schule von 1946 ging der Verlag Volk und Wissen auch dazu über, eigene Schullehrbücher zu entwickeln. ${ }^{68}$ Der Fokus lag zu Beginn auf den Fächern Deutsch, Geschichte und Geografie. Mit der Einführung der neuen Lehrpläne für das Schuljahr 1951/52 „standen für einen Teil der Fächer [erstmals] [...] mit dem Lehrplan abgestimmte Lehrbücher bereit" (Geißler 2000: 288). ${ }^{69}$ Ferner verfolgte der Verlag eine Editionspolitik, die insbesondere sowjetische Pädagogen und Psychologen in das Blickfeld der Lehrkräfte rückte (Anonymus 1985). Auf der Grundlage des Lehrplanwerkes von 1959 entstanden in der Zeit „,von 1959 bis 1965 insgesamt 143 obligatorische Schulbücher für die Oberschule, davon 71 Lehrbücher, 19 Arbeits- und Übungsbücher, 44

66 Seit 1959 unter der Bezeichnung Deutsche Lehrerzeitung herausgegeben.

67 Der Verlag Volk und Wissen führte für die verschiedenen Fachbereiche Sektionen, die sich aus Abteilungen für die jeweiligen Lehrplanfächer zusammensetzten. Beispielsweise bestand die Sektion Naturwissenschaften aus den Abteilungen Biologie, Chemie, Physik, Mathematik und Sonderschulen. Anfangs arbeiteten die Redaktionen der Abteilungen gleichzeitig an den Schulbüchern und an den Fachzeitschriften. Diese Arbeitsweise ging zu Lasten der Buchredakteure und führte in den 1962 Jahren zu einer Trennung der Buch- und Zeitschriftenredaktion (Kummer, Interview vom 28.12.2016).

68 Beispielsweise erschienen 1949 die Fibel Lesen und Lernen von Robert Alt und Johannes Feuer sowie als 1000. Verlagserscheinung der neu entwickelte Atlas zur Erd-und Länderkunde. Ein Jahr später entstanden unter der Mitwirkung von Paul Wandel die ersten Lehrbücher für den Geschichtsunterricht (Anonymus 1985).

69 Infolge der an den Lehrplänen geäußerten Kritik wurde auch „die Übereinstimmung von Lehrplan und Lehrbuch überprüft" (Geißler 2000: 289). 
Stoffsammlungen und 9 Nachschlagewerke“ (Günther \& Uhlig 1969: 126).70 Auch veröffentlichte der Verlag eine große Anzahl von Monografien, die teilweise in den Publikationsreihen Pädagogische Wissenschaft und sozialistische Schule (11 Bände bis 1962) sowie Diskussionsbeiträge zu Fragen der Pädagogik (42 Bände bis 1965) erschienen (ebd.). Mit der Einführung neuer präzisierter Lehrpläne ab 1965/66 wurden auch neue Lehrbücher vom Verlag herausgegeben. Das Gesetz über das einheitliche sozialistische Bildungssystem von 1965 regelte dazu im $\$ 23$ (2), dass „die Lehrbücher und die Lehrmittel [...] entsprechend dem Inhalt der Lehrpläne zu gestalten“ sind (Rockstuhl 2006: 39). Die neuen Lehrbücher ,zeichneten sich durch eine größere Variabilität ihrer didaktischen Struktur aus und forderten in stärkerem Maße die selbständige Aneignung des Stoffes" (Günther \& Uhlig 1969: 176). Diesem Ziel folgte auch die Herausgabe von Nachschlagewerken für Schülerinnen und Schüler. Für die Lehrkräfte erarbeitete der Verlag Unterrichtshilfen, ,in denen für jedes Unterrichtsfach und jede Klasse der Gehalt der präzisierten Lehrpläne inhaltlich und didaktisch aufbereitet wurde" (ebd.).

„Zur weiteren Ausgestaltung der sozialistischen Schule gab der Verlag in enger Zusammenarbeit mit der Akademie der Pädagogischen Wissenschaften der DDR und der Praxis für alle Fächer Fachmethodiken heraus und - im Zusammenhang mit der Einführung überarbeiteter bzw. neuer Lehrpläne für alle Fächer und Klassen - ,Unterrichtshilfen ${ }^{\varsigma}$ und natürlich neue Schulbücher, die den höheren Anforderungen an den Unterricht in der sozialistischen Schule entsprechen." (Anonymus 1985: 387, Hervorhebung im Original)

Das Gesetz, über das einheitliche sozialistische Bildungssystem von 1965 regelte dazu im \23 (3), dass für die Lehrkräfte

„methodische und fachwissenschaftliche Materialien bereitzustellen [sind], die ihnen die Planung, Vorbereitung und Durchführung des Unterrichts mit hohem Niveau ermöglichen und erleichtern“ (Rockstuhl 2006: 39).

\section{Das Schulfach Biologie}

Sektion Schulbiologie in der Biologischen Gesellschaft (in) der DDR

Nach der Gründung der BRD und der DDR entstand für die gesamtdeutschen Vertretungen der Biologie eine bivalente Situation. Von politischer Seite gefördert, setzten sich ab 1957 mit Otto Schwarz (1900-1983) an der Spitze einige ostdeut-

70 Die in den Abteilungen des Verlages entwickelten Schullehrbücher wurden zur Genehmigung bei der Verlagsleitung vorgelegt. Gab es nichts zu beanstanden, beantragte der Verlag bei der jeweiligen Fachabteilung des MfV eine Druckgenehmigung (Kummer, Interview vom 28.12.2016). 
sche Biologen für einen autonomen Fachverband ein (Zabel 2007), was zur Trennung der gesamtdeutschen Verbände der Biologen beitrug (Höxtermann \& Höxtermann 2007). ${ }^{71}$ Zur Gruppe um Otto Schwarz und Werner Rothmaler (1908-1962) gehörte auch Erwin Zabel (*1928), dessen Aufgabe es sein sollte „die Interessen der Lehrer im Vorbereitungskomitee zu vertreten“" (Zabel 2007: 219). Am 10. April 1959 erfolgte im Plenarsaal der Humboldt-Universität in Berlin die Gründung der Biologischen Gesellschaft in der DDR (Biologische Gesellschaft der DDR o. J.). Bis dato dem Ministerium für Hoch- und Fachschulwesen zugeordnet, erfuhr sie während der ersten zehn Jahre „einen vielschichtigen Bedeutungswandel“ (Höxtermann 1997: 52). Dies zeigte sich auch in der 1972 vollzogenen Umbenennung der Biologischen Gesellschaft in der DDR in die Biologische Gesellschaft der DDR (ebd.). Ab 1. Juli 1969 gehörte die Biologische Gesellschaft der Deutschen Akademie der Wissenschaften zu Berlin an, die in Folge des SMAD-Befehls Nr. 187 am 1. Juli 1946 ihre Arbeit aufnahm. Im Statut (\$2, Abs. 1) formulierte die Gesellschaft neben anderen Aufgabenfeldern einen konkreten Bildungsauftrag.

„Die Biologische Gesellschaft stellt sich die Aufgabe [...] bei der allseitigen Bildung und Erziehung der Bürger der Deutschen Demokratischen Republik gemäß $\ 78$, Abs. 2 des Gesetztes über das einheitliche sozialistische Bildungssystem vom 25. Februar 1965 [...] mitzuarbeiten und Unterstützung zu geben bei der Ausarbeitung und Begutachtung von bildungspolitischen Dokumenten und Materialien." (Biologische Gesellschaft der DDR o. J.: 2)

Die Gesellschaft unterstützte folglich Bildungs- und Erziehungsprozesse, beteiligte sich an der Erstellung von Lehrwerken und stellte sich die Aufgabe, in Fragen des Biologieunterrichtes beratend zu wirken (Kupke 1974). Der erste Präsident der Biologischen Gesellschaft wurde ihr Initiator Otto Schwarz (Höxtermann \& Höxtermann 2007). Der aus Weimar stammende Botaniker und damalige Rektor der Friedrich-Schiller-Universität in Jena, der durch 192 Gründungsmitglieder gewählt wurde, übte dieses Amt bis zum 1. Oktober 1963 aus (Markert 2006; Hoßfeld 2007a). Die Anwesenden wählten nach einer vorausgegangenen strittigen Diskussion auch die Schulbiologin Anni Kupke (1892-1979) sowie die Schulbiologen Horst Luthardt (*1930), Albert Pietsch (1889-1979) und Erich Taubert (1920-2003) ins Präsidium. Die Diskussion über die Aufnahme fand insbesondere durch das Engagement von Erwin Zabel und Werner Rothmaler ein Ende (Kupke 1959a; Zabel 2007). Bereits auf der ersten Präsidiumssitzung der Biologischen Gesellschaft am 3. Juli 1959 wurde der Aufbau einer Arbeitsgruppe Schulbiologie

71 Nach Kummer (2016b: 139) sollten auf Vorschlag der Akademie der Wissenschaften der DDR „die verschiedenen Wissenschaften Fachgesellschaften bilden“ um „den Austausch neuer Erkenntnisse, Möglichkeiten gemeinsamen Forschens und persönlicher Kontakte [zu] verbessern und [zu] erweitern“. 
beschlossen (Kupke 1959b, 1974). ${ }^{72}$ Ihre Aufgaben sollten darin bestehen, „nach Abstimmung mit dem MfV Einfluß auf die Weiterbildung der Biologielehrer, auf die Lehrpläne für den Biologieunterricht, die Studienpläne des Fernstudiums im Fach Biologie und die Durchführung von Exkursionen zu nehmen" (Kupke 1959b: 383; vgl. auch Kupke 1974).

Das für die Biowissenschaften bedeutende Jahr 1959 - die Jubiläen der Werke Pbilosophie zoologique (Lamarck 1809), On the Origin of Species (Darwin 1859), Die Welträtsel (Haeckel 1899) sowie Haeckels 40. Todestag - waren Anlass, die 1. Tagung der Biologischen Gesellschaft unter dem Titel Arbeitstagung zu Fragen der Evolution durchzuführen (Schwarz 1960). Diese fand vom 20. bis 24. Oktober 1959 in Jena statt. Der Wissenschaftshistoriker Georg Uschmann (1913-1986) betonte in seinem Vortrag, dass „die Wahl Jenas als Ort unserer Arbeitstagung über Fragen der Evolution [...] durch den genius loci bestimmt [wurde] und [...] bewußt an eine Tradition an[knüpft], die in dieser Stadt durch ERNST HAECKEL ihr charakteristisches Gepräge erhielt" (1960: 1, Hervorhebung im Original). Ein Jahr später veröffentlichte der Verlag Gustav Fischer die Vorträge in einem Sammelband. Dieser enthielt wissenschaftstheoretische, -historische sowie rein evolutionsbiologisch argumentierende Artikel und bot noch vorhandenen Vertretern des Lyssenkoismus eine gewisse Plattform (Hoßfeld 2007a). Die Tagung verhalf den Kreis der mitarbeitenden Schulbiologen zu vergrößern, da ,sie [...] bei den teilnehmenden Schulbiologen, besonders bei denen aus dem Thüringer Bereich, den Wunsch nach Bildung von Arbeitszirkeln“" weckte (Kupke 1974: 10). Es konstituierte sich unter der Leitung von Erich Taubert die regionale Arbeitsgruppe Schulbiologie Thüringen. Diese führte teilweise in kleineren Gruppen in Erfurt, Gera und Suhl Weiterbildungen durch. Aufgrund der Initiative der Mitglieder wurden „die Thüringer Schulbiologen bereits als ,Zentrum“ ihres Faches und ,Musterbeispiel' für die Arbeit der Gesellschaft angesehen" (Höxtermann 1997: 51). Weitere Gruppen fanden sich auch in Berlin, Potsdam sowie Leipzig zusammen.

Die erste Tagung der Schulbiologen fand im Jahr 1960 unter der Leitung von Albert Pietsch im Rahmen der Jahreshauptversammlung der Biologischen Gesellschaft an der Humboldt-Universität in Berlin statt. In Folge dieser Aktivitäten wurde auf der 2. Generalversammlung in Dresden im Oktober 1963 durch das Präsidium der Biologischen Gesellschaft der Arbeitsgruppe Schulbiologie der Status einer Sektion zuerkannt. Albert Pietsch, der in diesem Jahr den Ehrentitel Verdienter Lehrer des Volkes erhielt (Hubrich 2013), übernahm den Vorsitz. Noch im selben Jahr fand in Berlin und Potsdam vom 5. bis 9. Dezember eine Tagung der Sektion zum Thema Die Bedentung und die Aufgabe des Biologieunterrichts für die Erriehung und Bildung des Menschen in der sozialistischen Gesellschaft statt. Diese stand im Kontext schulpolitischer Inhalte des 6. Parteitages der SED und war eingebettet in

72 Der Biologischen Gesellschaft gehörten später 11 Sektionen an. Das Präsidium setzte sich aus je zwei Vertretern der Sektionen und der Präsidentin bzw. dem Präsidenten zusammen (Kummer 2016b). 
die Zeit der Ausarbeitung des Gesetzes über das einheitliche sozialistische Bildungssystem. Die Teilnehmerinnen und Teilnehmer der Tagung formulierten Empfehlungen zur Verbesserung des Biologieunterrichtes sowie der Ausbildung der Lehrkräfte und schickten diese an das MfV, das Deutsche Zentralinstitut für Lehrmittel (DZL), das Staatliche Kontor für Unterrichtsmittel und Schulmöbel (SKUS) sowie den Verlag Volk und Wissen (Prehn 1964; Anonymus 1964).

Im Jahr 1964 fanden in Erfurt die 1. Schulbiologentage unter Einbindung von Fachlehrerinnen und Fachlehrern sowie Wissenschaftlerinnen und Wissenschaftlern aus dem In- und Ausland statt. Es wurde eine Mitgliederversammlung durchgeführt, ein Arbeitsprogramm beschlossen und eine Sektionsleitung mit Erich Taubert aus Weimar als Vorsitzenden gewählt (Kupke 1974). Im Verlauf der Mitgliederversammlung lag der Fokus auf der Stellung der Schulbiologie in der DDR (Merbold 1964). In der Folgezeit richteten die Verantwortlichen im Turnus von einem Jahr Schulbiologentage an wechselnden Orten aus, die sich dadurch zu einer zentralen Veranstaltungsform und einem Ort der Weiterbildung für Lehrkräfte entwickelten. Nach einer Konsolidierungsphase wurde 1966 auf den 3. Schulbiologentagen in Berlin ein neues Arbeitsprogramm vorgestellt, das eindeutig den „Biologieunterricht zum Arbeitsgegenstand der Sektion“ erklärte (Baer 1967a: 403). Die neu gewählte Sektionsleitung erhielt den Auftrag, mit dem MfV eine Vereinbarung zu treffen, die es Biologielehrerinnen und -lehrern ermöglichen sollte, an den Tagungen teilnehmen zu können (Kirsch 1966).

Heinz-Werner Baer (1927-2009), der von 1966 bis 1971 den Vorsitzender der Sektion übernahm, klärte in seiner Eröffnungsansprache auf den 4. Schulbiologentagen 1967 die inhaltliche Ausrichtung der Sektion. Er betonte, dass der „Arbeitsgegenstand der Sektion [...] die Schulbiologie und ihre Fachdidaktik“ ist und „die Sektion Schulbiologie [...] damit insbesondere die Belange des BiologieFachunterrichts und die Interessen der Biologielehrer und Fachdidaktiker" vertritt (Baer 1968: 5). Die Veranstaltungen sollten dabei helfen, dass sich „die Gemeinschaftsarbeit von Lehrern und Lehrerbildnern, von Forschern und nach Qualifizierung strebenden Kollegen der Praxis" weiterentwickelt und vertieft (ebd.: 6). In diese Zeit fielen auch die Vorbereitungen der Gründung regionaler Gruppen in Neubrandenburg, Schwerin, Leipzig, Dresden und Berlin. Das Thema der 4. Schulbiologentage in Rostock lautete Neubestimmung des Inhalts und der Gestaltung des Biologieunterrichts und bezog sich auf das Gesetz über das einheitliche sozialistische Bildungssystem (ebd.). Zwischen der Biologischen Gesellschaft und dem MfV wurde eine Vereinbarung erarbeitet, die dazu diente, vermehrt Biologielehrerinnen und lehrer in die Arbeit der Sektion einzubinden (Baer 1967a). Die Themen der folgenden Tagungen entsprachen zumeist der Einführung der neuen Lehrpläne und sollten „mit ihren Ergebnissen ihrer Realisierung dienen“ (Baer 1971: 4). Auch die 
im Jahr 1971 in Karl-Marx-Stadt ${ }^{73}$ durchgeführten 8. Schulbiologentage ${ }^{74}$ zum Thema Evolution und Genetik ${ }^{75}$ verfolgten dieses Ziel. Die besondere Bedeutung dieser Tagung begründete sich auch dahingehend, dass ein großer Teil der Schülerinnen und Schüler ,im 10. Schuljahr mit dem Unterricht über die Evolution und Genetik den Abschluß ihrer Schulbildung auf dem Gebiet der Biologie“ erhielt (ebd.).

Zwischen 1971 und 1984 übernahm Erwin Zabel von der Pädagogischen Hochschule in Güstrow, dessen Laufbahn als Neulehrer begann, den Vorsitz der Sektion (Zabel 2002). Er sah das Ziel der Veranstaltungen u. a. darin, „Erfahrungen auszutauschen und Ergebnisse wissenschaftlicher Untersuchungen zu diskutieren" (Zabel 1974: 3). Nach eigenen Angaben setzte er sich als Vorsitzender die Ziele, den Ernst-Haeckel-Schülerpreis weiter zu profilieren und ein Symposium einzurichten, „das vor allem der Erörterung theoretischer Fragen dienen und insbesondere die Biologie-Didaktiker ansprechen sollte" (Zabel 2007: 224). Neben den bestehenden Kontakten in das sozialistische Ausland war es auch gelungen, die westdeutschen Biologie-Didaktiker Ulrich Kattmann und Roland Hedewig einzuladen. Die Themen der 9. und 10. Schulbiologentage stellten insofern eine Zäsur dar, da sie nicht mehr wie bisher „mit der Einführung von Schwerpunktstoffgebieten des neuen Lehrplans konform" gingen (Baer 1975: 8). Sie fokussierten auf das Experiment und die Unterrichtsmittel im Biologieunterricht, die jahrgangsübergreifend und von allgemeingültigem Interesse waren. Mit der 12. Tagung im Jahr 1975 konnte ein Rekord aufgestellt werden, denn sie war mit 441 Teilnehmern die größte Veranstaltung in der Geschichte der Schulbiologentage (Zabel 2007). Sie wurde gemeinsam mit Schulbiologinnen und -biologen aus Polen durchgeführt und fand in Cottbus zum Thema Landeskultur und Umweltschutz. im Biologieunterricht statt. Das Thema war von allgemeinem Interesse, da sich aufgrund der ökonomischen Entwicklung in den 1960er Jahren „das Aufgabenspektrum des Naturschutzes auf Fragen des Umweltschutzes erweitert" hatte (Behrens \& Hoffmann 2013: 523). ${ }^{76}$ Die 13. Schulbiologentage im Jahr 1976 stellten erneut

73 Anlässlich des Karl-Marx-Jahres wurde die Stadt Chemnitz durch Otto Grotewohl (1894-1964) am 10. Mai 1953 in Karl-Marx-Stadt umbenannt. Die Rückbenennung in erfolgte am 1. Juni 1990 im Zuge der deutschen Wiedervereinigung.

74 Geplanten, organisierten und gestaltetet wurde die Tagung durch die Lehr- und Forschungsgruppe Methodik der Sektion Biologie der Friedrich-Schiller-Universität Jena (Leitung der Sektion Schulbiologie der Biologischen Gesellschaft in der DDR 1971). Wie in vorhergehenden Tagungen übernahm auch das Sekretariat der Biologischen Gesellschaft „einen großen Teil der organisatorischen und gesellschaftlichen Vorbereitungen“ (Baer 1971: 5).

75 Mit dem Thema Evolution und Genetik wurden Fachdisziplinen ausgewählt, ,die in dem ab September 1971 [...] einzuführenden Lehrplan der Klasse 10 im Mittelpunkt stehen“ (Baer 1971: 4).

76 Anfang der 1960er Jahre wurden Vorschläge für ein Landeskulturgesetz erarbeitet, das mit Beschluss vom 14. Mai 1970 das Naturschutzgesetz von 1954 ablöste. Einige wesentliche Grundsätze und Aufgaben des neuen Gesetzes waren die Gestaltung und Pflege der Landschaft, der Schutz der heimatlichen Natur, die Nutzung und der Schutz des Bodens, der Gewässer und 
das Thema Unterrichtsmittel in den Fokus der Auseinandersetzung. Auf dieser Tagung debattierten die Anwesenden auch über Grundsätze bei der Gestaltung neuer Unterrichtsmittel sowie über Probleme bei der Gestaltung von Schullehrbüchern und Unterrichtshilfen (Fehling 1976). Ferner sollte durch eine „Erhöhung der Effektivität der kommunistischen Erziehung der Kinder und Jugendlichen und des Niveaus ihrer Ausbildung" ein Beitrag zur Vorbereitung des 9. Parteitages der SED geleistet werden (ebd.: 440). Die Wahl des Themas und des Austragungsortes der 15. Schulbiologentage im Jahr 1978 war auch politisch motiviert. Sie stand im Kontext der Vorbereitung des 8. Pädagogischen Kongresses und des 30. Jahrestages der DDR (Fehling \& Kurze 1978). Auch die 19. Schulbiologentage dienten der Orientierung hin zu einer kommunistischen Erziehung, wie sie auf dem 8. Pädagogischen Kongress sowie dem 10. Parteitag der SED gefordert wurde (Heinzel 1981).

Im Anschluss an die 21. Tagung 1984 in Erfurt bestimmte die Wahlversammlung der Sektion Schulbiologie eine neue Leitung (Gehlhaar 1985a). In dessen Folge führte bis 1990 Ingrid Heinzel (*1939), Leiterin der Biologiemethodik an der Pädagogischen Hochschule Dr. Theodor Neubauer in Erfurt/Mühlhausen und später an der Friedrich-Schiller-Universität in Jena, den Vorsitz der Sektion. Die Teilnehmer der 22. Schulbiologentage von 1986 strebten an, „das marxistischleninistische Tätigkeitskonzept für den naturwissenschaftlichen Unterricht [...] zu erörtern" (Gehlhaar 1985b: 430). Erstmalig integrierten die Veranstalter auch eine Posterdiskussion (Neupert 1987). Auf der Wahlversammlung der Sektion während der 23. Schulbiologentage in Halle im Jahr 1988 lag die Mitgliederzahl bei 371 (Gehlhaar 1988).

In der Gesamtschau zählte die Sektion Schulbiologie innerhalb der Biologischen Gesellschaft (in) der DDR zu den mitgliederstärksten Gruppen. Die Vorträge und Themen auf den jährlichen Schulbiologentagungen betonten die vielfältige inhaltliche Arbeit an der Schnittstelle von Fachdidaktik, Fachwissenschaft und wissenschaftstheoretischen Diskursen im und um den Biologieunterricht (Tab. 9). Durch Veröffentlichungen in Tagungsbänden, den Mitteilungen der Sektion Schulbiologie 77 und der Zeitschrift Biologie in der Schule wurden die Ergebnisse adressatenorientiert aufbereitet und einem breiten Leserkreis zugänglich gemacht. Ferner erstreckte sich die Arbeit der Sektion auch auf die Verleihung des ErnstHaeckel-Scbülerpreises, die Ausrichtung von Symposien zu theoretischen Fragen der Biologiedidaktik, die Vermittlung von Weiterbildungskursen sowie die Durchführung von Exkursionen. Mit der Auflösung der Biologischen Gesellschaft im Jahr 1990, die bei ihrer Auflösung etwa 1500 Mitglieder in 11 Sektionen aufwies, endete nachfolgend auch die Geschichte der Sektion Schulbiologie (Zabel 2002, 2007).

der Wälder, die Reinhaltung der Luft, der Schutz vor Lärm sowie die Nutzbarmachung und schadlose Beseitigung der Abprodukte (Anonymus 1969a; Behrens \& Hoffmann 2013).

77 Die Mitteilungen der Sektion Schulbiologie der Biologischen Gesellschaft erschienen in zwangloser Folge und wurden den Mitgliederinnen und Mitgliedern der Sektion zugesandt. 
Tabelle 9: Themen der Schulbiologentage (verändert nach Zabel 2007)

\begin{tabular}{|c|c|c|c|}
\hline Nr. & Jahr & Ort & Thema \\
\hline 1 & 1964 & Erfurt & Mikrobiologie in Wissenschaft, Technik und Unterricht \\
\hline 2 & 1965 & Leipzig & $\begin{array}{l}\text { Anthropologie und Gesundheitserziehung in Forschung und } \\
\text { Unterricht }\end{array}$ \\
\hline 3 & 1966 & Berlin & $\begin{array}{l}\text { Probleme der allgemeinen und angewandten Entomologie und ihre } \\
\text { Bedeutung für den Fachunterricht }\end{array}$ \\
\hline 4 & 1967 & Rostock & $\begin{array}{l}\text { Zur Neubestimmung des Inhalts und der Gestaltung des } \\
\text { Biologieunterrichts }\end{array}$ \\
\hline 5 & 1968 & Frankfurt (Oder) & Biologieunterricht und naturwissenschaftliche Bildung \\
\hline 6 & 1969 & Dresden & $\begin{array}{l}\text { Selbständigen Schülertätigkeit im Biologieunterricht und in der } \\
\text { außerschulischen Arbeit }\end{array}$ \\
\hline 7 & 1970 & Güstrow & $\begin{array}{l}\text { Zum Inhalt und zur Gestaltung des Physiologie- und } \\
\text { Ökologieunterrichts in der allgemeinbildenden Schule der DDR }\end{array}$ \\
\hline 8 & 1971 & Karl-Marx-Stadt & Evolution und Genetik im Biologieunterricht \\
\hline 9 & 1972 & Potsdam & Das Experiment im Biologieunterricht \\
\hline 10 & 1973 & Halle (Saale) & $\begin{array}{l}\text { Unterrichtsmittel im Biologieunterricht - erkenntnistheoretische, } \\
\text { wahrnehmungspsychologische und methodische Aspekte }\end{array}$ \\
\hline 11 & 1974 & Schwerin & $\begin{array}{l}\text { Die Gestaltung des Lernens im Biologieunterricht als zunehmend } \\
\text { schöpferischer Prozess }\end{array}$ \\
\hline 12 & 1975 & Cottbus & $\begin{array}{l}\text { Landeskultur und Umweltschutz im Biologieunterricht (gemeinsam } \\
\text { mit Polen) }\end{array}$ \\
\hline 13 & 1976 & $\begin{array}{l}\text { Neubranden- } \\
\text { burg }\end{array}$ & $\begin{array}{l}\text { Methoden, Verfahren und Einsatz von Unterrichtsmitteln zur } \\
\text { Führung des Erkenntnisprozesses im Biologieunterricht }\end{array}$ \\
\hline 14 & 1977 & Leipzig & $\begin{array}{l}\text { Die Gestaltung des Aneignungsprozesses im Biologieunterricht } \\
\text { unter besonderer Berücksichtigung des Festigens und Anwendens }\end{array}$ \\
\hline 15 & 1978 & Berlin & $\begin{array}{l}\text { Ergebnisse und Entwicklungstendenzen in den Biowissenschaften } \\
\text { und die Gestaltung des Biologieunterrichts als erkenntnisintensiver, } \\
\text { erziehungswirksamer Prozess }\end{array}$ \\
\hline 16 & 1979 & Magdeburg & $\begin{array}{l}\text { Literarische Arbeitsmittel und ihr Einsatz für die biologische Bildung } \\
\text { und Erziehung der Schüler }\end{array}$ \\
\hline 17 & 1980 & Rostock & Grundlegendes Wissen und Fachkoordination im Biologieunterricht \\
\hline 18 & 1981 & Güstrow & $\begin{array}{l}\text { Beitrag der Arbeitsgemeinschaften nach Rahmenprogramm und des } \\
\text { fakultativen Biologieunterrichts zur Entwicklung sozialistischer } \\
\text { Persönlichkeiten }\end{array}$ \\
\hline 19 & 1982 & Mühlhausen & Erziehungswirksame Gestaltung des Biologieunterrichts \\
\hline 20 & 1983 & Jena & Leistungsermittlung und Leistungsbewertung im Biologieunterricht \\
\hline 21 & 1984 & Erfurt & $\begin{array}{l}\text { Der Beitrag des Biologieunterrichts zur Förderung einer gesunden } \\
\text { Lebensweise }\end{array}$ \\
\hline 22 & 1985 & Köthen & Vortragsveranstaltung \\
\hline 23 & 1986 & $\begin{array}{l}\text { Neubranden- } \\
\text { burg }\end{array}$ & $\begin{array}{l}\text { Wirksame Gestaltung der Lehrer- und Schülertätigkeit im } \\
\text { Biologieunterricht }\end{array}$ \\
\hline 24 & 1987 & Sonneberg & Der Mensch als soziales Wesen \\
\hline 25 & 1988 & Halle (Saale) & Weiterentwicklung des Biologieunterrichts \\
\hline 26 & 1989 & Schwerin & Vortragsveranstaltung \\
\hline 27 & 1990 & Magdeburg & Auflösung der Sektion \\
\hline
\end{tabular}


Ausbildung und Biologiedidaktik

Die erste Fachmethodik mit dem Titel Zur Methodik und Praxis des Biologieunterrichts an der Grundschule erschien in der Reihe Lernen und Lehren bereits im Jahr 1948. Der Verfasser Willi Lemke (1893-1973) beteiligte sich 1945 beim Aufbau einer Abteilung Volksbildung in der Gemeinde Stansdorf und übernahm die Leitung einer Schule. Auch bei der Beschaffung und Ausbildung neuer Lehrkräfte wirkte er maßgeblich mit. Er leitete Umschulungskurse für Biologie- und Heimatkundelehrerinnen und -lehrer und plante Kurse für Neulehrerinnen und -lehrer. Ferner war er bis etwa 1954 Lehrbuchautor und Herausgeber der ersten Biologieschulbücher. ${ }^{78}$ Seine unmittelbare Beschäftigung mit dem Biologieunterricht endete im Jahr 1954, als er von Otto Schwarz als Oberassistent und Kustos an das Herbarium Haussknecht der Friedrich-Schiller-Universität Jena berufen wurde. Neben Rothmaler war Lemke „entscheidend für das Zustandekommen und die Weiterentwicklung aller botanischen Bestimmungsbücher [...] [des] Verlages verantwortlich“ (Anonymus 1973: 251; vgl. auch Anonymus 1963).

Unter dem Titel Didaktik des Biologieunterrichts erschien im Jahr 1962 ein erstes umfangreiches Lehrbuch. Es wurde von Albert Uhlig (1896-1965) und einem Autorenkollektiv herausgegeben. Die Biologiedidaktik ${ }^{79}$ dieser Zeit gliederte sich in die allgemeine, spezielle sowie vergleichende und historische Biologie-Didaktik (In- und Ausland). Die allgemeine Biologie-Didaktik umfasste den theoretischen Anteil mit den Schwerpunkten Gegenstand, Stoff, Wert, Repräsentation, Methoden und Lehrkraft. Aus diesen Bereichen ergaben sich Überlegungen zu Stoffkreisen, Stoffgebieten, Stoffeinheiten und Unterrichtseinheiten, die sich auf die spezielle Gestaltung der unterrichtlichen Arbeit auswirkten. Die spezielle BiologieDidaktik umfasste mit der unterrichtlichen und außerunterrichtlichen Arbeit den praktischen Teil. Die Gestaltung der unterrichtlichen Arbeit verband Theorie und Praxis mit speziellen und allgemeinen Betrachtungen sowie Überlegungen zur Unter,- Mittel und Oberstufe (Grönke \& Kirsch 1969). Als Sektion Schulbiologie gehörte die Biologiedidaktik bzw. Biologiemethodik der Biologischen Gesellschaft der DDR an (Berck \& Graf 2010).

78 Er verfasste fast vollständig die von 1950 an herausgegebene erste Serie neuer Lehrbücher der demokratischen Schule (Anonymus 1963).

79 Allgemein waren die Aufgabenfelder der Biologiedidaktik ein Bestandteil der Wissenschaften in der DDR, zu denen die Naturwissenschaften und Gesellschaftswissenschaften zählten.

Innerhalb der Gesellschaftswissenschaften verortete sich die Pädagogik, zu der die Disziplinen Familien-, Vorschul-, Schul-, Hochschul- und Erwachsenenpädagogik sowie Jungpioniere (JP) und Freie Deutsche Jugend (FDJ) gehörten. Der Bereich der Schulpädagogik befasste sich mit Sonderschulen, allgemeinbildenden Schulen und Berufsschulen. Die Pädagogik der allgemeinbildenden Schulen setzte sich aus den Aspekten Geschichte der Pädagogik, Didaktik sowie allgemeine Pädagogik zusammen. Die Didaktik wiederum untergliederte sich in generelle und spezielle Didaktik bzw. Fachdidaktik, in der die Biologie-Didaktik verortet war (Grönke \& Kirsch 1969). 
In den 1960er Jahren attestierte Hagen (1964) einen unzureichenden Biologieunterricht, der den Anforderungen der Gesellschaft und der Wissenschaften nicht gerecht wird. Er forderte daher von den Biologielehrerinnen und -lehrern den Willen zur Weiterqualifikation sowie ,eine bessere Organisation der Lehrerfortbildung“ (ebd.: 32). Das Zentralinstitut für Weiterbildung der Lehrer und Erzieher gab erstmalig 1964 ,Themenvorschläge für die fachliche Weiterbildung der Biologielehrer mit abgeschlossener Ausbildung heraus“" (Tille 1993b: 268, Hervorhebung im Original). Die Qualifizierung erfolgte über die Teilnahme an zentralen Veranstaltungen und ,vor allem im Selbststudium wissenschaftlicher Literatur“ (ebd.). Auf pädagogische Literatur konnten Lehrerinnen und Lehrer in der Pädagogischen Zentralbibliothek in Berlin und Leipzig zugreifen. Diese Einrichtung stellte Ende der 1960er Jahre etwa 600000 Titel leihweise zur Verfügung (Anonymus 1969b). Auch im Verlag Volk und Wissen erschienen insbesondere in den 1960er Jahren mehrere methodische Schriften, mit dem Ziel die Lehrkräfte bei ihren Unterrichtsvorbereitungen zu unterstützen. Dazu gehörten beispielsweise die Bücher:

- Mikroskopie für Lehrer und Naturfreunde (1955) von Werner Schlüter,

- Biologische Versuche im Unterricht (1960) ${ }^{80}$ von Heinz-Werner Baer,

- Schulgartenpraxis (1962) von Karl Krüger und Ulrich Millat,

- Die Sicherung der Lernergebnisse im Biologieunterricht (1962) von Gerhard Dietrich,

- Der W ald und die Forstwirtschaft (1963) von Johannes Blanckmeister und Erwin Kienitz,

- Biologische Arbeitstechniken (1964) von Heinz-Werner Baer und Ottokar Grönke,

- Biologische Aufgabensammlung (1965) von Joachim Günther und Karl Scheiding,

- Wald, Hecke, Strand (1966) von Kurt Lobeck und Irmtraud Meincke,

- Praxis der Süßwasserbiologie (1966) von Alfred Schubert,

- Zum Problem der sexuellen Belehrung durch den Biologielehrer (1967) von Werner Kirsch,

- Biologie Arbeitsgemeinschaften, Exkursionen (1969) von Rudolf Hundt und Erhard Kreße sowie

- Mikrobiologisches Praktikum (1975) von Helga Schröder.

Neben den institutionellen Wegen der Ausbildung bot auch die Fachzeitschrift Biologie in der Schule Möglichkeiten für die Weiterbildung an. So veröffentlichte die Zeitschrift 1969 ein verbindliches Lehrprogramm zur Weiterbildung in Kursen für Lehrer im Fach Biologie und 1971 darauf aufbauende Studienhilfen für ausgewählte fachwissen-

80 Ab der 7. stark bearbeiteten Auflage erschien das Werk von Heinz-Werner Baer mit neuem Einband unter dem Titel Biologische Schulexperimente. 
schaftliche und didaktisch-methodische Themen des Lehrprogramms. ${ }^{81} \mathrm{Im}$ Verlauf der Entwicklung des Ausbildungssystems war die Ausbildung von Lehrerinnen und Lehrern im Fach Biologie an ein zweites Fach gebunden. Ein Blick auf das Ausbildungsjahr 1973/74 verdeutlicht exemplarisch die Möglichkeiten und Besonderheiten der Fachrichtung. Erstens war die Auswahl an Kombinationen begrenzt und an inhaltliche Nähe gebunden, zweitens konnte das Fach Biologie in Verbindung mit einer Ausbildung zum Pionierleiter erfolgen und drittens war für die Kombination Biologie und Körpererziehung eine Vielzahl von Ausbildungsstätten vorhanden (Tab. 10).

Tabelle 10: Ausbildungsmöglichkeiten für Fachlehrerinnen und -lehrer im Fachgebiet Biologie für das Studienjahr 1973/74 (verändert nach Richter 1972)

\begin{tabular}{|l|l|}
\hline Grundstudium & Hochschulen \\
\hline Biologie, Chemie & Friedrich-Schiller-Universität Jena \\
\hline Chemie, Biologie & Humboldt-Universität zu Berlin \\
& Karl-Marx-Universität Leipzig \\
& Pädagogische Hochschule „Karl Liebknecht“ Potsdam \\
& Pädagogisches Institut Köthen \\
\hline Biologie, Körpererziehung & Martin-Luther-Universität Halle \\
& Ernst-Moritz-Arndt-Universität Greifswald \\
& Pädagogische Hochschule „Karl Liebknecht“ Potsdam \\
& Pädagogische Hochschule „Liselotte Hermann“ Güstrow \\
& Pädagogische Hochschule „Nadeshda Konstantinowna \\
& Krupskaja“ Halle \\
& Pädagogisches Institut Köthen \\
& Pädagogisches Hochschule „Dr. Theodor Neubauer“ Er- \\
& furt/Mühlhausen \\
\hline Körpererziehung, Biologie & Martin-Luther-Universität Halle \\
& Friedrich-Schiller-Universität Jena \\
\hline Geographie, Biologie & Ernst-Moritz-Arndt-Universität Greifswald \\
\hline Pionierleiter, Biologie & Pädagogische Hochschule „Nadeshda Konstantinowna \\
& Krupskaja“ Halle \\
\hline
\end{tabular}

Da die biologischen Wissenschaften „weltweit große Fortschritte“ (Kummer 2016a: 124) machten und neue Erkenntnisse in die Lehrpläne aufgenommen werden mussten, publizierte der Verlag Volk und Wissen insbesondere in den 1970er Jahren unter dem Sammeltitel Fachinformationen für Lehrer vermehrt Werke, „die dem Lehrer das fachliche und methodische Erschließen des Lehrplaninhalts erleichtern sollten“ (Dietrich \& Kummer 1979: 418). ${ }^{82}$ Dazu zählten die Werke:

81 Das Lehrprogramm wurde erstellt vom Zentralinstitut für Weiterbildung der Lehrer und Erzieher beim MfV in der DDR (1969). Die Studienhilfen wurden ausgearbeitet vom Zentralinstitut für Weiterbildung der Lehrer und Erzieher Ludwigsfelde (1971).

82 Kummer (2016a: 125) spricht davon, dass sie „- für Biologie - die Herausgabe spezieller Fachliteratur für Lehrer im Schulbuchverlag durchgesetzt“ hat und zwar „mit Erfolg, wie die Absatzzahlen belegen“. 
- Beiträge zur Abstammungslehre (ab 1964) ${ }^{83}$ von Helmut Böhme, Rudolf Hagemann und Rolf Löther,

- Stoffwechsel der Pflanzen (1966) von Klaus Müntz,

- Pflanzen und Tiere tropischer Gebiete (1967) von Gerhard Grümmer,

- Biographien bedeutender Biologen (1977) von Werner Plesse und Dieter Rux,

- Der Mensch. Anatomie, Physiologie, Ontogenie (1979) von Karl Sommer sowie die Bände

- Exkursionsflora von Werner Rothmaler und Exkursionsfauna von Erwin Stresemann (Anonymus 1985, vgl. auch Kummer 2016a).

Im Jahr 1976 veröffentlichte der ehemalige Neulehrer Gerhard Dietrich (19271986) ${ }^{84}$ zusammen mit einem Autorenkollektiv ein weiteres Lehrbuch. Es erschien unter dem Titel Methodik - Biologieunterricht im Verlag Volk und Wissen. Eine Vereinbarung zwischen den sozialistischen Ländern hatte die Umbenennung der Biologiedidaktik in Biologiemethodik zur Folge. Die Begriffe waren jedoch inhaltlich identisch. Das Werk stellte ,als einzige geschlossene Abhandlung“ ein „lang erstrebte[s] Hochschullehrbuch“ dar, das versuchte, „eine zweckmäßige Verbindung von biologiemethodischer Theorie und Unterrichtspraxis herzustellen“ (Dietrich 1976. 19).

„Die ,Methodik Biologieunterricht' stellt einen Versuch dar, den erreichten Erkenntnisstand auf wesentlichen Gebieten der Biologiemethodik geschlossen darzustellen und damit den in der Schulpraxis wirkenden Biologielehrern und den Lehrerstudenten der Fachrichtung Biologie eine theoretische Hilfe für die Interpretation und Umsetzung der Biologielehrpläne zu geben." (Dietrich 1976: 9)

An allen Universitäten und an fünf Pädagogischen Hochschulen konnten sich elf eigenständige Lehrstühle für die Biologie-Didaktik etablieren (Zabel 2002; Berck \& Graf 2010). Sie widmeten sich spezifischen Forschungsschwerpunkten, was zur Effektivität der Arbeitsgruppen beitrug. Beispielsweise fokussierte Ingrid Heinzel in Jena auf Leistungsermittlung und -bewertung, Rudolf Hundt (1927-2016) in Halle auf Ökologie und Unterrichtsmittel sowie Ottokar Grönke (*1929) in Potsdam auf die Geschichte des Biologieunterrichtes und das Experimentieren (Tab. 11). Die Lehrstühle erarbeiteten parallel zur Grundlagenforschung die Lehrwerke

83 Trotz der Beteiligung führender Wissenschaftlerinnen und Wissenschaftler, lehnte der Verlag das Manuskript zunächst ab. Nach einiger Diskussion konnten dann aber doch Teil 1 und 2 im Jahr 1964 und Teil 3 zwei Jahre später erscheinen. Der Verkaufserfolg des 1. Teils auf der Buchmesse im Jahr 1965 führte schließlich auch zur höheren Auflagen für den 2. und 3. Teil und „später - nach anerkennenden Rezensionen auch im westlichen Ausland - erschienen Teile in einem Band mit ,Prachtausstattung" (Leineneinband mit Schutzumschlag)" (Kummer 2016a: 124).

84 Gerhard Dietrich wurde 1980 zum Generalsekretär der Akademie der pädagogischen Wissenschaften berufen. 
für den Biologieunterrichts und die Unterrichtshilfen für die Klassen 5 bis 12. Die Erarbeitung erfolgte arbeitsteilig, denn für die einzelnen Klassen war jeweils ein Lehrstuhl verantwortlich. Dabei war eine enge Zusammenarbeit mit dem Bereich Biologie der Akademie der Pädagogischen Wissenschaften (Leitung Christa PewsHocke) und der Abteilung Biologie des Verlages Volk und Wissen (Leitung Gertrud Kummer) kennzeichnend.

Tabelle 11: Lehrstühle für Biologiedidaktik der DDR (vgl. Zabel 2002; Berck \& Graf 2010)

\begin{tabular}{|c|c|c|}
\hline Einrichtung & $\begin{array}{l}\text { Lehrstuhlinhaberin und - } \\
\text { inhaber, wiss. Mitarbei- } \\
\text { terin und Mitarbeiter }\end{array}$ & Forschungsschwerpunkte \\
\hline \multicolumn{3}{|c|}{ Universitäten } \\
\hline Berlin & Manfred Kurze & Problemorientierter Biologieunterricht \\
\hline Greifswald & $\begin{array}{l}\text { Johannes Müller, Wulf- } \\
\text { Dieter Lepel }\end{array}$ & $\begin{array}{l}\text { Programmierter Unterricht, Begriffsbildung im Biologieun- } \\
\text { terricht, Strukturen des stofflichen Inhalts }\end{array}$ \\
\hline $\begin{array}{l}\text { Halle- } \\
\text { Wittenberg }\end{array}$ & $\begin{array}{l}\text { Rudolf Hundt, Wolfgang } \\
\text { Lerchner }\end{array}$ & $\begin{array}{l}\text { Unterrichtsmittelkomplexe für den Biologieunterricht, Um- } \\
\text { weltbildung und -erziehung, Urbane Ökosysteme }\end{array}$ \\
\hline Jena & $\begin{array}{l}\text { Joachim Günther, Ingrid } \\
\text { Heinzel }\end{array}$ & Aufgabenkultur und Aufgabengestaltung \\
\hline Leipzig & $\begin{array}{l}\text { Gerhard Dietrich, Karl- } \\
\text { Heinz Gehlhaar }\end{array}$ & $\begin{array}{l}\text { Fähigkeitsentwicklung im Biologieunterricht, Schülervorstel- } \\
\text { lungen }\end{array}$ \\
\hline Rostock & $\begin{array}{l}\text { Heinz Werner Baer, } \\
\text { Frank Horn, Karl Meiß- } \\
\text { ner }\end{array}$ & $\begin{array}{l}\text { Grundwissen aus der Zoologie, Fachübergreifende Bezie- } \\
\text { hungen, Umweltbildung unter regionalem Aspekt als didak- } \\
\text { tische Leitlinie sowie unter dem Blickwinkel der ästheti- } \\
\text { schen Erziehung im Biologieunterricht }\end{array}$ \\
\hline \multicolumn{3}{|c|}{ Pädagogische Hochschulen } \\
\hline $\begin{array}{l}\text { Erfurt- } \\
\text { Mühlhau- } \\
\text { sen }\end{array}$ & $\begin{array}{l}\text { Hans Fischer, Ingrid } \\
\text { Heinzel }\end{array}$ & Erziehung im Biologieunterricht \\
\hline Güstrow & Erwin Zabel & $\begin{array}{l}\text { Grundwissen aus der Botanik, Fakultativer Biologieunter- } \\
\text { richt, Lernkultur }\end{array}$ \\
\hline Halle & Manfred Matzke & Unterrichtsmittel im Biologieunterricht \\
\hline Köthen & $\begin{array}{l}\text { Hans-Joachim Schwier, } \\
\text { Ursula Pälchen }\end{array}$ & Schulbuchgestaltung für den Biologieunterricht \\
\hline Potsdam & Ottokar Grönke & $\begin{array}{l}\text { Geschichte des Biologieunterrichts und der Biologie- } \\
\text { Didaktik, Besonderheiten des Lernens in den Klassen } 11 \\
\text { und } 12 \text { (u. a. Experiment im Biologieunterricht) }\end{array}$ \\
\hline
\end{tabular}

Methodisch bestand seit den 1950er Jahren die Forderung, dass der Biologieunterricht so aufgebaut sein muss, „daß die Entwicklungslehre zur Grundlage des Unterrichts wird“ (Gottschalk 1954: 268). Neben der Vermittlung von Fachwissen sollte „die große ideologische und erzieherische Kraft, die gerade der Biologie innewohnt, so wirksam werden, daß in den Schülern ein wissenschaftliches Weltbild systematisch aufgebaut wird“ (Kupke 1954: 241). In den 1960er Jahren erfolgte eine Bestimmung der Funktion des Biologieunterrichtes im Rahmen sozialistischer Allgemeinbildung (Horn 1987). Mit der Einführung der „polytechnischen 
Das Bildungssystem der DDR und das Schulfach Biologie

Bildung wurden im [...] Biologieunterricht Schülerübungen verstärkt" und „der Experimentalunterricht zu einem Wesensmerkmal moderner naturwissenschaftlicher Bildung“ (Günther \& Uhlig 1969: 109). Im Unterschied zu anderen Fächern begegneten die Schülerinnen und Schüler im Unterrichtsfach Biologie einer Objektvielfalt in Form verschiedener Lebewesen. Zwar stand im Zentrum des Biologieunterrichtes der Umgang mit den Organismen, doch wurden diese der Erarbeitung allgemeiner Naturgesetze zu Grunde gelegt (Anonymus 1960). Biologische Sachverhalte sollten vom Standpunkt des dialektischen Materialismus aus dargelegt werden (Wernecke 1967b: 464). Ein Beitrag zur Vermittlung von Grundkenntnissen des Marxismus-Leninismus war inhärenter Bestandteil des Faches. Ende der 1980er Jahre erfolgte eine „Besinnung auf die Hauptfunktion des Biologieunterrichtes und damit auf seinen spezifischen Beitrag für die Persönlichkeitsentwicklung im Rahmen der in der Schule zu vermittelnden Allgemeinbildung“ (Horn 1987: 210). Noch 1989 betonte die damalige Ministerin für Volksbildung Margot Honecker auf dem 9. und letzten Pädagogischen Kongress: „In unserer Zeit verdient der Biologieunterricht große Aufmerksamkeit“ (1989: 48).

\section{Stundentafel}

Die Ferienzeiten waren für das gesamte Gebiet der DDR einheitlich gestaltet. Mit etwa acht Wochen Sommerferien (Juli bis August), eine Woche Herbstferien (Mitte Oktober), zwei Wochen Weihnachtsferien (Dezember/Januar), drei Wochen Winterferien (Februar) und eine Woche Frühjahrsferien (Mai) umfasste das Schuljahr 40 Unterrichtswochen (Rockstuhl 2006). Dadurch ergaben sich für das Fach Biologie bei einer Wochenstunde 40 Jahresstunden, bei zwei Wochenstunden 80 Jahresstunden und bei drei Wochenstunden 120 Jahresstunden. Da die Stundenanzahl im direkten Zusammenhang mit dem Umfang des Unterrichtsstoffes steht, lassen sich bereits aus der Entwicklung der Stundenvorgaben Rückschlüsse auf den Stellenwert des Faches innerhalb des Fächerkanons schließen. Die Einführung der Grundschule 1946 war mit deutlichen Veränderungen in der bis dahin gültigen Stundentafel verbunden. Das Fach Biologie wurde 1946 „noch nicht gesondert aufgeführt, sondern [war] Bestandteil des Faches Naturkunde" (Geißler 2000: 91). Als Einzelfach erschien es ein Jahr später. Es wurde jedoch lediglich in den Klassen 5 bis 7 für alle Schüler berücksichtigt. Durch die Differenzierung in Kern- und Kursunterricht erhielten Schüler der Kasse 8 in den Sprachkursen keinen Biologieunterricht. Die Schüler der mathematisch-naturwissenschaftlichen Kurse erhielten wahlweise zwei Stunden Biologie oder Chemie (DVV in der SBZ 1947).

Ab dem 1. September 1951 galt für die Grundschule eine neue Stundentafel. Als Begründung wurden die Erfüllung des Fünfjahresplans auf kulturellem Gebiet, die Hebung des wissenschaftlichen Niveaus im Unterricht und der Kampf gegen das Sitzenbleiben angeführt (Uhlig 1970). Mit dem Schuljahr 1951/52 und den damit einhergehenden neuen Lehrplänen setzte ab der Klasse 4 „mit je zwei Wo- 
chenstunden für Geschichte, Biologie und Erdkunde ein vorbereitender Fachunterricht ein“ (Geißler 2000: 288; vgl. auch Tille 1992).

Tabelle 12: Entwicklung der Stundentafel für das Fach Biologie

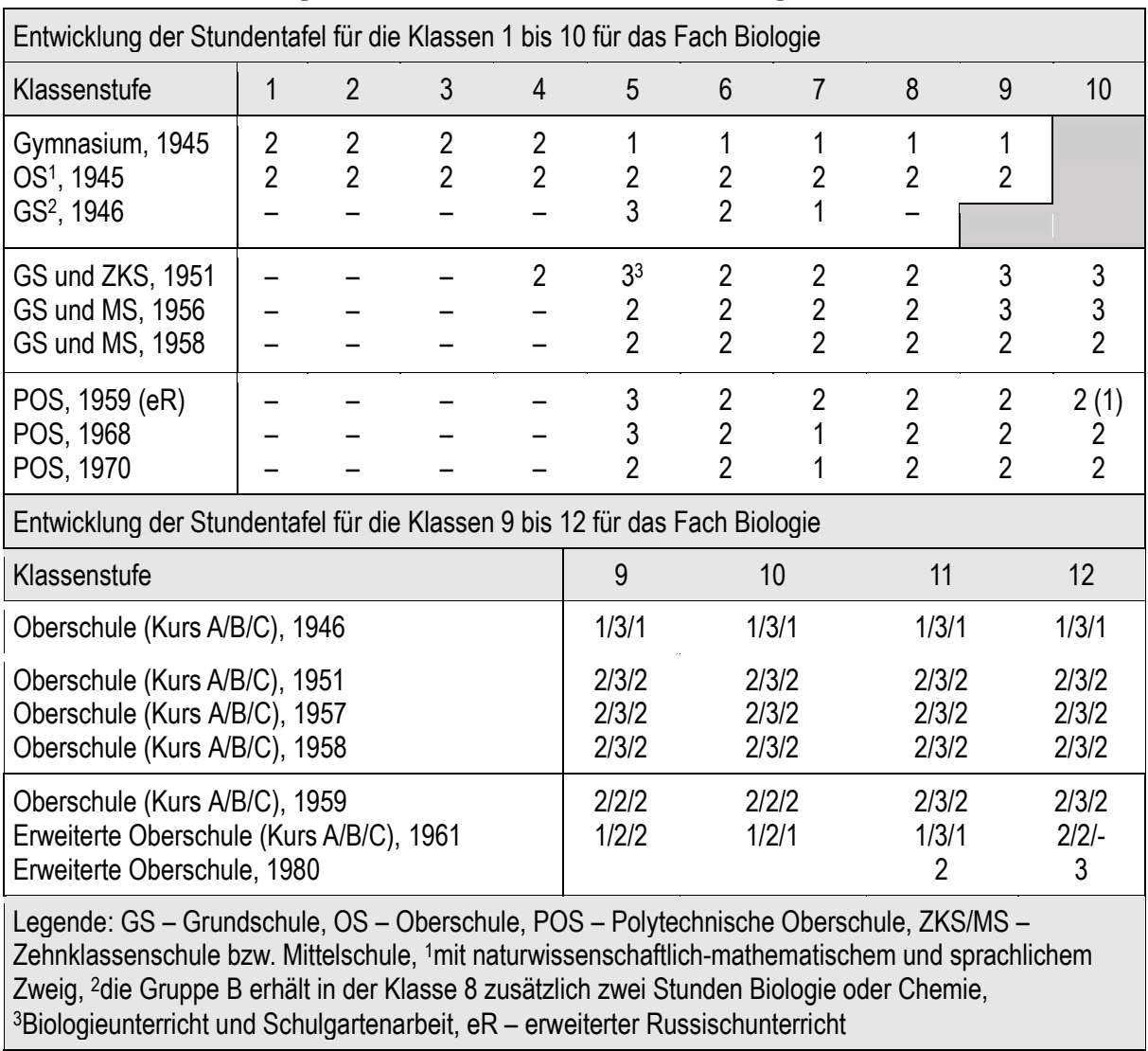

Die weitere Entwicklung zeigt, dass lediglich in den Klassen 6 und 8 die Stundenzahlen konstant blieben. Auf der 3. Parteikonferenz der SED im Jahr 1956 zeigte sich, dass „der naturwissenschaftliche Unterricht nicht mehr den neusten Erkenntnissen entsprach“ und einige Lehrplanthemen differenziert werden müssen, damit „die Landschulen [...] die speziellen Probleme des Dorfes behandeln“ können (Günther \& Uhlig 1969: 94). Auch die neue Stundentafel für das Schuljahr 1958/59 verfolgte neben der Durchsetzung der polytechnischen Bildung das Ziel, eine ,grundlegende Verbesserung des gesamten Unterrichts, besonders der naturwissenschaftlichen Fächer" voranzutreiben (ebd.: 109). In den Stundentafeln der Oberschule spiegelte sich das Kurssystem wider. Für den mathematischnaturwissenschaftliche Kurs standen stets mindestens zwei, teilweise drei Stunden im Fach Biologie zur Verfügung. Waren 1946 noch deutliche Unterschiede zwi- 
schen den Kursen vorhanden, so näherten sich die Stundenzahlen in den 1950er Jahren an (Tab. 12).

\section{Lehrplankonzeption}

In den ersten Biologielehrplänen, die in der ersten Phase der Lehrplanrunde entstanden, waren die Themen für die Jahrgänge 5 und 6 noch nach heimatkundlichphänologischen Kriterien angeordnet. Die Behandlung der Organismen erfolgte hier monografisch in einer morphologisch-ökologisch-ökonomischen Betrachtungsweise. Auf der unmittelbaren Beobachtung und der Sippenkenntnis lag groBer Wert. Die Behandlung von Blütenpflanzen und Wirbeltieren erfolgte zu Beginn, aufgrund von Bekanntheitsgrad, Größe und Lebensweltbezug. Ziel war es, an ausgewählten Organismen Anpassungen erst beschreiben und später erklären zu können (Tille 1992). Bereits die zweite Auflage von 1947 brachte deutliche Veränderungen mit sich. Angaben zu empfohlenen Unterrichtsstunden, die bei der Planungsarbeit und bei der Gewichtung des Stoffes unterstützten und im Lehrplan von 1946 noch vorhanden waren, entfielen. Auch inhaltliche Änderungen traten auf. Während sich im Lehrplan von 1946 die Menschenkunde auf alle Klassen der Grundschule erstreckte, wurde diese Disziplin nun ausschließlich in den Klassen 7 und 8 behandelt (Tab. 13). Der Unterrichtsgegenstand Lebensgemeinschaft $W$ ald, der im Lehrplan von 1946 in Klasse 7 thematisiert wurde, fand einzig im Kursunterricht statt. Auch die Abstammung des Menschen sollte nur im Kursunterricht vermittelt werden. Besonders markant waren die Stoffübersichten zur Pflanzenkunde (Klasse 7) und Tierkunde (Klasse 8), die sich „wie Inhaltsverzeichnisse von Lehrbüchern der Botanik und Zoologie für die Oberschulklassen 9 und 10, ergänzt durch ganz speziellen Stoff“" (Tille 1992a: 324), gliederten.

Tabelle 13: Lehrplankonzeption von 1947

\begin{tabular}{|c|c|c|}
\hline \multicolumn{2}{|c|}{ Klasse } & Inhaltliche Vorgaben \\
\hline \multicolumn{2}{|c|}{ 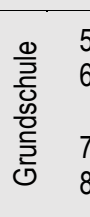 } & $\begin{array}{l}\text { Pflanzen im Garten; Bäume; Vögel; Tiere in Haus, Hof, Wald und Feld } \\
\text { Pflanzen im Garten, am Wegesrand, auf der Wiese; Felder im Jahresverlauf; Tiere und } \\
\text { Pflanzen des Waldes; Tiere des Süßwassers und des Meeres; Insekten } \\
\text { Entwicklungsgeschichte und Systematik der Pflanzen; Menschenkunde } \\
\text { Entwicklungsgeschichte und Systematik der Tiere; Menschenkunde }\end{array}$ \\
\hline $\begin{array}{l}\frac{\Phi}{2} \\
\frac{\bar{D}}{0} \\
\frac{\omega}{\Phi} \\
\frac{0}{0}\end{array}$ & $\begin{array}{r}9 \\
10 \\
11 \\
12\end{array}$ & $\begin{array}{l}\text { Botanik } \\
\text { Zoologie } \\
\text { Menschenkunde } \\
\text { Fragen der Ökologie; Erblehre; Abstammungslehre; Die Abstammung des Menschen; die } \\
\text { Lehre von der Entstehung des Lebens auf der Erde }\end{array}$ \\
\hline
\end{tabular}


Die Biologielehrpläne für die Grundschule von 1951 bis 1953 enthielten am Ende des Jahresplanes eine Zusammenstellung der zu vermittelnden Kenntnisse und Erkenntnisse sowie Fähigkeiten und Fertigkeiten. Eine „Tendenz zur Orientierung an der wissenschaftlichen Systematik" war bei der Stoffauswahl und anordnung erkennbar und der Stoff „vorwiegend nach den großen Organismengruppen zusammengestellt" (Tille 1992b: 383; vgl. auch MfV 1951). $\mathrm{Da}$ „ein hohes Bildungsniveau vorrangig in vielen Einzelerkenntnissen gesehen“ wurde, entstand ein Stoff-Zeit-Problem, das zum exemplarischen Lernen führte (ebd.: 384). Wie im Lehrplan von 1946 verteilten die Verantwortlichen die Menschenkunde auf die Klassenstufen 5 bis 8 (Tab. 14). Die Vererbungs- und Abstammungslehre gewann enorm an Bedeutung, da für sie nun die gesamte zweite Hälfte des 8. Schuljahres zur Verfügung stand. Geprägt waren die Lehrpläne auch durch eine Ideologisierung der Abstammungslehre (Tille 1992b). Mitte der 1950er Jahre „setzte eine breit angelegte Diskussion zur Neugestaltung des Biologieunterrichts an der Grundschule ein" (Tille 1992c: 427). Es standen Fragen des systematischen Aufbaus, der Fasslichkeit und der Veranschaulichung der Inhalte im Vordergrund. Ziel war es, „den Anforderungen der Volkswirtschaft, insbesondere der Landwirtschaft, durch eine entsprechende polytechnische Bildung gerecht [zu] werden" (ebd.). Das führte teilweise zu unterschiedlichen Lehrplänen und Lehrbüchern für die Stadt- und Landschulen und zu einer „'Verlandwirtschaftlichung des Biologieunterrichts“ (ebd.: 428, Hervorhebung im Original; Dietrich \& Kummer 1979).

Tabelle 14: Lehrplankonzeption von 1951

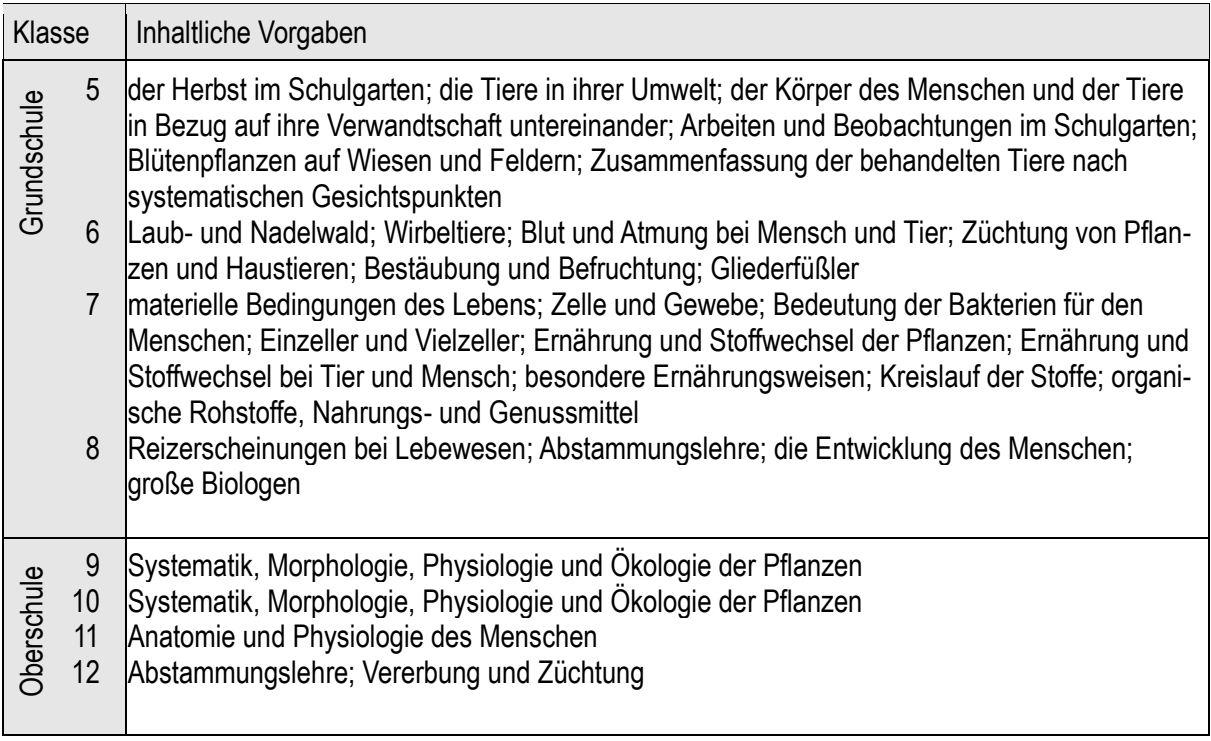


Ab 1959 löste schrittweise die zehnjährige POS die achtjährige Grundschule ab. Für den der Biologieunterricht standen „,drei große Ziele“ im Fokus: „Herausbilden eines wissenschaftlichen Weltbildes [...]; Erarbeitung von Kenntnissen über die wissenschaftlichen Grundlagen der Biologie und [...] Erziehung zu einer neuen, einer sozialistischen Moral“ (Dietrich \& Kummer 1979: 413). Das Lehrplanwerk bestimmte das Niveau der einzelnen Disziplinen von den Zielvorstellungen aus und legte von dort aus Anteil, Umfang, Stellung und Inhalt fest (Günther \& Uhlig 1969). Verschiedentlich waren noch „differenzierte Stoffe für Stadt- und Landschulen vorgesehen", doch die Schwerpunkte des Biologielehrgangs trafen [...] für Stadt- und Landschulen gleichermaßen zu“" (Dietrich \& Kummer 1979: 414). Zur „Diskussion über das Fach Biologie gehörten Anschaulichkeit, Faßlichkeit, Wissenschaftlichkeit und Systematik des Unterrichts, die Behandlung von Lebensgemeinschaften, die Verbindung von Theorie und Praxis, die weltanschauliche Bildung und Erziehung“ (Tille 1993a: 134). Es „entstand ein grundsätzlich linear aufgebauter Lehrplan, der das Prinzip der Anschaulichkeit berücksichtigte“ (ebd.: 135; vgl. auch MfV 1959, 1961; Tab. 15). An den zentral erarbeiteten Lehrplänen beteiligten sich Biologiedidaktikerinnen und Biologiedidaktiker sowie Lehrerinnen und Lehrer. Nach der Bestätigung durch das MfV waren diese interpretierbar und verbindlich zugleich. Parallel erfolgten Untersuchungen auf ihre Wirksamkeit.

Tabelle 15: Lehrplankonzeption ab 1959

\begin{tabular}{|c|c|}
\hline Klasse & Inhaltliche Vorgaben \\
\hline 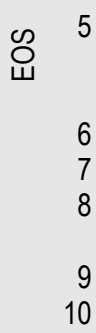 & $\begin{array}{l}\text { Herbstarbeiten im Schulgarten und auf den Feldern der LPG; im Wald, im Park und in Kleinge- } \\
\text { hölzen; Herbstarbeiten im Schulgarten; heimische Tiere; Garten, Wald und Flur im Frühling } \\
\text { und Sommer } \\
\text { Blütenpflanzen der Heimat; im Schulgarten; Wirbeltiere } \\
\text { Zellenlehre; Mikrobiologie; mehrzellige wirbellose Tiere } \\
\text { Anatomie und Physiologie der Pflanze; Anatomie und Physiologie des Menschen; der Wald als } \\
\text { Biozönose } \\
\text { Biologie und Landwirtschaft; Anatomie und Physiologie des Menschen } \\
\text { Züchtung von Pflanzen und Tieren; Entwicklungslehre }\end{array}$ \\
\hline $\begin{array}{rr}0 & 9 \\
0 & 10 \\
& 11 \\
& 12\end{array}$ & $\begin{array}{l}\text { Biozönose; Überblick über das System des Organismen } \\
\text { Biozönose; Überblick über das System des Organismen; Anatomie und Physiologie des Men- } \\
\text { schen } \\
\text { Anatomie und Physiologie des Menschen; ausgewählte Gebiete aus der Physiologie der } \\
\text { Pflanzen und Tiere } \\
\text { die Lehre von der Evolution der Organismen }\end{array}$ \\
\hline
\end{tabular}


Fortschritte in den Biowissenschaften, speziell in der Molekularbiologie, führten in den 1950er und 1960er Jahren zu einer weltweiten ${ }^{85}$ und

„oft kontrovers geführte Diskussion zu der Frage, welche Konsequenzen daraus für die Zielsetzung und den Inhalt (das ,Bildungsgut") des Biologieunterrichts abgeleitet werden sollten. [...] Auf diesem Hintergrund erfolgten im Zeitraum von 1964 bis etwa 1972 in Abstimmung mit dem Bereich Biologie-Didaktik der Universität Rostock [...] Untersuchungen zum Grundwissen für den Biologieunterricht [...]. Ziel war dabei auch, stärker zwischen Elementen des Inhalts, die sicher und anwendungsbereit beherrscht werden müssen, und solchen zu unterscheiden, die nur informativ mitgeteilt werden sollten." (Zabel 1998: 46)

Zwischen 1966 und 1971 wurden schließlich schrittweise präzisierte Lehrpläne gültig, „die eine eindeutig höhere Qualität aufwiesen“ (Dietrich \& Kummer 1979: 416). In diesen Plänen

„sollte das Grundwissen exakter bestimmt und präziser ausgewiesen, die stofflichen Anforderungen stärker nach dem grundlegendem Wissen und Können festgelegt, Ausgangs- und Endniveau innerhalb eines Teillehrgangs genauer bestimmt, notwendige Schülertätigkeiten ausgewiesen sowie methodische Hinweise zur Intensivierung des Lernens eingefügt werden“ (Tille 1993b: 268).

Dabei „gab es eine intensive Diskussion zur inhaltlichen ,Linienführung dieser Pläne“ (Zabel 1998: 2). Allgemein spielte in dieser Zeit „die Leitlinienproblematik eine zunehmend größere Rolle“, in deren Ergebnis sechs „fachinnere stoffliche Leitlinien und dazugehörige Aspekte [...] in die Diskussion eingeführt" und in das Lehrbuch Methodik Biologieunterricht (1976) aufgenommen wurden (ebd.: 5f.; vgl. dazu auch Dietrich 1976: 47f.):

- Einheitlichkeit und Mannigfaltigkeit der lebenden Natur,

- Evolution der lebenden Natur,

- Erkennbarkeit der lebenden Natur,

- Gesellschaftliche Bedingtheit der Anwendung von Erkenntnissen über die lebende Natur,

- Methoden und Arbeitsverfahren.

Grundlegend gliederte sich die Lehrplankonzeption der präzisierten Pläne für das Fach Biologie in vier Stufen. Die erste Stufe umfasste morphologische Betrach-

85 Zabel (1998: 46) resümiert, dass „das 1959 von amerikanischen Wissenschaftlern entwickelte BSCS-Curriculum (Biologie [sic] Science Curriculum Study) mit seinen drei Versionen für Klasse 10 (der ökologischen Version, der entwicklungsgeschichtlichen Version und molekularbiologischen Version) und das nachfolgende englische Nuffield-Projekt (1962) [...] das Denken und Handel“ damals stark beeinflusste. 
tungen ohne Zellbau in den Klassenstufen 5 und 6 sowie mit Zellbau in den Klassenstufen 7 und 10. Die zweite Stufe beinhaltete physiologische Betrachtungen mit den Themen Mensch in Klasse 8 und Pflanze in Klasse 9. Die dritte Stufe war auf allgemeinbiologische Betrachtungen ausgerichtet mit den Disziplinen Ökologie in Klasse 9 und Genetik sowie Phylogenetik in Klasse 10. Allgemeinbiologische Betrachtungen kamen auch in den EOS zum Tragen und umfassten die Fachbereiche Systematik und Ökologie in Klasse 11 sowie Physiologie und Genetik in Klasse 12 (Grönke \& Kirsch 1969; vgl. auch MfV 1968a, 1968b, 1969; Dietrich et al. 1979; Tab. 16).

Tabelle 16: Lehrplankonzeption ab 1965

\begin{tabular}{|c|c|}
\hline Klasse & Inhaltliche Vorgaben \\
\hline $\begin{array}{r}5 \\
6 \\
7 \\
8 \\
9 \\
10\end{array}$ & $\begin{array}{l}\text { Wirbeltiere; Samenpflanzen } \\
\text { Samenpflanzen; Wirbellose } \\
\text { Mikroskopieren, Zellenlehre; Einzeller; blütenlose Pflanzen } \\
\text { Anatomie, Physiologie und Hygiene des Menschen } \\
\text { Pflanzenphysiologie; Ökologie } \\
\text { Genetik; Abstammungslehre; Züchtung von Pflanzen und Tieren; Wiederholung, Systematisie- } \\
\text { rung, Ausblick }\end{array}$ \\
\hline œ 11 & $\begin{array}{l}\text { aus der Systematik der Pflanzen und Tiere; Ökologie; Physiologie der Pflanzen und Tiere } \\
\text { Physiologie der Pflanzen und Tiere; Genetik; die Bedeutung der Biologie für die Gesellschaft }\end{array}$ \\
\hline
\end{tabular}

Obwohl die präzisierten Pläne ,inhaltlich [...] einen relativ abgeschlossenen Lehrgang der systematischen Behandlung von Lebewesen nach taxonomischen Gesichtspunkten“ bildeten, ,klagten viele Fachlehrer [...] über Stoff-Zeit-Probleme“ (Tille 1993c: 387f.). Nach der Einführung der präzisierten Lehrpläne stand fest, „daß es entgegen der ursprünglichen Planung in absehbarer Zeit keine weitere Lehrplanentwicklungsphase geben wird“" (Tille 1993d: 430). Hier griff schlicht die Tatsache aus dem Gesetz über das einheitliche sozialistische Bildungssystem der DDR von 1965, das im \23 (1) regelte, den „Bildungsinhalt für einen längeren Zeitraum festzulegen“ (Rockstuhl 2006: 39).

In der zweiten Hälfte der 1980er Jahre wurde ein neues Lehrplanwerk ausgearbeitet, das nur noch für die Klassen 5, 6 und 10 Gültigkeit erlangte. Die Verantwortlichen kamen ,,in der Absicht des Abbaus eines einseitigen deduktiven Vorgehens [...] vom erklärenden wieder auf den beschreibenden und umschreibenden, den formal vergleichenden und klassifizierenden Unterricht zurück“ (Tille 1993d: 432). Ziel der Biologielehrpläne war es die Artenkenntnis zu erweitern sowie Inhalte aus den Themenfeldern Vererbung und Evolution der Organismen verständlicher zu vermitteln (Honecker 1989). Das Konzept, den Evolutionsgedanken von Klasse 5 an anzubahnen und in Klasse 10 abschließend und zusammenfassend zu behandeln, wurde beibehalten und durchgehend vertieft (Horn 1989; vgl. auch MfV 1985, 1989). Der Lehrplan für die Klassenstufe 5 bis 7 beruhte auf 
dem Strukturansatz Taxonomie und integrierte allgemeinbiologische Themen (Berck \& Graf 2010). In Klasse 8 lag der Fokus auf der Menschenkunde. In den Klassenstufen 9 bis 12 forderte der Stoffplan die Behandlung von Themen aus der Allgemeinen Biologie (Tab. 17).

Tabelle 17: Lehrplankonzeption ab 1988

\begin{tabular}{|c|c|}
\hline Klasse & Inhaltliche Vorgaben \\
\hline $\begin{array}{ll}\text { œ } & 5 \\
\text { ㅇ } & 6\end{array}$ & $\begin{array}{l}\text { Wirbeltiere; Samenpflanzen } \\
\text { Samenpflanzen; die Zelle; einzellige und mehrzellige wirbellose Tiere; Überblick über Bau und } \\
\text { Lebenserscheinung bei Tieren (Systematisierung) } \\
\text { Pilze; Bakterien und Blaualgen; Algen, Moos- und Farnpflanzen; Systematisierung; Überblick } \\
\text { über Bau und Lebenserscheinung von Organismengruppen, über Beziehungen zwischen } \\
\text { Organismengruppen (Systematisierung) } \\
\text { der Mensch als höchstentwickeltes Lebewesen; Stoff- und Energiewechsel; Sinnes- und } \\
\text { Nervenfunktionen; Hormone; Fortpflanzung und Individualentwicklung; Körperhaltung und } \\
\text { Bewegung, funktionelle Einheit des Organismus } \\
\text { Pflanzen und ihre Lebensprozesse; Beziehungen zwischen den Organismen und ihrer Umwelt } \\
\text { Vererbung; Evolution der Organismen }\end{array}$ \\
\hline $\begin{array}{ll}\text { œ } & 11 \\
\text { ㅇ } & 12\end{array}$ & $\begin{array}{l}\text { Systematik; Ökologie } \\
\text { Physiologie; Genetik }\end{array}$ \\
\hline
\end{tabular}





\section{Unterrichtsmedien im Biologieunterricht}

„Die Unterrichtsmittel stellen für die Schüler die anschauliche Basis für den Erwerb von Kenntnissen und Erkenntnissen, für das Erfassen von Zusammenhängen, Gesetzmäßigkeiten und Theorien biologischer Strukturen und Funktionen dar und sind damit eine wichtige Grundlage für die fachliche und weltanschauliche Bildung." (Baer 1975: 8)

\section{Lehrpläne und Lehrbücher im Überblick}

Die Vermittlung einschlägiger evolutionsbiologischer Inhalte erfolgte in den Klassen 8, 10 und 11/12 (Tab. 18). Jedoch variierten die Zeitspanne, die Inhalte sowie die zur Verfügung gestellten Medien. Das folgende Kapitel informiert über formale Aspekte epochal bedeutsamer Lehrpläne, Unterrichtshilfen und obligatorischer sowie fakultativer Schullehrbücher. Bei der Auswahl fand der Grad der Bearbeitung Berücksichtigung. Dieser wurde durch die Abteilung Biologie (Schulbuch) des Verlages Volk und Wissen vorgegeben und war aus der Auflagenkennzeich- 
nung ersichtlich (vgl. Abteilung Biologie [Schulbuch] des volkseigenen Verlages Volk und Wissen 1975). Folgende Kennzeichnungen lagen den Lehrbuchausgaben zu Grunde:

- E Neuerscheinung

- SN Stark bearbeiteter Nachdruck

- BN Bearbeiteter Nachdruck

- DN Durchgesehener Nachdruck

- UN Unveränderter Nachdruck

Konkret bedeutet dies, dass Lehrbücher mit der Kennzeichnung E und SN inhaltliche Neuerungen enthielten. Die Lehrbücher mit der Bezeichnung BN und DN verfügten zwar über Korrekturen in unterschiedlichem Ausmaß, jedoch wurden inhaltliche Veränderungen nicht vorgenommen. Materialien mit der Bezeichnung UN kamen unverändert zur Auflage (ebd.).

Tabelle 18: Epochal bedeutsame Lehrpläne, Lehrbücher und methodische Hilfen mit evolutionsbiologischen Inhalten im Fach Biologie der Klassenstufen 8, 10 und 11/12 auf dem Gebiet der SBZ/DDR von 1945 bis 1989

\begin{tabular}{|c|c|c|}
\hline Klasse & Lehrbücher & Lehrpläne, Direktiven und methodische Hilfen \\
\hline 8 & $\begin{array}{l}\text { - } \quad \text { Lehrbuch der Biologie für das } \\
\text { 7. und 8. Schuljahr (1946) } \\
\text { - } \quad \text { Lehrbuch der Biologie für das } \\
\text { 8. Schuljahr (1951, 1953, 1957) }\end{array}$ & $\begin{array}{l}\text { - Lehrpläne für die Grund- und Oberschulen in der } \\
\text { sowjetischen Besatzungszone Deutschlands } \\
\text { (1946, 1947) } \\
\text { - Lehrplan für Grundschulen, Biologie (1951, 1952, } \\
\text { 1953) } \\
\text { - Direktive zur Arbeit mit dem Lehrplan für das Fach } \\
\text { Biologie (1955) }\end{array}$ \\
\hline 10 & 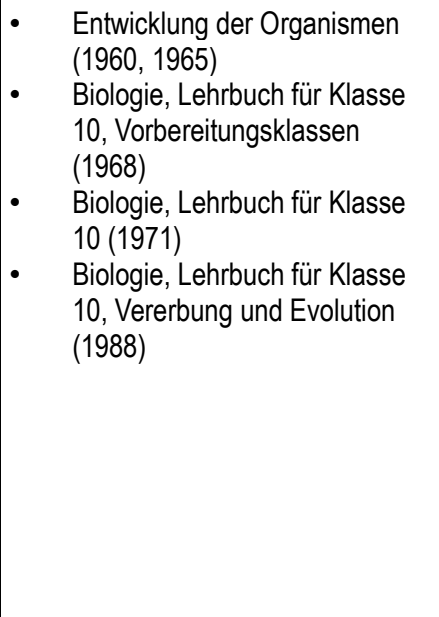 & $\begin{array}{ll}\text { - } & \text { Lehrplan für Zehnjahrschulen, Biologie (1951) } \\
\text { - } & \text { Lehrplan der zehnklassigen allgemeinbildenden } \\
\text { - } & \text { Metytechnischen Oberschulen (1959) } \\
\text { im Fach Biologie (1959) } \\
\text { - } \quad \text { Lehrplan für das Fach Biologie Klassen } 7 \text { bis } 10 \\
\text { - } \quad \text { Lehrplan für den Biologieunterricht der } \\
\text { Vorbereitungsklassen } 9 \text { und } 10 \text { zum Besuch der } \\
\text { - } \quad \text { Erweiterten Oberschule (1967, 1968) } \\
\text { - } \quad \text { Unterrichntshilfen Biologie, 10. Klasse (1971) } \\
\text { - } \quad \text { Lehrplan Biologie (1988) } \\
\text { - } \quad \text { Unterrichtshilfen Biologie, 10. Klasse (1988) } \\
\quad \text { allger Lehrplan der zehnklassigen } \\
\text { Erläuterungen den Lehrplanes Biologie (1989) }\end{array}$ \\
\hline
\end{tabular}




\begin{tabular}{|c|c|c|}
\hline $11 / 12$ & $\begin{array}{l}\text { - Lehrbuch der Biologie für das } \\
\text { 12. Schuljahr (1952, 1957) } \\
\text { Biologie IV, Ein Lehrbuch für } \\
\text { die erweiterte Oberschule, } 12 . \\
\text { Klasse, Die Lehre von der } \\
\text { Evolution der Organismen } \\
\text { (1964 in zwei Bd., ab } 1965 \text { in } \\
\text { einem Band) }\end{array}$ & $\begin{array}{l}\text { - Lehrpläne für die Grund- und Oberschulen in der } \\
\text { sowjetischen Besatzungszone Deutschlands } \\
\text { (1946, 1947) } \\
\text { - Lehrplan für Oberschulen, Biologie (1951, 1953, } \\
\text { 1954) } \\
\text { Biologieunterricht Oberschule, vorläufiger Lehrplan } \\
\text { (1956) } \\
\text { - Lehrplan der zwölfklassigen erweiterten } \\
\text { - Oberschule für das Schuljahr 1959/60 (1959) } \\
\text { Lehrplan der zwölfklassigen erweiterten } \\
\text { Oberschule (1961) } \\
\text { Anweisung zur Korrektur des Lehrplans Biologie } \\
\text { der Erweiterten Oberschule (1967) }\end{array}$ \\
\hline
\end{tabular}

Nach Kummer (2009) enthielten die ersten Schullehrbücher für das Fach Biologie der 1940er und 1950er Jahre Auszüge aus Fachbüchern (u. a. aus dem Fischer Verlag). Kummer, die ab 1962 kommissarisch die Buchredaktion des Verlages Volk und Wissen übernahm, wurde knapp ein Jahr später als Abteilungsleiterin bestätigt (Kummer 2009, 2016a). Die bisherige Abteilungsleiterin für Biologie Hannelore Ploog, geb. Willbrandt (1923-2003) übernahm die Zeitschriftenredaktion. Gertrud Kummer war seit 1961 Mitglied der Sektion Schulbiologie der Biologischen Gesellschaft (in) der DDR. Es gelang ihr u. a. auf Tagungen verschiedener Sektionen und in weiterführenden Gesprächen, Fachdidaktikerinnen und didaktiker sowie Fachwissenschaftlerinnen und -wissenschaftler für die Lehrbucharbeit zu gewinnen. Diese steuerten ihrer Kompetenz entsprechende Lehrbuchkapitel bei und korrigierten die Kapitel ihrer Kollegen. Für das Gesamtwerk wurden externe Gutachter ${ }^{86}$ beauftragt. Entsprechend der Spezifika des Biologieunterrichtes lautete der Anspruch an das Biologielehrbuch der DDR, den Schüler an das originale Objekt heranzuführen und ihn in seinen Erkundungen zu unterstützen. Das Schulbuch sollte vielseitigen Ansprüchen Rechnung tragen: in die Arbeit mit Büchern einführen, Anleitung für praktische Schülerarbeiten sein, zum Gebrauch im Unterricht dienen, Möglichkeiten der Wiederholung des Stoffes eröffnen sowie bei Vorbereitungen auf Prüfungen helfen (ebd.). Die Biologielehrbücher der DDR setzten sich aus Elementen und Darstellungsformen zusammen. Als Elemente galten Sachinformationen, Merkstoffe, Aufgaben, Bestimmungstabellen und Leiteinrichtungen, die durch Darstellungsformen, wie Texte, Abbildungen, Tabellen und Symbole wiedergegeben wurden (Pälchen 1974).

86 Gutachter waren u. a. die Biologiedidaktiker Gerhard Dietrich aus Leipzig und Wolfgang Lerchner, der bei Rudolf Hundt im Wissenschaftsbereich Methodik des Biologieunterrichts in Halle (Saale) als wissenschaftlicher Mitarbeiter tätig war. Lerchner beteiligte sich auch an der Entwicklung der Unterrichtshilfen Biologie (Kummer, Interview vom 28.12.2016). 


\section{Klasse 8}

Grundsätzlich zeigt sich, dass für die Klassenstufe 8 ausschließlich die Lehrpläne von 1946 bis 1955 konkrete Inhaltsangaben zur Evolutionsbiologie enthielten. Aus den Ergebnissen dieser Schriftbelege kann von einer zumindest administrativen Vorgabe der Behandlung adäquater Inhalte im Unterricht ausgegangen werden (Abb. 8). Unterschiede bestanden hingegen in der Inhaltsauswahl, in der Struktur und im Umfang, die im weiteren Verlauf der Arbeit näher erläutert werden. Mit dem Lehrplan der zehnklassigen allgemeinbildenden polytechnischen Oberschulen, der am 1. September 1959 in Kraft trat, wurde die Vermittlung evolutionsbiologischer Inhalte für die Klassenstufe 8 eingestellt. Auch in den folgenden Lehrplänen von 1966 bis 1989 blieb eine Vermittlung dieser Fachdisziplin in Klasse 8 aus.

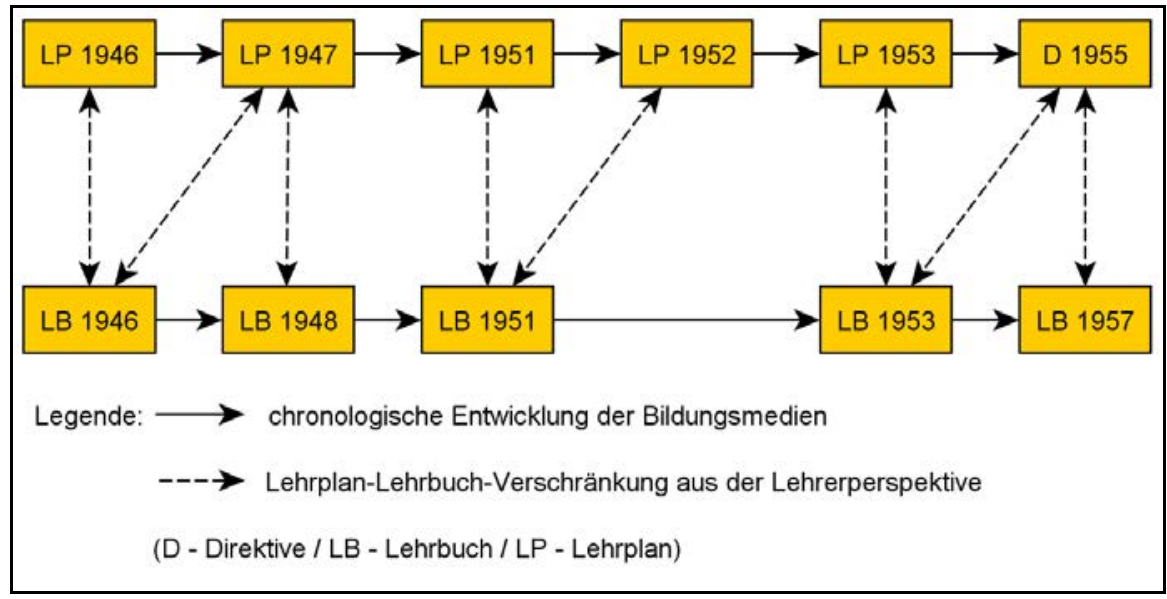

Abbildung 8: Entwicklung der Lehrplan-Lehrbuch-Verschränkung für die 8. Klasse

Nach Ende des Zweiten Weltkrieges erschienen am 1. Juli 1946 die Lehrpläne für die Grund- und Oberschulen in der Sowjetischen Besatzungszone Deutschlands. Diese hatten bis 1950/51 den Status von Rahmenlehrplänen. Die Deutsche Zentralverwaltung für Volksbildung verwies darauf, „daß die Aufstellung der Stoffpläne aufgrund der Lehrpläne der DVV eine durch ,eingehende Beratung in Klassen- und Gesamtkonferenzen‘ zu lösende ,Aufgabe der einzelnen Schulen' sei“" (Geißler et al. 1996: 150), was einen großen Entscheidungsspielraum der Lehrenden implizierte (vgl. Gropengießer \& Kattmann 2006). Den Lehrkräften war es vorbehalten, aus den Inhaltsangaben der Lehrpläne auszuwählen, da ,gewisse Pensen verbindlich, andere dagegen austauschbar" waren (DZfV in der SBZ 1946: 4). Im Jahr 1947 erschien die 2. Auflage der Lehrpläne für die Grund- und Oberschulen in der Sowjetischen Besatzungszone Deutschlands. Der Status eines Rahmenlehrplans blieb erhalten. Veränderungen betrafen das Vorwort und die Stoffverteilung. Das Vorwort zur 2. 
Auflage von 1947 enthielt als Motive für die Neubearbeitung „1. Eine sinnvolle Abstimmung der Lehrstoffe aufeinander; 2. die Beseitigung der Stoffüberbürdung; 3. die Beseitigung von Verfrühungen" (DVV in der SBZ 1947: 4). Gleichzeitig wies die DVV kontradiktorisch darauf hin, dass „die erste Fassung der Lehrpläne nur wenig geändert" wurde (DVV in der SBZ 1947: 4).

Das erste Schulbuch für die Klassenstufe 8 auf dem Gebiet der SBZ erschien ebenfalls 1946 unter dem Titel Lehrbuch der Biologie für das 7. und 8. Schuljabr (Abb. 9). Die ersten beiden Auflagen dieses Schülerbuches gab die biologische Lehrfachkommission der DVV in der SBZ heraus. Als Herausgeber der 3. durchgesehenen Auflage von 1948 zeichneten Fritz Löbel und Wilhelm Maschke. Das Buch ging ,im Stoffumfang weit über das Maß hin-
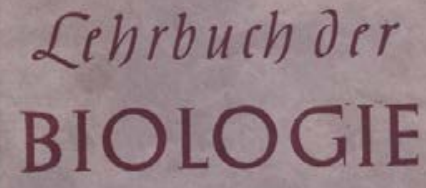

Abbildung 9: Lehrbuch Klasse 8 (1946) aus, das in einem Schuljahr bewältigt werden konnte“, so dass die Lehrkräfte „aus der Gesamtheit des im Lehrbuch dargebotenen Stoffes selbst auswählen“ mussten (Dietrich \& Kummer 1979: 406).

„Die ersten Biologielehrbücher für die Grundschule stellten einen wichtigen Fortschritt dar. In ihrer methodischen Gestaltung zeigten sie manche Ansätze, die weiterentwickelt zu werden verdienten. Dazu gehörten die vielen Beobachtungsaufgaben und die Anleitungen zu leichten, von den Schülern selbständig zu bewältigenden Experimenten, die Hinweise auf die biologischen Erscheinungen in der Umgebung der Schüler, die betonte Beachtung des Prinzips der Anschaulichkeit in Text und Bild und - wenn auch mit gewissen Einschränkungen - die starke emotionale Ausrichtung des Inhalts und der sprachlichen Darstellungsweise." (Dietrich \& Kummer 1979: 406)

Ausgearbeitet vom DPZI gab das MfV im Jahr 1951 einen neuen Lehrplan unter dem Titel Lehrplan für Grundschulen, Biologie, 5. bis 8. Schuljahr heraus (vgl. MfV 1951a). Dies geschah im Rahmen der zweiten Lehrplanrunde. Mit inhaltlichen Veränderungen folgte bereits ein Jahr später eine 2. Auflage. Ebenfalls im Jahr 1951 konnten die Lehrkräfte und Schülerinnen und Schüler der Klassenstufe 8 auf eine Neuausgabe des Schulbuches unter dem Titel Lebrbuch der Biologie zurückgreifen. Es wurde in Zusammenarbeit mit einem Autorenkollektiv von Willi Lemke herausgegeben und wies im Vergleich zum Vorgängermodell eine modifizierte inhaltliche Struktur auf. 
Zum 1. September 1953 gültig, veröffentlichte die Hauptabteilung Unterricht und Erziehung des MfV einen überarbeiteten Lehrplan unter dem Titel Lehrplan für Grundscbulen, Biologie, 5. bis 8. Schuljahr. Die Grundlage für die Überarbeitung bildete der Lehrplan von 1952. Im selben Jahr erschien ein Schulbuch mit inhaltlichen Kürzungen als 4. durchgesehene Ausgabe der Auflage von 1951. Dem Umbau des Lehrplanes lag „die Tatsache einer zu großen Stoffülle“ (Gottschalk 1954: 270) zu Grunde. Bei der Überarbeitung achteten die Autorinnen und Autoren darauf, Stoffeinheiten nicht in andere Schuljahre zu verlagern, um Schwierigkeiten bei der Benutzung der Lehrbücher und der Durchführung der Prüfungen zu vermeiden (Boje 1953). Den notwendig gewordenen Kürzungen lag ferner die Prämisse zu Grunde, „daß alle im Lehrplan geforderten Stoffeinheiten [...] im Lehrbuch nachgelesen und durchgearbeitet werden können“ (ebd.: 337). Da das Lehrbuch mehr Stoff enthielt als der Lehrplan vorgab, betonte das MfV, dass der Lehrplan „für Stoffauswahl und Stoffverteilung maßgebend ist, nicht aber das Lehrbuch, das in einzelnen Abschnitten über die Thematik hinausgeht oder eine abweichende Anordnung der Abschnitte aufweist" (MfV 1953a: 3).

Ergänzend und mit Korrekturen versehen, veröffentlichte die Hauptabteilung Unterricht und Erziehung des MfV der DDR zum Schuljahr 1955/56 eine Direktive zur Arbeit mit dem Lehrplan für das Fach Biologie. Ausgewiesenes Erziehungs- und Bildungsziel war die Erziehung der Jugend zu Patrioten sowie die Verbesserung der wissenschaftlichen Bildung, um die Anforderungen der Volkswirtschaft zu erfüllen (Kühn 1955). Albert Pietsch ${ }^{87}$ (1959) argumentierte, dass sich insbesondere durch Entwicklungen in der Landwirtschaft neue Forderungen an den Biologieunterricht ergaben. Die Direktive enthielt für die Klassen der Mittelstufe entsprechende Korrekturen des Lehrplans, die in einer „stärkere[n] Berücksichtigung landwirtschaftlicher Themen ohne jede Erweiterung des Gesamtstoffumfangs" (Kühn 1955: 409) mündeten.

Unter Beibehaltung des Titels Lehrbuch der Biologie für das 8. Schuljahr erschien im Jahr 1957 eine stark überarbeitete Neuausgabe des Lehrbuches von 1953. Das Schulbuch wurde von Herbert Arnold, Herbert Bach (1926-1996), Hans-Günter Gießmann (1922-2014), Helmut Nestler (*1932) sowie Georg Uschmann verfasst. Mit Herbert Bach, der am Institut für Anthropologie der Friedrich-SchillerUniversität in Jena tätig war, beteiligte sich ein ausgebildeter Anthropologe an der Lehrbucherarbeitung (Hoßfeld 2005). Ferner brachten sich mit dem Wissenschaftshistoriker Georg Uschmann vom Ernst-Haeckel-Haus in Jena sowie dem Geologen und Paläontologen Helmut Nestler von der Universität Greifswald weitere Wissenschaftler ein. Hans-Günter Gießmann, der als Facharzt für Physiologie am Physiologischen Institut der Humboldt-Universität in Berlin arbeitete, erstellte die im Schülerbuch aufgenommenen humanbiologischen Aspekte.

87 Albert Pietsch arbeitete seit 1953 als Professor an der Pädagogischen Hochschule in Potsdam (Biologiedidaktik). 


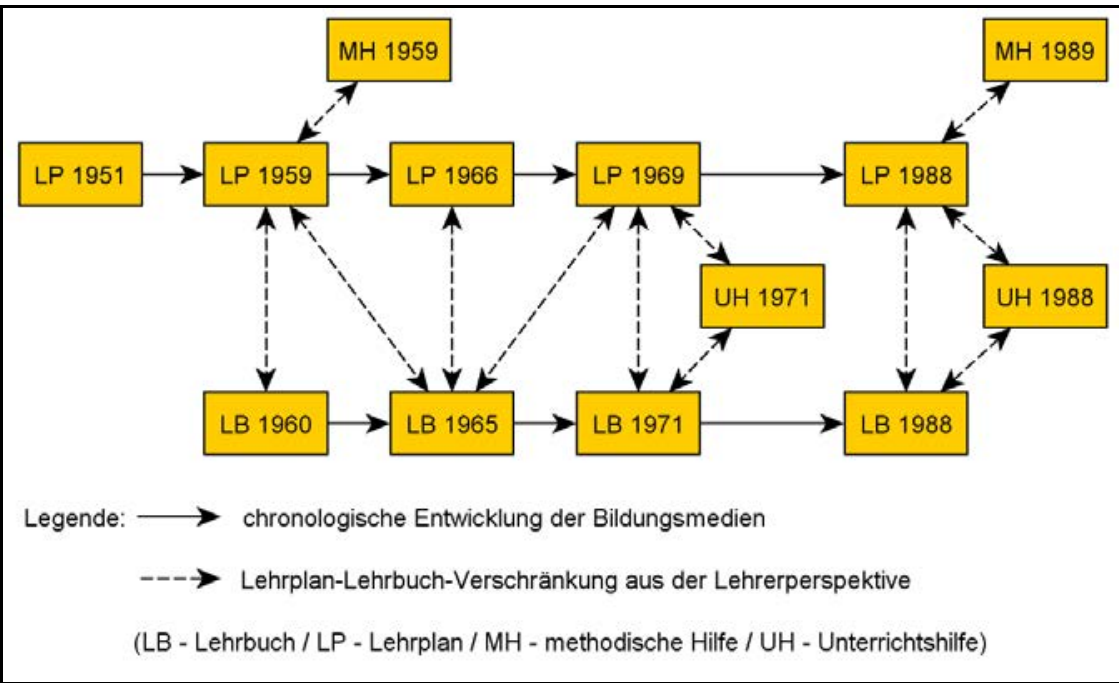

Abbildung 10: Entwicklung der Lehrplan-Lehrbuch-Verschränkung für die 10. Klasse

Klasse 10

In der 10. Klassenstufe fanden erstmals, ausgearbeitet vom DPZI und herausgegeben sowie bestätigt vom MfV im Jahr 1951 im Lehrplan für die Zehnklassenschule, evolutionsbiologische Themen Beachtung (Abb. 10). Ein adäquates Schulbuch für diese Schulform konnte im Rahmen der historischen Quellenforschung nicht ausfindig gemacht werden. Es ist davon auszugehen, dass die Lehrkräfte für die unterrichtliche Arbeit Alternativlösungen, wie Unterrichtsvorschläge aus der Zeitschrift Biologie in der Schule und Schulbücher aus der Klassenstufe 8 und 12, nutzten. Dagegen enthielt der Lehrplan für Oberschulen von 1951, der bereits 1954 ersetzt wurde, für die Klasse 10 keine evolutionsbiologischen Inhalte. Am 1. September 1956 trat für die 1955 eingeführte Mittelschule ein neuer Lehrplan in Kraft. Die Evolutionsbiologie wurde nicht behandelt. Stoffliche Schwerpunkte für die Klasse 10 waren ,grundlegende Erkenntnisse der ,Mitschurinschen“ Biologie und der Züchtung von Pflanzen und Tieren“ (Tille 1992c: 429). Spezielle Lehrbücher für die Mittelschule gab es nicht. Stattdessen verwies der Lehrplan auf Lehrbücher der Oberschule sowie Sekundärliteratur (MfV 1956). Im Jahr 1957 erschien ein neuer Lehrplan für Mittelschulen. Im Mittelpunkt des Stoffgebietes Die Züchtung von Pflanzen und Tieren standen die Vererbung erworbener Eigenschaften und die Vererbung als Eigenschaft des ganzen Organismus. Der Neodarwinismus wurde dagegen als pseudowissenschaftliche Grundlage der menschenfeindlichen 
Rassendiskriminierung dargestellt. ${ }^{88}$ Doch zeigt die Aufnahme der Mendel'schen Regeln, der Chromosomentheorie der Vererbung, der Mutationstheorie von de Vries und des Genbegriffs nach Johannsen auch „eine erstaunlich tolerante Haltung" der Lehrplanautorinnen und -autoren ,in der Anfangsphase der ,Entstalinisierung"“ (Tille 1992c: 429).

Im Schuljahr 1959 wurde auf der Grundlage der Beschlüsse des 4. Plenums des ZK der SED der Lehrplan für die zehnklassige allgemeinbildende POS eingeführt (Krombholz \& Lengert 1959). Anders als der Lehrplan der zwölfklassigen EOS, der in der Klassenstufe 12 evolutionsbiologische Inhalte vorsah, enthielt dieser für die Klasse 10 entsprechende Themen. Der Lehrplan galt „als verbindliche Grundlage für den Unterricht" (MfV 1959a: 11) und war bei seiner Einführung mit Übergangsregeln verbunden. Diese sollten spätestens nach dem Schuljahr von 1960/61 auslaufen, so dass nach dem neuen Lehrplan unterrichtet werden konnte. Die Lehrkräfte konnten entsprechend früher ihren Unterricht darauf einstellen (MfV 1959a). Der Lehrplan stand im Kontext der Zielformulierungen des 5. Parteitages der SED. Infolge dessen lag der Fokus darauf, „die Schule eng mit dem Leben zu verbinden und mit den Erfordernissen der sozialistischen Entwicklung in Einklang zu bringen“ (ebd.: 11). Mit dem neuen Lehrplan war eine Aufforderung an alle Lehrkräfte verbunden, ihre Erfahrungen entweder der Abteilung Allgemeinbildung des MfV oder dem DPZI mitzuteilen (ebd.). Die daraus folgenden Änderungen mündeten 1966 in einen „Nachdruck des Lehrplans von 1959 unter Berücksichtigung aller seit 1959 durchgeführten verbindlichen Veränderungen“ (MfV 1966: 1). Neben dem Lehrplan von 1959 standen den Lehrkräften für die Vermittlung der Unterrichtsinhalte auch Methodische Hinweise zur Arbeit mit dem Lehrplan, herausgegeben von der Sektion Unterrichtsmethodik und Lehrpläne des DPZI, zur Verfügung. Diese stellten einen „Vorschlag für die Aufgliederung der Stoffgebiete“ (DPZI 1959: 3) und somit auch der evolutionsbiologischen Inhalte dar. Die Schüler erhielten die Lehrhefte Anatomie, Physiologie und Hygiene des Menschen sowie Die Züchtung. Für die im Lehrplan geforderten evolutionsbiologischen Inhalte stand jedoch anfangs kein Lehrbuch bzw. Lehrheft zur Verfügung (Anonymus 1959). Stattdessen wurden entsprechende Hinweise zur Unterrichtsgestaltung in der Zeitschrift Biologie in der Schule veröffentlicht (vgl. Vogelbein 1959).

Unter dem Titel Entwicklung der Organismen, Lebrbuch für Biologie stand ab 1960 für die Vermittlung der Lehrplaninhalte ein adäquates Lehrbuch der Biologie für die 10. Klassenstufe zur Verfügung (Abb. 11). Das Lehrbuch war thematisch mit der Intention umgrenzt, eine Unabhängigkeit von Lehrplanänderungen zu erreichen sowie den Schülern eine engere Beziehung zum Lehrbuch zu ermöglichen

88 Hoßfeld (2012a: 55) betont, „dass der Rasse(n)begriff auf Menschen angewendet, wissenschaftlich unseriös und politisch-ideologisch skandalös ist“. Bereits seit den 1950er Jahren „sind Überlegungen zu Rassenunterschieden in der wissenschaftlichen Literatur [...] obsolet“ (ebd.). 
(Anonymus 1960). Da das Lehrbuch die „schwierigsten Probleme der Biologie" behandelte, wurden ,stärker als bei anderen Lehrbüchern Fachwissenschaftler als Autoren herangezogen" (Pädagogisches Institut Mühlhausen und Abteilung des volkseigenen Verlages Volk und Wissen 1960: 428). Als Autoren zeichneten Herwart Ambrosius (*1925), Herbert Arnold, Dieter Bernhardt, Wolfgang Crome (1927-1967), Gunter Friedrich, Klaus Kloss (1934-2004), Wilhelm Linke, Helmut Nestler, Joachim Nitschmann (1925-2016), Wolfgang Padberg, Georg Uschmann und Irmtraut Wessely. Die Verfasser des Lehrbuches wirkten zum Großteil an wissenschaftlichen Einrichtungen. Ambrosius arbeitete zwischen 1954-1962 als wissenschaftlicher Oberassistent und Lehrbeauftragter am Zoologischen Institut der Mathematisch-

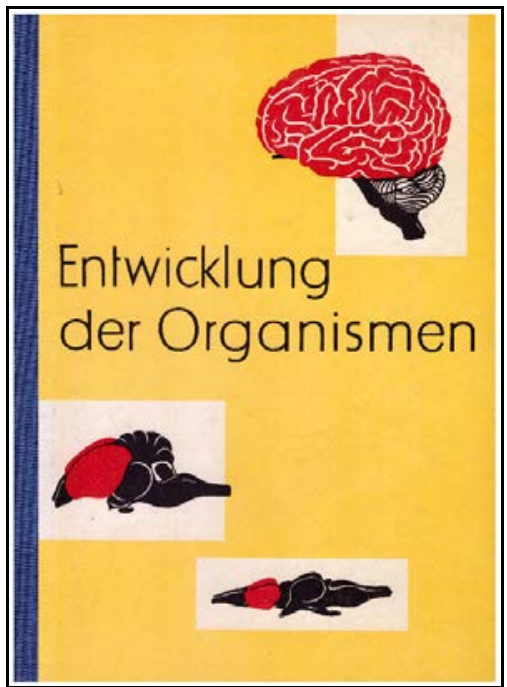

Abbildung 11: Lehrbuch Klasse 10 (1960)

Naturwissenschaftlichen Fakultät in Leipzig (vgl. auch Emmerich 2001). Uschmann war seit 1959 Direktor des Ernst-Haeckel-Hauses in Jena (Hoßfeld 2005). Crome arbeitete als Kustos für Arachniden und Myriapoden am Museum für Naturkunde zu Berlin (Anonymus 2011). Der Biologe Kloss war als Assistent bei Rothmaler am Institut für Agrarbiologie der Ernst-Moritz-Arndt-Universität in Greifswald tätig (vgl. Zott \& Zott 2010). Nitschmann arbeitete am Zoologischen Institut der Humboldt-Universität zu Berlin zu den Schwerpunkten Embryologie und Entwicklungsphysiologie. Linke war an der Zentralen Lehrstätte für Naturschutz in Müritzhof bei Waren tätig und Wessley arbeitete am Institut für Pädagogik der Ernst-Moritz-Arndt-Universität in Greifswald zu den Schwerpunkten Schulbiologie, Pflanzengeographie und -taxonomie. Ferner arbeitete an der Entwicklung „,neben vielen Wissenschaftlern und Lehrern vor allem ein Kollektiv des Pädagogischen Instituts Mühlhausen unter der Leitung von Hans Fischer mit" (MfV 1961: 3).

Bereits mit seinem Erscheinen wurden dem Lehrbuch Schwächen attestiert, die sich aus der Eile der Entwicklung, dem Mangel an praktischer Überprüfung sowie neuen Erkenntnissen ergaben. Daher überprüften Dozentinnen und Dozenten des Pädagogischen Instituts Mühlhausen, Lehrerinnen und Lehrer der Bezirke Erfurt und Suhl sowie Mitarbeiterinnen und Mitarbeiter der Abteilung Biologie des Verlages Volk und Wissen die Lehrbücher (Pädagogisches Institut Mühlhausen und Abteilung des volkseigenen Verlages Volk und Wissen 1960). Von mehreren Autoren verfasst, legte der Verlag Volk und Wissen unter der Redaktion von Manfred Gemeinhardt und Gertrud Kummer im Jahr 1965 ein neues 
Lehrbuch auf. Dieses enthielt unter Beibehaltung des Titels inhaltliche Veränderungen. Am Prozess der Begutachtung beteiligten sich Wissenschaftlerinnen und Wissenschaftler aus verschiedenen Bereichen der Biologie, Didaktikerinnen und Didaktiker sowie Lehrerinnen und Lehrer (Bach et al. 1967).

In Weiterführung der Beiträge zur Abstammungslehre bot das Werk Allgemeine Biologie zusätzlich eine methodische Aufbereitung des Stoffes für den Unterricht. Im Jahr 1966 in Moskau herausgegeben lag es 1969 in deutscher Fassung vor. Neue Erkenntnisse in den Biowissenschaften und deren Einbindung in die Lehrpläne bedingten eine „möglichst umfassende Information der Unterrichtenden" (Beljajew 1969: 10). Vordergründig für die Arbeit der Lehrkräfte gedacht, lieferte es „Anregungen dafür, wie komplizierte Sachverhalte der Genetik oder der Evolution (bspw. Faktoren der Evolution) für Schüler der 10. Klasse verständlich dargeboten werden können " (ebd.). Die Redaktion sah auch die Möglichkeit, interessierten Schülern das Buch an die Hand zu geben. Eine reichhaltige Visualisierung bot entsprechende Möglichkeiten an (Abb. 12).

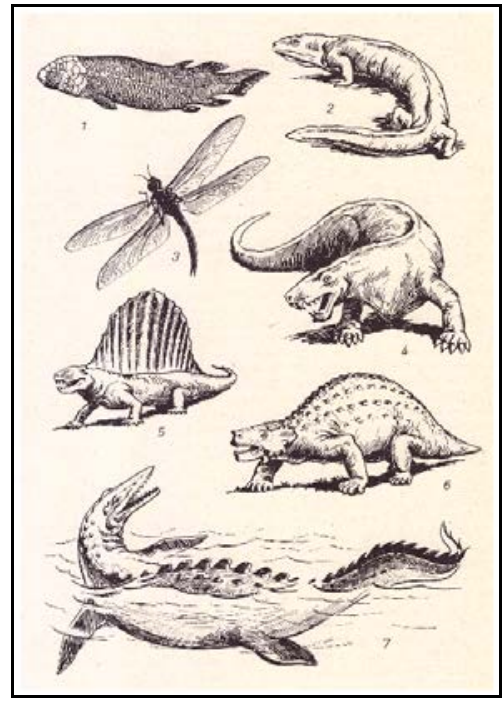

Abbildung 12: Vertreter der zweiten Hälfte des Paläozoikums (Beljajew et al. 1969)

Mit der Einführung der Vorbereitungsklassen der EOS im Schuljahr 1967/68 erschien ein präzisierter Lehrplan für das Fach Biologie, der aufgrund von Übergangsregelungen für die jeweiligen 10. Klassen mit dem Schuljahr 1971/72 in Kraft trat. Verantwortlich für die Entwicklung war die Abteilung Unterricht des MfV. Zusätzlich wurden Ursula Schleiden und Helmut Fritsch zur Durchführung der Arbeiten an das MfV abgeordnet (Wernecke 1967a). Dieser Plan konkretisierte als Arbeitsgrundlage die Zielstellungen und Aufgaben der staatsbürgerlichen Erziehung aus dem Programm der SED und den Materialien des 11. Plenums für das Fach Biologie (Wernecke 1967b). Mit der Verlagerung der Evolutionsbiologie von Klasse 12 in Klasse 10 wurde auch die weltanschaulich-erzieherischen Wirkung dieser Fachdisziplin diskutiert und ihr zentraler Stellenwert im Biologieunterricht neu bestimmt (Wernecke 1967a). Das entsprechende Lehrbuch erschien 1968 unter dem Titel Biologie, Lebrbuch für Klasse 10, Vorbereitungsklassen (Abb. 13). Wie bereits beim Lehrbuch von 1965, übernahmen Manfred Gemeinhardt und Gertrud Kummer vom Verlag Volk und Wissen die Redaktion.

Mit dem Schuljahr 1971/72 wurde auch für alle Klassen und Fächer der POS ein neues Lehrplanwerk zur „Grundlage des Erziehungs- und Bildungsprozesses“ (Wernecke 1971: 305). Die damit verbundenen Zielstellungen standen im Kontext 
der gesellschaftlichen Entwicklung und der Entwicklung sozialistischer Persönlichkeiten (ebd.). Bereits im Jahr 1969 erschien für die Klasse 10 der neue Lehrplan unter dem Titel Lehrplan für Biologie Klassen 9 und 10. Parallel dazu stand für den Unterricht ab 1971 das Schulbuch Biologie, Lebrbuch für Klasse 10 zur Verfügung (Abb. 13). Auch bei diesem Lehrbuch zeichneten sich Gemeinhardt und Kummer für die Redaktion verantwortlich. Mit den Autoren Herbert Bach, Jochen Helms, Irmtraut Meincke (geb. Wessely), Johannes Müller, Werner Plesse, Dietrich Rothacker und Michael Teile brachten erneut Fachkräfte aus den Bereichen der Wissenschaft und der Praxis ihre Kenntnisse ein. Beispielhaft seien hier genannt der Invertebraten-Paläontologe Jochen Helms, der Wissenschaftsphilosoph Werner Plesse sowie der am Institut für Pflanzenzüchtung in Groß Lüsewitz (Kreis Rostock) der Deutschen Akademie für Landwirtschaftswissenschaften der DDR tätige Agrarwissenschaftler und Spezialist für Wildkartoffeln Dietrich Rothacker.

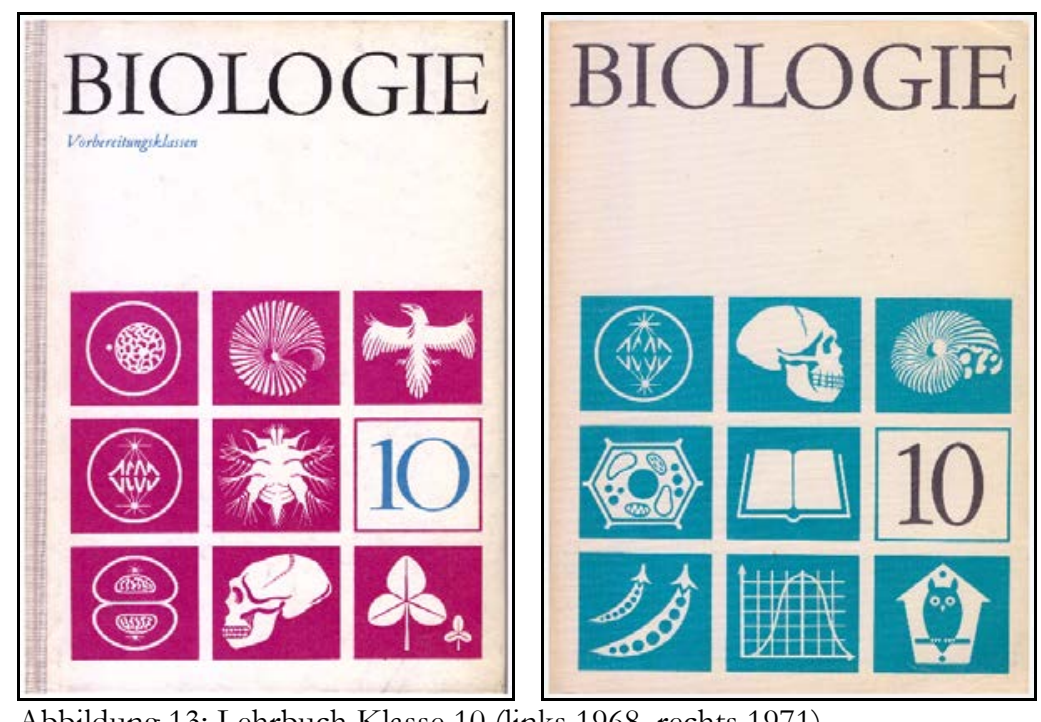

Abbildung 13: Lehrbuch Klasse 10 (links 1968, rechts 1971)

Mitte der 1970er Jahre eingeführt, diente als fakultatives Schulbuch der Titel Biologie in Übersichten (Abb. 14). Für die Kapitel Stammesentwicklung des Menschen und Stammesentwicklung der Organismen zeichnete Hiltrud Graef als Autorin. Das Buch enthielt den Unterrichtsstoff für die Klassen 5 bis 10 und war für die Hand des Schülers ab Klasse 8 vorgesehen. Es orientierte primär auf das grundlegende Wissen und stellte die Inhalte konzentrierter als im Lehrbuch dar. Die inhaltliche Struktur folgte ,nicht der pädagogischen-methodischen Grundkonzeption des Lehrplans, sondern fachsystematischen und -logischen Prinzipien“ (Dorber \& Dorber 1976: 484). Als besonders geeignet galt das Buch „für die immanente 
Wiederholung und die Prüfungsvorbereitung in Klasse 10“ (Graef et al. 1985: 14). Bei den Darstellungsformen bevorzugten die Autoren Schematisierungen und übernahmen zahlreiche Abbildungen aus dem Biologielehrbuch (Abb. 14). Die Einbindung in den Unterricht oblag anfangs den Vorstellungen der Lehrkräfte, da die Aufgaben der Schulbücher und die Unterrichtshilfen den Wissensspeicher nicht berücksichtigten (Dorber \& Dorber). Ab dem 1. September 1977 stand der Titel „an jeder Schule als Klassensatz zur Verfügung“" (Graef et al. 1985: 14).
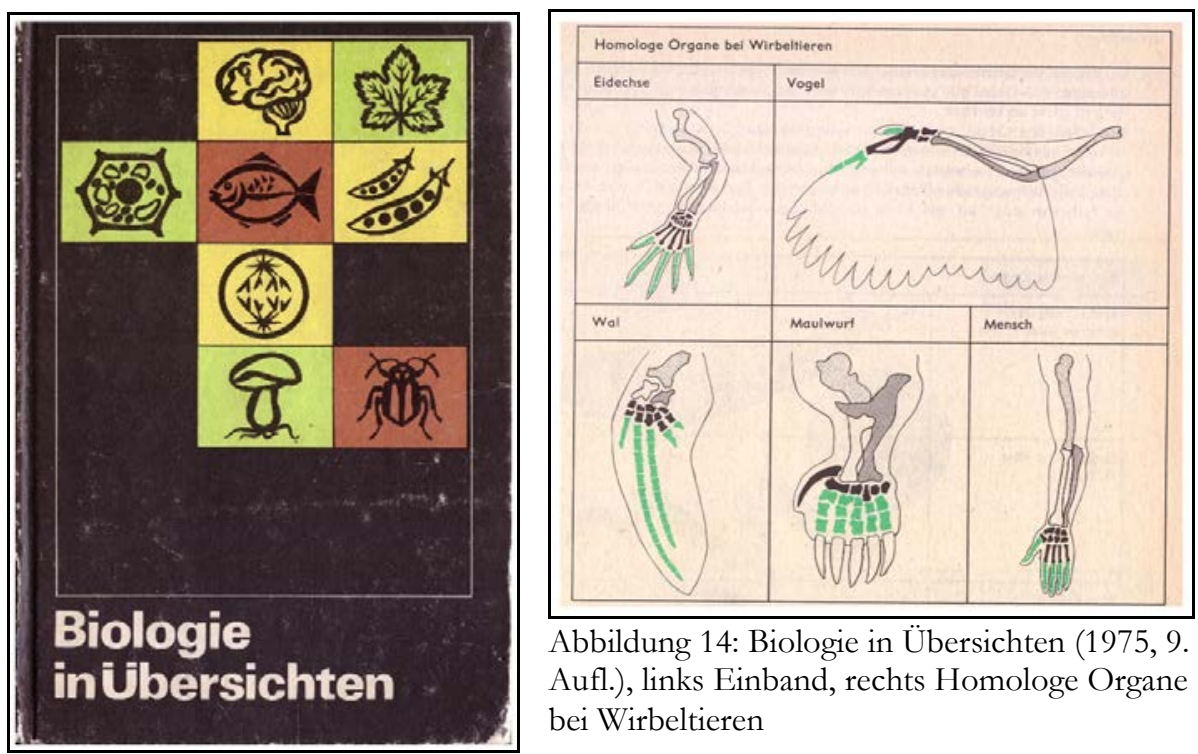

Abbildung 14: Biologie in Übersichten (1975, 9. Aufl.), links Einband, rechts Homologe Organe bei Wirbeltieren

Ein neuer Lehrplan, der sich an Aussagen des 11. Parteitages der SED orientierte, erschien mit Wirkung zum 1. September 1988 unter dem Titel Lehrplan Biologie Klassen 5 und 10 (Kaiser 1988). Der Ausarbeitung ging eine öffentliche Diskussion in der Zeitschrift Biologie in der Scbule voraus. Inhaltlich von einem bewährten Konzept ausgehend, sollten von Klasse 5 an Möglichkeiten zur Vertiefung des Evolutionsgedankens umfassender genutzt werden (Horn \& Kaiser 1986; Horn 1987). Das entsprechende Schulbuch wurde ebenfalls im Jahr 1988 unter dem Titel Biologie, Lehrbuch für Klasse 10, Vererbung und Evolution herausgegeben (Abb. 15). Unter der Leitung von Karl Sommer (1940-2017) übernahmen Ilse König und Gertrud Kummer die Redaktion. Ferner arbeitete Frank Horn (wissenschaftlicher Mitarbeiter am Institut für mathematischen und naturwissenschaftlichen Unterricht der APW in Berlin) am Lehrbuch mit. Als Autoren zeichneten Gertrud Kummer, Manfred Matzke (*1934), Hans-Joachim Schwier, Karl Sommer, Horst Theuerkauf (1932-2015) sowie Herbert Ullrich (*1932). Der Biologe und Anthropologe Herbert Ullrich vom Zentralinstitut für Alte Geschichte und Archäologie der Akademie der Wissenschaften sei beispielhaft für die Einbindung wissen- 
schaftlicher Fachkräfte genannt. Bei den didaktisch-methodischen Überlegungen brachte u. a. der Biologiedidaktiker Matzke von der Pädagogischen Hochschule in Halle-Köthen sein Wissen ein (vgl. Körnig 2009). Der Lehrer Theuerkauf war als Bildautor tätig. Kummer berichtet (2009: 15; Interview vom 28.12.2016), wie sie im Rahmen einer Lehrerdelegation in Ungarn auf Horst Theuerkauf aufmerksam wurde, ,,der - in Ermangelung von Fotos in den Schulbüchern - für seinen Unterricht Dias in hoher Zahl anfertigt hatte". Aus dieser Begegnung erwuchs eine gemeinsame Arbeit an den Lehrbüchern der 1970er Jahre, die in den späteren Lehrbuchserien fortgesetzt und intensiviert wurde.

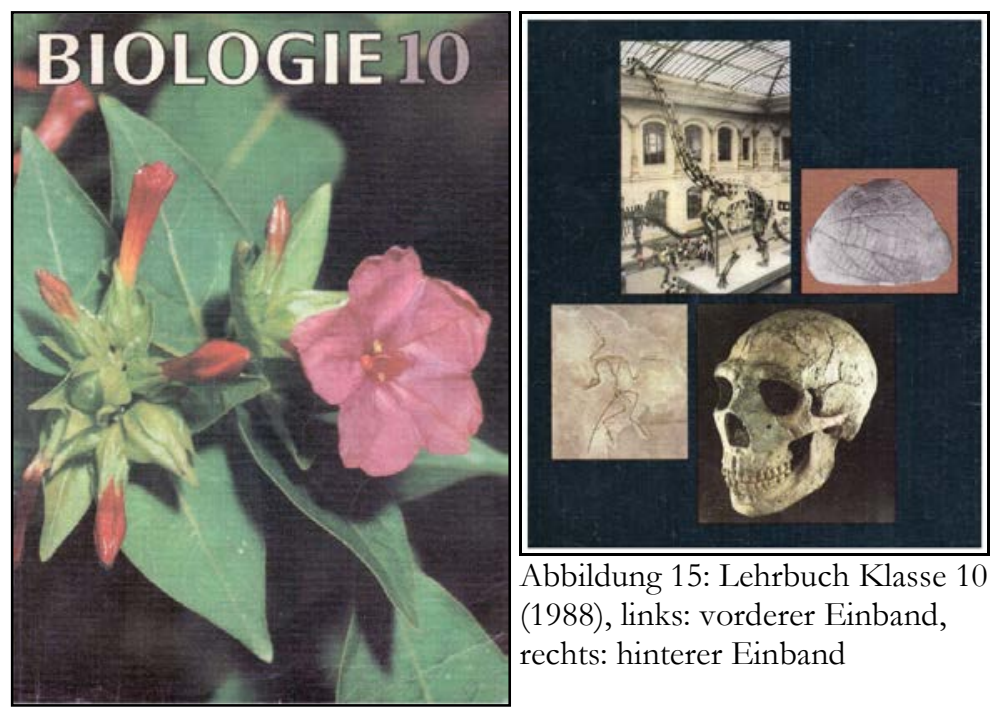

Die Akademie der pädagogischen Wissenschaften der DDR gab ergänzend 1989 Inhaltliche und methodische Erläuterungen heraus. Diese sollten „das Lehrplanverständnis vertiefen helfen, indem Funktion und Ziele des Biologieunterrichtes, Auswahl und Anordnung des Inhalts sowie Hauptmerkmale der didaktisch-methodischen Konzeption des Gesamtlehrgangs dargestellt und erläutert" wurden (Horn 1989: 7). Zu dem Lehrplan von 1971 stand den Lehrkräften auch der Titel Unterrichtshilfen Biologie Klasse 10 zur Verfügung. Dietrich und Kummer (1979: 418) resümieren, dass „zusammen mit den Lehrplänen und Lehrbüchern - erstmalig in der DDR und unseres Wissens bis heute einmalig in der Welt - für jedes Fach für jede Klassenstufe ,Unterrichtshilfen' herausgegeben wurden“. Ihm kam die Aufgabe zu, „auf theoretischer Basis konkrete Hinweise und Vorschläge für die Behandlung der im Lehrplan ausgewiesenen Stoffeinheiten und für die Durchführung der einzelnen Unterrichtsstunden zu unterbreiten“ (Dietrich 1976: 9). Die Unterrichtshilfen enthielten Vorbemerkungen und Stoffverteilungspläne zu den einzelnen Stoffeinheiten, die ,in Stoffabfolge und Gliederung dem Lehrplan und 
dem Lehrbuch“ folgten (Graef et al. 1985: 12). Sie „beschränkten sich jedoch bei der didaktisch-methodischen Aufbereitung des Stoffs nicht exakt auf das in den Lehrplänen präzis ausgewiesene Wissen“ (Tille 1993c: 388). Die Stoffverteilungspläne listeten Stunden, Themen und Stoffschwerpunkte, Stoffverbindungen sowie Unterrichtsmittel auf. Ferner verfügte die Unterrichtshilfe über detailliert Stundenentwürfe. Diese enthielten Stundenziele, Unterrichtsmittel, Hinweise zur technischen Vorbereitung, die stoffliche Gliederung, methodische Hinweise, Tafelübersichten, Beispiele für Hausaufgaben und Arbeitsblätter (Abb. 16 und 17). Einerseits bot der Titel umfangreiche Hilfen bei der Unterrichtsvorbereitung und -durchführung, andererseits betonten die Autoren, die Unterrichtshilfe:

„lediglich als Empfehlungen zu betrachten. Eine schematische Übernahme kann nicht zum Erfolg führen. Jeder Biologielehrer muß die Unterrichtshilfen für seine konkrete Situation in der Klasse und oftmals sogar für Parallelklassen durchdenken und spezifisch variieren. [...] Nur so wird er die im Lehrplan der 10. Klassen gestellten Anforderungen optimal erfüllen können." (Graef et al. 1985: 12)

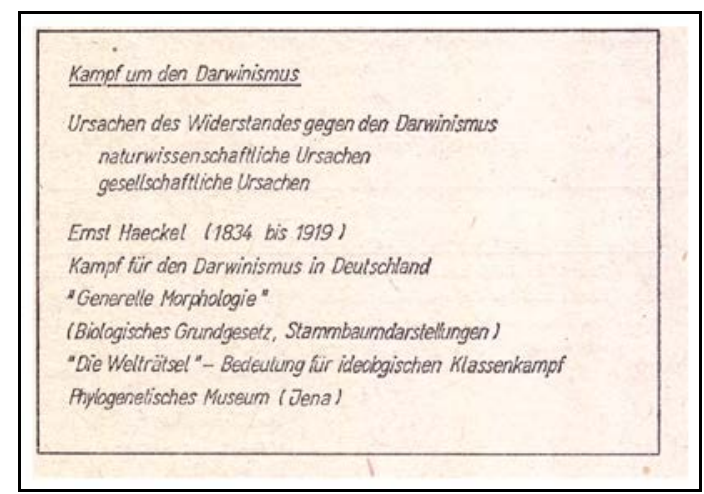

Abbildung 16: Tafelbild (Graef et al. 1985)

Auch zum Lehrplan von 1988 stand den Lehrkräften eine Neuauflage der Unterrichtshilfen zur Verfügung. Diese „soll[t]en dem Lehrer bei der schöpferischen Umsetzung des Lehrplans helfen“ (Graef et al. 1989: 12). Die Stoffabfolge entsprach weiterhin dem Lehrplan. Auf Stundenentwürfe verzichteten die Autorinnen und Autoren jedoch. Auch für die Hand der Lehrkräfte und als Arbeitsgrundlage für den Unterricht konzipiert, erschien im Jahr 1990 eine Handreichung zur Arbeit mit den Lebrplänen Biologie Klasse 5 bis 10. 


\section{Klasse 12}

Im Schuljahr 1946 verfügten die Lehrkräfte auch für die 12. Klassenstufe über den bereits erwähnten Lehrplan für die Grund- und Oberschulen in der sowjetischen Besatzungszone Deutschlands (Abb. 18). Das Lehrbuch Landwirtschaftliche Biologie für die Oberschule aus dem Jahr 1946, von Willy Matthes (Karl-Marx-Schule in Leipzig) bearbeitet, deckte jedoch nur in Teilen die Lehrplaninhalte ab. Für die Vermittlung evolutionsbiologischer Inhalte musste daher auf Alternativen zurückgegriffen werden.

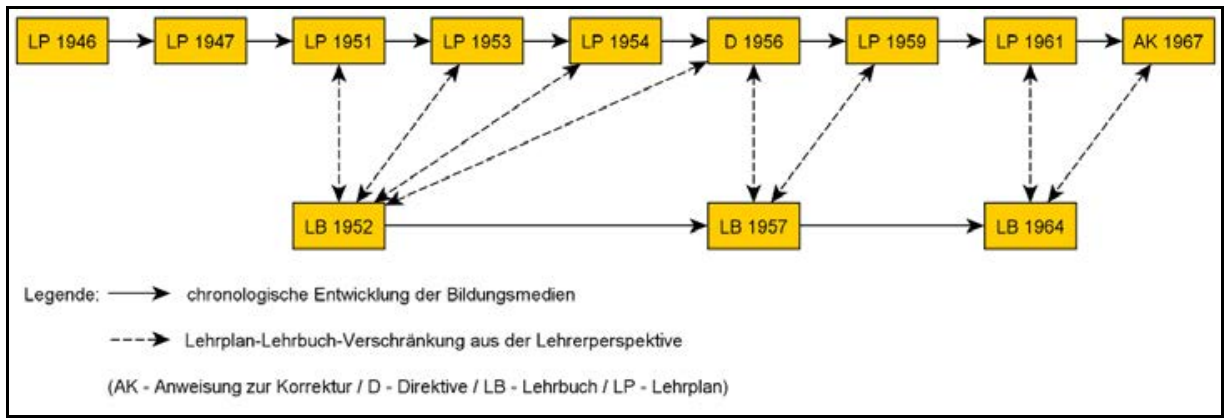

Abbildung 18: Entwicklung der Lehrplan-Lehrbuch-Verschränkung für die 12. Klasse

In der zweiten Lehrplanrunde erschienen unter dem Titel Lehrplan für Oberschulen in den Jahren 1951, 1953 und 1954 neue Lehrpläne mit evolutionsbiologischen Inhalten (vgl. MfV 1951b). Der Verlag Volk und Wissen gab dazu 1952 das Lehrbuch der Biologie für das 12. Schuljabr heraus (Abb. 19), das ,in Zusammenarbeit von Methodikern und führenden Wissenschaftlern geschaffen wurde“ (Anonymus 1952: 249). Zu diesem Autorenkollektiv gehörten HansEckhard Gruner, Heinz Reinhard, Karl Renatus, Werner Rothmaler, Georg Schneider und Georg Uschmann. Nicht nur der in Jena aktive Georg Schneider, sondern auch der Botaniker Werner Rothmaler von der MartinLuther-Universität in Halle-Wittenberg vertrat zu dieser Zeit Ansichten des Lyssenkoismus (Laitko 2010). Dies zeigte sich letztlich im Schülerbuch, das über entsprechende Inhalte verfügte. Da das Lehrbuch erst im Oktober erschien, veröffentlichte bereits im Juni die Zeitschrift Biologie in der Schule zwei Lehrbuchkapitel (Anonymus 1952). Ziel war es, den Biologielehrerinnen und -lehrern die Arbeit zu

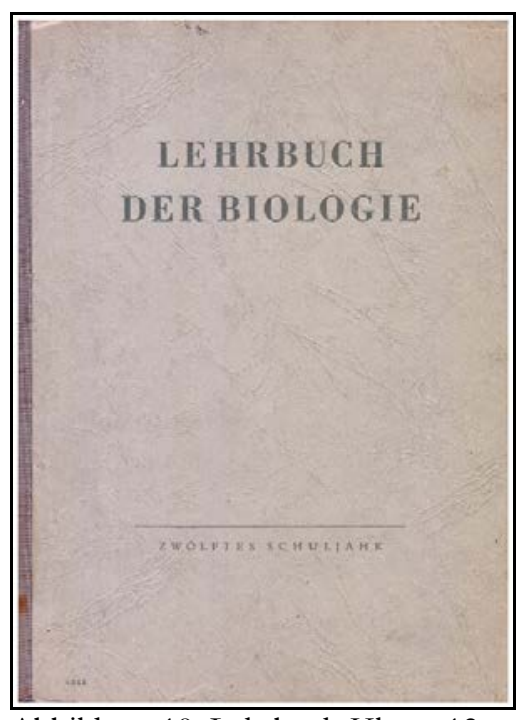

Abbildung 19: Lehrbuch Klasse 12 (1952) 
erleichtern. Das Lehrbuch wies gegenüber dem Lehrplan eine abweichende Stoffanordnung auf, „welche eine methodisch vorteilhaftere unterrichtliche Behandlung der betreffenden Stoffgebiete“ (MfV 1952a: 433) ermöglichen sollte. Diese neue Stoffanordnung berücksichtigten die Autoren bei der nächsten Lehrplanrunde im Jahr 1953 (ebd.). Auch inhaltlich erfuhr der Stoffplan von 1953 eine Angleichung an das Lehrbuch von 1952 (Kühn 1953).

Am 1. September 1954 trat ein Lehrplan für die Klassen 9 bis 12 an Oberschulen in Kraft. Das ausgewiesene Ziel war die „Erziehung von Patrioten“ (MfV 1954: 3). Der Unterricht fand in Kursen (A, C sowie B) statt, wobei „der Biologieunterricht in den mathematisch-naturwissenschaftlichen Klassen (B) $[\ldots]$ im Besonderen das Ziel [hatte], die Schüler auf ein naturwissenschaftliches Fachstudium [an] einer Fachschule oder Hochschule nach der 10. bzw. 12. Klasse vorzubereiten“ (MfV 1954: 5).

Mit Wirkung zum 1. September 1956 veröffentlichte die Hauptabteilung Unterricht und Erziehung des MfV die Direktive Biologieunterricht Oberschule. Der Unterricht fand weiterhin in den Kursen A, B und C statt. Der Titel stellte jedoch „keinen neuen Lehrplan, sondern eine Übergangslösung bis zum Erscheinen eines neuen Lehrplans dar“ (MfV 1958: 3). Dennoch wurde der „zu behandelnde Stoff und seine Gliederung [...] durch die Direktive festgelegt" (ebd.). Da die Lehrbücher noch an den alten Lehrplan angepasst waren, enthielt das Vorwort die Bemerkung, dass der Umfang und die Anforderungen der Lehrbücher über die Anforderungen der Direktive hinausgehen. Ein weiterer Hinweis ließ die Möglichkeit offen, Umstellungen innerhalb der Hauptkapitel vorzunehmen.

Ein neues Lehrbuch erschien mit einigen inhaltlichen Kürzungen im Jahr 1957. Das äußere Design änderte sich nicht. Als Autoren zeichneten Werner Rothmaler und Georg Uschmann. Der Titel Lehrbuch der Biologie für das 12. Schuljahr blieb erhalten und wurde um den Untertitel Die Entstehung des Lebens auf der Erde und seine Entwicklung bis zum Menschen, Zur Geschichte der Abstammungslebre erweitert. In diesem Jahr erhielten die Schüler auch das Lehrheft Züchtung von Pflanzen und Tieren. Als Verfasser zeichneten der Agrarbiologe Heinz Kress (1913-1996) und der Veterinärmediziner Ekkehard Wiesner $(* 1924)$. Kress war in dieser Zeit Professor mit Lehrauftrag für Methoden der sowjetischen Pflanzenzüchtung und Saatzucht-Leiter an der Forschungsstelle für Agrobiologie bei Güstrow (vgl. Catalogus Professorum Rostochiensium 2014). Wiesner wirkte im Bereich Tierzucht und Tierernährung an der Veterinärmedizinischen Fakultät der HumboldtUniversität zu Berlin (Gerber 2008).

Veränderungen im Schulwesen, wie die neue Stundentafel vom 4. Mai 1959, bedingten die Ausarbeitung eines neuen Lehrplans (Steinheit 1959). Unter dem Titel Lehrplan der zuölfkelassigen erweiterten Oberscbule für das Schuljahr 1959/60 diente der Übergangslehrplan lediglich für das Schuljahr 1959/60. Als Zusammenfassung aller bis dato gültigen Pläne verstanden, entsprach dieser „der vielfach erhobenen Forderung nach der Herausgabe eines gültigen Dokuments für die Unterrichtsar- 
beit an den erweiterten Oberschulen" (MfV 1959b: 4). Da jedoch noch das alte Lehrbuch an die Schulen ausgeliefert wurde, konnten die Lehrkräfte in der Zeitschrift Biologie in der Schule Beiträge zu veränderten Lehrplaninhalten wahrnehmen (Anonymus 1959). Zusätzlich erhielten die Schüler das Lehrheft Die Züchtung, das auch den Schülern der 10. Klasse der allgemeinbildenden polytechnischen Oberschule zur Verfügung stand (ebd.).

Im Jahr 1961 gab das MfV eine neue Stundentafel sowie einen neuen Lehrplan unter dem Titel Lehrplan der zwölffelassigen erweiterten Oberschule heraus. Die Veränderungen standen im Kontext der Beschlüsse des 5. Parteitages der SED sowie der Ziele des 6. Pädagogischen Kongresses (vgl. MfV 1961: o. S.). Ziel war es, „das Lernen und die sozialistische Erziehung entsprechend den Anforderungen unserer sich entwickelnden sozialistischen Gesellschaft zu verbessern" (ebd.). Die Einführung der Pläne und Stundentafeln erfolgte schrittweise, beginnend mit den 9. Klassen im Schuljahr 1960/61 bis zum Schuljahr 1963/64 für die Klasse 12. Zur Orientierung wurden die Pläne und Stundentafeln jedoch bereits 1961 veröffentlicht. Der Unterricht fand weiterhin in Kursen statt.
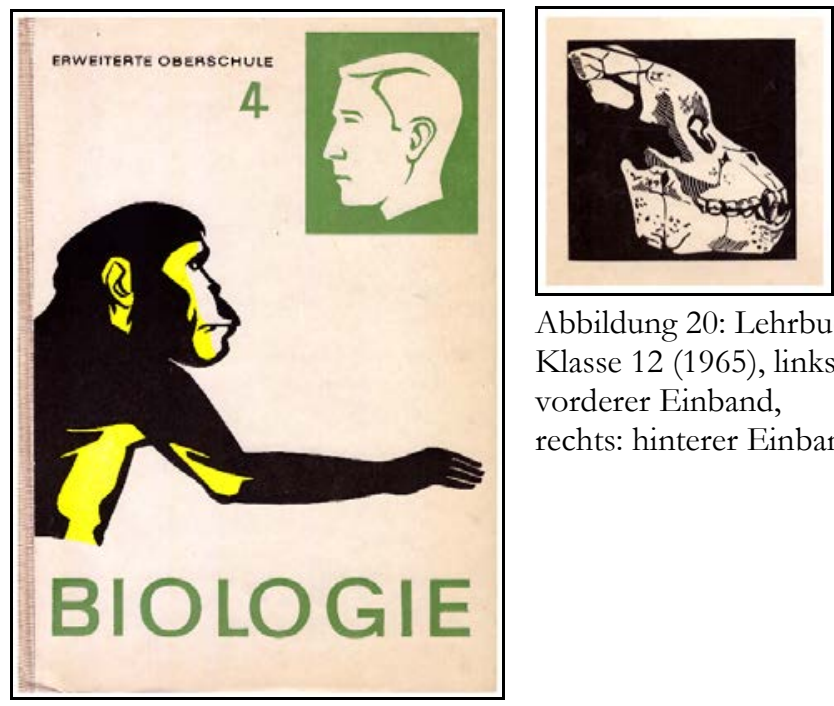

Abbildung 20: Lehrbuch

Klasse 12 (1965), links:

vorderer Einband, rechts: hinterer Einband

Im Jahr 1964 noch in zwei Bänden aufgelegt ${ }^{89}$, erschien ab 1965 das Lehrbuch Biologie IV, ein Lebrbuch für die erveiterte Oberschule, 12. Klasse, Die Lebre von der Evolution der Organismen in einem Band (Abb. 20). Gertrud Kummer übernahm die Redaktion und die Autoren Herbert Bach, Dieter Bernhardt, Wolfgang Crome, Rolf

89 Grundsätzlich erfolgte die Auslieferung der Lehrbücher Ende Juli. Die Fertigstellung des Lehrbuches verzögerte sich jedoch. Daher wurden zuerst die bereits vorliegenden Inhalte gedruckt, um die unterrichtliche Arbeit abzusichern. Die fehlenden Kapitel erschienen dann als zweiter Band (Interview mit Gertrud Kummer vom 29.12.2016). 
Löther, Helmut Nestler und Martin Zacharias trugen zur Erstellung des Buches bei. Auch diese Lehrbucherarbeitung unterstützten Wissenschaftler verschiedener Fachgebiete (Abb. 21). Beispielhaft seien hier genannt Dieter Bernhardt, Philosoph am Institut für Philosophie der Universität Greifswald (Bernhardt 1965), Rolf Löther (*1933), Biophilosoph und Biohistoriker sowie Martin Zacharias (1927-1988), Genetiker am Institut für Kulturpflanzenforschung Gatersleben in der Abteilung Genetik und Zytologie (Müntz \& Wobus 2013).

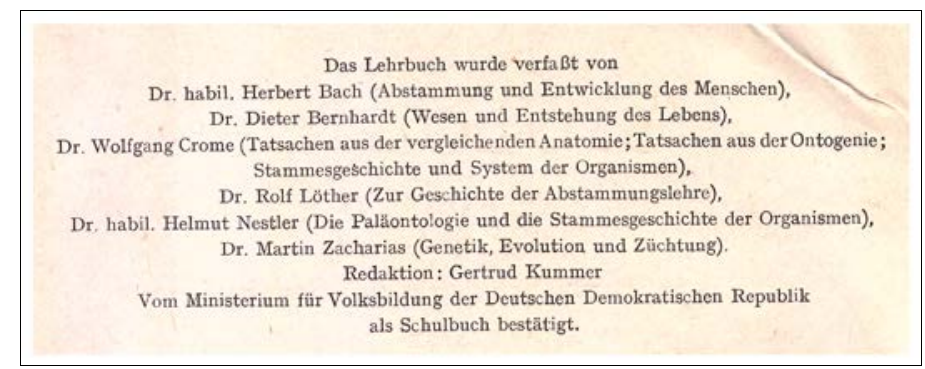

Abbildung 21: Verfasser und ihre Fachdisziplinen, Lehrbuch Klasse 12 (Bach et al. 1965)

Mit Wirkung zum 1. September 1967 veröffentlichte das MfV eine Anweisung zur Korrektur des Lehrplans Biologie der Erweiterten Oberschule, die den 1961 eingeführten Lehrplan für die 12. Klasse im A- und B-Zweig ersetzte und dem 1965 eingeführten Lehrbuch der Biologie IV entsprach (vgl. MfV 1967a: 1). Als Grund für die Veränderungen führte das MfV die „Entwicklung der biologischen Wissenschaften in den letzten Jahren“ (MfV 1967a: 1) an. Im Schuljahr 1970/71 entfielen evolutionsbiologische Inhalte in den Lehrplanwerken und Lehrbüchern für die 12. Klasse und wurden in die Klasse 10 verlagert. Diese inhaltliche Ausrichtung blieb bis zum Zusammenschluss beider deutscher Staaten erhalten.

\section{Von der Faustskizze zum Schülerarbeitsmittel}

Bereits 1945 nahm im Bereich der Volksbildung die Zentralbild- und Lehrmittelstelle ihre Arbeit auf. Als Nachfolgeinstitution wirkten von 1946 bis 1950 die Zentralbildstelle (ZBS), von 1950 bis 1954 das Zentralinstitut für Film und Bild in Unterricht, Erziehung und Wissenschaft (ZFB) sowie von 1954 bis 1962 das Deutsche Zentralinstitut für Lehrmittel (DZL). Übergeordnete Einrichtung war von 1946 bis 1949 die DVfV und nach 1949 das MfV. Die Arbeit und Aufgaben des DZL übernahm zwischen 1962 bis 1970 die Sektion Unterrichtsmittel. Diese unterstand dem DPZI. Als Nachfolger der Sektion Unterrichtsmittel wirkte im Zeitraum von 1970 bis 1990 das Institut für Unterrichtsmittel (IU). Es wurde auch unter der Bezeichnung Institut für audiovisuelle Unterrichtsmittel geführt. Die ebenfalls im Jahr 1970 gegründete Akademie der Pädagogischen Wissenschaften 
(APW) fungierte als übergeordnete Institution (Tab. 19). Für die Entwicklung von Unterrichtsmedien stand das Institut für Unterrichtsmittel der APW den Lehrkräften und ihren Ideen als Ansprechpartner zur Verfügung. Das Institut veröffentlichte die Schriftenreihe Unterrichtsmittel, in der beispielsweise die Themen Didaktische Möglichkeiten und Wirkungen des Schulfernsehens (1971, Heft 41), Anschauung und Anschauungsmittel (1973, Heft 43) und Audiovisuelle Unterrichtsmittel und erziehungswirksamer Unterricht (1979, Heft 45) erörtert wurden. Im Zeitraum 1970 bis 1990 bestand für Lehrfilme, wissenschaftliche Filme bzw. Hochschulfilme das Institut für Film, Bild und Ton (FBT). Es war dem Ministerium für Hoch- und Fachschulwesen untergeordnet. Ferner existierte von 1972 bis 1990 das Zentralinstitut für Schulfunk und Fernsehen mit dem MfV als übergeordnete Institution. Im Jahr 1948 gründete sich unter der Bezeichnung Staatliches Kontor für Unterrichtsmittel und Schulmöbel (SKUS) eine Vertriebsorganisation für Schulbedarf, Lehr- und Lernmittel sowie Schulbücher (vgl. SKUS Lehrmittel GmbH o. J.). Ferner bestanden in den Kreisen, Städten und Stadtbezirken der DDR Filmbild-, Schulbild- bzw. Kreisfilmstellen (DEFA-Stiftung o. J.b).

Tabelle 19: Institutionen für Unterrichtsmedien (verändert nach DEFA-Stiftung o. J.a)

\begin{tabular}{|l|l|l|}
\hline Zeit & Institution für Unterrichtsmedien & Übergeordnete Institution \\
\hline 1945 & Zentralbild- und Lehrmittelstelle & - \\
\hline $1946-1950$ & Zentralbildstelle & $\begin{array}{l}\text { Zentralverwaltung für Volksbildung, } \\
\text { ab 1949 Ministerium für Volksbildung }\end{array}$ \\
\hline $1950-1954$ & $\begin{array}{l}\text { Zentralinstitut für Film und Bild in Unterricht, } \\
\text { Erziehung und Wissenschaft }\end{array}$ & Ministerium für Volksbildung \\
\hline $1954-1962$ & Deutsches Zentralinstitut für Lehrmittel & Ministerium für Volksbildung \\
\hline $1962-1970$ & Sektion Unterrichtsmittel & $\begin{array}{l}\text { Deutsches Pädagogisches } \\
\text { Zentralinstitut }\end{array}$ \\
\hline $1970-1990$ & Institut für Unterrichtsmittel & $\begin{array}{l}\text { Akademie der Pädagogischen } \\
\text { Wissenschaften }\end{array}$ \\
\hline
\end{tabular}

Die Aussage von Lothar Herrmann, einem Fachlehrer für Biologie und Chemie, in den Mitteilungen der Sektion Scbulbiologie veröffentlicht, zeigt exemplarisch die Probleme auf, die in den Anfangsjahren im Schuldienst in der SBZ auftraten.

„Als wir im Dezember 1945 den Unterricht in unserer antifaschistischdemokratischen Schule aufnahmen, waren für Lehrer und Schüler Tafel und Kreide bzw. Griffel meist die einzigen verfügbaren bzw. organisierbaren Lern- und Lehrmittel. 1947 halfen uns die neuverlegten ,Faustskizzen für den naturkundlichen Unterricht' von A. GÜRTLER, biologische Skizzen während des Unterrichts an der Wandtafel anzufertigen.“ (Herrmann 1974: 252, Hervorhebung im Original) 
Die im Zitat benannten Faustskizzen wurden im Verlag Ernst Wunderlich aus Leipzig verlegt und enthielten beispielsweise Zeichnungen der Vordergliedmaßen von Mensch, Affe, Schwein, Rind und Pferd (Gürtler 1947). Diese konnten vergleichend anatomisch betrachtet und als Beleg für die Abstammung genutzt werden. Wenn auch die Ausstattung an den Schulen und in allen Fachbereichen mangelhaft war, so wurde doch die biologische Sammlung bereits nach 1945 in der SBZ für alle Schulen gefordert (Grönke 1969). Aufgrund der schwierigen materiellen Situation an den Schulen in der Zeit nach dem Krieg, ist aber davon auszugehen, dass eine Umsetzung dieser Forderung nur bedingt erfolgen konnte. Es fehlte an Einrichtungsgegenständen, Materialien für die Schüler, Personal und anderen, für den Schulbetrieb essentiellen, Rahmenbedingungen. Die Schwierigkeiten benennend, betonte Paul Wandel in seiner Rede auf dem 2. Pädagogischen Kongress 1947 in Leipzig:

„Es ist gelungen, ein geordnetes Schulleben zu entfalten, obwohl 800000 Kinder mehr eingeschult werden mussten als 1939, viele Schulen mit all ihren Einrichtungen für den Schulunterricht unbrauchbar waren und fast $80 \%$ der Lehrer und die ganze Schulverwaltung ersetzt werden mußten.“ (Wandel o. J.: 3)

Im Jahr 1951 gab die Hauptabteilung Unterricht und Erziehung des MfV für die Grundschulen ein Mindestausstattungsplan heraus (Schulze-Marx 1954). Noch 1953 kritisierte Ottokar Grönke (1954: 140, Hervorhebung im Original), Assistent in der Abteilung Biologie-Methodik an der PH Potsdam, dass ,in der Schule [...] $\mathrm{B}$ ild t a feln für Themen, bei denen ohne Lebendanschauung gearbeitet werden muß, dringend gebraucht" werden.

$\mathrm{Zu}$ den ersten Unterrichtsmedien, die im Unterricht beim Thema Evolutionsbiologie zum Einsatz kamen, gehörten die im Jahr 1954 erschienenen Lehrtafeln Anthropologie (Geisenhainer 1959). Im Rahmen eines Entwicklungsplanes wurde die Entwicklung neuer Lehrmittel für das Fach Biologie vorangetrieben (DZfL 1957). Dazu zählten Anschauungstafeln mit Lebensbildern fossiler Pflanzen und Tiere (u. a. Devon-Süßwasserlandschaft, Steinkohlewald, Braunkohlewald) und Lehrtafeln sowie Anschauungsbilder zur Anthropogenie (u. a. Skelettvergleiche zwischen Menschenaffen und Menschen der Neuzeit, Schädelfunde, Rekonstruktionen der Köpfe der Vor-, Ur- und Altmenschen). Die Grundausrüstung für den Biologieunterricht im Allgemeinen legte die Nomenklatur von 1959 fest. Diese war einerseits als staatliche Forderung, andererseits aber als Empfehlung zu verstehen, da etwa die Hälfte der Gegenstände selbst hergestellt oder beschafft werden konnte (Grönke 1969). Für evolutionsbiologische Inhalte wurden im Schuljahr 1959 in Ergänzung zur Bildreihe Anthropologie drei Anschauungstafeln und vier Anschauungsbilder zur Verfügung gestellt. Die Anschauungstafeln dienten vorrangig der Darstellung der physischen Entwicklung des Menschen. Sie zeigten das Skelett eines rezenten Menschen sowie eines Gorillas im Vergleich (Abb. 22), 
die Entwicklung der Schädelform sowie die Entwicklung des Werkzeuggebrauchs. Die Anschauungsbilder stellten die gesellschaftliche Entwicklung des Menschen dar. Sie zeigten Australopithecinen in ihrem Lebensraum, die Fundstätte des Sinanthropus, den Lebensraum der Ehringsdorfer sowie Jungpaläolithiker in einer Siedlung. Adäquate inhaltliche Hinweise stellte im Jahr 1959 die Zeitschrift Biologie in der Schule den Lehrkräften zur Verfügung (Geisenhainer 1959).

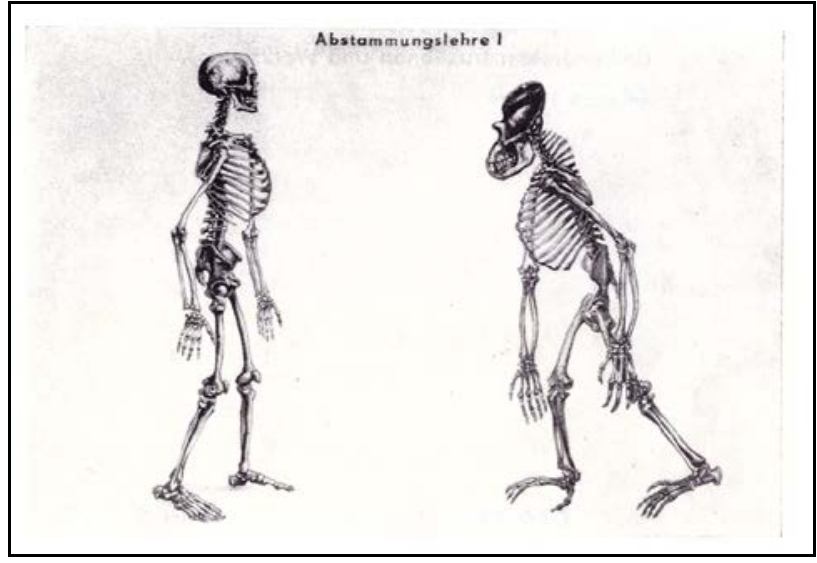

Abbildung 22: Skelettvergleich Gorilla-Mensch (SKUS 1974)

Das Deutsche Zentralinstitut für Lehrmittel gab 1962 das Werk Lehrmittel für den Biologieunterricht heraus. Dieses erschien als Loseblattsammlung in zwei Kunstledermappen mit Reißmechanik, bis 1971 in fünf Ergänzungen. Sie dienten als „Anleitung zur Verwendung der für den Biologieunterricht zur Verfügung stehenden industriell gefertigten Lehrmittel“" (DZL 1962: o. S.). Ziel war es, durch Erläuterungstexte und Hinweise die Lehrkräfte bei der Auswahl der Unterrichtsmedien zu beraten. Alle angegebenen Modelle, Geräte und Anschauungstafeln konnten beim SKUS in Leipzig bezogen werden. Darüber hinaus bestand die Möglichkeit Filme, Lichtbildreihen und Magnettonbänder bei den zuständigen Kreisstellen für Unterrichtsmittel auszuleihen (ebd.).

Insgesamt standen im Jahr 1962 für das Sachgebiet Abstammungs- und Entwicklungslehre zehn Anschauungstafeln, ein Unterrichtsfilm sowie sechs Lichtbildreihen und im Jahr 1969 (komplettiertes Verzeichnis) 15 Anschauungstafeln, ein Unterrichtsfilm, 13 Lichtbildreihen sowie Schädelrekonstruktionen als Modelle zur Verfügung (Tab. 20). Im Gegensatz zu einigen anderen Sachgebieten wurden keine Magnettonbänder als Lehrmittel angeboten. Für die Vielzahl der Lichtbildreihen (Dias) zum Sachgebiet Abstammungslehre standen den Lehrkräften Beihefte zur Verfügung (Windelband 1966a; 1966b; 1966c; 1967). Herausgegeben vom Deutschen Pädagogischen Zentralinstitut enthielten diese Hefte Bilderläuterungen und me- 
thodische Hinweise zur Verwendung im Unterricht. Ergänzend veröffentlichte die Zeitschrift Biologie in der Schule im Mai 1962 einen Beitrag zur Herstellung und Verwendung der Bildrolle. Diese galt in dieser Zeit als geeignetes Anschauungsmittel für Entwicklungsvorgänge (Prehn 1962). Ein weiteres zeittypisches Mittel war die Manipermhafttafel, die insbesondere bei der Behandlung der Anthropogenese, so Krüger (1962), vielseitige Möglichkeiten im Unterricht bot.

Tabelle 20: Unterrichtsmedien für das Sachgebiet Abstammungs- und Entwicklungslehre von 1962 und 1969 (verändert nach DZL 1962; vgl. Windelband et al. 1969)

\begin{tabular}{|c|c|c|}
\hline & 1962 & 1969 \\
\hline Modelle & - & Schädelrekonstruktionen \\
\hline $\begin{array}{l}\text { An- } \\
\text { schauungstaf } \\
\text { eln }\end{array}$ & $\begin{array}{l}\text { Mitteldevonische Landschaft, Stein- } \\
\text { kohlewald, Braunkohlewald, Vor- } \\
\text { mensch, Urmensch, Altmensch, } \\
\text { Eiszeitmensch, Abstammungslehre I, } \\
\text { Abstammungslehre II, Abstam- } \\
\text { mungslehre III }\end{array}$ & $\begin{array}{l}\text { Mitteldevonische Landschaft, Steinkohlewald, } \\
\text { Braunkohlewald, Vormensch, Urmensch, Alt- } \\
\text { mensch, Eiszeitmensch, Abstammungslehre I, } \\
\text { Abstammungslehre II, Abstammungslehre III, } \\
\text { Tiere der Kreidezeit I, Tiere der Kreidezeit II, } \\
\text { Jurameer, Zwischeneiszeitliche Landschaft, } \\
\text { Proconsul }\end{array}$ \\
\hline $\begin{array}{l}\text { Unterrichts- } \\
\text { filme }\end{array}$ & $\begin{array}{l}\text { Die Entstehung der Eiweiße und } \\
\text { Koazervate }\end{array}$ & Die Entstehung der Eiweiße und Koazervate \\
\hline $\begin{array}{l}\text { Lichtbildrei- } \\
\text { hen }\end{array}$ & $\begin{array}{l}\text { Paläozoologie I (Übersichtsreihe), } \\
\text { Paläozoologie II (Ammoniten), } \\
\text { Paläozoologie III (Saurier), Paläobo- } \\
\text { tanik, Abstammungslehre I, Ab- } \\
\text { stammungslehre II }\end{array}$ & $\begin{array}{l}\text { Paläozoologie I (Übersichtsreihe), Paläozoologie } \\
\text { II (Ammoniten), Paläozoologie III (Saurier), } \\
\text { Paläobotanik, Abstammungslehre I, Abstam- } \\
\text { mungslehre II, Abstammungslehre I: Geschichte } \\
\text { der Organismen, Abstammungslehre II: Über- } \\
\text { gangsformen, Abstammungslehre III: Verglei- } \\
\text { chende Morphologie und Anatomie, Abstam- } \\
\text { mungslehre IV: Ontogenie, Abstammungslehre V: } \\
\text { Biogeographie, } \\
\text { Abstammungslehre VI: Anthrophogenese, Mitose }\end{array}$ \\
\hline
\end{tabular}

Der Ansicht folgend, „daß die großen pädagogischen Potenzen des Fernsehens zu nutzen sind, ohne dabei auf die notwendige Führung durch den unterrichtenden Lehrer zu verzichten" (Kirsch 1967: 428), strahlte ab 1964 der Deutsche Fernsebfunk (DFF) Schülerprogramme aus. Die Anpassung der Sendungen an Lehrplan und Klassenstufe erfolgte in Kooperation durch die Arbeitsgruppe Schulfernsehen beim Wissenschaftlichen Rat des MfV und den Verantwortlichen des DFF. Die ersten biologischen Sendungen erschienen im Schuljahr 1965/66 (ebd.). Zwar wurden die Themen durch die Untergruppe Biologie der Arbeitsgruppe Schulfernsehen vorläufig auf die Klassenstufen 5 und 6 zugeschnitten, dennoch „handelt[e] es sich vorwiegend um Probleme der Entwicklungslehre und der Anwendung biologischer Erkenntnisse in der Praxis“ (ebd.: 430). Die Zeitschrift Biologie in der Schule veröffentlichte in den folgenden Jahren regelmäßig Beiträge zu Fernsehsendungen und ihren methodisch-didaktischen Einsatzmöglichkeiten. Hier fanden auch Sendungen aus 
dem Bereich der Evolutionsbiologie, wie Die Alten aus Bilzingsleben, Berücksichtigung (Schubert 1989a). Über Inhalt, Aufbau, Gestaltung und Nutzen der Sendungen informierte das Zentralinstitut für Schulfunk und Schulfernsehen der Pädagogischen Hochschule in Potsdam. Es gab Informationen und Empfeblungen zur Arbeit mit den Sendungen des Bildungsfernsehens der DDR für das Fach Biologie heraus. Eine der letzten produzierten Sendungen Übrigens ist der Darwin ganz famos stellte „anschauliches Material über Darwins Naturbeobachtungen, speziell seine Reise mit der Beagle und dort insbesondere seine Eindrücke auf den Galapagos-Inseln bereit" (Schubert 1989b: 15).

Im Jahr 1966 stand für die Lehrkräfte der Titel Biologische Aufgabensammlung aus dem Verlag Volk und Wissen zur Verfügung. Als Autoren zeichneten Joachim Günther und Karl Scheiding. Das Buch enthielt u. a. 46 Aufgabenstellungen zum Thema Abstammungslebre. Ziel war es, „den Unterricht zu intensivieren und bei den Schülern die Freude an der Natur und die Achtung vor dem Leben zu vertiefen" (Günther \& Scheiding 1966: 10). Die Auswahl der Aufgaben orientierte daher auf eine produktive Aneignung des Stoffes. Dabei sollte auch explizit Sekundärliteratur zu Rate gezogen werden, wie das Schülerleseheft Lebensspuren im Stein (1963) von Helmut Nestler aus der Reihe Bücher für den Scbüler (Abb. 23)..$^{90}$
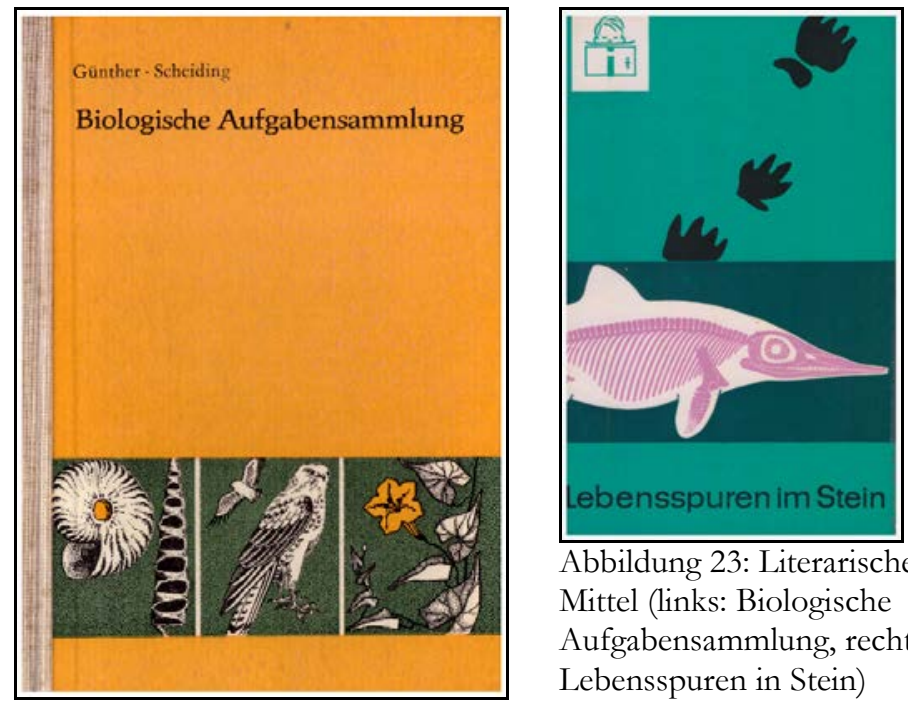

Abbildung 23: Literarische

Mittel (links: Biologische

Aufgabensammlung, rechts:

Lebensspuren in Stein)

90 In der Reihe Bücher für den Scbüler sind auf dem Gebiet der Biologie (teilweise aus dem Russischen übersetzt) weitere Schülerlesehefte erschienen: u. a. Getarnte Tiere, Vom Leben der Tiere und Schütz̨t die Natur. 
Tabelle 21: Evolutionsbiologische Unterrichtsmedien aus den Gesamtbedarfsplänen für Unterrichtsmittel von 1971 und 1972 (verändert nach MfV 1971; MfV 1972)

\begin{tabular}{|c|c|c|c|c|c|c|c|c|c|}
\hline \multirow[t]{2}{*}{ Unterrichtsmittel } & \multicolumn{9}{|c|}{ Verwendung in den Klassen } \\
\hline & 5 & 6 & 7 & 8 & 9 & 10 & 11 & 12 & \\
\hline$\frac{\text { Filme }}{\text { Entstehung der Eiweiße und Koazervate (s/w; } 10 \mathrm{~min})}$ & - & - & - & - & - & 2 & - & - & \\
\hline $\begin{array}{l}\text { Lichtbildreihen } \\
\text { Entwicklung der Organismen (farbig; etwa } 18 \text { Bilder) } \\
\text { Natürliche und künstliche Auslese (farbig; etwa } 18 \text { Bilder) } \\
\text { Paläozoologie III: Saurier (s/w; } 14 \text { Bilder) } \\
\text { Abstammungslehre I: Geschichte der Organismen (farbig; } 21 \\
\text { Bilder) } \\
\text { Abstammungslehre II: Übergangsformen (farbig; Auswahl) } \\
\text { Abstammungslehre III: Morphologie und vergleichende } \\
\text { Anatomie (farbig; } 20 \text { Bilder) } \\
\text { Abstammungslehre IV: Ontogenese (farbig; } 8 \text { bis } 9 \text { Bilder) } \\
\text { Abstammungslehre Vl: Anthropogenese (farbig; } 11,13 \text { bis } 28 \\
\text { Bilder) }\end{array}$ & $\begin{array}{l}- \\
- \\
-\end{array}$ & $\begin{array}{l}- \\
- \\
- \\
-\end{array}$ & $\begin{array}{l}- \\
- \\
-\end{array}$ & $\begin{array}{l}- \\
- \\
-\end{array}$ & $\begin{array}{l}- \\
- \\
-\end{array}$ & $\begin{array}{l}1 \\
1 \\
- \\
3\end{array}$ & $\begin{array}{l}- \\
- \\
- \\
- \\
-\end{array}$ & $\begin{array}{l}- \\
- \\
-\end{array}$ & \\
\hline $\begin{array}{l}\text { Tonbildreihen } \\
\text { Geschichte der Entwicklungslehre (farbig; etwa } 24 \text { Bilder) }\end{array}$ & - & - & - & - & 1 & 1 & - & & \\
\hline $\begin{array}{l}\text { Anschauungstafeln / Anschauungsbilder } \\
\text { Mitteldevonische Landschaft } \\
\text { Steinkohlewald } \\
\text { Paläozoologie I (Jurameer) } \\
\text { Tiere der Kreidezeit I } \\
\text { Tiere der Kreidezeit II } \\
\text { Braunkohlewald } \\
\text { Zwischeneiszeitliche Landschaft } \\
\text { Abstammungslehre I: Skelettvergleich Gorilla - Mensch } \\
\text { Abstammungslehre II: Schädelvergleiche } \\
\text { Abstammungslehre III: Lebendrekonstruktionen und } \\
\text { Werkzeuge } \\
\text { Vormensch (Australopithecus) } \\
\text { Urmensch (Sinanthropus) } \\
\text { Altmensch (Ehringsdorfer) } \\
\text { Eiszeitmensch (Homo sapiens diluvialis) } \\
\text { Horde der Proconsulaffen }\end{array}$ & $\begin{array}{l}- \\
- \\
- \\
3 \\
3 \\
- \\
- \\
- \\
- \\
-\end{array}$ & $\begin{array}{l}- \\
- \\
- \\
- \\
- \\
- \\
- \\
- \\
-\end{array}$ & $\begin{array}{l}2 \\
2 \\
- \\
- \\
- \\
- \\
- \\
- \\
- \\
-\end{array}$ & $\begin{array}{l}- \\
- \\
- \\
- \\
- \\
- \\
- \\
- \\
- \\
-\end{array}$ & $\begin{array}{l}- \\
- \\
- \\
- \\
- \\
- \\
- \\
- \\
- \\
-\end{array}$ & $\begin{array}{l}1 \\
1 \\
1 \\
1 \\
1 \\
1 \\
1 \\
1 \\
1 \\
1\end{array}$ & $\begin{array}{l}2 \\
2 \\
- \\
- \\
- \\
- \\
- \\
- \\
- \\
-\end{array}$ & $\begin{array}{l}- \\
- \\
- \\
- \\
- \\
- \\
- \\
- \\
- \\
-\end{array}$ & \\
\hline $\begin{array}{l}\text { Schädelnachbildung } \\
\text { Sinanthropus } \\
\text { Cro Magnon } \\
\text { La Chapelle } \\
\text { Schimpanse (weibl.) } \\
\text { Steinheim }\end{array}$ & $\begin{array}{l}- \\
- \\
- \\
-\end{array}$ & $\begin{array}{l}- \\
- \\
- \\
- \\
-\end{array}$ & $\begin{array}{l}- \\
- \\
- \\
-\end{array}$ & $\begin{array}{l}- \\
- \\
- \\
- \\
-\end{array}$ & $\begin{array}{l}- \\
- \\
- \\
- \\
-\end{array}$ & $\begin{array}{l}2 \\
2 \\
2 \\
2 \\
2\end{array}$ & $\begin{array}{l}- \\
- \\
- \\
- \\
-\end{array}$ & $\begin{array}{l}- \\
- \\
- \\
- \\
-\end{array}$ & \\
\hline
\end{tabular}


Auch die Sektion Schulbiologie der Biologischen Gesellschaft lieferte Impulse zur Verbesserung und Erweiterung der Ausstattung an den Schulen. Die Gesellschaft veranstaltete im Jahr 1968 im Rahmen der 5. Schulbiologentage eine Ausstellung selbst hergestellter Unterrichtsmittel. Ein entsprechender Aufruf zur Beteiligung erging in der Zeitschrift Biologie in der Schule (Baer 1967b). In dieser Zeit vollzog sich hinsichtlich der Unterrichtsmedien für den Biologieunterricht ein Trend von der Schau- zur Arbeitssammlung (Baer \& Grönke 1969).

Ab 1967 veröffentlichte das MfV parallel zu den Lehrplänen für einzelne Klassenstufen und Fächer in Verfügungen und Mitteilungen Bedarfspläne für Unterrichtsmittel (MfV 1971). Im Jahr 1971, nach dem 7. Pädagogischen Kongress, gab das MfV für die Klassen 1 bis 10 einen Gesamtbedarfsplan für Unterrichtsmittel heraus, mit der Intention einer „Zusammenfassung und inhaltliche[n] Weiterentwicklung“ (ebd.: 3). Im Bedarfsplan teilte die Unterrichtsmedien nach drei Verbindlichkeitsstufen ein und ordnete diese den Klassenstufen zu (Tab. 21). Erarbeitet durch das Institut für Unterrichtsmittel der APW und durch die Ministerin für Volksbildung Margot Honecker bestätigt, galt er „als verbindliche staatliche Grundlage für die Ausrüstung der Oberschulen im Zeitraum 1971 bis 1975“ (MfV 1971: 3). Ziel war es, „,bis 1975 einen annähernd gleichen Ausstattungsstand an allen Oberschulen zu erreichen“ (ebd.). Die Bereitstellung der Materialien erfolgte durch das SKUS und wurde zum Teil aus dem zentralen Staatshaushalt finanziert (ebd.). Simon (1971: 334) resümiert, dass der Unterrichtsmittel-Bedarfsplan des Faches Biologie auf Bedarfsanalysen basierte und bereits Erfahrungen mit dem neuen Lehrplan berücksichtigte. Die Lehrkräfte sollten bei ihrer Unterrichtsplanung Bedarfsplan, Lehrplan, Lehrbuch und Unterrichtshilfe als Einheit wahrnehmen (ebd.). Fachzeitschriften veröffentlichten im Jahr 1971 Fachbedarfspläne, in denen „die Zuordnung der Unterrichtsmittel eines Unterrichtsfaches zu den Stoffeinheiten der Lehrpläne vorgenommen und damit die Verwendbarkeit der einzelnen Unterrichtsmittel in Abhängigkeit von den Zielen und Inhalten der verschiedenen Lehrplanthemen charakterisiert" wurde (MfV 1971: 7). Auch für die Klassen 11 und 12 lag im Jahr 1972 ein Gesamtbedarfsplan für Unterrichtsmittel vor (Tab. 21).

Während „Ermittlungen ergaben, daß etwa fünf Jahre nach Einführung der Lehrpläne von 1959 nur etwa 25 \% aller Schulen einen Fachraum für Biologie besaßen" (Tille 1993b: 268), wurde in Folge der Anforderungen des Lehrplanwerkes von 1971 mit dem Schuljahr 1973/74 erstmals an allen Schulen ein Biologiefachraum geplant (Sehmrau 1976; Abb. 24). Zwar differierte die Gestaltung der Fachräume, doch mit Blick auf die Ausstattung mit verbindlichen Unterrichtsmitteln und Arbeitsmaterialien attestierten die Verantwortlichen im Rahmen einer Überprüfung Fortschritte (vgl. ebd.: 338). Mit dem Hinweis, dass ,zur Erfüllung des neuen Lehrplanes in hoher Qualität [...] der erkenntnistheoretische und psychologische Wert und der didaktisch-methodische Einsatz der verfügbaren Unterrichtsmittel von außerordentlich großer Bedeutung [ist] ", betonte Heinz-Werner Baer (1975: 8) auf den 10. Schulbiologentagen 1973 in Halle, den Stellenwert der 
Unterrichtsmittel für die Lehrplanumsetzung. Den Unterrichtsmitteln kam eine Doppelfunktion zu, da sie als ,eine wichtige Grundlage für die fachliche und weltanschauliche Bildung“" (ebd.) angesehen wurden.

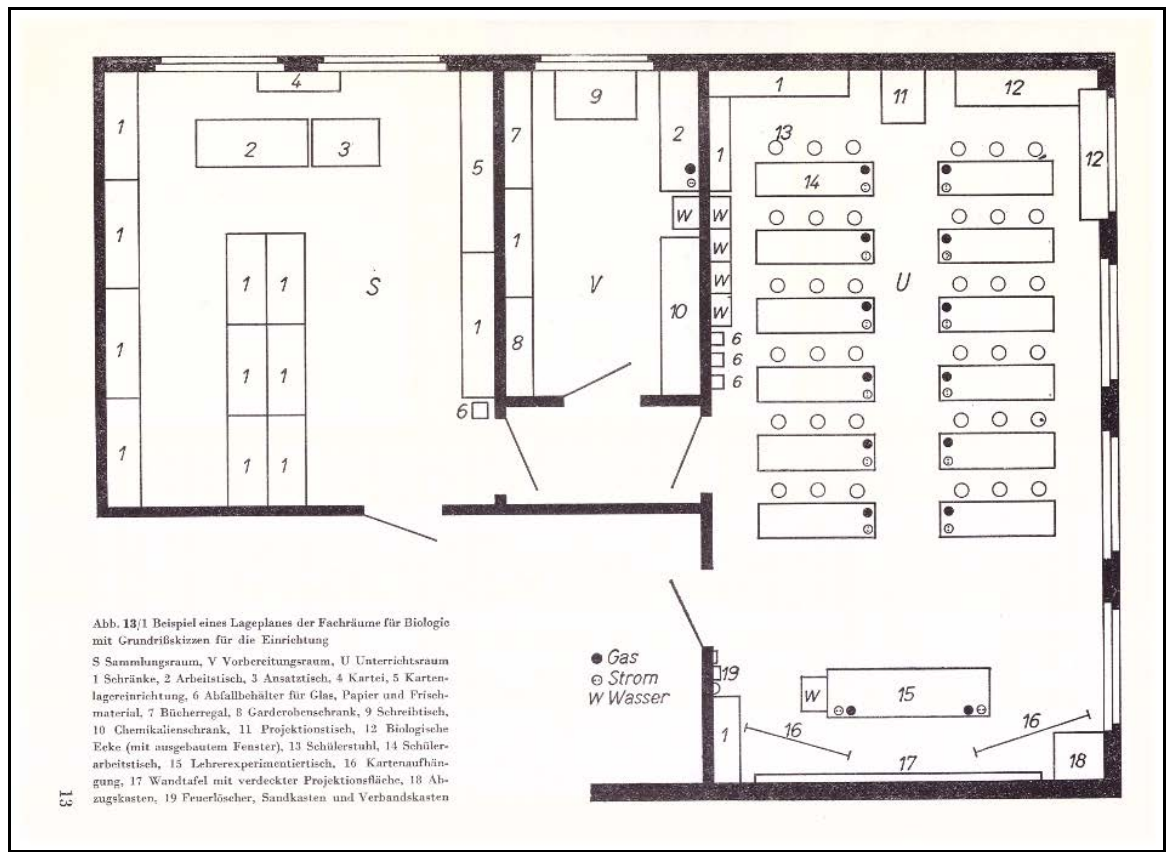

Abbildung 24: Lageplan für einen Biologiefachraum (Baer et al. 1969)
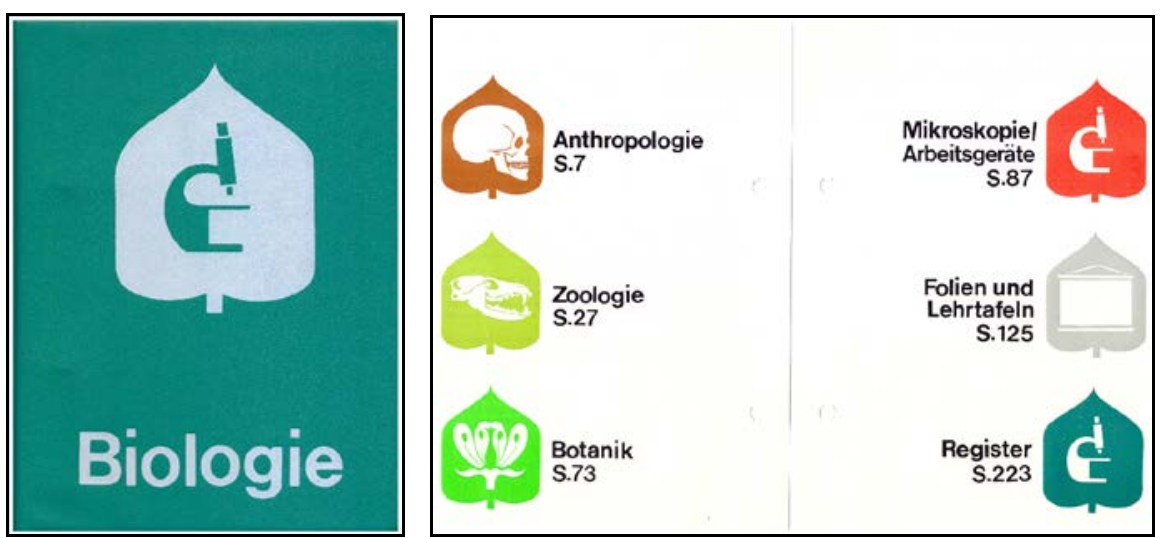

Abbildung 25: Katalog Biologie, Unterrichtsmittel (links: Einband, rechts: Inhalt) 
Das SKUS in Leipzig führte eine ständige Ausstellung in ihren Geschäftsräumen durch und gab den Katalog Biologie, Unterrichtsmittel heraus (Abb. 25). Dieser enthielt Angaben über verbindliche und empfohlene Unterrichtsmittel sowie die Klassenstufe. Bei „der Zusammenstellung dieses Kataloges war der vom Ministerium für Volksbildung herausgegebene Gesamtbedarfsplan die Grundlage“" (SKUS 1974: 3).

In Anlehnung an den 8. Pädagogischen Kongress veröffentlichte das MfV im Jahr 1980 einen neuen Gesamtaustattungsplan für Unterrichtsmittel (Tab. 22). Dieser enthielt alle, für die Unterrichtsarbeit an der zehnklassigen allgemeinbildenden POS als erforderlich angesehenen, Unterrichtsmittel und technischen Geräte. Bisherige Unterrichtsmittel unterlagen einer Prüfung ihrer Praxistauglichkeit (vgl. MfV 1980: 5). Dies hatte Streichungen und Neuaufnahmen zur Folge. Der Plan galt als „,verbindliche Grundlage für die Vervollkommnung und Erhaltung der Ausstattung einer jeden Schule“ (ebd.). Für einen erziehungswirksamen und problemorientierten Unterricht im Stoffgebiet Abstammungslehre empfahlen Cassube und Windelband (1980: 12) in der Zeitschrift Biologie in der Schule ergänzend dazu die Einbindung populärwissenschaftlicher Lektüre.

Tabelle 22: Evolutionsbiologische Unterrichtsmedien aus dem Gesamtausstattungsplan für Unterrichtsmittel der zehnklassigen allgemeinbildenden polytechnischen Oberschule der DDR von 1980 (verändert nach MfV 1980)

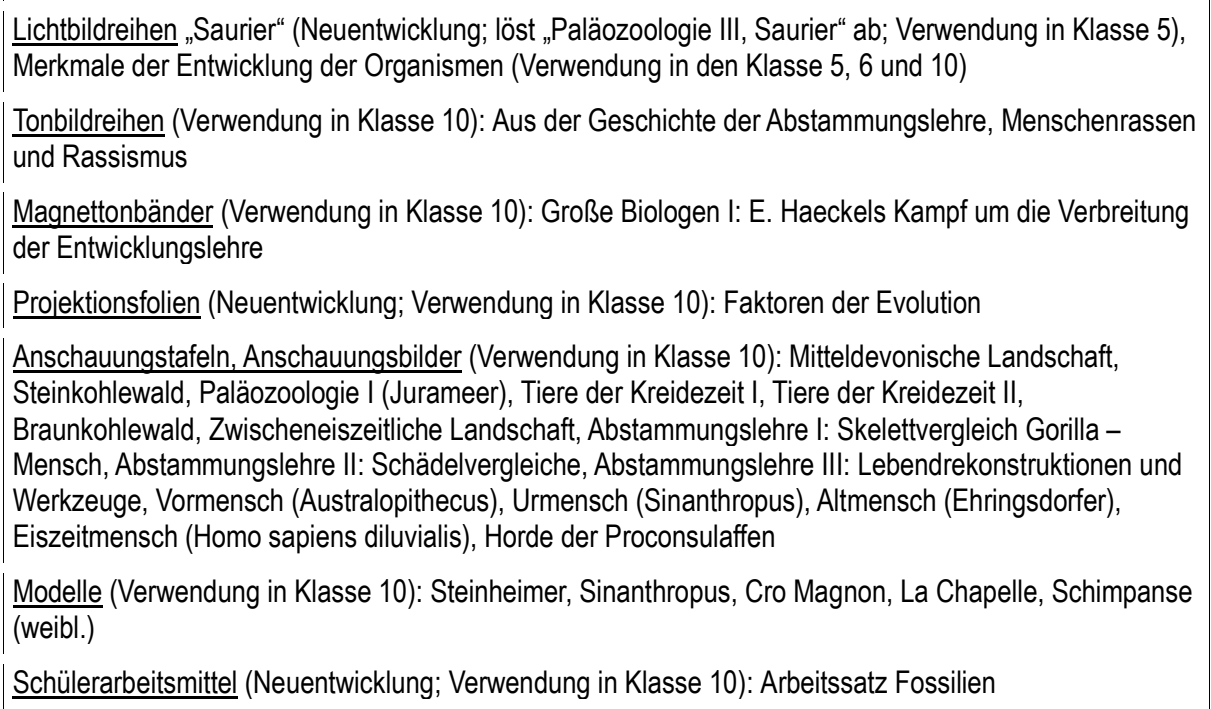

Da neben den bisher genannten Unterrichtsmedien gerade auch originale Begegnungen außerhalb des Klassenraumes zum Kompetenzerwerb beitragen (Gesang et al. 2012), sollen dazu noch einige Überlegungen das Kapitel abschließen. Insbesondere naturkundliche Einrichtungen arbeiten in den Bereichen Wissenschaft 
und Forschung und investieren in die Bildung der Öffentlichkeit (Wagner 2008). Auch die Natur- und Heimatkundemuseen sowie die botanischen und zoologischen Gärten in der DDR galten für das Fach Biologie im Allgemeinen und für evolutionsbiologische Inhalte im Speziellen als geeignete Lernorte für den außerschulischen Unterricht. Im Jahr 1963 erschien das Nachschlagewerk Handbuch der Museen und wissenschaftlichen Sammlungen der DDR, das die Einrichtungen nach Bezirken geordnet vorgestellte. Als Herausgeber zeichnete der Prähistoriker Heinz Arno Knorr (1909-1996). Hinsichtlich der Qualität der Exponate und der Effektivität des kurzen Weges boten lokale Museen erste Anlaufstellen. Eine herausragende Rolle kam dem Naturkundemuseum in Berlin, als größte Einrichtung seiner Art auf dem Gebiet der DDR, zu. Hier konnte auch der Urvogel Archaeopteryx besichtigt werden, der vielfach in den Schullehrbüchern abgebildet war (Stephan 1975; Abb. 26). Bei dem sogenannten Berliner Exemplar, einem im Wesentlichen vollständigen Skelett aus den Solnhofener Plattenkalken, sind Konturen der Federn erhalten geblieben (Simpson 1984). Ein weiteres Wahrzeichen des Museums hatte einst eine deutsche Expedition aus dem damaligen Deutsch-Ostafrika, dem heutigen Tansania, mitgebracht. Es ist dies das weltweit größte aufgestellte Skelett eines Dinosauriers (Brachiosaurus brancal). Nach einer Studie von Taylor (2009) ist Brachiosaurus brancai neuerdings in eine eigene Gattung Giraffatitan zu stellen.
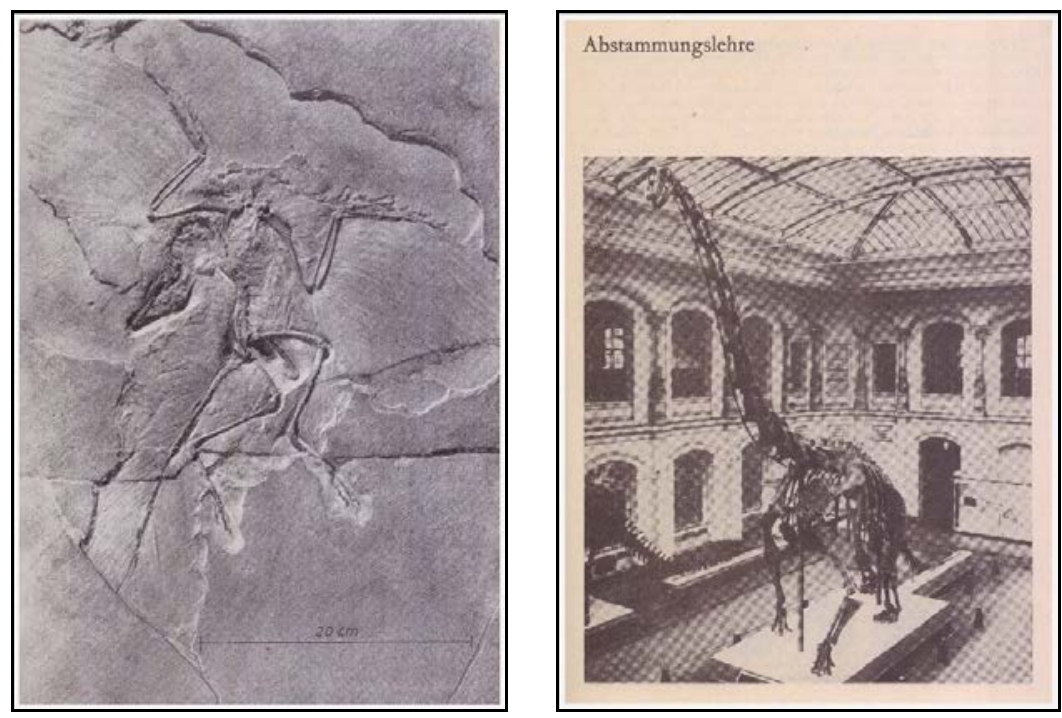

Abbildung 26: links: Abdruck eines Urvogels aus dem Solnhofener Plattenkalk, Lehrbuch Klasse 10 (Ambrosius et al. 1964); rechts: Blick in den Sauriersaal des Naturkundemuseums zu Berlin, Lehrbuch Klasse 10 (Bach et al. 1987) 
Hundt und Kreße (1969: 261) zeigen für den Unterricht in der DDR, dass „,bei Besichtigung durch Arbeitsgemeinschaften bzw. Interessenzirkel [...] das Problem der Abstammung bzw. der Evolution der Wirbeltiere spezieller behandelt [...] werden" kann. So empfahlen die Autoren, in Klasse $10 \mathrm{im}$ Stoffgebiet Die Entwicklung der Organismen in der Stoffeinheit Allgemeine Merkmale der Entwicklung die Stunde Homologe Organe: Gliedmaßenskelett der Wirbeltiere im Naturkundemuseum in Berlin vertiefend zu behandeln. In ihrem Titel Arbeitsgemeinschaften - Exkursionen (1969) skizzierten sie Lehrwege für den Museumsgang und stellten mögliche Abläufe der Besichtigung dar. Über ausgewählte Bereiche, wie den Sauriersaal des Museums, konnten sich die Schüler bereits im Lehrbuch einen ersten Eindruck verschaffen. Auch die Autoren der Unterrichtshilfen legten einen Besuch von musealen Einrichtungen nahe.

„Besonders wichtig ist es, die entsprechenden Sammlungen in den naturkundlichen Museen für den Unterricht in Klasse $10 \mathrm{zu}$ nutzen. Neben den örtlichen Möglichkeiten sollten vor allem solche Einrichtungen wie z. B. das Naturkundemuseum Berlin, das Phyletische Museum und das HaeckelMuseum Jena, das Museum für Ur- und Frühgeschichte in Weimar, das Museum für Völkerkunde in Leipzig besucht werden." (Graef et al. 1985: 14)

Nach Baer (1959: 354) wurde bei der Behandlung evolutionsbiologischer Inhalte der Besuch entsprechender Museen als ,geradezu notwendig“ erachtet. Im Rahmen der Vorbereitung für die Jugendweihe standen Museen mit evolutionsbiologischen Themen in der Ausstellung ebenso im Fokus, so dass diese Einrichtungen eine Zunahme der Besucherzahlen verzeichnen konnten (ebd.; Fischer et al. 2008). 



\title{
Der Lehrplan für Biologie
}

\begin{abstract}
„Zur Vergangenheit unseres Bildungswesens gehören auch 45 Jahre Biologieunterricht in der DDR. Als einen wesentlichen Maßstab für die Qualität dieses Unterrichtsfaches kann man die Lehrplaninhalte heranziehen." (Tille 1992: 321)
\end{abstract}

\section{Klasse 8}

Der Rahmenlehrplan von 1946 und 1947

Der Begriff Darwinismus nahm im Vorwort des Rahmenlehrplans von 1946 eine zentrale Stellung ein. Die Autoren betonten, dass „der Biologieunterricht [...] den Schülern Kenntnisse vermitteln [sollte], welche für das Verständnis [...] der Grundlagen des Darwinismus erforderlich sind“ (DZfV in der SBZ 1946: 3). Geprägt durch die Folgen des Krieges und des Nationalsozialismus sowie im Bemühen um eine Neuausrichtung der Denkweise und des damit verbunden Handelns wurde diese Zielformulierung weiter spezifiziert. 
„Der Schüler hat besonders die Erkenntnis mitzunehmen, daß Darwins Lehre vom Kampfe ums Dasein nicht auf das Zusammenleben der Menschen übertragbar ist, daß blutige Auseinandersetzungen zwischen einzelnen Menschen, Menschengruppen und Völkern mit dieser Lehre nicht zu rechtfertigen sind, daß vielmehr alle Kräfte auf die Hebung des Gemeinwohles und die Verwirklichung wahrer Humanität zu richten sind.“ (DZfV in der SBZ 1946: 3)

Für den Kursunterricht im mathematisch-naturwissenschaftlichen Zweig der Klasse 8 standen mit zwei Wochenstunden 80 Jahresstunden zur Verfügung. Der Stoffplan gliederte sich in die Kapitel Überblick, über das System des Pflanzen- und Tierreichs (5 h), Bestimmungsübungen (5 h), Fortpflanzung im Pflanzen- und Tierreich (8 h), Einfübrung in die Vererbungslebre (13 h), Die Schädlinge und ibre Bekämpfung (4 h), Die Leistungen der Pflanzen $(11 \mathrm{~h})$, Die Wirbeltiere $(20 \mathrm{~h})$, Die Abstammung und Entwicklung des Menschen (10 h), Die Rassen bei den Pflanzen, Tieren und Menschen - Die Irrlebren des Nationalsozialismus (4 h). Mit $10 \mathrm{~h}$ betrug der Anteil evolutionsbiologischer Inhalte $12,5 \%$. Außer den Stundenangaben enthielten die Kapitel nur wenige inhaltliche Erläuterungen. Eine Feinstruktur der evolutionsbiologischen Inhalte existierte nicht. Die Inhaltsanalyse entlang der Hauptkategorien verdeutlicht, dass im Stoffplan einzig die Kategorie Hominisation vertreten war. Die Raumanalyse des Lehrplans zeigt entsprechend mit $100 \%$ für die Kategorie Hominisation eine klare Präferenz.

Auch in der 2. Auflage des Lehrplans, der 1947 veröffentlicht wurde, zeigten sich im Vorwort die Nachwirkungen des Nationalsozialismus. Dies spiegelte sich in den Formulierungen zu den Aufgaben der Lehrerinnen und Lehrer und der Neuinterpretation der Darwinistischen Lehre wider.

„Schließlich soll der Schüler imstande sein, selbständig über die letzten Fragen des Lebens nachzudenken. Dazu muß er sich auf ein gut fundiertes Wissen über die stammesgeschichtliche Entwicklung alles Lebendigen stützen können. Darüber hinaus wird es noch einige Zeit am Platze sein, daß der Lehrer ihn aufklärt über die Irrlehren des Nationalsozialismus, daß nämlich Darwins Lehre vom Kampf ums Dasein nur für Beziehungen zwischen Tieren und Pflanzen [...] zu rechtfertigen ist." (DVV in der SBZ 1947: 5)

Der Stoffplan der 2. Auflage von 1947 gliederte sich mit je $40 \mathrm{~h}$ in Kern- und Kursunterricht. Weitere Stundenangaben gab der Stoffplan nicht vor, so dass der Anteil der Fachinhalte unbestimmt blieb und die Lehrkräfte über entsprechende Freiheiten in der Unterrichtsplanung verfügten. Der Kernunterricht gliederte sich in die Kapitel Tierkunde und Menschenkunde. Diese Kapitel verfügten über Stoffangaben sowie Gesichtspunkte für die Stoffauswahl und -behandlung. Explizit evolutionsbiologische Kapitel kamen zwar nicht mehr vor, doch integrierten die Autoren vereinzelt entsprechende Inhalte. So fanden beispielsweise im Kapitel Tier- 
kunde die Gesichtspunkte Urvögel und Homologie der Gliedmaßen Berücksichtigung. Der Stoffplan für den Kursunterricht war weniger strukturiert und forderte die evolutionsbiologischen Inhalte Entwicklungs- und Stammesgeschichte sowie der vorgeschicbtliche Mensch.

Die Lehrpläne für die Grundschule von 1951 bis 1953

Die Vorworte der Lehrpläne für Grundschulen von 1951 und 1952 waren in ihrem Wortlaut identisch und blieben auch im Lehrplan von 1953 gültig. Sie gliederten sich in das Wesen des Faches, erkenntnistheoretische und erzieherische Aufgaben sowie in die Unterrichtsziele. Es wurde betont, dass „,in der sozialistischen Gesellschaft [...] die Biologie auf der Grundlage des dialektischen Materialismus ihre allseitige Entfaltung und Weiterentwicklung zu einer schöpferischen Wissenschaft“ erfährt (MfV der DDR 1952: 4). Die Schüler sollten erkennen, „wie die Leitsätze des dialektischen Materialismus auf die Erforschung der organischen Natur ausgedehnt werden“ (MfV der DDR 1952: 4). Der Abschnitt Die sittliche Erziehung enthielt konkrete Angaben zur Evolutionsbiologie.

„Die Erkenntnisse aus der Entwicklungs-, Vererbungs-, und Abstammungslehre bilden wissenschaftliche Grundlagen für die Erziehung zu einem kämpferischen Humanismus. Die Schüler müssen befähigt werden, reaktionäre und unwissenschaftliche Anschauungen entschieden zu bekämpfen.“ (MfV der DDR 1951: 4)

Als Unterrichtsziele formulierten die Autoren „Kenntnisse vom natürlichen System, von der Onto- und Phylogenese der Pflanzen und Tiere“ sowie „die Erkenntnis der Gesetzmäßigkeit der Entwicklung der lebenden Organismen und des dialektischen Charakters dieser Entwicklung“" (MfV der DDR 1951: 4).

Der Stoffplan des Lehrplans Biologie von 1951 gab bei zwei Wochenstunden für das Schuljahr 66 Jahresstunden inhaltlich vor. Die Grobstruktur des Lehrplans von 1951 gliederte sich in die Kapitel Reizerscheinungen der Lebewesen $(22 \mathrm{~h})$, Abstammungslebre (30 h), Die Entwicklung des Menschen (10 h) sowie Große Biologen (4 h). Jedes Kapitel enthielt den zu behandelnden Stoff mit Stundenangaben sowie Bemerkungen zum Stoff. Zusätzlich waren Sonderstunden für Besichtigungen, Exkursionen und Prüfungsvorbereitungen ohne weitere inhaltliche Angaben mit einem Umfang von $14 \mathrm{~h}$ vorgesehen. Davon sollten $6 \mathrm{~h}$ für Prüfungsvorbereitungen und $8 \mathrm{~h}$ für Exkursionen verwendet werden (Fritsch \& Lemke 1952). Mit $42 \mathrm{~h}$ nahm der Anteil evolutionsbiologischer Inhalte 63,6 \% am Gesamtjahrgang ein. Hinsichtlich evolutionsbiologischer Inhalte sollten die Schüler am Ende des Schuljahres „die Grundzüge der [...] stammesgeschichtlichen Entwicklung“ (MfV 1951a: 16) erkannt haben, einschließlich der „Entstehung des Menschen aus dem Tierreich, seine Entwicklung zum heutigen Menschen und seine Stellung als Glied und Umgestalter der Natur“ (MfV 1951a: 16). Dadurch, dass die Schüler „den reaktionären „Neodarwinismus' als eine Verfälschung des Darwinismus“ (MfV 
1951a: 16) erkennen und ablehnen sollten, vollzog sich im Lehrplan ein Paradigmenwechsel. Propagiert wurde „die Weiterentwicklung des Darwinismus zum schöpferischen Darwinismus durch Mitschurin und Lyssenko" (MfV 1951a: 16, Hervorhebung im Original). Diese Lernziele blieben auch in den folgenden Lehrplänen von 1952 und 1953 im Grundtenor gleich. Die Feinstruktur evolutionsbiologischer Inhalte gliederte sich in die Bereiche (MfV 1951a):

- Abstammungslehre

- Beweise für die Abstammungslehre (16 h)

- Versteinerungskunde

- Vergleichende Anatomie

- System, Übergangsformen

- Entwicklungsgeschichte

- Tier- und Pflanzengeographie

- Die Entstehung der Arten; Darwinismus (8 h)

- Veränderlichkeit der Lebewesen

- Auslese

- Artbildung durch Züchtung

- Stammesentwicklung der Tiere und Pflanzen (6 h)

- Entstehung des Lebens

- Stammbaum

- Das System

- Die Entwicklung des Menschen $(10 \mathrm{~h})$

- Entwicklung innerhalb der Affenreihe

- Menschwerdung

- Vormensch, Urmensch, Altmensch

- Die menschliche Gesellschaft

- Große Biologen (2 h für evolutionsbiologische Inhalte)

Dieser Stoffplan zeigte hinsichtlich Gliederung und Umfang deutliche Unterschiede zu den Lehrplänen von 1946 bzw. 1947. Die Inhalte ordneten die Autoren in der Reihenfolge: Paläontologie - Neontologie - Paläontologie Neontologie - Theorien - Biogenese - Systematik - Hominisation - Theorien. Dabei nahmen die Kategorien Theorien und Hominisation mit einem Umfang von je $10 \mathrm{~h}$ fast die Hälfte (48 \%) der evolutionsbiologischen Themen ein. Belege für Evolutionsprozesse aus der Paläontologie bzw. der Neontologie umfassten mit jeweils $8 \mathrm{~h}$ insgesamt $38 \%$. Weitere $6 \mathrm{~h}(14 \%)$ waren für die Kategorien Systematik und Biogenese vorgesehen. Die Stundenangaben ermöglichten hier keine getrennte Darstellung der Kategorien (Abb. 27). 


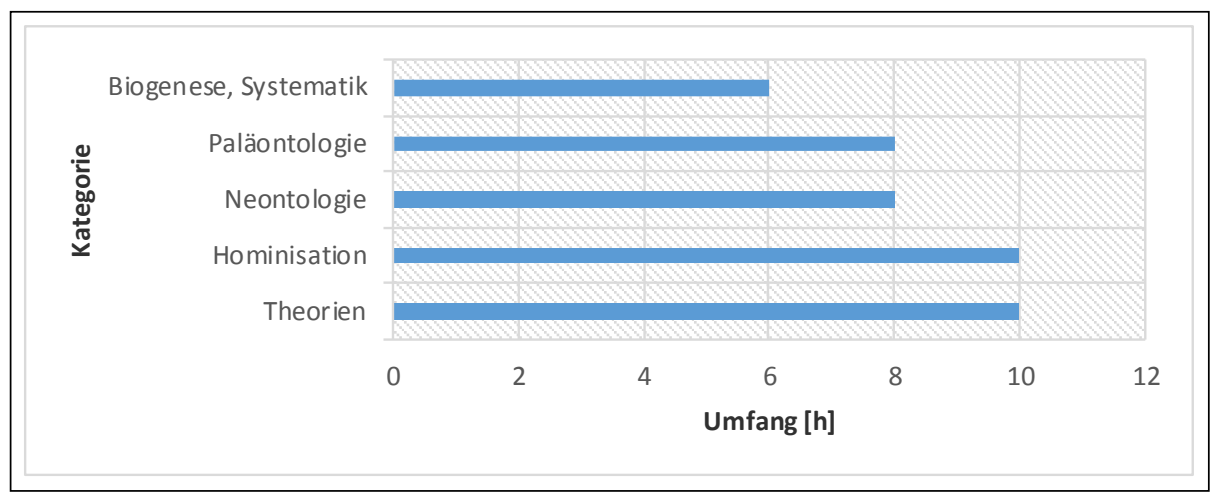

Abbildung 27: Stundenumfang für Kategorien, Lehrplan Klasse 8 (1951)

Der Biologielehrplan von 1952 für das 8. Schuljahr (zwei Wochenstunden) legte 58 Jahresstunden inhaltlich fest. Der Stoffplan gliederte sich in die Kapitel Reizerscheinungen bei Lebewesen (22 h), Abstammungslebre (27 h), Die stammesgeschichtliche Entwicklung des Menschen (5 h) sowie Die Geschichte der beiden grundlegenden Theorien in der Biologie, der Abstammungslehre und der Zellenlehre (4 h). Jedes Kapitel enthielt weiterhin den zu behandelnden Stoff mit Stundenangaben sowie Bemerkungen zum Stoff. Die restlichen 22 Stunden standen den Lehrkräften als Sonderstunden zur Verfügung und gewährten den Lehrkräften einen planerischen Spielraum. In Anlehnung an den Lehrplan von 1951 konnten die Lehrkräfte abschließend auch diesem Dokument Erwartungen und Lernziele entnehmen (MfV 1952b).

Mit $34 \mathrm{~h}$ nahm der Anteil evolutionsbiologischer Inhalte am Gesamtjahrgang 58,6 \% ein. Die Feinstruktur evolutionsbiologischer Inhalte entsprach dem Lehrplan von 1951. Veränderungen betrafen Formulierungen einzelner Inhalte sowie deren Stundenumfang. Es entfielen $13 \mathrm{~h}$ auf das Thema Beweise für die Richtigkeit der Abstammungslehre, je $7 \mathrm{~h}$ auf die Themen Die Entstehung der Arten sowie Stammesentwicklung der Tiere und Pflanzen, $5 \mathrm{~h}$ auf das Thema Die Stammesgeschichtliche Entwicklung des Menschen und $2 \mathrm{~h}$ auf das Thema Die Geschichte der beiden grundlegenden Theorien in der Biologie. Als neues didaktisches Element war für jedes Kapitel $1 \mathrm{~h}$ für Wiederholungen und Leistungskontrollen vorgesehen.

Der Unterschied zum Lehrplan von 1951 wird im Vergleich der Vorgaben deutlich. Die Autoren gliederten den Stoff in der Reihenfolge: Paläontologie Neontologie - Paläontologie - Neontologie - Didaktisches - Theorien - Didaktisches - Biogenese - Systematik - Didaktisches - Hominisation - Didaktisches Theorien. Die Kategorie Theorien nahm weiterhin mit einem Umfang von $8 \mathrm{~h}$ (24\%) fast ein Viertel der evolutionsbiologischen Themen ein. Ebenso blieb der Anteil der Belege für Evolutionsprozesse mit $36 \%$ hoch. Der Anteil der Kategorie Paläontologie stieg auf $7 \mathrm{~h}(21 \%)$ an und mit insgesamt $6 \mathrm{~h}(18 \%)$ nahm auch der Stellenwert der Kategorien Systematik und Biogenese zu. Mit 4 h (12\%) inte- 
grierten die Verantwortlichen erstmals die Kategorie Didaktisches im Lehrplan. Im Gegenzug reduzierte sich der Umfang der Kategorie Neontologie auf $5 \mathrm{~h}$ $(15 \%)$. Der größte Einschnitt erfolgte bei der Kategorie Hominisation, die nunmehr nur noch mit 4 h (12\%) vertreten war (Abb. 28).

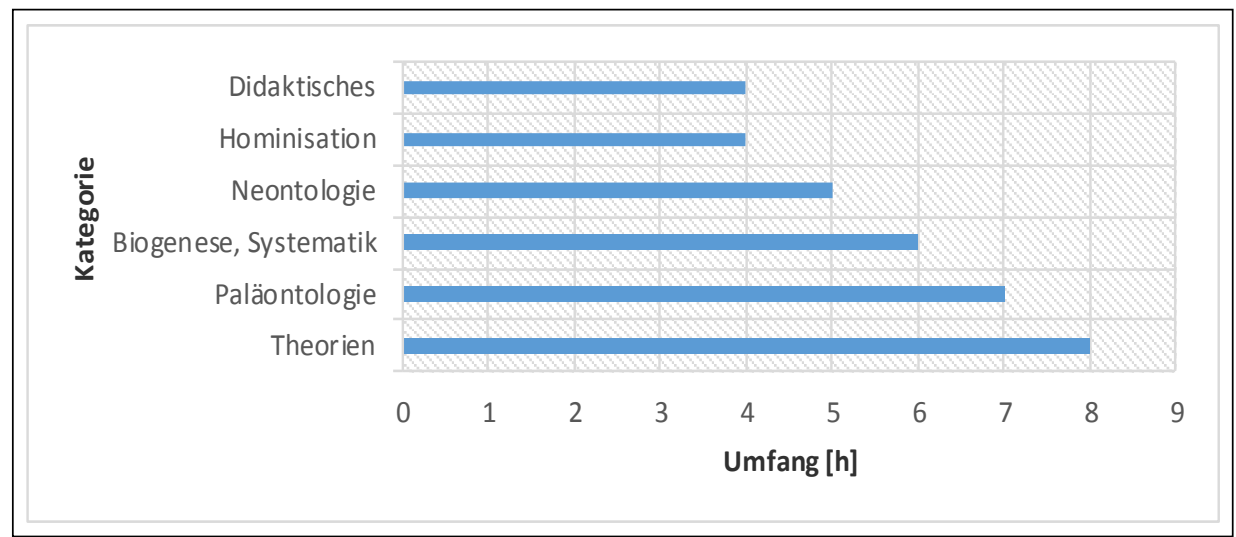

Abbildung 28: Stundenumfang für Kategorien, Lehrplan Klasse 8 (1952)

Der Lehrplan von 1953 gab für das 8. Schuljahr (zwei Wochenstunden) 68 h inhaltlich vor. Die Grobstruktur gliederte sich in die Kapitel Reizerscheinungen bei Lebewesen (25 h) sowie Abstammungslehre und Weiterentwicklung der Organismen durch den Menschen $(33 \mathrm{~h})$. Am Ende des Schuljahres war im Umfang von $8 \mathrm{~h}$ eine Gesamtwiederholung vorgesehen. Der Stoffplan des Lehrplans von 1953 enthielt neben den Stoff- und Stundenangaben Bemerkungen zum Stoff. Für Exkursionen und Prüfungsvorbereitungen standen 4 Stunden zur Verfügung. Der Anteil evolutionsbiologischer Inhalte lag bei $33 \mathrm{~h}(48,5 \%)$. Die Feinstruktur evolutionsbiologischer Inhalte gliederte sich in die Kapitel (MfV 1953a):

- Beweise für die Richtigkeit der Abstammungslehre (12 h)

- Versteinerungskunde

- Vergleichende Anatomie

- Darwinismus (5 h)

- Schöpferische Biologie (4 h)

- Anwendung in der Praxis $(4 \mathrm{~h})$

- Stammesentwicklung der Pflanzen, Tiere und des Menschen (6 h)

$\circ$ Entstehung des Lebens

- Stammbaum

- Das System

- Geschichte der Abstammungslehre (2 h) 
Die Inhaltsanalyse des Stoffplans zeigt folgende Gliederung: Paläontologie Neontologie - Theorien - Biogenese - Systematik - Theorien. Nicht mehr vorhanden waren Inhalte aus der Kategorie Hominisation. Die Raumanalyse verdeutlicht klare Präferenzen (Abb. 29). Mit einem Umfang von 15 h (45\%) lag der Schwerpunkt der Darstellung im Bereich der Kategorie Theorien. Konstant blieb der Anteil der Kategorie Paläontologie mit 8 h (24\%). Dagegen reduzierte sich der Stellenwert der Kategorie Neontologie mit 4 h auf $12 \%$. Dennoch hatten Belege für Evolutionsprozesse mit insgesamt $36 \%$ weiterhin eine hohe Relevanz. Erstmals stellte der Lehrplan Inhalte aus den Kategorien Systematik mit $4 \mathrm{~h}$ (12 $\%)$ und Biogenese mit 2 h (6\%) getrennt dar.

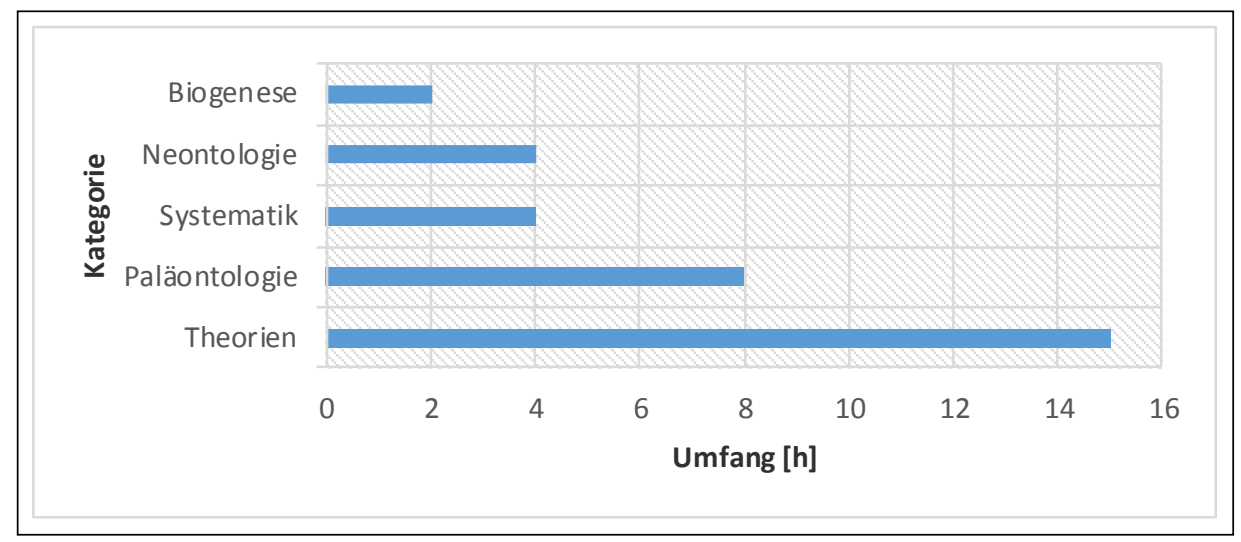

Abbildung 29: Stundenumfang für Kategorien, Lehrplan Klasse 8 (1953)

\section{Die Direktive von 1955}

Eine Direktive, die 1955 erschien, enthielt Hinweise, die sich auf bestimmte Abschnitte des Lehrplans bezogen und dessen Bemerkungen erweiterten (MfV 1955). Erklärtes Ziel der Direktive für die Mittelstufe von 1955 war es, „die Schüler [...] besser für die künftige Arbeit in vielen neuen landwirtschaftlichen Berufen [...] vorzubereiten“ (MfV 1955: 1). Besondere Berücksichtigung fand in diesem Zusammenhang die polytechnische Bildung, die Auseinandersetzung mit den Unterrichtsinhalten in der freien Natur sowie die Aneignung und Festigung der Kenntnisse mittels geeigneter Methoden. Spezielle Hinweise auf Veränderungen evolutionsbiologischer Inhalte enthielt das Vorwort nicht.

Der Stoffplan der Direktive von 1955 gab für den 8. Jahrgang (zwei Wochenstunden) $60 \mathrm{~h}$ inhaltlich vor. Die Stoffübersicht enthielt ausschließlich Themen, denen inhaltliche Veränderungen zu Grunde lagen sowie Stundenangaben. Die Grobstruktur gliederte sich in die Kapitel Reizerscheinungen der Lebewesen (18 h) sowie Abstammungslebre und Weiterentwicklung der Organismen durch den Menschen (30 h). Zusätzlich sah die Direktive $12 \mathrm{~h}$ für praktische Arbeiten im Schulgarten vor. 
Diese Unterrichtsstunden ergaben sich durch die „Herausnahme einiger Stoffe aus dem bisherigen Plan“ (MfV 1955: 13). Die restlichen 20 Stunden dienten für Exkursionen, Wiederholungen und Prüfungsvorbereitungen. Der Anteil evolutionsbiologischer Inhalte umfasste $30 \mathrm{~h}(50 \%)$ und gliederte sich in die Kapitel (MfV 1955):

- Darwinismus (5 h)

- Kurze Lebensbeschreibung Darwins

- Grundgedanken

- Kritische Betrachtung des Begriffs, Kampf ums Dasein

- Beweise für die Richtigkeit der Abstammungslehre (10 h)

- Beweise aus der Versteinerungskunde

- Beweise aus der vergleichenden Anatomie

- Stammesentwicklung der Pflanzen, Tiere und des Menschen (8 h)

- Stammbäume

- Das natürliche System

- Stammesgeschichtliche Entwicklung

- Abstammung und Entwicklung des Menschen

- Mitschurin-Biologie und ihre Anwendung in der Praxis (7 h)

Die Direktive forderte bei der Behandlung des Themas Darvinismus neben Inhalten zu Leben und Werk Darwins auch eine kritische Auseinandersetzung mit dem Begriff Kampf ums Dasein. Die Behandlung des Themas Beweise aus der Versteinerungskunde basierte laut Direktive auf dem Lehrbuchkapitel Tiere und Pflanzen in der erdgeschichtlichen Entwicklung. An dieser Stelle wurde die Lehrplan-LehrbuchVerschränkung ausdrücklich betont. Die Direktive forderte die Behandlung des Themas Beweise aus der vergleichenden Anatomie auf die fünfstrahligen Extremitäten der Wirbeltiere zu beschränken. Stammbäume, das natürliche System, die stammesgeschichtliche Entwicklung sowie die Abstammung und Entwicklung des Menschen waren die Themen, die bei der Behandlung des Themas Stammesentwicklung der Pflanzen, Tiere und des Menschen Berücksichtigung fanden. Im Kapitel Mitschurin-Biologie und ibre Anwendung in der Praxis sollten grundlegende Erkenntnisse der Lehre Mitschurins und die Methoden der Mitschurin'schen Biologie behandelt werden.

Die Inhaltsanalyse verdeutlicht folgende Gliederung des Stoffplans: Theorien - Paläontologie - Neontologie - Systematik - Hominisation - Theorien. Der Stellenwert der Kategorie Theorien blieb mit einem Umfang von $12 \mathrm{~h}(40 \%)$ weiterhin hoch. Die Relevanz von Indizien für Evolutionsprozesse stieg auf $34 \%$. Hierbei nahm die Kategorie Neontologie mit nur 2 h (7\%) einen deutlich geringeren Anteil ein. Wieder aufgenommen wurde die Kategorie Hominisation, die jedoch strukturell durch eine Stundenangabe mit der Kategorie Systematik abgebildet war. Beiden kam ein Umfang von 8 h $(27 \%)$ zu. Die Kategorien Biogenese sowie Didaktisches forderte der Stoffplan nicht (Abb. 30). 


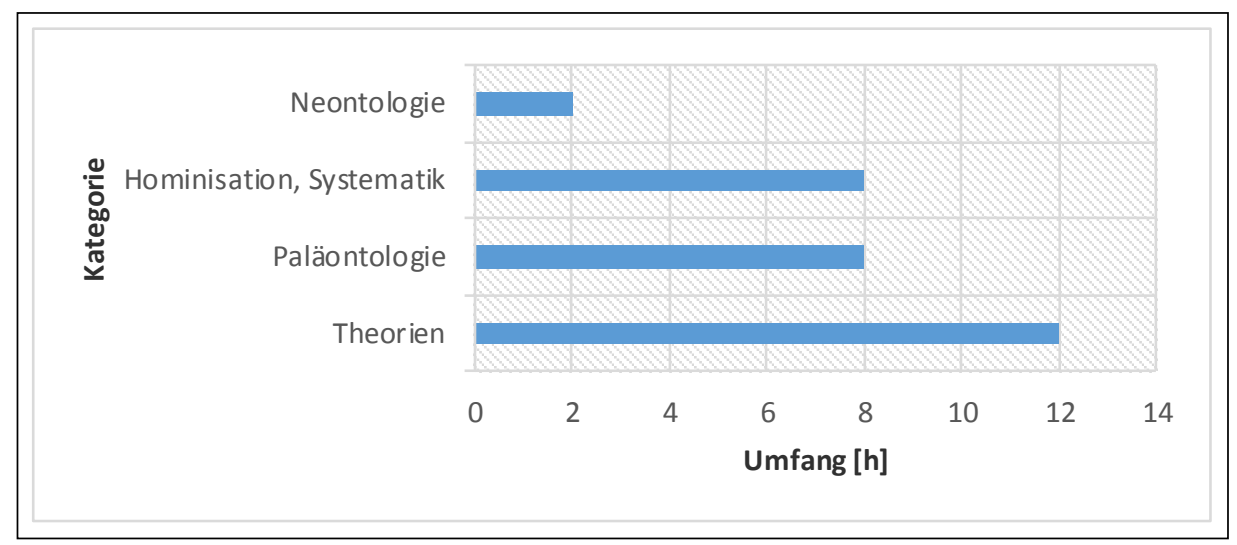

Abbildung 30: Stundenumfang für Kategorien, Direktive Klasse 8 (1955)

Die Lehrpläne von 1959 bis 1989

Mit der Ausgabe des Lehrplans von 1959 wurde die Behandlung der Evolutionsbiologie im Jahrgang 8 eingestellt. Dennoch betonten die Autoren in den Vorworten der Lehrpläne, dass diese Thematik von Klasse 5 an angebahnt werden sollte.

„Von Anfang an sind den Schülern Beispiele für die Variabilität der Organismen zu geben. Die Behandlung der Tiere und Pflanzen berücksichtigt entsprechend dem Auffassungsvermögen der Schüler in zunehmendem Maße entwicklungsgeschichtliche Zusammenhänge, wobei die Schüler viele Beispiele für die Entwicklung [...] kennenlernen.“ (MfV 1959a: 1)

Im Jahr 1968 erschien der Präzisierte Lehrplan für Biologie, Klasse 8. Dieser trat am 1. September 1969 für den Unterricht in der zehnklassigen allgemeinbildenden Oberschule in Kraft. Das Thema für diese Jahrgangsstufe lautete Anatomie, Physiologie und Hygiene des Menschen. Das Vorwort für die Klasse 8 enthielt keine Hinweise auf evolutionsbiologische Inhalte. Erst etwa zwanzig Jahre später, im Jahr 1989, erschien für die Klasse 8 ein neuer Lehrplan. Dieser sollte im Jahr 1991 in Kraft treten. Er enthielt wieder Angaben, inwieweit der Evolutionsgedanke im Unterricht dieser Klassenstufe eingebunden werden sollte.

„Dabei wird unter Nutzung von Vorleistungen herausgearbeitet, daß alle Menschen in wesentlichen Merkmalen übereinstimmen und gegenüber den Säugetieren Besonderheiten aufweisen. Die Schüler gewinnen einen ersten Einblick in die Entwicklungsgeschichte des Menschen." (MfV 1989: 10)

In den Erläuterungen zum Lehrplan von 1989 wurden ,inhaltliche Voraussetzungen für das Verständnis des Evolutionsgedankens“ (Horn 1989: 18) detailliert dargestellt mit dem Ziel, Grundlagen für das Verständnis der Evolutionsbiologie zu schaffen. 


\section{Zusammenfassung}

Im Zeitraum 1945 bis 1955 vollzogen sich der erste Strukturwandel im Schulsystem der DDR sowie die erste und zweite Phase der Lehrplanentwicklung. Die Inhaltsanalyse der Vorworte offenbarte eine Verknüpfung evolutionsbiologischer Inhalte mit ideologischen Orientierungen (Typ 1) in den Lehrplänen von 1946, 1951, 1952 und 1953 (Tab. 23). Dennoch sind diese Orientierungen differenziert zu betrachten, da sie eine Verschiebung im gesellschaftlichen Wertesystem erkennen lassen. Formulierte der Lehrplan von 1946 die „Verwirklichung wahrer Humanität" (DVV in SBZ 1946: 3) als Ziel, so wurde nach Gründung der DDR 1949 in den 1950er Jahren bereits ein „kämpferischer Humanismus“ (MfV 1951a: 4) angestrebt. Tille (1992b: 384) spricht von einer ,ausgeprägte[n] Ideologisierung“ des Lehrplankapitels Abstammungslehre und Weiterentwicklung der Organismen durch den Menschen. Die Fokussierung auf den Schöpferischen Darwinismus (Mitschurin, Lyssenko) und die gleichzeitige Ausblendung von Vertretern der klassischen Genetik (Mendel, Morgan, Weismann) ist „ein typisches Beispiel für die Einseitigkeit der ideologischen Bildung und Erziehung“ (ebd.; vgl. auch Porges et al. 2016; Porges et al. 2017). Im Vorwort der Direktive von 1955 fehlten dagegen Verweise auf evolutionsbiologische Inhalte und somit traten weder ein Sach- noch ein Ideologiebezug auf (Typ 4). Die späteren Lehrpläne von 1959 und 1989 formulierten zwar, wie der Evolutionsgedanke einzubinden bzw. anzubahnen ist, enthielten jedoch für die Vermittlung der Evolution kein eigenes Kapitel. Die Vorworte orientierten in diesem Kontext rein sachbezogen (Typ 2). Im Vorwort des Lehrplans von 1968 (Typ 3) kamen evolutionsbiologische Themen nicht zur Sprache.

Tabelle 23: Sach- und Ideologiebezug, Lehrpläne Klasse 8

\begin{tabular}{|llll|}
\hline \multicolumn{1}{|c|}{ ja } & \multicolumn{1}{c|}{ Ideologiebezug } \\
Sachbezug & ja & Typ 1: 1946, 1951, 1952, 1953 & Typ 2: 1959, 1989 \\
& nein & Typ 3:- & Typ 4: 1955 \\
\hline
\end{tabular}

Die deskriptive Analyse der Grobstruktur zeigte, dass die Lehrplänen zwischen 1947 und 1955 Fachinhalte aus der Humanbiologie (1947), der Systematik der Tiere (1947), der Evolutionsbiologie (1946 bis 1955), der Zytologie (1951, 1952), der Reizphysiologie (1951 bis 1955), der Züchtungsbiologie (1951 bis 1955) und der Genetik (1955) forderten. Ihre Struktur veränderte sich in diesem Zeitraum durch Hinzunahme, Zusammenlegung und Weglassen von Fachdisziplinen. Der Lehrplan von 1947 fasste evolutionsbiologische Aspekte und die Systematik der Tiere in einem Kapitel zusammen. In den Lehrplänen von 1951 bis 1953 bildeten Fachinhalte aus der Evolutionsbiologie und der Züchtungsbiologie ein eigenes Kapitel und der Lehrplan von 1955 bildete Inhalte aus der Evolutionsbiologie, der 
Züchtungsbiologie sowie der Genetik in einem Kapitel gemeinsam ab. Aspekte des Lyssenkoismus integrierten die Lehrpläne von 1951 bis 1955 (Abb. 31).

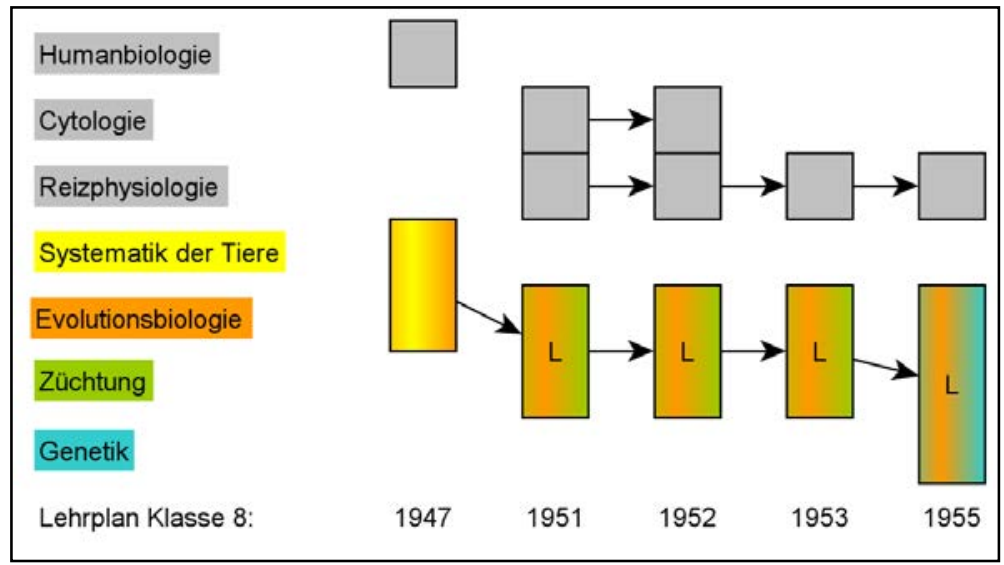

Abbildung 31: Lehrpläne Klasse 8, Entwicklung der Grobstruktur (L: Lyssenkoismus)

Die deskriptive Analyse evolutionsbiologischer Lehrplanvorgaben belegte auch deutliche Unterschiede zwischen den Rahmenlehrplänen der 1940er Jahre und den Lehrplänen der 1950er Jahre. Gab der Lehrplan von 1946 noch alle Jahresstunden inhaltlich vor, verfügten die späteren administrativen Vorgaben über Sonderstunden für Prüfungsvorbereitungen, Exkursionen und Wiederholungen. Bedingt durch lokale Möglichkeiten, zum Beispiel das Phyletische Museum in Jena sowie die jeweiligen Rahmenbedingungen an den Schulen, konnten die Lehrkräfte den Unterricht entsprechend individualisieren. Hinsichtlich evolutionsbiologischer Themen forderte der Lehrplan von 1946 eine Behandlung ausschließlich für den mathematisch-naturwissenschaftlichen Zweig. Da die Grundschule nach der 8. Klasse endete, realisierte der Stoffplan die Zielvorgabe aus dem Vorwort des Lehrplans für die Schüler der A und C Kurse nicht.

Parallel zum Strukturwechsel im Schulsystem und in Folge der 2. Lehrplanrunde forderten die Lehrpläne der 1950er Jahre eine detaillierte Behandlung evolutionsbiologischer Inhalte. Diese waren für alle Schüler verbindlich. Auch stieg der Stundenumfang für evolutionsbiologische Inhalte deutlich. Trotz Kürzungen und Korrekturen blieb die Gliederung des Lehrplans von 1951 auch im Lehrplan von 1952 erhalten. Strukturelle Veränderungen vollzogen die Autoren dagegen im Lehrplan von 1953 sowie in der Direktive von 1955. Der Begriff Abstammungslehre blieb jedoch stets zentraler Bestandteil relevanter Kapitelüberschriften in den Lehrplänen bzw. Direktiven von 1946 bis 1955 (Tab. 24). 
Tabelle 24: Lehrpläne Klasse 8, Kapitelüberschriften und Stundenumfang

\begin{tabular}{|llcccc|}
\hline Jahr & Kapitel & WS [h] & JSS [h] & JSE [h] & rH [\%] \\
1946 & Die Abstammung und Entwicklung des Menschen & $\begin{array}{c}2 \\
\text { (Kurs B) }\end{array}$ & 80 & 10 & 12,5 \\
1951 & $\begin{array}{l}\text { Abstammungslehre / Die Entwicklung des Men- } \\
\text { schen / Große Biologen }\end{array}$ & 2 & 66 & 42 & 63,6 \\
1952 & $\begin{array}{l}\text { Abstammungslehre / Die stammesgeschichtliche } \\
\text { Entwicklung des Menschen / Die Geschichte der } \\
\text { beiden grundlegenden Theorien in der Biologie }\end{array}$ & 2 & 58 & 34 & 58,6 \\
1953 & $\begin{array}{l}\text { Abstammungslehre und Weiterentwicklung der } \\
\text { Organismen durch den Menschen }\end{array}$ & 2 & 68 & 33 & 48,5 \\
$\begin{array}{l}\text { Abstammungslehre und Weiterentwicklung der } \\
\text { Organismen durch den Menschen }\end{array}$ & 2 & 60 & 30 & 50,0 \\
Legende: JSE - Jahresstunden für Evolutionsbiologie, JSS - Jahresstunden für Stoffangaben, rH - \\
relative Häufigkeit, WS - Wochenstunden im Fach Biologie
\end{tabular}

Die Inhaltsanalyse verdeutlichte ferner Veränderungen in der inhaltlichen Ausrichtung der Lehrpläne (Tab. 25). Der Lehrplan von 1946 forderte nur die Kategorie Hominisation. In den Lehrplänen von 1951 und 1955 fehlte die Kategorie Didaktisches und im Lehrplan von 1953 die Kategorie Hominisation. Ausschließlich der Lehrplan von 1952 enthielt Inhalte aus allen Kategorien. Wiederholt fassten die Autoren auch zwei Kategorien zusammen, wie in den Lehrplänen von 1951 und 1952 (Biogenese und Systematik) sowie 1955 (Systematik und Hominisation).

Tabelle 25: Lehrpläne Klasse 8, Verteilung der Kategorien

\begin{tabular}{|c|c|c|c|c|c|}
\hline Kategorie & 1946 & 1951 & 1952 & 1953 & 1955 \\
\hline Biogenese & - & the & $x^{2}$ & + & - \\
\hline Theorien & - & + & + & + & + \\
\hline Paläontologie & - & + & + & + & + \\
\hline Neontologie & - & + & + & + & the \\
\hline Hominisation & + & the & the & - & the \\
\hline Systematik & - & + & + & + & + \\
\hline Didaktisches & - & - & + & - & - \\
\hline
\end{tabular}

Ferner verdeutlichte auch die Raumanalyse, dass die Lehrpläne ab 1951 eine differenzierte Behandlung evolutionsbiologischer Inhalte forderten. Der Vergleich der Lehrpläne zeigte, dass der Stellenwert der Kategorie Neontologie zwischen 1951 und 1955, insbesondere nach 1952, abnahm. Dabei unterlagen diejenigen Inhalte 
einer Streichung, die bei der Behandlung „erfahrungsgemäß die größeren Schwierigkeiten bereitet[en]“ (Boje 1953: 339). Da jedoch der relative Anteil der Kategorie Paläontologie anstieg, sank der Stellenwert von Indizien für Evolutionsprozesse seit 1951 lediglich um $5 \%$ Prozent. Der Kategorienkomplex Biogenese, Systematik und Hominisation unterlag Schwankungen, besonders in den Jahren 1946 mit einem hohen und 1953 mit einen geringen prozentualen Anteil (Abb. 32). Diese Veränderungen erklären sich durch die Aufnahme bzw. Streichung der Kategorie Hominisation. Die Gründe für die Streichung im Lehrplan von 1953 lagen in den notwendigen Kürzungen nach der zweiten Lehrplanrunde. Die Wahl fiel auf das Kapitel Die stammesgeschichtliche Entwicklung des Menschen. Hier sahen die Autoren große methodische Schwierigkeiten aufgrund eines wissenschaftlichen Disputes zur Stellung des Australopithecus. Ziel war es, die Schüler nicht mit ungesicherten Tatsachen zu konfrontierten (Boje 1953). Da aufgrund der Kürzungen im Lehrplan von 1953 auch das Lehrbuch von 1953 keine adäquaten Inhalte mehr enthielt, fand ein entsprechender Unterricht an vielen Schulen nicht statt (Kühn 1955).

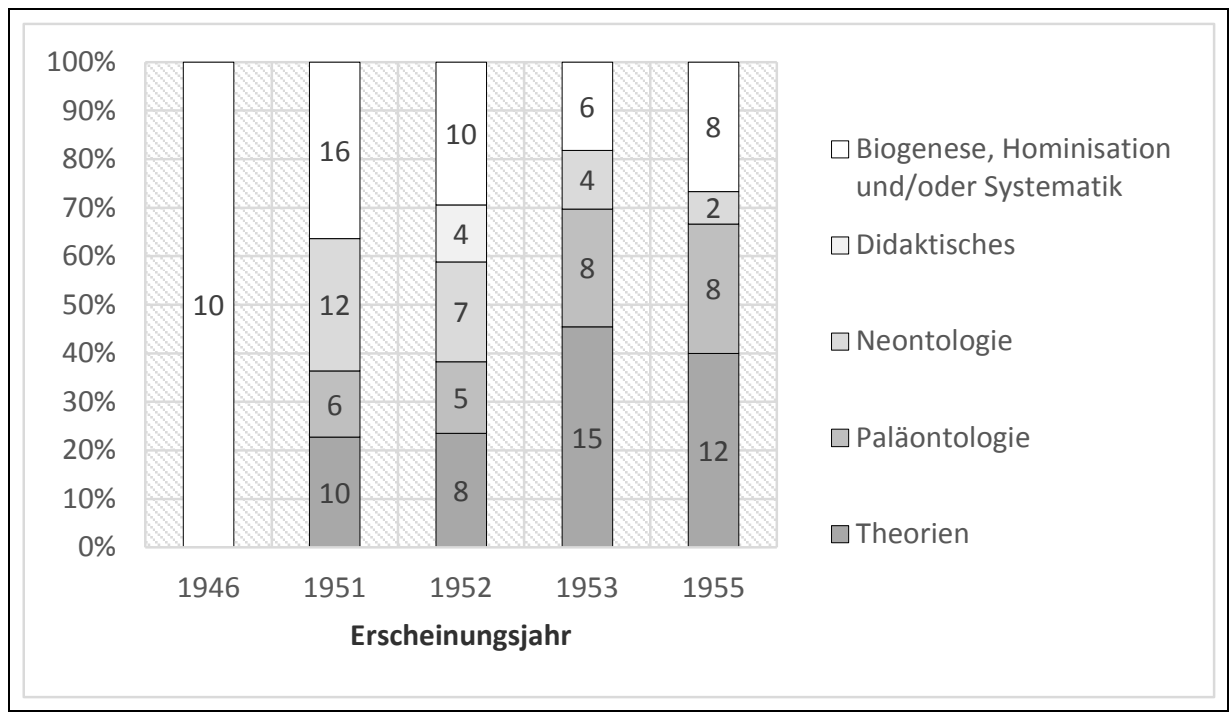

Abbildung 32: Relative Häufigkeit der Kategorien, Lehrpläne Klasse 8

Der erneute Anstieg des Kategorienkomplexes Biogenese, Systematik und Hominisation in der Direktive von 1955 erklärt sich durch die Wiederaufnahme der Kategorie Hominisation als Lehrplaninhalt. Das Thema Die Abstammung und Entwicklung des Menschen erfuhr hier eine genaue Bestimmung, um den Lehrbuchkürzungen entgegenzuwirken. Die Streichung des Inhalts Entstehung des Lebens in der Direktive von 1955 sollte durch Einfügungen bei der Behandlung des Themas Die Abstammung und Entwicklung des Menschen erfolgen. Die Raumanalyse zeigte weiter, 
dass der Kategorie Theorien ein größer werdender Stellenwert innerhalb der evolutionsbiologischen Themen zukam. Insbesondere vollzogen die Autoren mit dem Lehrplan von 1953 durch die Hinzunahme des Lehrplaninhalts Schöpferische Biologie eine Schwerpunktverlagerung. Die Reduktion in der Direktive von 1955 ergab sich durch die Streichung des Lehrplaninhalts Geschichte der Abstammungslebre, der ,aus Gründen der Faßlichkeit beziehungsweise aus Zeitgründen in der Mittelstufe nicht mehr behandelt" wurde (Kühn 1955: 412).

\section{Klasse 10}

Der Lehrplan für die Zehnklassenschule von 1951

Das Vorwort des Lehrplans für die Zehnklasseschule von 1951 war in Vorbemerkungen sowie erzieherische Aufgaben und Ziele gegliedert. Hinsichtlich der Evolutionsbiologie wurde „die Kenntnis der Abstammungslehre einschließlich der Lehre von der Abstammung des Menschen“ (MfV 1951c: 3) verlangt. Weitere Hinweise zur Evolutionsbiologie enthielt das Vorwort nicht.

Insgesamt gab der Lehrplan für den 10. Jahrgang (drei Wochenstunden) $102 \mathrm{~h}$ inhaltlich vor. Er gliederte sich in die Kapitel Anatomie und Physiologie des Menschen (66 h) sowie Allgemeine Biologie (36 h). Die übrigen $18 \mathrm{~h}$ waren als Sonderstunden für Wiederholung, Vorbereitung und Durchführung der Abschlussprüfungen vorgesehen. Der Stoffplan enthielt Stoff- und Stundenangaben sowie Bemerkungen zum Stoff. Der Anteil evolutionsbiologischer Inhalte betrug $18 \mathrm{~h}(15 \%)$ und gliederte sich in die Kapitel (MfV 1951c):

- Die Entstehung des Lebens auf der Erde und seine Entwicklung bis zum Menschen

- Die Entstehung des Lebens auf der Erde (3 h)

- Von Kohlenwasserstoffen zu den Urorganismen

- Kennzeichen des Lebens

- Irrdeutungen der Lebensentstehung

- Die Entwicklung der Arten; Beweise für die Abstammungslehre (9 h)

- Abstammung und Entwicklung des Menschen (6 h)

Der Stoff gliederte sich entsprechend in der Reihenfolge: Biogenese - Paläontologie - Neontologie - Hominisation. Die Kategorien Paläontologie und Neontologie wurden durch eine Stundenangabe zusammen abgebildet. Ihnen kam mit 9 h $(50 \%)$ der größte Stellenwert zu. Die Kategorie Hominisation umfasste mit 6 h einen Anteil von $33 \%$ und die Kategorie Biogenese mit 3 h einen Anteil von $17 \%$. Für die Kategorien Theorien, Systematik sowie Didaktisches forderte der Lehrplan keine Inhalte (Abb. 33). 


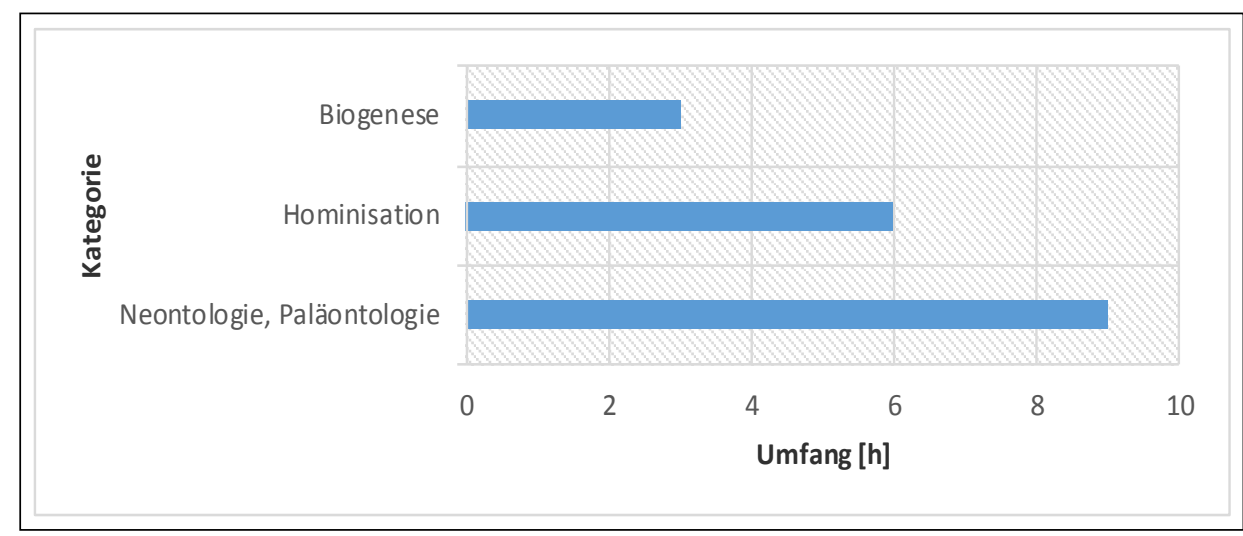

Abbildung 33: Stundenumfang für Kategorien, Lehrplan Klasse 10 (1951)

Der Übergangslehrplan von 1959 und der Lehrplan von 1960

Einen zentralen Stellenwert nahm die Evolutionsbiologie im Lehrplan von 1959 ein. Die Variabilität der Organismen sollte an Beispielen von Klasse 5 an verdeutlicht werden. Im Zentrum des Biologieunterrichtes der 10. Klasse stand die Entwicklungslehre (MfV 1959a). Die Zielformulierung, dass „bei der Behandlung der Phylogenese des Menschen [...] die besondere Bedeutung der Arbeit für die Menschwerdung“ (MfV 1959a: 28) herauszustellen ist, ließ eine Anlehnung an Friedrich Engels Schrift Anteil der Arbeit an der Menschwerdung des Affen in seinem Werk Dialektik der Natur erkennen. Dies galt umso mehr, da die Ansichten von Marx und Engels im Unterkapitel Geschichte der Entwicklungslebre behandelt werden sollten. Grundlegend forderte der Lehrplan die Lehrkräfte auf, im Unterricht „biologische und gesellschaftliche Entwicklungsfaktoren zu zeigen“ (MfV 1959a: 28).

Der Lehrplan von 1959 für die zehnklassige allgemeinbildende POS stellte eine Übergangsregelung für das Schuljahr 1959/60 dar. Dieser gab bei zwei Wochenstunden 56 Stunden inhaltlich vor. Er gliederte sich in die Kapitel Anatomie und Physiologie des Menschen (15 h), Entwicklungslehre (20 h) sowie Züchtung von Pflanzen und Tieren $(21 \mathrm{~h})$. Die restlichen $24 \mathrm{~h}$ standen als Sonderstunden zur freien Verfügung. Evolutionsbiologische Inhalte umfassten mit 20 h ein Drittel des Stoffs (35,7\%). Eine Feinstruktur gab der Lehrplan nicht vor. Er forderte jedoch, die Kenntnisse aus Klasse $8 \mathrm{zu}$ wiederholen sowie diese um die Inhalte aus dem neuen Lehrplan für Klasse 10 zu ergänzen.

Mit dem Schuljahr 1960/61 trat ein neuer Lehrplan für die POS in Kraft. Dieser wurde zur Orientierung bereits mit dem Übergangslehrplan von 1959 herausgegeben. Er gab für den 10. Jahrgang (zwei Wochenstunden) weiterhin $56 \mathrm{~h}$ inhaltlich und $24 \mathrm{~h}$ als Sonderstunden vor. Die Autoren gliederten den Lehrplan in die Kapitel Züchtung von Pflanzen und Tieren (21 h) sowie Entwicklungslehre (35 h). 
Den Lehrkräften standen im Stoffplan ausführliche Angaben über die zu behandelnden Inhalte zur Verfügung. Jedoch fehlten weitere Stundenangaben. Dies ermöglichte den Lehrkräften mehr Freiräume in ihren Entscheidungen, bot aber auch weniger Orientierung für die Unterrichtsplanung. Der Anteil evolutionsbiologischer Inhalte stieg auf $35 \mathrm{~h}(62,5 \%)$ an und setzte sich aus acht Kapiteln zusammen (MfV 1959a):

- Aufgaben und Methoden der entwicklungsgeschichtlichen Forschung

- Tatsachen aus der Paläontologie

- Übergangsformen

- Vergleichende Anatomie

- Vergleichende Embryologie

- Das natürliche System und die Geschichte der Organismen

- Geschichte der Entwicklungslehre

- Entstehung des Lebens auf der Erde

Der Stoffplan gliederte sich in die Kategorien Theorien - Paläontologie Neontologie - Systematik - Hominisation - Theorien - Biogenese. Einzig die Kategorie Didaktisches fehlte. Da jedoch nur eine Stundenangabe für den gesamten Themenkomplex vorhanden war, können Aussagen zum Stellenwert der Kategorien mittels Raumanalyse nicht getroffen werden.

Der Nachdruck von 1966

Im Jahr 1966 erschien ein Nachdruck des Lehrplans unter Berücksichtigung aller verbindlichen Veränderungen. Im Vorwort formulierten die Autoren fachliche Zielvorstellungen. Hinsichtlich evolutionsbiologischer Themen forderte der Lehrplan, anhand vieler Beispiele einen inhaltlichen Zugang ab Klasse 7 zu gewährleisten. In Klasse 10 sollten ,bei der Behandlung der Abstammungslehre die bereits bekannten Einzelerscheinungen unter entwicklungsgeschichtlichen Gesichtspunkten zusammengefasst und systematisiert" werden (MfV 1966: 3). Der Lehrplan verknüpfte evolutionsbiologische Inhalte auch mit gesellschaftlichen Werten. So galt der Inhalt Der Kampf um die Anerkennung der Entwicklungslehre als geeignete Möglichkeit, „die Schüler zu kritischer und parteilicher Stellungnahme zu erziehen“ (MfV 1966: 4). Ein weiterer inhaltlich-gesellschaftlicher Schwerpunkt lag auf der Vermittlung der „Gleichwertigkeit aller Menschenrassen“ (MfV 1966: 4). Grundlegendes Ziel war es auch, den Schülern die Bedeutung der Erkenntnisse für die materialistische Weltanschauung zu vermitteln. Gleichfalls sollten die Lehrkräfte zeigen, „daß es noch ungeklärte Probleme gibt und daß zu einigen offenen Fragen verschiedene wissenschaftliche Hypothesen existieren“ (MfV 1966: 21). Der zentrale Gedanke der Stoffeinheit war jedoch letzten Endes die Erkenntnis, „daß die Entwicklung der Organismen eine Tatsache ist" (MfV 1966: 21).

Inhaltlich gab der Lehrplan von 1966 für den Jahrgang 10 (zwei Wochenstunden) $56 \mathrm{~h}$ vor. Die restlichen $24 \mathrm{~h}$ standen als Sonderstunden zur Verfügung. Der 
Lehrplan enthielt Stundenangaben sowie ausführliche Bemerkungen zum Stoff unter der Überschrift Die Lehre von der Entwicklung der Organismen (56 h). Dieses eine Kapitel beinhaltete auch Themen aus der Genetik und der Züchtung. Der Anteil evolutionsbiologischer Inhalte umfasste $38 \mathrm{~h}(67,9 \%)$ und gliederte sich in die Abschnitte (MfV 1966):

- Tatsachen aus der Entwicklungslehre (9 h)

- Aus der Paläontologie

- Aus der vergleichenden Anatomie

- Aus der vergleichenden Physiologie

- Aus der Ontogenie

- Geschichte der Organismen (14 h)

- Wesen und Entstehung des Lebens

- Abstammung und Entwicklung des Menschen

- Stammesgeschichte und natürliches System der Organismen

- Zur Geschichte der Entwicklungslehre (8 h)

- Faktoren der stammesgeschichtlichen Entwicklung (7 h)

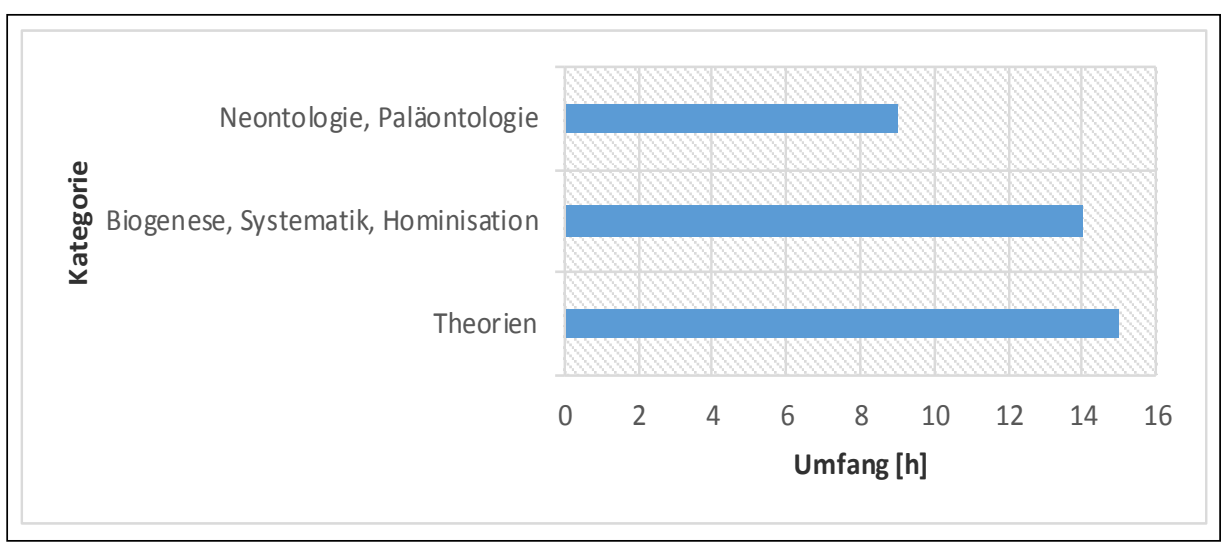

Abbildung 34: Stundenumfang für Kategorien, Lehrplan Klasse 10 (1966)

Der Stoffplan gliederte sich folglich in die Bereiche: Paläontologie - Neontologie - Biogenese - Hominisation - Systematik - Theorien. Aufgrund der Stundenangaben im Lehrplan ist eine Raumanalyse nur durch das Zusammenlegen einzelner Kategorien möglich, dennoch sind Präferenzen erkennbar (Abb. 34). Den größten Stellenwert nahm die Kategorie Theorien mit 15 h (39 \%) ein. Die Kategorien Biogenese, Systematik und Hominisation waren zusammen mit $14 \mathrm{~h}(37 \%)$ und die Kategorien Paläontologie und Neontologie mit 9 h (37\%) abgebildet. Außer der Kategorie Didaktisches wurden somit alle Kategorien behandelt. 
Der Lehrplan für die Vorbereitungsklassen von 1967

Das Vorwort des Lehrplans von 1967 für die Vorbereitungsklassen enthielt in Verbindung mit dem Biologieunterricht drei Erziehungs- und Orientierungsaussagen. Ziel war es erstens, den Schülern „Kenntnisse und Erkenntnisse auf dem Gebiet des Marxismus-Leninismus“ (MfV 1967b: 6) zu vermitteln, zweitens „ihr sozialistisches Staatsbewußtsein und ihre Liebe zur DDR zu entwickeln“ (ebd.: 7) und drittens zur „Parteinahme für die Sache des Sozialismus“ (ebd.) zu erziehen. Beim dritten Ziel wurden evolutionsbiologische Inhalte als Beispiele angeführt und exemplarisch aufgezeigt, wie sich Fachinhalte mit Erziehungszielen verknüpfen lassen.

„So sollen die Schüler z. B. an der Geschichte der Abstammungslehre erkennen, daß die eigentlichen Ursachen für den Widerstand der herrschenden Klasse gegen die Verbreitung der Evolutionstheorie darin lagen, daß diese Theorie den Kampf der Arbeiterklasse gegen die Besitz und Machtverhältnisse im Imperialismus ideologisch stärkte und die marxistische Weltanschauung unterstützte.“ (MfV 1967b: 7)

Insgesamt planten die Autoren für den 10. Jahrgang (zwei Wochenstunden) $52 \mathrm{~h}$ inhaltlich und $28 \mathrm{~h}$ als Sonderstunden. Der Lehrplan gliederte sich in die Kapitel Genetik (16 h), Abstammungslebre (29 h), Tier- und Pflanzenzüchtung als Weiterfübrung der Evolution durch den Menschen (4 h) sowie Die Biosphäre und der Mensch (3 h). Stundenangaben sowie ausführliche Bemerkungen zum Stoff standen den Lehrkräften mit dem Stoffplan zur Verfügung. Mit $29 \mathrm{~h}$ betrug der Anteil evolutionsbiologischer Inhalte 55,8 \% und gliederte sich in die Kapitel (MfV 1967b):

- Einführung in die Abstammungslehre (1 h)

- Faktoren der stammesgeschichtlichen Entwicklung (4 h)

- Mutation

- Selektion

- Zusammenwirken der Evolutionsfaktoren

- Evolution und Ontogenese (1 h)

- Homologie und Analogie (3 h)

- Der fossile Befund (3 h)

- Fossilien und ihre Bildung

- Übersicht über Organismen verschiedener Erdzeitalter

- Übergangs- oder Zwischenformen

- Entwicklungsreihen

- Die Abstammung des Menschen (6 h)

- Die Stellung des Menschen im System der Organismen

- Fossile Zeugnisse der Menschwerdung 
- Die biologischen und gesellschaftlichen Faktoren der Menschwerdung

- Arteinheit der heutigen Menschenrassen

- Stammesgeschichte und System der Organismen (2 h)

- Zur Geschichte der Abstammungslehre (5 h)

- Auffassungen über die Entwicklung der Organismen vor Darwin

- Die wissenschaftliche Begründung der Entwicklungslehre durch Charles Darwin

- Kampf um die Verbreitung des Entwicklungsgedankens

- Die Entstehung des Lebens auf der Erde (4 h)

- Wesen des Lebens

- Entstehung des Lebens

Inhaltlich gliederte sich der Stoffplan folglich in die Kategorien: Didaktisches Theorien - Neontologie - Paläontologie - Hominisation - Systematik - Theorien - Biogenese. Aufgrund der Stundenangaben können die Kategorien Theorien und Neontologie nicht getrennt einer Raumanalyse unterzogen werden. Sie nahmen zusammengefasst mit $13 \mathrm{~h}(45 \%)$ den höchsten Stellenwert ein (Abb. 35). Die Kategorie Hominisation wies mit $6 \mathrm{~h}(21 \%)$ ebenfalls einen hohen Stellenwert auf. Die restlichen Stunden standen für die Kategorien Biogenese 4 h (14\%), Paläontologie 3 h (10\%), Systematik 2 h (7\%) und Didaktisches 1 h (3\%) zur Verfügung.

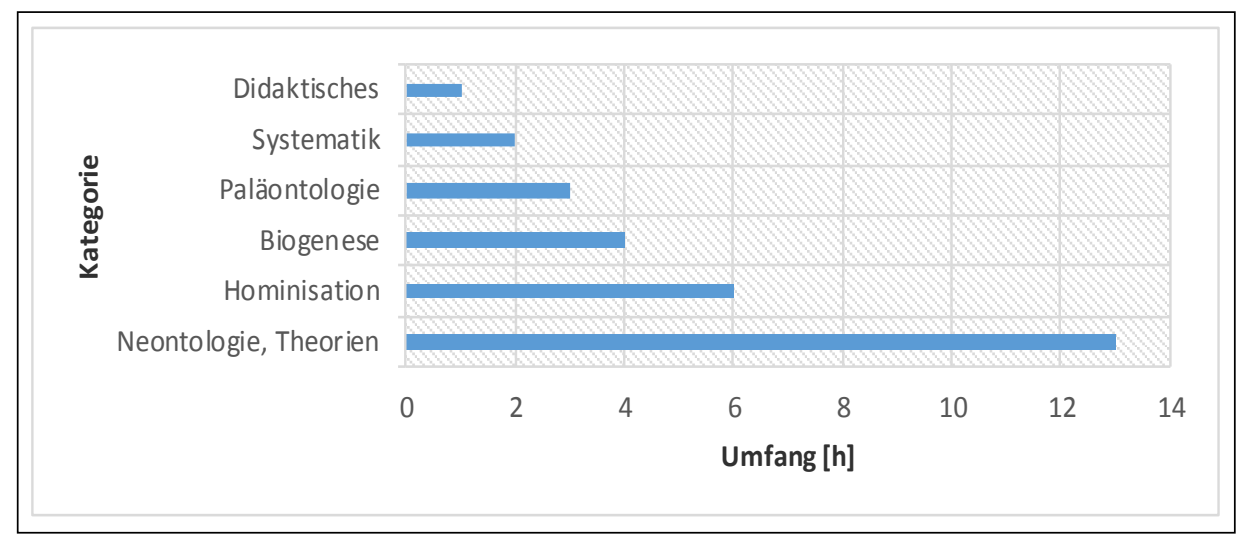

Abbildung 35: Stundenumfang für Kategorien, Lehrplan Klasse 10 (1967)

Der Lehrplan von 1969

Im Jahr 1969 erschien ein neuer Lehrplan für die POS. Das Vorwort gliederte sich in Ziele und Aufgaben sowie Hinweise zur methodischen und organisatorischen Gestaltung des Unterrichts. Im ersten Abschnitt bestimmten die Autoren das zu vermittelnde 
grundlegende Fachwissen und die zu entwickelnden Fähigkeiten. Auch „der spezifische Beitrag des Biologieunterrichtes zur Erziebung sozialistischer Staatsbürger" (MfV 1969: 38, Hervorhebung im Original) wurde betont. Die Schüler sollten erkennen, „daß die eigentlichen Ursachen für den Widerstand der herrschenden Klassen gegen die Verbreitung der Abstammungslehre darin lagen, daß diese Theorie den Kampf der Arbeiterklasse gegen die Ausbeuterordnung ideologisch stärkte" (MfV 1969: 39). Die Verbindung ideologischer Aussagen mit fachwissenschaftlichen Inhalten war auch im zweiten Teil des Vorworts klar erkennbar.

„Das Stoffgebiet ,2. Abstammungslehre` muß ein Schwerpunkt sowohl weltanschaulicher und politisch-ideologischer Systematisierung und Wertung der Erkenntnisse durch die Schüler als auch selbständigen Lernens der Schüler sein.“ (MfV 1969: 40)

Der Lehrplan gab für den 10. Jahrgang (zwei Wochenstunden) $56 \mathrm{~h}$ inhaltlich und $24 \mathrm{~h}$ als Sonderstunden vor. Mit $22 \mathrm{~h}$ betrug der Anteil evolutionsbiologischer Inhalte 39,3\%. Aus den Kapiteln Genetike (19 h), Abstammungslehre (22 h), Züchtung von Pflanzen und Tieren $(7 \mathrm{~h})$ sowie Wiederbolung, Systematik, Ausblick (8 h) setzte sich die Grobstruktur zusammen. Weiterhin enthielt der Stoffplan Stundenangaben sowie ausführliche Bemerkungen zum Stoff. Sechs Themenfelder lagen der Feinstruktur evolutionsbiologischer Inhalte zu Grunde (MfV 1969):

- Theorie der Stammesentwicklung (5 h)

- Stammesentwicklung der Pflanzen und Tiere (5 h)

- Aus der Geschichte der Abstammungslehre $(3 \mathrm{~h})$

- Die Entstehung des Lebens auf der Erde $(2 \mathrm{~h})$

- Die Stammesentwicklung des Menschen (5 h)

- Wiederholung und Systematisierung $(2 \mathrm{~h})$

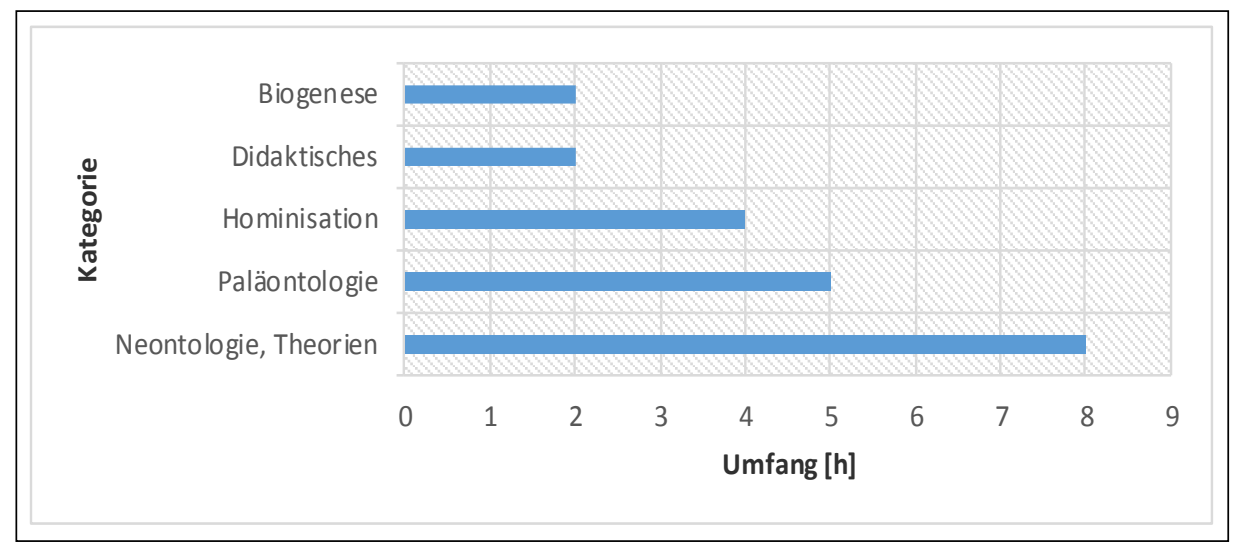

Abbildung 36: Stundenumfang für Kategorien, Lehrplan Klasse 10 (1969) 
Der Stoffplan gliederte sich in die Kategorien: Theorien - Neontologie - Paläontologie - Theorien - Biogenese - Hominisation - Didaktisches. Mit 8 h (36\%) wurden die Kategorien Theorien und Neontologie zusammen ausgewiesen. Sie nahmen den höchsten Stellenwert ein. Den Kategorien Paläontologie und Hominisation kam mit je 5 h (23\%) ebenfalls ein hoher Stellenwert zu (Abb. 36). Dagegen umfassten die Kategorien Biogenese und Didaktisches lediglich jeweils $2 \mathrm{~h}$ $(9 \%)$.

\section{Der Lehrplan von 1988}

Im Jahr 1988 erschien ein neuer Lehrplan. Die Struktur des Vorworts gliederte sich in Ziele und Aufgaben sowie Hinweise zur didaktisch-methodischen und organisatorischen Gestaltung des Biologieunterrichts und war dem Lehrplan von 1969 ähnlich. Inhaltlich zeigten sich jedoch deutliche Änderungen, da ideologische Aussagen kaum noch eine Rolle spielten. Der erste Abschnitt fasste die Themen der jeweiligen Klassenstufen zusammen. Grundlegende Aussagen der Lehre Darwins sowie deren Weiterentwicklung unter Beachtung der Vererbung kamen als evolutionsbiologische Inhalte zur Sprache. Auch forderte der Lehrplan, die Artentstehung, das Zusammenwirken der Evolutionsfaktoren, die Richtungen der Evolution (Höherentwicklung ${ }^{91}$, Spezialisierung, Rückbildung), die Entstehung des Lebendigen sowie die Stammesentwicklung der Organismen zu behandeln. Im Rahmen der Vermittlung zur Abstammung und Entwicklung des Menschen sollten die Schüler „ihre Überzeugungen vom unwissenschaftlichen und menschenfeindlichen Charakter des Rassismus" festigen (MfV 1988: 10f.).

Für den Jahrgang 10 (zwei Wochenstunden) waren $56 \mathrm{~h}$ inhaltlich und $24 \mathrm{~h}$ als Sonderstunden geplant. Mit $29 \mathrm{~h}$ betrug der Anteil evolutionsbiologischer Inhalte $51,8 \%$. Die Grobstruktur gliederte sich in die Kapitel Vererbung (24 h) und Evolution der Organismen $(29 \mathrm{~h})$. Die restlichen $3 \mathrm{~h}$ standen zur freien Verfügung. Der Lehrplan enthielt nur wenig differenzierte Stundenangaben, jedoch ausführliche Bemerkungen zum Stoff. Aus drei Kapiteln setzte sich die Feinstruktur evolutionsbiologischer Inhalte zusammen (MfV 1988):

- Grundlagen der Evolution der Organismen (14 h)

- Entstehung des Lebens und Stammesentwicklung der Organismen (10 h)

- Abstammung und Entwicklung des Menschen (5 h)

Die Inhalte gliederten die Autoren in die Kategorien: Theorien - Neontologie Biogenese - Paläontologie - Hominisation. Den höchsten Stellenwert nahmen die Kategorien Theorien und Neontologie ein (Abb. 37). Sie waren zusammen abgebildet und umfassten $14 \mathrm{~h}$ (38\%). Auch die Kategorien Paläontologie und Bioge-

91 Der Begriff ,Höherentwicklung` ist mit heutigen Erkenntnissen der Evolutionsbiologie nicht mehr vereinbar. 
nese fasste der Lehrplan mit 10 h (34 \%) zusammen. Die Kategorie Hominisation sollte in $5 \mathrm{~h}(17 \%)$ vermittelt werden.

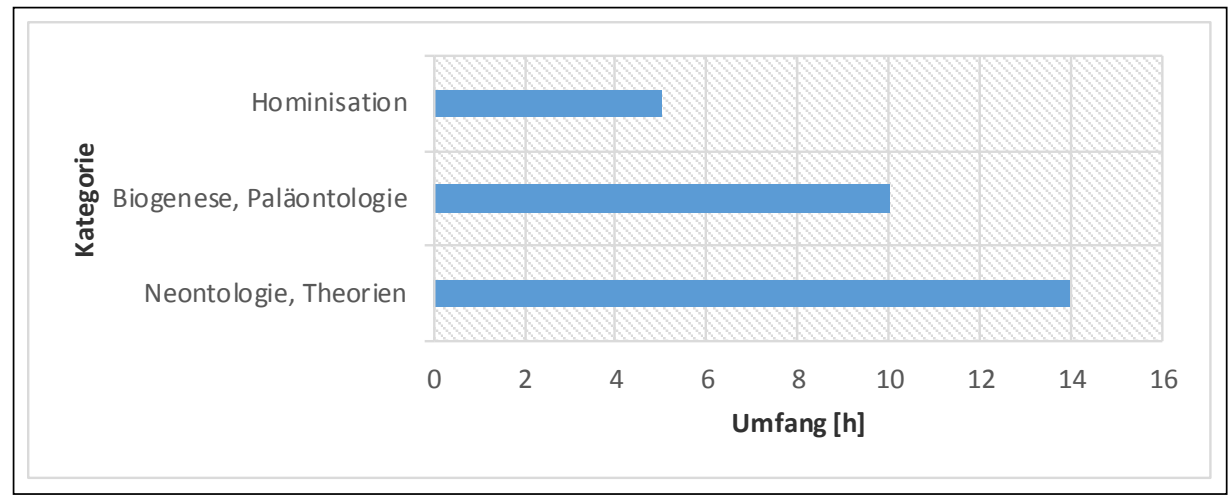

Abbildung 37: Stundenumfang für Kategorien, Lehrplan Klasse 10 (1988)

\section{Zusammenfassung}

Der zweite Strukturwandel im Schulsystem der DDR, das Gesetz über das einheitliche sozialistische Bildungssystem sowie die Phasen zwei bis fünf der Lehrplanentwicklung bildeten die Rahmenbedingungen, unter denen die Lehrpläne mit evolutionsbiologischen Inhalten für die Klassenstufe 10 entstanden. Diese gesellschaftlichen und schulpolitischen Veränderungen beeinflussten die Darstellung der Fachdisziplin Evolutionsbiologie in den Lehrplänen. Grundlegend war der Lehrplan von 1951 rein sachbezogen ausgerichtet (Typ 2), während die Autoren in den folgenden Lehrplänen evolutionsbiologische Inhalte für ideologische Orientierungen nutzten (Tab. 26). Die Lehrpläne von 1959 und 1966 verknüpften evolutionsbiologische Inhalte jedoch nur latent mit ideologischen Zielen. Hier wurde die Vermittlung eines materialistischen Weltbildes als „das Hauptziel aller Erziehungs- und Bildungsarbeit der sozialistischen Schule" (Heichler 1963: 471) avisiert. In diesem Kontext nahm die Evolutionsbiologie als „Höhepunkt und systematischen Abschluß“ (ebd.) eine exponierte Stellung ein. Ein klarer Bezug zum Marxismus und eine Ideologisierung evolutionsbiologischer Fachinhalte vollzogen sich dagegen in den Lehrplänen von 1967 und 1969. Das im Jahr 1969 in Folge des Gesetzes über das einheitliche sozialistische Bildungssystem ausgearbeitete Lehrplanwerk setzte neue Maßstäbe in der Verbindung von Fachinhalten mit ideologischen Zielsetzungen. Das Bildungssystem wurde als „ein Teilsystem des gesellschaftlichen System des Sozialismus" (Günther 1969: 14, Hervorhebung im Original) verstanden. Dem neuen Lehrplanwerk lag als Konzept die sozialistische Allgemeinbildung zu Grunde (Drefenstedt \& Neuner 1969). In diesem Kontext verlangte das gesellschaftliche System, „alle Lebensbereiche mit der sozialistischen Ideologie zu durchdringen“ 
(Günther 1969: 14). Im Lehrplan von 1988, der fünften Lehrplanrunde, waren ideologische Bemerkungen dann kaum mehr enthalten. Lediglich das Thema Rassismus als unerwünschte Erscheinungsform menschlichen Handelns kam in Verbindung mit evolutionsbiologischen Inhalten zu Sprache.

Tabelle 26: Sach- und Ideologiebezug, Lehrpläne Klasse 10

\begin{tabular}{|lcll|}
\hline \multicolumn{1}{c|}{ ja } & \multicolumn{1}{c|}{ Ideologiebezug } \\
Sachbezug & ja & Typ 1: 1959, 1966, 1967, 1969, 1988 & Typ 2: 1951 \\
& nein & Typ 3: - & Typ 4: - \\
\hline
\end{tabular}

Die deskriptive Analyse der Grobstruktur zeigte, dass in den Lehrplänen zwischen 1951 und 1988 Fachinhalte aus den Bereichen der Humanbiologie (1951), der Evolutionsbiologie (1951 bis 1988), der Genetik (1951 bis 1988) und der Züchtungsbiologie (1951 bis 1969) vertreten waren. Die Gliederung der Vorgaben veränderte sich in diesem Zeitraum durch Weglassen, Zusammenlegen und Trennen der Fachdisziplinen. Dabei standen die Inhalte aus der Evolutionsbiologie, Genetik und Züchtung in wechselnden gegenseitigen Beziehungen zueinander. In den Lehrplänen von 1951 und 1959 waren Fachinhalte aus der Züchtung sowie Genetik in einem Kapitel und im Lehrplan von 1966 alle drei Themenfelder zusammengefasst. In den Jahren 1967 und 1969 wurde jedes Themenfeld eigenständig behandelt, 1988 entfielen züchtungsbiologische Inhalte (Abb. 38). Die Anordnung der Stoffgebiete folgte im Lehrplan von 1966 der historischen Entwicklung der Wissenschaften, da die Abstammungslehre vor der Genetik behandelt wurde. Den Lehrplänen von 1969 und 1988 lag dagegen eine Wissenschaftslogik zu Grunde, die die Genetik als theoretische Grundlage vor evolutionsbiologische Überlegungen setzte (Wernecke 1971).

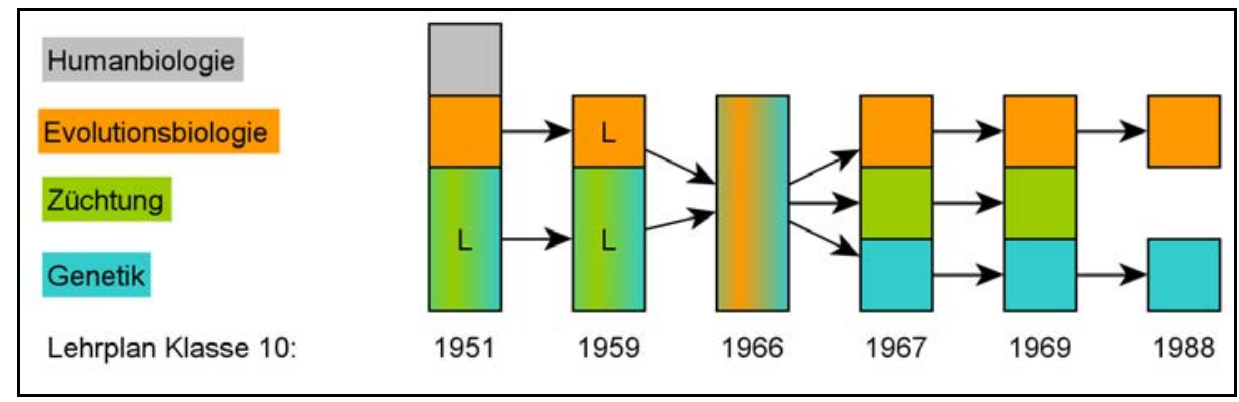

Abbildung 38: Lehrpläne Klasse 10, Entwicklung der Grobstruktur (L: Lyssenkoismus)

Die deskriptive Analyse verdeutlichte ferner Modifikationen in der Struktur evolutionsbiologischer Inhalte. Mit dem Lehrplan von 1969 wurden „grundsätzliche 
Veränderungen in der Zielorientiertheit vorgenommen“, so dass „die biologischen Gesetzmäßigkeiten [...] stärker in den Vordergrund" traten (Dietrich 1972: 16). Die Anordnung der Inhalte im Lehrplan zielte ,auf das theoretische Verständnis der Zusammenhänge" (Wernecke 1971: 309). Um dies zu erreichen, stellten die Autoren die Theorie der Stammesentwicklung an den Anfang des Lehrgangs und strafften die Geschichte der Abstammungslehre. Der neue Gehalt des Lehrplans lag aber in der "geschlossenen wissenschaftlichen Behandlung“" (Dietrich 1972: 17) der Stoffeinheit Genetik. Dies führte zu Veränderungen im Stundenumfang für die jeweiligen Stoffgebiete. Der Stundenumfang für evolutionsbiologische Inhalte stieg bis 1966, reduzierte sich 1969 und nahm 1988 erneut zu. Auch der Einsatz biologischer Begriffe lässt drei Phasen erkennen. In den Lehrplänen von 1951 bis 1966 war der Begriff Entwicklung zentral, in den Lehrplänen von 1967 und 1969 der Begriff Abstammungslebre und im Lehrplan von 1988 wurde erstmals das Wort Evolution als zentraler Begriff gewählt (Tab. 27).

Tabelle 27: Lehrpläne Klasse 10, Kapitelüberschriften und Stundenumfang

\begin{tabular}{|c|c|c|c|c|c|}
\hline Jahr & zentraler Begriff & WS [h] & JSS [h] & JSE [h] & $\mathrm{rH}[\%]$ \\
\hline 1951 & $\begin{array}{l}\text { Die Entstehung des Lebens auf der Erde } \\
\text { und seine Entwicklung bis zum Menschen }\end{array}$ & 3 & 102 & 18 & 17,6 \\
\hline 1959 (Ü) & Entwicklungslehre & 2 & 56 & 20 & 35,7 \\
\hline 1960 & Entwicklungslehre & 2 & 56 & 35 & 52,5 \\
\hline 1966 (ND) & $\begin{array}{l}\text { Die Lehre von der Entwicklung der } \\
\text { Organismen }\end{array}$ & 2 & 56 & 38 & 67,9 \\
\hline 1967 (VK) & Abstammungslehre & 2 & 52 & 29 & 55,8 \\
\hline 1969 & Abstammungslehre & 2 & 56 & 22 & 39,3 \\
\hline 1988 & Evolution der Organismen & 2 & 56 & 29 & 51,8 \\
\hline
\end{tabular}

Die Inhaltsanalyse belegte, dass ausschließlich der Lehrplan von 1967 (Vorbereitungsklassen) alle Kategorien enthielt. Meist fehlte die Kategorie Didaktisches, die nur 1967 und 1969 vorhanden war. Die Kategorie Systematik fehlte in den Lehrplänen von 1951 und 1969 und die Kategorie Theorien im Lehrplan von 1951, da Aussagen zur schöpferischen Biologie in einem eigenen Kapitel zu Züchtung und Genetik behandelt wurden. Aufgrund der wechselnden Verflechtung der Inhalte ist eine vergleichende Darstellung der Raumanalyse nicht möglich. In den Lehrplänen ab 1967 griffen die Kategorien Theorien und Neontologie ineinander. In den Lehrplänen von 1951, 1966 und 1988 wiesen die Kategorien Paläontologie 
und Neontologie und im Lehrplan von 1959/60 alle Kategorien eine Stundenangabe auf (Tab. 28). Dennoch zeigte sich, dass über die Jahre der Schwerpunkt auf Inhalten aus der Kategorie Theorien lag.

Tabelle 28: Lehrpläne Klasse 10, Verteilung der Kategorien

\begin{tabular}{|c|c|c|c|c|c|c|c|}
\hline Kategorie & 1951 & 1959 (Ü) & 1960 & 1966 (ND) & 1967 (VK) & 1969 & 1988 \\
\hline Biogenese & + & + & +2 & $y^{2}$ & + & + & s \\
\hline Theorien & - & t & + & + & t. & +2 & + \\
\hline Paläontologie & + & +1 & + & +2 & + & + & is \\
\hline Neontologie & - & $\sqrt{2}=$ & + & 4 & 4 & -1 & +2 \\
\hline Hominisation & + & t. & + & 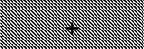 & + & + & + \\
\hline Systematik & - & is & t. & S & + & - & - \\
\hline Didaktisches & - & - & - & - & + & + & - \\
\hline
\end{tabular}

\section{Klasse 12}

Der Rahmenlehrplan von 1946

Das Vorwort aus dem Rahmenlehrplan von 1946 wurde bereits für die Klassenstufe 8 erläutert. Es galt ebenso für den 12. Jahrgang. Der Lehrplan von 1946 wies für die Kurse A und C 40 h (eine Wochenstunde) und für den Kurs B 80 h (zwei Wochenstunden) aus. Weitere Stundenangaben standen den Lehrkräften nicht zur Verfügung. Für die 12. Klasse gab der Lehrplan das Thema Allgemeine Biologie vor. In der 2. Auflage von 1947 waren die Inhalte des Stoffplans strukturierter. Der Stundenumfang stieg für den Kurs B auf 120 h (drei Wochenstunden) an. Neben den Themen Fragen der Ökologie und Erblehre enthielten beide Ausgaben die evolutionsbiologischen Kapitel Abstammungslehre, Die Abstammung des Menschen sowie Die Lehre von der Entstehung des Lebens auf der Erde. Während die beiden letztgenannten Kapitel keine näheren Erläuterungen aufwiesen, verfügte das erstgenannte Kapitel über inhaltlichen Zusatzinformationen (Tab. 29). In den Kursen A und C der 2. Ausgabe sollte im Kapitel Abstammung des Menschen auch Engels, Anteil der Arbeit an der Menschwerdung des Affen vermittelt werden. Der gleichnamige Artikel aus dem Buch Dialektik der Natur von Friedrich Engels war entsprechend lehrplanrelevant. Somit war an dieser Stelle bereits eine Verknüpfung fachwissenschaftlicher Inhalte mit gesellschaftlich-politischen Ansichten erkennbar. Die Inhaltsanalyse verdeutlicht in der Gesamtschau folgende Gliederung des Stoffplans: Theorien - 
Neontologie - Paläontologie - Neontologie - Theorien - Hominisation - Biogenese.

Tabelle 29: Inhalte mit evolutionsbiologischem Hintergrund, Lehrplan Klasse 12 (verändert nach DZfV in der SBZ 1946)

Abstammungslehre
Der Artbegriff bei Linné / Die Grundlage der modernen Lehre von den Arten / Das
Natursystem als Abbild des Evolutionsprozesses der organischen Welt / Die Veränderlichkeit
der Kulturpflanzen und Haustiere / Der Kampf ums Dasein / Die natürliche Zuchtwahl / Die
Entstehung neuer Arten bei Tieren und Pflanzen / Die Beweise für die Evolution der
organischen Welt aus dem Gebiet der vergleichenden Anatomie, Embryologie, Paläontologie
und Biogeographie
(Gruppe B zusätzlich: Die Entwicklung der Abstammungslehre. Darwin, Linné, Cuvier,
Geoffroy Saint-Hilaire, Lamarck, Goethe)
Zeitgenossen und Fortsetzer Darwins: Wallace, Huxley, McDougall, Haeckel, Weismann,
Timirjasew, Mitschurin, Lyssenko
Abstammung des Menschen
Stammbaum des Menschen
Die Lehre von der Entstehung des Lebens auf der Erde

Der Lehrplan für Oberschulen von 1953

Der Lehrplan für Oberschulen von 1953 sah für den Jahrgang 12 im Kurs B (drei Wochenstunden) insgesamt $96 \mathrm{~h}$ inhaltlich und $24 \mathrm{~h}$ als Sonderstunden vor. Für die Kurse A und C (zwei Wochenstunden) forderte der Lehrplan $64 \mathrm{~h}$ inhaltlich und $16 \mathrm{~h}$ als Sonderstunden. Unter der Überschrift Allgemeine Biologie gliederte er sich für alle Kurse in vier Bereiche: Die Entstebung des Lebens auf der Erde und die Entwicklung der Organismen bis zum Menschen (Kurs B 30 h, A/C 18 h), Die Entwicklungslehre im Rahmen der gesellschaftlichen Entwicklung (Kurs B 33 h, A/C 24 h), Die schöpferische Weiterentwicklung der Organismen durch den Menschen (Kurs B 21 h, A/C $14 \mathrm{~h}$ ) sowie Wiederholung des Jahresstoffes (Kurs B 12 h, A/C 8 h). Im Stoffplan standen den Lehrkräften differenzierte Stunden- sowie ausführliche Inhaltsangaben zur Verfügung. Evolutionsbiologische Inhalte umfassten mit $63 \mathrm{~h}$ im Kurs B 65,5\% und mit $42 \mathrm{~h}$ in den Kursen A und C 65,6\%. Die Autoren gliederten sie in folgende Abschnitte (MfV 1953b):

- Die Entstehung des Lebens auf der Erde und die Entwicklung der

Organismen bis zum Menschen (Kurs B 30 h, A/C 18 h)

- Die Entstehung des Lebens auf der Erde

- Die Abstammungslehre und Beweise für ihre Richtigkeit

- Die Entstehung der Arten und die Grundlagen der systematischen Gliederung

- Abstammung und Entwicklung des Menschen

- Die Entwicklungslehre im Rahmen der gesellschaftlichen Entwicklung (Kurs B 33 h, A/C 24 h) 
- Die Theorien von der Konstanz der Arten und deren Unwissenschaftlichkeit

- Die Entwicklungslehre Lamarcks

- Die wissenschaftliche Begründung der Entwicklungslehre durch Darwin

- Der Kampf Haeckels um die Anerkennung des Darwinismus

- Die Weiterentwicklung des Darwinismus zur schöpferischen Mitschurinschen Biologie

- Kritik an den unwissenschaftlichen Theorien der formalen Genetik

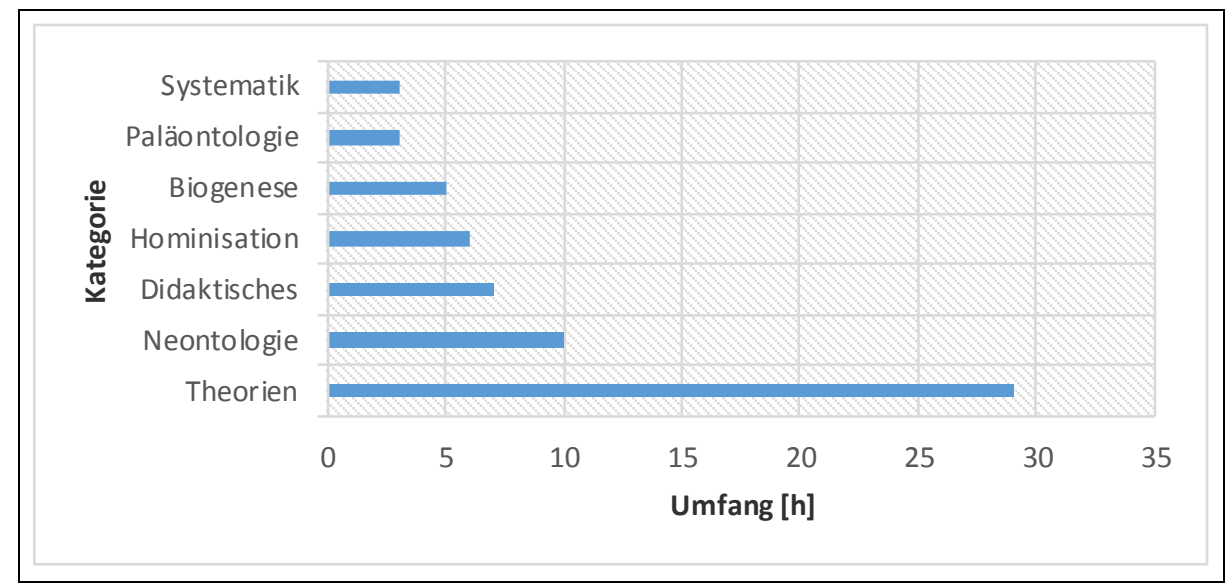

Abbildung 39: Stundenumfang für Kategorien, Lehrplan Klasse 12 Kurs B (1953)

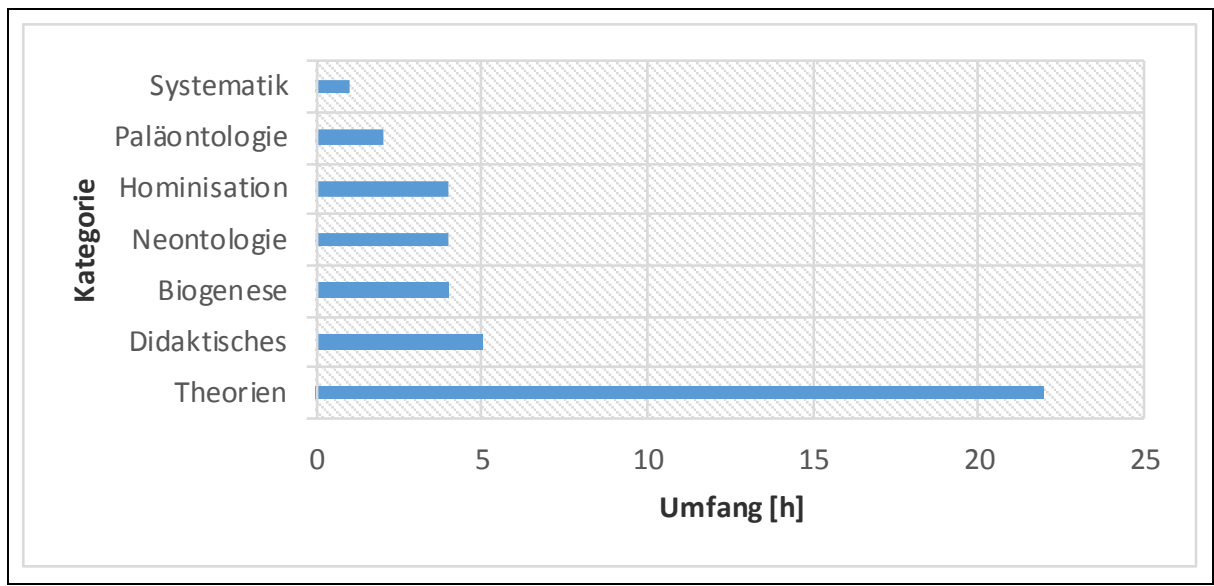

Abbildung 40: Stundenumfang für Kategorien, Lehrplan Klasse 12 Kurs A, C (1953) 
Die Inhaltsanalyse des Stoffplans verdeutlicht, dass alle Kategorien vorhanden und in folgender Reihenfolge abgebildet waren: Biogenese - Didaktisches - Paläontologie - Neontologie - Didaktisches - Systematik - Hominisation - Didaktisches - Theorien - Didaktisches - Theorien. Mit 29 h (46\%) im Kurs B und 22 h (46 \%) in den Kursen A und C nahm die Kategorie Theorien den höchsten Stellenwert ein (Abb. 39, 40). Der Umfang der anderen Kategorien fiel vergleichsweise gering aus.

\section{Der Lehrplan von 1954}

Das Vorwort des Lehrplans von 1954 enthielt in Verbindung mit evolutionsbiologischen Themen ideologische Aussagen. Es stand die materielle Natur von Erscheinungen und Vorgängen, ihr dialektischer Charakter und die wissenschaftliche Erklärung der Naturvorgänge im Vordergrund (MfV 1954). Auch die Rassendiskriminierung sowie der Missbrauch wissenschaftlicher Erkenntnisse wurden angesprochen. Ebenso orientierte der Lehrplan an der Auslegung des Darwinismus durch Lyssenko. Die Schüler sollten ,zur Anerkennung der Leistungen der internationalen, insbesondere der russischen und sowjetischen biologischen Wissenschaft erzogen werden“ (ebd.: 4). Der Lehrplan forderte hier eine Würdigung der Personen Mitschurin und Lyssenko. Damit verbunden war die Vermittlung der Entwicklung des Darwinismus zum schöpferischen Darwinismus sowie die Anerkennung ihrer „Forschungsergebnisse [...] als Grundlage der modernen Biologie“ (ebd.).

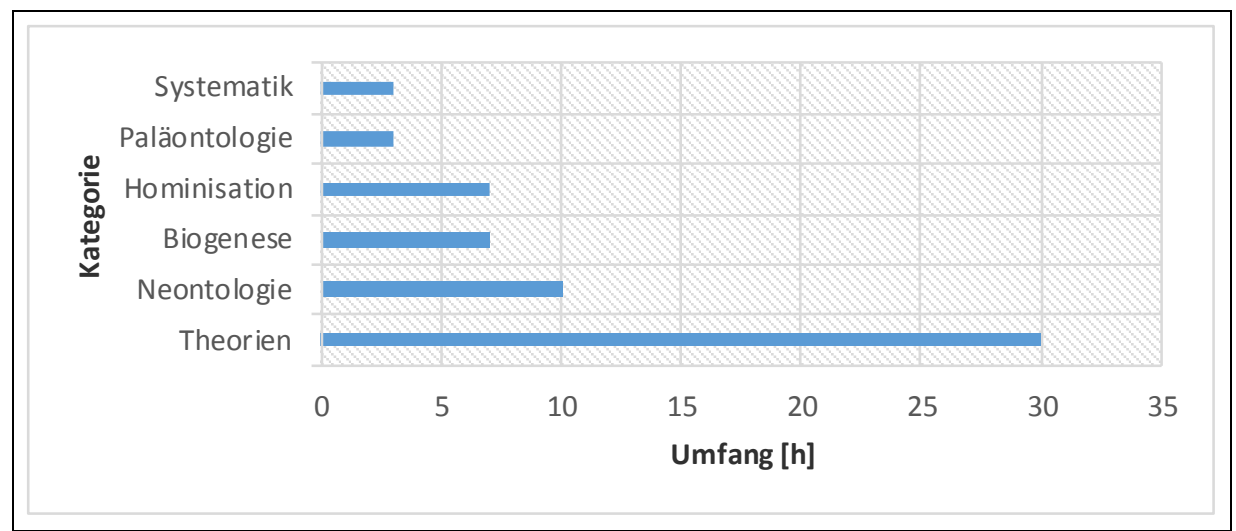

Abbildung 41: Stundenumfang für Kategorien, Lehrplan Klasse 12 Kurs B (1954)

Die Jahresstunden sowie die allgemeine Struktur des Lehrplans von 1954 stimmen mit dem Lehrplan von 1953 überein. Der Umfang evolutionsbiologischer Inhalte reduzierte sich jedoch durch Veränderungen in den detaillierten Inhalts- und Stundenangaben. Für die Kurse A und C standen 40 h und für den Kurs B 60 h (beide $62,5 \%$ ) zur Verfügung. Die Autoren gliederten die Inhalte folgenderma- 
Ben: Biogenese - Paläontologie - Neontologie - Systematik - Hominisation Theorien auf. Im Gegensatz zum Lehrplan von 1954 fehlte die Kategorie Didaktisches. Doch zeigte der Stoffplan ähnliche Präferenzen (Abb. 41, 42). Die Kategorie Theorien nahm mit 30 h (50\%) im Kurs B und 22 h (55\%) in den Kursen A und $\mathrm{C}$ den höchsten Stellenwert ein. Der Umfang der anderen Kategorien fiel deutlich geringer aus.

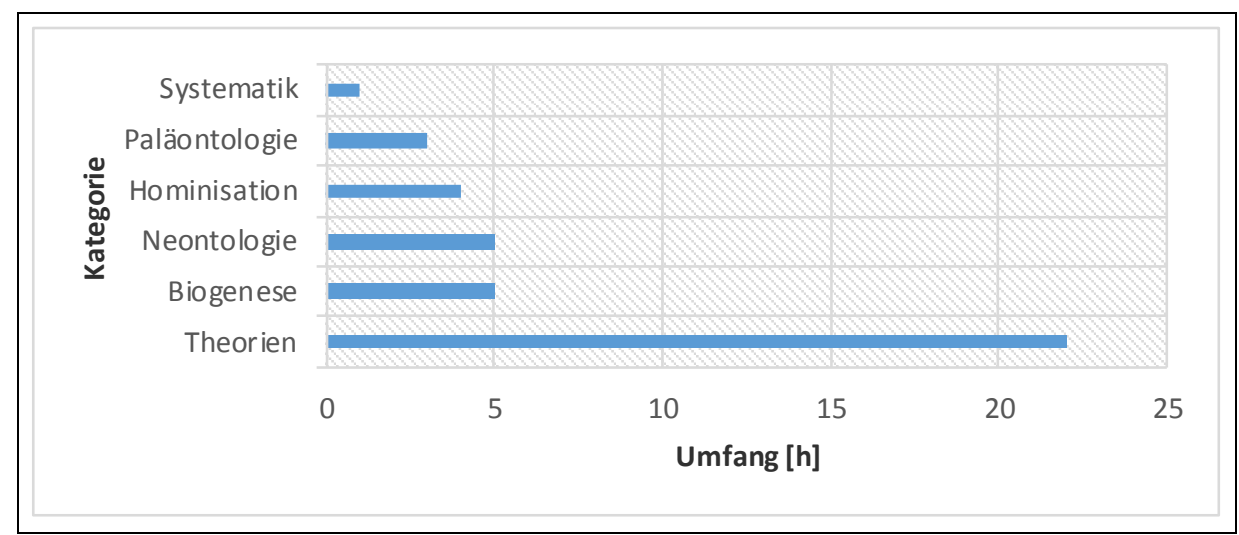

Abbildung 42: Stundenumfang für Kategorien, Lehrplan Klasse 12 Kurs A, C (1954)

Die Direktive von 1956

Die Direktive von 1956 enthielt Kürzungen und Änderungen mit dem Ziel, den Lehrkräften mehr Möglichkeiten für Bildungs- und Erziehungsarbeit zu verschaffen. Drei Aspekte standen dabei im Vordergrund. Erstens war die Auseinandersetzung mit und in der belebten Natur zu fördern, zweitens ein Beitrag zur polytechnischen Bildung zu leisten und drittens sollte der Biologieunterricht „stärker als bisher [...], den Schülern die Grundlagen einer wissenschaftlich begründeten materialistischen Weltanschauung [...] vermitteln" (MfV 1958: 5). Dabei wurde die dritte Forderung „,besonders durch die Behandlung wesentlicher Stoffgebiete aus der allgemeinen Biologie und der Abstammungs- und Entwicklungslehre in der 12. Klasse verwirklicht" (ebd.).

Inhaltlich gab die Direktive im Kurs B (drei Wochenstunden) 78 h vor. Die Sonderstunden umfassten $42 \mathrm{~h}$. Für die Kurse A und C (zwei Wochenstunden) forderte die Vorgabe inhaltlich $52 \mathrm{~h}$. Die restlichen $28 \mathrm{~h}$ standen als Sonderstunden zur Verfügung. Unter der Überschrift Allgemeine Biologie gliederte sich die Direktive von 1956 in vier Kapitel: Die Entstehung des Lebens auf der Erde und die Entwicklung der Organismen bis zum Menschen (Kurs B 32 h, A/C 18 h), Zur Geschicbte der Abstammungslebre (Kurs B 12 h, A/C 7 h), Weiterentwicklung der Organismen durch den Menschen (Kurs B 27 h, A/C 21 h) sowie Zur Entwicklung der Vererbungslehre (Kurs B 7 h, A/C 6 h). Alle Kapitel enthielten Stundenangaben sowie umfassende Anmerkungen zum Stoff. Die evolutionsbiologische Themen umfassten im Kurs B $44 \mathrm{~h}$ 
(56,4 \%) und in den Kursen A und C 25 h (48,1\%). Ihr Anteil war folglich in den mathematisch-naturwissenschaftlichen Klassen um 8,3 \% höher. Die Feinstruktur der evolutionsbiologischen Kapitel unterschied sich nur im zweiten Themenkomplex vom Lehrplan von 1954 (MfV 1958):

- Zur Geschichte der Abstammungslehre (Kurs B 12 h, A/C 7 h)

- Die Theorien von der Konstanz der Arten

- Die Abstammungslehre Lamarcks

- Die wissenschaftliche Begründung der Entwicklungslehre durch Darwin

- Der Kampf um die Anerkennung des Darwinismus

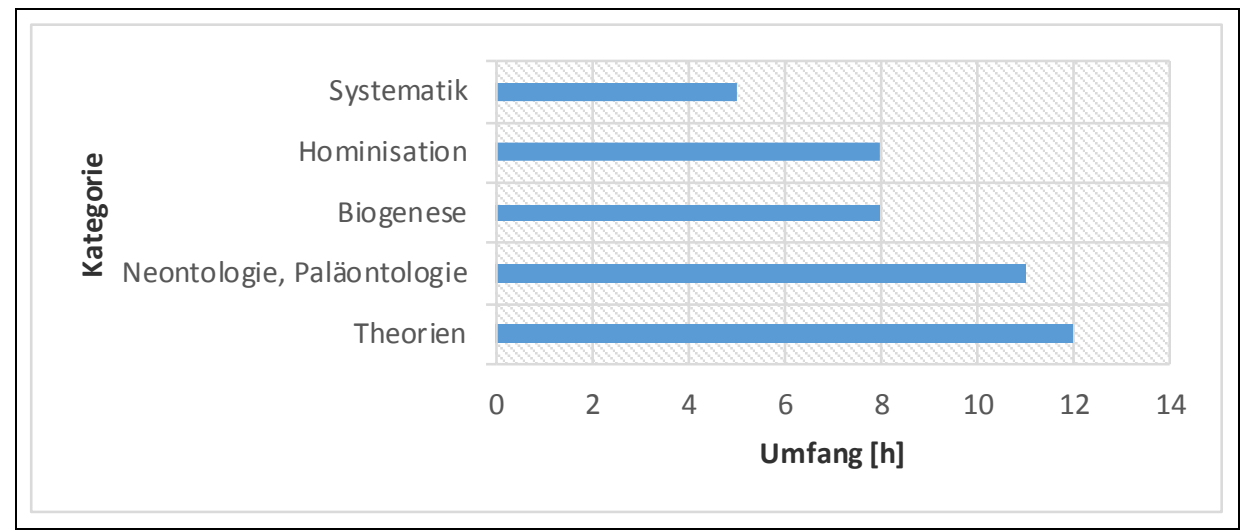

Abbildung 43: Stundenumfang für Kategorien, Lehrplan Klasse 12 Kurs B (1956)

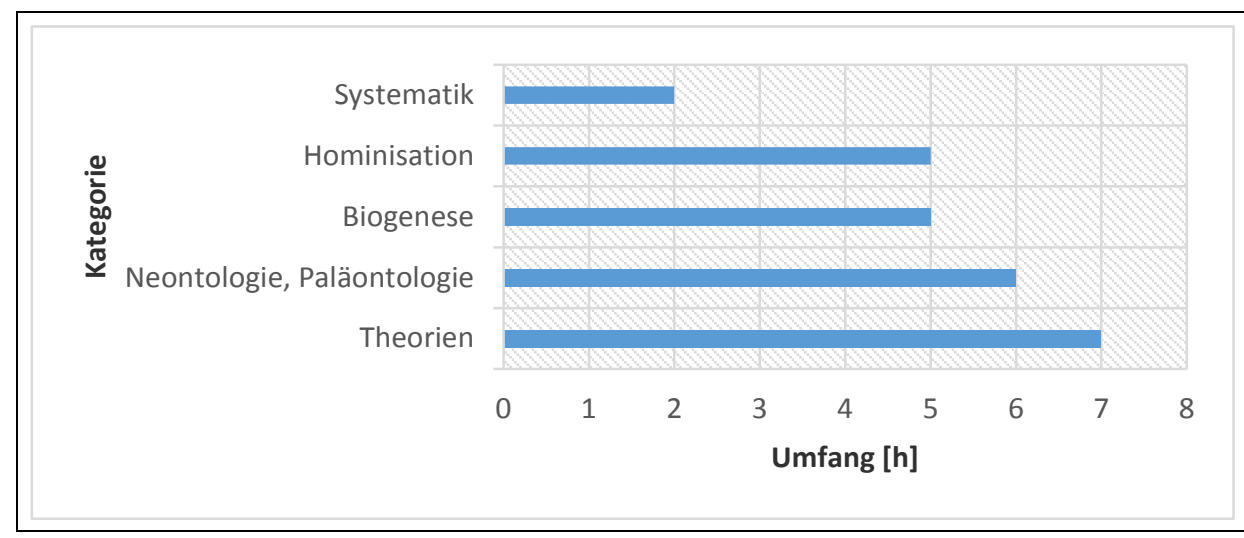

Abbildung 44: Stundenumfang für Kategorien, Lehrplan Klasse 12 Kurs A, C (1956)

Die Autoren gliederten die evolutionsbiologischen Themen folgendermaßen: Biogenese - Paläontologie - Neontologie - Systematik - Hominisation - Theorien. Die grundlegende Struktur wurde folglich beibehalten. Außer Didaktisches bildete 
die Direktive alle Kategorien ab. Dass eine ausgewogene Verteilung der Kategorien vorhanden war, zeigen entsprechende Raumanalysen (Abb. 43, 44). Dennoch nahm die Kategorie Theorien mit $12 \mathrm{~h}$ im Kurs B (27\%) und $7 \mathrm{~h}$ im Kurs A bzw. C $(28 \%)$ den höchsten Stellenwert ein. Mit einer Stundenangabe bildete der Stoffplan die Kategorien Paläontologie und Neontologie ab. Diese Themenfelder umfassten mit $11 \mathrm{~h}$ im Kurs B $25 \%$ und $6 \mathrm{~h}$ im Kurs A bzw. C $24 \%$. Einen Umfang von je $8 \mathrm{~h}$ im Kurs B (18\%) und je $5 \mathrm{~h}$ im Kurs A bzw. C (20\%) gab die Direktive für die Kategorien Hominisation und Biogenese vor. Die Kategorie Systematik umfasste $5 \mathrm{~h}$ im Kurs B (11 \%) und $2 \mathrm{~h}$ in den Kursen A und C (8\%).

Der Übergangslehrplan von 1959

Die Unterrichtsinhalte folgten einem geschichtlichen Ablauf und versetzten den Schüler in die Lage, „den wissenschaftlichen und politisch-ideologischen Kampf um die Anerkennung der Entwicklungslehre [mitzuerleben]“ (MfV 1959b: 281). Bei der Behandlung der Entwicklung des Menschen sollte:

„das Verhältnis zwischen biologischen und gesellschaftlichen Entwicklungsfaktoren verdeutlicht, [...] die Rolle der Arbeit bei der Menschwerdung aufgezeigt $[. .$.$] [und] [...] Irrwege und Fehlurteile idealistischer Welt-$ und Lebensdeutungen klargestellt [werden]“ (ebd.).

Inhaltlich sah der Übergangslehrplan von 1959 für den Kurs B (drei Wochenstunden) $78 \mathrm{~h}$ und für die Kurse A und C (zwei Wochenstunden) $52 \mathrm{~h}$ vor. Dadurch blieb auch die Anzahl der Sonderstunden erhalten. Der Lehrplan gliederte sich in fünf Bereiche: Entstehung und Entwicklung der Deszendenztheorie (Kurs B 26 h, A/C 18 h), Abstammung des Menschen (Kurs B 8 h, A/C 6 h), Entstehung des Lebens auf der Erde (Kurs B 8 h, A/C 4 h), Weiterentwicklung der Organismen durch den Menschen (Kurs B $32 \mathrm{~h}, \mathrm{~A} / \mathrm{C} 34 \mathrm{~h}$ ) sowie Wiederbolung und Vorbereitung der Reifeprïfung (nur Kurs B mit 4 h). Evolutionsbiologische Themen umfassten im Kurs B $42 \mathrm{~h}$ $(53,8 \%)$ und in den Kursen A und C je 28 h (53,8 \%). Folgende Feinstruktur lag ihnen zu Grunde (MfV 1959b):

- Entstehung und Entwicklung der Deszendenztheorie (Kurs B 26 h, A/C $18 \mathrm{~h})$

- Gegenüberstellung von Ontogenie und Phylogenie

- Theorie von der Konstanz der Arten

- Erste Vertreter des Entwicklungsgedankens

- Abstammungslehre Lamarcks

- Entwicklungslehre Darwins

- Kampf um die Anerkennung des Darwinismus

- Zusammenstellung der Beweise für die Deszendenztheorie

- Abstammung des Menschen (Kurs B 8 h, A/C 6 h)

- Vergleich zwischen Tier und Mensch 
- Stufenfolge der Entwicklung des Menschen

- Zeitpunkt, Ort und Ursachen der Menschwerdung

- $\quad$ Entstehung des Lebens auf der Erde (Kurs B 8 h, Kurs A/C 4 h)

- Unterschiede zwischen Lebenden und Leblosem

- Idealistische Theorien der Entstehung des Lebens

- A. I. Oparins dialektisch-materialistische Theorie

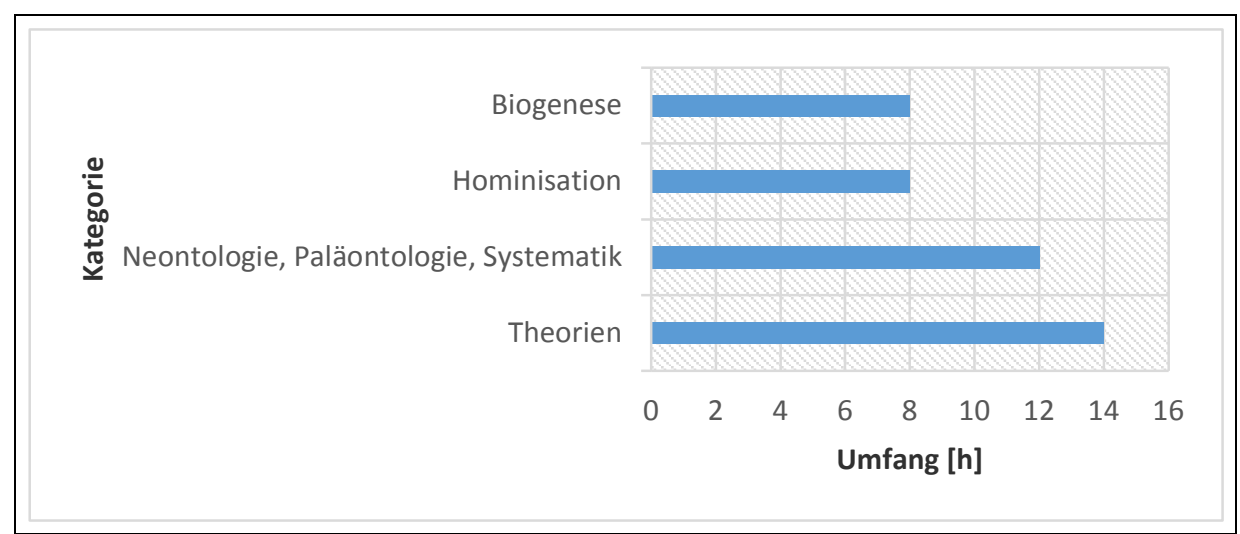

Abbildung 45: Stundenumfang für Kategorien, Lehrplan Klasse 12 Kurs B (1959)

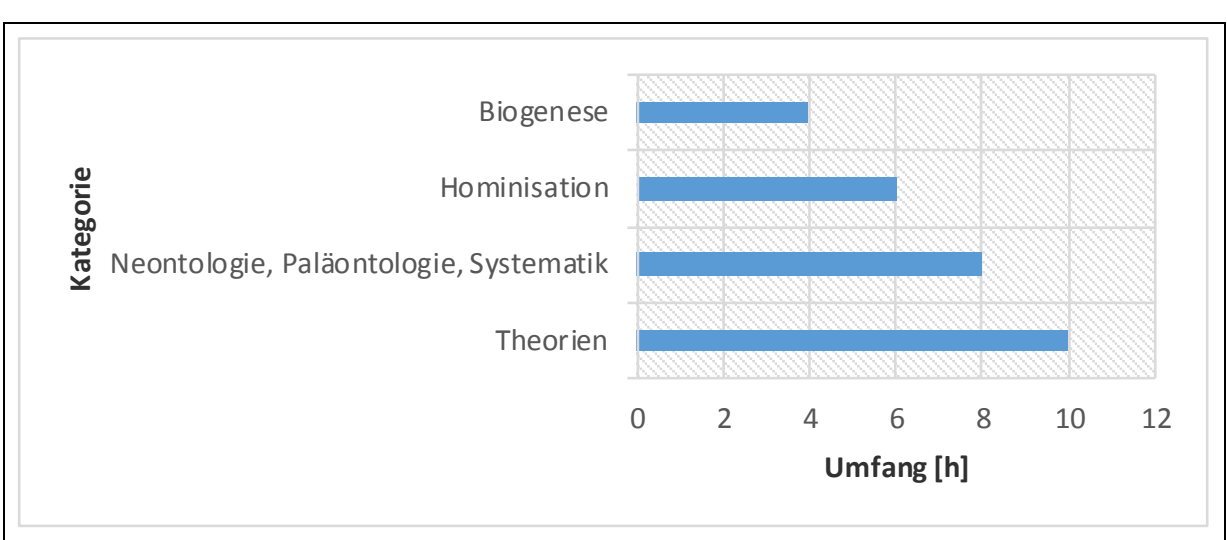

Abbildung 46: Stundenumfang für Kategorien, Lehrplan Klasse 12 Kurs A, C (1959)

Der Vorgabe zeigte folgende Gliederung: Theorien - Paläontologie - Neontologie - Systematik - Hominisation - Biogenese. Auf Inhalte aus der Kategorie Didaktisches verzichteten die Autoren. Dass eine ausgewogene Verteilung der Themenkomplexe vorhanden war, belegen entsprechende Raumanalysen (Abb. 45, 46). Etwa ein Drittel der Inhalte umfasste die Kategorie Theorien. Mit 14 h (33\%) im Kurs B und 10 h (36\%) in den Kursen A und C nahm sie den größten Stellenwert ein. Bedingt durch die Stundenangabe bildeten die Kategorien Paläontologie, 
Neontologie sowie Systematik in der Vorgabe eine Stoffeinheit (Belege für Evolutionsprozesse). Auch diese nahm mit 12 h (29\%) im Kurs B und 8 h (29\%) in den Kursen A und C einen Umfang von ca. einem Drittel ein. Die Kategorie Hominisation wies einen Umfang von $8 \mathrm{~h}(19 \%)$ im Kurs B und $6 \mathrm{~h}(22 \%)$ in den Kursen A und $\mathrm{C}$ auf. Mit $8 \mathrm{~h}$ im Kurs B und $4 \mathrm{~h}$ in den Kursen A und C umfasste die Kategorie Biogenese $19 \%$ bzw. $14 \%$.

Der Lehrplan von 1961

Verbindungen zwischen sozialistischer Ideologie und evolutionsbiologischen Inhalten waren auch im Vorwort des Lehrplans von 1961 enthalten. Die Autoren argumentierten, dass die Biowissenschaften einer differenzierten, vom jeweiligen Gesellschaftssystem ausgehenden, Wirkung unterliegen. Betont wurde, „daß sich die biologische Wissenschaft erst im Sozialismus voll entfalten und ihre humanistischen Aufgaben voll erfüllen kann“ (MfV 1961: B/3). Anhand „unwissenschaftlicher Rassentheorien“ (MfV 1961: B/3) begründete der Lehrplan den „Mißbrauch der biologischen Wissenschaft im Imperialismus“ (MfV 1961: B/3). Daraus resultierte der „Kampf gegen jegliche Rassendiskriminierung“ (MfV 1961: B/3) als Erziehungsziel.

Für die Kurse A und B (zwei Wochenstunden) gab der Lehrplan 42 Jahresstunden inhaltlich vor. Die Sonderstunden umfassten $38 \mathrm{~h}$. Das Thema des Jahrgangs lautete Die Lehre von der Evolution der Organismen. Es gliederte sich in fünf Kapitel: Tatsachen aus der Entwicklung der Organismen (8 h), Geschichte der Organismen (10 h), Faktoren der stammesgeschichtlichen Organismen (3 h), Zur Geschichte der Entwicklungslehre (8 h) sowie Weiterentwicklung der Organismen durch den Menschen (13 h). Ein umfangreicher Stoffverteilungsplan mit Stundenangaben und Bemerkungen zum Stoff stand den Lehrkräften zur Verfügung. Mit 29 h lag der Anteil für evolutionsbiologische Themen bei $69 \%$. Diese gliederten die Autoren in die Kapitel (MfV 1961):

- Tatsachen aus der Entwicklung der Organismen (8 h)

- Tatsachen aus der Paläontologie

- Tatsachen aus der vergleichenden Anatomie

- Tatsachen aus der Ontogenie

- Geschichte der Organismen (10 h)

- Entstehung der lebenden Materie

- Stammesgeschichte der Organismen

- Abstammung und Entwicklung des Menschen

- Faktoren der stammesgeschichtlichen Entwicklung (3 h)

- Zur Geschichte der Entwicklungslehre (8 h)

- Theorie von der Konstanz der Arten

- Erste Vertreter des Entwicklungsgedankens

- Lamarcks Entwicklungstheorie 
- Die wissenschaftliche Begründung der Entwicklungslehre durch Charles Darwin

- Kampf um Durchsetzung der Entwicklungslehre

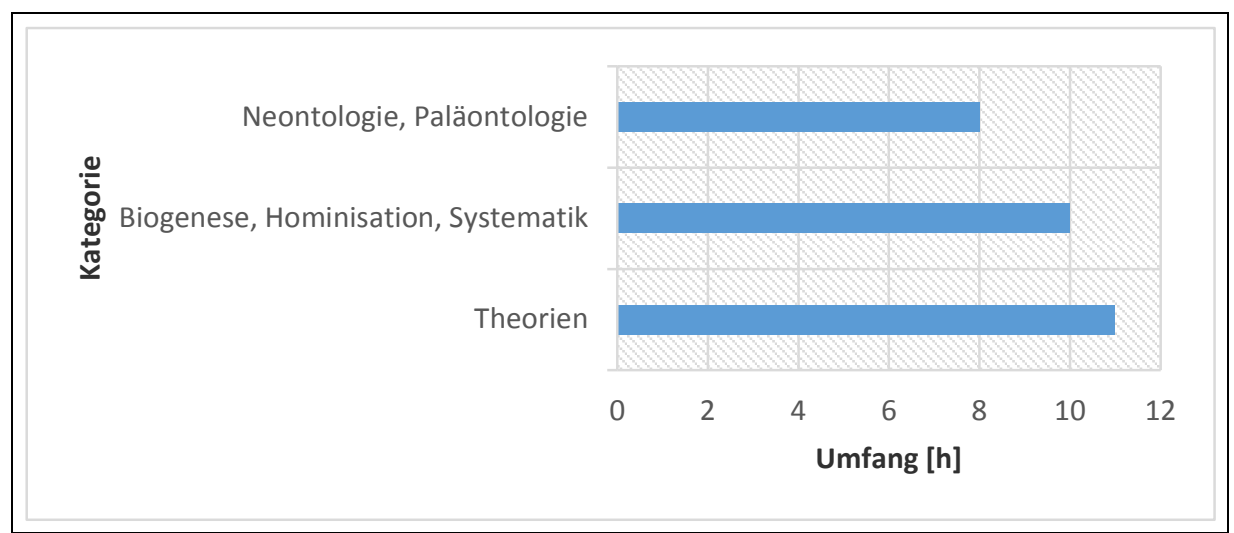

Abbildung 47: Stundenumfang für Kategorien, Lehrplan Klasse 12 Kurs A, B (1961)

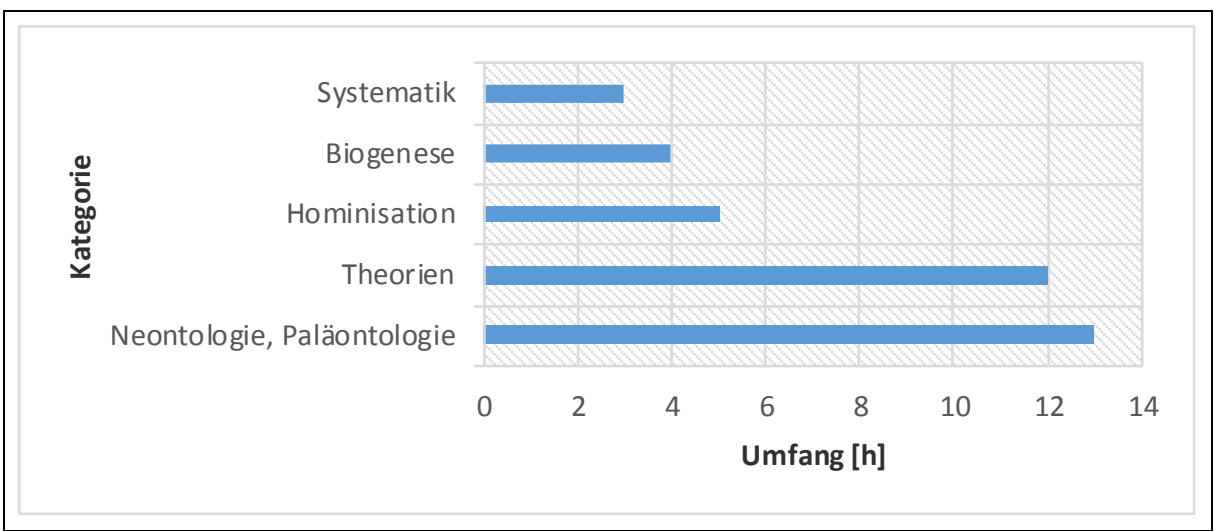

Abbildung 48: Stundenumfang für Kategorien, Lehrplan Klasse 10/11 Kurs C (1961)

Mit einem Umfang von insgesamt 50 h verteilten sich die Inhalte im Kurs C auf andere Jahrgänge. Das erste Kapitel sowie die ersten beiden Unterkapitel aus Kapitel zwei sollten in Kasse 10 und die übrigen Inhalte in Klasse 11 behandelt werden. Dem Stoffplan der Kurse A und B lag die Gliederung Paläontologie Neontologie - Biogenese - Systematik - Hominisation - Theorien zu Grunde. Die Raumanalyse zeigt, dass zu je ca. einem Drittel die Kategorie Theorien mit 11 h (38 \%), die Kategorien Biogenese, Systematik und Hominisation mit 10 h (34 \%) sowie die Kategorien Paläontologie und Neontologie mit 8 h (28\%) behandelt werden sollten. Eine ähnliche prozentuale Verteilung enthielt der Lehrplan für den Kurs C (Abb. 47, 48). 
Die Korrektur von 1967

Das kurze Vorwort enthielt lediglich Gründe für die Neuerung sowie wenige sachbezogene Ausführungen. Ideologische Bemerkungen enthielt die Korrektur nicht. In den Kursen A und B standen weiterhin 42 Jahresstunden für Fachinhalten zur Verfügung, so dass bei zwei Wochenstunden $38 \mathrm{~h}$ als Sonderstunden verblieben. Das Thema des Jahrgangs blieb erhalten, jedoch veränderte sich die Grobstruktur: Abstammungslebre (22 h) sowie Genetik, Evolution und Züchtung (20 h). Stundenangaben und umfangreiche Inhaltsangaben konnten dem Stoffverteilungsplan entnommen werden. Mit $25 \mathrm{~h}$ reduzierte sich der Anteil evolutionsbiologischer Inhalte leicht auf 59,5\%. Diese gliederten sich in die Kapitel (MfV 1967a):

- Tatsachen aus der Entwicklung der Organismen (8 h)

- Aus der Paläontologie

- Aus der vergleichenden Anatomie

- Aus der Ontogenie

- Geschichte der Organismen (10 h)

- Wesen und Entstehung des Lebens

- Stammesgeschichte der Organismen

- Abstammung und Entwicklung des Menschen

- Zur Geschichte der Abstammungslehre (4 h)

- Faktoren der Evolution (3 h)

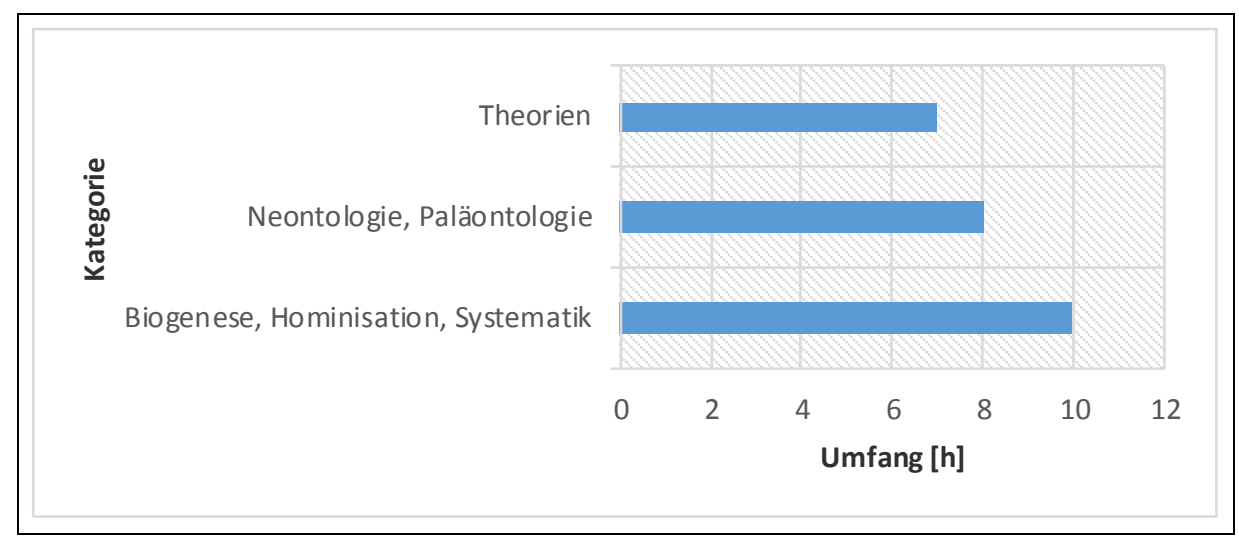

Abbildung 49: Stundenumfang für Kategorien, Lehrplan Klasse 12 Kurs A, B (1967)

Die evolutionsbiologischen Themen folgten der Gliederung: Paläontologie Neontologie - Biogenese - Systematik - Hominisation - Theorien. Keine Aufnahme in die Vorgabe fand die Kategorie Didaktisches. Zum Lehrplan von 1961 veränderten sich die Präferenzen leicht (Abb. 49). Aufgrund der Stundenangabe bildeten die Kategorien Biogenese, Systematik und Hominisation weiterhin eine 
Einheit. Diese umfasste mit $10 \mathrm{~h}$ einen Anteil von $40 \%$. Auch die Kategorien Paläontologie und Neontologie blieben als Themenkomplex mit einem Umfang von $8 \mathrm{~h}(32 \%)$ erhalten. Der Umfang der Kategorie Theorien verringerte sich auf $7 \mathrm{~h}(28 \%)$.

\section{Zusammenfassung}

In den Untersuchungszeitraum fallen alle strukturellen Veränderungen des Schulsystems der SBZ/DDR sowie die Phasen eins bis vier der Lehrplanentwicklung. Die Inhaltsanalyse der Vorworte belegte in den untersuchten Lehrplänen zwischen 1946 und 1961 eine Verflechtung evolutionsbiologischer Inhalte mit ideologischen Orientierungen (Typ 1). Hierbei sind Rückwirkungen gesellschaftlichpolitischer Veränderungen auf die Lehrpläne erkennbar. Orientierte der Lehrplan von 1946 evolutionsbiologische Inhalte am Humanismus, so waren entsprechende Fachinhalte im Lehrplan von 1954 am sowjetischen Vorbild (Lyssenkoismus) ausgerichtet. In den folgenden Lehrplänen dienten evolutionsbiologische Themen dazu, den Schüler zu einer materialistischen Weltanschauung sowie zur sozialistischen Ideologie zu erziehen. Dagegen wies die Anweisung zur Korrektur (1967) eine rein sachbezogene Orientierung auf (Tab. 30).

Tabelle 30: Sach- und Ideologiebezug, Lehrpläne Klasse 12

\begin{tabular}{|lcll|} 
& \multicolumn{2}{c|}{ Ideologiebezug } & \multicolumn{1}{c|}{ nein } \\
Sachbezug & ja & Typ 1: 1946, 1954, 1956, 1959, 1961 & Typ 2: 1967 \\
& nein & Typ 3: - & Typ 4: - \\
\hline
\end{tabular}

Die deskriptive Analyse der Grobstruktur zeigte, dass in den Lehrplänen zwischen 1946 und 1967 Fachinhalte aus den Bereichen der Ökologie (1946), der Evolutionsbiologie (1946 bis 1967), der Genetik (1946 bis 1967) und der Züchtungsbiologie (1946 bis 1967) vertreten waren. Die Grobstruktur veränderte sich, wie in den Lehrplänen für die Klassenstufe 10, durch Weglassen, Zusammenlegen und Trennen der genannten Fachdisziplinen. Die Inhalte aus der Evolutionsbiologie, Genetik und Züchtung standen in wechselnden gegenseitigen Beziehungen zueinander. Der Lehrplan von 1946 fasste Fachinhalte aus der Züchtung und der Genetik in einem Kapitel zusammen. Auch waren Kapitel mit evolutionsbiologischen ökologischen Inhalten vorhanden. Die Lehrpläne von 1953 und 1954 enthielten ein Kapitel mit Inhalten aus den drei Themenfeldern (Evolutionsbiologie, Genetik, Züchtung) sowie ein eigenständiges Kapitel zur Züchtung. Im Lehrplan von 1956 schließlich wurde jedes Themenfeld klar abgegrenzt. Die Abhandlung evolutionsbiologischer Inhalte in einem eigenen Kapitel blieb bis 1967 bestehen. Die Pläne von 1959 und 1961 stellten darüber hinaus Aspekte der Genetik sowie der 
Züchtungsforschung in einem Kapitel dar und im Lehrplan von 1967 waren Aspekte aus allen drei Fachdisziplinen vorhanden (Abb. 50).

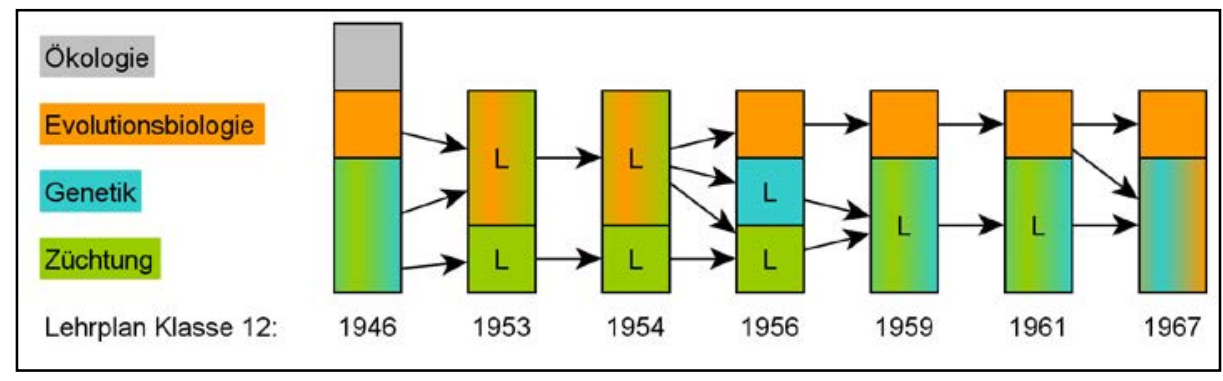

Abbildung 50: Lehrpläne Klasse 12, Entwicklung der Grobstruktur (L: Lyssenkoismus)

Tabelle 31: Lehrpläne Klasse 12, Kapitelüberschriften und Stundenumfang

\begin{tabular}{|c|c|c|c|c|c|c|c|c|c|c|c|c|c|}
\hline \multirow[t]{2}{*}{ Jahr } & \multirow[t]{2}{*}{ zentraler Begriff } & \multicolumn{3}{|c|}{$\begin{array}{l}\text { WS im } \\
\text { Kurs [h] }\end{array}$} & \multicolumn{3}{|c|}{ JSS im Kurs [h] } & \multicolumn{3}{|c|}{ JSE im Kurs [h] } & \multicolumn{3}{|c|}{ rH im Kurs [\%] } \\
\hline & & A & C & B & A & C & B & A & C & B & $A$ & C & B \\
\hline 1946 & Abstammungslehre & 1 & 1 & 3 & 40 & 40 & 80 & k. A. & k. A. & k. A. & - & - & - \\
\hline 1953 & Entwicklungslehre & 2 & 2 & 3 & 64 & 64 & 96 & 42 & 42 & 63 & 65,6 & 65,6 & 65,5 \\
\hline 1954 & Entwicklungslehre & 2 & 2 & 3 & 64 & 64 & 96 & 40 & 40 & 60 & 62,5 & 62,5 & 62,5 \\
\hline 1956 & Entwicklungslehre & 2 & 2 & 3 & 52 & 52 & 78 & 25 & 25 & 44 & 48,1 & 48,1 & 56,4 \\
\hline 1959 & Entwicklungslehre & 2 & 2 & 3 & 52 & 52 & 78 & 28 & 28 & 42 & 53,8 & 53,8 & 53,8 \\
\hline 1961 & $\begin{array}{l}\text { Die Lehre von der } \\
\text { Evolution der } \\
\text { Organismen }\end{array}$ & 2 & 0 & 2 & 42 & 0 & 42 & 42 & 0 & 42 & 69,0 & 0 & 69,0 \\
\hline 1967 & $\begin{array}{l}\text { Die Lehre von der } \\
\text { Evolution der } \\
\text { Organismen }\end{array}$ & 2 & 0 & 2 & 42 & 0 & 42 & 25 & 0 & 25 & 59,5 & 0 & 59,5 \\
\hline \multicolumn{14}{|c|}{$\begin{array}{l}\text { Legende: A - neusprachlicher Zweig, B - mathematisch-naturwissenschaftlicher Zweig, C - altsprach- } \\
\text { licher Zweig, JSE - Jahresstunden für Evolutionsbiologie, JSS - Jahresstunden für Stoffangaben, } \mathrm{k} \text {. } \\
\text { A. - keine Angaben, } \mathrm{rH} \text { - relative Häufigkeit, WS - Wochenstunden im Fach Biologie }\end{array}$} \\
\hline
\end{tabular}

Die deskriptive Analyse evolutionsbiologischer Inhalte belegte, dass sich, wie auch bereits für die Klassenstufe 8 aufgezeigt, die inhaltliche Struktur der Rahmenlehrpläne der 1940er Jahre von denen der 1950er Jahre wesentlich unterschied. Dagegen zeigten die Lehrpläne von 1953 und 1954 in Gliederung und Stundenumfang deutliche Übereinstimmungen auf. Zwar bestanden auch zwischen dem Lehrplan von 1963 und der Korrektur von 1967 strukturelle Gemeinsamkeiten, doch mit 
der Einführung der Genetik als Lehrplaninhalt veränderte sich die Reihenfolge sowie der Stundenumfang evolutionsbiologischer Inhalte. Während im Strukturverlauf der Lehrpläne zwischen 1953 und 1956 erst Forschungsergebnisse und danach ihre Geschichte dargestellt wurden, folgte die Struktur im Übergangslehrplan von 1959 einem historischen Ablauf. Ziel war es, Stoffüberschneidungen und Wiederholungen zu vermeiden, das Interesse der Schüler zu wecken, selbstständiges Schlussfolgern zu fördern sowie den politisch-ideologischen Kampf nachvollziehbar zu gestalten (Steinheit 1959). Ebenso veränderte sich die Begriffsverwendung. Stand 1946 der Begriff Abstammungslehre im Vordergrund, so wurde in den Lehrplänen von 1951 bis 1959 der Begriff Entwicklungslehre angewandt und ab 1961 dann von Evolution der Organismen gesprochen (Tab. 31).

Tabelle 32: Lehrpläne Klasse 12, Verteilung der Kategorien

\begin{tabular}{|c|c|c|c|c|c|c|c|}
\hline Kategorie & 1946 & 1953 & 1954 & 1956 & 1959 & 1961 & 1967 \\
\hline Theorien & the & + & + & + & + & + & + \\
\hline Paläontologie & th & + & + & + & + & 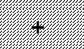 & the \\
\hline Neontologie & $x^{-2}$ & + & + & +2 & th & + & + \\
\hline Biogenese & the & + & + & + & + & 5 & 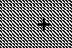 \\
\hline Systematik & - & + & + & + & th & 3 & 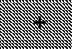 \\
\hline Hominisation & the & + & + & + & + & 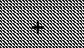 & 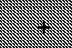 \\
\hline Didaktisches & - & + & - & - & - & - & - \\
\hline
\end{tabular}

Die Inhaltsanalyse verdeutlichte einen Strukturwechsel zwischen dem Lehrplan von 1946 und den darauf folgenden Lehrplänen (Tab. 32). Ausschließlich der Lehrplan von 1953 forderte Inhalte aus allen Kategorien. Ab 1954 fehlte die Kategorie Didaktisches. Einzelne Lehrpläne fassten Kategorien als Einheit zusammen (1951 und 1952 die Kategorien Biogenese und Systematik sowie 1955 die Kategorien Systematik und Hominisation). Zwar ist aufgrund der Verflechtungen der Kategorien eine vergleichende Darstellung der Raumanalysen nicht möglich, dennoch belegten die Ergebnisse, dass der Kategorie Theorien stets ein hoher Stellenwert innerhalb der evolutionsbiologischen Themen zukam. Als Sonderfall stellte Die Weiterentwicklung des Darwinismus zur schöpferischen Mitschurinschen Biologie im Lehrplan von 1953 ,den wichtigsten Abschnitt des gesamten Biologieunterrichts der allgemeinbildenden Schule dar“ (Kühn 1953: 342). 


\title{
Das Biologielehrbuch für die Klasse 8
}

\begin{abstract}
„Wenn auch verschiedene Einwände gegen Darwins Lehre erhoben wurden, und manche seiner Gedanken eine Umdeutung erfuhren, die keineswegs im Sinne ihres Schöpfers lag, so ist doch ihr wesentlicher Kern und Inhalt unberührt geblieben." (Löbel \& Maschke 1948: 197)
\end{abstract}

\section{Design}

Umfang und Textformat

Der Umfang der Lehrbücher von 1946, 1951, 1953 und 1957 für den Jahrgang 8 lag stets über 100 Seiten. Neben evolutionsbiologischen Themen nahmen die Autoren in die Schulbücher weitere Fachinhalte in unterschiedlichem Ausmaß auf. Infolgedessen traten signifikante Veränderungen im absoluten und relativen Umfang evolutionsbiologischer Inhalte auf (Abb. 51). Während der relative Umfang evolutionsbiologischer Inhalte zwischen den Auflagen von 1946 und 1951 deutlich anstieg, sank er in den folgenden Ausgaben leicht. Konkret umfasste das 
Lehrbuch von 1946 mit 129 Seiten für die Klasse 7 und mit 106 Seiten für die Klasse 8 insgesamt 236 Seiten. Auf 19 Seiten standen evolutionsbiologische Inhalte zur Verfügung. Dies entsprach einem Anteil von 17,9 \% für den 8. Jahrgang. Das Lehrbuch von 1951 war ausschließlich für die 8. Klasse konzipiert und mit einem Umfang von 131 Seiten ausgestattet. Davon enthielten 89 Seiten $(67,9 \%)$ evolutionsbiologische Inhalte. In der 4. bearbeiteten Auflage von 1953 reduzierte sich in Folge einer Lehrplanveränderung der Umfang des Lehrbuches auf 104 Seiten und der Anteil der evolutionsbiologischen Inhalte auf 63 Seiten (60,6\%). Im Lehrbuch von 1957 stieg der Umfang dann wieder an. Von 126 Seiten zeigten 74 Seiten $(58,7 \%)$ evolutionsbiologische Inhalte.

Die Autoren statteten alle Lehrbücher mit einem Inhalts- und ein Sachverzeichnis aus. Ein Glossar oder ein Literaturverzeichnis stand den Schülern nicht zur Verfügung. Farbige Unterlegungen von Texten waren in den Lehrbüchern nicht vorhanden. In geringem Maße kamen im Lehrbuch von 1946 Fettdruck und Sperrschrift sowie verschiedene Schriftgrößen vor. In den Lehrbüchern von 1951 und 1953 fand zur Hervorhebung einzelner Wörter oder Wortgruppen neben Fettdruck auch Kursivschrift Anwendung. Darüber hinaus kam eine kleinere Schriftgröße u. a. für in den Text eingebundene Aufgabenstellungen zum Einsatz. Das Schülerbuch von 1957 enthielt Personennamen in Sperrschrift und für einzelne Wörter oder Wortgruppen Fettdruck sowie Kursivschrift.

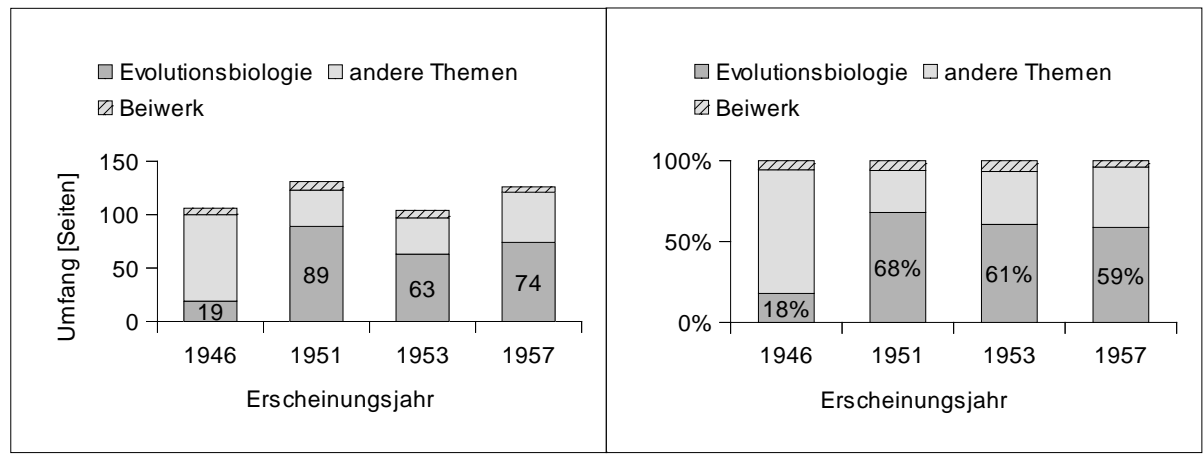

Abbildung 51: links: absoluter Umfang, rechts: relativer Umfang in den Lehrbüchern für die Klasse 8

Einband und Abbildungen

Das Lehrbuch von 1946 beinhaltete die Jahrgänge 7 und 8. Mit einem Hardcover ausgestattet, visualisierte der Einband ein Waldbiotop mit je einem Vertreter der Plantae, Fungi und Animalia aus der Domäne der Eukarya. Eine evolutionsbiologische Abbildung zeigte der Buchdeckel nicht. Vielmehr ließ sich ein ökologischer Strukturansatz ableiten. Die äußere Gestaltung informierte über das Schulfach. 
Eine Angabe zu den Jahrgangsstufe(n) konnte dem Einband nicht entnommen werden. Aufgrund der Maße 16,5 cm x 23,4 cm x 1,5 cm war das Format für Schüler handlich. Auch die Auflagen von 1951, 1953 und 1957 verfügten über ein Hartcover. Äußerlich schlicht und ohne grafische Darstellungen konnte ausschließlich das Fach und der Jahrgang dem Einband entnommen werden. Das Format änderte sich zum Vorgängermodell nur wenig.

Alle Abbildungen im Text lagen von 1946 bis 1957 in schwarz/weiß bzw. in Graustufen vor. Die Anzahl der eingesetzten Abbildungen korrelierte mit dem Umfang der Bücher. Das Verhältnis der Bilder pro Seite für das Stoffgebiet $A b$ stammungslebre lag in der Lehrbuchausgabe von 1946 mit 1,5 Bilder/Seite über den Werten von 1951 mit 1,0 Bilder/Seite, 1953 mit 0,9 Bilder/Seite sowie 1957 mit 1,1 Bilder/Seite (Abb. 52). Konkret waren im Lehrbuch von 1946 für die Klassen 7 und 8 insgesamt 255 Abbildungen in den Text eingebunden. Davon bezogen sich 108 Abbildungen auf Fachinhalte der 8. Klassenstufe und 28 Abbildungen auf Inhalte zur Evolutionsbiologie (25,9\%). Zusätzlich lagen acht Farbtafeln ohne Bezug zu evolutionsbiologischen Themen als Anhang bei.

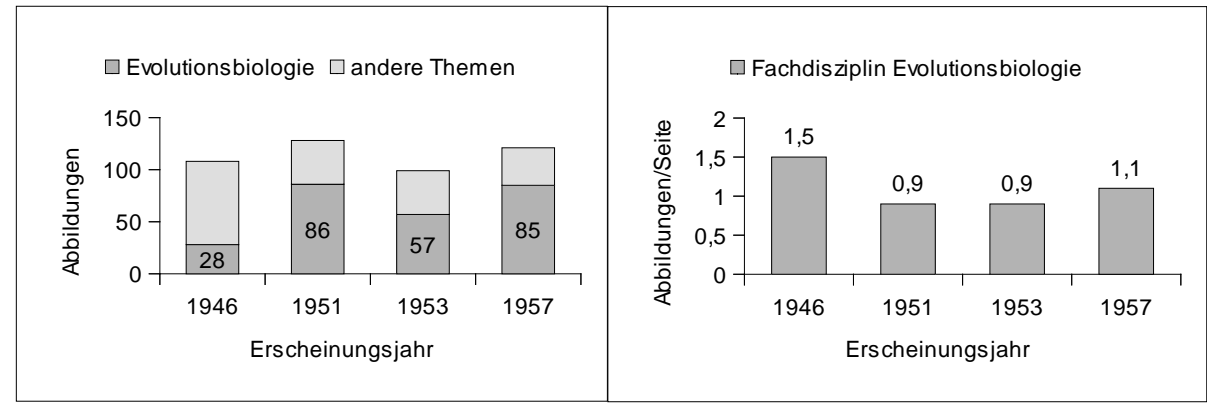

Abbildung 52: links: Anzahl der Abbildungen, rechts: Abbildungen pro Seite in den Lehrbüchern für die Klasse 8

Die Anzahl der Abbildungen im Text stieg in der Ausgabe von 1951 deutlich auf 128 an. Davon enthielten 86 Abbildungen (67,2\%) evolutionsbiologische Inhalte. Im Lehrbuch von 1953 reduzierten die Autoren die Abbildungen im Text auf 99. Die Rücknahmen betrafen auch evolutionsbiologische Darstellungen, von denen das Lehrbuch nunmehr 57 Abbildungen (57,6 \%) enthielt. In der Ausgabe von 1957 stieg die Anzahl der Abbildungen im Text auf 121 erneut an. Davon waren auf 85 Abbildungen (70,2 \%) Inhalte zur Evolutionsbiologie dargestellt. Ergänzend besaß das Lehrbuch von 1957 vier Kunstdrucktafeln mit evolutionsbiologischen Inhalten. Diese stellten verschiedene Fossilien sowie eine Landschaft aus dem Tertiär dar (Kategorie Paläontologie). 
Tabelle 33: äußeres und inneres Design, Lehrbücher Klasse 8

\begin{tabular}{|c|c|c|c|c|}
\hline Auflage & 1946 & 1951 & 1953 & 1957 \\
\hline \multicolumn{5}{|l|}{ Umfang: } \\
\hline absoluter Umfang [Seiten] & 106 & 131 & 104 & 126 \\
\hline Textumfang & 100 & 123 & 97 & 121 \\
\hline davon Evolutionsbiologie & 19 & 89 & 63 & 74 \\
\hline davon andere Themen & 81 & 34 & 34 & 47 \\
\hline Beiwerk & 6 & 8 & 7 & 5 \\
\hline relativer Umfang Evolutionsbiologie am Buch [\%] & 17,9 & 67,9 & 60,6 & 58,7 \\
\hline relativer Umfang Evolutionsbiologie am Text [\%] & 19,0 & 72,4 & 64,9 & 61,2 \\
\hline \multicolumn{5}{|l|}{ Beiwerk: } \\
\hline Inhaltsverzeichnis & ja & ja & ja & ja \\
\hline Glossar & nein & nein & nein & nein \\
\hline Literaturhinweise & nein & nein & nein & nein \\
\hline Sachverzeichnis & ja & ja & ja & ja \\
\hline \multicolumn{5}{|l|}{ Paratext: } \\
\hline$\overline{\text { Abbildungen im Text }}$ & 108 & 128 & 99 & 121 \\
\hline davon Evolutionsbiologie & 28 & 86 & 57 & 85 \\
\hline relativer Umfang [\%] & 25,9 & 67,2 & 57,6 & 70,2 \\
\hline Abbildungen pro Textseite & 1,1 & 1,0 & 1,0 & 1,0 \\
\hline davon Evolutionsbiologie & 1,5 & 1,0 & 0,9 & 1,1 \\
\hline Gestaltung & $s / w$ & $s / w$ & $s / w$ & $s / w$ \\
\hline Farbtafeln & 8 & 0 & 0 & 0 \\
\hline Kunstdrucktafeln & 0 & 0 & 0 & 4 \\
\hline davon Evolutionsbiologie & 0 & 0 & 0 & 4 \\
\hline \multicolumn{5}{|l|}{ Kategoriale Zuordnung der Abbildungen: } \\
\hline Theorien & 1 & 16 & 16 & 17 \\
\hline Biogenese & 0 & 1 & 1 & 0 \\
\hline Neontologie & 5 & 9 & 4 & 6 \\
\hline Paläontologie & 5 & 32 & 32 & 37 \\
\hline Systematik & 1 & 4 & 4 & 4 \\
\hline Hominisation & 16 & 24 & 0 & 21 \\
\hline Didaktisches & 0 & 0 & 0 & 0 \\
\hline Gesamt & 28 & 86 & 57 & 85 \\
\hline
\end{tabular}

Der Schwerpunkt der Visualisierung lag im Lehrbuch von 1946 auf der Kategorie Hominisation und in den Lehrbüchern von 1951, 1953 und 1957 im Bereich der Kategorie Paläontologie (Tab. 33). Zwar behandelte das Lehrbuch von 1953 die Kategorie Hominisation nicht, dennoch verdeutlicht die Summe und der Mittelwert aller Abbildungen aus den relevanten Büchern von 1946 bis 1957 den hohen Stellenwert der Visualisierung für diese Kategorie. Während der Kategorie Theorien im Lehrbuch von 1946 nur eine Abbildung zugeordnet werden kann, war seit den 1950er Jahren auch diese Kategorie mit einer Vielzahl von Abbildungen vertreten. 


\section{Struktur}

Das Lehrbuch von 1946

Die Inhalte aus dem Schulbuch von 1946 für die Klassenstufe 8 gliederten sich in zehn Kapitel. Davon enthielten zwei evolutionsbiologische Inhalte. Diese waren im Lehrbuch durch andere Kapitel voneinander getrennt und bildeten keinen eigenen Themenkomplex. Die Feinstruktur evolutionsbiologischer Inhalte gliederte sich wie folgt:

- Einführung in die Abstammungslehre (9 Seiten; 47,4 \%)

$\circ$ Einleitung

- Zeugnisse für die Abstammung

- Darwins Erklärung

- Abstammung und Entwicklung des Menschen (10 Seiten; 52,6 \%)

- Die Eiszeit in Europa

- Alter und Ursprung des Menschen

- Menschenaffen in der Tertiärzeit

- Vor- oder Affenmenschen

- Urmenschen (Neandertaler)

- Altmenschen

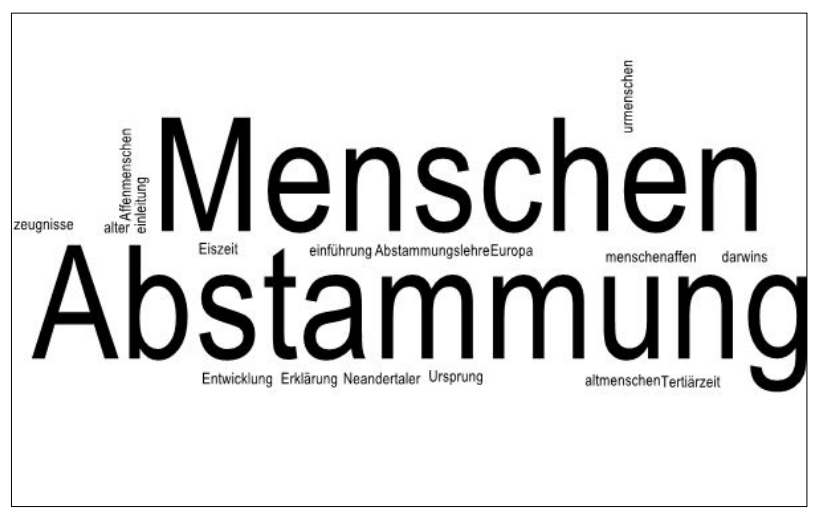

Abbildung 53: Biologische Begriffe im Inhaltsverzeichnis, Lehrbuch Klasse 8 (1946)

Eine Frequenzanalyse der evolutionsbiologischen Kapitelüberschriften zeigt Präferenzen für die biologischen Begriffe Menschen und Abstammung (Abb. 53). Die Inhaltsanalyse der Texte verdeutlicht die Gliederung: Theorien - Paläontologie Biogenese - Paläontologie - Neontologie - Paläontologie - Neontologie - Theorien - Systematik - Hominisation. In einem Kapitel behandelt, wies die Kategorie Hominisation die längste zusammenhängende Textpassage auf (Abb. 54). Inhalte 
aus der Kategorie Paläontologie kamen dagegen an drei Stellen und Themen aus den Kategorien Neontologie sowie Theorien jeweils an zwei Stellen im Lehrbuch vor. Mit 185,5 Zeilen (53 \%) nahm die Kategorie Hominisation den größten Anteil innerhalb der Fachdisziplin Evolutionsbiologie ein. Hier lag der Schwerpunkt der Vermittlung, was auch den Vorgaben des Lehrplans entsprach. Die fünf weiteren Kategorien machten die restlichen $47 \%$ aus. Davon umfassten die Kategorien Theorien 79,5 Zeilen (23\%), Neontologie 53,5 Zeilen (15\%), Paläontologie 21,5 Zeilen (6\%), Systematik 6,5 Zeilen (2\%) und Biogenese 2 Zeilen (1\%).

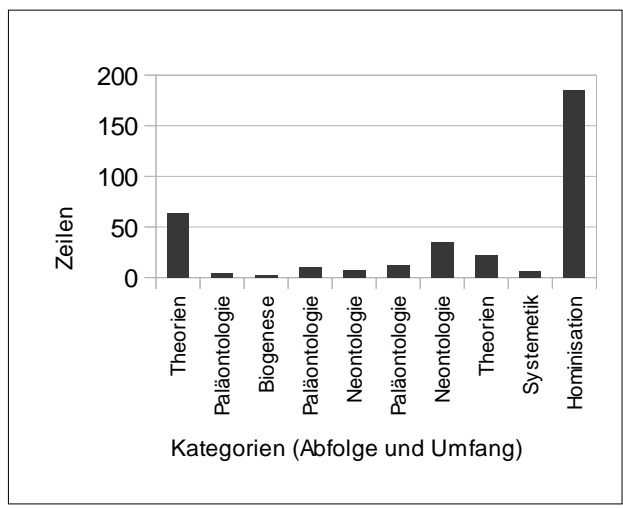

Abbildung 54: Kategorien im Lehrbuch Klasse 8 (1946)

Das Lehrbuch von 1951

Die Inhalte der Ausgabe von 1951 waren in die vier Themenbereiche Reizerscheinungen der Lebewesen, Abstammungslebre, Entwicklung des Menschen sowie Große Biologen gegliedert. Die drei Letztgenannten wiesen evolutionsbiologische Inhalte auf, bildeten jedoch keinen in sich geschlossenen Themenkomplex bzw. waren keinem gemeinsamen evolutionsbiologischem Begriff zugeordnet. Die Feinstruktur evolutionsbiologischer Inhalte gliederte sich in die Kapitel:

- Abstammungslehre

- Grundtatsachen (29 Seiten; 32,6\%)

- Tiere und Pflanzen in der erdgeschichtlichen Entwicklung

- Übereinstimmungen im Körperbau

- Zwischenformen

- Übereinstimmungen in der Keimesentwicklung

- Besonderheiten in der Verbreitung von Tieren und Pflanzen

- Die Entstehung der Arten (18 Seiten; 20,2\%)

- Veränderungsfähigkeit der Arten, Formen der Abänderungen

- Auslese

- Artbildung durch Züchtung 
- Die Stammesentwicklung der Tiere und Pflanzen (13 Seiten; 14,6 \%)

- Entstehung des Lebens auf der Erde

- Stammbaum

- Das System

- $\quad$ Entwicklung des Menschen (20 Seiten; 22,5 \%)

- Entwicklung innerhalb der Affenreihe

- Menschwerdung

- Der heutige Mensch

- Der Mensch als Umgestalter der Natur

- Große Biologen (auf 9 Seiten evolutionsbiologische Inhalte; 10,1 \%)

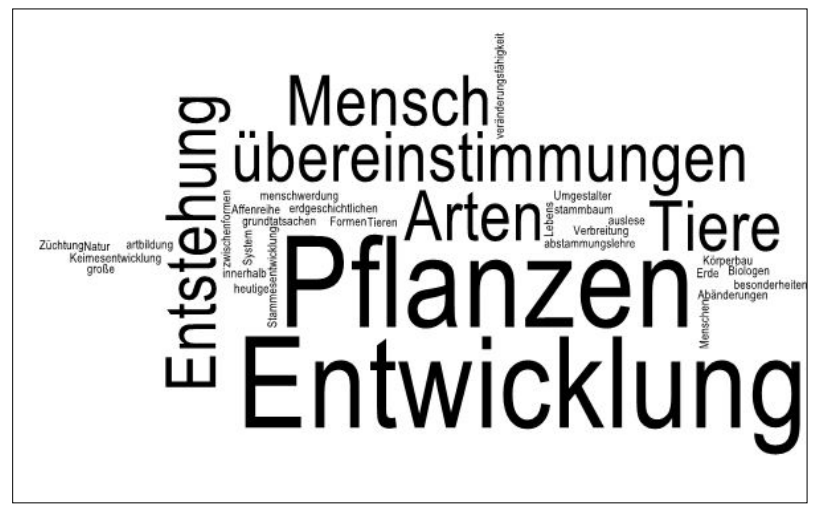

Abbildung 55: Biologische Begriffe im Inhaltsverzeichnis, Lehrbuch Klasse 8 (1951)

Die Frequenzanalyse des Inhaltsverzeichnisses betont, dass die Begriffe Entwicklung und Pflanzen im Vordergrund standen (Abb. 55). Den Lehrbuchtexten lag die Gliederung Paläontologie - Neontologie - Paläontologie - Neontologie - Theorien - Biogenese - Systematik - Hominisation - Systematik - Theorien zu Grunde. Die Kategorie Theorien wies dabei die längste zusammenhängende Textpassage auf. Inhalte aus den $\mathrm{Ka}$ tegorien Paläontologie, Neontologie, Systematik und Theorien wurden jeweils an zwei Stellen im Lehrbuch behandelt (Abb. 56). Anders als im Lehrbuch von 1946 kam der Kategorie Theorien mit 847,5 Zeilen (53\%) der größte Stellenwert zu. Weiterhin umfassten die Kategorien Hominisation 461 Zeilen (18,5\%), Paläontologie

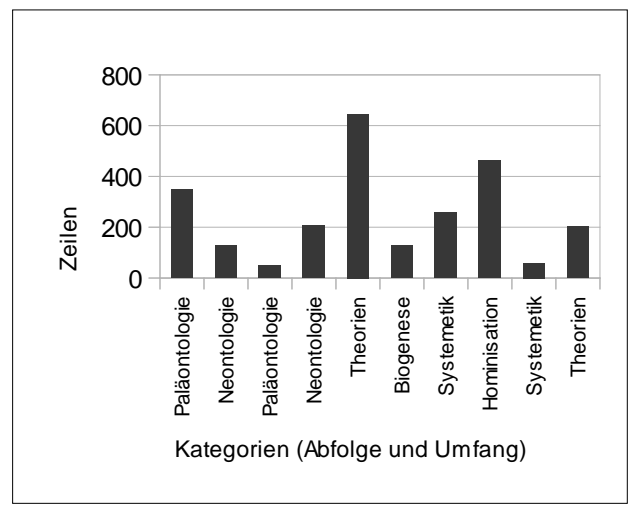

Abbildung 56: Kategorien im Lehrbuch Klasse 8 (1951) 
399 Zeilen (16 \%), Neontologie 334 Zeilen (13,4\%), Systematik 317 Zeilen $(12,7 \%)$ und Biogenese 129,5 Zeilen (5,2\%).

Das Lehrbuch von 1953

Das Lehrbuch von 1953 ähnelte in seiner Struktur dem Lehrbuch von 1951. Es enthielt lediglich an einigen Stellen Kürzungen, wodurch sich der absolute und relative Umfang der Kapitel veränderte. So fehlte der Themenbereich Entwicklung des Menschen und die Unterkapitel Übereinstimmungen in der Keimesentwicklung sowie Besonderbeiten in der Verbreitung von Tieren und Pflanzen aus dem Themenbereich $A b$ stammungslehre. Im Lehrbuch befand sich dazu der Hinweis:

„Entsprechend dem ab 1.9.1953 verbindlichen Stoffplan sind folgende Abschnitte der 1. bis 3. Auflage in der vorliegenden Ausgabe nicht enthalten: Übereinstimmung in der Keimesentwicklung[,] Besonderheiten in der Verbreitung von Tieren und Pflanzen[,] Entwicklung des Menschen." (Lemke 1956: 2)

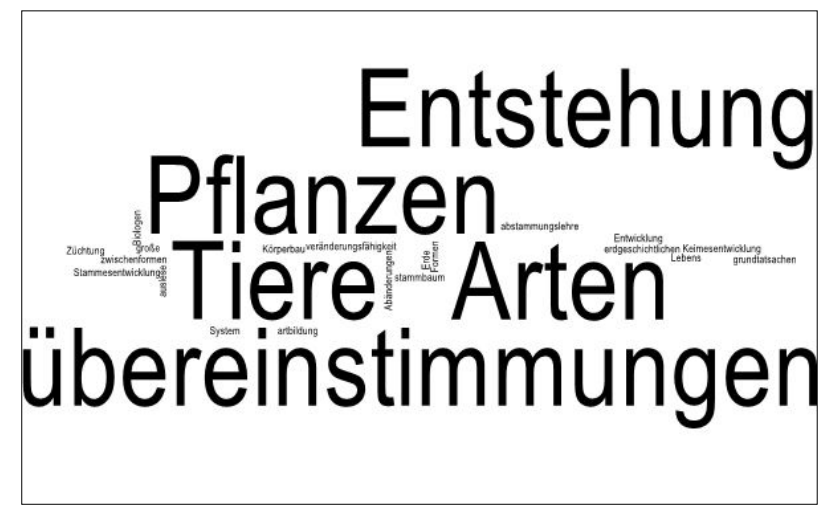

Abbildung 57: Biologische Begriffe im Inhaltsverzeichnis, Lehrbuch Klasse 8 (1953)

Die Frequenzanalyse des Inhaltsverzeichnisses zeigte Präferenzen für die Begriffe Entstehung, Pflanzen, Tiere und Arten (Abb. 57). Die Inhaltsanalyse der Texte des Lehrbuches von 1953 zeigt die Gliederung Paläontologie - Neontologie - Paläontologie - Theorien - Biogenese - Systematik - Theorien auf. Weiterhin bildeten Themen aus der Kategorie Theorien die längste zusammenhängende Textpassage. Inhalte aus den Kategorien Paläontologie und Theorien behandelte das Lehrbuch jeweils an zwei Stellen (Abb. 58). Der Kategorie Theorien kam mit 854 Zeilen $(46,4 \%)$ unverändert der größte Stellenwert zu. Auch die Kategorie Paläontologie nahm mit 409,5 Zeilen (22,2\%) einen großen Raum am Text ein. Die Kategorie Systematik umfasste 319 Zeilen (17,9\%) und die Kategorien Neontologie und Biogenese je 129,5 Zeilen (7 \%). 


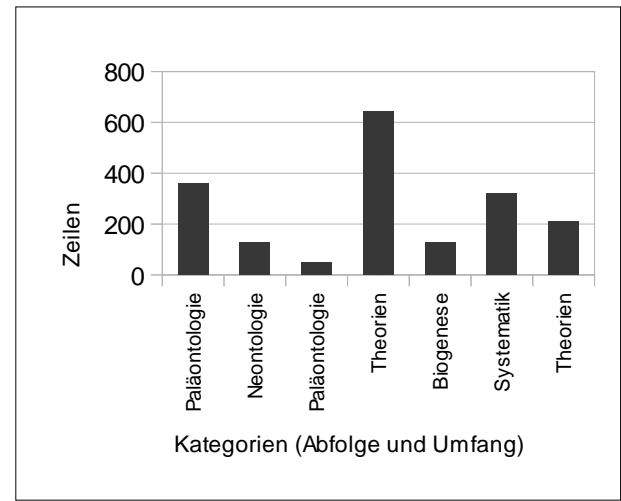

Abbildung 58: Kategorien im Lehrbuch Klasse 8 (1953)

Das Lehrbuch von 1957

Die Inhalte der Ausgabe von 1957 gliederten sich in acht Kapitel. Die Struktur war heterogen. Das erste Kapitel Sinnesorgane und Nervensystem des Menschen enthielt keine evolutionsbiologischen Inhalte. Abschließend behandelte das Lehrbuch das Kapitel Die Veränderung der Natur durch den Menschen, aus dem Bereich der Züchtungsbiologie. Die Feinstruktur evolutionsbiologischer Inhalte stellte sich wie folgt dar:

- Charles Darwin und seine Lehre (16 Seiten; 21,6\%)

- Der Entwicklungsgedanke vor Darwin

- Charles Robert Darwin

- Die Verbreitung des Darwinismus

- $\quad$ Beweise aus der Versteinerungskunde (29 Seiten; 39,2\%)

- Die Erde hat eine Geschichte

- Überblick über die Erdgeschichte

- Die Zeitbestimmung der Geologie

- Die Fossilien

- Aus der Geschichte der Erde

- Der Steinkohlewald

- Die Kreidezeit und ihre Saurier

- Die Braunkohlenzeit

- Die Eiszeit

- Die Entwicklung der Lebewesen

- Übersicht über die Entwicklung der Lebewesen

- Beispiele aus der Entwicklung der Lebewesen

- Die Leitfossilien

- Die Arbeit des Paläontologen

- Wo finden wir Fossilien 
- Beweise aus der vergleichenden Anatomie (4 Seiten; 5,4 \%)

- Das natürliche System der Lebewesen (6 Seiten; 8,1 \%)

- Bildliche Darstellung der Verwandtschaft von Lebewesen (4 Seiten; $5,4 \%$ )

- Die Abstammung des Menschen (15 Seiten; 20,3 \%)

- Die heutige Menschheit

- Mensch und Tier

- Zeugnisse von früheren Menschen

- Die eiszeitlichen Menschengruppen

- Der eiszeitliche Homo sapiens

- Die Neandertaler

- Die Pithecanthropus-Gruppe

- Die Australopithecinen-Gruppe

- Die Verwandtschaft der eiszeitlichen Menschengruppen

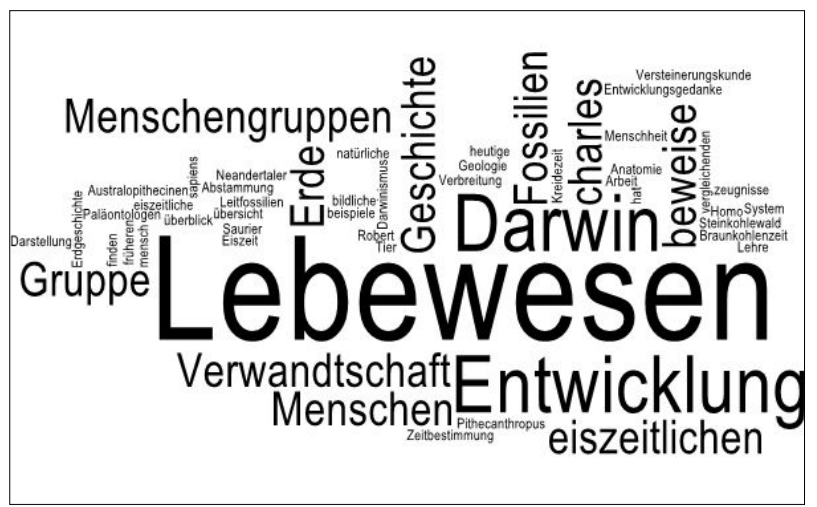

Abbildung 59: Biologische Begriffe im Inhaltsverzeichnis, Lehrbuch Klasse 8 (1957)

Die Frequenzanalyse der Kapitelüberschriften ergab, dass im Lehrbuch von 1957 der biologische Begriff Lebewesen den größten Stellenwert einnahm. An zweiter Stelle folgten die Worte Darwin sowie Entwicklung (Abb. 59). Im Lehrbuch von 1957 verdeutlicht die Inhaltsanalyse der Texte die Gliederung Theorien - Paläontologie - Biogenese - Paläontologie - Neontologie - Systematik - Hominisation. Die Kategorie Paläontologie wies hier die längste zusammenhängende Textpassage auf. Ausschließlich die Kategorie Paläontologie wurde an zwei Stellen im Lehrbuch behandelt (Abb. 60). Anders als in den Vorgängermodellen kam im Lehrbuch von 1957 der Kategorie Paläontologie mit 668,5 Zeilen (38,4 \%) der größte Stellenwert zu. Weiterhin umfassten die Kategorien Theorien 389,5 Zeilen $(22,4 \%)$, Systematik 331 Zeilen (19 \%), Hominisation 275,5 Zeilen (15,8\%), Neontologie 70 Zeilen (4\%) und Biogenese lediglich 5 Zeilen (0,3\%). 


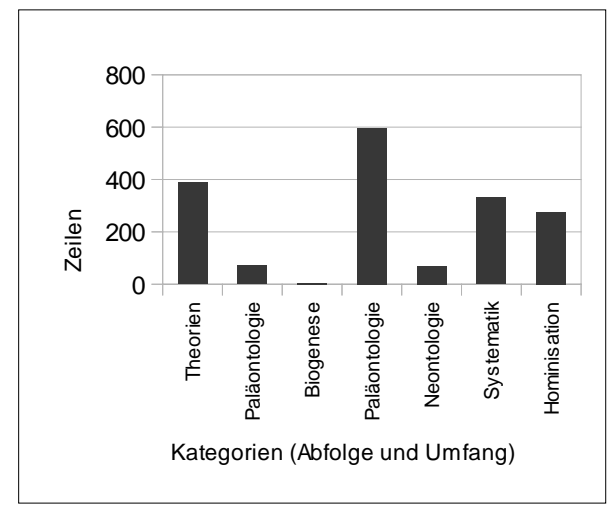

Abbildung 60: Kategorien im Lehrbuch Klasse 8 (1957)

\section{Zusammenfassung}

Die Strukturanalyse der Lehrbuchkapitel von 1946 bis 1957 lässt eine Gliederung der Inhalte in drei Bereiche erkennen, deren Reihenfolge bzw. Zusammensetzung die Autoren mit jeder Lehrbuchausgabe veränderten. Inhalte aus der Kategorie Hominisation behandelten die Lehrbücher von 1946, 1951 und 1957 in einem eigenen Kapitel. Alle anderen Kategorien fassten die Schulbücher von 1946, 1951 und 1953 unter dem Begriff Abstammungslehre zusammen. Zusätzlich wurde Leben und Werk historischer Personen in den Lehrbüchern von 1951 und 1953 in einem eigenen Kapitel behandelt. Das Lehrbuch von 1957 gliederte die Inhalte schließlich in mehrere Kapitel auf. In den Lehrbüchern von 1946 und 1957 begannen die evolutionsbiologischen Inhalte mit der Kategorie Theorien, während entsprechende Inhalte in den Lehrbüchern von 1951 und 1953 in der Mitte sowie am Ende des Lehrbuches zur Sprache kamen (Tab. 34).

Der Umfang der evolutionsbiologischen Kapitel innerhalb der Ausgabe von 1946 war annähernd gleich. Während neben dem Umfang auch die weitere Gliederung der Kapitel in den Lehrbüchern von 1951 und 1953 ausgeglichen blieb, variierten die Kapitel in Umfang und Gliederung im Lehrbuch von 1957 stark. Dem Kapitel Grundtatsachen, das am Anfang der Lehrbücher von 1951 und 1953 stand, kam der größte Stellenwert zu. Auch im Lehrbuch von 1957 lag mit dem Kapitel Beweise aus der Versteinerungskunde der Schwerpunkt auf Indizien für Evolutionsprozesse (Tab. 34).

Die kategoriale Raumanalyse der Lehrbuchtexte belegt, dass die Lehrbuchausgabe von 1946 auf die Kategorie Hominisation fokussierte. Die Lehrbücher von 1951 und 1953 enthielten dagegen umfangreiche Texte zur Kategorie Theorien. Insbesondere im Lehrbuch von 1953 stieg der relative Anteil der Kategorie Theorien durch die Streichung des Kapitels Entwicklung des Menschen an. Das Lehrbuch von 1957 zeigte eine Präferenz für Inhalte aus der Kategorie Paläontologie 
(Abb. 61). Zwischen 1946 und 1957 nahm der Umfang paläontologischer und systematischer Themen zu und der Umfang neontologischer Inhalte ab.

Tabelle 34: Strukturierung der Lehrbücher für die Klasse 8

\begin{tabular}{|c|c|c|c|c|c|c|}
\hline Ausgabe & Kapitel & $\begin{array}{l}\text { Anzahl der } \\
\text { Unterkapitel }\end{array}$ & $\begin{array}{l}\text { absoluter } \\
\text { Umfang } \\
\text { [Seiten] }\end{array}$ & $\begin{array}{l}\text { relativer } \\
\text { Umfang } \\
{[\%]}\end{array}$ & Stellenwert & Kategorie \\
\hline \multirow[t]{3}{*}{1946} & 1 & 3 & 9 & 47,5 & 2 & $\mathrm{~T}, \mathrm{P}, \mathrm{N}, \mathrm{B}, \mathrm{S}$ \\
\hline & 2 & 6 & 10 & 52,6 & 1 & \\
\hline & 2 & 9 & 19 & 100,0 & & \\
\hline \multirow[t]{6}{*}{1951} & 1 & 5 & 29 & 32,6 & 1 & $\mathrm{P}, \mathrm{N}$ \\
\hline & 2 & 3 & 18 & 20,2 & 3 & $T$ \\
\hline & 3 & 3 & 13 & 14,6 & 4 & $B, S$ \\
\hline & 4 & 4 & 20 & 22,5 & 2 & $\mathrm{H}$ \\
\hline & 5 & 0 & 9 & 10,1 & 5 & $S, T$ \\
\hline & 5 & 15 & 89 & 100,0 & & \\
\hline \multirow[t]{5}{*}{1953} & 1 & 3 & 23 & 36,5 & 1 & $P, N$ \\
\hline & 2 & 3 & 18 & 28,6 & 2 & $T$ \\
\hline & 3 & 3 & 13 & 20,6 & 3 & $B, S$ \\
\hline & 4 & 0 & 9 & 14,3 & 4 & $S, T$ \\
\hline & 4 & 9 & 63 & 100,0 & & \\
\hline \multirow[t]{7}{*}{1957} & 1 & 3 & 16 & 21,6 & 2 & T \\
\hline & 2 & 7 & 29 & 39,2 & 1 & $P, B$ \\
\hline & 3 & 0 & 4 & 5,4 & 5 & $\mathrm{~N}$ \\
\hline & 4 & 0 & 6 & 8,1 & 4 & S \\
\hline & 5 & 0 & 4 & 5,4 & 5 & S \\
\hline & 6 & 6 & 15 & 20,3 & 3 & $\mathrm{H}$ \\
\hline & 6 & 16 & 74 & 100,0 & & \\
\hline
\end{tabular}

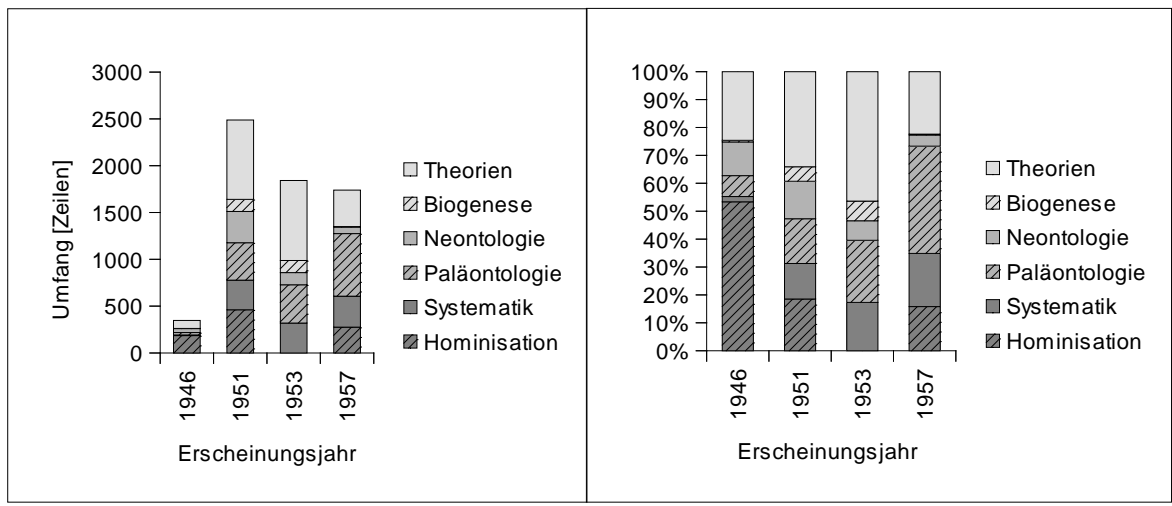

Abbildung 61: links: absoluter Umfang, rechts: relativer Umfang der Kategorien in den Lehrbüchern der Klasse 8 


\section{Fachinhalte}

\section{Evolutionstheorien}

Die Ergebnisse einer Frequenzanalyse im Lehrbuchtext zeigen, dass von 1946 bis 1957 die Anzahl der aufgenommenen Personen in den Lehrbüchern anstieg. Dadurch nahm auch die Breite der Inhalte zu. Konkret nannte das Lehrbuch von 1946 fünf und das Lehrbuch von 1957 bereits 17 historische Persönlichkeiten. Ferner erhöhte sich die Häufigkeit der Nennungen der jeweiligen Personen, was eine Zunahme der thematischen Intensität bedeutet (Tab. 35).

Tabelle 35: Frequenzanalyse (Gesamt), Lehrbücher Klasse 8

\begin{tabular}{|lrrrr|}
\hline Auflage & 1946 & 1951 & 1953 & 1957 \\
Anzahl der Personen & 5 & 10 & 11 & 17 \\
Summe der Nennungen & 13 & 97 & 93 & 117 \\
\hline
\end{tabular}

Dabei kam, von der Häufigkeit der Nennungen ausgehend, in allen Lehrbüchern Darwin der höchste Stellenwert zu - ähnlich der Darwin-Industrie in der Wissenschaftsgeschichte. Während Buffon, Cuvier, Lamarck und Linné im Lehrbuch von 1946 zumindest einmal genannt wurden, fand Haeckel erst ab 1951 Erwähnung. Charakteristisch für die Lehrbücher von 1951 und 1953 war darüber hinaus der hohe Stellenwert von Mitschurin und Lyssenko. Durch die Neustrukturierung im Lehrbuch von 1957 verlagerten sich diese Inhalte in ein eigenes züchtungsbiologisches Kapitel. Auf die Verdienste von Alfred Russell Wallace wurde lediglich im Lehrbuch von 1957 hingewiesen (Abb. 62).

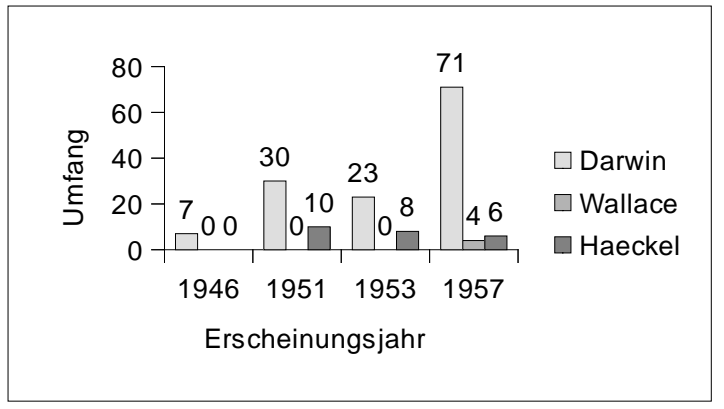

Abbildung 62: Darwin, Wallace und Haeckel in den Lehrbüchern für die Klasse 8

\section{Darwinismus}

Alle Lehrbücher für die 8. Klassenstufe enthielten über die bloße Nennung der Person Darwin Angaben aus dessen Leben und Werk. Dabei traten deutliche Unterschiede in Umfang, Tiefe und Anordnung der inhaltlichen Auseinandersetzung 
auf (Tab. 36). Das Lehrbuch von 1946 enthielt biografische Angaben im Kapitel Einleitung und einen kurzen theoretischen Einblick im Kapitel Darwins Erklärung. Im Kapitel Große Biologen behandelten die Lehrbücher von 1951 und 1953 Darwin biografisch. Über Auszüge aus seinen Theorien konnten sich die Schüler im Kapitel Die Entstebung der Arten informieren. Die künstliche und natürliche Auslese sowie eine kritische Auseinandersetzung mit dem Begriff Kampf ums Dasein bildeten die Schwerpunkte. Die Kritik, in der Direktive von 1955 gefordert, lehnte sich inhaltlich an den ,russischen Nationalstil in der Reaktion auf Darwin“ (Todes 2009: 221) an. Das Schülerbuch von 1957 stellte Darwins Leben und Werk zusammenhängend im Kapitel Die Begründung der wissenschaftlichen Abstammungslehre dar. Über die Verbreitung des Darwinismus durch Ernst Haeckel informierten alle Schulbücher für die Klasse 8 der 1950er Jahre. Im Lehrbuch von 1957 nahmen die Autoren auch Angaben über Thomas Henry Huxley, die Brüder Wladimir O. Kowalewsky und Alexander O. Kowalewsky sowie Kliment Arkadjewitsch Timirjasew auf. Insgesamt nahm die Informationsdichte zwischen 1946 und 1957 $\mathrm{zu}$.

Tabelle 36: Darwins Leben und Werk, Lehrbücher Klasse 8

\begin{tabular}{|l|l|l|l|l|}
\hline Auflage & Vor der Reise & Reisebeschreibung & Nach der Reise & Theoretische Aspekte \\
\hline 1946 & Herkunft & Südamerika & Auswertung & $\begin{array}{l}\text { Überproduktion, } \\
\text { Variabiliät, natürliche } \\
\text { Selektion }\end{array}$ \\
\hline $1951 / 53$ & $\begin{array}{l}\text { Herkunft, Medizin- } \\
\text { und } \\
\text { Theologiestudium, } \\
\text { Vorlieben }\end{array}$ & $\begin{array}{l}\text { Grund der Reise, } \\
\text { Madeira, Kerguelen- } \\
\text { Inseln }\end{array}$ & $\begin{array}{l}\text { Auswertung, Tauben- } \\
\text { zucht }\end{array}$ & $\begin{array}{l}\text { Variabilität bei erbli- } \\
\text { chen Eigenschaften, } \\
\text { Auslese (natürliche, } \\
\text { künstliche), Kampf } \\
\text { ums Dasein }\end{array}$ \\
\hline 1957 & $\begin{array}{l}\text { Herkunft, } \\
\text { Schulbildung, } \\
\text { Vorlieben, } \\
\text { Medizinstudium, } \\
\text { erste } \\
\text { Entdeckungen, } \\
\text { Theologiestudium }\end{array}$ & $\begin{array}{l}\text { Grund der Reise, } \\
\text { Vermittlung, Dauer, } \\
\text { Anschauungen Lyells, } \\
\text { Exkursionen in Süd- } \\
\text { amerika, Grundfinken } \\
\text { auf Galapagos, } \\
\text { Korallenriffe, Ankunft }\end{array}$ & $\begin{array}{l}\text { Reisetagebuch, Land- } \\
\text { sitz in Down, Entwurf } \\
\text { des Artenbuches, } \\
\text { Darwins Arbeitsweise, } \\
\text { Pflanzensamen, Tau- } \\
\text { benzucht, } \\
\text { Wallace, Vorlage des } \\
\text { Manuskripts, Auflage } \\
\text { des Artenbuches }\end{array}$ & $\begin{array}{l}\text { Variabiliät, Überpro- } \\
\text { duktion, natürliche } \\
\text { Zuchtwahl, Kampf } \\
\text { ums Dasein, Gesetze } \\
\text { der Abänderung, } \\
\text { Beweise }\end{array}$ \\
\hline
\end{tabular}

Auch die vorhandenen Abbildungen verdeutlichen eine Zunahme der Informationsdichte. Die Lehrbücher von 1946, 1951, 1953 und 1957 enthielten je eine Fotografie, auf der Darwin als alter Mann um 1880 zu sehen war (Abb. 63). Zusätzlich integrierten die Autoren im Lehrbuch von 1957 Fotografien aus dem Archiv des Ernst-Haeckel-Hauses in Jena. Diese zeigten u. a. Darwin als jungen Mann um 1849, die Beagle in der Mündung des Santa-Cruz, Grundfinkenarten von den Galapagos-Inseln, Darwins Landhaus und eine Handzeichnung Haeckels vom Stamm- 
baum des Menschen. Nach einer Vorlage von Georg Uschmann angefertigt, zeichnete eine Karte die Reise Darwins detailliert nach. Sie nahm einen Raum von zwei Seiten ein und enthielt Datums- und Ortsangaben der Route. Eine schematische Darstellung verschiedener Taubenrassen verdeutlichte in den Ausgaben der 1950er Jahre „die Möglichkeit einer starken Veränderung von Tierarten“ (Arnold et al. 1957: 44). Als „Beispiel für die Wirkung der Auslese“ (ebd.: 46) enthielten die Lehrbücher die Abbildung einer Kerguelenfliege.

Tabelle 37: Ankerbeispiele für die fünf Darwin'schen Theorien, Lehrbücher Klasse 8

\begin{tabular}{|c|c|c|c|c|c|}
\hline Auflage & Veränderlichkeit & $\begin{array}{l}\text { Abstammungs- } \\
\text { lehre }\end{array}$ & Gradualismus & Aufspaltung & Selektionslehre \\
\hline 1946 & $\begin{array}{l}\text { „Unter den Nach- } \\
\text { kommen aller } \\
\text { Lebewesen gibt } \\
\text { es Formen, die } \\
\text { sich in ganz } \\
\text { geringem, oft } \\
\text { nicht oder kaum } \\
\text { feststellbaren } \\
\text { Maße von ihren } \\
\text { Eltern unter- } \\
\text { scheiden.“ } \\
\text { (Löbel \& Masch- } \\
\text { ke 1948: 197) }\end{array}$ & $\begin{array}{l}\text { "Sie sind aber } \\
\text { geeignet, uns } \\
\text { eine Vorstellung } \\
\text { von der Entwick- } \\
\text { lung, der Ab- } \\
\text { stammung, zu } \\
\text { vermitteln." } \\
\text { (Löbel \& Masch- } \\
\text { ke 1948: 197) }\end{array}$ & - & - & $\begin{array}{l}\text { "Der Kampf ums } \\
\text { Dasein führt die } \\
\text { natürliche Ausle- } \\
\text { se, die Selektion, } \\
\text { durch.“ } \\
\text { (Löbel \& Masch- } \\
\text { ke 1948: 197) }\end{array}$ \\
\hline $1951 / 53$ & $\begin{array}{l}\text { „Kein Lebewesen } \\
\text { ist einem ande- } \\
\text { ren in allen } \\
\text { Einzelheiten } \\
\text { gleich.“ (Lemke } \\
\text { 1953: 59) }\end{array}$ & $\begin{array}{l}\text { „In diesem Buch } \\
\text { beweist Darwin, } \\
\text { daß die heute } \\
\text { lebenden Orga- } \\
\text { nismen von } \\
\text { einfacher gebau- } \\
\text { ten Lebewesen } \\
\text { abstammen." } \\
\text { (Lemke 1953: 59) }\end{array}$ & - & \begin{tabular}{|l} 
„So weisen diese \\
Inseln heute \\
eigentümliche \\
Pflanzenarten \\
auf, die es sonst \\
nirgends gibt." \\
(Lemke 1953: 67)
\end{tabular} & $\begin{array}{l}\text { "Darwin erkannte, } \\
\text { daß auch in der } \\
\text { freien Natur eine } \\
\text { Auslese stattfin- } \\
\text { det." (Lemke } \\
\text { 1953: } 66)\end{array}$ \\
\hline 1957 & \begin{tabular}{|l|} 
"Auch die einzel- \\
nen wildlebenden \\
Pflanzen und \\
Tiere der glei- \\
chen Art unter- \\
scheiden sich \\
immer wieder \\
durch kleinere \\
Abweichungen." \\
(Arnold et al. \\
1957: 45)
\end{tabular} & $\begin{array}{l}\text { „Daraus läßt sich } \\
\text { meist erkennen, } \\
\text { welche Pflanzen } \\
\text { und Tiere mitei- } \\
\text { nander verwandt } \\
\text { sind." (Arnold et } \\
\text { al. 1957: 47) }\end{array}$ & \begin{tabular}{|l|} 
"So entstehen \\
im Laufe langer \\
Zeiträume \\
neue Arten." \\
(Arnold et al. \\
1957: 48) \\
\end{tabular} & \begin{tabular}{|l} 
„Dagegen entwi- \\
ckelten sich auf \\
abgelegenen \\
Inseln oder auf \\
Kontinenten [...] \\
besondere For- \\
men.“ (Arnold et \\
al. 1957: 47)
\end{tabular} & $\begin{array}{l}\text { "Durch ,natürliche } \\
\text { Zuchtwahl" } \\
\text { werden günstige } \\
\text { Abweichungen } \\
\text { ausgelesen." } \\
\text { (Arnold et al. } \\
\text { 1957: 48) }\end{array}$ \\
\hline
\end{tabular}

Im Zusammenhang mit der Darstellung der Darwin'schen Theorien enthielten alle Lehrbücher Informationen über die Veränderlichkeit der Arten, ihrer Abstam- 
mung sowie zu dem Prinzip der natürlichen Auslese. In den Lehrbüchern von 1951, 1953 und 1957 standen den Schülern auch Aussagen zur Theorie der Aufspaltung der Arten zur Verfügung. Ausschließlich im Lehrbuch von 1957 integrierten die Autoren Formulierungen, die auf die Theorie des Gradualismus hinwiesen. In der Gesamtschau nahm somit von 1946 bis 1957 die Darstellung der Darwin'schen Theorien in ihrer Komplexität zu (Tab. 37).

\section{Neodarwinismus vs. Schöpferischer Darwinismus}

Wie bereits dargelegt, enthielt das Lehrbuch von 1946 in den relevanten Kapiteln keine Inhalte über Leben und Werk von Mitschurin und Lyssenko. In den Lehrbüchern von 1951 und 1953 wurden dagegen im Kapitel Große Biologen beide Personen vorgestellt. Das Kapitel Die Entstehung der Arten informierte auf acht Seiten über die Züchtungsverfahren der sowjetischen Forscher, „durch die es möglich ist, bei Pflanzen und Tieren neue Eigenschaften zu erzielen, die die Stammform nicht besitzt" (Lemke 1951: 77, Hervorhebung im Original). Mit der Auslagerung der Züchtung als eigenständiges Kapitel im Lehrbuch von 1957 entfiel die Behandlung von Mitschurin und Lyssenko innerhalb der evolutionsbiologischen Kapitel (Abb. 64). Alle Lehrbücher für die Klasse 8 enthielten dagegen keine Angaben zum Neodarwinismus bzw. zur Person August Weismann und ebenso keine Inhalte der Synthetischen Theorie. Die Betonung des Schöpferischen Darwinismus wurde somit durch Aufnahme bzw. Nichtaufnahme spezifischer Inhalte beeinflusst.

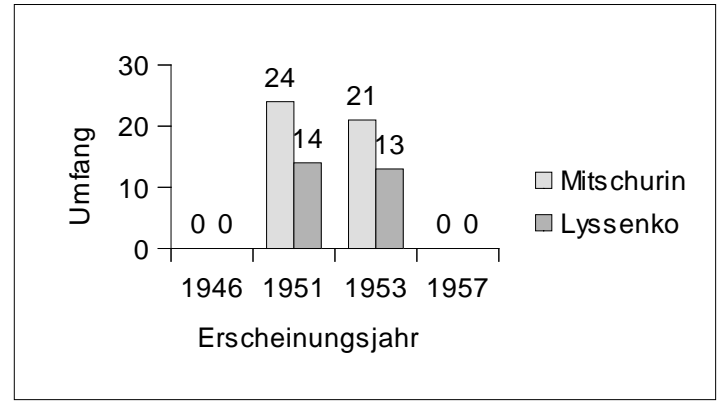

Abbildung 64: Mitschurin und Lyssenko in den Lehrbüchern für die Klasse 8

Neontologie

\section{Homologie und Analogie}

Angaben zu Homologien waren in allen Lehrbüchern vorhanden, wenn auch in unterschiedlicher Tiefe. Das Lehrbuch von 1946 bot eine vergleichende Darstellung von Gehirnen und Handskeletten verschiedener Wirbeltiere (Vertebrata) in Text und Abbildung, ohne den Begriff Homologie zu integrieren (Abb. 65). Auch 
außerhalb der evolutionsbiologischen Kapitel des Lehrbuches wurde der Stellenwert von Erkenntnissen aus der vergleichenden Anatomie für die Abstammungslehre betont.

„Wir können aber durchaus schon Verständnis dafür erlangen, welche groBe Bedeutung die vergleichend-anatomische Wissenschaft hat, besonders für die Abstammungslehre. Eine vergleichende Betrachtung hat in vielen Fällen gezeigt, in welchem Grade manche Tiere miteinander, ve r w a nd t ${ }^{\text {‘ }}$ sind oder anders ausgedrückt, wie ihre Abstammung ist. Gerade die vergleichende Anatomie hat manche Belege dafür gebracht, daß es eine Abstammung, eine Entwicklung gibt.“ (Löbel \& Maschke 1948: 204, Hervorhebung im Original)

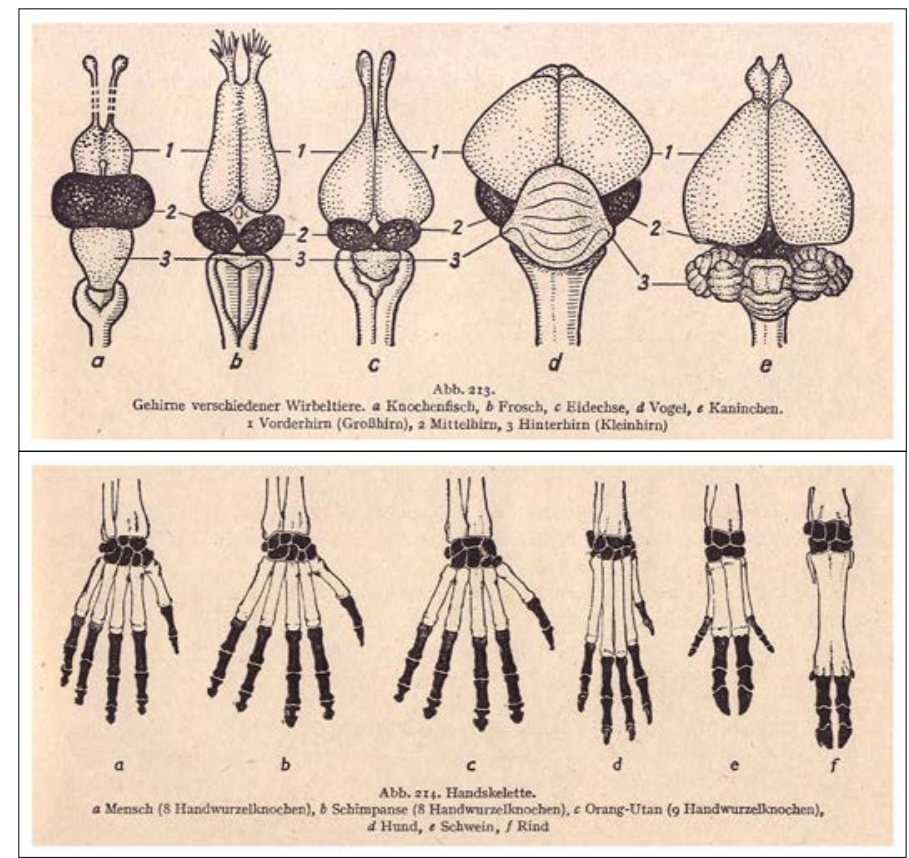

Abbildung 65: oben: Gehirne verschiedener Wirbeltiere, unten: Handskelette (Löbel \& Maschke 1948)

In den Lehrbüchern von 1951 und 1953 erfolgten eine Definition dieses Begriffs und eine nähere Erläuterung anhand von Beispielen. Als homologe Organe wurden die vorderen Gliedmaßen von Wirbeltieren (Landsalamander, Meeresschildkröte, Vogel, Fledermaus, Wal, Maulwurf und Mensch) sowie die Gliedmaßen von Insekten (Insecta; Gottesanbeterin, Laufkäfer, Heuschrecke, Honigbiene, Maulwurfsgrille, Wandelndes Blatt) im Text vorgestellt und mit einer Grafik veranschaulicht. Ferner kamen Hornbildungen der Haut sowie Haarformen im Text 
vor. Auch das Lehrbuch von 1957 verglich im Text die Vordergliedmaßen von Wirbeltieren und visualisierte diese in Form einer Abbildung. Anders als in der Lehrbuchausgabe von 1951 fehlten in dieser Darstellung die Vordergliedmaßen der Meeresschildkröte (Cheloniidae) und des Krokodils (Crocodylia). Eine Nennung des Begriffs homolog erfolgte nicht.

Angaben zu Analogien waren nicht in allen Lehrbüchern vorhanden. So enthielten die Lehrbücher von 1946 und 1957 dazu keine Informationen. Dagegen definierten die Lehrbücher von 1951 und 1953 den Begriff analoge Organe. Auch erfolgte im Text eine nähere Erläuterung anhand der Vordergliedmaßen von Maulwurf (Talpidae) und Maulwurfsgrille (Gryllotalpidae) sowie der Wurmgestalt von Regenwurm (Lumbricidae), Mehlwurm (Larve des Mehlkäfers, Tenebrio molitor), Aal (Anguilla), Blindwühle (Gymnophiona) und Doppelschleiche (Amphisbaenia). Eine schematische Darstellung zeigte die ähnliche Körperform der genannten Beispiele.

\section{Rudimente und Atavismen}

Angaben zu Rudimenten waren in allen Lehrbüchern vorhanden, wenn auch in unterschiedlicher Tiefe. Das Lehrbuch von 1946 bot Informationen über das Skelett von Walen (Cetacea). Davon sollten Rückschlüsse auf die Abstammung gezogen werden. Eine Darstellung des Knochengerüstes des Grönlandwals (Balaena mysticeta) diente der Visualisierung (Abb. 66). Der Begriff Rudimente fand keine Verwendung. Die Lehrbücher von 1951 und 1953 definierten den Begriff Rudimente und stellten die Rückbildung der Gliedmaßen bei Eidechsen (Lacertidae) vor. Eine schematische Darstellung zeigte Zauneidechse (Lacerta agilis), Erzschleiche (Chalcides spec.), Scheltopusik (Pseudopus apodus) und Blindschleiche (Anguis fragilis). Auch die Augen des Maulwurfes (Talpidae) und des Olmes (Proteidae) sowie der Wurmfortsatz und die Ohrmuskeln beim Menschen führten die Autoren als rudimentäre Organe an. Im Lehrbuch von 1957 erfolgte ebenfalls die Nennung und Definition des Begriffs. Die Behandlung fiel jedoch deutlich kürzer aus. Als Beispiele listete das Schulbuch die Griffelbeine der Pferde (Equus) und den dritten Finger bei Vögeln (Aves) auf.

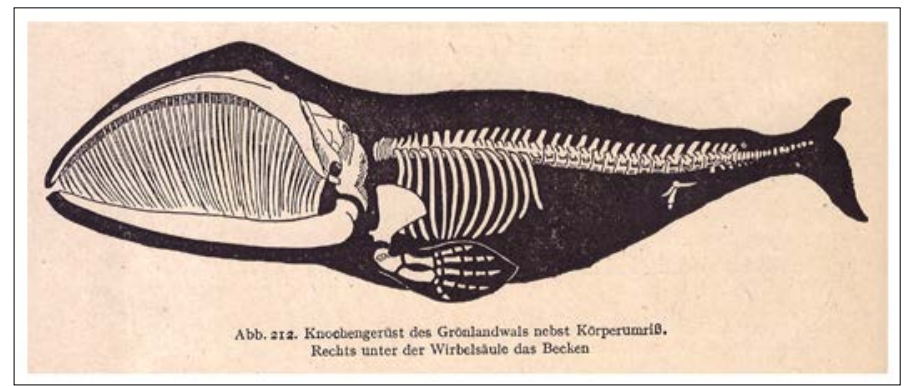

Abbildung 66: Knochengerüst des Grönlandwals (Löbel \& Maschke 1948) 
Angaben zu Atavismen bot ausschließlich das Lehrbuch von 1946, wenn auch in geringem Umfang. Als Beispiel wurde die Missbildung eines Pferdefußes in Text und Abbildung dargelegt.

\section{Biogeografie}

Die Lehrbücher von 1946 und 1953 enthielten zur Biogeografie keine Informationen. Einige wenige Bemerkungen zu dieser Thematik bot das Lehrbuch von 1957 im Zusammenhang mit Darwins Weltreise (Kategorie Theorien) an. Allein das Lehrbuch von 1951 informierte ausführlich über Verbreitungsgebiete von Tieren und Pflanzen. Hier wurden sowohl weit verbreitete Lebensformen als auch solche mit einem engen Verbreitungsgebiet vorgestellt. Im Lehrbuch kamen die Besonderheiten in der Tier- und Pflanzenwelt von Australien und Madagaskar sowie die geologischen Gründe dafür zur Sprache. Die Autoren betonten, dass „die Eigenart der Tier- und Pflanzen Australiens [...] bedingt [ist] durch die frühzeitige Trennung dieses Kontinents von den übrigen Festländern der Erde“ (Lemke 1951: 64). Das Schnabeltier (Ornithorbynchus anatinus) und ein Paradiesvogel (Paradisaeidae) waren als Abbildungen vorhanden. Ferner begründete das Schülerarbeitsmittel die einstige Verbindung von Gewässern (Baikalsee, Kaspisches Meer, Gardasee) zu anderen Meeren anhand verwandter Tierarten. Letztlich wurde das Fazit gezogen, „daß sich die betreffenden Arten aus gemeinsamen Vorfahren entwickelt haben“ (ebd.: 65, Hervorhebung im Original).

\section{Embryologie und Ontogenie}

Während die Lehrbücher von 1946 und 1951 Angaben zur Embryologie enthielten, kamen entsprechende Informationen in den Lehrbüchern von 1953 und 1957 nicht vor. Das Lehrbuch von 1946 stellte die Individualentwicklung der Scholle (Pleuronectes platessa) aus der Ordnung der Plattfische (Pleuronectiformes) dar. Der Fokus der Darlegung lag auf der Verlagerung des linken Auges. Abbildungen visualisierten den Organismus und ausgewählte Entwicklungsstufen (Abb. 67).

Von der befruchteten Eizelle ausgehend, verwies das Lehrbuch von 1951 auf Ähnlichkeiten in der Keimesentwicklung von einzelligen Lebewesen, Geißelträgern, Volvoxkugeln bis hin zu den Hohltieren (Coelenterata). Eine Abbildung der Keimesentwicklung eines Säugereies veranschaulichte die im Text beschriebenen Stadien. Ferner behandelte das Lehrbuch von 1951 Übereinstimmungen von Wirbeltierembryonen. Eine Abbildung zeigte Embryonen eines Vogels, eines Affen sowie eines Menschen. Diese diente als Grundlage für eine vergleichende Betrachtung im Text. In deren Verlauf stellten der Kopf, die Augen, die Anlage der Wirbelsäule, die Faltenbildungen, die Kiemenspalten sowie das Herz Vergleichskriterien dar. Die weitere differenzierte Entwicklung der Wirbeltiere behandelnd, deutete der Text die Abstammung des Menschen von einem affenähnlichen Vorfahren an, mit dem Hinweis, dass ,der menschliche Embryo [...] lange Zeit dem der 
Menschenaffen [ähnelt]“ (Lemke 1951: 61). Von diesen Informationen ausgehend, stellte das Lehrbuch die biogenetische Grundregel vor und zitierte Ernst Haeckel mit den Worten: „Die Keimesgeschichte eines jeden Tieres ist eine kurze Wiederholung seiner Stammesentwicklung" (Lemke 1951: 61, Hervorhebung im Original). Keine Erwähnung fand die Tatsache, dass Ernst Haeckel seine Erkenntnisse als biogenetisches Grundgesetz formulierte. Dies kann im Sinne einer didaktischen Reduktion verstanden werden. Zur Sprache kam, dass während der Keimesentwicklung die Entwicklungsstufen der Stammesgeschichte nicht vollständig, sondern „nur in abgekürzter und veränderter Form“ (Lemke 1951: 61) erkennbar sind. Die Möglichkeiten, Verwandtschaftsbeziehungen aufzuklären, erörterte der Text ausführlich anhand der Entwicklung zweiseitig-symmetrischer Kleinfische bei Plattfischen und einer vergleichenden Darstellung niederer Krebse und ihrer Naupliuslarven. Bereits Charles Darwin lieferte mit seiner Monografie zur Systematik der Rankenfußkrebse (Cirripedia) einen Beitrag zum Nachweis der Evolution. Obwohl er den Lohn seiner zeitaufwändigen Cirripedien-Forschung hinterfragte, resümierte er in seiner Autobiografie (Darwin 2008: 127), „meine Arbeit [...] hat, so meine ich, ihren Wert, da ich nicht nur etliche neue, bemerkenswerte Formen beschrieb, sondern auch die Homologien zwischen verschiedenen Körperteilen ausfindig machen konnte“.

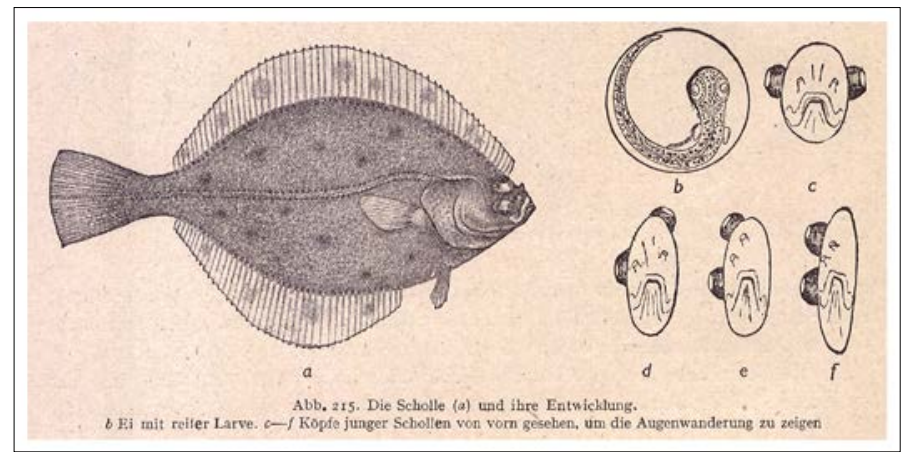

Abbildung 67: Die Scholle und ihre Entwicklung (Löbel \&

Maschke 1948)

\section{Paläontologie}

\section{Systemtabellen und Phylogenie}

Angaben zu Erdzeitaltern (Ären bzw. Äonen) zeigte das Lehrbuch von 1946 in einer Abbildung über Das erdgeschichtliche Auftreten der großen Pflanzen- und Tiergruppen sowie des Menschen. Neben Zeitangaben enthielt die Übersicht entsprechende Formationen (auch Systeme bzw. Perioden) der Zeitalter. Eine weitere Abbildung lieferte einen Überblick über die Geschichte des Lebens auf der Erde vom Kambrium bis 
zum Diluvium mit ausgewählten Angaben zu Organismen. Dieser fehlten Angaben zu Erdzeitaltern und Zeit (Abb. 68).

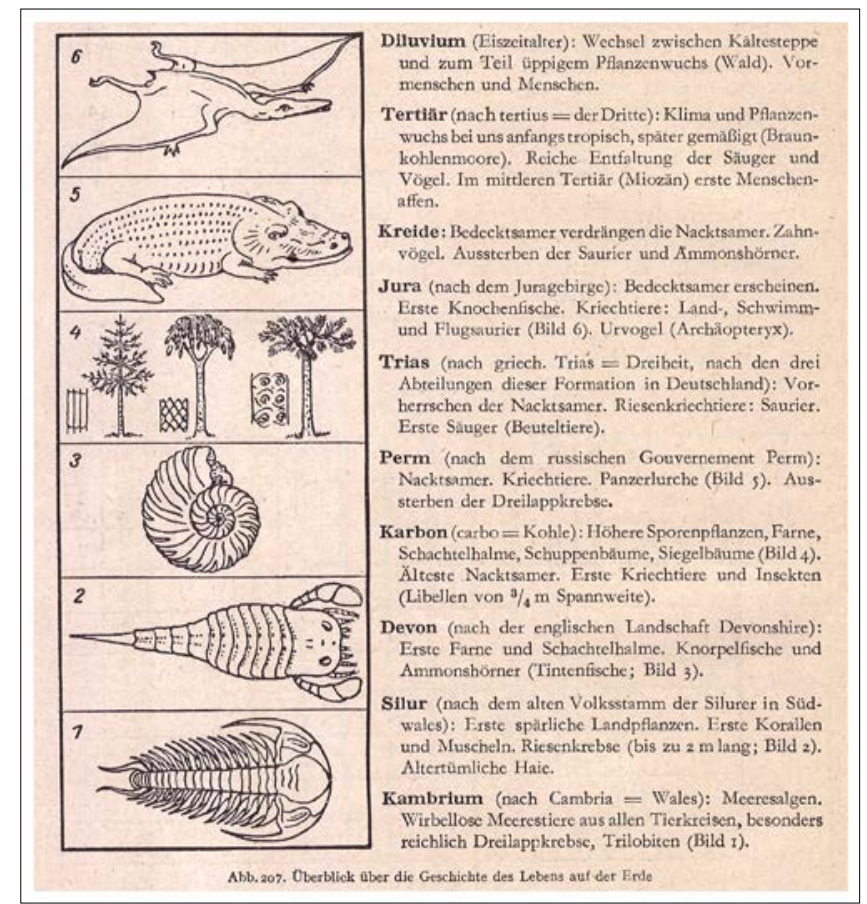

Abbildung 68: Überblick über die Geschichte des Lebens (Löbel \& Maschke 1948)

In den Lehrbüchern von 1951 und 1953 zeigte eine Tabelle die vier Erdzeitalter Urzeit, Altzeit, Mittelzeit und Neuzeit, deren zeitlichen Beginn sowie entsprechende Formationen, die durch anschließende Textpassagen und Abbildungen näher erläutert wurden. Fehlten im Lehrbuch von 1951 noch die Formationen der Urzeit, so waren diese mit Algonikum und Archaikum im Lehrbuch von 1953 angegeben. Das Lehrbuch von 1957 stellte unter dem Begriff Erdzeitalter fünf Ären (Erd-Neuzeit, Erd-Mittelalter, Erd-Altertum, Erd-Frühzeit, Erd-Urzeit) sowie dazugehörige Formationen und Zeitangaben anhand einer Abbildung vor. In allen Lehrbüchern fehlte die Formation Ordovizium (Tab. 38). ${ }^{92}$ Die Lehrbücher von 1951 und 1953 beschrieben das Leben im Steinkohlewald, in der Jurazeit, im Braunkohlewald und in der Eiszeit detailliert. Das Lehrbuch von 1957 legte den Steinkohlewald, die Kreidezeit und ihre Saurier, die Braunkohlezeit und die Eiszeit ausführlich in Text und Bild dar.

92 Obwohl Charles Lapworth (1842-1920) den Begriff Ordovizium bereits 1879 einführte, hat sich diese Schlussfolgerung erst später bewahrheitet (Kutschera 2009b: 176). 
Tabelle 38: Systemtabelle, Lehrbücher Klasse 8 (*vgl. dazu Kutschera 2015)

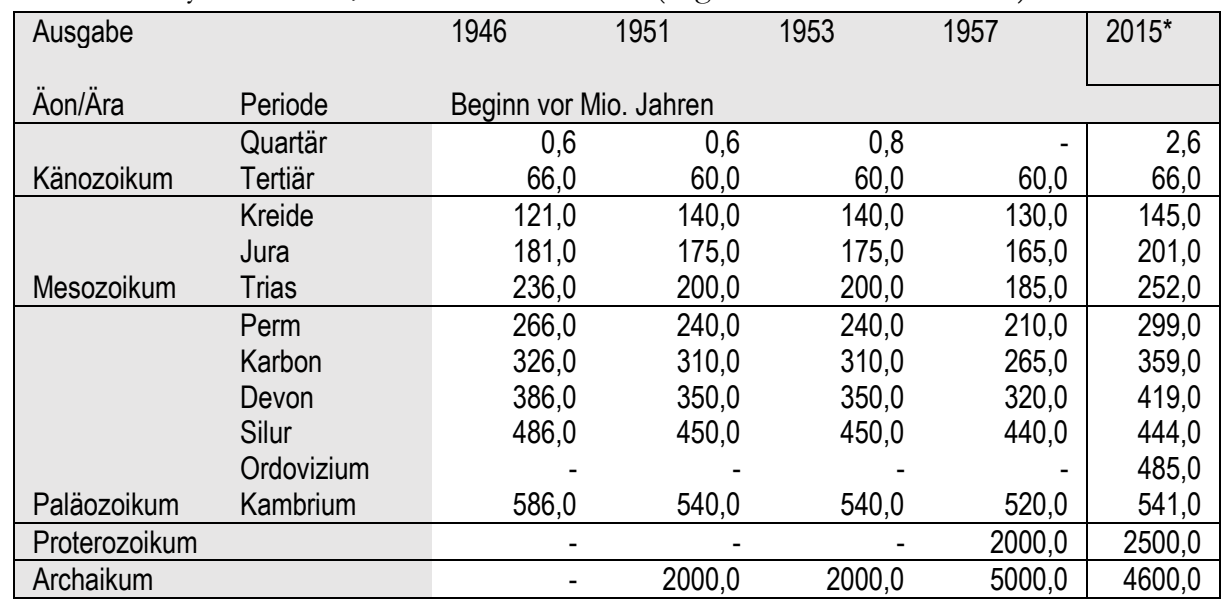

Entwicklungsreiben und Zwischenformen

Das Lehrbuch von 1946 ging in Text und Bild auf die Evolution von Lungenfischen (Dipnoi) und Pferden (Equus) ein. Bei den Darstellungen zur Evolution der Lungenfische lag der Fokus auf der Entwicklung der Flossen und der Körperform. Bei den Erläuterungen zur Evolution der Pferde wurden die Größenzunahme und die Veränderungen im Fußskelett thematisiert (Abb. 69).

In den Lehrbüchern aus den Jahren 1951 und 1953 gingen die Autoren von „den großen Entwicklungslinien der Tier- und Pflanzenwelt"، aus (Lemke 1951: 50). Dem didaktischen Prinzip vom Allgemeinen zum Speziellen folgend, stellten sie die Entwicklungsreihen von Elefant, Pferd und Sumpfdeckelschnecke vor. Der Text verwies darauf, dass lückenlose Entwicklungsreihen durch die Paläontologie nicht für alle Tiere und Pflanzen erbracht werden können. Die Schullehrbücher visualisierten für die Schülerinnen und Schülern folgende Entwicklungsreihen: Elefantenschädel, Pferde mit Schädel, Zähnen und Vorderfüßen sowie Gehäuse der Sumpfdeckelschnecke (Viviparidae). Das Lehrbuch von 1957 enthielt ebenfalls Informationen zur Evolution der Elefanten (Elephantidae) und der Pferde. Visualisiert wurde die Entwicklungsreihe der Elefanten. In der Gesamtschau war die Darstellung der Entwicklung der Pferde allen Ausgaben gemeinsam (Tab. 39)

Die Lehrbuchausgabe von 1946 beinhaltete Angaben zum Urvogel sowie eine Abbildung eines Urvogels aus dem Solnhofener Schiefer. In der Ausgabe von 1948 wurde diese Abbildung durch einen Fund aus dem lithographischen Schiefer bei Eichstedt ersetzt. Auch das Lehrbuch von 1957 stellte in Text und Abbildung sowie in einer Kunstdrucktafel den Urvogel als Zwischenform vor. Ausführlichere Informationen enthielten die Lehrbücher von 1951 und 1953 (Tab. 40). Zwischen Tier und Pflanze stehend, wurden Geißelträger vorgestellt und die Schlussfolgerung gezogen, „daß ähnliche Lebewesen die Stammformen für die Entwicklung 
des Tier- und Pflanzenreiches waren“ (Lemke 1951: 57). Am Beispiel der Kugelalge Volvox betonte das Lehrbuch, dass zwischen Ein- und Mehrzellern Zwischenformen existieren. Vom Körperbau und der Lebensweise der Lungenfische ausgehend, zeigte der Text den Übergang der Wirbeltiere vom Wasser- zum Landleben auf. Als Beleg für die Abstammung der Vögel von den Kriechtieren diente die Entdeckung des Urvogels. Ferner wurden die Merkmale von Kloakentieren vorgestellt und die Schlussfolgerung gezogen, „daß sich auch die Säugetiere aus den Kriechtieren entwickelt haben“ (Lemke 1951: 58). In den Text eingebunden war eine Abbildung, die eine Gesteinsplatte mit Resten des Urvogels aus dem Solnhofener Schiefer sowie dessen Rekonstruktion darstellte.

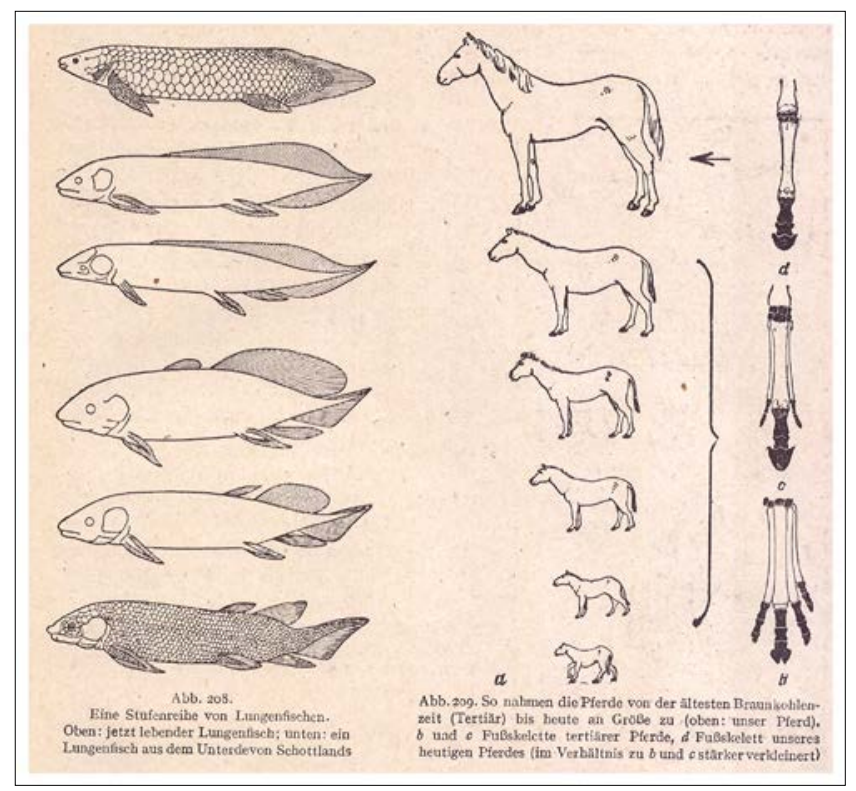

Abbildung 69: Die Evolution der Lungenfische und Pferde (Löbel \& Maschke 1948)

Tabelle 39: Entwicklungsreihen, Lehrbücher Klasse 8

\begin{tabular}{|l|l|l|l|l|}
\hline Auflage & Lungenfische & $\begin{array}{l}\text { Sumpfdeckel- } \\
\text { schnecke }\end{array}$ & Pferde & Rüsseltiere \\
\hline 1946 & $\begin{array}{l}\text { 1. Zusammen- } \\
\text { fließen unpaari- } \\
\text { ger Flossen, } \\
\text { Rückbildung } \\
\text { paariger Flos- } \\
\text { sen, Körperform } \\
\text { wird aalähnlicher }\end{array}$ & $\begin{array}{l}\text { 1. mehrzehige } \\
\text { Vorfahren }\end{array}$ & - \\
\hline
\end{tabular}




\begin{tabular}{|c|c|c|c|c|}
\hline $1951 / 53$ & - & $\begin{array}{l}\text { 1. kegelförmige } \\
\text { Gehäuse, glatte } \\
\text { und stark gewölb- } \\
\text { te Rundungen / } \\
\text { 2. kleinere } \\
\text { Schneckenhäu- } \\
\text { ser mit Leisten- } \\
\text { bildung / 3. } \\
\text { stärkere Leisten } \\
\text { und Größenzu- } \\
\text { nahme }\end{array}$ & $\begin{array}{l}\text { 1. fünfzehig, wenig } \\
\text { größer als ein } \\
\text { Fuchs / } 2 \text {. } \\
\text { vierzehig, unteres } \\
\text { Tertiär / } 3 \text {. } \\
\text { dreizehig, schaf- } \\
\text { groß, jüngeres } \\
\text { Tertiär / 4. Rückbil- } \\
\text { dung der Seitenze- } \\
\text { hen, Umwandlung } \\
\text { des Gebisses }\end{array}$ & $\begin{array}{l}\text { 1. kleiner Säuger, Tertiär, ähn- } \\
\text { lich dem Tapir, Unpaarzeher, } \\
\text { Schädel schweineähnlich / } 2 \text {. } \\
\text { verlängerter Schädel, jüngere } \\
\text { Schicht des Tertiär, je ein } \\
\text { Schneidezahn rechts und links / } \\
\text { 3. mittleres Tertiär, Schneide- } \\
\text { zähne im Unter- und Oberkiefer } \\
\text { verlängert / 4. untere Schneide- } \\
\text { zähne gehen zurück, obere } \\
\text { werden länger, Umbildung zu } \\
\text { Stoßzähnen, Nase und Oberlip- } \\
\text { pe verlängern sich zum Rüssel }\end{array}$ \\
\hline 1957 & - & - & $\begin{array}{l}\text { 1. Hundegröße, } \\
\text { mehrere Zehen am } \\
\text { Boden / 2. Größen- } \\
\text { zunahme, Verkür- } \\
\text { zung der Seitenze- } \\
\text { hen / 3. Hauspferd } \\
\text { mit Mittelzehe }\end{array}$ & $\begin{array}{l}\text { 1. ähnelt im Körperbau einem } \\
\text { Elefanten, Unterschiede im } \\
\text { Schädelbau, langer Unterkiefer } \\
\text { mit zwei Stoßzähnen, kurzer } \\
\text { Rüssel, kurze obere Stoßzähne / } \\
\text { 2. kürzerer Unterkiefer, schwä- } \\
\text { chere Stoßzähne, Rüssel länger, } \\
\text { Stoßzähne des Oberkiefers } \\
\text { länger / 3. ähnelt heutigen } \\
\text { Elefanten stark, kurzer Unterkie- } \\
\text { fer, Stoßzzähne des Oberkiefers } \\
\text { lang, schlanker langer Rüssel }\end{array}$ \\
\hline
\end{tabular}

Tabelle 40: Zwischenformen und Entwicklungsreihen, Lehrbücher Klasse 8

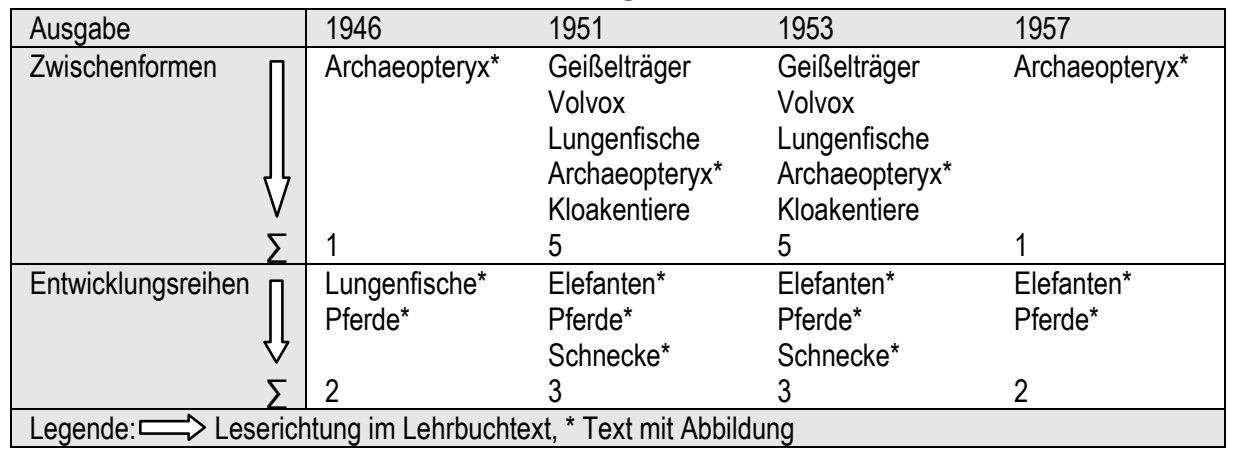

Fossilisation und Altersbestimmung

Das Lehrbuch von 1946 enthielt einzig die Aussage: „Die ,Fossilien“, ,Versteinerungen“ können sich nur von Formen erhalten haben, die „Hartgebilde“ besaßen“ (Biologische Lehrfachkommission bei der DVV in der SBZ 1946: 191). Dagegen boten die Lehrbücher von 1951, 1953 und 1957 ausführliche Informationen über die Entstehung von Fossilien (Fossilisation) an. Es wurden Fossilarten vorgestellt (Knochenfunde, Versteinerungen, Abdrücke) und Beispiele benannt. Zusätzlich 
standen den Schülern in den Lehrbüchern von 1951 und 1953 Informationen über Einschlüsse zur Verfügung. Über Formen der Konservierung informierte nur das Lehrbuch von 1957. Angaben zu Fundorten waren in den Lehrbüchern von 1951/53 fallweise und im Lehrbuch von 1957 zu jeder vorgestellten Fossilart vorhanden. Die Lehrbücher von 1951/53 wiesen weniger Abbildungen auf als das Lehrbuch von 1957 (Tab. 41).

Tabelle 41: Fossilisation, Lehrbücher Klasse 8

\begin{tabular}{|c|c|c|c|}
\hline Auflage & $\begin{array}{l}\text { Fossilisation, } \\
\text { Fossilienarten }\end{array}$ & Beispiele ( ${ }^{*}$ mit Abbildung) & Fundorte \\
\hline 1946 & Versteinerungen & - & - \\
\hline \multirow[t]{4}{*}{$1951 / 53$} & Knochenfunde & Auerochse, Dronte, europäische Wildpferde, Moa* & $\begin{array}{l}\text { Kieslager von Flüs- } \\
\text { sen, Sandgruben }\end{array}$ \\
\hline & Versteinerungen & $\begin{array}{l}\text { Schnecken, Muscheln, Seeigel, Korallen, Kalk- } \\
\text { schwämme }\end{array}$ & - \\
\hline & Abdrücke & $\begin{array}{l}\text { Blätter, Insekten (Libelle*), Quallen, Kriechspuren, } \\
\text { Fährte eines ausgestorbenen Tieres* }\end{array}$ & - \\
\hline & Einschlüsse & $\begin{array}{l}\text { Käfer, Geradflügler, Wanzen, Termiten, Motten, } \\
\text { Blattläuse, Kleinschmetterlinge, Tausendfüßler, } \\
\text { Spinnen, Asseln, Fadenwürmer, kleine Schne- } \\
\text { cken, Haare von Säugern, Federn von Vögeln, } \\
\text { Eidechse, Blüten* }\end{array}$ & $\begin{array}{l}\text { Küstengebiet der } \\
\text { Ostsee }\end{array}$ \\
\hline \multirow[t]{4}{*}{1957} & Knochenfunde & $\begin{array}{l}\text { Höhlenbär*, Höhlenhyäne, Geweihstangen von } \\
\text { Hirschen und Rehen, Stoßzähne vom Mammut* }\end{array}$ & $\begin{array}{l}\text { Hermannshöhle bei } \\
\text { Rübeland (Harz), } \\
\text { Sand- und Kiesgru- } \\
\text { ben }\end{array}$ \\
\hline & Abdrücke & $\begin{array}{l}\text { Farnwedel*, Insekten (Libelle)*, Quallen, Tierfähr- } \\
\text { ten*, Spuren von Krebsen und Insekten, Kriech- } \\
\text { spuren von Würmern }\end{array}$ & $\begin{array}{l}\text { Zwickau, Solnhofen } \\
\text { (Fränkische Alb) }\end{array}$ \\
\hline & Versteinerungen & $\begin{array}{l}\text { Donnerkeil }{ }^{\star} \text {, Schnecken }{ }^{*} \text {, Trilobit* }{ }^{*} \text {, versteinerter } \\
\text { Wald }\end{array}$ & Rügen \\
\hline & $\begin{array}{l}\text { Konservierung } \\
\text { (Bernstein, Eis) }\end{array}$ & $\begin{array}{l}\text { Insekten, Vogelfedern, Haare von Säugetieren, } \\
\text { Blüten*, Mammut }\end{array}$ & Sibirien \\
\hline
\end{tabular}

Ergänzend enthielt das Lehrbuch von 1957 zum Thema Fundstellen das Kapitel Wo finden wir Fossilien?. Es wurden Fossilien aus den Orten bzw. Gebieten Rügen (Seeigel, Schwämme, Kleinlebewesen, Seelilien), Rüdersdorf bei Berlin (Muscheln), Thüringer Becken (Schnecken, Knochen, Zähne), Solnhofen (Seelilien, Fische, Quallen, Urvogel) sowie Boll und Holzmaden in Württemberg (Ichthyosaurus) genannt. Als schematische Darstellungen waren ein versteinerter Seeigel, ein Seeigel (Echinoidea) und eine Seelilie (Crinoidea) sowie eine schwimmende rezente Tintenschnecke (Coleoidea) abgebildet. Eine Fotografie aus Othenio Abels Tierwelt 
der Vorzeit zeigte einen mit Haut erhaltenen Ichthyosaurier. Kutschera (2008: 22) betont, dass diese Tiergruppe ,zu den am besten erforschten ausgestorbenen Reptilien [zählt], da zahlreiche sehr gut erhaltene versteinerte Skelette [...] gefunden wurden." Am Ende des Kapitels konnten die Schüler auf einer Kunstdrucktafel aus den Photographischen Werkstätten Fritz. Seebeck einen Fisch aus den Solnhofener Plattenkalken, Trockenrisse auf Gestein und einen Ammoniten (Ammonoidea) betrachten. Eine zweite Tafel, bereitgestellt durch das Geologisch-Paläontologische Museum Berlin, zeigte den Urvogel Archaeopteryx aus den Solnhofener Plattenkalken. Das im Jahr 1877 gefundene Exemplar konnte, so die Beschriftung der Tafel, im Museum für Naturkunde der Humboldt-Universität zu Berlin betrachtet werden.

Die Bedeutung von Leitfossilien erläuterten die Lehrbücher von 1951 und 1953. Auch das Lehrbuch von 1957 ging auf den Begriff Leitfossil und seine Bedeutung für die Verwendung ,in der freien Natur“" (Arnold et al. 1957: 75) ein. Die Darstellung erfolgte jedoch auf insgesamt einer Seite ausführlicher als in den Vorgängermodellen. Als Beispiele wurden neben Ammoniten, Muscheln, Schnecken und Blättern für das Erd-Altertum Dreilapper (Trilobita) und für die ErdMittelzeit Säugetiere (Mammalia) benannt. Zusätzlich enthielt das Lehrbuch von 1957 Informationen zur Altersbestimmung mittels Atomzerfall. 


\title{
Das Biologielehrbuch für die Klasse 10
}

\author{
„Die Auseinandersetzungen über \\ Darwins Auffassungen dauerten über \\ ein Jahrhundert an und sind auch in der \\ Gegenwart noch nicht abgeschlossen." \\ (Kummer et al. 1988: 75)
}

\section{Design}

Umfang und Textformat

Das Schulbuch von 1960 umfasste 100 Seiten. Mit 93 Seiten nahmen evolutionsbiologische Inhalte einen Anteil von $93 \%$ ein. Verzeichnisse u. ä. stand dem Schüler auf den übrigen sieben Seiten zur Verfügung. Ab 1965 integrierten die Autoren in die Lehrbücher auch genetische und züchtungsbiologische Themen, was sich auf den Umfang auswirkte. Im Lehrbuch von 1965 stieg der Lehrbuchumfang auf 176 Seiten an. Mit 108 Seiten (61,4 \%) blieb der absolute Umfang für evolutionsbiologische Inhalte jedoch annähernd gleich. Das Lehrbuch für die Vorbereitungsklassen der EOS von 1968 wies mit 160 Seiten ebenfalls einen hohen Umfang auf. Eine starke Reduktion auf 65 Seiten (40,6 \%) erfuhren hingegen die darin enthaltenen evolutionsbiologischen Themen, da dem Stoffgebiet Genetik 
nun ein größerer Raum beigemessen wurde. Im Lehrbuch von 1971 stieg der Umfang weiter auf 192 Seiten an. Mit 76 Seiten (39,6 \%) fiel der Raum, im Vergleich zum Lehrbuch von 1965, für evolutionsbiologische Themen gering aus. Eine deutliche Reduzierung auf 144 Seiten erfuhr das Lehrbuch von 1988. Der Umfang für evolutionsbiologische Themen blieb dagegen mit 69 Seiten (47,9 \%) annähernd erhalten. Es zeigt sich, dass der relative Umfang für evolutionsbiologische Darstellungen im Schülerbuch nach 1960 stark sank und in den Lehrbüchern von 1968, 1971 und 1988 annähernd konstant blieb (Abb. 70).

Das Lehrbuch von 1960 war mit einem Inhalts- und einem Sachverzeichnis ausgestattet. Neben diesen wies das Schülerbuch von 1965 erstmals auch ein Glossar auf. Die Ausgabe von 1968 verfügte zusätzlich über Literaturhinweise, die im Lehrbuch von 1971 nicht wieder aufgenommen wurden. Im Lehrbuch von 1988 entfiel auch das Glossar. In der Ausstattung mit Beiwerk waren somit die Lehrbücher von 1960 und 1988 sowie die Lehrbücher von 1965 und 1971 gleich. Das Lehrbuch von 1968 für die Vorbereitungsklassen enthielt in der Gesamtschau die meisten Hilfestellungen für Schüler.

Das Textformat der Lehrbücher von 1960 und 1965 war durch Fettdruck bzw. Kursivschrift ausgewählter Begriffe bzw. Wortgruppen charakterisiert. Zusatzinformationen waren durch eine kleinere Schriftgröße sowie Namen durch Großbuchstaben kenntlich gemacht. Im Lehrbuch von 1968 fand weiterhin eine kleinere Schriftgröße für Zusatzinformationen Verwendung. Dennoch war der Text homogener gestaltet, da Kursivschrift, Fettdruck und Großbuchstaben nur noch vereinzelt zur Anwendung kamen. Das Lehrbuch von 1971 enthielt weder Fettdruck noch eine andere Schriftgröße. Erhalten blieb dagegen der Einsatz von Kursivschrift und Großbuchstaben. Ein kleiner schwarzer Stern kennzeichnete Abschnitte als Zusatzinformation. Zusätzlich enthielten die Seiten Randmarken, denen der jeweilige Hauptabschnitt des Lehrbuches entnommen werden konnte. Das Schema eines Ammoniten, das auch auf dem Einband zu sehen war, diente hierbei als Markierung der Seiten, die evolutionsbiologische Inhalte vermittelten. Auch im Lehrbuch von 1988 kamen Randmarken zum Einsatz. Das Schema eines Hominidenschädels diente hier als Symbol. Ein kleines Viereck markierte erneut Textstellen, die Zusatzinformationen zum Stoff lieferten. Merksätze und Aufgabenstellungen wurden durch Fettdruck und Kursivschrift hervorgehoben. Weitere Schriftgrößen sowie Großbuchstaben kamen nicht zum Einsatz.

Die Lehrbücher von 1960 und 1965 enthielten Aufgabenstellungen und Fragen. Am Ende des Lehrbuches von 1968 befand sich ein Fragenkatalog, deren Reihenfolge der Logik des Textflusses folgte. Mit Nummern markierte relevante Textstellen erleichterten die Suche nach Antworten. Auch das Lehrbuch von 1971 verknüpfte Fragen und Textstellen auf diese Weise. Erstmals, wenn auch nur vereinzelt, integrierten die Verfasser im Lehrbuch von 1971 Merksätze und grenzten diese durch eine farbige Linie vom übrigen Text ab. Das Lehrbuch von 1988 enthielt dann häufig in den Textfluss integrierte Merksätze. Diese waren mit einem 
Symbol markiert und durch Fettdruck hervorgehoben. Aufgabenstellungen wurden mit farbigen Linien abgegrenzt und als Kursivschrift abgedruckt.

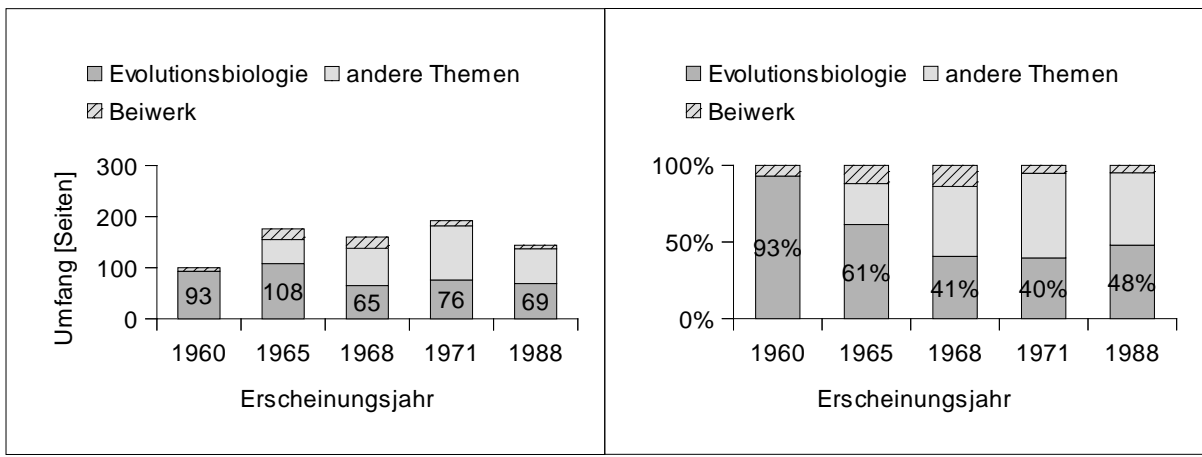

Abbildung 70: oben: absoluter Umfang, unten: relativer Umfang in den Lehrbüchern für die Klasse 10

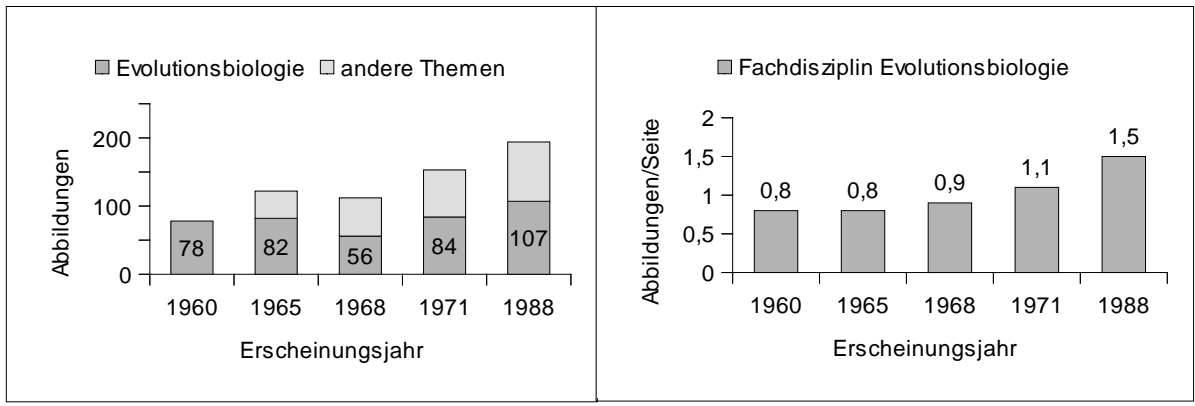

Abbildung 71: links: Anzahl der Abbildungen, rechts: Abbildungen pro Seite in den Lehrbüchern für die Klasse 10

Einband und Abbildungen

Mit einem Hardcover ausgestattet, war das Format der Auflagen von 1960 bzw. 1965 den Lehrbüchern der Klassenstufe 8 ähnlich und für den Schüler handlich. Der äußeren Gestaltung konnte der Titel entnommen werden. Angaben zum Fach und zur Klassenstufe enthielt der Einband nicht. Farbig gestaltet, visualisierten die Buchdeckel der Lehrbücher drei Applikationen. Diese stellten aus dem Bereich der vergleichenden Anatomie die Gehirne verschiedener Wirbeltiere dar (Kategorie Neontologie). Im Wechsel der Hintergrundfarbe von gelb (1960) zu blau (1965) bestand der einzige Unterschied des äußeren Designs. Mit einem Rotton auf dem Einband der Auflage von 1968 setzte sich die Farbverwendung in der äußeren Gestaltung der Schulbücher fort. Auf dem Buchdeckel visualisierten schematische Darstellungen Themen aus verschiedenen Bereichen der Evoluti- 
onsbiologie: Urvogel, Ammonit (beide Kategorie Paläontologie), Hominidenschädel (Kategorie Hominisation) sowie Naupliuslarve (Kategorie Neontologie). Dem handlichen Format konnte das Fach, die Klassenstufe sowie der Hinweis Vorbereitungsklassen entnommen werden. Auch die äußere Gestaltung der Auflage von 1971 wies durch Schematisierungen auf Lehrbuchinhalte hin. Mit einem Hominidenschädel und einem Ammoniten enthielt sie u. a. Abbildungen, die evolutionsbiologische Aspekte visualisierten. Den Vorgängermodellen im Format ähnlich blieb das Buch für den Schüler handlich. Weiterhin informierte der Einband über das Fach und die Klassenstufe. Die Gestaltung orientierte sich an der Auflage von 1968. Statt roter wurde jedoch blaue Farbe eingesetzt. Mehrfarbig präsentierte sich dagegen der Einband des Lehrbuches von 1988. Erstmals befanden sich auf der Rückseite Abbildungen mit Bezug zur Evolutionsbiologie. Diese stellten Fossilarten, wie Abdrücke (Blatt, Archaeopteryx) und Knochenfunde (Hominidenschädel, Saurierskelett) dar. Die Abbildungen entsprachen den Kategorien Paläontologie und Hominisation. Auf der Vorderseite des Einbandes wurde mit der Darstellung der japanischen Wunderblume ein Bezug zu genetischen Inhalten hergestellt. Auch bei diesem Schülerbuch konnte die Klasse und das Unterrichtsfach dem Einband entnommen werden. Im Format traten keine Veränderungen auf.

Während der Textumfang für evolutionsbiologische Inhalte seit 1968 reduziert wurde und reduziert blieb, stieg die Anzahl der Abbildungen in den Lehrbüchern an. Auch die Anzahl der Abbildungen pro Seitenanzahl im evolutionsbiologischen Textteil zwischen 1960 und 1988 nahm stetig zu. Waren 1960 noch 0,8 Abbildungen pro Seite vorhanden, so stieg dieser Wert im Jahr 1988 auf 1,5 Abbildungen pro Seite (Abb. 71). Das Lehrbuch von 1988 enthielt schließlich wiederholt bis zu drei Seiten keinen Textanteil (außer Bildunterschriften), sondern lediglich Abbildungen. In der methodischen Reihe Unterrichtshilfen Biologie Klasse 10 von 1985 begründeten die Autoren Gründe die notwendige Anzahl der Abbildungen.

„Für die Behandlung der Stoffgebiete in Klasse 10 können nur wenige Objekte als Originale beschafft werden, es müssen verstärkt Abbildungen eingesetzt werden. Diese Tatsache wurde bei der Lehrbuchentwicklung besonders beachtet. Es wurden zahlreiche Fotos aufgenommen und viele Probleme an Schemadarstellungen erläutert.“ (Graef et al. 1985: 14)

Konkret enthielt das Lehrbuch von 1960 insgesamt 78 schwarz/weiß Abbildungen im Text, vier Farbtafeln und vier Kunstdrucktafeln. Alle wiesen ausschließlich Informationen aus Bereichen der Evolutionsbiologie auf. Auf den Farbtafeln waren Gehirne, Vordergliedmaßen verschiedener Wirbeltiere (Abb. 72) und der Stammbaum der Wirbeltiere abgebildet. Die Kunstdrucktafeln enthielten Abbildungen verschiedener Fossilien sowie Kulturgegenstände von Hominiden. Ferner war das Lehrbuch mit einem vorderen und hinterm Vorsatz ausgestattet. Beide stellten den Stammbaum der Hominiden und den Stammbaum der Pferde sche- 
matisch dar. Im Lehrbuch von 1965 blieb die Gestaltung des vorderen Vorsatzes erhalten. Verändert wurde dagegen der hintere Vorsatz. Dieser enthielt eine Weltkarte, die Reiseroute der Beagle und Abbildungen fossiler Tiere auf den jeweiligen Kontinenten. Im Lehrbuchtext integrierten die Autoren 122 schwarz/weiß Abbildungen. Davon enthielten 82 Abbildungen Inhalte aus Bereichen der Evolutionsbiologie $(67,2 \%)$. Zusätzlich kamen drei Farbtafeln und vier Kunstdrucktafeln in dem Schülerbuch vor. Die Inhalte der Farbtafeln und der Großteil der Kunstdrucktafeln glichen denen aus dem Lehrbuch von 1960.

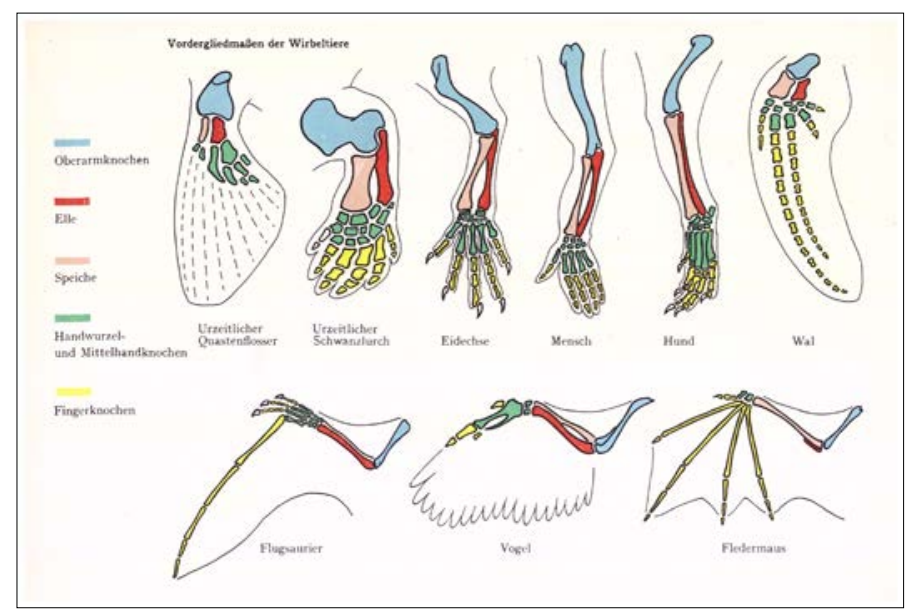

Abbildung 72: Vordergliedmaßen der Wirbeltiere (Ambrosius et al. 1964)

Das Lehrbuch von 1968 (Vorbereitungsklassen) enthielt 112 Abbildungen in schwarz/weiß bzw. Graustufen. Der absolute und relative Anteil evolutionsbiologischer Darstellungen sank im Vergleich zum Vorgängermodell auf 56 Abbildungen $(50 \%)$. Der vordere Vorsatz stellte den Aufbau der DNS und die identische Replikation schematisch dar. Der hintere Vorsatz zeigte den Stammbaum der Wirbeltiere (ausgestorbene und lebende Tierformen) vom Silur bis zum Quartär in den Lebensräumen Wasser, Land und Luft. Beide Vorsätze waren farblich (Vierfarbdruck). Farbtafeln bzw. Kunstdrucktafeln enthielt das Lehrbuch nicht.

Im Lehrbuchtext von 1971 stieg die Anzahl der Abbildungen auf 153 an. Davon enthielten 84 Abbildungen (54,9 \%) Inhalte aus Bereichen der Evolutionsbiologie. Die Abbildungen waren nun neben schwarz und weiß zum Teil mit einem gelben Farbton versehen. Nach Kummer (2009: 15; Interview vom 28.12.2016) führten Neuentwicklungen in der Druckindustrie dazu, dass zwei Druckfarben 
eingesetzt werden konnten. ${ }^{93}$ Ziel war es, die entscheidenden Inhalte in den Abbildungen farbig hervorzuheben. Um Verwechslungen mit der Natur zu vermeiden, wählte die Buchredaktion eine neutrale Farbe. Grün (Pflanzen) und Rot (Blut) kamen dafür nicht in Frage. ${ }^{94}$ Zusätzlich enthielt das Werk elf Drucktafeln, wovon drei evolutionsbiologische Themen darstellten (Urvogel, verkieselter Torf aus Urwaldpflanzen, Stammesentwicklung des Menschen). Von den elf Drucktafeln wurden acht Seiten im Vierfarbdruck als Anhang genehmigt, um „neben Schemadarstellungen auch einige Originalabbildungen in die Bücher aufnehmen zu können" (Kummer 2009: 15). Auf die Forderung der Herausgeberin standen alle Abbildungen beim dazugehörigen Text, wurden Zeichnungen zu einem Kapitel von einem Künstler geschaffen und Schemadarstellungen direkt beschriftet. Diese Neuerungen erhöhten die Übersichtlichkeit für die Schüler (vgl. ebd.). Die Abbildungen waren nun nicht mehr nummeriert, sondern durch ihre Position und einen Verweis im Text (Seitenzahl) mit diesem inhaltlich verbunden. Vorsätze enthielt das Lehrbuch nicht mehr. Der evolutionsbiologische Themenbereich wurde mit einer Aufnahme aus dem Sauriersaal des Museums für Naturkunde Berlin eingeleitet, wodurch ein regionaler bzw. nationaler Bezug entstand. Konkret zeigte die Abbildung das Skelett eines Brachiosaurus brancai.

Erstmals durchgängig vielfarbig präsentierte sich das Lehrbuch für die Klassenstufe 10 von 1988. Zwar wurden nur vier Farben eingesetzt, aber durch Farbmischung erschien „das Bild in natürlichen Farben und auch die Fotos konnten dem Text direkt zugeordnet werden“" (Kummer 2009: 15). Die Abbildungen enthielten keine Nummerierung. Sie waren aber vereinzelt durch Verweise im Text mit diesem inhaltlich verbunden. Insgesamt summierte sich die Anzahl der im Text eingebundenen Abbildungen auf 194. Davon stellten 107 Abbildungen (55,2 \%) Inhalte aus Bereichen der Evolutionsbiologie dar. Die vordere Innenseite des Einbandes zeigte den Stammbaum der Hominiden und die hintere Innenseite die Etappen der Anthropogenese mit Abbildungen von Schädeln und Werkzeugen.

Die kategoriale Zuordnung der Abbildungen belegt den hohen Stellenwert der Kategorie Paläontologie in allen Lehrbüchern. Inhalte aus dieser Kategorie waren stets mit einer hohen Anzahl an Abbildungen vertreten. In den Lehrbüchern von 1960, 1968 und 1971 bildete die Mehrzahl der Abbildungen Inhalte aus dieser Kategorie ab. Dagegen lag in den Lehrbüchern von 1965 und 1988 der Schwerpunkt auf der Kategorie Theorien bzw. auf der Kategorie Hominisation (Tab. 42).

93 Kummer und Dietrich (1979: 418) resümieren, dass „Dank der Erfolge in der Volkswirtschaft und der Entwicklung in der polygrafischen Industrie“ die Lehrbücher mehrfarbig gedruckt wurden. Konkret wurde das Lehrbuch für die Klasse 5 vierfarbig und die Lehrbücher für alle anderen Jahrgänge zweifarbig gedruckt.

94 Die Herausgeberin Gertrud Kummer formulierte im Interview vom 28.12.2016 einen Grundsatz ihrer Arbeit: „Was gute beschriftete Abbildungen an Wissen liefern, wird im Text nicht wiederholt." Allgemein war sie bestrebt, den Text zu reduzieren und mehr Abbildungen zu integrieren. 
Tabelle 42: äußeres und inneres Design, Lehrbücher Klasse 10

\begin{tabular}{|c|c|c|c|c|c|}
\hline Auflage & 1960 & 1965 & 1968 & 1971 & 1988 \\
\hline \multicolumn{6}{|l|}{ Umfang: } \\
\hline absoluter Umfang [Seiten] & 100 & 176 & 160 & 192 & 144 \\
\hline Textumfang & 93 & 155 & 138 & 182 & 137 \\
\hline davon Evolutionsbiologie & 93 & 108 & 65 & 76 & 69 \\
\hline davon andere Themen & 0 & 47 & 73 & 106 & 68 \\
\hline Beiwerk & 7 & 21 & 22 & 10 & 7 \\
\hline relativer Umfang Evolutionsbiologie am Buch [\%] & 93,0 & 61,4 & 40,6 & 39,6 & 47,9 \\
\hline relativer Umfang Evolutionsbiologie am Text [\%] & 100,0 & 69,7 & 47,1 & 41,8 & 50,4 \\
\hline \multicolumn{6}{|l|}{ Beiwerk: } \\
\hline Inhaltsverzeichnis & ja & ja & ja & ja & ja \\
\hline Glossar & nein & ja & ja & ja & nein \\
\hline Literaturhinweise & nein & nein & ja & nein & nein \\
\hline Sachverzeichnis & & ja & ja & ja & ja \\
\hline \multicolumn{6}{|l|}{ Paratext: } \\
\hline$\overline{\text { Abbildungen im Text }}$ & 78 & 122 & 112 & 153 & 194 \\
\hline davon Evolutionsbiologie & 78 & 82 & 56 & 84 & 107 \\
\hline relativer Umfang [\%] & 100,0 & 67,2 & 50,0 & 54,9 & 55,2 \\
\hline Abbildungen pro Textseite & 0,8 & 0,8 & 0,8 & 0,8 & 1,4 \\
\hline davon Evolutionsbiologie & 0,8 & 0,8 & 0,9 & 1,1 & 1,6 \\
\hline Gestaltung & $\mathrm{s} / \mathrm{w}$ & $\mathrm{s} / \mathrm{w}$ & $s / w$ & s/w/gelb & farbig \\
\hline integrierte Tafeln & 8 & 7 & 0 & 11 & 0 \\
\hline davon Evolutionsbiologie & 8 & 7 & 0 & 3 & 0 \\
\hline \multicolumn{6}{|l|}{ Kategoriale Zuordnung der Abbildungen: } \\
\hline Theorien & 25 & 29 & 10 & 10 & 24 \\
\hline Biogenese & 4 & 4 & 4 & 4 & 3 \\
\hline Neontologie & 11 & 9 & 13 & 14 & 4 \\
\hline Paläontologie & 26 & 26 & 15 & 34 & 33 \\
\hline Systematik & 0 & 0 & 0 & 0 & 0 \\
\hline Hominisation & 12 & 14 & 14 & 22 & 43 \\
\hline Didaktisches & 0 & 0 & 0 & 0 & 1 \\
\hline Gesamt & 78 & 82 & 56 & 84 & 107 \\
\hline
\end{tabular}

\section{Struktur}

Das Lehrbuch von 1960

Die Inhalte der Ausgabe von 1960 waren, wie der Titel Entwicklung der Organismen nahelegte, im Fachbereich Evolutionsbiologie zu verorten und in sechs heterogen strukturierte Kapitel gegliedert. Folgende Feinstruktur lag dem Lehrbuch zu Grunde:

- $\quad$ Einleitung (2 Seiten; 2,2 \%)

- Tatsachen aus der Entwicklungslehre (27 Seiten; 29,3\%)

- Tatsachen aus der Paläontologie

- Übergangsformen 
- Tatsachen aus der vergleichenden Anatomie

- Tatsachen aus der vergleichenden Physiologie

- Tatsachen aus der Embryologie

- Tatsachen aus der Tier- und Pflanzengeographie

- Faktoren der stammesgeschichtlichen Entwicklung (12 Seiten; 12,9\%)

- Die Geschichte der Organismen (25 Seiten; 26,9\%)

- Die Entstehung der lebenden Materie

- Die Geschichte der Organismen

- Die Stammesgeschichte des Menschen

- Zur Geschichte der Entwicklungslehre (19 Seiten; 20,4 \%)

- Die Entwicklungslehre Lamarcks

- Charles Darwin und seine Lehre

- Die Verbreitung des Darwinismus

- $\quad$ Anhang (8 Seiten; 8,6 \%)

- Beziehungen zwischen Stammbaum und System

- Übersicht über das System der Organismen

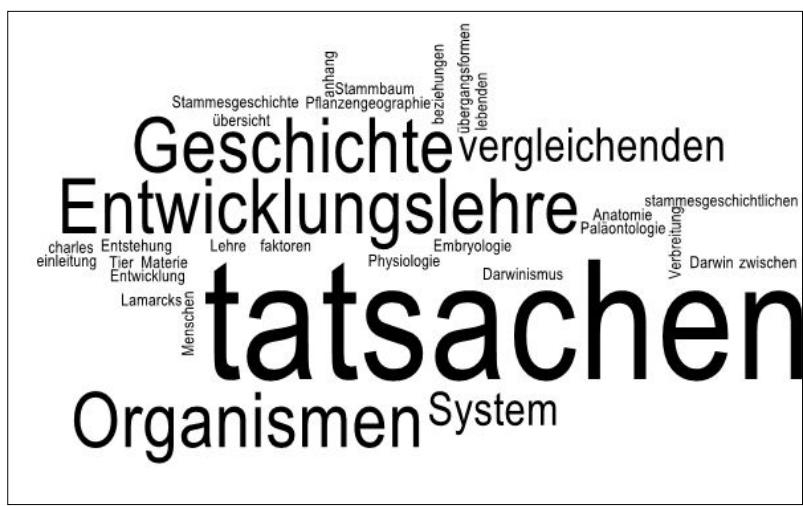

Abbildung 73: Biologische Begriffe im Inhaltsverzeichnis, Lehrbuch Klasse 10 (1960)

Eine Frequenzanalyse der Kapitelüberschriften zeigt eine zentrale Stellung des biologischen Begriffs Tatsachen. Weitere zentrale Begriffe waren Entwicklungslehre, Geschichte und Organismen (Abb. 73). Die Inhaltsanalyse der Texte des Lehrbuches von 1960 zeigt die Gliederung Didaktisches - Paläontologie - Neontologie Theorien - Biogenese - Paläontologie - Hominisation - Theorien auf. Dabei umfasste die Kategorie Theorien die längste zusammenhängende Textpassage (Abb. 74). Themen aus den Kategorien Paläontologie und Theorien wurden an zwei verschiedenen Stellen im Lehrbuch behandelt. Dass Textinhalten aus der Kategorie Theorien mit 979 Zeilen (36,1 \%) der größte Stellenwert zukam, zeigt die Raum Analyse. In der Reihenfolge des Umfangs umfassten die Kategorien 
Paläontologie 512 Zeilen (18,9 \%), Neontologie 437,5 Zeilen (16,1\%), Systematik 282 Zeilen (10,4 \%), Hominisation 239,5 Zeilen (8,8 \%), Biogenese 193,5 Zeilen (7,1\%) sowie Didaktisches 69 Zeilen (2,5\%).

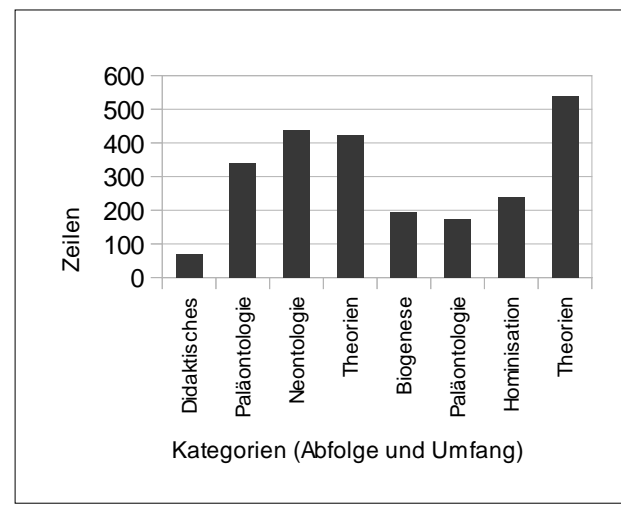

Abbildung 74: Kategorien im Lehrbuch

Klasse 10 (1960)

Das Lehrbuch von 1965

Die Inhalte im Lehrbuch von 1965 waren in elf heterogen strukturierte Kapitel gegliedert. Im Gegensatz zum Vorgängermodell enthielt das Schülermaterial hier auch Kapitel zur Genetik und Züchtungsbiologie. So behandelte das neunte Kapitel Grundlagen der Vererbung und das elfte und letzte Kapitel Die Züchtung von Pflanzen und Tieren. Den evolutionsbiologischen Kapiteln lag folgende Feinstruktur zu Grunde:

- $\quad$ Einleitung (2 Seiten; 1,9\%)

- Tatsachen beweisen die Entwicklungslehre (25 Seiten; 23,1 \%)

- Aus der Paläontologie

- Entwicklungsreihen

- Übergangsformen

- Aus der vergleichenden Anatomie

- Aus der vergleichenden Physiologie

- Aus der Embryonal- und Jugendentwicklung

- Aus der Tier- und Pflanzengeographie

- Wesen und Entstehung des Lebens (18 Seiten; 16,7 \%)

- Das Wesen des Lebens

- Stoffliche Zusammensetzung der Lebewesen

- Eigenschaften des Lebens

- Das Leben als spezifische Bewegungsform der Materie

- Die historische Entwicklung der Kenntnis vom Leben 
- Die Entstehung des Lebens

- Überholte Vorstellungen von der Entstehung des Lebens

- Die Organismen verschiedener Erdzeitalter (10 Seiten; 9,3 \%)

- Abstammung und Entwicklung des Menschen (14 Seiten; 13\%)

- Die Stellung des Menschen im Organismenreich

- Fossile Zeugnisse der Menschwerdung

- Die heutigen Menschenrassen

- Weitere biologische Entwicklungsvorgänge beim heutigen Menschen

- Stammesgeschichte und System der Organismen (2 Seiten; 1,9\%)

- Übersicht über das natürliche System der Organismen (7 Seiten; 6,5 \%)

- Zur Geschichte der Entwicklungslehre (17 Seiten; 15,7 \%)

- Die Entwicklungslehre Lamarcks

- Charles Darwin und seine Lehre

- Die Verbreitung des Darwinismus

- Faktoren der stammesgeschichtlichen Entwicklung (13 Seiten; $12 \%$ )

- Die Selektion

- Die Mutation

- Die Isolation

- Populationswellen

- Die Kombination

- Das Zusammenwirken der Evolutionsfaktoren

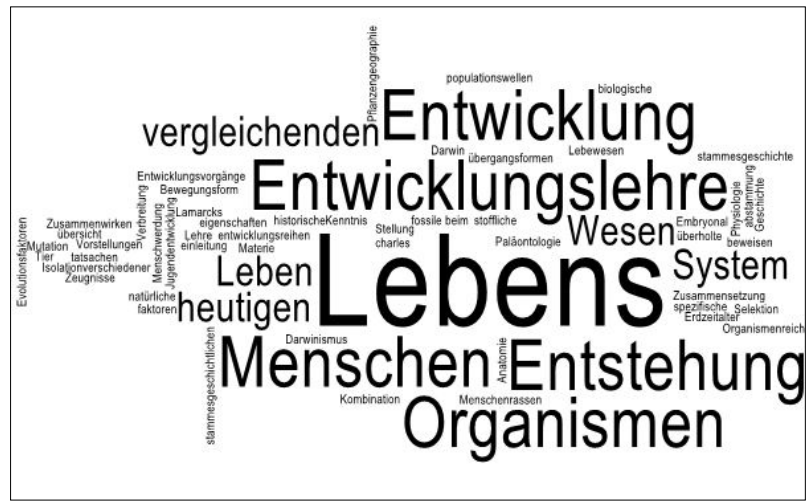

Abbildung 75: Biologische Begriffe im Inhaltsverzeichnis, Lehrbuch Klasse 10 (1965)

Die Frequenzanalyse der Kapitelüberschriften ergab, dass das Wort Lebens den zentralen biologischen Begriff darstellte. Dies ergab sich aus dem erstmals aufgenommenen, umfangreichen und eigenständigen Kapitel zur Biogenese. Weitere zentrale biologische Begriffe lauteten Entstehung, Entwicklung, Entwicklungslehre sowie Menschen und Organismen (Abb. 75). 
Die Inhaltsanalyse der Texte des Lehrbuches von 1965 verdeutlicht die Gliederung Didaktisches - Paläontologie - Neontologie - Biogenese - Paläontologie - Hominisation - Systematik - Theorien. Anders als im Lehrbuch von 1960 umfasste die Kategorie Biogenese die längste zusammenhängende Textpassage (Abb. 76). An zwei verschiedenen Stellen im Lehrbuch wurde die Kategorie Paläontologie aufgenommen. Darüber hinaus zeigt die Raum Analyse, dass Textinhalten aus der Kategorie Biogenese mit 630 Zeilen

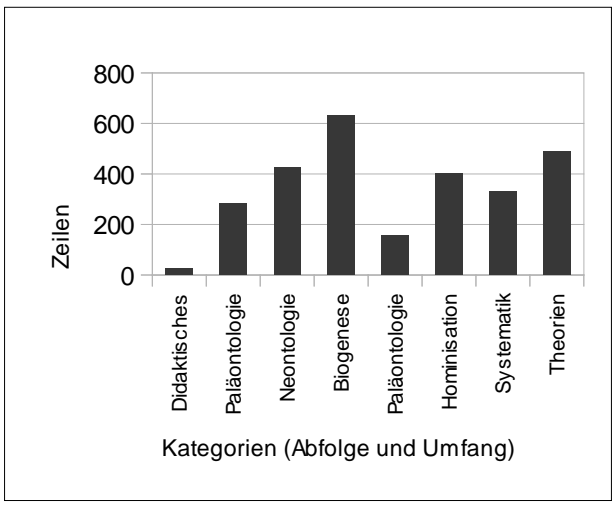

Abbildung 76: Kategorien im Lehrbuch Klasse 10 (1965) (23\%) der größte Stellenwert zukam.

In der Reihenfolge des Umfangs umfassten die Kategorien Theorien 488,5 Zeilen (17,8 \%), Paläontologie 438,5 Zeilen (16,0 \%), Neontologie 423,5 Zeilen (15,5\%), Hominisation 400,5 Zeilen (14,6\%), Systematik 329 Zeilen (12\%) sowie Didaktisches 27 Zeilen (1\%).

\section{Das Lehrbuch von 1968}

Das Lehrbuch von 1968 enthielt Kapitel mit Inhalten aus der Genetik, Evolutionsbiologie, Züchtung und Ökologie. Alle Inhalte aus dem Bereich der Evolutionsbiologie waren unter dem Begriff Abstammungslehre zusammengefasst und wurden zu Beginn auf einer Seite (1,5\%) eingeleitet. Diesem evolutionsbiologischen Kapitel lag folgende Feinstruktur zu Grunde:

- Faktoren der stammesgeschichtlichen Entwicklung (2 Seiten; 3,1 \%)

- Mutation

- Selektion

- Das Zusammenwirken der Evolutionsfaktoren (4 Seiten; 6,2 \%)

- Evolution und Ontogenese (3 Seiten; 4,6 \%)

- Homologie und Analogie (6 Seiten; 9,2\%)

- Homologe Organe

- Analoge Organe

- Rudimentäre Organe

- Der fossile Befund (11 Seiten; 16,9\%)

- Die Fossilien und ihre Entstehung

- Die Gliederung der Erdgeschichte

- Übergangsformen

- Entwicklungsreihen

- Abstammung und Entwicklung des Menschen (14 Seiten; 21,5 \%) 
- Die Stellung des Menschen im Organismenreich

- Fossile Zeugnisse der Menschwerdung

- Die heutigen Menschenrassen

- Stammesgeschichte und System der Organismen (2 Seiten; 3,1 \%)

- Das natürliche System der Organismen

- Die binäre Nomenklatur

- Stammbaum und System

- Zur Geschichte der Abstammungslehre (9 Seiten; 13,8 \%)

- Die Entstehung des Lebens auf der Erde (13 Seiten; $20 \%$ )

- Das Wesen des Lebens

- Die Entstehung des Lebens

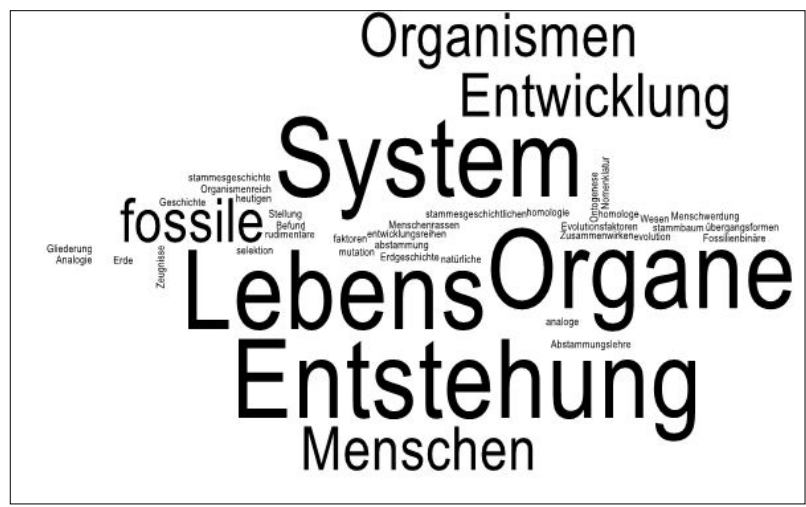

Abbildung 77: Biologische Begriffe im Inhaltsverzeichnis, Lehrbuch Klasse 10 (1968)

Eine Frequenzanalyse biologischer Begriffe aus den Kapitelüberschriften im Lehrbuch von 1968 belegt, dass Präferenzen für verschiedene Wörter vorlagen (Abb. 77). Die Inhaltsanalyse der Texte des Lehrbuches von 1968 zeigt die Gliederung Didaktisches - Theorien Neontologie - Paläontologie - Hominisation - Systematik - Theorien Biogenese auf. Inhalte aus der Kategorie Biogenese umfassten, wie bereits im Lehrbuch von 1965, die längste zusammenhängende Textpassage (Abb. 78). Die kategoriale Raum Analyse verdeutlicht, dass Textinhalten aus der Kategorie Theorien mit 561 Zeilen (29,6 \%) der größte Stellenwert zukam. Des Weiteren umfassten in der Reihen-

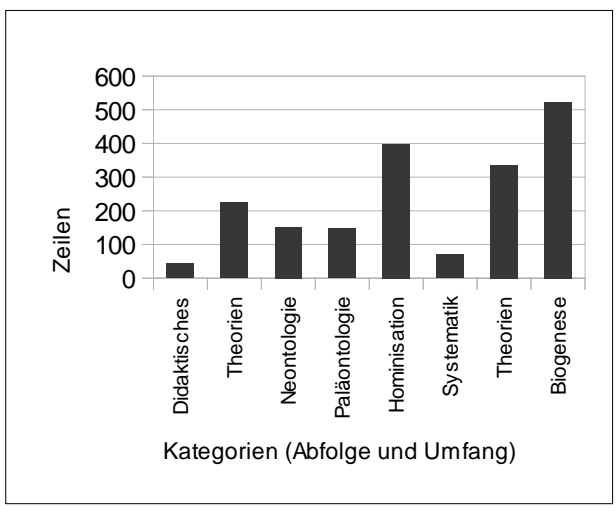

Abbildung 78: Kategorien im Lehrbuch Klasse 10 (1968) 
folge des Umfangs die Kategorien Biogenese 523 Zeilen (27,6 \%), Hominisation 398 Zeilen (21,0 \%), Neontologie 152 Zeilen (8,0 \%), Paläontologie 174,5 Zeilen (7,8 \%), Systematik 71 Zeilen (3,7\%) sowie Didaktisches 43 Zeilen (2,3\%).

Das Lehrbuch von 1971

Die Inhalte aus dem Lehrbuch von 1971 waren in vier Kapitel gegliedert: Genetik, Abstammungslehre, Tier- und Pflanzenzüchtung als Weiterfübrung der Evolution durch den Menschen sowie Die Biosphäre und der Mensch. Dem Forschungsinteresse folgend, wird an dieser Stelle ausschließlich die Struktur des zweiten Kapitels näher betrachtet. Dieses wurde mit einer Abbildung im Umfang von einer Seite (1,3\%) eingeleitet (Kategorie Didaktisches). Folgende Feinstruktur lag diesem Kapitel zu Grunde:

- Theorie der Stammesentwicklung (16 Seiten; 21,1 \%)

- Genetische Verhältnisse in Populationen

- Isolation und Artneubildung

- Individual- und Stammesentwicklung

- Stammesentwicklung der Pflanzen und Tiere (17 Seiten; 22,4 \%)

- Entstehung der Fossilien

- Entwicklung der Organismen in den verschiedenen Erdzeitaltern

- Übergangsformen

- Aus der Geschichte der Abstammungslehre (11 Seiten; 14,5 \%)

- Die Schaffung naturwissenschaftlicher Voraussetzungen für die wissenschaftliche Abstammungslehre

- Die unmittelbaren Vorläufer der wissenschaftlichen Abstammungslehre

- Wissenschaftliche Abstammungslehre

- Der Kampf um die Durchsetzung des Darwinismus

- Die Entstehung des Lebens auf der Erde (10 Seiten; 13,2 \%)

- Kennzeichen der Lebewesen

- Die Entstehung des Lebens

- Die Stammesentwicklung des Menschen (18 Seiten; 23,7 \%)

- Tier und Mensch

- Die heutigen Menschenrassen

- Wiederholung und Systematisierung (3 Seiten; 3,9\%)

- Höherentwicklung und Spezialisierung

- Zeitlicher Ablauf der Stammesentwicklung

- Übergangsformen 


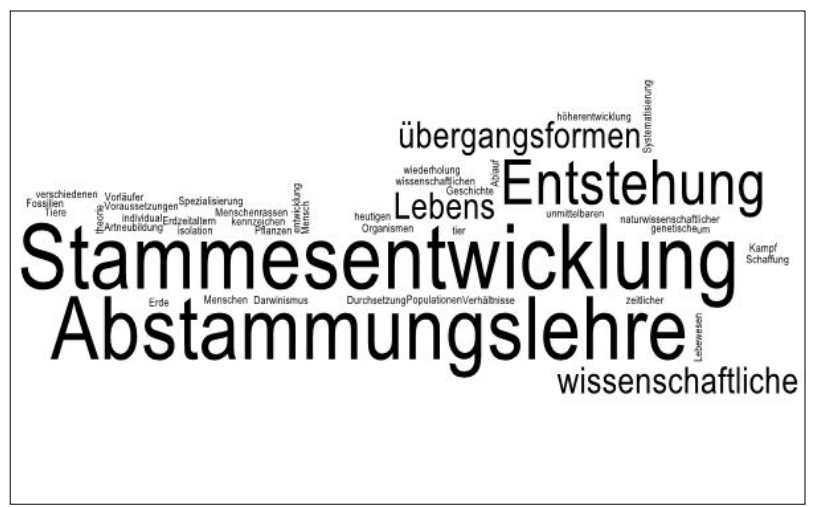

Abbildung 79: Biologische Begriffe im Inhaltsverzeichnis, Lehrbuch Klasse 10 (1971)

Die biologischen Begriffe Stammesentwicklung sowie Abstammungslehre waren, entsprechend einer Frequenzanalyse, zentrale Worte der Kapitelüberschriften im Lehrbuch von 1971 (Abb. 79). Die Inhaltsanalyse der Lehrbuchtexte verdeutlicht die Gliederung Theorien - Neontologie - Paläontologie - Theorien - Biogenese Hominisation - Didaktisches. Inhalte aus der Kategorie Hominisation umfassten, im Unterschied zu den Vorgängermodellen, die längste zusammenhängende Textpassage (Abb. 80). Neu war die Aufnahme von Inhalten aus der Kategorie Didaktisches am Ende des Lehrbuches und das Fehlen von Inhalten aus der Kategorie Systematik. Inhalte aus der Kategorie Theorien wurden erneut an zwei verschiedenen Stellen im Lehrbuch behandelt. Die Raum Analyse verdeutlicht, dass Textinhalten aus der Kategorie Theorien mit 442 Zeilen $(27,4 \%)$ weiterhin der größte Stellenwert zukam. In der weiteren Reihenfolge umfassten die Kategorien Hominisation 391 Zeilen (24,2 \%), Biogenese 331 Zeilen (20,5\%), Paläontologie 242 Zeilen (15,0\%), Neontologie 138 Zeilen $(8,5 \%)$ sowie Didaktisches 72 Zeilen $(4,5 \%)$.

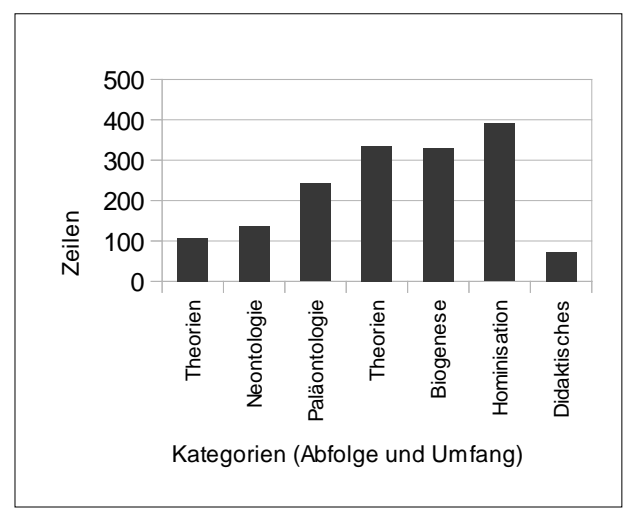

Abbildung 80: Kategorien im Lehrbuch Klasse 10 (1971)

Das Lehrbuch von 1988

Die Inhalte aus dem Lehrbuch von 1988 waren in zwei Hauptabschnitte gegliedert und den Begriffen Genetik bzw. Evolution der Organismen zugeordnet. Dem Forschungsinteresse folgend, wurde lediglich der zweite Abschnitt betrachtet. Diesem 
war eine Einleitung im Umfang von einer Seite (1,4\%) vorangestellt, die Inhalte aus der Kategorie Didaktisches aufwies. Alle weiteren Inhalte waren in drei Bereiche gegliedert. Der erste Themenkomplex setzte sich aus den Kapiteln Wissenschaftliche Abstammungslehre von Charles Darwin, Auffassungen Lamarcks über die Herkunft der Arten, Die Verbreitung der Abstammungslehre Darwins durch Ernst Haeckel, Bestätigung und Weiterentwicklung der Abstammungslehre Darwins, Evolutionsfaktoren, Evolutionsrichtungen sowie Aufgaben zur Wiederholung zusammen. Mit 22 Seiten umfassten diese Kapitel 31,9 \% und beinhalteten Informationen aus den Kategorien Theorien, Neontologie sowie Didaktisches. Der zweite Bereich gliederte sich in die Kapitel Entstehung des Lebens, Stammesentwicklung der Organismen, Fossilien, Etappen der Stammesentwicklung, Wirbeltiere mit Merkmalen von zwei Wirbeltierklassen sowie Aufgaben zur Wiederholung. Diese Kapitel besaßen einen Umfang von 21 Seiten (30,4 \%) und beinhalteten Aussagen aus den Kategorien Biogenese, Paläontologie sowie Didaktisches. Der dritte Bereich setzte sich aus den Kapiteln Abstammung und Entwicklung des Menschen, Herausbildung des Menschen aus tierischen Vorfahren, Entwicklung bis zum Menschen der Gegenwart, Menschenrassen und ibre Entstehung sowie Aufgaben zur Wiederholung zusammen. Mit 25 Seiten umfassten diese Kapitel 36,2 \% und beinhalteten Angaben aus den Kategorien Hominisation sowie Didaktisches. Das Ergebnis einer Frequenzanalyse der Kapitelüberschriften zeigt eine Präferenz für die Begriffe Menschen, Abstammungslehre und Wiederholung (Abb. 81).

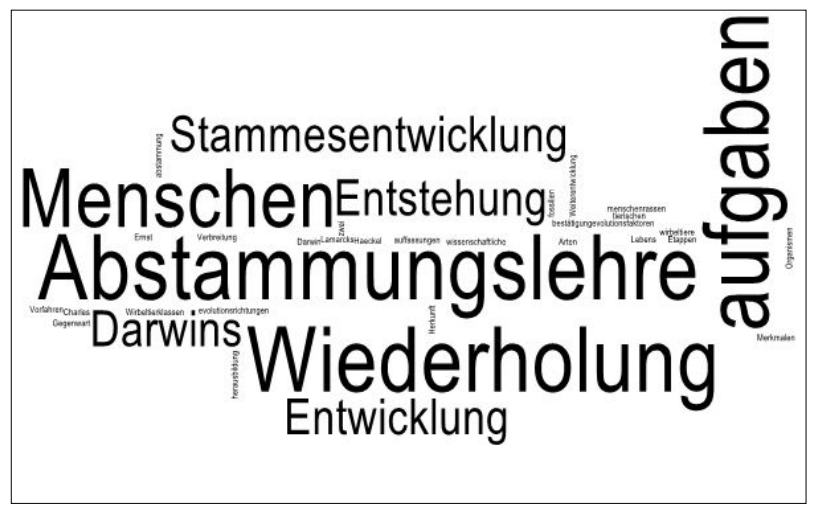

Abbildung 81: Biologische Begriffe im Inhaltsverzeichnis, Lehrbuch Klasse 10 (1988)

Die Inhaltsanalyse der Lehrbuchtexte von 1988 belegt die Gliederung Didaktisches - Theorien - Neontologie - Didaktisches - Biogenese - Paläontologie Didaktisches - Hominisation - Didaktisches. Inhalte aus der Kategorie Hominisation umfassten, wie bereits im Lehrbuch von 1971, die längste zusammenhängende Textpassage (Abb. 82). Ebenso fehlten Inhalte aus der Kategorie Systematik. Die Kategorie Didaktik wurde an vier verschiedenen Stellen im Lehrbuch aufgenommen. Inhalte aus der Kategorie Theorien behandelte das Lehrbuch, im Ge- 
gensatz zu den Vorgängermodellen von 1960, 1968 und 1971, zusammenhängend. Die Raum Analyse verdeutlicht einen weiteren Unterschied $\mathrm{zu}$ den Lehrbüchern von 1960 bis 1971. Es zeigt sich, dass nun Textinhalten aus der Kategorie Hominisation mit 493 Zeilen (35,0 \%) der größte Stellenwert zukam. In weiterer Reihenfolge umfassten die Kategorien Theorien 335 Zeilen (23,8 \%), Paläontologie 195 Zeilen (13,8 \%), Didaktisches 145 Zeilen (10,3\%), Neontologie 135 Zei- Abbildung 82: Kategorien im Lehrbuch len $(9,6 \%)$ sowie Biogenese 106 Zeilen Klasse 10 (1988) $(7,5 \%)$.

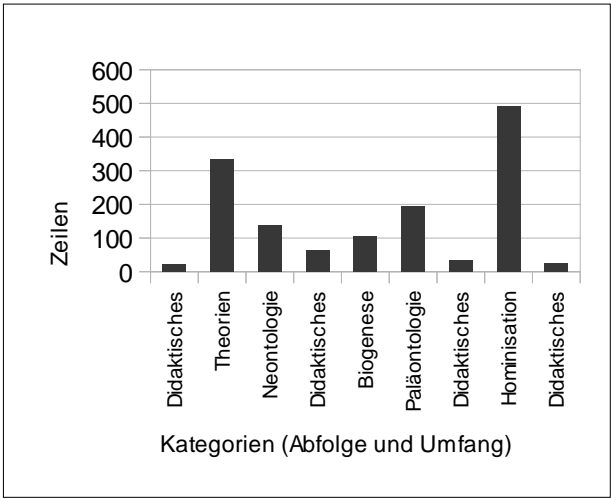

\section{Zusammenfassung}

Die Raumanalyse belegt einen hohen Stellenwert der Kategorie Theorien. Deutlich wird auch, dass der Umfang von Inhalten aus der Kategorie Hominisation zwischen 1960 und 1988 stetig zunahm. Dagegen stand den Schülern die Kategorie Systematik, zumindest innerhalb evolutionsbiologischer Kapitel, ab 1971 nicht mehr zur Verfügung. Während die Lehrbücher von 1960 und 1988 die Kategorie Biogenese nur in geringem Umfang behandelten, präferierte insbesondere im Lehrbuch von 1968 diese Kategorie (Abb. 83).

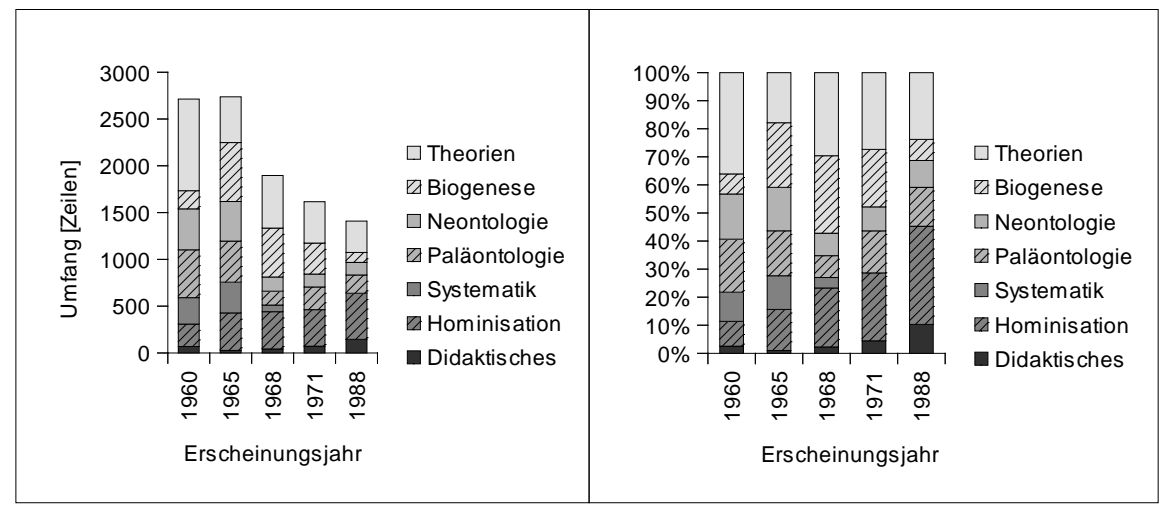

Abbildung 83: links: absoluter Umfang, rechts: relativer Umfang der Kategorien in den Lehrbüchern der Klasse 10 
Tabelle 43: Strukturierung der Lehrbücher für die Klasse 10

\begin{tabular}{|c|c|c|c|c|c|c|}
\hline Ausgabe & Kapitel & $\begin{array}{l}\text { Anzahl der } \\
\text { Unterkapitel }\end{array}$ & $\begin{array}{l}\text { absoluter } \\
\text { Umfang } \\
\text { [Seiten] }\end{array}$ & $\begin{array}{l}\text { relativer } \\
\text { Umfang } \\
{[\%]}\end{array}$ & Stellenwert & Kategorie \\
\hline \multirow[t]{6}{*}{1960} & $E$ & 0 & 2 & 2,2 & 6 & $D$ \\
\hline & 1 & 6 & 27 & 29,0 & 1 & $\mathrm{P}, \mathrm{N}$ \\
\hline & 2 & 0 & 12 & 12,9 & 4 & $T$ \\
\hline & 3 & 3 & 25 & 26,9 & 2 & $\mathrm{~T}, \mathrm{~B}, \mathrm{P}, \mathrm{H}$ \\
\hline & 4 & 3 & 19 & 20,4 & 3 & $\mathrm{~T}$ \\
\hline & 5 & 2 & 8 & 8,6 & 5 & $S$ \\
\hline$\Sigma$ & 6 & 14 & 93 & 100,0 & & \\
\hline \multirow[t]{9}{*}{1965} & $E$ & 0 & 2 & 1,9 & 9 & $\mathrm{D}$ \\
\hline & 1 & 7 & 25 & 23,1 & 1 & $\mathrm{P}, \mathrm{N}$ \\
\hline & 2 & 7 & 18 & 16,7 & 2 & $B$ \\
\hline & 3 & 0 & 10 & 9,3 & 6 & $P$ \\
\hline & 4 & 4 & 14 & 13,0 & 4 & $\mathrm{H}$ \\
\hline & 5 & 0 & 2 & 1,9 & 8 & $S$ \\
\hline & 6 & 0 & 7 & 6,5 & 7 & $S$ \\
\hline & 7 & 3 & 17 & 15,7 & 3 & $\mathrm{~T}$ \\
\hline & 8 & 6 & 13 & 12,0 & 5 & $\mathrm{~T}$ \\
\hline$\sum$ & 9 & 27 & 108 & 100,0 & & \\
\hline \multirow[t]{10}{*}{1968} & $E$ & 0 & 1 & 1,5 & 9 & $\mathrm{D}$ \\
\hline & 1 & 2 & 2 & 3,1 & 8 & $T$ \\
\hline & 2 & 0 & 4 & 6,2 & 6 & $T$ \\
\hline & 3 & 0 & 3 & 4,6 & 7 & $\mathrm{~N}$ \\
\hline & 4 & 3 & 6 & 9,2 & 5 & $\mathrm{~N}$ \\
\hline & 5 & 4 & 11 & 16,9 & 3 & $P$ \\
\hline & 6 & 3 & 14 & 21,5 & 1 & $\mathrm{H}$ \\
\hline & 7 & 3 & 2 & 3,1 & 8 & $S$ \\
\hline & 8 & 0 & 9 & 13,8 & 4 & $T$ \\
\hline & 9 & 2 & 13 & 20,0 & 2 & B \\
\hline$\Sigma$ & 10 & 17 & 65 & 100,0 & & \\
\hline \multirow[t]{7}{*}{1971} & $E$ & 0 & 1 & 1,3 & 7 & $\mathrm{D}$ \\
\hline & 1 & 3 & 16 & 21,1 & 3 & $\mathrm{~T}, \mathrm{~N}$ \\
\hline & 2 & 3 & 17 & 22,4 & 2 & $P$ \\
\hline & 3 & 4 & 11 & 14,5 & 4 & $T$ \\
\hline & 4 & 2 & 10 & 13,2 & 5 & B \\
\hline & 5 & 2 & 18 & 23,7 & 1 & $\mathrm{H}$ \\
\hline & 6 & 3 & 3 & 3,9 & 6 & $D$ \\
\hline$\Sigma$ & 7 & 17 & 76 & 100,0 & & \\
\hline \multirow[t]{5}{*}{1988} & $E$ & 0 & 1 & 1,4 & 4 & $\mathrm{D}$ \\
\hline & 1 & 7 & 22 & 31,9 & 2 & $\mathrm{~T}, \mathrm{~N}, \mathrm{D}$ \\
\hline & 2 & 6 & 21 & 30,4 & 3 & $B, P, D$ \\
\hline & 3 & 5 & 25 & 36,2 & 1 & $\mathrm{H}, \mathrm{D}$ \\
\hline & 4 & 18 & 69 & 100,0 & & \\
\hline
\end{tabular}


Mit drei umfangreichen und zwei vom Umfang geringeren Kapiteln war das Lehrbuch von 1960 heterogen strukturiert. Dabei lag der Schwerpunkt auf dem Kapitel Tatsachen aus der Entwicklungslehre, das sich am Anfang des Lehrbuches befand. Bis auf zwei waren alle Kapitel im Lehrbuch von 1965 weiter strukturiert, variierten jedoch deutlich im Umfang. Der Fokus lag, wie bereits im Lehrbuch von 1960, auf dem Kapitel Tatsachen beweisen die Entwicklungslehre. Die Kapitel im Lehrbuch von 1968 unterschieden sich deutlich in Umfang und Gliederung. Im Vordergrund standen, im Unterschied zu den Vorgängermodellen, die Kapitel Abstammung und Entwicklung des Menschen sowie Die Entstebung des Lebens auf der Erde. Den größten Umfang im Lehrbuch von 1971 wies das Kapitel Die Stammesentwicklung des Menschen auf. Eine homogene Struktur kennzeichnete das Lehrbuch von 1988. Alle drei Kapitel umfassten etwa ein Drittel des Lehrbuches. Eine Verflechtung von Inhalten aus verschiedenen Kategorien war für die Kapitel typisch. Im Vergleich zu den Vorgängermodellen integrierten die Autoren auch die Aufnahme von Zusammenfassungen am Ende eines jeden Kapitels (Tab. 43).

\section{Fachinhalte}

Evolutionstheorien

Das Ergebnis einer Frequenzanalyse im Text aufgenommener historischer Personen in den Lehrbüchern für die Klasse 10 zwischen 1965 und 1968 belegt deutliche Unterschiede in der thematischen Breite. Der Vielzahl der genannten Personen in den Ausgaben von 1960 und 1965 stand eine Reduktion entsprechender Inhalte ab 1968 gegenüber. Ebenso differierte in den Lehrbüchern die Häufigkeit der Nennungen der jeweiligen Personen und somit die thematische Intensität (Tab. 44). Den höchsten Stellenwert nahm, wie auch in den Lehrbüchern für den 8. Jahrgang, die Person von Darwin ein (Abb. 84). Ebenfalls ein hoher Stellenwert kam Ernst Haeckel und Jean de Baptiste Lamarck zu.

Tabelle 44: Frequenzanalyse (Gesamt), Lehrbücher Klasse 10

\begin{tabular}{|lrrrrr|}
\hline Auflage & 1960 & 1965 & 1968 & 1971 & 1988 \\
Anzahl der Personen & 37 & 40 & 19 & 19 & 15 \\
Summe der Nennungen & 181 & 196 & 115 & 95 & 112 \\
\hline
\end{tabular}

\section{Darwinismus}

Alle Lehrbücher enthielten Aussagen zum Darwinismus und zu Darwin. Die Inhalte der Lehrbücher von 1960 und 1965 glichen in großen Teilen den Inhalten aus dem Lehrbuch der 8. Klasse von 1957. Umfangreich informierte sie über Darwins Leben vor und nach seiner Reise, Erlebnisse während seiner Reise sowie einige theoretische Ansichten aus seinem Werk. Im Lehrbuch von 1968 lag der Schwerpunkt dagegen auf Darwins Theorien. Dieser Bereich erfuhr dann in den 
Lehrbüchern von 1971 und 1988 eine starke Reduzierung. Gleichfalls reduziert wurden ab 1968 die Inhalte aus Darwins Leben vor und nach der Reise. Als Schwerpunkt erhalten blieben Eindrücke von der Fahrt mit der Beagle sowie seinen Exkursionen in Südamerika und auf dem Galapagos-Archipel. Während die Autoren Darwin als Begründer einer wissenschaftlichen Evolutionstheorie in allen Lehrbüchern vorstellten, fand Wallace lediglich in den Schulbüchern von 1960 und 1965 in diesem Kontext Erwähnung (Tab. 45; Abb. 84).

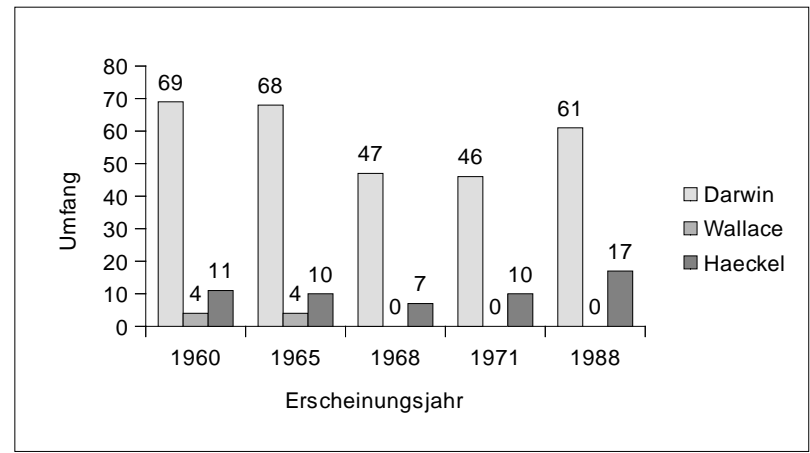

Abbildung 84: Darwin, Wallace und Haeckel in den Lehrbüchern der Klasse 10

Tabelle 45: Darwins Leben und Werk, Lehrbücher Klasse 10

\begin{tabular}{|c|c|c|c|c|}
\hline Aufl. & Vor der Reise & Reisebeschreibung & Nach der Reise & Theoretische Aspekte \\
\hline $1960 / 65$ & $\begin{array}{l}\text { Herkunft, } \\
\text { Schulbildung, } \\
\text { Vorlieben, } \\
\text { Medizinstudium, } \\
\text { erste } \\
\text { Entdeckungen, } \\
\text { Theologiestudium }\end{array}$ & $\begin{array}{l}\text { Grund der Reise, } \\
\text { Vermittlung, Dauer, } \\
\text { Anschauungen } \\
\text { Lyells, Exkursionen } \\
\text { in Südamerika, } \\
\text { Grundfinken auf } \\
\text { Galapagos }\end{array}$ & $\begin{array}{l}\text { Reisetagebuch, Landsitz } \\
\text { in Down, Entwurf des } \\
\text { Artenbuches, Darwins } \\
\text { Arbeitsweise, Pflanzen- } \\
\text { samen, Taubenzucht, } \\
\text { Wallace, Vorlage des } \\
\text { Manuskripts, Auflage des } \\
\text { Artenbuches }\end{array}$ & $\begin{array}{l}\text { Variabilität, Überpro- } \\
\text { duktion, natürliche } \\
\text { Zuchtwahl, Kampf } \\
\text { ums Dasein, Gesetze } \\
\text { der Abänderung, } \\
\text { Beweise }\end{array}$ \\
\hline 1968 & Vorlieben, Studium & $\begin{array}{l}\text { Anschauungen } \\
\text { Lyells, Exkursionen } \\
\text { in Südamerika und } \\
\text { auf den Galapagos- } \\
\text { Inseln }\end{array}$ & $\begin{array}{l}\text { Arbeitsweise, Tauben- } \\
\text { zucht, Auflage des Arten- } \\
\text { buches, weitere Werke }\end{array}$ & $\begin{array}{l}\text { Variabilität, Überpro- } \\
\text { duktion, natürliche } \\
\text { und künstliche } \\
\text { Zuchtwahl, Kampf } \\
\text { ums Dasein, Gesetze } \\
\text { der Abänderung, } \\
\text { Beweise, Verbreitung } \\
\text { der Lebewesen }\end{array}$ \\
\hline 1971 & & & $\begin{array}{l}\text { Taubenzucht, Auflage des } \\
\text { Artenbuches }\end{array}$ & $\begin{array}{l}\text { Zuchtwahl (künstli- } \\
\text { che, natürliche, } \\
\text { geschlechtliche) }\end{array}$ \\
\hline 1988 & $\begin{array}{l}\text { Herkunft, Studium, } \\
\text { Vorlieben }\end{array}$ & & $\begin{array}{l}\text { Taubenzucht, Auflage des } \\
\text { Artenbuches, Auseinan- } \\
\text { dersetzungen }\end{array}$ & $\begin{array}{l}\text { Zuchtwahl (künstli- } \\
\text { che, natürliche) }\end{array}$ \\
\hline
\end{tabular}


Aussagen zu den fünf Darwin'schen Theorien waren in den Schulbüchern nur zum Teil vorhanden. Diese wurden jedoch nicht immer als Gedanken Darwins benannt, sondern häufig an anderen Stellen im Lehrbuch behandelt. Zum Beispiel enthielt das Lehrbuch von 1971 innerhalb der Kategorie Theorien keine Aussagen über die Vervielfachung der Arten. Innerhalb anderer Kategorien fand jedoch durch Stammbäume die Aufspaltung der Arten eine Darstellungsform. Auch die Ausgabe von 1988 enthielt in der Kategorie Theorien dazu keine Aussagen. Ausführlich erläuterten die Autoren hingegen in allen Lehrbüchern die Selektionstheorie. Die übrigen Theorien standen den Schülern meist nur latent in einem Satz zur Verfügung (Tab. 46).

Tabelle 46: Ankerbeispiele für die fünf Darwin'schen Theorien, Lehrbücher Klasse 10

\begin{tabular}{|c|c|c|c|c|c|}
\hline Aufl. & |Veränderlichkeit & $\begin{array}{l}\text { Abstammungsleh- } \\
\text { re }\end{array}$ & Gradualismus & Aufspaltung & Selektionslehre \\
\hline 1960 & \multirow{3}{*}{ 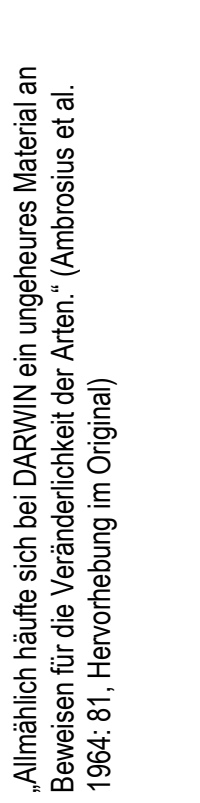 } & \multirow{3}{*}{ 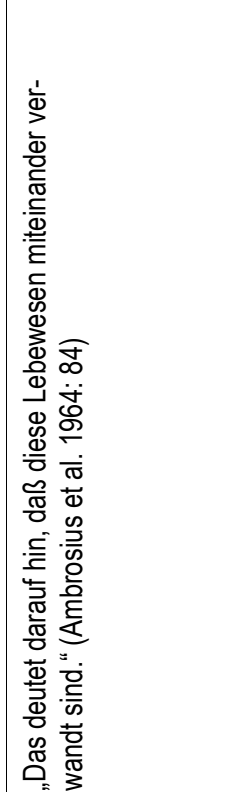 } & \multirow{3}{*}{ 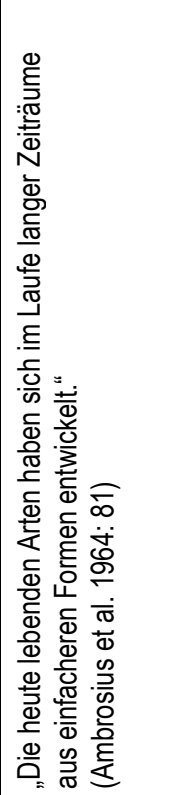 } & \multirow[b]{2}{*}{ 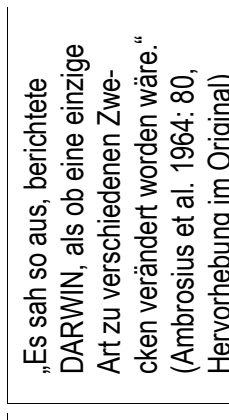 } & \multirow{3}{*}{ 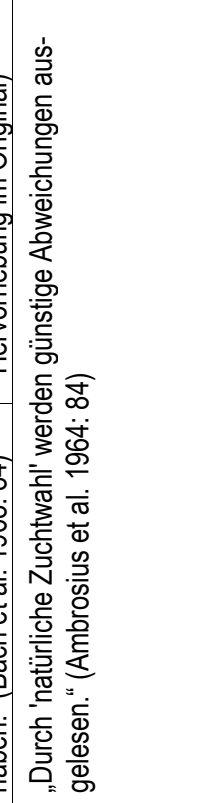 } \\
\hline 1965 & & & & & \\
\hline 1968 & & & & 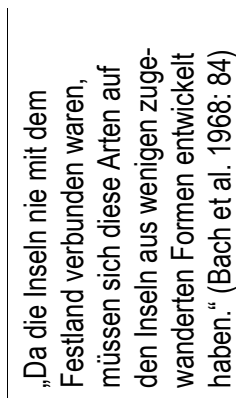 & \\
\hline
\end{tabular}




\begin{tabular}{|c|c|c|c|c|c|}
\hline 1971 & 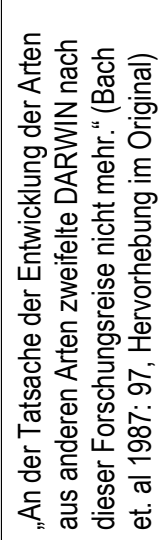 & 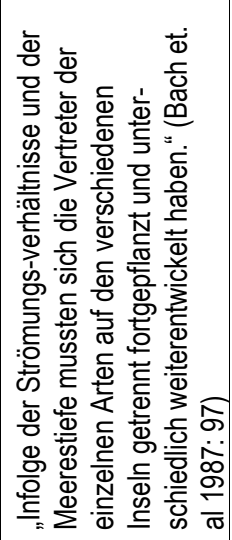 & 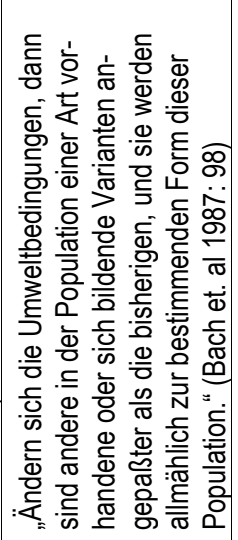 & - & 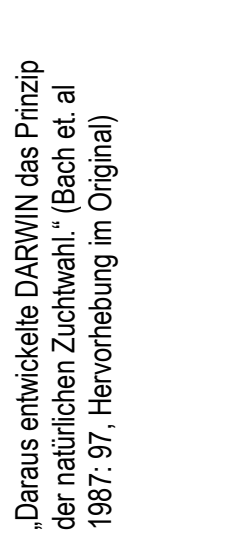 \\
\hline 1988 & 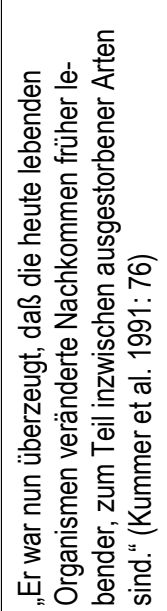 & & 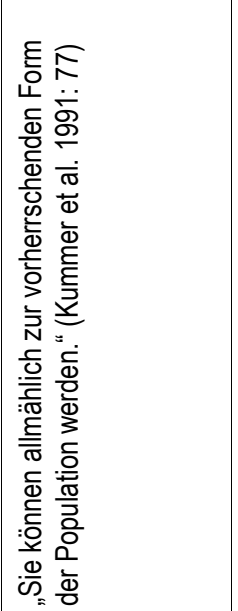 & - & 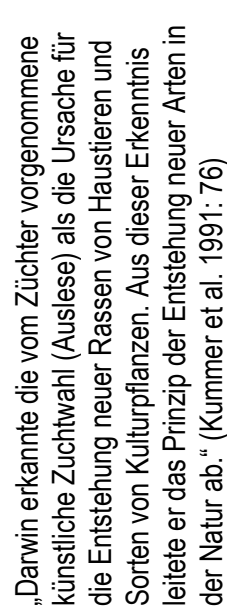 \\
\hline
\end{tabular}

Das Lehrbuch von 1960 enthielt Abbildungen von Darwin um 1849 und 1880. Auch die Beagle und ein Teil seiner Reiseroute (Südamerika) wurden dargestellt. Ebenso waren Grundfinkenarten, Darwins Landhaus in Down sowie eine Kerguelenfliege abgebildet. In der Ausgabe von 1965 entfiel die Abbildung der Reiseroute. Das Lehrbuch von 1968 enthielt erneut die Route sowie eine Abbildung von Darwin um 1849. Im der Ausgabe von 1971 stand den Schülern eine Fotografie von Darwin um 1880 und die Reiseroute (Südamerika) zur Verfügung. Das Lehrbuch von 1988 zeigte das Titelblatt aus Darwins Werk On the Origin of Species in englischer und deutscher Ausgabe, ein Darwin-Portrait um 1880, die Beagle sowie die gesamte Reiseroute. 


\section{Neodarwinismus vs. Schöpferischer Darwinismus}

Auf die Arbeiten Mitschurins wurde im Rahmen der Kategorie Theorien nur im Lehrbuch von 1960 hingewiesen. Im Kapitel Faktoren der stammesgeschicbtlichen Entwicklung propagierten die Verantwortlichen die Mitschurin'sche Biologie, jedoch ohne ein theoretisches Fundament anzubieten:

„Die MITSCHURINsche Genetik ist gegenwärtig noch in voller Entwicklung und verfügt zur Zeit noch nicht über ein abgerundetes Theoriensystem. Jedoch läßt sich schon jetzt sagen, daß die umfassende Lösung von Vererbung, Veränderung und Entwicklung nur von ihr aus zu finden sein wird.“ (Ambrosius et al. 1964: 37, Hervorhebung im Original)

Damit verbunden war die Vermittlung von einer Vererbung erworbener Eigenschaften: „Heute wird bei der Züchtung [...] in zunehmendem Maße auch die gerichtete Veränderung (Vererbung erworbener Eigenschaften) untersucht" (Ambrosius et al. 1964: 45). Gleichzeitig lehnten die Autorinnen und Autoren in der Ausgabe von 1960 Grundzüge der Keimplasmatheorie ab. August Weismann wurde dabei nicht erwähnt (Abb. 85).

„Viele Wissenschaftler wandten sich mit Recht gegen solche einseitigen Auffassungen. Sie wiesen darauf hin, daß die Keimzellen nicht unabhängig von den Körperzellen existieren und daß an den Veränderungen des Erbplasmas auch äußere Einflüsse mitwirken“ (Ambrosius et al. 1964: 35).

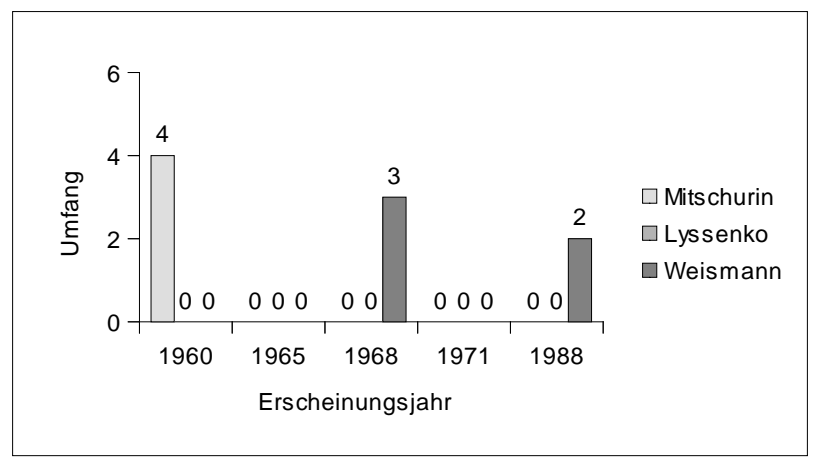

Abbildung 85: Mitschurin, Lyssenko und Weismann in den Lehrbüchern der Klasse 10

Im Lehrbuch von 1965 verlagerten die Autorinnen und Autoren Angaben zum Schöpferischen Darwinismus in das Kapitel zur Genetik. Ab 1968 verschwanden entsprechende Inhalte dann ganz aus den Lehrbüchern für die 10. Klasse. Obwohl der Schöpferische Darwinismus in den Büchern präsent war, fehlten Angaben zur Person Lyssenko in allen Lehrbüchern (Abb. 85). Auch Abbildungen aus diesem Bereich wurden nicht in die Bücher integriert. 
Während die Lehrbücher von 1965 und 1971 keine Angaben zu zum Neodarwinismus enthielten, würdigten die Lehrbücher von 1968 und 1988 Weismanns Einsatz für die Lehren Darwins und sein wissenschaftlicher Beitrag für die Weiterentwicklung des Darwinismus. Die Autoren betonten, dass

„August Weismann [...] sich bedingungslos hinter die Lehre Darwins [stellte], da er aufgrund seiner Untersuchungen und Erkenntnisse über die Vermehrung durch Zellteilung von ihrer Richtigkeit überzeugt war" (Kummer et al. 1988: 83).

Der Begriff Neodarwinismus kam in keinem der Lehrbücher vor. Auch Abbildungen waren nicht vorhanden.

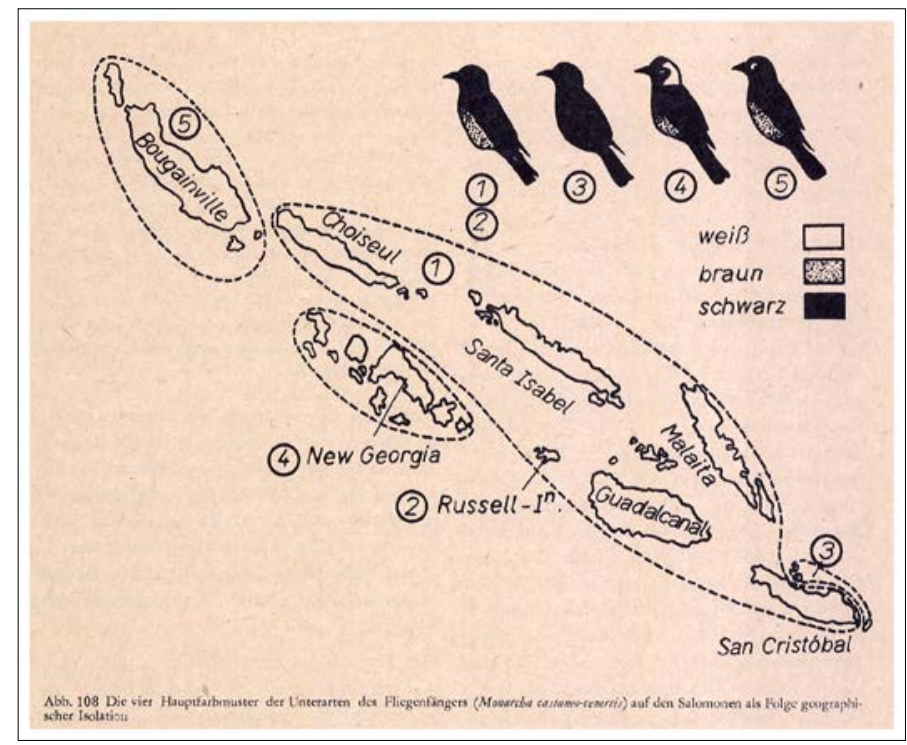

Abbildung 86: Die vier Hauptfarbmuster des Fliegenfängers auf den Salomonen als Folge geografischer Isolation (Bach et al. 1967)

\section{Synthetische Evolutionstheorie}

Die genetische Variabilität durch Mutation und Rekombination, eine Aussage der Synthetischen Evolutionstheorie, behandelten die Lehrbücher mit unterschiedlicher Schwerpunktsetzung. Im Lehrbuch von 1960 hieß es dazu: „Mutationen haben sicherlich große Bedeutung für die Aufspaltung der Arten. Die Mutationstheorie reicht aber zur Klärung der Stammesgeschichte allein nicht aus" (Ambrosius et al. 1964: 40). Im Lehrbuch von 1965 wurden dagegen Mutationen als „der primäre Evolutionsfaktor“" (Bach et al. 1967: 126) und im Lehrbuch von 1968 als die „einzige Quelle der Evolution“ (Bach et al. 1968: 39) angesehen. Das Lehr- 
buch von 1971 verwies zwar auf Mutationen als Ursache genetischer Variabilität, behandelte diese Inhalte aber, ebenso wie die Rekombination, in der vorgelagerten Stoffeinheit Genetik. Erst im Lehrbuch von 1988 stellten die Verfasser genetische Variation durch Mutation und Neukombination in der Stoffeinheit Evolution der Organismen ohne Akzentuierung dar. Im Zusammenhang mit der Behandlung der Evolutionsfaktoren erläuterten die Lehrbücher von 1960, 1965, 1971 und 1988 auch die Speziation durch geografische Isolation (Abb. 86). Das Schülerbuch von 1988 visualisierte in diesem Kontext Darwin-Finken auf den Galapagos-Inseln sowie geographische Rassen einer Fliegenschnäpperart. Ebenso wie die Rekombination wurde dieses Postulat des Synthetischen Darwinismus im Lehrbuch für die Vorbereitungsklassen von 1968 nicht aufgenommen. Keines der Lehrbücher wies auf Begründer der Synthetischen Evolutionstheorie hin.

\section{Neontologie}

\section{Homologie und Analogie}

Die Lehrbücher von 1960 bis 1988 informierten über homologe Organe. Alle Ausgaben erläuterten den Sachverhalt am Beispiel der Vordergliedmaßen der Wirbeltiere und am Beispiel der Laubblattumbildungen von Pflanzen. Dabei wurden die Vordergliedmaßen im Textverlauf stets zuerst behandelt. Weitere Belege waren die Atmungsorgane sowie der Blutkreislauf der Wirbeltiere (1960, 1965), die Entwicklung von Lunge und Schwimmblase (1960, 1965, 1968), Gehirne von Wirbeltieren (1960, 1965, 1971) sowie Blütenteile (1971). Außer in der Ausgabe von 1971 standen die Belege von Pflanzen an letzter Stelle. Der Umfang der angeführten Beispiele variierte. Enthielten die Ausgaben von 1960 und 1965 je sechs Beispiele, so waren es 1968 drei, 1971 vier und 1988 zwei. Dagegen veränderte sich der Umfang der Visualisierung im Verhältnis zu den genannten Belegen kaum. Die Lehrbücher von 1960, 1965, 1968 und 1988 veranschaulichten alle und das Lehrbuch von 1971 die Hälfte der Belege mit Abbildungen. Art und Inhalt der Abbildungen variierte jedoch, was am Beispiel der Vordergliedmaßen der Wirbeltiere verdeutlicht werden kann.

Die Lehrbücher von 1960 und 1965 enthielten eine Abbildung (Schema), die die Vordergliedmaßen von neun Wirbeltieren zeigte (urzeitlicher Quastenflosser, urzeitlicher Schwanzlurch, Eidechse, Mensch, Hund, Wal, Flugsaurier, Vogel, Fledermaus). Das Lehrbuch von 1968 verfügte ebenfalls über eine Abbildung (Schema). Auf dieser waren die Vordergliedmaßen auf acht Wirbeltiere reduziert und in einer anderen Reihung sowie Auswahl abgebildet (Mensch, Hund, Eidechse, Wal, Maulwurf, Vogel, Fledermaus, Quastenflosser). Im Lehrbuch von 1971 standen den Schülern zwei Abbildungen, ein Schema und eine Fotografie präparierter Skelette, zur Verfügung. Die Darstellung war erneut in Reihung sowie Auswahl verändert und nun auf sieben Vordergliedmaßen reduziert (Eidechse, Vogel, Wal, Fledermaus, Maulwurf, Katze, Mensch). Auch das Lehrbuch von 
1988 enthielt eine Schematisierung, die eine veränderte Reihenfolge und Auswahl präsentierte (Abb. 87). Sie stellte nur noch die Vordergliedmaßen von drei Wirbeltieren dar (Eidechse, Fledermaus, Wal).

Außer im Lehrbuch von 1971 enthielten die Lehrbücher auch Angaben über analoge Organe. Der Sachverhalt wurde anhand der Beispiele Vogel- und Insektenflügel $(1960,1965,1968,1988)$, Vordergliedmaßen von Maulwurf und Maulwurfsgrille (1960, 1965, 1968), Atmungsorgane von Insekten und landlebenden Wirbeltieren (1968), Ranken (1968) sowie Stacheln und Dornen (1988) erläutert. Visualisierungen waren lediglich im Lehrbuch von 1968 integriert.

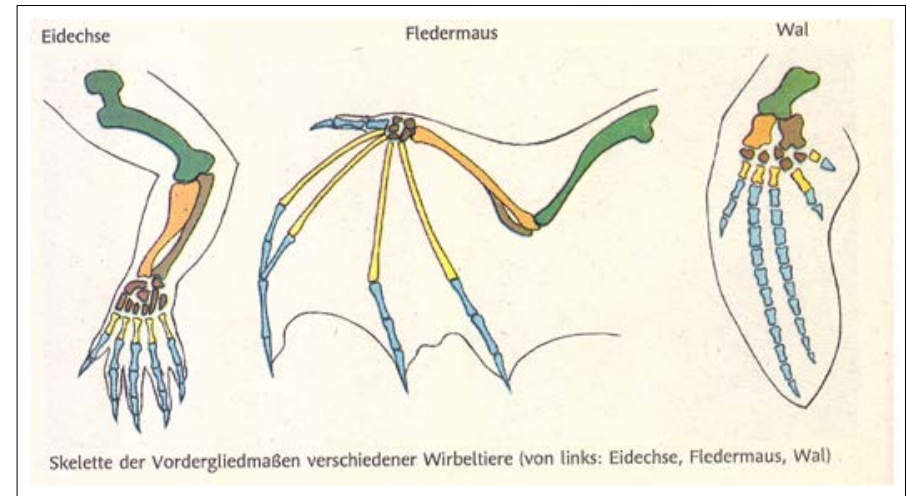

Abbildung 87: Homologe Organe (Kummer et al. 1988)

\section{Rudimente und Atavismen}

Auch zu Rudimenten bzw. Rückbildungen enthielten alle Lehrbücher Informationen. Dabei diente das Skelett der Wale (Cetacea) in allen Ausgaben als Beleg. Die Blüten der Braunwurzgewächse (1960, 1965, 1968), Blätter bei Kakteen (1988), Blindarmfortsatz des Menschen (1971, 1988), fehlende Blätter zur Assimilation des Kohlenstoffs bei Pflanzen (1971), Fußskelette diverser Huftiere (1968, 1971, 1988) bzw. von Pferden (1965), das Skelett von Blindschleichen (1968, 1971), von Schlangen (1968, 1971, 1988), der Verdauungskanal bei Bandwürmern (1988) sowie die unteren Wirbelknochen der menschlichen Wirbelsäule $(1960,1965)$ waren weitere Beispiele. Im Gegensatz zu den Homologien visualisierten die Lehrbücher nicht alle, sondern lediglich ein bis zwei Belege. Dies waren 1960 das Fußskelett des Pferdes und die Blüte der Braunwurzgewächse (Scrophulariaceae), 1965 das Fußskelett des Pferdes, 1968 und 1971 das Skelett von Walen sowie die Fußskelette diverser Huftiere und 1988 das Skelett von Walen. Außer in der Ausgabe von 1988 führten die Autoren Belege von Pflanzen stets zuletzt an. Keines der Lehrbücher enthielt Informationen über Atavismen. 


\section{Biogeografie}

Die Lehrbücher von 1960 und 1965 enthielten neben der Darstellung der Isolation als Evolutionsfaktor (Kategorie Theorien) jeweils ein eigenständiges Kapitel, das Tatsachen aus der Biogeografie vorstellte. Als Beispiele, jedoch ohne Visualisierungen, benannten die Ausgaben von 1960 und 1965 Folgeerscheinungen der Eiszeit (Gemsenrassen, Hochgebirgspflanzen) und der Inselbildung (GalapagosInseln, Australien). Während im Lehrbuch von 1968 derartige Inhalte fehlten, wurde in den Lehrbüchern von 1971 und 1988 diese Thematik erneut aufgenommen, jedoch im Zusammenhang mit der Darstellung der Evolutionstheorien bzw. dem Evolutionsfaktor Isolation behandelt.

\section{Embryologie und Ontogenie}

Ausschließlich die Lehrbücher von 1960, 1965 und 1968 behandelten Belege aus der Embryologie in einem eigenständigen Kapitel. Der Großteil der Texte und Abbildungen blieb hierbei konstant. Erläuterungen zum Biogenetischen Grundgesetz von Ernst Haeckel orientierten sich an den Entwicklungsstufen des menschlichen Embryos und an den Ähnlichkeiten in der Ausbildung von Larvenstadien bei Wirbellosen (Invertebrata). In den Text eingebunden war eine Abbildung, die die Entwicklungsstufen von Fisch, Schildkröte, Vogel und Mensch darstellte. Ebenso visualisierten die Lehrbücher die Larve der Entenmuschel und die Larven von Ringelwürmern und Weichtieren. Die Ausgabe von 1968 enthielt darüber hinaus eine schematische Darstellung von Morula, Blastula und Gastrula. In den Lehrbüchern von 1971 und 1988 verlagerten die Autoren Haeckels Biogenetische Grundgesetz in ein Kapitel, das die Geschichte der Evolutionstheorien dargelegte (Kategorie Theorien).

Paläontologie

\section{Systemtabellen und Phylogenie}

Alle Lehrbücher beinhalteten Systemtabellen. Hierbei traten Unterschiede in der zeitlichen Datierung auf (Tab. 47). Die größten Differenzen bestanden bei den geologischen Erdzeitaltern (Äonen) Proterozoikum und Archaikum. Teilweise verzichteten die Autoren hier auf Zeitangaben, wie in den Ausgaben von 1960, 1968 und 1971. Weitere Veränderungen betrafen die Namen und die Zuordnung der Zeitalter (Äonen/Ären) und Perioden. So wurde beispielsweise im Lehrbuch von 1960 das Ordovizium noch als erster Zeitabschnitt des Silurs gelistet. Auch der Umfang der Systemtabellen variierte. Neben Zeitalter, System und Zeitangaben enthielt das Lehrbuch von 1965 auch Abteilungen. Ebenso informierte die Systemtabelle in dieser Ausgabe über die Entwicklung der Organismen im jeweiligen System. Auch das Lehrbuch von 1988 ordnete in einer Tabelle die Entwick- 
lung der Organismen den Erdzeitaltern und Zeitangaben zu. Angaben über Perioden standen den Schülern nicht zur Verfügung.

Tabelle 47: Systemtabelle, Lehrbücher Klasse 10 (*vgl. dazu Kutschera 2015)

\begin{tabular}{|c|c|c|c|c|c|c|c|}
\hline Ausgabe & & 1960 & 1965 & 1968 & 1971 & 1988 & $2015^{*}$ \\
\hline Äon/Ära & Periode & \multicolumn{6}{|c|}{ Beginn vor Mio. Jahren } \\
\hline \multirow{4}{*}{ Känozoikum } & Quartär & 0,6 & 1,0 & 1,0 & 1,5 & - & 2,6 \\
\hline & Tertiär & 60,0 & 70,0 & 70,0 & 65,0 & 70,0 & 66,0 \\
\hline & Kreide & 140,0 & 135,0 & 135,0 & 135,0 & - & 145,0 \\
\hline & Jura & 175,0 & 180,0 & 180,0 & 190,0 & - & 201,0 \\
\hline \multirow[t]{6}{*}{ Mesozoikum } & Trias & 200,0 & 220,0 & 220,0 & 225,0 & 250,0 & 252,0 \\
\hline & Perm & 240,0 & 270,0 & 270,0 & 280,0 & - & 299,0 \\
\hline & Karbon & 310,0 & 350,0 & 350,0 & 350,0 & - & 359,0 \\
\hline & Devon & 350,0 & 400,0 & 400,0 & 400,0 & - & 419,0 \\
\hline & Silur & 450,0 & 430,0 & 430,0 & 440,0 & - & 444,0 \\
\hline & Ordovizium & - & 490,0 & 490,0 & 500,0 & - & 485,0 \\
\hline Paläozoikum & Kambrium & 540,0 & 600,0 & 600,0 & 600,0 & 600,0 & 541,0 \\
\hline Proterozoikum & & $>2000,0$ & 1900,0 & 3400,0 & - & 4000,0 & 2500,0 \\
\hline Archaikum & & - & 3500,0 & - & 4500,0 & 5000,0 & 4600,0 \\
\hline
\end{tabular}

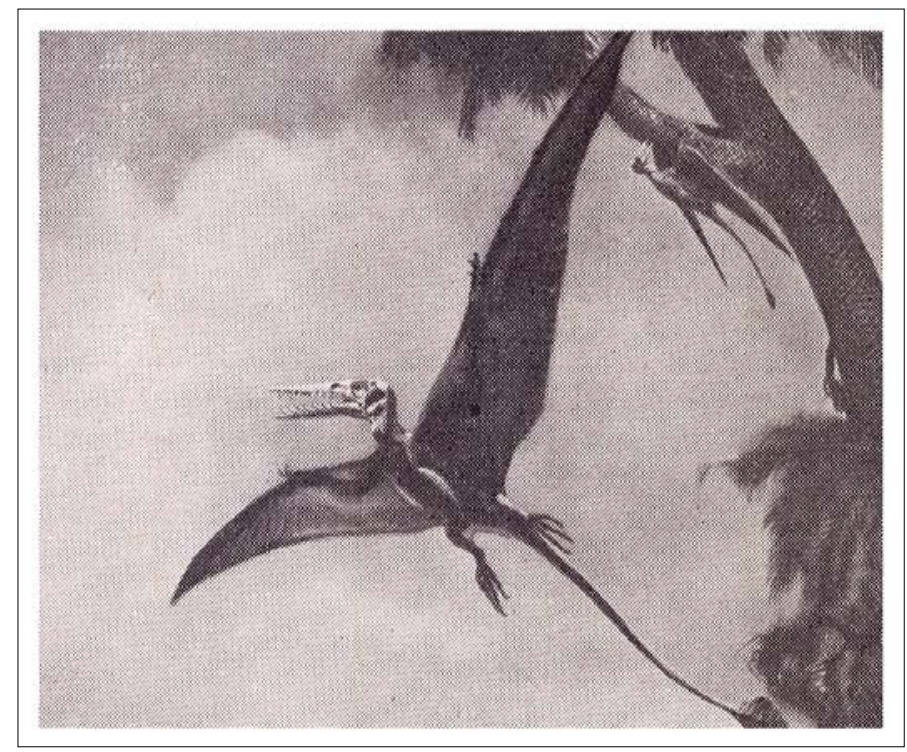

Abbildung 88: Flugsaurier (Bach et al. 1968)

Weitere Informationen aus der Paläobiologie enthielt der Lehrbuchtext aller Schulbücher. Dabei war, im Verhältnis zum Textumfang, eine reichhaltige Bebilderung für diese Thematik typisch (Abb. 88). Das Lehrbuch von 1988 präsentierte hier auf neun Seiten 16 Abbildungen (teilweise über eine Seite) und lediglich ein- 
einhalb Seiten Text. Einige Abbildungen fanden in mehreren Lehrbüchern Verwendung. Die Abbildung eines Landsauriers aus dem Buch Tiere der Urzeit war in allen Lehrbüchern integriert und ab 1988 auch farbig (Abb. 89).

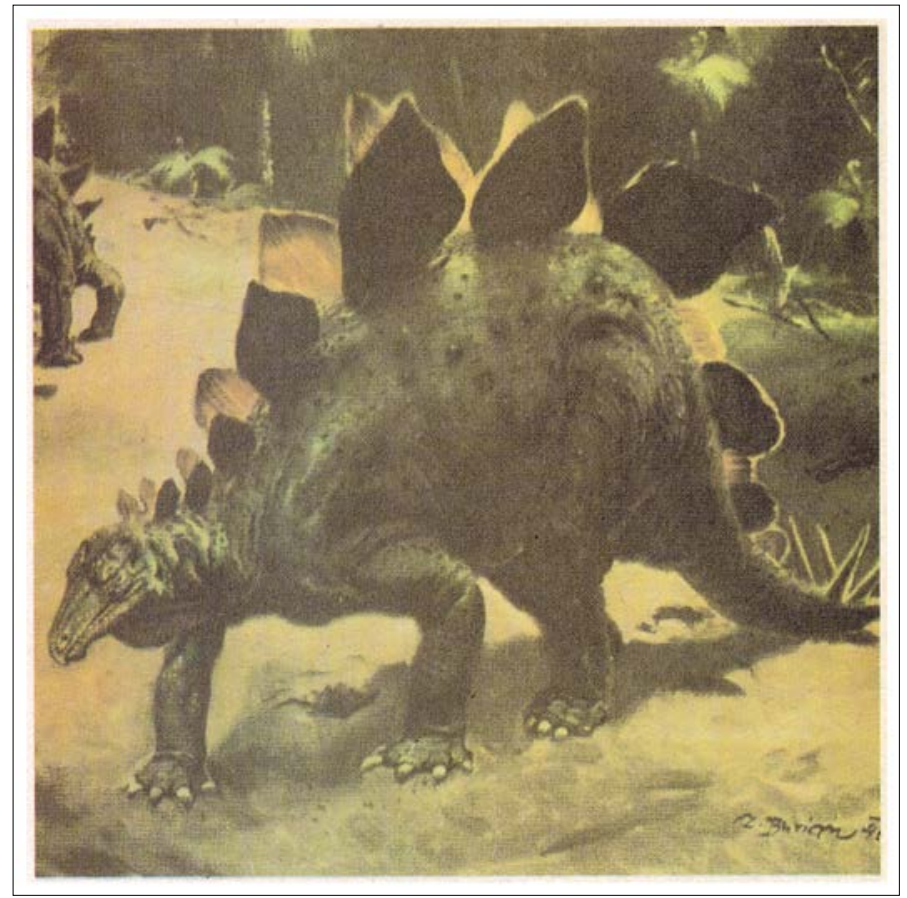

Abbildung 89: Landsaurier (Sommer et al. 1988)

\section{Entwicklungsreihen und Zwischenformen}

In den Lehrbüchern von 1960 bis 1971 thematisierten die Autoren die Entwicklung des Pferdes (Equus), insbesondere des Pferdefußskeletts. Die Lehrbücher von 1960 und 1965 enthielten ferner die Entwicklung des Zapfens der Nadelbäume (Coniferales) als Beispiel für Entwicklungsreihen. Für alle Belege waren Abbildungen vorhanden. Die Lehrbücher von 1965 und 1968 fassten die Informationen in einem Unterkapitel zusammen. In den Lehrbüchern von 1960 und 1971 waren die Angaben dagegen in andere Kapitel integriert. Im Lehrbuch von 1988 stand den Schülern keine Information über Entwicklungsreihen zur Verfügung. Somit fand eine fortschreitende Reduzierung des Informationsgehaltes und des Umfangs statt (Tab. 48). 
Tabelle 48: Entwicklungsreihen, Lehrbücher Klasse 10

\begin{tabular}{|l|l|l|}
\hline Aufl. & Zapfen der Nadelbäume & Pferde \\
1960 & $\begin{array}{l}\text { 1. Erdaltertum, kätzchenartige } \\
\text { Blütenstände der Cordaiten / } \\
\text { 2. ständige Vereinfachung der } \\
\text { Blüten }\end{array}$ & $\begin{array}{l}\text { 1. Eozän, Schulterhöhe 25 cm, Urpferd, bewohnte nordameri- } \\
\text { kanische Sumpfwälder, Backenzähne mit niedriger Krone, } \\
\text { Vorderfüße mit vier Zehen am Boden, fünfte verkümmert, } \\
\text { Hinterfüße mit drei Zehen am Boden / 2. größer, Rückbildung } \\
\text { der Zehen fortgeschritten, dreizehig, Hauptlast auf Mittelzehe / } \\
\text { 3. hohe und harte Zahnkrone, weitere Rückbildung der Seiten- } \\
\text { zehen, Spitzengänger, Schulterhöhe 150 cm }\end{array}$ \\
& $\begin{array}{l}\text { 1. Erdaltertum, kätzchenartige } \\
\text { Blütenstände der Cordaiten / } \\
\text { 2. ständige Vereinfachung der } \\
\text { Blüten }\end{array}$ & $\begin{array}{l}\text { 1. Eozän, Schulterhöhe 25 cm, Urpferd (Hyracotherium), } \\
\text { bewohnte nordamerikanische Sumpfwälder, Backenzähne mit } \\
\text { niedriger Krone, Vorderfüße mit vier Zehen am Boden, fünfte } \\
\text { verkümmert, Hinterfüße mit drei Zehen am Boden / 2. }\end{array}$ \\
& $\begin{array}{l}\text { Mesohippus, größer, Rückbildung der Zehen fortgeschritten, } \\
\text { dreizehig, Hauptlast auf Mittelzehe / 3. hohe Zahnkrone, weite- } \\
\text { re Rückbildung der Seitenzehen, Größenzunahme }\end{array}$ \\
\hline 1968 & - & $\begin{array}{l}\text { 1. Tertiär, Schulterhöhe 25 cm, Urpferd, bewohnte nordameri- } \\
\text { kanische Sumpfwälder, Backenzähne mit niedriger Krone, } \\
\text { Vorderfüße mit vier von fünf Zehen am Boden, Hinterfüße mit } \\
\text { drei Zehen am Boden / 2. dreizehig / 3. hohe Zahnkrone, } \\
\text { weitere Rückbildung der Seitenzehen }\end{array}$ \\
\hline 1971 & - & $\begin{array}{l}\text { 1. Urpferd, Schulterhöhe 25 cm, bewohnte nordamerikanische } \\
\text { Sumpfwälder, Füße angepasst, Pflanzenfresser / 2. weitere } \\
\text { Ausbreitung, Anpassungen des Gebisses an trockene Step- } \\
\text { pengräser }\end{array}$ \\
\hline 1988 & - & - \\
\hline
\end{tabular}

Tabelle 49: Zwischenformen und Entwicklungsreihen, Lehrbücher Klasse 10

\begin{tabular}{|c|c|c|c|c|c|}
\hline Ausgabe & 1960 & 1965 & 1968 & 1971 & 1988 \\
\hline \multirow[t]{5}{*}{ Zwischenformen } & $A^{*}$ & $A^{*}$ & $A^{*}$ & $A^{*}$ & $Q^{*}$ \\
\hline & $Q^{*}$ & $Q^{*}$ & $Q^{*}$ & $Q^{*}$ & $A^{*}$ \\
\hline & $\mathrm{N}^{*}$ & $\mathrm{~N}^{*}$ & & & $S^{*}$ \\
\hline & $F^{*}$ & $F^{*}$ & & & \\
\hline & 4 & 4 & 2 & 2 & 3 \\
\hline \multirow[t]{3}{*}{ Entwicklungsreihen } & $\mathrm{P}^{*}$ & $\mathrm{P}^{*}$ & $\mathrm{P}^{*}$ & $\mathrm{P}^{*}$ & \\
\hline & $Z^{*}$ & $Z^{*}$ & & & \\
\hline & 2 & 2 & 1 & 1 & 0 \\
\hline
\end{tabular}

Alle Lehrbücher für die Klasse 10 enthielten ein Unterkapitel über Zwischenformen. In diesem wurden die Beispiele Urvogel und Quastenflosser (Latimeria) dargestellt. Die Lehrbüchern von 1960 und 1965 beschrieben auch Nacktsprosser (Psilophyton) sowie Farnsamer (Pteridospermen) und das Lehrbuch von 1988 das Schnabeltier (Ornithorbynchus anatinus) als Übergangsformen. Alle vorgestellten 
Beispiele veranschaulichten die Autoren mindestens durch eine Abbildung (Tab. 49; Abb. 90, 91).

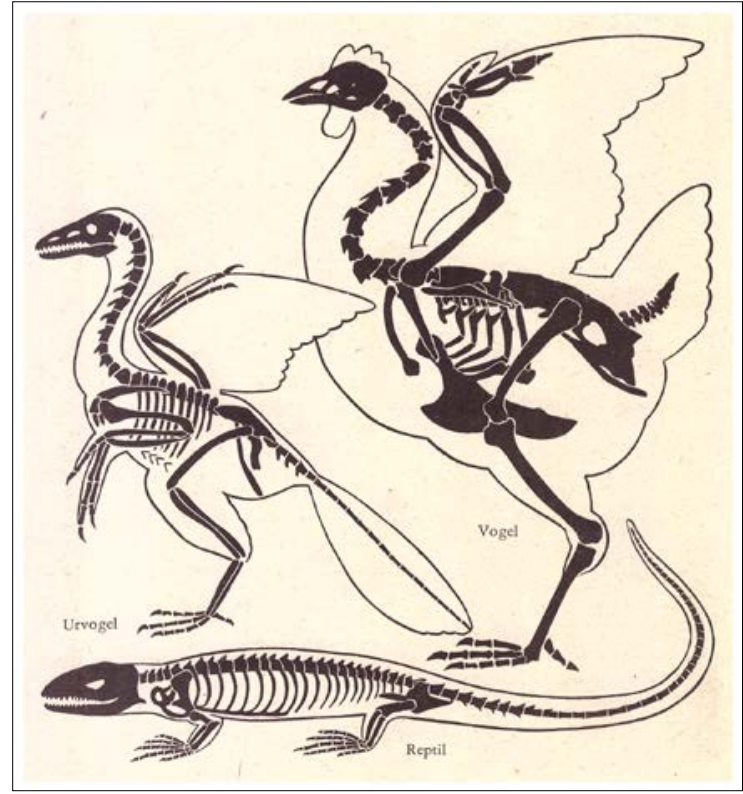

Abbildung 90: Der Urvogel und Merkmale von Reptilien und Vögeln (Bach et al. 1987)

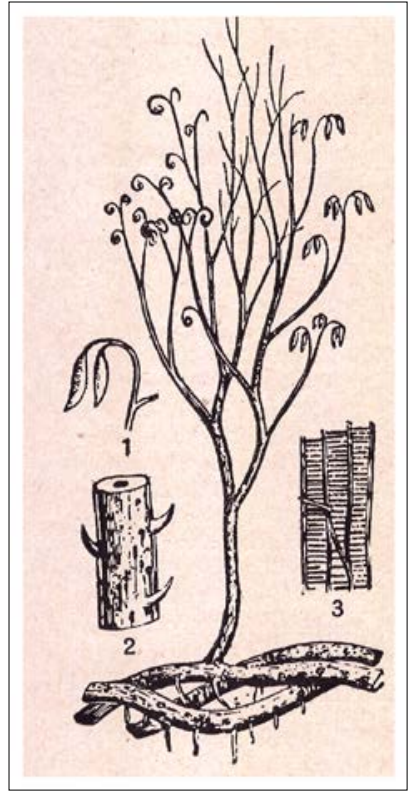

Abbildung 91: Nacktsprosser (Ambrosius et al. 1964)

\section{Fossilisation und Altersbestimmung}

Alle Lehrbücher enthielten Informationen über die Entstehung von Fossilien (Fossilisation). Ferner wurden Einschlüsse, Versteinerungen und Abdrücke in allen Lehrbüchern als Fossilienarten erläutert. Beispiele waren im Text und/oder als Abbildung integriert. Jedoch wiesen die Lehrbücher von 1971 und 1988 deutlich mehr Abbildungen als die Vorgängermodelle auf. Die Erläuterungen zu den jeweiligen Fossilienarten enthielten in Einzelfällen Angaben zu Fundorten. Zusätzlich verfügten die Lehrbücher von 1960 und 1965, ähnlich dem Lehrbuch für die 8. Klasse von 1957, über das Kapitel Wo finden wir Fossilien?. In diesem Kontext wurden Fossilien aus den Orten bzw. Gegenden Norddeutschland (Seeigel, Donnerkeil, Schwämme, Kleinlebewesen, Seelilie), Rüdersdorf bei Berlin (Muscheln) und Thüringer Becken (Muscheln, Schnecken, Seelilien) vorgestellt und fallweise visualisiert (Tab. 50). Das Lehrbuch von 1971 nannte Fundorte innerhalb des Themas Fossilisation, jedoch ohne spezifische Fossilien zuzuordnen. Aufgrund der Modifikationen in den Angaben nahm der Umfang der Darstellung stetig zu (1960/65/68 je zwei Seiten, 1971 drei Seiten, 1988 fünf Seiten). 
Tabelle 50: Fossilisation, Lehrbücher Klasse 10

\begin{tabular}{|c|c|c|c|}
\hline Auflage & $\begin{array}{l}\text { Fossilisation, } \\
\text { Fossilienarten }\end{array}$ & Beispiele (*mit Abbildungen) & Fundorte \\
\hline \multirow[t]{5}{*}{ 1960/65 } & Konservierung & Mammut, Nashorn & Sibirisches Eis \\
\hline & Einschlüsse (Bernstein) & Mücken, Blattläuse, Käfer & - \\
\hline & Hartteile & $\begin{array}{l}\text { Knochen (Urpferdchen), Zähne, } \\
\text { Schalen, Gehäuse }\end{array}$ & Geiseltal (Halle) \\
\hline & Versteinerungen & - & - \\
\hline & Abdrücke & Kriechspur eines Sauriers* & - \\
\hline \multirow[t]{5}{*}{1968} & Mumifizierung & Mammut, Nashorn & Sibirisches Eis \\
\hline & Einschlüsse (Bernstein) & Insekten & - \\
\hline & Hartteile & Knochen, Zähne & - \\
\hline & Versteinerungen & Belemnitrest ${ }^{*}$, Ammonit $^{*}$ & - \\
\hline & Abdrücke & Fährte eines Sauriers* & - \\
\hline \multirow[t]{5}{*}{1971} & Mumifizierung & - & \multirow{5}{*}{$\begin{array}{l}\text { Bergbau, Steinbrüche, } \\
\text { Ausschachtungen, Polareis }\end{array}$} \\
\hline & Einschlüsse (Bernstein) & Insekt* & \\
\hline & Abdrücke & Fährte*, Blatt* & \\
\hline & Versteinerungen & $\begin{array}{l}\text { Knochen, Schneckengehäuse, } \\
\text { Seeigel*, Korallenstock*, Torf aus }^{*} \text { Urlandpflanzen* (Verkieselung) }^{*}\end{array}$ & \\
\hline & Inkohlungen & Farn* & \\
\hline \multirow[t]{5}{*}{1988} & Knochenfunde & $\begin{array}{l}\text { Skelett eines Höhlenbären, } \\
\text { menschlicher Unterkiefer }\end{array}$ & - \\
\hline & Versteinerungen & $\begin{array}{l}\text { Schneckengehäuse, Seeigel }{ }^{*} \text {, } \\
\text { Muschelschalen*, Donnerkeile* }\end{array}$ & Ostseeküste \\
\hline & Abdrücke & $\begin{array}{l}\text { Federn des Urvogels, } \\
\text { Saurierfährte }^{*} \text {, Laubblatt* }\end{array}$ & - \\
\hline & Einschlüsse & $\begin{array}{l}\text { Insekten (Bernstein) }{ }^{*} \text {, Mammut } \\
\text { (Eis) }\end{array}$ & Sibirien \\
\hline & Inkohlungen & Braunkohle*, Farn* & - \\
\hline
\end{tabular}

Die Thematik der Altersbestimmung von Fossilien war zwischen 1960 und 1988 einer starken Reduktion unterworfen. Die Lehrbücher von 1960 und 1965 enthielten dazu eigene Abschnitte, die die Altersbestimmung mittels Atomzerfall und Leitfossilien erläuterten. Dagegen wiesen die Verfasser im Lehrbuch von 1968 im Kapitel Gliederung der Erdgeschichte lediglich auf „besondere Fossilien“ (Bach et al. 1968: 54-57) hin, die Zeitabschnitte kennzeichnen und „Aussagen über das Alter der Gesteine“ (ebd.: 57) ermöglichen. Im Lehrbuch von 1971 fand einzig im Kapi- 
tel Die Schaffung naturwissenschaftlicher Voraussetzungen für die wissenschaftliche Abstammungslehre der Begriff Leitfossilien in Verbindung mit den Erkenntnissen des Engländers William Smith Erwähnung: „Im 18. Jahrhundert stellte der englische Bauingenieur William SMITH (1769 bis 1839) fest, dass bestimmte Fossilien für bestimmte Erdschichten charakteristisch sind (Leitfossilien), es also gewisse Beziehungen zwischen der Erdgeschichte und der Geschichte der Organismen gibt" (Bach et al. 1987: 94, Hervorhebung im Original). Das Lehrbuch von 1988 enthielt zu Altersbestimmungen keine Angaben. 


\section{Das Biologielehrbuch für die Klasse 12}

„Wir können die Bedeutung der Ab-
stammungslehre für die Biologie und für
die wissenschaftliche Weltanschauung
erst ganz verstehen, wenn wir auch ihre
Geschichte kennen." (Bach et al.
1967: 94)

\section{Design}

Umfang und Textformat

Das Schulbuch von 1952 umfasste 160 Seiten. Davon waren auf 108 Seiten $(67,5 \%)$ evolutionsbiologische Inhalte vorhanden. Zwar fiel der Umfang des Lehrbuches von 1957 mit 114 Seiten geringer aus, doch umfassten evolutionsbiologische Inhalte weiterhin 108 Seiten (94,7 \%). Der Reduktion lag eine Änderung des Lehrplans zu Grunde, die mit der Auslagerung einzelner Kapitel in ein Lehrheft verbunden war. Im Lehrbuch von 1965 stieg der Textumfang deutlich auf 246 Seiten an. Davon enthielten 142 Seiten (57,7\%) evolutionsbiologische Inhalte. Durch diese Modifikationen nahm der Textumfang für evolutionsbiologi- 
sche Inhalte zwar zu, dennoch verringerte sich der prozentuale Anteil am Lehrbuch (Abb. 92).

Das Beiwerk der Lehrbücher von 1952 und 1957 bestand aus einem Inhaltsund Sachverzeichnis. Neben diesen enthielt das Lehrbuch von 1965 auch ein Glossar und somit im Vergleich mehr Hilfestellungen für Schüler.

Von 1952 bis 1965 stieg die Anzahl der verwendeten Textformate. Schlüsselbegriffe bzw. Wortgruppen waren in allen Lehrbücher von 1952, 1957 und 1965 durch Fettdruck bzw. Kursivschrift kenntlich gemacht, wodurch sie sich vom übrigen Text abhoben und ein schnelleres Erfassen der zentralen Inhalte erleichterten. Des Weiteren waren Personennamen in den Lehrbüchern von 1957 und 1965 in Großbuchstaben abgedruckt. Im Lehrbuch von 1965 waren darüber hinaus Zusatzinformationen durch eine kleinere Schriftgröße vom Kerntext getrennt sowie Aufgabenstellungen und Fragen im Anschluss an jedes Unterkapitel in den Textverlauf integriert.

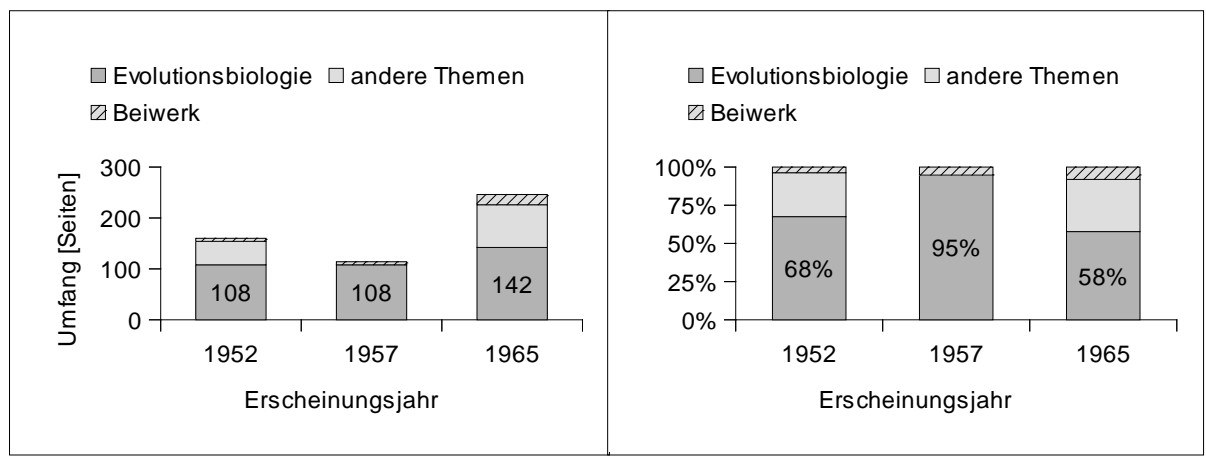

Abbildung 92: links: absoluter Umfang, rechts: relativer Umfang in den Lehrbüchern für die Klasse 12

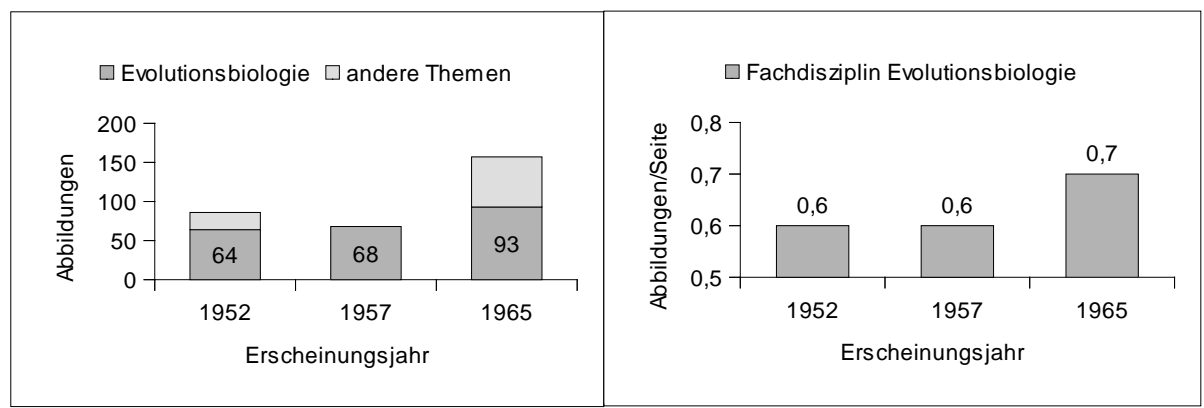

Abbildung 93: links: Anzahl der Abbildungen, rechts: Abbildungen pro Seite in den Lehrbüchern für die Klasse 12 
Einband und Abbildungen

Die Einbände der Auflagen von 1952, 1957 und 1965 waren Hardcover und im Format den Lehrbüchern der Klassenstufe 8 und 10 ähnlich. Der äußeren Gestaltung der Lehrbücher von 1952 und 1957 konnte das Fach und die Klassenstufe entnommen werden. Die Einbände waren in einem Grünton gehalten und enthielten keine Abbildungen. Dem Einband der Auflage von 1965 konnte lediglich das Schulfach entnommen werden. Jedoch wies eine Ziffer auf dem Einband indirekt auf die 12. Klassenstufe hin. Die Gestaltung war farbig (grün, gelb) und enthielt das Schema eines Menschenkopfes sowie eines Menschenaffen. Auf der Rückseite befand sich ein Hominidenschädel. Die Abbildungen vermittelten somit konsequent Inhalte aus der Kategorie Hominisation.

Die Anzahl der Abbildungen nahm in den Lehrbüchern von 1952 bis 1965 zu. Dabei stieg der Umfang der Abbildungen pro Seite von 0,6 (Ausgaben 1952 und 1957) auf 0,7 (Ausgabe 1965) leicht an (Abb. 93). Im Lehrbuch von 1952 standen den Schülern 86 schwarz/weiß Abbildungen zur Verfügung. Davon informierten 64 Abbildungen (74,4\%) über evolutionsbiologische Aspekte. Die Ausgabe von 1957 enthielt 68 Abbildungen in schwarz/weiß. Alle diese Abbildungen wiesen, ebenso wie die Texte, ausschließlich Informationen zur Evolutionsbiologie auf. Beiden Lehrbüchern lagen keine Farb- oder Kunstdrucktafeln bei. Im Lehrbuch von 1965 waren dagegen die Vorsätze grafisch gestaltet. Der vordere Vorsatz zeigte den Stammbaum der Tiere und der hintere Vorsatz den Stammbaum der Pflanzen. Im Text integrierten die Autoren 157 Abbildungen in schwarz/weiß. Mit 93 Abbildungen umfassten Inhalte aus Bereichen der Evolutionsbiologie 59,2 \%. Zusätzlich lagen vier Farbtafeln und vier Kunstdrucktafeln bei. Alle Farbdrucke und drei der Kunstdrucke

(Archaeopteryx, Hominidenschädel, Schnitzereien und Werkzeuge) entsprachen den Beilagen aus dem Lehrbuch für die Klasse 10 von 1960. Neu aufgenommen wurde eine Darstellung aus dem Buch Aus Jabrmillionen (1962) von Arno Hermann Müller (Abb. 94).

Die kategoriale Zuordnung der Abbildungen belegt, dass der Schwerpunkt der Visualisierung in den Lehrbüchern von 1952 und 1957 auf der Kategorie Neontologie lag. An zweiter und dritter Stelle folgten die Kategorien Theorien und Paläontologie. Im Lehrbuch von 1965 verlagerte sich die

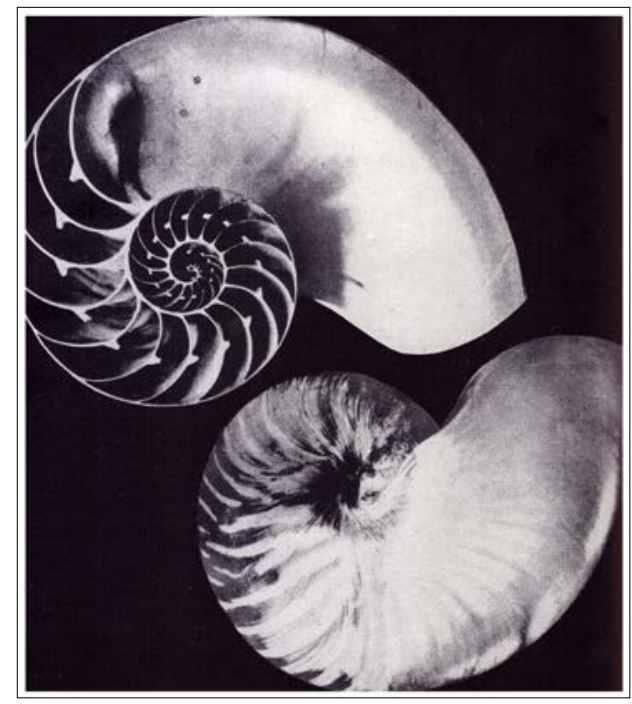

Abbildung 94: Nautilus pompilius aus dem Indik (Bach et al. 1967) 
Ausrichtung. Die Mehrzahl der Abbildungen zeigte Inhalte aus der Kategorie Theorien. Dennoch blieb die Visualisierung der Kategorien Neontologie und Paläontologie hoch. Hinzu kam eine umfangreiche Visualisierung von Inhalten aus der Kategorie Hominisation (Tab. 51).

Tabelle 51: äußeres und inneres Design, Lehrbücher Klasse 12

\begin{tabular}{|c|c|c|c|}
\hline Auflage & 1952 & 1957 & 1965 \\
\hline $\begin{array}{l}\text { Umfang: } \\
\text { absoluter Umfang [Seiten] } \\
\begin{array}{l}\text { Textumfang } \\
\quad \text { davon Evolutionsbiologie } \\
\quad \text { davon andere Themen }\end{array} \\
\begin{array}{l}\text { Beiwerk } \\
\text { relativer Umfang Evolutionsbiologie am Buch [\%] } \\
\text { relativer Umfang Evolutionsbiologie am Text [\%] }\end{array}\end{array}$ & $\begin{array}{c}160 \\
154 \\
108 \\
46 \\
6 \\
67,5 \\
70,1 \\
\end{array}$ & $\begin{array}{c}114 \\
108 \\
108 \\
0 \\
6 \\
94,7 \\
100,0 \\
\end{array}$ & $\begin{array}{l}246 \\
226 \\
142 \\
84 \\
20 \\
57,7 \\
62,8 \\
\end{array}$ \\
\hline $\begin{array}{l}\text { Beiwerk: } \\
\text { Inhaltsverzeichnis } \\
\text { Glossar } \\
\text { Literaturhinweise } \\
\text { Sachverzeichnis }\end{array}$ & $\begin{array}{l}\text { ja } \\
\text { nein } \\
\text { nein } \\
\text { ja }\end{array}$ & $\begin{array}{l}\text { ja } \\
\text { nein } \\
\text { nein } \\
\text { ja }\end{array}$ & $\begin{array}{l}\text { ja } \\
\text { ja } \\
\text { nein } \\
\text { ja }\end{array}$ \\
\hline $\begin{array}{l}\text { Paratext: } \\
\begin{array}{l}\text { Abbildungen im Text } \\
\quad \text { davon Evolutionsbiologie }\end{array} \\
\quad \text { relativer Umfang [\%] } \\
\begin{array}{l}\text { Abbildungen pro Textseite } \\
\quad \text { davon Evolutionsbiologie }\end{array} \\
\begin{array}{l}\text { Gestaltung } \\
\text { Farbtafeln } \\
\text { Kunstdrucktafeln } \\
\quad \text { davon Evolutionsbiologie }\end{array}\end{array}$ & $\begin{array}{c}86 \\
64 \\
74,4 \\
0,6 \\
\\
0,6 \\
\text { s/w } \\
\\
\\
0 \\
0 \\
0\end{array}$ & $\begin{array}{c}68 \\
68 \\
100,0 \\
0,6 \\
0,6 \\
s / w \quad \\
0 \\
0 \\
0\end{array}$ & $\begin{array}{c}157 \\
93 \\
59,2 \\
0,7 \\
\\
\text { s/w } \\
0,7 \\
\\
\\
4 \\
4 \\
8\end{array}$ \\
\hline $\begin{array}{l}\text { Kategoriale Zuordnung der Abbildungen: } \\
\text { Theorien } \\
\text { Biogenese } \\
\text { Neontologie } \\
\text { Paläontologie } \\
\text { Systematik } \\
\text { Hominisation } \\
\text { Didaktisches } \\
\text { Gesamt }\end{array}$ & $\begin{array}{r}15 \\
4 \\
25 \\
16 \\
0 \\
0 \\
4 \\
0 \\
64\end{array}$ & $\begin{array}{r}19 \\
3 \\
25 \\
17 \\
0 \\
4 \\
0 \\
68\end{array}$ & $\begin{array}{r}31 \\
6 \\
22 \\
16 \\
0 \\
0 \\
18 \\
0 \\
93\end{array}$ \\
\hline
\end{tabular}

\section{Struktur}

Das Lehrbuch von 1952

Die Inhalte im Lehrbuch von 1952 gliederten sich in die vier Bereiche Die Entstebung des Lebens auf der Erde und seine Entwicklung bis zum Menschen, Die Abstammungslehre im Rahmen der gesellschaftlichen Entwicklung, Die Weiterentwicklung der Organismen 
durch den Menschen sowie Das natürliche System der Organismen. Evolutionsbiologische Inhalte standen den Schülern in den ersten beiden Abschnitten zur Verfügung. Eine umfangreiche Feinstruktur diente zur Orientierung:

- Die Entstehung des Lebens auf der Erde und seine Entwicklung bis zum Menschen

- Die Entstehung des Lebens auf der Erde (8 Seiten; 7,4 \%)

- Die Abstammungslehre und Beweise für ihre Richtigkeit (34 Seiten; $31,5 \%)$

- Beweise aus der Paläontologie

- Beweise aus der vergleichenden Anatomie

- Beweise aus der Serologie

- Beweise aus der Ontogenie

- Beweise aus der Tier- und Pflanzengeographie

- Die Entstehung der Arten und die Grundlagen der systematischen Gliederung der Organismen (3 Seiten; 2,8 \%)

- Die Abstammung und Entwicklung des Menschen (7 Seiten; 6,5 \%)

- Die Abstammungslehre im Rahmen der gesellschaftlichen Entwicklung

- Die Lehre von der Konstanz der Arten (7 Seiten; 6,5 \%)

- Die Entwicklungslehre Lamarcks (7 Seiten; 6,5\%)

- Die wissenschaftliche Begründung der Entwicklungslehre durch Darwin (11 Seiten; 10,2\%)

- Der Kampf Haeckels um den Darwinismus (9 Seiten; 8,3 \%)

- Die Weiterentwicklung des Darwinismus zur schöpferischen Biologie (22 Seiten; 20,4\%)

- Kliment Arkadjewitsch Timirjasew

- Wassili Robertowitsch Wiljams

- Iwan Wladimirowitsch Mitschurin

- Trofim Denissowitsch Lyssenko

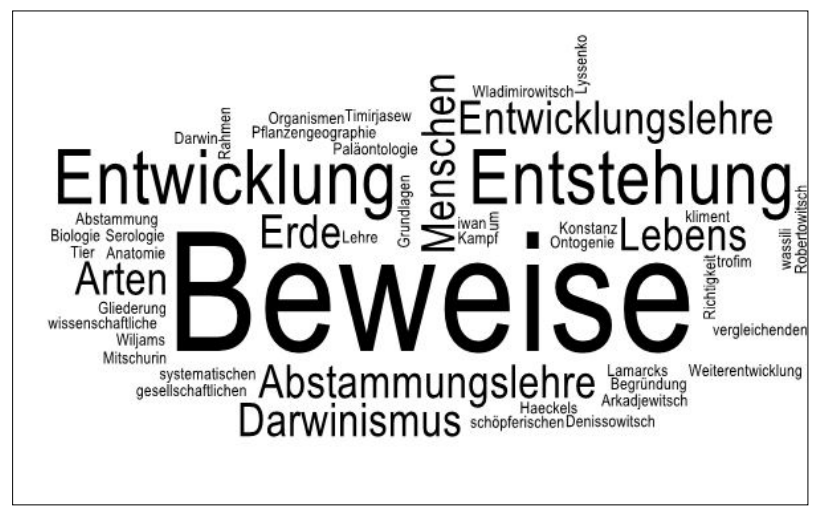

Abbildung 95: Biologische Begriffe im Inhaltsverzeichnis, Lehrbuch Klasse 12 (1952) 
Das Ergebnis einer Frequenzanalyse biologischer Begriffe der Kapitelüberschriften belegt, dass im Lehrbuch von 1952 den Worten Bewveise sowie Entstehung und Entwicklung der größte Stellenwert zukam (Abb. 95). Hier treten inhaltliche Übereinstimmung mit den Ergebnissen der kategorialen Zuordnung der Abbildungen auf.

Die Inhaltsanalyse der Texte im Lehrbuch von 1952 verdeutlicht die Gliederung Biogenese - Paläontologie - Neontologie - Paläontologie - Neontologie Systematik - Hominisation - Theorien. An letzter Stelle behandelt, umfasste die Kategorie Theorien die längste zusammenhängende Textpassage (Abb. 96). Paläontologische und neontologische Inhalte standen den Schülern an zwei Stellen zur Verfügung. Die Darstellung der anderen Kategorien erfolgte hingegen in sich geschlossen. Dass die Kategorie Theorien mit 2173,5 Zeilen (56,9\%) den größten Umfang einnahm und somit den inhaltlichen Schwerpunkt des Lehrbuches bildete, verdeutlicht die Raum Analyse der Lehrbuchtexte. In weiterer Reihenfolge umfassten die Kategorien Neontologie 631 Zeilen $(16,5 \%)$, Biogenese 314,5 Zeilen $(8,2 \%)$, Paläontologie 296 Zeilen $(7,7 \%)$, Hominisation 256,5 Zeilen Abbildung 96: Kategorien im Lehrbuch (6,7\%), Systematik 125 Zeilen (3,3\%) Klasse 12 (1952) sowie Didaktisches 23 Zeilen (0,5\%).

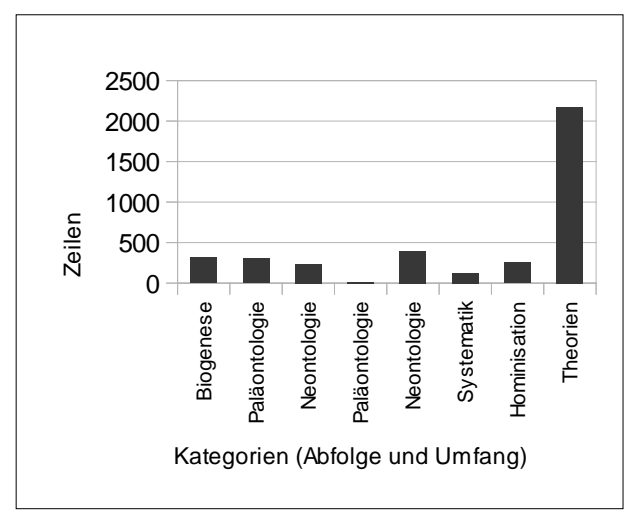

Das Lehrbuch von 1957

Mit der Ausgabe von 1957 veränderte sich die inhaltliche und strukturelle Ausrichtung in Teilen des Lehrbuches. Im Klappentext befand sich ein entsprechender Hinweis.

„Infolge einer Lehrplanänderung sind in diesem Buch die [...] Abschnitte ,Die Weiterentwicklung des Darwinismus zur schöpferischen Biologie‘ und ,Die Weiterentwicklung der Organismen durch den Menschen' nicht mehr vorhanden. Dafür wurde in den 12. Klassen das Lehrheft ,Die Züchtung von Pflanzen und Tieren' [...] benötigt." (Rothmaler \& Uschmann 1957: 2)

Dies erklärt den reduzierten Umfang zum Vorgängermodell. Der erste Themenbereich Die Entstebung des Lebens auf der Erde und seine Entwicklung bis zum Menschen glich in Struktur, Umfang und inhaltlich-kategorialer Ausrichtung dem Lehrbuch von 1952. Der relative Umfang unterschied sich dagegen vom Vorgängermodell, da Veränderungen in den anderen Kapiteln auftraten. So nahmen die Kapitel Die 
Entstebung des Lebens auf der Erde 8,3\%, Die Abstammungslebre und Beweise für ibre Ricbtigkeit 35,4 \%, Die Entstehung der Arten und die Grundlagen der systematischen Gliederung der Organismen 3,1 \% sowie Die Abstammung und Entwicklung des Menschen 7,3 \% ein. Der zweite Themenkomplex Zur Geschichte der Abstammungslehre enthielt grundlegende Veränderungen. Lediglich die Ausrichtung der Inhalte (Kategorie Theorien) stimmte mit der Lehrbuchausgabe von 1952 überein. Nach einer Hinführung im Umfang von sechs Seiten (6,3\%) enthielt der Themenbereich vier Kapitel. Die ersten drei Kapitel Die Entwicklungslebre Lamarcks mit einem Umfang von fünf Seiten (5,2\%), Cwiver und Geoffroy Saint-Hilaire mit einem Umfang von drei Seiten (3,1\%) sowie Die wissenschaftliche Begründung der Entwicklungslehre durch Darwin mit einem Umfang von zwölf Seiten (12,5\%) waren nicht weiter strukturiert. Das vierte Kapitel Die Kämpfe um den Darwinismus gliederte sich dagegen in fünf Unterkapitel Thomas Henry Huxley, Ernst Haeckel, Wladimir Onufriewitsch Kowalewsky, Alexander Onufriewitsch Kowalewsky sowie Kliment Arkadjewitsch Timirjasew. Es umfasste 18 Seiten (18,8 \%). Das Ergebnis einer Frequenzanalyse biologischer Begriffe aus den Kapitelüberschriften im Lehrbuch von 1957 zeigt Übereinstimmungen mit dem Lehrbuch von 1952. Erneut nahm der Begriff Beweise eine zentrale Stellung ein (Abb. 97).

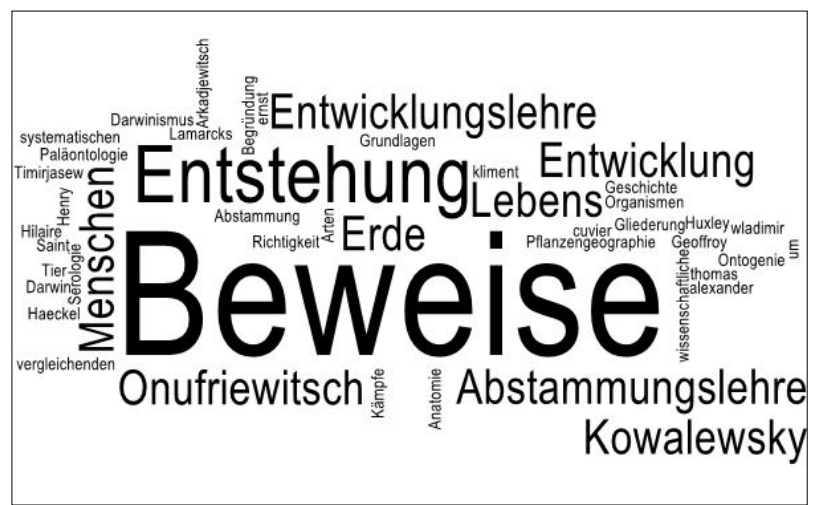

Abbildung 97: Biologische Begriffe im Inhaltsverzeichnis, Lehrbuch Klasse 12 (1957)

Die Inhaltsanalyse der Lehrbuchtexte von 1957 zeigt, dass die Gliederung Biogenese - Paläontologie - Neontologie - Paläontologie - Neontologie - Systematik Hominisation - Theorien aus dem Lehrbuch von 1952 beibehalten wurde. Trotz Veränderungen im Umfang wies die Kategorie Theorien dabei die längste zusammenhängende Textpassage auf (Abb. 98). Die Raumanalyse verdeutlicht, dass der Textumfang dieser Kategorie auf 1467 Zeilen (46,8 \%) reduziert wurde. Dennoch nahmen entsprechende Inhalte den größten Umfang am Lehrbuch ein. In weiterer Reihenfolge umfassten die Kategorien Neontologie 626,5 Zeilen (20,0 \%), Biogenese 324,5 Zeilen (10,3\%), Paläontologie 294,5 Zeilen (9,4 \%), Hominisation 
277,5 Zeilen (8,8 \%), Systematik 124 Zeilen (4,0\%) sowie Didaktisches 23 Zeilen $(0,6 \%)$.

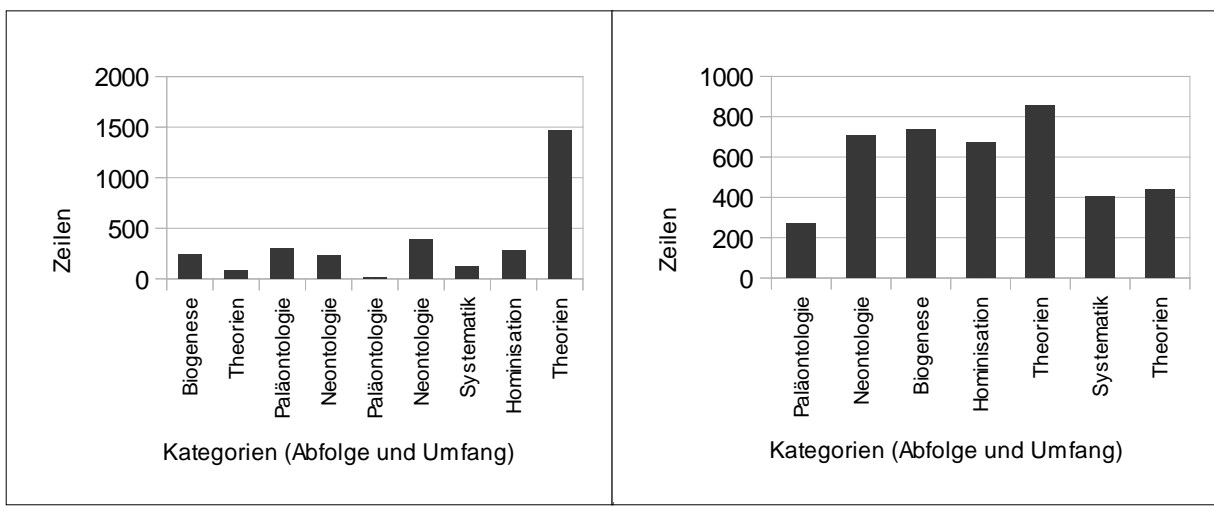

Abbildung 98: Kategorien im Lehrbuch Klasse 12 (1957)

Abbildung 99: Kategorien im Lehrbuch Klasse 12 (1965)

\section{Das Lehrbuch von 1965}

Die Inhalte im Lehrbuch von 1965 gliederten sich in elf Kapitel. Das achte Kapitel behandelte die Grundlagen der Vererbungslehre und die letzten beiden Kapitel befassten sich mit Inhalten zur Pflanzen- und Tierzüchtung. Wie schon in den Lehrbüchern von 1952 und 1957 umfassten Inhalte aus der Kategorie Theorien die längste zusammenhängende Textpassage und bildete weiterhin den inhaltlichen Schwerpunkt des Lehrbuches (Abb. 99). Im Vergleich zu seinen Vorgängermodellen reduzierte sich jedoch mit 1293 Zeilen (31,6 \%) der relative Umfang der Texte aus dieser Kategorie deutlich. Der Grund dafür lag in der ausgewogenen Kapitelstruktur. Die anderen Kategorien wiesen folgenden Umfang auf: Biogenese 736,5 Zeilen (18,0 \%), Neontologie 708,5 Zeilen (17,3\%), Hominisation 674 Zeilen (16,5\%), Systematik 406 Zeilen (9,9\%) sowie Paläontologie 271 Zeilen (6,6 \%). Die Inhaltsanalyse evolutionsbiologischer Lehrbuchtexte von 1965 verdeutlicht die Gliederung Paläontologie - Neontologie - Biogenese - Hominisation - Theorien - Systematik - Theorien. Die Feinstruktur der evolutionsbiologischen Inhalte stellte sich wie folgt dar:

- Die Paläontologie und die Stammesgeschichte der Organismen (13 Seiten; $9,2 \%)$

- Tatsachen aus der vergleichenden Anatomie (21 Seiten; 14,9\%)

- Entwicklung der Lunge

- Blutkreislauf und Atmungsorgane der Wirbeltiere

- Das Gehirn der Wirbeltiere 
- Homologe Organe

- Analoge Organe

- Konvergenzen und Divergenzen

- Rudimentäre Organe

- Homologe, analoge und rudimentäre Organe bei Pflanzen

- Tatsachen aus der Ontogenie (11 Seiten; 7,8 \%)

- Die Biogenetische Grundregel

- Die stammesgeschichtliche Bedeutung der Gastrula

- Vergleich von Wirbeltierembryonen

- Wesen und Entstehung des Lebens (22 Seiten; 15,6 \%)

- Das Wesen des Lebens

- Stoffliche Zusammensetzung des Lebens

- Eigenschaften des Lebens

- Das Leben als spezifische Bewegungsform der Materie

- Die historische Entwicklung der Kenntnis vom Leben

- Die Entstehung des Lebens

- Die weitere Entwicklung der Urorganismen

- Überholte Vorstellungen von der Entstehung des Lebens

- Abstammung und Entwicklung des Menschen (22 Seiten; 15,6 \%)

- Die Stellung des Menschen im System der Organismen

- Die Abstammung des Menschen

- Die Menschwerdung war nicht nur ein biologischer Vorgang

- Die biologische Entwicklung des Homo sapiens

- Die heutigen Menschenrassen

- Biologische Entwicklungsvorgänge beim heutigen Menschen

- Zur Geschichte der Abstammungslehre (27 Seiten; 19,1\%)

- Schöpfung oder Entwicklung

- Die naturwissenschaftliche Voraussetzungen für die Abstammungslehre

- Vorläufer der wissenschaftlichen Abstammungslehre

- Die Begründer der wissenschaftlichen Abstammungslehre

- Die Verbreitung und Weiterentwicklung des Darwinismus

- Stammesgeschichte und System der Organismen (10 Seiten; 7,1 \%)

- Faktoren der stammesgeschichtlichen Entwicklung (15 Seiten; 10,6 \%)

- Die Selektion

- Die Mutation

- Die Isolation

- Populationswellen

- Die Kombination

- Das Zusammenwirken der Evolutionsfaktoren 
Im Ergebnis einer Frequenzanalyse der Kapitelüberschriften zeigt sich, dass im Lehrbuch von 1965 der biologische Begriff Entwicklung eine zentrale Stellung einnahm. Weitere zentrale Begriffe in diesem Schulbuch waren Lebens, Abstammungslebre, Menschen und Organe (Abb. 100).

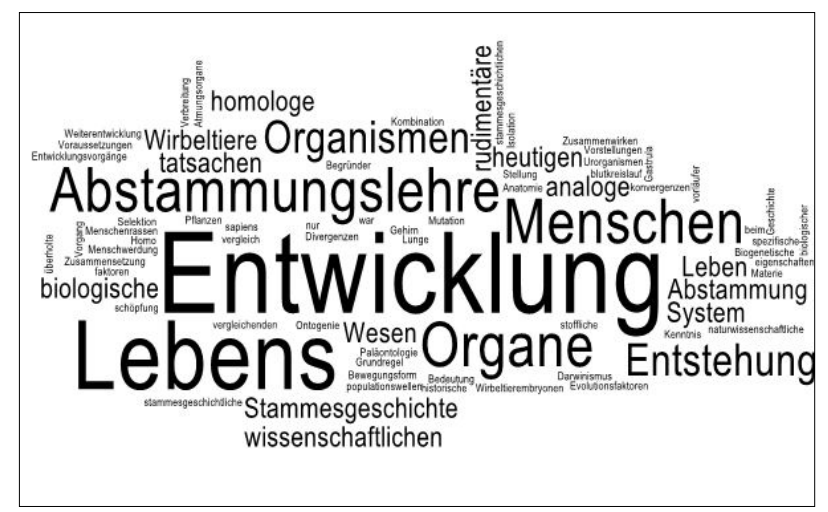

Abbildung 100: Biologische Begriffe im Inhaltsverzeichnis, Lehrbuch Klasse 12 (1965)

\section{Zusammenfassung}

Die Struktur der Kapitel im Lehrbuch von 1952 folgte im ersten Themenkomplex einem Verlauf, der von der Entstehung des Lebens bis zur Entwicklung des Menschen führte. Der zweite Themenkomplex diente der Darstellung wissenschaftsgeschichtlicher Aspekte. Dabei war aufgrund der Oberbegriffe bereits zu erkennen, dass umfangreiche Darstellungen zum Thema Schöpferischer Darwinismus folgten. Umfang als auch Gliederung der Kapitel verdeutlichten, dass den Kapiteln Die Abstammungslehre und Beweise für ibre Richtigkeit sowie Die Weiterentwicklung des Darvinismus zur schöpferischen Biologie der größte Stellenwert zukam. Lediglich diese Kapitel waren untergliedert und nahmen einen deutlich größeren Raum ein als die übrigen Kapitel. Die Struktur der Kapitel im Lehrbuch von 1957 folgte, wie bereits im Lehrbuch von 1952, im ersten Themenkomplex einem Verlauf, der die Phylogenese nachzeichnete. Der zweite Themenkomplex stellte zwar weiterhin Wissenschaftsgeschichte dar, die Inhalte waren jedoch neu ausgerichtet. Umfang und Gliederung der Kapitel belegen, dass den Kapiteln Die Abstammungslebre und Bewveise für ibre Richtigkeit sowie Die Kämpfe um den Darwinismus der größte Stellenwert zukam. Wie bereits im Lehrbuch von 1952 waren nur diese untergliedert und umfassten einen deutlich größeren Raum als die anderen Kapitel. Die Kapitelstruktur im Lehrbuch von 1965 folgte im Gegensatz zu den Vorgängermodellen einem anderen Verlauf. Hier befanden sich Indizien für Evolutionsprozesse am Anfang des Lehrbuches. Die Anzahl der Unterkapitel im Lehrbuch von 1965 belegt eine differenzierte Gliederung der Inhalte. Umfang als auch Gliederung der 
Kapitel verdeutlichen eine ausgewogene Verteilung der Inhalte. Den größten Raum nahm dabei das Kapitel Zur Geschichte der Abstammungslehre ein (Tab. 52).

Tabelle 52: Strukturierung, Lehrbücher Klasse 12

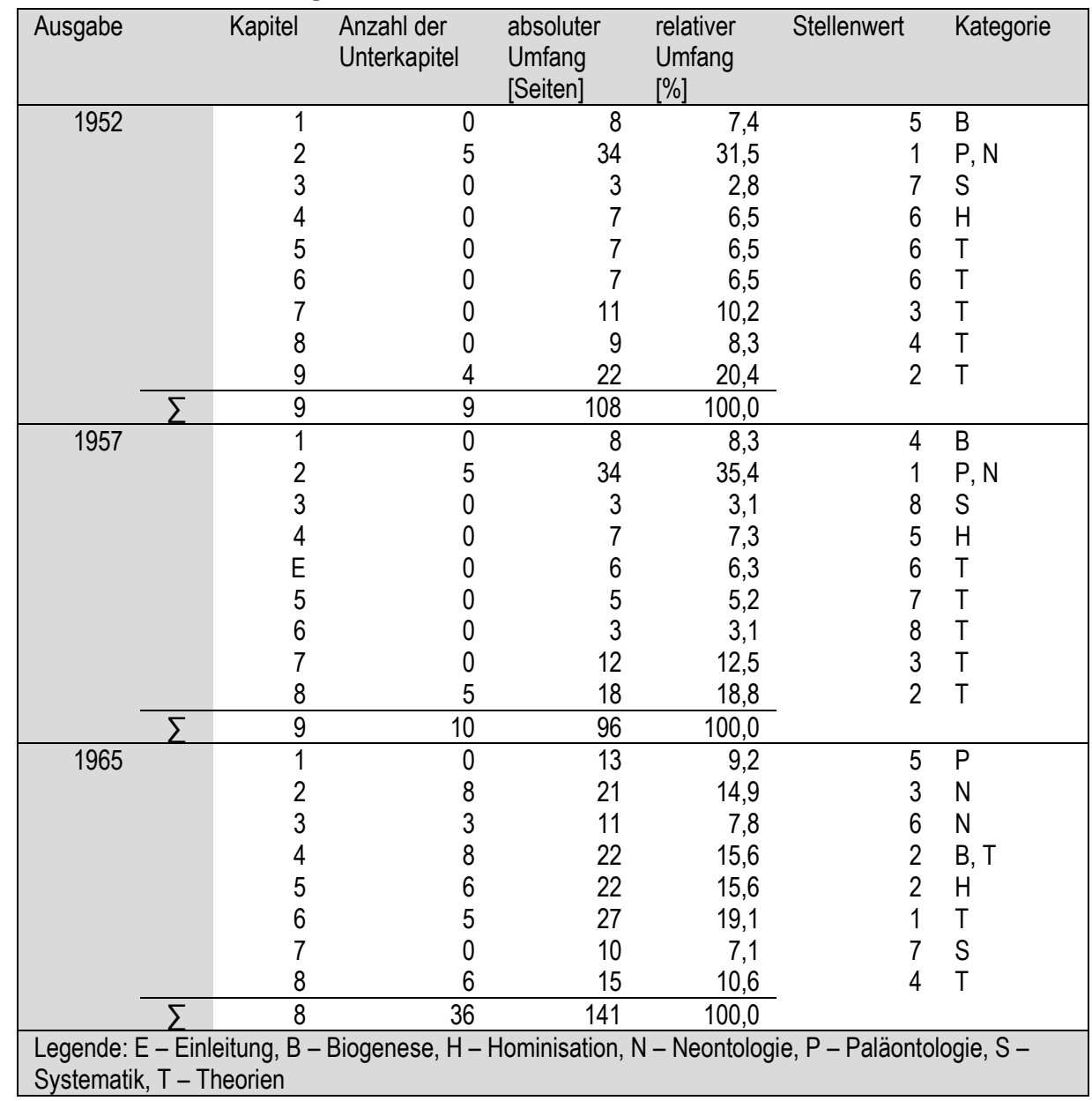

Die kategoriale Raumanalyse belegt, einen hohen Stellenwert der Kategorie Theorien in allen drei Lehrbüchern. Jedoch nahm der absolute sowie relative Umfang von 1952 bis 1965 ab. Im Gegenzug dazu nahm der Umfang der Kategorien Biogenese sowie Hominisation insbesondere im Lehrbuch von 1965 deutlich zu. Die Kategorien Neontologie und Paläontologie variierten dagegen im Umfang nur wenig (Abb. 101). 


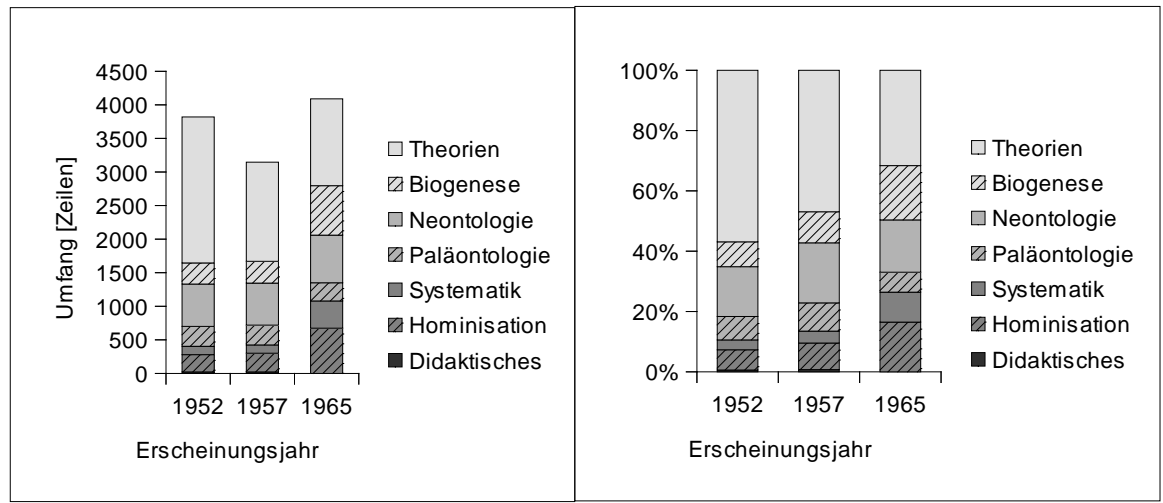

Abbildung 101: links: absoluter Umfang, rechts: relativer Umfang der Kategorien in den Lehrbüchern der Klasse 12

\section{Fachinhalte}

\section{Evolutionstheorien}

Mit 75 Personen in den Ausgaben von 1952 und 1957 sowie 70 Personen in der Ausgabe von 1965 behandelten die Lehrbücher für die Klasse 12 deutlich mehr historische Persönlichkeiten als die Schülerbücher für die Klassen 8 und 10. Die Zahlen belegen, dass trotz inhaltlicher Veränderungen eine Konstanz in der thematischen Breite für entsprechende Inhalte erhalten blieb. Dagegen verringerte sich die thematische Intensität, da sich die Häufigkeit von 582 Nennungen (Ausgabe 1952) auf 294 Nennungen (Ausgabe 1965) reduzierte (Tab. 53). Sowohl die thematische Breite als auch die Intensität fielen jedoch deutlich höher aus als in den Lehrbüchern für die Klassen 8 und 10. Den höchsten Stellenwert nahm in allen Lehrbüchern für die Klasse 12, wie bereits in den Lehrbüchern der unteren Jahrgänge, die Person Charles Darwin ein. Ebenfalls einen hohen Stellenwert, wenngleich in der Frequenz deutlich geringer als Darwin, kam dem Biologen Ernst Haeckel zu (Abb. 102). Während Alfred Russell Wallace in den Lehrbüchern von 1952 und 1957 mit einigen wenigen Nennungen vertreten war, nahmen die Autoren entsprechende Inhalte zu seiner Person im Lehrbuch von 1965 nicht mehr auf (Abb. 103). Eine weitere Eigenschaft der Lehrbücher bestand darin, dass eine Vielzahl von Personen nur einmal genannt wurde.

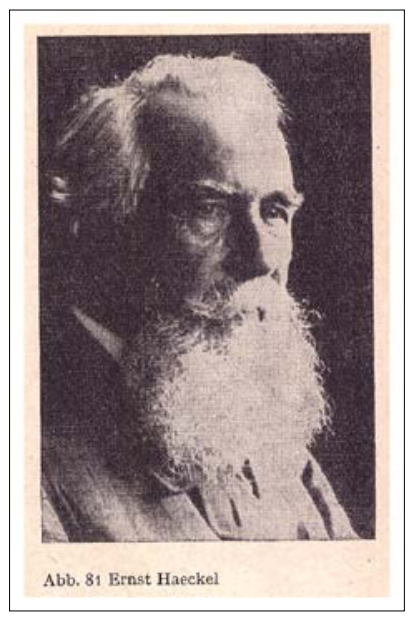

Abbildung 102: Ernst Haeckel (Bach et al. 1967) 
Tabelle 53: Frequenzanalyse (Personen), Lehrbücher Klasse 12

\begin{tabular}{|lrrr|}
\hline Auflage & 1952 & 1957 & 1965 \\
Anzahl der Personen & 75 & 75 & 70 \\
Summe der Nennungen & 582 & 469 & 294 \\
\hline
\end{tabular}

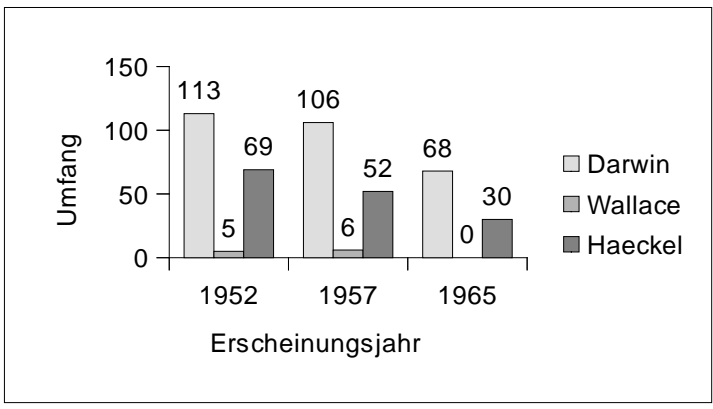

Abbildung 103: Darwin, Wallace und Haeckel in den Lehrbüchern der Klasse 12

\section{Darwinismus}

Die Lehrbücher von 1952, 1957 und 1965 enthielten Aussagen zu Darwins Leben und Werk. Hierbei wurden die vier thematischen Blöcke: Darwins Leben vor seiner Reise, die Fahrt mit der Beagle, Darwins Leben nach seiner Reise sowie Inhalte aus Darwins Werk über die Entstehung der Arten in allen Lehrbüchern behandelt. Weitgehende Übereinstimmung bestand zwischen den Lehrbüchern von 1952 und 1957. Das Lehrbuch von 1965 unterschied sich jedoch deutlich in Umfang und Tiefe der Inhalte von seinen Vorgängermodellen (Tab. 54). Allen Lehrbüchern gemeinsam war, dass die Behandlung der Inhalte einem chronologischen Verlauf folgte. Ein Charakteristikum der Lehrbücher bestand darin, dass sie den Einfluss des Darwinismus auf teleologische Erklärungen in der Biologie betonten und in diesem Kontext die Sichtweise von Karl Marx aufzeigten. Am ausführlichsten wurde dies im Lehrbuch von 1965 behandelt. Rolf Löther, im Lehrbuch von 1965 zuständig für das Kapitel Zur Geschichte der Abstammungslehre, formulierte:

„Die teleologische Erklärungsweise von Naturerscheinungen geriet notwendig mit dem Vordringen der menschlichen Erkenntnis, mit der Frage nach den Ursachen und Gesetzmäßigkeiten, dem ,Warum?‘, in Widerspruch und erwies sich als starkes Hemmnis des wissenschaftlichen Fortschritts." (Bach et al. 1967: 112)

Von den fünf Darwin'schen Theorien stand in den Lehrbüchern von 1952 bis 1965 insbesondere die Selektionstheorie im Vordergrund der Abhandlungen. Zwar waren auch die übrigen Theorien zum Teil in den Lehrbüchern vertreten, jedoch deuteten die Verfasser diese zumeist nur in einem Satz an (Tab. 55). 
Tabelle 54: Darwins Leben und Werk, Lehrbücher Klasse 12

\begin{tabular}{|c|c|c|c|c|}
\hline Klasse & Vor der Reise & Reisebeschreibung & Nach der Reise & Theoretische Aspekte \\
\hline 1952 & \multirow[t]{2}{*}{$\begin{array}{l}\text { Herkunft, } \\
\text { Schulbildung, } \\
\text { Vorlieben, } \\
\text { Medizinstudium, } \\
\text { erste } \\
\text { Entdeckungen, } \\
\text { Theologiestudium }\end{array}$} & \multirow[t]{2}{*}{$\begin{array}{l}\text { Grund der Reise, Ver- } \\
\text { mittlung, Vorbereitung, } \\
\text { Anschauungen Lyells, } \\
\text { Exkursionen in Süd- } \\
\text { amerika, Grundfinken } \\
\text { auf Galapagos, Koral- } \\
\text { lenriffe, Ankunft }\end{array}$} & \multirow[t]{2}{*}{$\begin{array}{l}\text { Reisetagebuch, Notiz- } \\
\text { bücher, Malthus, Ent- } \\
\text { wurf des Artenbuches, } \\
\text { weitere Veröffentlichun- } \\
\text { gen, Darwins Arbeits- } \\
\text { weise, Wallace, Vorlage } \\
\text { des Manuskripts, Aufla- } \\
\text { ge des Artenbuches, } \\
\text { ideologische Auseinan- } \\
\text { dersetzungen, Landsitz } \\
\text { in Down, die letzten } \\
\text { Werke }\end{array}$} & $\begin{array}{l}\text { Zuchtwahl (künstli- } \\
\text { che, natürliche, } \\
\text { geschlechtliche), } \\
\text { Kampf ums Dasein, } \\
\text { Kreuzung der Indivi- } \\
\text { duen, Gesetze der } \\
\text { Abänderung, Bas- } \\
\text { tardbildung, Verbrei- } \\
\text { tung, Verwandtschaft } \\
\text { der Lebewesen, } \\
\text { Gesetze der Variabili- } \\
\text { tät }\end{array}$ \\
\hline 1957 & & & & $\begin{array}{l}\text { Zuchtwahl (künstli- } \\
\text { che, natürliche, } \\
\text { geschlechtliche), } \\
\text { Kampf ums Dasein, } \\
\text { gemeinsame Ab- } \\
\text { stammung }\end{array}$ \\
\hline 1965 & $\begin{array}{l}\text { Herkunft, } \\
\text { Schulbildung, } \\
\text { Vorlieben, } \\
\text { Medizinstudium, } \\
\text { Theologiestudium }\end{array}$ & $\begin{array}{l}\text { Grund der Reise, Ver- } \\
\text { mittlung, Exkursionen in } \\
\text { Südamerika, Grundfin- } \\
\text { ken auf Galapagos }\end{array}$ & $\begin{array}{l}\text { Auswertung der Reise, } \\
\text { Auflage des Artenbu- } \\
\text { ches, ideologische } \\
\text { Auseinandersetzungen, } \\
\text { Darwinismus und } \\
\text { Teleologie }\end{array}$ & $\begin{array}{l}\text { Zuchtwahl (künstli- } \\
\text { che, natürliche, } \\
\text { geschlechtliche), } \\
\text { Kampf ums Dasein }\end{array}$ \\
\hline
\end{tabular}

Tabelle 55: Ankerbeispiele für die fünf Darwin'schen Theorien, Lehrbücher Klasse 12

\begin{tabular}{|c|c|c|c|c|c|}
\hline Klasse & $\begin{array}{l}\text { eränder- } \\
\text { hkeit }\end{array}$ & Abstammungslehre & $\begin{array}{l}\text { Gradualismu } \\
\mathrm{s}\end{array}$ & $\begin{array}{l}\text { Aufspal- } \\
\text { tung }\end{array}$ & Selektionslehre \\
\hline 1952 & \multirow{2}{*}{\multicolumn{2}{|c|}{$\begin{array}{l}\text { „Wenn man diese Abstufung und } \\
\text { Verschiedenartigkeit der Struktur } \\
{[\ldots . .] \text { sieht, so kann man sich wirk- }} \\
\text { lich vorstellen, daß infolge einer } \\
\text { ursprünglichen Armut an Vögeln } \\
\text { [...] die eine Spezies hergenom- } \\
\text { men und zu verschiedenen Zwe- } \\
\text { cken modifiziert worden sei."“ } \\
\text { (Gruner et al. 1953: 75) }\end{array}$}} & \multicolumn{3}{|c|}{$\begin{array}{l}\text { „Im Laufe der Zeit werden so aus Varietäten echte Arten, } \\
\text { wobei die verbindenden Zwischenglieder allmählich durch } \\
\text { Selektion vernichtet werden." (Gruner et al. 1953: 79) }\end{array}$} \\
\hline 1957 & & & \multicolumn{3}{|c|}{$\begin{array}{l}\text { „DARWIN erklärt also die Entstehung der Arten durch die } \\
\text { natürliche Zuchtwahl (Auslese) zahlreicher, nacheinander } \\
\text { auftretender, für sich allein unbedeutender günstiger } \\
\text { Abänderungen.“ (Rothmaler \& Uschmann 1957: 81, } \\
\text { Hervorheb. i. O.) }\end{array}$} \\
\hline 1965 & \multicolumn{3}{|c|}{$\begin{array}{l}\text { „Daraus schloß er, daß sie von amerikanischen } \\
\text { Vorfahren abstammen [...] und und sich unter den } \\
\text { neuen Lebensverhältnissen im Laufe der } \\
\text { Generationen änderten.“ (Bach et al. 1967: 109) }\end{array}$} & - & $\begin{array}{l}\text { „Die natürliche Zuchtwahl [...] } \\
\text { wurde von DARWIN als wesent- } \\
\text { liche Ursache für die Entwick- } \\
\text { lung der Arten erkannt." (Bach et } \\
\text { al. 1967: 110, Hervorheb. i. O.) }\end{array}$ \\
\hline
\end{tabular}




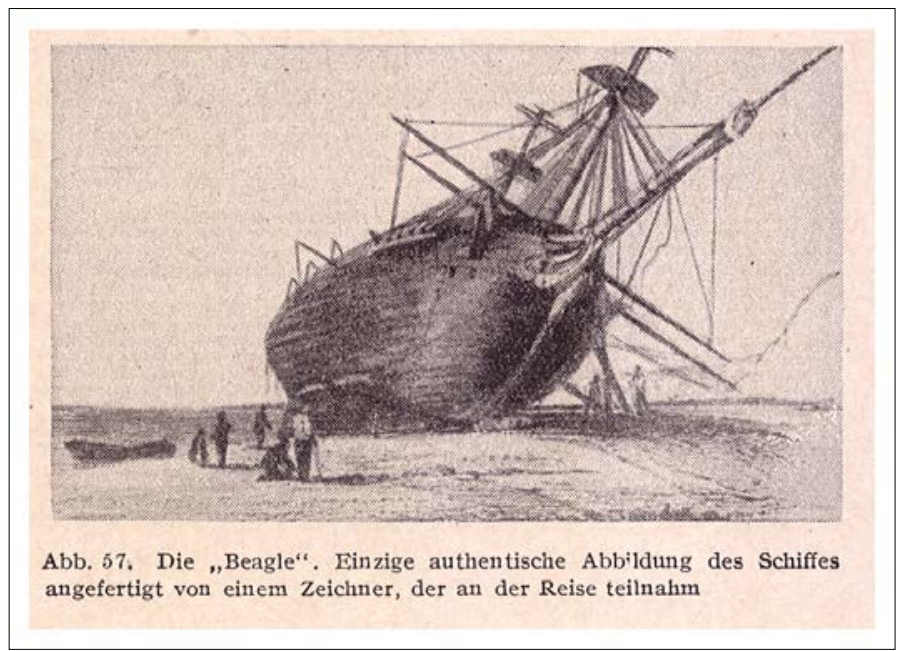

Abbildung 104: Das Forschungsschiff Beagle (Gruner et al. 1953)

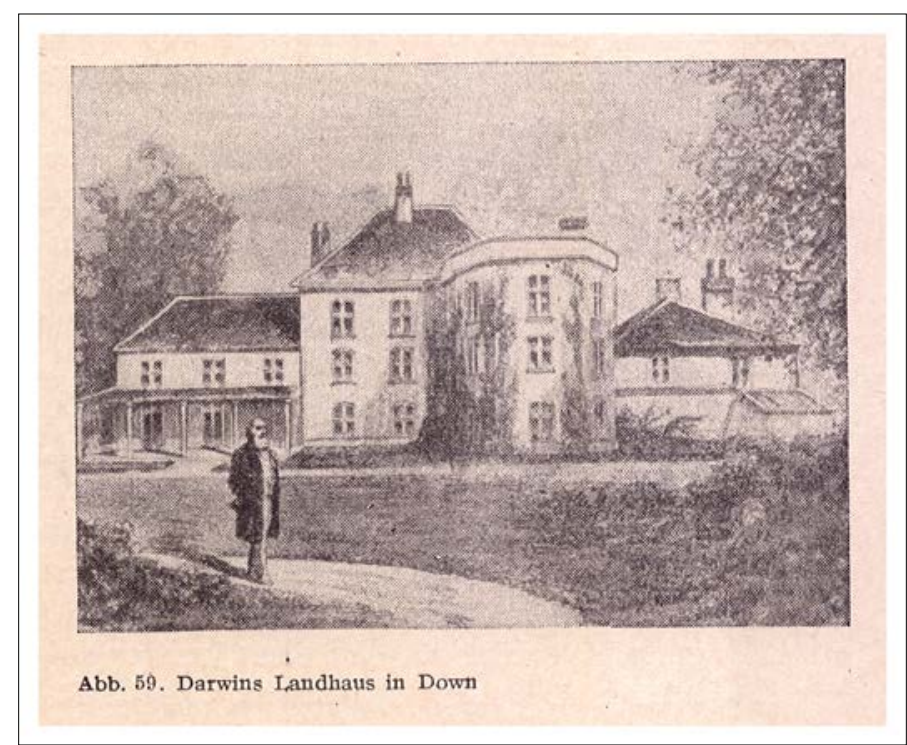

Abbildung 105: Darwins Landhaus (Gruner et al. 1953)

Auch der Inhalt und der Umfang der Visualisierung veränderten sich. Charles Darwin wurde in den Lehrbüchern von 1952 und 1957 als alter Mann (um 1880) und im Lehrbuch von 1965 als junger Mann (um 1849) abgebildet. Alle drei Lehrbücher enthielten eine Darstellung des Forschungsschiffes Beagle (Abb. 104). Im Lehrbuch von 1957 stand den Schülern die Reiseroute (auf zwei Seiten) und im Lehrbuch von 1965 ein Teil der Reiseroute (Südamerika) zur Verfügung. Die 
Lehrbücher von 1957 und 1965 zeigten ebenso eine Abbildung der DarwinFinken. Weiterhin waren in der Ausgabe von 1965 das Schema einer Kerguelenfliege mit verkümmerten Flügeln, Schutztrachten bei Tieren (Blattähnliche Insekten) sowie das Geweih eines Riesenhirsches abgebildet. Die Lehrbücher von 1952 und 1957 enthielten einen Auszug aus einem Brief an Ernst Haeckel sowie eine Abbildung von Charles Darwins Landhaus in Down (Abb. 105).

\section{Neodarwinismus vs. Schöpferischer Darwinismus}

Im Lehrbuch von 1952 erfuhr der Neodarwinismus von August Weismann eine Wertung durch die Darlegung der Ansichten Ernst Haeckels. Dieser lehnte die Weismann'sche Theorie ab, die ,nach ihm zur, Irrlehre der Präformation und zur vitalistischen Teleologie‘ zurück" führte (Gruner et al. 1953: 89). Auch das Lehrbuch von 1957 enthielt in geringem Umfang Aussagen über Weismann und den Neodarwinismus. Hier wurde dessen wissenschaftliche Gültigkeit jedoch weder be- noch widerlegt. Dennoch wies die vorhandene Aussage latent auf eine Ablehnung hin.

„Aufgrund der zu dieser Zeit bestehenden Meinungsverschiedenheiten um den ,Neodarwinismus' des Zoologen AUGUST WEISMANN (1834 bis 1914) hatte ein Redner überhaupt an der Richtigkeit der Entwicklungslehre gezweifelt.“ (Rothmaler \& Uschmann 1957: 86, Hervorhebung im Original)

Stattdessen enthielt das Lehrbuch von 1952 umfangreiche Angaben über Mitschurin, Lyssenko und den schöpferischen Darwinismus (Abb. 106). Wie bereits dargelegt, behandelte diese Ausgabe die Theorien Mitschurins und Lyssenkos ausführlich in zwei Unterkapiteln. In diesen wurde die Vererbung erworbener Eigenschaften postuliert und u. a. das Verfahren der Jarowisation beschrieben. Fotografien zeigte die sowjetischen Biologen mit ihrer Unterschrift (Abb. 107). Das Bild von Lyssenko, das ihn mit einem Roggen (Secale cereale) in den Händen zeigte, integrierten die Autoren auch in den folgenden Lehrbücher der 1950er Jahre für die Klassenstufe 8.

Im Lehrbuch von 1965 fand dagegen ein Paradigmenwechsel statt. Die Angaben zum Neodarwinismus waren umfangreicher, visualisierten August Weismann (Abb. 108) und belegten nunmehr seinen Beitrag, der in der Wiederlegung der Lehre von den erworbenen Eigenschaften bestand.

„WEISMANNs Kritik an den zeitgenössischen Vorstellungen über 'Vererbung erworbener Eigenschaften' trug wesentlich zur Überwindung naiver Annahmen über das Vererbungsgeschehen bei.“ (Bach et al. 1967: 119, Hervorheb. i. O.) 


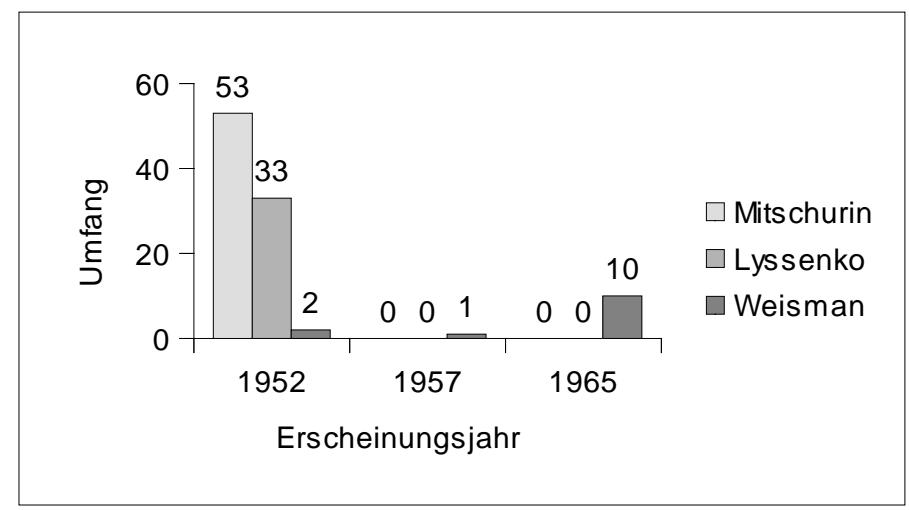

Abbildung 106: Mitschurin, Lyssenko und Weismann in den Lehrbüchern für die Klasse 12

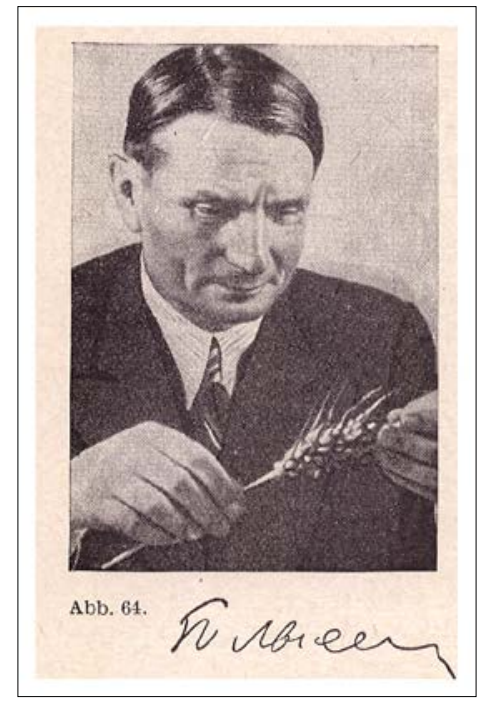

Abbildung 107: Trofim D.

Lyssenko (Gruner et al. 1953)

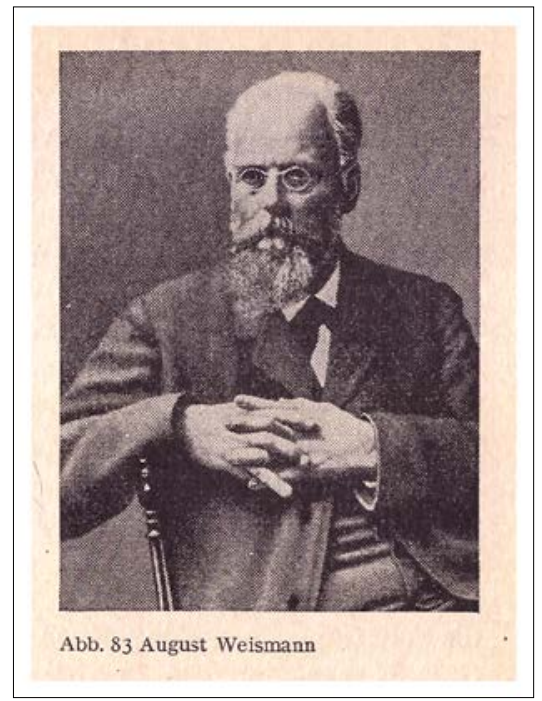

Abbildung 108: August Weismann (Bach et al. 1967)

\section{Synthetischer Darwinismus}

Ausschließlich das Lehrbuch von 1965 enthielt Postulate des Synthetischen Darwinismus. Das Kapitel Faktoren der stammesgeschichtlichen Entwicklung erläuterte die genetische Variation durch Mutation und Rekombination. Mutationen wurden hier ,als der primäre Evolutionsfaktor“ (Bach et al. 1967: 191) bezeichnet. Mutationen bei der Fruchtfliege (Drosophila melanogaster), flugunfähige Insekten von den Kerguelen-Inseln, Varianten des Marienkäfers aus Ostasien (Harmonia axyridis) 
sowie Blätter der Küchenschelle (Pulsatilla spec.) dienten den Erläuterungen als Beispiele. Die Autoren gingen auch auf die Speziation durch geografische Isolation ein. Der Fliegenfänger auf den Salomonen-Inseln diente hierbei in Text und Abbildung als Beispiel.

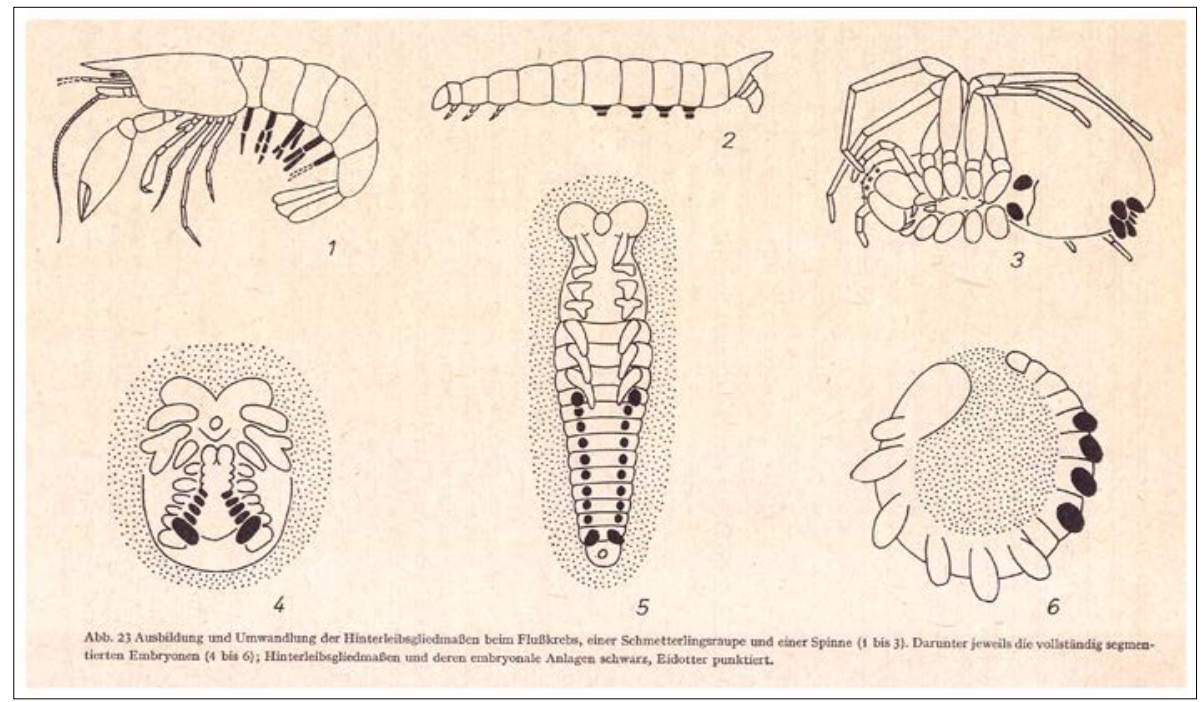

Abbildung 109: Ausbildung und Umwandlung der Hinterleibsgliedmaßen beim Flusskrebs, einer Schmetterlingsraupe und einer Spinne (Bach et al. 1967)

\section{Neontologie}

\section{Homologie und Analogie}

Den Fragen der Homologie kam in den Lehrbüchern der 1950er Jahre eine ,auBerordentliche Bedeutung“ (Rothmaler 1952: 341) zu. In allen Lehrbüchern wurde der Begriff homolog verwendet und definiert. Die Auswahl der Beispiele und der Umfang der Darstellung differierten jedoch. Für die Begriffserklärung verwiesen die Autoren in den Lehrbüchern von 1952/57 exemplarisch darauf, dass „die oberen Nagezähne der Nagetiere und die Stoßzähne der Elefanten den Schneidezähnen anderer Säuger homolog [sind]" (Gruner et al. 1967: 25). Das Schulbuch von 1965 führte als homologe Organe „die Hinterleibsbeine des Flusskrebses, die stummelförmigen Nachschieber der Schmetterlingsraupen und die Atemorgane und Spinnwarzen der Webspinnen" (Bach et al. 1967: 28) als Beispiele an (Abb. 109). Die Lehrbücher von 1952/57 informierten anschließend ausführlich (zwölf Seiten) über die vergleichende Anatomie der Wirbeltiere (Vertebrata). Es wurden die Gehörknöchelchen der Säugetiere, die Ausbildung der Wirbelsäule, die fünfstrahlige Extremität, die Krallen, Hufe und Nägel, das Blutgefäßsystem, die Lunge 
und Schwimmblase, das Gehirn sowie die Körperdecke der Wirbeltiere als „,reichhaltiges Beweismaterial für die Abstammungslehre“ (Gruner et al. 1953: 27) beschrieben, vergleichend dargestellt und illustriert. Darüber hinaus enthielten die Lehrbücher Informationen und eine Abbildung zu den Mundwerkzeugen von Insekten. Die Ausführungen im Lehrbuch von 1965 fielen mit fünf Seiten geringer aus. Beschrieben und vergleichend dargestellt wurde das Gliedmaßenskelett sowie die Schwimmblase und Lunge der Wirbeltiere. Diese Beispiele waren visualisiert. Eine Farbtafel veranschaulichte die Vordergliedmaßen der Wirbeltiere. Darüber hinaus stellten die Autoren in einem weiteren Kapitel homologe Organe bei Pflanzen vor (Laub- und Blütenblätter, Blattranken und -dornen) und illustrierten diese Beispiele.

Die Lehrbücher von 1952 und 1957 definierten den Begriff Analogie. Sie erläuterten und visualisierten Analogien am Beispiel der Ranken sowie Dornen bei Pflanzen. Auch kamen im Text die Kiemen der Fische (Pisces) und Krebse (Crustacea) als analoge Organe zur Sprache. Im Lehrbuch von 1965 stand den Schülern zu diesem Inhalt ein eigenes Kapitel zur Verfügung. Dieses enthielt eine Begriffsdefinition. Anhand der Flügel bei Vögeln (Aves) und Insekten (Insecta) sowie Grabschaufeln des Maulwurfes (Talpidae) und der Maulwurfsgrille (Gryllotalpidae) wurden analoge Organe am Beispiel näher erläutert. Letzteres war auch als Abbildung vorhanden.

\section{Rudimente und Atavismen}

Die Lehrbücher von 1952 und 1957 enthielten viele Beispiele für rudimentäre Organe, die als „sehr aufschlußreich für stammesgeschichtliche Betrachtungen“ (Gruner et al. 1952: 35) in die Ausführungen aufgenommen wurden. Eine Begriffsdefinition stand den Schülern jedoch nicht zur Verfügung. Als Beispiele dienten Rudimente bei Pflanzen (Blätter und Blütenbestandteile), Rückbildungen bei Insektenflügeln, Lichtsinnesorgane höhlenbewohnender Tiere, Rückbildungserscheinungen am Skelett von Wirbeltieren sowie rudimentäre Bildungen beim Menschen. Visualisiert war die Flügelreduktion bei Lausfliegen (Hippoboscidae) sowie Schulter- und Beckengürtel der Blindschleiche (Anguis fragilis). Das Lehrbuch von 1965 behandelte rudimentäre Organe in einem eigenen Unterkapitel und definierte, anders als in den Vorgängermodellen, den biologischen Begriff Rudimente. Den Erläuterungen dienten Rückbildungserscheinungen am Skelett von Wirbeltieren, Extremitäten der Huftiere, rudimentäre Bildungen beim Menschen, flugunfähige Laufvögel und flugunfähige Käfer als Beispiele. Eine Abbildung zeigte das Skelett eines Grönlandwals (Balaena mysticetus).

Die Lehrbücher von 1952 und 1957 definierten am Ende des Kapitels Beweise aus der vergleichenden Anatomie den Begriff Atavismus. Als Beispiele nannten die Autoren Pelorien bei zweiseitig-symmetrischen Blüten, überzählige Zehen bei Pferden und überzählige Brustwarzen, starke Behaarung sowie Halsfisteln beim Menschen. Visualisiert war die Extremität eines Perdes mit überzähligem Huf. Diese 
Abbildung wurde auch in das Lehrbuch von 1965 aufgenommen und im Text dargelegt. Weitere Beispiele für „'Rückschläge' der Entwicklung“ (Bach et al. 1967: 36), im Kapitel Rudimentäre Organe behandelt, waren beidseitig gefärbte Schollen (Pleuronectidae), künstlich erzeugte vierflüglige Taufliegen (Drosopbilidae) sowie Fischsaurier und Wale (Cetacea). Abbildungen standen hier nicht zur Verfügung.

\section{Biogeografie}

Die Lehrbücher von 1952 und 1957 enthielten jeweils ein eigenständiges Kapitel zum Thema Beweise aus der Tier- und Pflanzengeographie. Von Darwins Beobachtungen in Südamerika ausgehend, wurde das Auftreten typischer Lebensformen durch geografische Isolation erläutert. Neben Südamerika stellten die Lehrbücher ausgewählte Organismen Australiens vor. Auch auf Besonderheiten von Inseln, isolierten Inseln, Inselgruppen, große Seen, den Einfluss der Eiszeit und des Menschen gingen die Autoren ein. Abbildungen waren jedoch nicht vorhanden. Im Zusammenhang mit den Darstellungen zu Darwins Reise mit der Beagle (Kategorie Theorien) kamen weitere Inhalte zur Sprache. Das Lehrbuch von 1965 enthielt hingegen keine Angaben neontologischer Forschungen aus dem Bereich der Biogeografie. Lediglich im Zusammenhang mit den Darstellungen zu Darwins Weltreise wurden flugunfähige Insekten als Folgeerscheinung der auf den Inseln herrschenden Bedingungen erwähnt.

\section{Embryologie und Ontogenie}

Die Lehrbücher von 1952 und 1957 stellten im Kapitel Beweise aus der Ontogenie dar, dass Verwandtschaftsverhältnissen durch ontogenetische Beobachtungen geklärt werden können. Die Erläuterungen gingen hierbei in Text und Bild von den Naupliuslarven verschiedener Krebse aus (Abb. 110). In diesem Kontext wurde die Leistung von Fritz Müller herausgestellt, der 1864 eine typische Larve bei einer Reihe von Krebsen nachwies. Die Autoren verglichen auch die Larven von Ringelwürmern und Weichtieren. Eine schematische Abbildung verdeutlichte den Vergleich. In den Erläuterungen zur Entwicklung der Gastrula sollten die Schüler „die große Rolle, die die Gastrula in der Entwicklung spielt" (Gruner et al. 1953: 40) erkennen und diese als gemeinsame Phase fast aller vielzelligen Tiere (Metazoa) verstehen. Anschließend stellte das Schülerbuch das Biogenetische Grundgesetz von Haeckel vor, definierte es und legte seine Grenzen dar. Die Prozesse Paligenese und Cenogenese wurden in diesem Zusammenhang erläutert. Für die Beziehung zwischen Phylogenese und Ontogenese konnten die Schüler auf umfangreich dargelegte Beispiele zurückgreifen (u. a. das Auftreten der Chorda, Kiemenspalten, Jugendformen der Plattfische, Anlagen der Vorderbeine bei der Blindschleiche). 


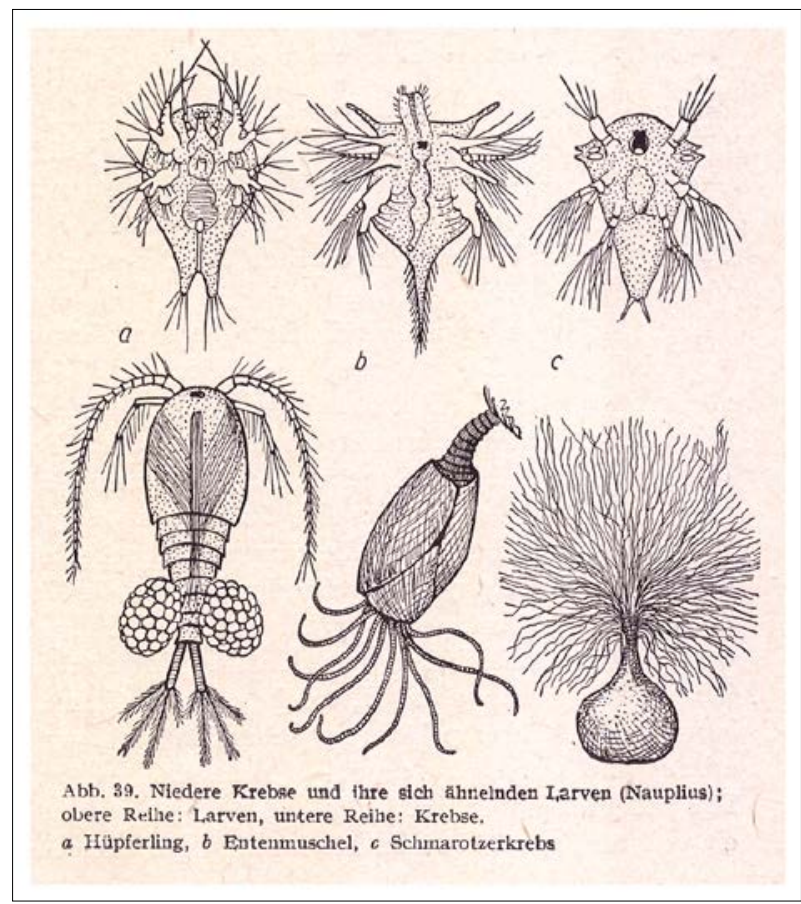

Abbildung 110: Niedere Krebse und sich ähnelnde Larven (Gruner et al. 1953)

Das Lehrbuch von 1965 erläuterte ausführlich die Biogenetische Grundregel am Beispiel der Entwicklungsweise der Frösche und Kröten in Text und Bild. In diesem Kontext wurde die Definition Haeckels von 1866 in den Text aufgenommen. Die Autoren betonten jedoch, dass ,Jahrzehnte hindurch [...] HAECKELS Erkenntnis fälschlicherweise als „Biogenetisches Grundgesetz bezeichnet“ wurde (Bach et al. 1967: 40, Hervorhebung im Original). In diesem Zusammenhang kamen im Textverlauf auch Beispiele zur Sprache, deren stammesgeschichtliche Zusammenhänge durch Haeckels Regel nicht erklärt werden können, wie fehlende Zähne im Schnabel von Vogelembryonen und das Puppenstadium der Insekten. Weitere durch Text und Bild dargestellte Fälle, bei denen die Biogenetische Grundregel Verwandtschaftsverhältnisse klärt, waren Vergleiche der Larven bei Ringelwurm, Meeresschnecke und Seestern sowie der Naupliuslarven bei Hüpferling, Entenmuschel, Seepocke und Wurzelfüßer. In einem zweiten Abschnitt erläuterte auch das Lehrbuch von 1965 die stammesgeschichtliche Bedeutung der Gastrula ausführlich in Text und Bild. Die Darlegungen fokussierten, von der befruchteten Eizelle ausgehend, auf die Entwicklungsstadien bis zur Gastrula. Anschließend wurde der Bau der Coelenterata und Bilateria verglichen sowie die weitere Entwicklung zu Protostomia und Deuterostomia. In einem dritten und letzten Ab- 
schnitt erfolgte schließlich ein Vergleich der Wirbeltierembryonen von Fisch, Schildkröte, Vogel und Mensch (Abb. 111).

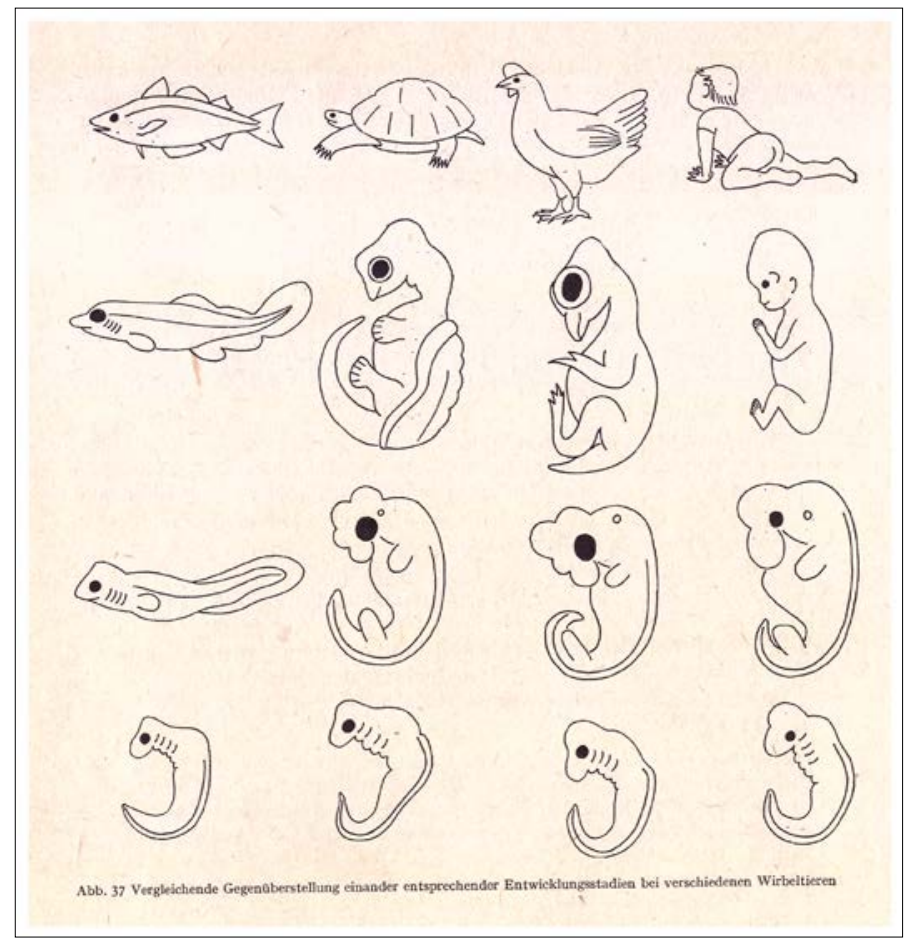

Abbildung 111: Entwicklungsstadien verschiedener Wirbeltiere (Bach et al. 1967)

\section{Paläontologie}

\section{Systemtabellen und Phylogenie}

Die Lehrbücher von 1952 und 1957 enthielten Informationen zur Erdgeschichte und den geologischen Formationen bzw. Perioden (Tab. 56). Die Darstellung folgte dem chronologischen Verlauf von der Urzeit über das Erdmittelalter bis zur Neuzeit. Der Text enthielt Angaben zu den Perioden, den Zeitangaben und den vorherrschenden Organismen. Zahlreichen Abbildungen zeigten charakteristische Fossilien (Trilobit, Armfüßer, Einzelkoralle, Grapholiten, Psilophyten, Ammonit, Nummuliten, Seelilie, Belemnit). Auch eine Landschaft (Steinkohlevegetation) war als Abbildung vorhanden. Eine Systemtabelle kam in den Lehrbüchern nicht vor. Im Lehrbuch von 1965 fand einzig eine spiralförmige Darstellung einer Systemtabelle Eingang (Abb. 112). Diese informierte über Erdzeitalter (Ären), Perioden, Zeitangaben und Lebensformen. Der Lehrbuchtext enthielt keine Angaben. Wei- 
tere Abbildungen waren nicht vorhanden. Dies spiegelte sich auch im Umfang der Darstellung wider (1952/57 je sieben Seiten, 1965 eine Seite).

Tabelle 56: Systemtabelle, Lehrbücher Klasse 12 (*vgl. dazu Kutschera 2015)

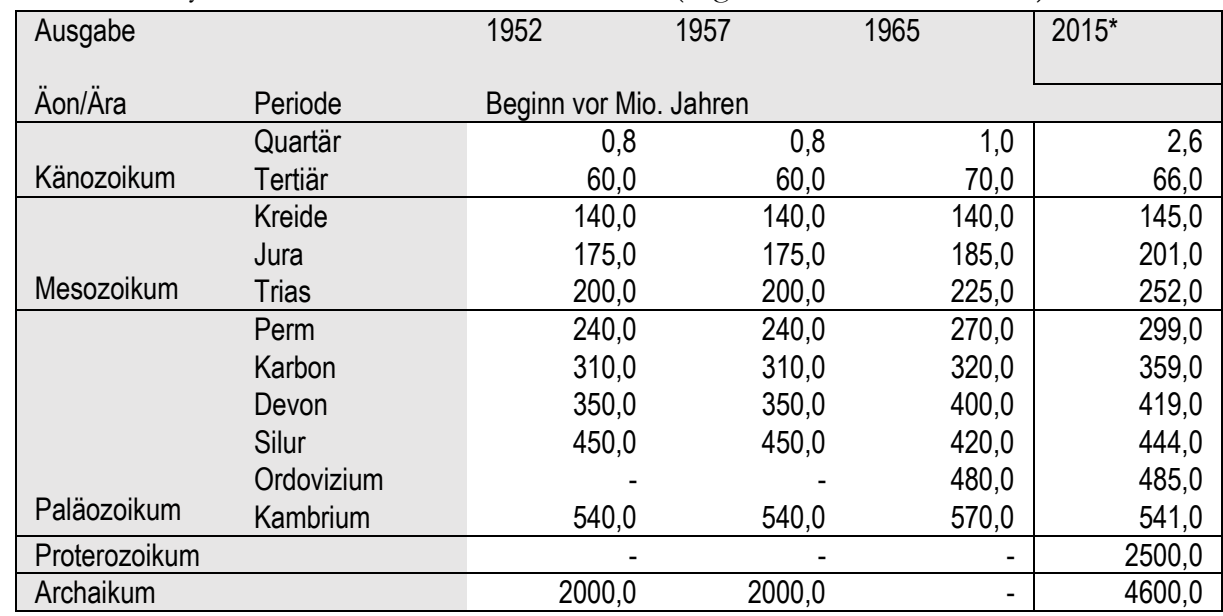

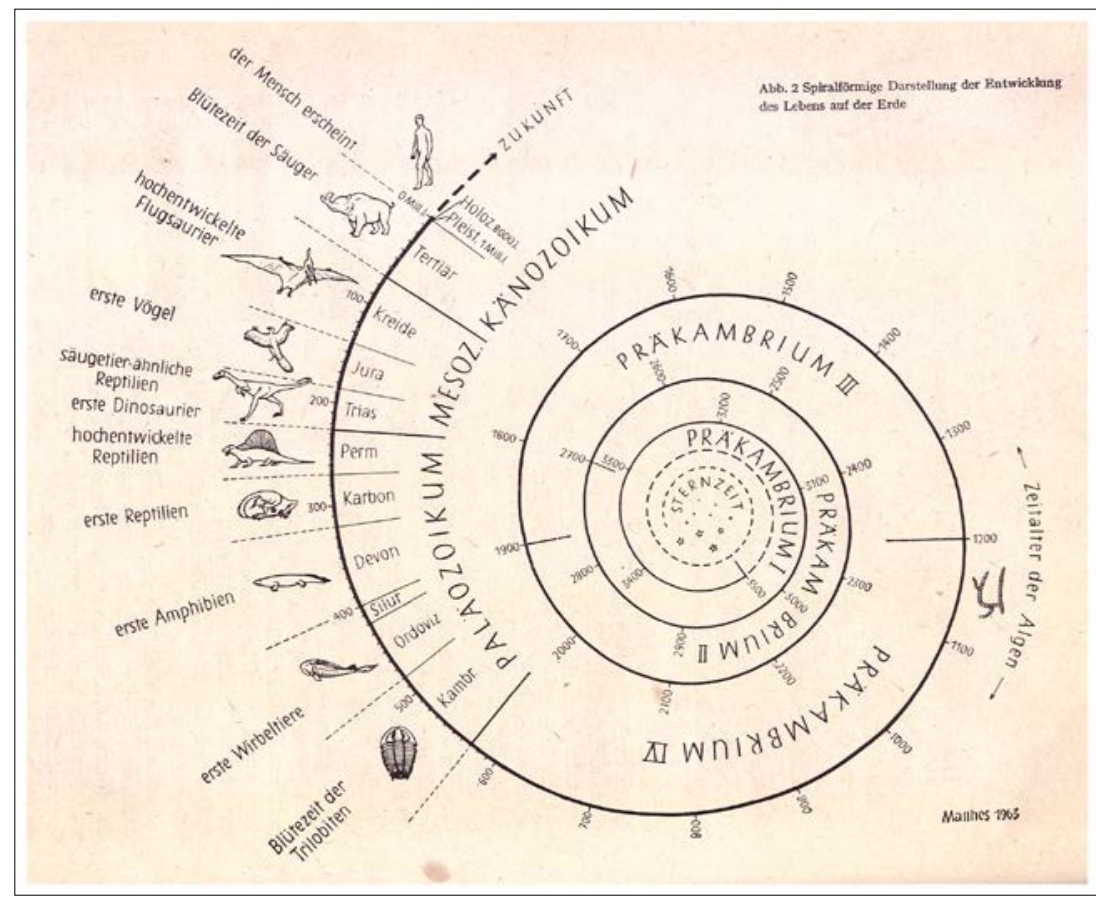

Abbildung 112: Die Entwicklung des Lebens auf der Erde (Bach et al. 1967) 


\section{Entwicklungsreihen und Zwischenformen}

In den Lehrbüchern von 1952 und 1957 stellten die Autoren vier Entwicklungsreihen vor. Zu Beginn wurde auf die Entwicklung der Nacktsamern sowie dem Entstehen des Zapfens der Nadelbäume eingegangen. Anschließend folgten ausführliche Darstellungen zu den Entwicklungsreihen von Pferden (Equus) und Rüsseltieren (Proboscidea). Auch über die Entwicklung der Ammoniten (Ammonoidea) informierte das Schülerbuch. Die Veränderungen des Pferdefußes, des Elefantenschädels und der Lobenlinien der Ammoniten waren als Abbildungen eingebunden. Der Umfang und die Tiefe der Darstellung von Entwicklungsreihen nahmen im Lehrbuch von 1965 zu. Das Lehrbuch bot ebenfalls Informationen über die Entwicklung der Pferde und Rüsseltiere. Visualisiert war die Entwicklung der vorderen Pferdegliedmaßen, des Pferdeschädels sowie des Mammutschädels. Darüber hinaus enthielt das Lehrbuch ausführliche Texte und detaillierte Abbildungen zur Entwicklung der Cephalopoden am Beispiel der Nautiloidea (Abb. 113; Tab. 57).



Abbildung 113: Die Entfaltung der Nautiloide im Alt-Paläozoikum (Bach et al. 1967) 
Tabelle 57: Entwicklungsreihen, Lehrbücher Klasse 12

\begin{tabular}{|c|c|c|c|c|}
\hline Klasse & $\begin{array}{l}\text { Zapfen der } \\
\text { Nadelbäume }\end{array}$ & Pferde & Rüsseltiere & Cephalopoden (*Ausschnitt) \\
\hline $1952 / 57$ & $\begin{array}{l}\text { Entwicklung } \\
\text { aus einer } \\
\text { zusammeng } \\
\text { esetzten } \\
\text { Ähre }\end{array}$ & $\begin{array}{l}\text { 1. Tertiär, Wald, Hunde- } \\
\text { größe, vierzehig, } \\
\text { kurzkronige Mahlzähne, } \\
\text { abgesetzte Vormahlzäh- } \\
\text { ne, kurzer Schädel / } 2 \text {. } \\
\text { dreizehig, Reduzierung } \\
\text { der Seitenzehen, Busch- } \\
\text { steppe / 3. Einhufer, } \\
\text { Mahlzähne mit langer } \\
\text { Krone, Mahlgebiß setzt } \\
\text { sich von Schneidezähnen } \\
\text { ab, Steppe, langer Schä- } \\
\text { del }\end{array}$ & $\begin{array}{l}\text { Umstellung der } \\
\text { Backenzähne, } \\
\text { Umbildung der } \\
\text { Schneidezähne, } \\
\text { Vergrößerung } \\
\text { des Körpers, } \\
\text { Nase und Ober- } \\
\text { lippe zu Rüssel, } \\
\text { Entwicklung der } \\
\text { Säulenbeine }\end{array}$ & Ständige Differenzierung \\
\hline 1965 & - & $\begin{array}{l}\text { 1. Tertiär, Wald, Schulter- } \\
\text { höhe } 25 \mathrm{~cm} \text {, mehrzehig, } \\
\text { kurzkronige Mahlzähne, } \\
\text { abgesetzte Vormahlzäh- } \\
\text { ne, kurzer Schädel / } 2 \text {. } \\
\text { Reduzierung der Seiten- } \\
\text { zehen, Buschsteppe / } 3 . \\
\text { Mahlzähne mit langer } \\
\text { Krone, Mahlgebiß setzt } \\
\text { sich von Schneidezähnen } \\
\text { ab, Steppe, langer Schä- } \\
\text { del, größerer Körper }\end{array}$ & $\begin{array}{l}\text { Umstellung der } \\
\text { Backenzähne, } \\
\text { Umbildung der } \\
\text { Schneidezähne, } \\
\text { Nase und Ober- } \\
\text { lippe zu Rüssel, } \\
\text { Entwicklung der } \\
\text { Säulenbeine }\end{array}$ & $\begin{array}{l}\text { Nautiloidea: 1. primitiver } \\
\text { Molluskentyp / 2. Oberkamb- } \\
\text { rium, Plectronoceras, kleine } \\
\text { Form, dichtstehende Kam- } \\
\text { merscheidewände, Gehäuse } \\
\text { schwach gekrümmt, Sipho am } \\
\text { Kammerrand, Siphonalduten } \\
\text { aufgebläht und kurz / 3. frühes } \\
\text { Ordovizium, Mannigfaltigkeit } \\
\text { an Bauplänen und Formen, } \\
\text { Auftreten der Endoceratiden } \\
\text { (Siphonalduten lang und } \\
\text { kalkige Stütze des Sipho, } \\
\text { Querböden im Sipho, Sipho } \\
\text { randlich oder zentral) / 4. } \\
\text { mittleres Ordovizium, Riesen- } \\
\text { exemplare bis } 5 \text { m lang, Sipho } \\
\text { randlich / * }\end{array}$ \\
\hline
\end{tabular}

Tabelle 58: Zwischenformen und Entwicklungsreihen, Lehrbücher Klasse 12

\begin{tabular}{|c|c|c|c|}
\hline Ausgabe & 1952 & 1957 & 1965 \\
\hline \multirow{5}{*}{ Zwischenformen } & Urvogel* & Urvogel $^{*}$ & Urvogel $^{*}$ \\
\hline & Stummelfüßer & Stummelfüßer & \\
\hline & Augentierchen & Augentierchen & \\
\hline & Farne & Farne & \\
\hline & 4 & 4 & 1 \\
\hline \multirow[t]{5}{*}{ Entwicklungsreihen } & Cephalopoden & Cephalopoden & Pferde* \\
\hline & Pferde* & Pferde* & Elefanten* \\
\hline & Elefanten* & Elefanten* & Cephalopoden* \\
\hline & Zapfen* & Zapfen* & \\
\hline & 4 & 4 & 3 \\
\hline
\end{tabular}


Die Lehrbücher von 1952, 1957 und 1965 behandelten den Urvogel (Archaeopteryx) als Zwischenform (Tab. 58). Die Darstellung erfolgte im Zusammenhang mit Informationen über Entwicklungsreihen. Dabei wurden Vogel- und Reptilienmerkmale kurz beschrieben. Die Lehrbücher von 1952 und 1957 enthielten die Abbildung einer Gesteinsplatte und das Lehrbuch von 1965 die Abbildung einer Rekonstruktion (Abb. 114). Im Kapitel Beweise aus der vergleichenden Anatomie stellten die Lehrbücher von 1952 und 1957 auch die rezenten Gattungen Peripatus und Euglena sowie fossile Samenfarne als Zwischenformen vor.

\section{Fossilisation und Altersbestimmung}

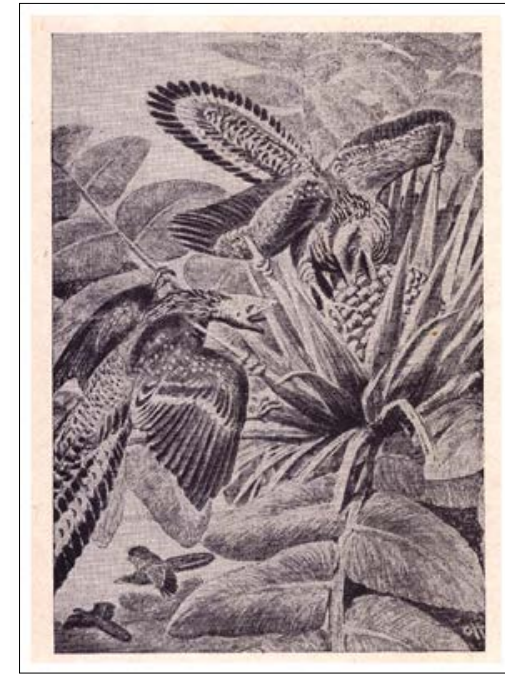

Abbildung 114: Rekonstruktion des Urvogels Archaeopteryx (Bach et al. 1967)

Die Lehrbücher von 1952, 1957 und 1965 enthielten Angaben über die Bildung von Fossilien (Fossilisation). Unterschiede bestanden in den Angaben zu Fossilarten. Alle Lehrbücher erläuterten die Entstehung von Abdrücken und Steinkernen. Die Ausgaben von 1952 und 1953 informierten auch über die Fossilisation ganzer Tiere und Körperteile sowie über Einschlüsse. Angaben zu Mumifizierungen und Versteinerungen konnten die Schüler im Lehrbuch von 1957 nutzen. Zum Teil erläuterten die Verfasser Fossilarten, ohne Beispiele zu benennen. Auch die Visualisierung der Lehrbücher unterschied sich. Für die angeführten Beispiele stand den Schülern in den Lehrbüchern von 1952 und 1957 keine Abbildung zur Verfügung. Das Lehrbuch von 1965 visualisierte dagegen alle Belege. Fallweise waren Angaben zu Fundorten vorhanden (Tab. 59).

Alle Lehrbücher stellten den Zerfall radioaktiver Elemente als Möglichkeit der Altersbestimmung von geologischen Schichten vor. Ebenso waren Angaben zu Leitfossilien in den Lehrbüchern von 1952 und 1957 integriert. Im Lehrbuch von 1965 wurde in Verbindung mit den Leistungen von William Smith im Kapitel Die naturwissenschaftlichen Voraussetzungen für die Abstammungslehre der Begriff Leitfossil in einem anderen Zusammenhang (Kategorie Theorien) benannt. 
Tabelle 59: Fossilisation, Lehrbücher Klasse 12

\begin{tabular}{|c|c|c|c|}
\hline Klasse & $\begin{array}{l}\text { Fossilisation, } \\
\text { Fossilienarten }\end{array}$ & Beispiele ( ${ }^{*}$ mit Abbildung) & Fundorte \\
\hline \multirow[t]{5}{*}{$1952 / 57$} & $\begin{array}{l}\text { ganze Tiere mit } \\
\text { Weichteilen }\end{array}$ & diluviale Mammute & Sibirisches Eis \\
\hline & Teile des Körpers & $\begin{array}{l}\text { Skelettreste, Zähne, Schuppen, } \\
\text { Schalen, Gehäuse, Panzer, Blät- } \\
\text { ter, Holzreste, Früchte }\end{array}$ & - \\
\hline & Einschlüsse (Bernstein) & Ameisen, Fliegen, Käfer, Spinnen & - \\
\hline & Abdruck & - & - \\
\hline & Steinkern & - & - \\
\hline \multirow[t]{4}{*}{1965} & Mumifizierung & Mammutrest ${ }^{*}$, Nashorn* & Sibirisches Eis, Starunia \\
\hline & Versteinerung & - & - \\
\hline & Abdruck & Libelle (Jura) ${ }^{*}$ & Solnhofen \\
\hline & Steinkern & Schnecken (mittlere Trias) ${ }^{*}$ & - \\
\hline
\end{tabular}





\title{
Fazit
}

\author{
„Aber Scbulbucbforschung, die nur die \\ Schulbücher selbst im Blick hat - ist \\ keine Schulbuchforschung." (Menck 2014: \\ 342, Hervorhebung im Original)
}

Das allgemeinbildende Schulsystem, die Lehrplanentwicklung und die Ausbildung der Lehrkräfte zeigten sich in ihrer Entwicklung abhängig von den jeweiligen gesellschaftlich-politischen Gegebenheiten in der SBZ/DDR und standen unter dem Einfluss des Ost-West-Konflikts. Für den Unterricht bedeutsam erkannten die Verantwortlichen die Entwicklung, institutionelle Einbettung und Bereitstellung von Unterrichtsmedien. Diese sollten im Spannungsfeld von Erziehung und Unterricht gleichermaßen einer fachlichen und weltanschaulichen Bildung der Schülerinnen und Schüler dienen. Zentraler Bestandteil der Lehrerhandreichungen und Unterrichtsmedien im Fach Biologie waren evolutionsbiologische Inhalte, die - häufig als Entwicklungs- oder Abstammungslehre bezeichnet - in den Abschlussklassen vermittelt wurden. Die Auswertung der Dokumente zeigte die Bedeutung evolutionsbiologischer Inhalte für den gesamten Erziehungs- und Bildungsprozess. Ihre Darstellung hinsichtlich Design, Struktur und Inhalt folgte dabei divergenten Ansätzen und Präferenzen. Modifikationen in den Lehr- und 
Lernmaterialien entstanden hierbei als Folge gesellschaftlicher und wissenschaftlicher Diskurse und Entwicklungen.

Die Verschränkung qualitativer und quantitativer Methoden erwies sich in dieser Arbeit als hilfreich. So konnten Merkmalsausprägungen der gewählten historischen Schriftzeugnisse aufgezeigt, interpretiert und kontextualisiert werden. Die Grenzen dieser Methodik bestehen, wie bereits zu Beginn der Arbeit dargelegt, darin, dass die Analyse der Schriftzeugnisse zwar die inhaltliche Basis der Unterrichtsarbeit aufzeigt, jedoch einen Einblick in die tatsächliche Unterrichtsrealität nur bedingt ermöglicht. Offen bleiben auch Fragen nach dem Stellenwert und der Darstellung der Evolutionsbiologie nach 1989, der Dauer von Übernahme weiterer fachwissenschaftlicher Neuerungen in die Lehr- und Lernmaterialien sowie den Auswirkungen des Ost-West-Konfliktes auf die Darstellung evolutionsbiologischer Inhalte in der Bundesrepublik Deutschland. Hierbei liefert die vorliegende Arbeit insbesondere hinsichtlich vergleichender Studien notwendige Daten und Interpretationsansätze, die in diesem Kapitel abschließend und zusammenfassend dargelegt werden.

\section{Stellenwert}

Allgemein wird deutlich, dass die Aufnahme evolutionsbiologischer Inhalte in die Lehrpläne im Zusammenhang mit der strukturellen Entwicklung des Schulsystems stand (Tab. 60). Die Ergebnisse zeigen, dass der Fachdisziplin Evolutionsbiologie im Biologieunterricht auf dem Gebiet der SBZ/DDR in den Klassen 8, 10 und 12 ein hoher Stellenwert zukam. In allen Etappen des Strukturwandels der allgemeinbildenden Schulen belegen die Vorgaben aus den Lehrplänen sowie die Behandlung in den Lehrbüchern der entsprechenden Abschlussklassen ihre exponierte Stellung. Mit jedem Strukturwechsel im allgemeinbildenden Schulsystem wurden entsprechende Themen ans Ende des geforderten Abschlussniveaus verlagert. Bereits Ende der 1950er Jahre betonte Lengert, dass „letzten Endes [...] jede richtig verlaufende Biologiestunde ein Beitrag zum Verstehen der Abstammungslehre durch die Schüler sein" muss (1959: 92). Darüber hinaus war es erklärtes Ziel und Konzeption des Biologielehrgangs seit den 1960er Jahren, das Verständnis für die Evolution von Klasse 5 an anzubahnen und in der Klasse 10 abschließend und zusammenfassend zu behandeln (Neuner 1969). Dietrich (1972: 18) argumentierte auf der 8. Schulbiologentagung, dass, in allen Klassenstufen [...] systematisch Wissen für die spätere geschlossene Behandlung der Abstammungslehre im Sinne einer [...] Linienführung bereitgestellt" wird. So zielten beispielsweise die Lehrplanautoren im Stoffabschnitt Körperbau der einzelnen Wirbeltierklassen für das 5. Schuljahr darauf, Voraussetzungen im Wissen über das Gliedmaßenskelett für den Begriffsinhalt homologe Organe zu schaffen (ebd.). 
Tabelle 60: Lehrpläne mit evolutionsbiologischen Inhalten

\begin{tabular}{|l|c|c|c|c|c|}
\hline $\begin{array}{l}\text { Strukturwandel im } \\
\text { Schulsystem }\end{array}$ & 1945 bis 1950 & 1951 bis 1958 & \multicolumn{2}{|c|}{1959 bis 1990} \\
\hline $\begin{array}{l}\text { Phasen der } \\
\text { Lehrplanentwicklung }\end{array}$ & ab 1946 & ab 1951 & ab 1959 & ab 1964 & ab 1982 \\
\hline $\begin{array}{l}\text { Lehrpläne für die 8. } \\
\text { Klasse }\end{array}$ & 1946,1947 & $\begin{array}{c}1951,1952, \\
1953,1955\end{array}$ & - & - & - \\
\hline $\begin{array}{l}\text { Lehrpläne für die 10. } \\
\text { Klasse }\end{array}$ & - & 1951 & 1959 & $\begin{array}{c}1966,1967, \\
1969\end{array}$ & 1988 \\
\hline $\begin{array}{l}\text { Lehrpläne für die 12. } \\
\text { Klasse }\end{array}$ & 1946,1947 & $\begin{array}{c}1951,1953, \\
1954,1956\end{array}$ & 1959 & 1961,1967 & - \\
\hline
\end{tabular}

Während ab 1946 in den Abschlussklassen 8 und 12 evolutionsbiologische Inhalte in die Lehrpläne aufgenommen wurden, fanden 1951 entsprechende Inhalte auch Eingang in die Lehrpläne für die Zehnklassenschulen. Infolge der Anhebung des geforderten Abschlussniveaus auf zehn Jahre wurde die Fachdisziplin Evolutionsbiologie aus den Stoffplänen der Klasse 8 in die Klasse 10 verlagert. Mit der Ausformung der zehnklassigen allgemeinbildenden POS manifestierte sich die Behandlung der Evolutionsbiologie letztlich in der Abschlussklasse 10. Auch in den Vorbereitungsklassen für die EOS wurde in Klasse 10 die Evolutionsbiologie unterrichtet. Mit der Einführung neuer präzisierter Lehrpläne im Schuljahr 1970/71 und den damit verbundenen Debatten zur Linienführung entfiel diese Fachdisziplin dann auch in der Klassenstufe 12 zugunsten der Genetik (Abb. 115).

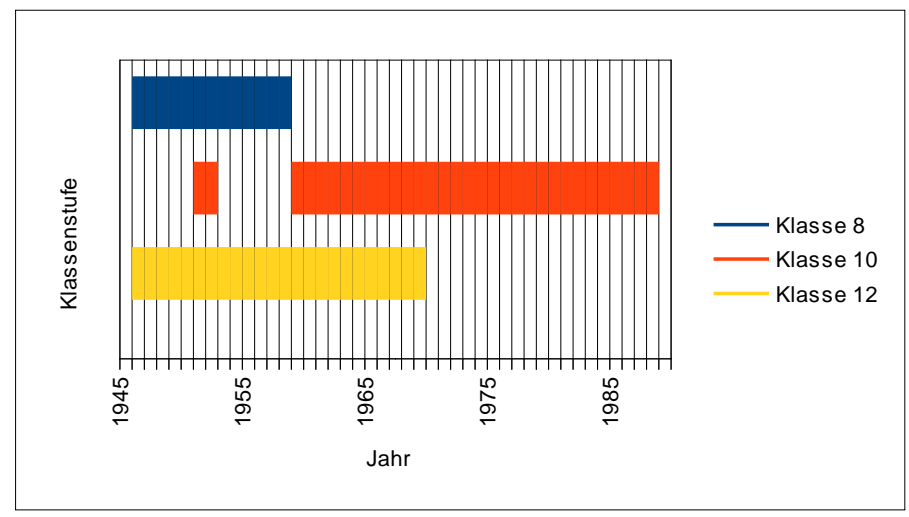

Abbildung 115: Evolutionsbiologie in den Jahrgängen 8, 10 und 12 
Ideologie und Evolutionsbiologie

In den Lehrplanvorworten verknüpften die Autorinnen und Autoren das Stoffgebiet Evolutionsbiologie meist mit ideologischen Aussagen (Tab. 61). Geprägt durch die jeweiligen politisch-gesellschaftlich Zustände unterlagen diese Erziehungs- und Orientierungsaussagen jedoch einem Wandel, der die Rolle von Lehrplänen bei gesellschaftlichen Transformationsprozessen verdeutlicht. Evolutionsbiologische Inhalte dienten einer Verhaltensorientierung und lieferten Identifikationspotenziale der jeweils herrschenden gesellschaftlich-politischen Ansichten. Im Bemühen um eine Vereinbarkeit mit dem dialektischen Materialismus unterlag diese Fachdisziplin dabei tiefgreifenden ideologischen Modifikationen, die zumeist nach einschneidenden politischen Veränderungen auftraten (z. B. Nachkriegszeit und Diskurs Schöpferischer Darwinismus).

Tabelle 61: Sach- und Ideologiebezug der Lehrpläne für die Klassen 8, 10 und 12

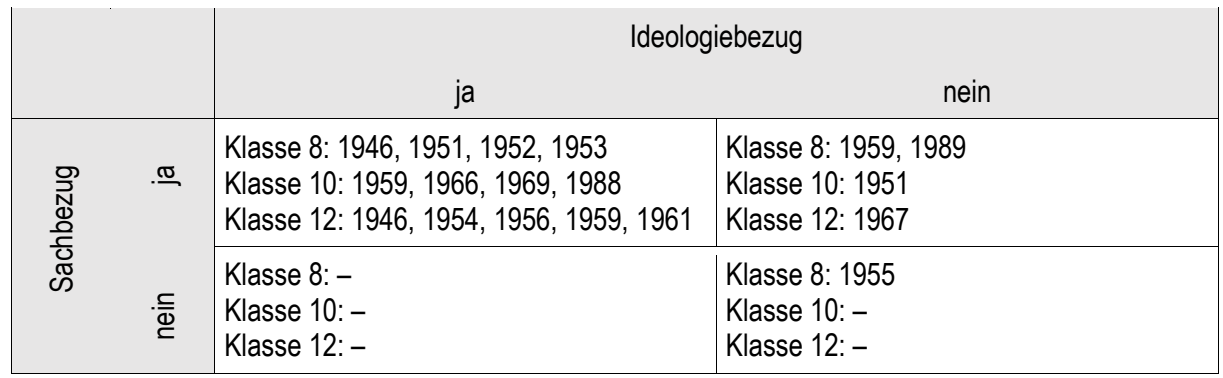

Der Wandel vollzog sich, wie gezeigt werden konnte, von einer humanistischen Orientierung in den Anfangsjahren, über eine Aufnahme des Schöpferischer Darwinismus in den 1950er Jahren, der Betonung einer materialistischer Sichtweise im Zusammenhang mit der Herausbildung eines sozialistischen Menschenbildes hin zu einer weitgehend ideologiefreien Darlegung. Stets integriert war darüber hinaus die Ablehnung einer menschenverachtenden Rassentheorie. Allgemein war die Linienführung im Biologieunterricht mit der Einführung der präzisierten Lehrpläne ab 1966 geprägt von "grundlegende[n] Aussagen des Marxismus-Leninismus“ (Dietrich 1976: 47). Für die Stoffpläne und Schullehrbücher war jedoch neben einer didaktischen Reduktion der Fachinhalte auch eine Reduktion ideologischer Aussagen typisch. Erarbeitet von Autorinnen und Autoren aus den Bereichen Schule, Fachdidaktik und Fachwissenschaft lag der Schwerpunkt auf der Vermittlung von Fachwissen. Letztlich kam der Fachdisziplin Evolutionsbiologie eine entscheidende Rolle beim Aufbau eines wissenschaftlichen Weltbildes zu.

Nach dem Zusammenschluss beider deutscher Staaten wurde von der Bildungspolitik der DDR Abstand genommen, was einen neuen Lehrplan erforderte und sich auch auf das Biologielehrbuch aus dem Verlag Volk und Wissen auswirkte (Porges 2016b). Das Ministerium für Bildung und Wissenschaft (1990: 3) argu- 
mentierte, dass sich dieses „Erfordernis [...] aus dem Zustand der Schule der DDR infolge einer weitgehend verfehlten, ideologisch-dogmatischen Bildungspolitik selbst ergibt". Hinsichtlich evolutionsbiologischer Bildungsinhalte orientierte der Lehrplan nunmehr auf einer Vielfalt von Auffassungen.

„Insgesamt soll der Lehrer bei der Vermittlung von Wissen über genetische und evolutive Sachverhalte mit den Schülern verschiedene Auffassungen der Entstehung des Lebens auf der Erde, über die Evolution der Organismen sowie über die Entwicklung des Menschen aus dem Tierreich erörtern. Das würde dazu beitragen, daß die Schüler - auf der Grundlage von Wissen - ihre eigenen weltanschaulichen Auffassungen entwickeln können." (Ministerium für Bildung und Wissenschaft 1990: 13, Hervorhebung im Original)

\section{Design}

Die Ergebnisse zeigen, dass sich das Design der Schulbücher in vielerlei Hinsicht, jedoch insbesondere in der Farbverwendung und der Integration von Abbildungen, wandelte. Das stets handliche Format änderte sich hingegen nicht. Grundsätzlich verhinderte die wirtschaftliche Notlage nach dem Krieg den Einsatz teurer Druckverfahren bei der Produktion von Schulbüchern. Dennoch waren bereits Farbtafeln im Schulbuches für die Klasse 8 von 1946 vorhanden. Für die Fachdisziplin Evolutionsbiologie erschien die erste Kunstdrucktafel im Lehrbuch für die Klasse 8 von 1957 und die erste Farbtafel im Lehrbuch für die Klasse 10 von 1960. Die farbige Gestaltung der Lehrbücher setzte jedoch erst deutlich später und in Folge des Gesetzes über das einheitliche sozialistische Bildungssystem der DDR von 1965 ein. Die an die Ausgabe der Lehrpläne gekoppelte neue Lehrbuchserie verfügte jetzt über einen Vier- bzw. Zweifarbdruck. Allgemein nahm die soziokulturelle Bedeutung von Farbe auch durch die Einführung des Farbfernsehens im Jahr 1969 zu. Räuber (1973: 129) legt dar, dass bei einer Zunahme der Bedeutung von Farbe im sozialen Zusammenleben Farben auch in der Gestaltung von Lehrbüchern Beachtung finden müssen. Diese progressive Rückwirkung gesellschaftlicher Veränderungen auf die Lehrbuchgestaltung zeigte sich auch in der neuen Lehrbuchserie, die sich ab den 1980er Jahren mehrfarbig und mit Farbfotos präsentierte. Auch war ein Trend zu mehr Abbildungen im Lehrbuch innerhalb evolutionsbiologischer Themen, insbesondere im Bereich der Hominisation und Paläontologie, erkennbar.

Als Hilfestellungen für den Schüler dienten stets Inhalts- und Sachverzeichnisse. Vereinzelt integrierten die Autoren auch ein Glossar und Literaturhinweise. Im Format blieben alle relevanten Schullehrbücher, die zwischen 100 und 246 Seiten umfassten, von 1946 bis 1989 unverändert handlich. Lediglich der Buchumschlag änderte sich Anfang der 1970er Jahre von einem Hard- zu einem Softcover.

Die Aufmachung der Einbände ließ bedingt Rückschlüsse auf den Inhalt der Lehrbücher zu, denn die Lehrbücher für die Klassenstufe 10 sowie das Lehrbuch 
für die Klassenstufe 12 von 1965 enthielten evolutionsbiologische Abbildungen. Hierbei fanden die Kategorien Hominisation, Neontologie und Paläontologie Beachtung. Doch spiegelten diese Abbildungen nicht die inhaltlichen Präferenzen der Texte wider. Eine Ausnahme bildete das Lehrbuch für die Klassenstufe 10 von 1988. Hier lag der Schwerpunkt auf der Kategorie Hominisation und adäquate Abbildungen präsentierte das Buch auf der Rückseite des Einbandes und auf den Vorsätzen (Porges \& Hoßfeld 2017).

\section{Inhaltsstruktur (Lehrplan-Lehrbuch-Verschränkung)}

In der DDR stellte der Lehrplan die „normative Basis“ des Unterrichtes dar (Stritzel 1989: 19). Mit Blick auf den Biologielehrplan bildete er jedoch „nicht nur die Grundlage für den Erwerb biologischer Kenntnisse und Erkenntnisse, sondern auch für zielgerichtete Fähigkeitsentwicklung und für die sozialistische Erziehung im Biologieunterricht" (Zentralinstitut für Weiterbildung der Lehrer und Erzieher beim Ministerium für Volksbildung der DDR 1969: 266). Neben dieser Planungsgrundlage wurden Lehrbücher und Unterrichtshilfen als Planungshilfen verstanden und auf der Basis von Lehrplänen erarbeitet. Im Kanon der Bildungsmedien galten Schullehrbücher hierbei als die „konkreteste Kodifizierung des in den Lehrplänen fixierten Inhalts“ (Neuner 1989: 411). Die Lehrkräfte in der DDR waren aufgefordert, die Möglichkeiten des Schulbuches im Kontext der Lehrplanvorgaben und der individuellen Situation in der Klasse zu nutzen.

„Erschließen Sie aus den im Fachlehrplan enthaltenen Aussagen, welche besonderen Potenzen der Schulbucheinsatz für die Entwicklung geistiger Fähigkeiten, von Methoden und Techniken geistiger Arbeit bietet! Prüfen Sie, auf welche Elemente des Könnens im Umgang mit dem Schulbuch es in Ihrem Fach, in Ihrer Klasse ankommt.“ (Stritzel 1989: 69)

Die Lehrpläne und Lehrbücher der jeweiligen Epochen stimmten in großen Teilen in ihrer Inhaltsstruktur überein. Davon ausgenommen waren die Lehrbücher und Rahmenlehrpläne der 1940er Jahre, die Lehrpläne der Zehnjahrschulen für die kein adäquates Lehrbuch zur Verfügung stand sowie die Materialien für die Klasse 10 von 1960. Dennoch lässt sich konstatieren, dass in Folge des Gesetzes über das einheitliche sozialistische Bildungssystem der DDR von 1965 die angestrebte Verbindung zwischen Lehrplan und Lehrbuch eine neue qualitative Stufe erreichte. Allgemein belegt die Inhaltsstruktur der Lehrpläne und Lehrbücher eine Lehrplan-LehrbuchVerschränkung, die unabhängig von zeittypischen Veränderungen grundlegenden Ordnungsprinzipien folgte (Tab. 62).

In allen Schullehrbüchern mit evolutionsbiologischem Inhalt traten Aspekte aus den Themenfeldern Evolutionstheorien, Paläontologie und Neontologie auf. Daneben spielten Fragen der Hominisation, Systematik und Biogenese eine bedeutende Rolle. Die Kategorie Hominisation war zumeist in einem eigenen Kapitel im hinteren Bereich der Materialien verortet, während die Kategorie Theorien 
häufig an zwei Stellen (historische und aktuelle Theorien) im Lehrbuch behandelt wurde. Neontologische und paläontologische Themen wurden zumeist gekoppelt behandelt, traten teilweise an verschieden Stellen auf und lokalisierten sich im vorderen Bereich der Materialien. Die Kategorie Neontologie folgte in der Regel der Kategorie Paläontologie. Informationen über die Entstehung des Lebens (Biogenese) verorteten die Autorinnen und Autoren stets an einer Stelle im Lehrbuch. Dabei lässt sich im Verlauf der Schulbuchgeschichte keine Konstante in der Anordnung erkennen. Entsprechende Inhalte befanden sich je nach Lehrbuch am Anfang, in der Mitte oder am Ende. Die Kategorie Systematik wurde stets im hinteren Bereich der Materialien angeordnet und war ebenso wie die Kategorie Didaktisches nicht in allen Materialien vorhanden (Tab. 62).

Tabelle 62: Vergleich der Inhaltsstruktur der Lehrbücher und Lehrpläne

\begin{tabular}{|c|c|c|}
\hline Klasse & Lehrplan & Lehrbuch \\
\hline 8 & $\begin{array}{l}\text { 1946: H } \\
\text { 1951: P, N, P, N, T, B, S, H, T } \\
\text { 1952: P, N, P, N, D, T, D, B, S, D, H, D, T } \\
\text { 1953: P, N, T, B, S, T } \\
\text { 1955: T, P, N, S, H, T }\end{array}$ & $\begin{array}{l}\text { 1946: T, P, B, P, N, P, N, T, S, H } \\
\text { 1951: P, N, P, N, T, B, S, H, S, T } \\
\text { - } \\
\text { 1953: P, N, P, T, B, S, T } \\
\text { 1957: T, P, B, P, N, S, H }\end{array}$ \\
\hline 10 & $\begin{array}{l}\text { 1951: B, P, N, H } \\
\text { 1960: T, P, N, S, H, T, B } \\
\text { 1966: P, N, B, H, S, T } \\
\text { 1967: D, T, N, P, H, S, T, B } \\
\text { 1969: T, N, P, T, B, H, D } \\
\text { 1988: T, N, B, P, H }\end{array}$ & $\begin{array}{l}\text { 1960: D, P, N, T, B, P, H, T } \\
\text { 1965: D, P, N, B, P, H, S, T } \\
\text { 1968: D, T, N, P, H, S, T, B } \\
\text { 1971: T, N, P, T, B, H, D } \\
\text { 1988: D, T, N, D, B, P, D, H, D }\end{array}$ \\
\hline 12 & $\begin{array}{l}\text { 1946: T, N, P, N, T, H, B } \\
\text { 1953: B, D, P, N, D, S, H, D, T, D, T } \\
\text { 1954: B, P, N, S, H, T } \\
\text { 1956: B, P, N, S, H, T } \\
\text { 1959: T, P, N, S, H, B } \\
\text { 1961: P, N, B, S, H, T } \\
\text { 1967: P, N, B, S, H, T }\end{array}$ & $\begin{array}{l}- \\
\text { 1952: B, P, N, P, N, S, H, T } \\
- \\
1957: B, P, N, P, N, S, H, T \\
- \\
- \\
1965: P, N, B, H, T, S, T\end{array}$ \\
\hline \multicolumn{3}{|c|}{$\begin{array}{l}\text { Legende: } \mathrm{B} \text { - Biogenese, D - Didaktisches, } \mathrm{H} \text { - Hominisation, } \mathrm{N} \text { - Neontologie, } \mathrm{P} \text { - Paläontologie, } \subseteq \\
\text { - Systematik, } \mathrm{T} \text { - Theorien }\end{array}$} \\
\hline
\end{tabular}

Inhaltliche Präferenzen

Die Darstellung der Fachdisziplin Evolutionsbiologie folgte in den Lehrbüchern verschiedenen Ansätzen und Präferenzen. Es zeigte sich jedoch, dass in allen Lehrbüchern die Kategorie Theorien besondere Beachtung fand, da sie vom Umfang stets an erster oder zweiter Stelle stand. Darüber hinaus bildete die Kategorie Hominisation insbesondere in den Materialien von 1946 und 1988, aufgrund einer 
starken Reduktion der übrigen Fachinhalte, das dominierende Element. Im Lehrbuch für die Klasse 8 von 1957 lag der Schwerpunkt schließlich auf der Kategorie Paläontologie und im Lehrbuch für die Klassenstufe 10 von 1965 auf der Kategorie Biogenese (Tab. 63).

Tabelle 63: Kategoriale Raumanalysen der Lehrbuchtexte zum Thema Evolutionsbiologie in den Biologielehrbüchern der Klassen 8, 10 und 12

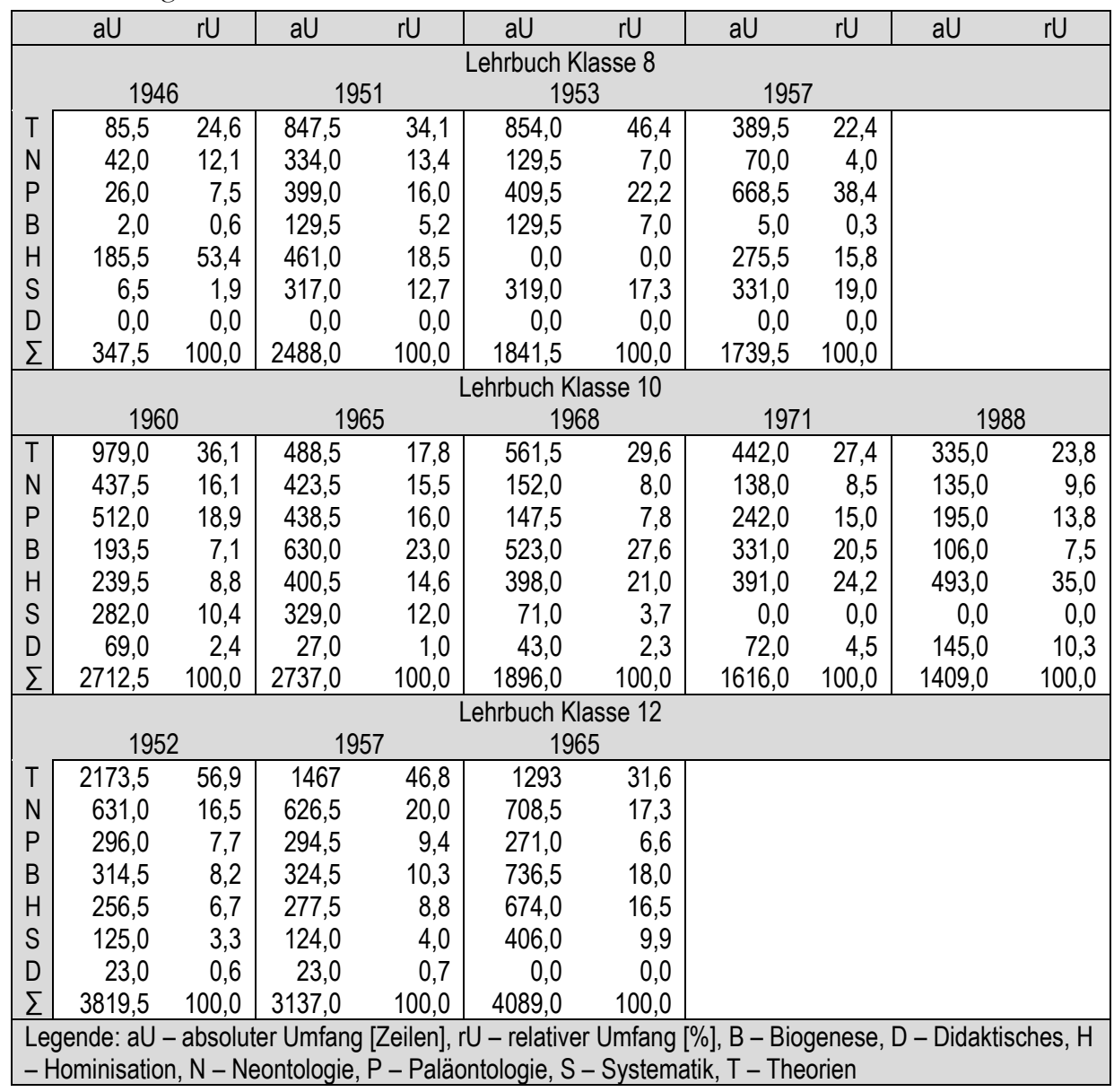

Besonders die Aufnahme von Evolutionstheorien spiegelte in den Lehr- und Lernmaterialien verzögert politisch-gesellschaftliche und wissenschaftliche Entwicklungen, Diskurse sowie Paradigmenwechsel wider. Allgemein war die Geschichte der Evolutionstheorien bereits seit 1945 unterrichtsrelevant. Insgesamt benennen die Lehrbücher 141 Personen, wovon inhaltlich Charles Darwin, Ernst Haeckel und Jean Baptiste Lamarck herausragten. In allen untersuchten Lehrbüchern wurde stets der Darwinismus, wenn auch im Umfang, in der Tiefe und in 
der Anordnung der inhaltlichen Auseinandersetzung variierend, behandelt. Darwins Leben vor seiner Reise mit der Beagle, seine Reisebeschreibungen, sein Leben nach der Forschungsreise und theoretische Aspekte seiner Arbeiten kamen als Subkategorien in allen Lehrbüchern vor. Innerhalb biologiehistorischer Darstellungen zu Darwins Hauptwerk On the Origin of Species (1859) stand von den fünf Darwin'schen Theorien (Mayr 2002) die Selektionstheorie im Vordergrund. In den 1950er Jahren fand zudem in den Lehr- und Lernmaterialien für die Klasse 8 eine kritische Auseinandersetzung mit dem Begriff Kampf ums Dasein statt (u. a. MfV 1955).

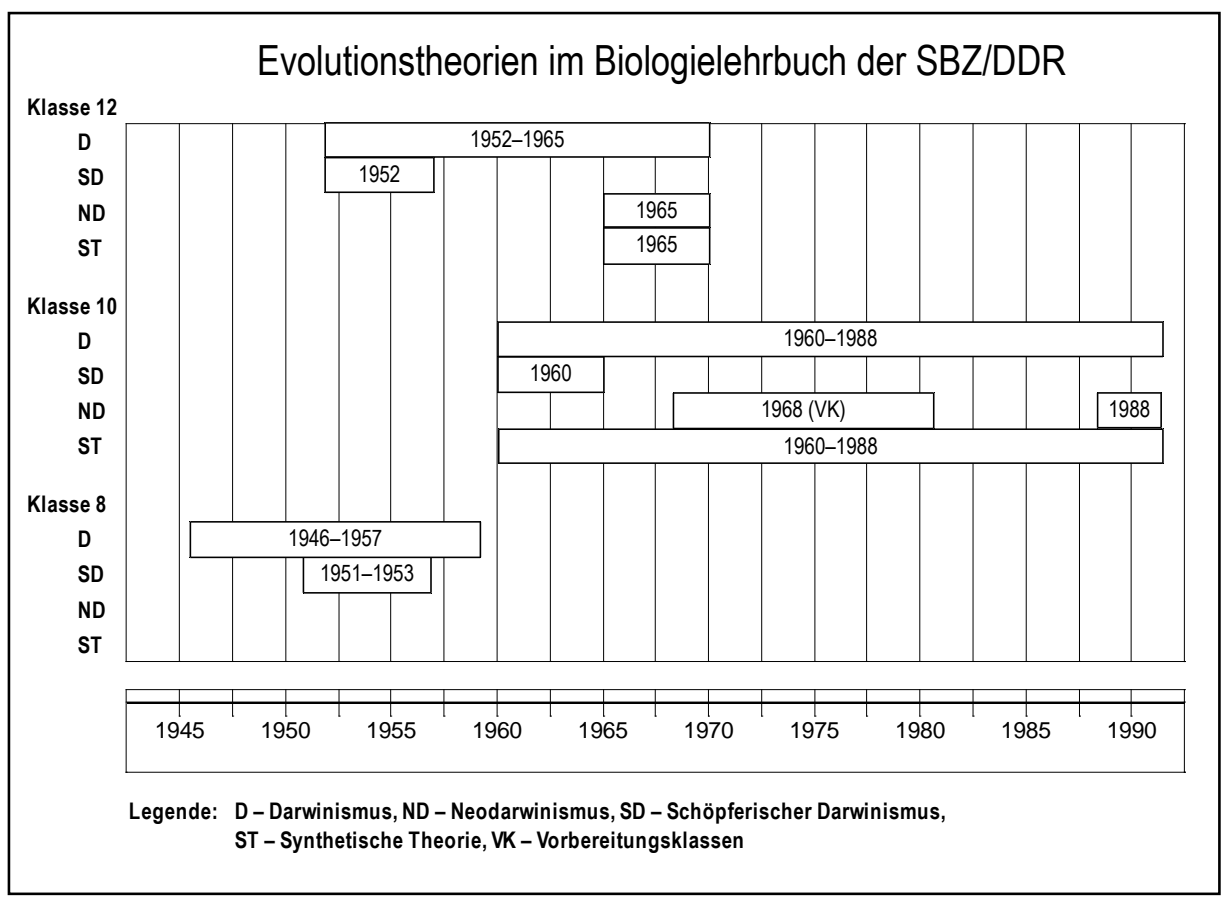

Abbildung 116: Evolutionstheorien in den Lehrbüchern der SBZ/DDR

Zwischen den frühen 1950er und den frühen 1960er Jahren war auch der Schöpferische Darwinismus ein fester Bestandteil der biologischen Lehr- und Lernmaterialien. Dies hatte zur Folge, dass Elemente der Synthetischen Theorie, die in den 1930er und 1940er Jahren entwickelt wurden (Reif et al. 2000), erst über 20 Jahre später Eingang in die Schulmaterialien fanden. Infolge seiner (politischen) Überwindung wurden dann vermehrt Erkenntnisse aus dem Bereich der Genetik aufgenommen. Ab 1965 entfiel schließlich der Schöpferische Darwinismus und die Autorinnen und Autoren nahmen Erkenntnisse des Neodarwinismus sowie des Synthetischen Darwinismus in die Lehrpläne und Lehrbücher auf (Abb. 116). Die Lehrbücher für die Klassen 10 und 12 von 1965 enthielten erstmals ein Kapitel 
zur Genetik (Grundlagen der Vererbungslehre) sowie Informationen über die Evolutionsfaktoren. Auch folgte die Anordnung der Stoffgebiete im Lehrplan und später im Lehrbuch nicht länger einer historischen Entwicklung der Wissenschaften, sondern ab 1967 einer wissenschaftslogischen Struktur. Grundlagen im Bereich der Genetik sollten ein Verständnis evolutiver Prozesse erleichtern. Dieses methodologische Umdenken korrespondierte mit dem endgültigen Ende der Lyssenko-Ära, das sich international im August 1965 mit dem Gregor Mendel Memorial Symposium (Brno) und dem Symposium on the Mutational Process (Prag) vollzog. Trotz aller Unterschiede im Detail, die zeit- und jahrgangstypisch waren und retrospektiv gesellschaftspolitische Strömungen und Autorenmeinungen erhellen, stellte Wissenschaftsgeschichte per se in den Schulmaterialien der SBZ/DDR eine Konstante dar. Eine Konstante, die es den Lehrkräften ermöglichte im Unterricht neben der aktuellen auch eine historische Perspektive einzunehmen (Porges et al. 2016; Porges et al. 2017; Porges \& Hoßfeld 2017).

Innerhalb neontologischer und paläontologischer Darstellungen nahmen die Autoren Modifikationen insbesondere in der Auswahl und Darstellung der Belege für Evolutionsprozesse vor. Im Themenbereich Neontologie boten alle Lehrbücher Informationen über Homologien und rudimentäre Organe an. Ein Schema, das das Knochengerüst eines Grönlandwales (Balaena mysticeta) zeigte, bildete hier ein wiederkehrendes Moment. Ferner dienten in allen Schulbüchern Angaben zu Fossilien und Zwischenformen als paläontologische Belege für den Ablauf der Evolution. Der Urvogel Archaeopteryx stellt hier ein stets genutztes Element dar, das als Schema, Rekonstruktion oder Abdruck visualisiert war (Porges \& Hoßfeld 2017).

Wie von Berck und Graf (2010) dargestellt, zeigte sich letztlich auch für die Geschichte des Biologieunterrichtes am Beispiel der Evolutionsbiologie auf dem Gebiet der SBZ/DDR von 1945 bis 1989, dass es „den“ Biologieunterricht nicht gab. In der Gesamtschau unterlag er vielmehr äußeren Einflüssen und einer progressiven Entwicklung, die in ein gesamtgesellschaftliches Bedingungsgefüge eingebettet war. In seinen vielfältigen Erscheinungsformen lassen dabei Naturwissenschaftler wie Ernst Haeckel, Georg Uschmann und Herbert Bach eine Traditionslinie der Universität Jena an den Inhalten des Biologieunterrichtes erkennen. Kontinuitäten in den Unterrichtsmedien, wie die Darstellungen aus Charles Darwins Leben und Werk, standen neben Diskontinuitäten, die sich aus fachwissenschaftlichen und fachdidaktischen, soziokulturellen sowie technischen Veränderungen ergaben. Dass die Autoren neben Evolutionstheorien auch paläontologische und neontologische Belege, Aspekte der Hominisation, Biogenese sowie die natürlichen Systematik in den Lehr- und Lernmaterialien publizierten, veranschaulicht den interdisziplinären Charakter der Fachdisziplin Evolutionsbiologie. Letztlich zeigte sich in den Unterrichtsmedien aber auch die Evolution der Evolutionstheorien selbst. 


\section{Literaturverzeichnis}

Abteilung Biologie (Schulbuch) des volkseigenen Verlages Volk und Wissen (Hrsg.) (1975): Schulbuchversorgung 1975/76 - Lehrbücher Biologie Klassen 5 bis 12. In: Biologie in der Schule, 24. Jg., H. 9, S. 394-395.

Akademie für Staats- und Rechtswissenschaft der DDR (Hrsg.) (1984): Unser Staat. DDR-Zeittafel 1949-1983. Berlin: Dietz.

Ambrosius, H., Arnold, H., Bernhardt, D., Crome, W., Friedrich, G., Kloss, K., Linke, W., Nestler, H., Nitschmann, J., Padberg, W., Uschmann, G. \& Wessely, I. (1964): Entwicklung der Organismen. Lehrbuch der Biologie. 5. Aufl. Berlin: Volk und Wissen.

Anonymus (1952): Aus dem Lehrbuch der Biologie für das 12. Schuljahr. In: Biologie in der Schule, 1. Jg., H. 6, S. 249-264.

Anonymus (1959): Die Biologielehrbücher für das Schuljahr 1959/60. In: Biologie in der Schule, 8. Jg., H. 9, S. 424-426.

Anonymus (1960): Um die Verbesserung der Biologielehrbücher. In: Biologie in der Schule, 9. Jg., H. 11, S. 497-504.

Anonymus (1963): Zum 70. Geburtstag vom Willi Lemke. In: Biologie in der Schule, 12. Jg., H. 11, S. 501-503.

Anonymus (1964): Nochmals zur internationalen Schulbiologentagung in Berlin. In: Biologie in der Schule, 13. Jg., H. 4, S. 187-188.

Anonymus (1969a): Landeskulturgesetz. Einige wesentlichen Grundsätze und Aufgaben aus dem Entwurf des Gesetzes über die planmäßige Gestaltung der sozialistischen Landeskultur der DDR. In: Berliner Zeitung, Jg. 25, Ausgabe 323, S. 3.

Anonymus (1969b): Liebe Kollegen! In: Biologie in der Schule, 18. Jg., H. 6, S. 274.

Anonymus (1973): Willi Lemke: 1893-1973. In: Biologie in der Schule, 22. Jg., H. 6, S. 250-251.

Anonymus (1976): Verfassung der Deutschen Demokratischen Republik. 4. Aufl. Berlin: Staatsverlag der DDR.

Anonymus (1985): 40 Jahre im Dienste unserer Schule. In: Biologie in der Schule, 34. Jg., H. 10, S. 386-388.

Anonymus (2011): Sammlungsgeschichte. Ehemalige Kustoden. Online im Internet unter http://www.naturkundemuseumberlin.de/sammlungen/zoologie/arachnida-myriapoda/ehemaligekustoden/ <16.11.2014>.

Arnold, H., Bach, H., Gießmann, H.-G., Nestler, H. \& Uschmann, G. (1957): Lehrbuch der Biologie für das 8. Schuljahr. Berlin: Volk und Wissen. 
Arnold, K.-H., Sandfuchs, U. \& Wiechmann, J. (Hrsg.) (2006): Handbuch Unterricht. Bad Heilbrunn: Klinkhardt.

Bach, H., Bernhardt, D., Crome, W., Löther, R. \& Nestler, H. (1964): Biologie IV. Ein Lehrbuch für die erweiterte Oberschule. 12. Klasse. Die Lehre von der Evolution der Organismen. Berlin: Volk und Wissen.

Bach, H., Bernhardt, D., Crome, W., Löther, R., Nestler, H. \& Zacharias, M. (1967): Biologie IV. Ein Lehrbuch für die erweiterte Oberschule. 12.

Klasse. Die Lehre von der Evolution der Organismen. 3. durchges. Aufl. Berlin: Volk und Wissen.

Bach, H., Bernhardt, D., Crome, W., Szigat, G., Dill, P., Friedrich, G., Kloß, K., Nestler, H., Nitschmann, J., Riemann, H., Uschmann, G., Meincke, I., Zacharias, M. \& Zachow, F. (1967): Entwicklung der Organismen. Lehrbuch der Biologie. durchges. Neuaufl. Berlin: Volk und Wissen.

Bach, H., Bernhardt, D., Kummer, G., Meincke, I., Müntz K., Nestler, H., Reinicke, H., Rothacker, D., Schmidt, G. \& Uschmann, G. (1968):

Biologie. Lehrbuch für Klasse 10. Vorbereitungsklassen. Berlin: Volk und Wissen.

Bach, H., Helms, J., Meincke, I., Müller, J., Plesse, W., Rothacker, D. \& Theile, M. (1987): Biologie. Lehrbuch für Klasse 10. 17. Aufl. Berlin: Volk und Wissen.

Baer, H.-W. (1959): Museen im Dienste des Biologieunterrichts. In: Biologie in der Schule, 8. Jg., H. 8, S. 354-363.

Baer, H.-W. (1967a): Zur Arbeit der Sektion Schulbiologie der Biologischen Gesellschaft in der DDR. In: Biologie in der Schule, 16. Jg., H. 8/9, S. 403-405.

Baer, H.-W. (1967b): Schulbiologentage 1968. In: Biologie in der Schule, 16. Jg., H. 12, S. 543-544.

Baer, H.-W. (1968): Zur Eröffnung der 4. Schulbiologentage. In: Mitteilungen der Sektion Schulbiologie der Biologischen Gesellschaft der DDR (Hrsg.): Zur Neubestimmung des Inhalts und der Gestaltung des Biologieunterrichts. Rostock: o. V., S. 5-7.

Baer, H.-W. (1971): Zur Eröffnung der 8. Schulbiologentage. In: Mitteilungen der Sektion Schulbiologie der Biologischen Gesellschaft der DDR (Hrsg.): Evolution und Genetik. Berlin: o. V., S. 3-5.

Baer, H.-W. (1975): Eröffnung der 10. Schulbiologentage 1973 in Halle. In: Biologische Gesellschaft der DDR Sektion Schulbiologie (Hrsg.): Unterrichtsmittel im Biologieunterricht. Erkenntnistheoretische, wahrnehmungspsychologische und methodische Aspekte. Halle (Saale): Abteilung Wissenschaftspublizistik der Martin-Luther-Universität, S. 7-9. 
Baer, H.-W., Dietrich, G., Götze, G., Grönke, O., Neubauer, M., Pietsch, A., Prehn, K.-G., Püschl, E., Schlüter, W., Schmeißer, G., Windelband, A. (1969): Biologische Arbeitstechniken für Lehrer und Naturfreunde. 2., erw. Aufl. Berlin: Volk und Wissen.

Baer, H.-W., Graef, H., Heichel, G., Heinzel, K., Hocke, C., Hundt, R., Kopprasch, G., Lobeck, K., Müller, J., Püschel, U., Stade, R. \& Theuerkauf, H. (1975): Biologie in Übersichten, Wissensspeicher für den Unterricht. 9. Aufl. Berlin: Volk und Wissen.

Baer, H.-W. \& Grönke, O. (Hrsg.) (1969): Biologische Arbeitstechniken für Lehrer und Naturfreunde. 2., erw. Aufl. Berlin: Volk und Wissen.

Barlow, N. (Hrsg.) (2008): Charles Darwin. Mein Leben. Frankfurt am Main etc.: Insel.

Baumgartner, G. \& Hebig, D. (Hrsg.) (1997): Biographisches Handbuch der SBZ/DDR 1945-1990. München: K. G. Sauer.

Bayrhuber, H. \& Kull, U. (1998): Linder Biologie. Lehrbuch für die Oberstufe. 21. Aufl. Hannover: Schroedel.

Beese, W. (2012): Die Marginalisierung der Naturwissenschaften an Thüringer Gymnasien - oder das Verhindern von Emergenz. Online im Internet unter http:/ / www.uni-

jena.de/unijenamedia/Downloads/faculties/bio_pharm/ag_didaktik/Bee se_+W_+_+Naturwissenschaftlicher+Unterricht.pdf $<13.11 .2014>$.

Beese, W. (2014): Evolution - Von Weltbildern und Zerrbildern. Vortrag. 20.

Tage des mathematischen und naturwissenschaftlichen Unterrichts.

Erfurt: 13.03.2014.

Behrens, H. \& Hoffmann, J. (Hrsg.) (2013): Naturschutz Geschichte(n). Lebenswege zwischen Ostseeküste und Erzgebirge. Friedland: Steffen.

Beljajew, D. K., Berg, G. L., Woronzew, N. N., Kerkis, J. J., Krasnowidowa, S. S., Nikoro, S. S., Salganik, R. I., Sokolowskaja, B. C. \& Christoljobowa N. B. (1969): Allgemeine Biologie. Berlin: Volk und Wissen.

Berck, K.-H. \& Graf, D. (2010): Biologiedidaktik. Grundlagen und Methoden. 4. Aufl. Wiebelsheim: Quelle \& Meyer.

Bernhardt, D. (1965): Einige philosophische Fragen der Entstehung des Lebens. Online im Internet unter http://digilib.phil.muni.cz/bitstream/handle/11222.digilib/106438/B_Ph ilosophica_12-1965-1_5.pdf (16.11.2014).

Biologische Gesellschaft der DDR Sektion Schulbiologie (Hrsg.) (1975):

Unterrichtsmittel im Biologieunterricht. Erkenntnistheoretische, wahrnehmungspsychologische und methodische Aspekte. Halle (Saale): Abteilung Wissenschaftspublizistik der Martin-Luther-Universität. 
Biologische Gesellschaft der Deutschen Demokratischen Republik (Hrsg.) (o. J.): Statut.

Biologische Gesellschaft in der Deutschen Demokratischen Republik (Hrsg.)

(1960): Arbeitstagung zu Fragen der Evolution. Zum Gedenken an

Lamarck - Darwin - Haeckel (20. bis 24. Oktober 1959 in Jena). Jena: G.

Fischer.

Biologische Lehrfachkommission bei der Deutschen Verwaltung für

Volksbildung in der Sowjetischen Besatzungszone (Hrsg.) (1946):

Lehrbuch der Biologie für das 7. und 8. Schuljahr. Berlin etc.: Volk und Wissen.

Böhme, H. (Hrsg.) (1964): Beiträge zur Abstammungslehre, Bd. 1. Berlin: Volk und Wissen.

Böhme, W., Dehlsen, M., Fischer, A., Jansen, H., König, G., Lange, M., Polit, R. \& Schütz G. (Hrsg.) (1973): Kleines politisches Wörterbuch. Berlin: Dietz.

Boje, L. (1953): Hinweise zu den überarbeiteten Stoffplänen der Grundschule im Fach Biologie. In: Biologie in der Schule, 2. Jg., H. 8, S. 337-339.

Borneleit, P. (2003): Lehrplan und Lehrplanerarbeitung, Schulbuchentwicklung und -verwendung in der DDR. In: ZDM, 35. Jg., H. 4, S. 134-145.

Brasseur, A. (2011): Einstellungen und Wissen zur Evolution und Wissenschaft in Europa. In: D. Graf (Hrsg.): Evolutionstheorie Akzeptanz und Vermittlung im europäischen Vergleich. Berlin etc.: Springer, S. 1-8.

Brunner, H., Jansohn, C., Lentzen, M. \& Mehl, D. (Hrsg.) (2009): Archiv für das Studium der neueren Sprachen und Literaturen, 246. Bd., 161. Jg., 2. Halbjahresbd. Berlin: Erich Schmidt.

Bucharin, N. I. (2001): Darwinismus und Marxismus. In: Hoßfeld, U. \& Brömer, R. (Hrsg.): Darwinismus und/als Ideologie. Berlin: Wissenschaft und Bildung, S. 127-155.

Buchsteiner, M. \& Strahl, A. (Hrsg.) (2013): Thünen-Jahrbuch, Bd. 8. Norderstedt: Books on Demand.

Butzmann, G. (Hrsg.) (1988): Meyers Jugendlexikon a - z. Leipzig: Bibliographisches Institut.

Campbell, N. A. (1997): Biologie. Heidelberg etc.: Spektrum.

Caroll, S. B. (2008a): Die Darwin-DNA. Wie die neueste Forschung die Evolutionstheorie bestätigt. Frankfurt am Main: S. Fischer.

Caroll, S. B. (2008b): Evo Devo. Das neue Bild der Evolution. Berlin: University Press. 
Cassube, M. \& Windelband, A. (1980): Populärwissenschaftliche Literatur für das Stoffgebiet „Abstammungslehre“ in Klasse 10. In: Biologie in der Schule, 29. Jg., H. 1, S. 12-20.

Catalogus Professorum Rostochiensium (2008): Heinz Kress. Online im Internet unter http://cpr.uni-rostock.de/metadata/cpr_person_00002387 (22.11.2014)

Choppin, A. (1992): Aspekte der Illustration und Konzeption von Schulbüchern. In: Fritzsche, K. P. (Hrsg): Schulbücher auf dem Prüfstand. Perspektiven der Schulbuchforschung und Schulbuchbeurteilung in Europa. Frankfurt am Main: Diesterweg, S. 137-150.

Darwin, C. (2004): Die Entstehung der Arten durch natürliche Zuchtwahl. Sonderausgabe. Hamburg: Nikol.

Darwin, C. (2008): Die Fahrt der Beagle. Tagebuch mit Erforschungen der Naturgeschichte und Geologie der Länder, die auf der Fahrt von HMS Beagle unter dem Kommando von Kapitän Fitz Roy, RN, besucht wurden. Frankfurt am Main: Fischer Taschenbuch.

Darwin, C. (2009): Die Abstammung des Menschen. Hamburg: Nikol.

Dawkins, R. (2007). Das Egoistische Gen. Jubiläumsausgabe. München: Spektrum.

Dawkins, R. (2009): Geschichten vom Ursprung des Lebens. Eine Zeitreise auf Darwins Spuren. Berlin: Ullstein.

Dawkins, R. (2010a): Die Schöpfungslüge. Warum Darwin recht hat. 2. Aufl. Berlin: Ullstein.

Dawkins, R. (2010b). Der erweiterte Phänotyp. Der lange Arm der Gene. Heidelberg: Spektrum.

DEFA-Stiftung (o. J.a): Struktur. Online im Internet unter http://www.defa.de/cms/DesktopDefault.aspx?TabID=1179 $<23.02 .2013>$.

DEFA-Stiftung (o. J.b): Staat: Ministerien: Film. Online im Internet unter http://www.defa.de/DesktopDefault.aspx?TabID=1008 <23.02.2013>.

Depaepe, M. \& Simon, F. (2003): Schulbücher als Quellen einer dritten Dimension in der Realitätsgschichte von Erziehung und Unterricht. Über neue Konzeptionen in der historisch-pädagogischen Schulbuchforschung. In: Wiater, W. (Hrsg.): Schulbuchforschung in Europa Bestandsaufnahme und Zukunftsperspektive. Bad Heilbrunn/Obb.: Klinkhardt, S. 65-77. 
Deschner, K. (2013): Schreiben um zu sein. Aphorismen. In: Gieselbusch, H., Röwer, G., Schmidt-Salomon, M. \& Strickstrock, F. (Hrsg.): Karlheinz Deschner und seine große Kriminalgeschichte des Christentums. Leben Werke Wirkungen. Zum Erscheinen von Band 10 im März 2013. Hamburg: Rowohlt, S. 57-62.

Detlev, F. (1993): Biologismus von oben. Das Menschenbild in Biologiebüchern. Duisburg: DISS.

Deutsche Verwaltung für Volksbildung in der Sowjetischen Besatzungszone Deutschlands (Hrsg.) (1947): Lehrpläne für die Grund- und Oberschulen in der Sowjetischen Besatzungszone Deutschlands. Biologie. 2. Aufl. Berlin: Volk und Wissen.

Deutsche Zentralverwaltung für Volksbildung in der Sowjetischen

Besatzungszone Deutschlands (Hrsg.) (1946): Lehrpläne für die Grundund Oberschulen in der Sowjetischen Besatzungszone Deutschlands. Biologie. Berlin: Volk und Wissen.

Deutsches Pädagogisches Zentralinstitut (Hrsg.) (1952): An alle Lehrer des Faches Biologie. In: Biologie in der Schule, 1. Jg., H. 4., S. 145-146.

Deutsches Pädagogisches Zentralinstitut (Hrsg.) (1959): Methodische Hinweise zur Arbeit mit dem Lehrplan im Fach Biologie. Berlin: Volk und Wissen.

Deutsches Pädagogisches Zentralinstitut Sektion Unterrichtsmethodik und Lehrpläne (Hrsg.) (1959): Methodische Hinweise zur Arbeit mit dem Lehrplan im Fach Biologie. Vorschläge zur Stoffverteilung im 1. und 2. Tertial. Erläuternde Bemerkungen zur Stoffverteilung. Berlin: Volk und Wissen.

Deutsches Zentralinstitut für Lehrmittel (Hrsg.) (1962): Lehrmittel für den Biologieunterricht. Berlin: Volk und Wissen.

Diamond, J. (2005): Geleitwort von Jared Diamond. In: Mayr, E.: Das ist Evolution. 3. Aufl. München: Goldmann, S. 7-13.

Diehl, E., Roßmann, G., Arlt, W., Benser, G., Fiedler, H., Gambke, H., Heitzer, H., Herrmann F.-J., Kanzig, H., Krusch, H.-J., Möschner, G., Otto, W., Reißig, K., Stöckigt, R., Trümpler, E. \& Wimmer, W. (1978): Geschichte der Sozialistischen Einheitspartei Deutschlands. Abriß. Berlin: Dietz.

Dietrich, G. (1972): Zu Problemen des Zielniveaus der 10. Klasse. In: Mitteilungen der Sektion Schulbiologie Heft 11, S. 15-21.

Dietrich, G. \& Kummer, G. (1979): 30 Jahre DDR - drei Jahrzehnte erfolgreiche Entwicklung des Biologieunterrichts. In: Biologie in der Schule, 28. Jg., H. 10, S. 401-421. 
Dietrich, G. (Hrsg.) (1976): Methodik Biologieunterricht (ausgearbeitet von einem Autorenkollektiv unter Ltg. Gerhard Dietrich, APW). Berlin: Volk und Wissen.

Dietrich, G. \& Grönke, O. (Hrsg.) (1979): Übersichten zur Biologie-Methodik. Potsdam: Pädagogische Hochschule.

Dobzhansky, T. (1973): Nothing in Biology Makes Sense Except in the Light of Evolution. In: The American Biology Teacher 35, S. 125-129.

Dorber, H. \& Dorber H. (1976): Erste Erfahrungen beim Einsatz des Wissensspeichers „Biologie in Übersichten“ im Unterricht. In: Biologie in der Schule, 25. Jg., H. 11., S. 484-487.

Dornhof, C. (1994): Lehrpläne der Sowjetischen Besatzungszone (SBZ) und der Deutschen Demokratischen Republik (DDR), Bd. 2. Berlin: Bibliothek für bildungsgeschichtliche Forschung.

Dreesmann, D., Graf, D. \& Witte, K. (Hrsg.) (2011): Evolutionsbiologie. Moderne Themen für den Unterricht. Heidelberg: Spektrum.

Drefenstedt, E. (1969): Die didaktische Konzeption des neuen Lehrwerks. In: Drefenstedt, E. \& Neuner, G. (Hrsg.): Lehrplanwerk und Unterrichtsgestaltung. Berlin: Volk und Wissen, S. 72-101.

Drefenstedt, E. \& Neuner, G. (1969): Zur Einführung. In: ders. (Hrsg.): Lehrplanwerk und Unterrichtsgestaltung. Berlin: Volk und Wissen, S. 9-12.

Drefenstedt, E. \& Neuner, G. (Hrsg.) (1969): Lehrplanwerk und Unterrichtsgestaltung. Berlin: Volk und Wissen.

Emmerich, F. (2001): Laudatio für Ehrenmitglied Herwart Ambrosius. In: Immunologische Nachrichten, Nr. 128., S. 4-5.

Engel, D. (2008): Die Zeitschrift "Biologie in der Schule" (1952-1989). Unveröffentlichte Examensarbeit, Jena.

Engels, E.-M. (2009): Charles Darwin: Person, Theorie, Rezeption. Zur Einführung. In: ders. (Hrsg.): Charles Darwin und seine Wirkung. Frankfurt am Main: Suhrkamp, S. 9-57.

Engels, E.-M. (Hrsg.) (2009): Charles Darwin und seine Wirkung. Frankfurt am Main: Suhrkamp.

Engels, F. (1966a): Die Entwicklung des Sozialismus von der Utopie zur Wissenschaft. In: Institut für Marxismus-Leninismus beim ZK der SED (Hrsg.): Karl Marx. Friedrich Engels. Ausgewählte Schriften in zwei Bänden, Bd. 2. Berlin: Dietz, S. 80-140.

Engels, F. (1966b): Dialektik der Natur. In: Institut für Marxismus-Leninismus beim ZK der SED (Hrsg.): Karl Marx. Friedrich Engels. Ausgewählte Schriften in zwei Bänden, Bd. 2. Berlin: Dietz, S. 51-67. 
Engels, F. (1966c): Anteil der Arbeit an der Menschwerdung des Affen. In: Institut für Marxismus-Leninismus beim ZK der SED (Hrsg.): Karl Marx. Friedrich Engels. Ausgewählte Schriften in zwei Bänden, Bd. 2. Berlin: Dietz, S. 68-79.

Engels, F. (1966d): Das Begräbnis von Karl Marx. In: Institut für MarxismusLeninismus beim ZK der SED (Hrsg.): Karl Marx. Friedrich Engels. Ausgewählte Schriften in zwei Bänden, Bd. 2. Berlin: Dietz, S. 152-154.

Fehling, G. (1976): „Methoden, Verfahren und Einsatz von Unterrichtsmitteln zur Führung des Erkenntnisprozesses im Biologieunterricht" - XIII. Schulbiologentage. In: Biologie in der Schule, 25. Jg., H. 10, S. 440-445.

Fehling, G. \& Kurze, M. (1978): XV. Schulbiologentage in Berlin vom 6. bis 9. Mai 1978 zum Thema „Ergebnisse und Entwicklungstendenzen in den Biowissenschaften und die Gestaltung des Biologieunterrichts als erkenntnisintensiver, erziehungswirksamer Prozess“. In: Biologie in der Schule, 27. Jg., H. 9, S. 366-370.

Fey, C.-C. (2015): Kostenfreie Online-Lehrmittel. Eine kritische Qualitätsanalyse. (= Beiträge zur historischen und systematischen Schulbuchforschung). Bad Heilbrunn: Julius Klinkhardt, S. 67-81.

Fey, C.-C. (2016): Zur Zulassungspraxis von Schulbüchern in Deutschland. In: Matthes, E. \& Schütze, S. (Hrsg.): Schulbücher auf dem Prüfstand (= Beiträge zur historischen und systematischen Schulbuchforschung). Bad Heilbrunn: Julius Klinkhardt, S. 67-81.

Fischer, J. (2004): Geschichte im Dienste der Politik. Die Darstellung des Zeitraums von 1933 bis 1945 in den Geschichtslehrplänen und schulbüchern der zehnklassigen allgemeinbildenden polytechnischen Oberschule in der DDR von 1959 bis 1989. (= Europäische Hochschulschriften, 981). Frankfurt am Main etc.: Peter Lang.

Fischer, M. S., Brehm, G. \& Hoßfeld, U. (2008): Das Phyletische Museum in Jena. Jena: Institut für spezielle Zoologie und Evolutionsbiologie mit Phyletischem Museum.

Freudig, D. \& Sauermost, R. (Red.) (1999): Lexikon der Biologie in fünfzehn Bänden, Bd. 1. Heidelberg: Spektrum.

Freudig, D. \& Sauermost, R. (Red.) (2000a): Lexikon der Biologie in fünfzehn Bänden, Bd. 5. Heidelberg: Spektrum.

Freudig, D. \& Sauermost, R. (Red.) (2000b): Lexikon der Biologie in fünfzehn Bänden, Bd. 4. Heidelberg: Spektrum.

Fritsch, H. \& Lemke, W. (1952): Die Entstehung der Arten (Darwinismus). Arbeitsplan für den Biologieunterricht im 8. Schuljahr, Lehrplankapitel BII. In: Biologie in der Schule, 1. Jg., H. 1, S. 21-26. 
Fritsche, K. P. (1992): Schulbuchforschung und Schulbuchbeurteilung im Disput. In: ders. (Hrsg.): Schulbücher auf dem Prüfstand. Perspektiven der Schulbuchforschung und Schulbuchbeurteilung in Europa. (= Schriftenreihe des Georg-Eckert-Instituts, 75). Frankfurt/Main: Diesterweg, S. 9-22.

Fritsche, K. P. (Hrsg.) (1992): Schulbücher auf dem Prüfstand. Perspektiven der Schulbuchforschung und Schulbuchbeurteilung in Europa. (= Schriftenreihe des Georg-Eckert-Instituts, 75). Frankfurt/Main: Diesterweg.

Fuchs, E., Kahlert, J. \& Sandfuchs, U. (Hrsg.) (2010): Schulbuch konkret. Kontexte Produktion Unterricht. Bad Heilbrunn: Klinkhardt.

Führ, C. \& Furck C.-L. (Hrsg.) (1998): Handbuch der deutschen Bildungsgeschichte, Bd. 6. 1945 bis zur Gegenwart, Teilbd. 2. Deutsche Demokratische Republik und neue Bundesländer. München: Beck.

Gehlhaar, K.-H. (1985a): Wahlversammlung der Sektion Schulbiologie. In: Biologie in der Schule, 34. Jg., H. 4, S. 141-142.

Gehlhaar, K.-H. (1985b): Vorinformation zu den XXII. Schulbiologentagen 1986. In: Biologie in der Schule, 34. Jg., H. 11, S. 430-431.

Gehlhaar, K.-H. (1988): Wahlversammlung der Sektion Schulbiologie. In: Biologie in der Schule, 37. Jg., H. 11, S. 431-432.

Geisenhainer, F. (1959): Lehrtafeln für die Entwicklungslehre. In: Biologie in der Schule, 8. Jg., H. 1., S. 18-23.

Geißler, G. (2000): Geschichte des Schulwesens in der Sowjetischen Besatzungszone und in der Deutschen Demokratischen Republik 1945 bis 1962. Frankfurt am Main: Peter Lang.

Geißler, G., Blask, F. \& Scholze, T. (1996): Geschichte, Struktur und Funktionsweise der DDR-Volksbildung, Bd. 1. Schule: Streng vertraulich! Die Volksbildung der DDR in Dokumenten. Berlin: Basisdr.

Gensel, P., Günther, S., Neye, H. \& Rumpf, D. (1999): Fossilien des Muschelkalks aus Weimars Umgebung. Weimar: Stadtmuseum.

Gerber, T. (2008): Persönlichkeiten aus Land- und Forstwirtschaft, Gartenbau und Veterinärmedizin. Biographisches Lexikon, Bd. 2., 3. Aufl. Berlin: Nora.

Gesang, K., Hild, S., Hoßfeld, U., Markert, M., Müller, H.-L. \& Prasse, J. (2012): Biologische Bildung an außerschulischen Lernorten in Thüringen. Bad Berka: Thillm (Thüringer Institut für Lehrerfortbildung, Lehrplanentwicklung und Medien). 
Gieselbusch, H., Röwer, G., Schmidt-Salomon, M. \& Strickstrock, F. (Hrsg.):

Karlheinz Deschner und seine große Kriminalgeschichte des

Christentums. Leben Werke Wirkungen. Zum Erscheinen von Band $10 \mathrm{im}$ März 2013. Hamburg: Rowohlt.

Glaubrecht, M. (2013): Am Ende des Archipels. Alfred Russel Wallace. Köln: Kiepenheuer \& Witsch.

Gottschalk, R. (1954): Das Ziel des Biologieunterrichts in den allgemeinbildenden Schulen. In: Biologie in der Schule, 3. Jg., H. 6, S. $267-271$.

Gould, S. J. (1999): Illusion Fortschritt. Die vielfältigen Wege der Evolution. Frankfurt am Main: Fischer Taschenbuch.

Graef, H., Günther, J., Kopprasch, G., Kummer, G., Meincke, I., Müller, J. \& Windelband, A. (1985): Unterrichtshilfen Biologie Klasse 10. 7. Aufl. Berlin: Volk und Wissen.

Graef, H., Günther, J., Kopprasch, G., Kummer, G., Meincke, I., Müller, J. \& Windelband, A. (1989): Unterrichtshilfen Biologie Klasse 10. Berlin: Volk und Wissen.

Graf, D. (2009): Evolution - das Rückgrat der Biologie. In: Der mathematische und naturwissenschaftliche Unterricht, Sonderheft Evolution, S. 3-4.

Graf, D. \& Lammers C. (2011): Evolution und Kreationismus in Europa. In: Graf, D. (Hrsg.): Evolutionstheorie - Akzeptanz und Vermittlung im europäischen Vergleich. Berlin etc.: Springer, S. 9-28.

Graf, D., Schmidt-Salomon, M., Storch, V., Voland, E. sowie rund 80 weitere Teilnehmer und Teilnehmerinnen des „Evokids-Kongresses 2015“ in der Hermann-Hoffmann-Akademie Gießen (2015): Resolution. Evolutionsunterricht in der Grundschule.

Graf, D. (Hrsg.) (2011): Evolutionstheorie - Akzeptanz und Vermittlung im europäischen Vergleich. Berlin etc.: Springer.

Griffhorn, B. \& Langlet, J. (2006): Einführung in die Selektionstheorie - So früh wie möglich! In: Praxis der Naturwissenschaften-Biologie in der Schule, 55. Jg., H. 6, S. 6-15.

Grönke, O. (1954): Neue Hilfsmittel für den Biologieunterricht. Bericht von der Leipziger Messe. In: Biologie in der Schule, 3. Jg., H. 3, S. 136-140.

Grönke, O. (1969): Biologische Sammlung. In: Baer, H.-W. \& Grönke, O. (Hrsg.): Biologische Arbeitstechniken für Lehrer und Naturfreunde. 2., erw. Aufl. Berlin: Volk und Wissen, S. 295-326. 
Grönke, O. \& Kirsch, W. (1969): Studienanleitung zur Methodik des Biologieunterrichts für das Fernstudium der Biologielehrer der Klassen 510. Potsdam: Pädagogische Hochschule.

Gropengießer, G. (2006): Unterrichtsplanung. In: Gropengießer, G. \& Kattmann, U. (Hrsg): Fachdidaktik Biologie. 7. Aufl. Köln: Aulis Deubner, S. 190-209.

Gropengießer, G. \& Kattmann, U. (Hrsg) (2006): Fachdidaktik Biologie. 7. Aufl. Köln: Aulis Deubner.

Groth (1952): Hinweis zum Lehrplan für Oberschulen - Biologie. In: Biologie in der Schule, 1. Jg., H. 10, S. 433.

Groth, D. (1983): Zur Neugestaltung des Biologieunterrichts der Grundschule in der antifaschistisch-demokratischen Schule (1945-1949). Dissertation, Potsdam.

Grümme,T., Krull, H.-P., Küttner, R. \& Nolte, M. (2010): Markl Biologie Oberstufe. Arbeitsbuch. Stuttgart: Ernst Klett.

Gruner, H.-E., Reinhard, H., Renatus, K., Rothmaler, W., Schneider, G. \& Uschmann, G. (1953): Lehrbuch der Biologie für das 12. Schuljahr. Berlin: Volk und Wissen.

Günther, J. \& Scheiding, K. (1966): Biologische Aufgabensammlung. Berlin: Volk und Wissen.

Günther, K.-H. (1969): Schule und Gesellschaft in der Deutschen Demokratischen Republik: Das gesamtgesellschaftliche System des Sozialismus in der Deutschen Demokratischen Republik und die Funktion des Bildungssystems. In: Drefenstedt, E. \& Neuner, G. (Hrsg.): Lehrplanwerk und Unterrichtsgestaltung. Berlin: Volk und Wissen, S. 13-22.

Günther, K.-H. \& Uhlig, G. (1969): Geschichte der Schule in der Deutschen Demokratischen Republik 1945 bis 1968. Berlin: Volk und Wissen.

Gürtler, A. (1947): Kindertümliche Faustskizzen und biologisch-schematische Zeichnungen für den naturkundlichen Unterricht. 1. Heft:

Tierwelt/Mineralien. Leipzig: Ernst Wunderlich.

Haeckel, E. (1909): Das Weltbild von Darwin und Lamarck. Festrede zur Hundertjährigen Geburtstagsfeier von Charles Darwin. Am 12. Februar 1909 gehalten im Volkshause zu Jena. 2. Aufl. Leipzig: Alfred Kröner.

Haeckel, E. (2009a): Kunstformen der Natur. 3. Aufl. Wiesbaden: marix.

Haeckel, E. (2009b): Die Welträtsel. Hamburg: Nikol.

Hagen, A. (1964): Einige Hinweise zur Verbesserung des Biologieunterrichts in den allgemeinbildenden Schulen. In: Biologie in der Schule, 13. Jg., H. 1, S. 32-33. 
Heberer, G. (2004): Nachwort. In: Die Entstehung der Arten durch natürliche Zuchtwahl. Sonderausgabe. Hamburg: Nikol, S. 679-687.

Heichler, G. (1963): Stoffplan „Entwicklungslehre“ - Klasse 10 der allgemeinbildenden polytechnischen Oberschule. In: Biologie in der Schule, 12. Jg., H. 11, S. 471-479.

Heinze, C. (2005): Das Schulbuch zwischen Lehrplan und Unterrichtspraxis. Zur Einführung in den Themenband. In: Matthes, E. \& Heinze, C. (Hrsg.): Das Schulbuch zwischen Lehrplan und Unterrichtspraxis. (= Beiträge zur historischen und systematischen Unterrichtspraxis). Bad Heilbrunn/Obb.: Julius Klinkhardt, S. 9-17.

Heinzel, I. (1981): Vorankündigung XIX. Schulbiologentage vom 8. bis 11.

Mai in Mühlhausen Thomas-Müntzer-Stadt. In: Biologie in der Schule, 30. Jg., H. 9, S. 390-391.

Henke, W. \& Rothe, H. (2003): Menschwerdung. Frankfurt am Main: Fischer.

Herbst, A., Ranke, W. \& Winkler, J. (Hrsg) (1994): So funktionierte die DDR. Lexikon der Organisationen und Institutionen, Bd. 2. Reinbek: Rowohlt Taschenbuch.

Herrmann, L. (1974): Beitrag unseres Tafelbildes als Mittel zur Aktivierung der Schüler im Biologieunterricht (dargestellt an Beispielen aus der 8. Klasse). In: Biologische Gesellschaft der DDR (Hrsg.): Mitteilungen der Sektion Schulbiologie der biologischen Gesellschaft der DDR, Heft 14, S. 252.

Högermann, C. \& Meißner, K. (Hrsg.) (2001): Biologie plus. Gymnasium Klassen 9/10. Thüringen. Berlin: Cornelsen.

Honecker, M. (1989): Unser sozialistisches Bildungssystem - Wandlungen, Erfolge, neue Horizonte. IX. Pädagogischer Kongress der DDR 13. bis 15. Juni 1989. Berlin: Dietz.

Horn, F. (1987): Standpunkte und ihre Umsetzung in den neuen Lehrplänen für die Klasse 5 bis 10. In: Biologie in der Schule, 36. Jg., H. 6, S. 209-215.

Horn, F. (1989): Der Lehrplan Biologie der zehnklassigen allgemeinbildenden polytechnischen Oberschule. Inhaltliche und methodische Erläuterungen. Berlin: Volk und Wissen.

Horn, F. \& Kaiser, G. (1986): Zur Weiterentwicklung des Biologieunterrichts in den Klassen 5 bis 10. In: Biologie in der Schule, 35. Jg., H. 2/3, S. 49-61.

Hoßfeld, U. (o. J.): Sammlung der Arbeitsgruppe Biologiedidaktik. Online im Internet unter http://www.uni-jena.de/Sammlung_Biologiedidaktik.html $<02.03 .2014>$. 
Hoßfeld, U. (2005): Geschichte der biologischen Anthropologie in Deutschland. Von den Anfängen bis in die Nachkriegszeit. (= Wissenschaftskultur um 1900, Bd. 2). Stuttgart: Franz Steiner.

Hoßfeld, U. (2007a): Traditionskultur in der Biologie. In: Hoßfeld, U.,

Mestrup, H. \& Kaiser, T. (Hrsg.): Hochschule im Sozialismus. Studien zur Friedrich-Schiller-Universität Jena (1945-1990), Bd. 1. Köln etc.: Böhlau, S. 1067-1085.

Hoßfeld, U. (2007b): Vom Christ zum Atheist: Der Kirchenaustritt Ernst Haeckels im Jahr 1910. In: Kutschera, U. (Hrsg.): Kreationismus in Deutschland. Fakten und Analysen. (= Naturwissenschaft und Glaube, Bd. 1). Berlin: Lit, S. 45-70.

Hoßfeld, U. (2009a): Quo vadis „Darwin-Industry“?: Tendenzen und Trends im Darwin-Jahr 2009. In: Brunner, H., Jansohn, C., Lentzen, M. \& Mehl, D. (Hrsg.): Archiv für das Studium der neueren Sprachen und Literaturen, 246. Bd., 161. Jg., 2. Halbjahresbd. Berlin: Erich Schmidt, S. 330-344.

Hoßfeld, U. (2009b): Darwin-Jahr 2009 - eine erste Bestandsaufnahme. In: Anzeiger des Vereins Thüringer Ornithologen, 6, S. 313-325.

Hoßfeld, U. (2012a): Biologie und Politik. Die Herkunft des Menschen. 2. ergänzte Aufl. Erfurt: Landeszentrale für politische Bildung Thüringen.

Hoßfeld, U. (2012b): Ernst Haeckel - der Gegenpapst aus Jena. Ein Thema aus der Geschichte des Thüringer Biologieunterrichtes. Vortrag. 18. Tage des mathematischen und naturwissenschaftlichen Unterrichts. Erfurt: 07.03.2012.

Hoßfeld, U. \& Breidbach, O. (2007): Biologie- und Wissenschaftsgeschichte in Jena: Das Ernst-Haeckel-Haus der Friedrich-Schiller-Universität. In: Hoßfeld, U., Mestrup, H. \& Kaiser, T. (Hrsg.): Hochschule im Sozialismus. Studien zur Friedrich-Schiller-Universität Jena (1945-1990), Bd. 2. Köln etc.: Böhlau, S. 1181-1206.

Hoßfeld, U. \& Breidbach, O. (2008): Ernst Haeckels Politisierung der Biologie. 2. Aufl. (= Blätter der Landeskunde Thüringen, Nr. 35). Erfurt: Landeszentrale für politische Bildung Thüringen.

Hoßfeld, U. \& Brömer, R. (2001): Darwinismus und/als Ideologie - Eine Einleitung. In: ders. (Hrsg.): Darwinismus und/als Ideologie. (= Verhandlungen zur Geschichte und Theorie der Biologie, Bd. 6.). Berlin: Wissenschaft und Bildung, S. 9-27.

Hoßfeld, U., Olsson, L. \& Levit, G. S. (2011): Evolutionäre Entwicklungsbiologie (Evo-Devo). In: Dreesmann, D., Graf, D. \& Witte, K. (Hrsg): Evolutionsbiologie. Moderne Themen für den Unterricht. Heidelberg: Spektrum, S. 151-179.

Hoßfeld, U. (Hrsg.) (2010): Absolute Ernst Haeckel. Freiburg: orange-press. 
Hoßfeld, U. \& Brömer, R. (Hrsg.) (2001): Darwinismus und/als Ideologie. (= Verhandlungen zur Geschichte und Theorie der Biologie, Bd. 6.). Berlin: Wissenschaft und Bildung.

Hoßfeld, U., Mestrup, H. \& Kaiser, T. (Hrsg.) (2007): Hochschule im

Sozialismus. Studien zur Friedrich-Schiller-Universität Jena (1945-1990), Bd. 1. Köln etc.: Böhlau.

Hoßfeld, U., Mestrup, H. \& Kaiser, T. (Hrsg.) (2007): Hochschule im Sozialismus. Studien zur Friedrich-Schiller-Universität Jena (1945-1990), Bd. 2. Köln etc.: Böhlau.

Höxtermann, E. \& Höxtermann, J. (2007): Otto Schwarz und die Gründung der Biologischen Gesellschaft in der DDR. In: Hoßfeld, U., Mestrup, H. \& Kaiser, T. (Hrsg.): Hochschule im Sozialismus. Studien zur FriedrichSchiller-Universität Jena (1945-1990), Bd. 2. Köln etc.: Böhlau, S. 1207-1232.

Höxtermann, E. (1997): Zur Profilierung der Biologie an den Universitäten der DDR bis 1968. Reprint 72. Max-Planck-Institute für Wissenschaftsgeschichte.

Hubrich, D. (2013): Verleihungsliste zum Ehrentitel „Verdienter Lehrer des Volkes“ von 1949 bis 1989. Online im Internet unter http://www.deutsche-gesellschaft-fuer-ordenskunde.de/DGOWP/wpcontent/uploads/2013/10/VL-VLdV-1949-1989.pdf <14.11.2014>.

Hundt, R. \& Kreße, E. (1969): Biologie, Arbeitsgemeinschaften-Exkursionen. Berlin: Volk und Wissen.

Institut für Marxismus-Leninismus beim ZK der SED (Hrsg.) (1966): Karl Marx. Friedrich Engels. Ausgewählte Schriften in zwei Bänden, Bd. 2. Berlin: Dietz.

Ivanov, M., Hrdličková, S. \& Gregorová, R. (o. J.): Illustrierte Fossilien Enzyklopädie. Eggolsheim: Dörfler.

Jahn, I. \& Schmitt, M. (2001): Carl Linnaeus (1707-1778). In: Jahn, I. \& Schmitt, M. (Hrsg.): Darwin \& Co. Eine Geschichte der Biologie in Portraits, Bd. 1. München: C.H.Beck, S. 9-30.

Jahn, I. \& Schmitt, M. (Hrsg.) (2001): Darwin \& Co. Eine Geschichte der Biologie in Portraits, Bd. 1. München: C.H.Beck.

Jahn, I. \& Schmitt, M. (Hrsg.) (2001): Darwin \& Co. Eine Geschichte der Biologie in Portraits, Bd. 2. München: C.H.Beck.

Jockel, S. (2014) Die Deutsche Nationalbibliothek im Überblick. Online im Internet unter http://www.dnb.de/DE/Wir/wir_node.html $<20.04 .2014>$.

Jordan, S. (2005): Einführung in das Geschichtsstudium. Stuttgart: Reclam. 
Junker, T. (2001): Charles Darwin (1809-1882). In: Jahn, I. \& Schmitt, M. (Hrsg.): Darwin und Co. Eine Geschichte der Biologie in Portraits, Bd. 1. München: C.H.Beck, S. 369-389.

Junker, T. (2009): Charles Darwin und die darwinsche Revolution. In: Praxis der Naturwissenschaften-Biologie in der Schule, 58. Jg., H. 3, S. 4-11.

Junker, T. (2011): Evolutionstheorie und Kreationismus. Ein aktueller Überblick. In: Graf, D. (Hrsg.): Evolutionstheorie - Akzeptanz und Vermittlung im europäischen Vergleich. Berlin etc.: Springer, S. 77-90.

Junker, T. \& Hoßfeld, U. (2009): Die Entdeckung der Evolution. Eine revolutionäre Theorie und ihre Geschichte. 2. durchges. Aufl. Darmstadt: Wissenschaftliche Buchgesellschaft.

Junker, T. \& Paul, S. (2009): Der Darwin Code: Die Evolution erklärt unser Leben. München: C.H.Beck.

Jürgens, U. (2006): Lehrwerke. In: Arnold, K.-H., Sandfuchs, U. \& Wiechmann, J. (Hrsg.) (2006): Handbuch Unterricht. Bad Heilbrunn: Klinkhardt, S. 404-413.

Kaiser, G. (1988): Schwerpunkte der Weiterentwicklung des

Biologieunterrichts. In: Biologie in der Schule, 37. Jg., H. 2/3, S. 63-74.

Kattmann, U. (2008): Evolution und Schöpfung. Kompakt. Unterricht Biologie 333.

Kattmann, U., Groß, J. \& Scheersoi, A. (2009): Evolution schafft Vielfalt. Seelze-Velber: Friedrich.

Kiper, H. (2001): Einführung in die Schulpädagogik. Weinheim etc.: 2001.

Kirchhöfer, D. (2009): Lehrer und Lehrerbildung. In: Markus, U. (Hrsg.): Was war unsere Schule wert? Volksbildung in der DDR. Berlin: Das neue Berlin, S. 137-162.

Kirsch, W. (1966): Schulbiologentage 1966. In: Biologie in der Schule, 15. Jg., H. 9, S. 376-380.

Kirsch, W. (1967). Biologieunterricht und Fernsehen. In: Biologie in der Schule, 16. Jg., H. 10, S. 427-432.

Kirschke, S. (1959): 100 Jahre Darwinismus. In: Neues Deutschland, 6. Juni, S. 9.

Knecht, P., Matthes, E., Schütze, S. \& Aamotsbakken, B. (Hrsg.) (2014):

Methodologie und Methoden der Schulbuch- und Lehrmittelforschung. (= Beiträge zur historischen und systematischen Schulbuchforschung). Bad Heilbrunn: Julius Klinkhardt. 
Knorre, D. von, Penzlin, H. \& Hertel, W. (2007): Der Lyssenkoismus und die Zoologie in Jena. In: Hoßfeld, U., Mestrup, H. \& Kaiser, T. (Hrsg.):

Hochschule im Sozialismus. Studien zur Friedrich-Schiller-Universität Jena (1945-1990), Bd. 2. Köln etc.: Böhlau.

Koch, M. (2012): Blick zurück in die Zukunft. Pädagogen diskutieren über Vorzüge und Nachteile des Schul- und Bildungssystems der DDR. In: Neues Deutschland, 7. September, S. 16.

Köhler, H. (2008): Datenhandbuch zur deutschen Bildungsgeschichte, Bd. 9. Schulen und Hochschulen in der Deutschen Demokratischen Republik. 1949-1989. Göttingen: Vandenhoeck \& Ruprecht.

Kolchinsky, E. I. (2001): Darwinismus und Marxismus in der Epoche des frühen Stalinismus. In: Hoßfeld, U. \& Brömer, R. (Hrsg.): Darwinismus und/als Ideologie. Berlin: Wissenschaft und Bildung, S. 157-166.

Körnig, G. (2009): Manfred Matzke - 75 Jahre. In: Mitteilungen der Deutschen Malakozoologischen Gesellschaft, Nr. 82, S. 71-73.

Kress, H. \& Wiesner, E. (1958): Die Züchtung von Pflanzen und Tieren. Lehrheft für den Biologieunterricht in den 12. Klassen. durchges. Neuaufl. Berlin. Volk und Wissen.

Krombholz, H. \& Lengert, W. (1959): Zum Biologielehrplan der allgemeinbildenden polytechnischen Oberschule. In: Biologie in der Schule, 8. Jg., H. 7, S. 309-316.

Krüger, D. \& Vogt, H. (2007): Es gibt nichts praktischeres als eine gute Theorie. In: ders. (Hrsg.): Theorien in der biologiedidaktischen Forschung. Ein Handbuch für Lehramtsstudenten und Doktoranden. Berlin etc.: Springer, S. 1-7.

Krüger, D. \& Vogt, H. (Hrsg.) (2007): Theorien in der biologiedidaktischen Forschung. Ein Handbuch für Lehramtsstudenten und Doktoranden. Berlin etc.: Springer.

Krüger, L. (1962): Die Verwendung der Manipermhafttafel bei der Behandlung der Anthropogenese. In: Biologie in der Schule, 11. Jg., H. 9, S. 424-425.

Krull, W. (2011): Geleitworte. In: Dreesmann, D., Graf, D. \& Witte, K. (Hrsg.): Evolutionsbiologie. Moderne Themen für den Unterricht. Heidelberg: Spektrum, S. 5-6.

Kuckartz, U. (2012): Qualitative Inhaltsanalyse. Methoden, Praxis, Computerunterstützung. Weinheim etc.: Beltz Juventa.

Kühn, O. (1953): Bemerkungen zur Überarbeitung des Stoffplans für Oberschulen. Biologie. 9. bis 12. Schuljahr. In: Biologie in der Schule, 2. Jg., H. 8, S. 340-343. 
Kühn, O. (1955): Hinweise zur Anwendung von Lehrplan und Direktive im Biologieunterricht der Grundschule. In: Biologie in der Schule, 4. Jg., H. 9, S. 408-412.

Kummer, G. (2009): Erinnerungen. Berlin: Unveröffentlichtes Manuskript. Kummer, G. (2016a): Von der Neulehrerin zur Redakteurin für Schulbücher. In: Jäkel, H. (Hrsg.): DDR unvergessen. Schkeuditz: GNN, S. 120-126.

Kummer, G. (2016b): Biologie in der DDR. In: Jäkel, H. (Hrsg.): DDR unvergessen. Schkeuditz: GNN, S. 139-146.

Kummer, G., Matzke, M., Schwier, H.-J., Sommer, K., Theuerkauf, H. \& Ullrich, H. (1988): Biologie. Lehrbuch für Klasse 10. Vererbung und Evolution. Berlin: Volk und Wissen.

Kupke, A. (1954): Bedeutung der Ministerratsverordnung vom 4. März 1954 für den Biologieunterricht. In: Biologie in der Schule, 3. Jg., H. 6, S. 241-247.

Kupke, A. (1959a): Zur Gründung der Biologischen Gesellschaft in der Deutschen Demokratischen Republik. In: Biologie in der Schule, 8. Jg., H. 5, S. 193-195.

Kupke, A. (1959b): Mitteilung der Biologischen Gesellschaft in der Deutschen Demokratischen Republik. In: Biologie in der Schule, 8. Jg., H. 8, S. 383.

Kupke, A. (1974): Zur Geschichte der Sektion Schulbiologie. In: Biologische Gesellschaft der DDR (Hrsg.): Mitteilungen der Sektion Schulbiologie der Biologischen Gesellschaft der DDR, H. 14, S. 6-15.

Kutschera, U. (o. J.): Über uns. Online im Internet unter http://www.evolutionsbiologen.de/ <21.12.2013>.

Kutschera, U. (2004): Streitpunkt Evolution. Darwinismus und Intelligentes Design. (= Naturwissenschaft und Glaube, Bd. 1). Berlin: Lit.

Kutschera, U. (2007a): Von Darwin zu Einstein: Der Evolutions- und Photonenglaube. In: ders. (Hrsg.): Kreationismus in Deutschland. Fakten und Analysen. (= Naturwissenschaft und Glaube, Bd. 1). Berlin: Lit, S. 13-44.

Kutschera, U. (2007b): Vorwort. In: ders. (Hrsg.): Kreationismus in Deutschland. Fakten und Analysen. (= Naturwissenschaft und Glaube, Bd. 1). Berlin: Lit, S. 5-7.

Kutschera, U. (2008): Evolutionsbiologie. 3. Aufl. Stuttgart: Eugen Ulmer. Kutschera, U. (2009a): Anti-Darwin-Propaganda 2009. Kooperative EvoEsoterik und der deutsche Intelligent Design-Kreationismus. In: Laborjournal 3, S. 18-21.

Kutschera, U. (2009b): Tatsache Evolution. Was Darwin nicht wissen konnte. München: dtv premium. 
Kutschera, U. (2010a): Erinnerungen an den Freiburger Evolutionsbiologen Günther Osche (1926-2009). In: Kaasch, M. \& Kaasch, J. (Hrsg.): Das Werden des Lebendigen. (= Verhandlungen zur Geschichte und Theorie der Biologie, Bd. 16.). Berlin: Wissenschaft und Bildung, S. 257-263.

Kutschera, U. (2010b): Was ist Kreationismus? Online im Internet unter http://www.youtube.com/watch?v=HQZacHdupyI <13.11.2014>.

Kutschera, U. (2012): Zehn Jahre Anti-Kreationismus. Das Geburtstagsvideo zum 10jährigen Jubiläum des AK Evolutionsbiologie. Online im Internet unter http://www.youtube.com/watch?v $=$ shbZR53T5Nw $<13.11 .2014>$.

Kutschera, U. (2014): Zum 100. Todestag. August Weismann (1834-1914) und das unsterbliche Leben. In: Biologie in unserer Zeit, 6. Jg., H. 44, S. 366-369.

Kutschera, U. (Hrsg.) (2007): Kreationismus in Deutschland. Fakten und Analysen. (= Naturwissenschaft und Glaube, Bd. 1). Berlin: Lit.

Kutschera, U. \& Hoßfeld, U. (2012): Zehn Jahre Arbeitskreis (AK) Evolutionsbiologie im Deutschen Biologenverband. In: Rudolstädter naturhistorische Schriften 18, S. 19-26.

Laitko, H. (2010): Hans Stubbe und das politische System der DDR. Ein prominentes, aber nichtspektakuläres Beispiel für das Wechselspiel von Naturwissenschaft und Politik in der DDR der 1950er und 1960er Jahre. In: Jahn, I. \& Wessel, A. (Hrsg.): Für eine Philosophie der Biologie. Festschrift to the 75th Birthday of Rolf Löther (= Berliner Studien zur Wissenschaftsphilosophie und Humanogenetik, Bd. 26). München: Kleine, S. $127-169$.

Lang, J. (1946): Die demokratische Einheitsschule. Leipzig: Volk und Buch.

Lässig, S. (2010): Wer definiert relevantes Wissen? Schulbücher und ihr gesellschaftlicher Kontext. In: Fuchs, E., Kahlert, J. \& Sandfuchs, U. (Hrsg.): Schulbuch konkret. Kontexte Produktion Unterricht. Bad Heilbrunn: Klinkhardt, S. 199-215.

Lefèvre, W. (2001): Jean Baptiste Lamarck (1744-1829). In: Jahn, I. \& Schmitt, M. (Hrsg.): Darwin \& CO. Eine Geschichte der Biologie in Portraits, Bd. 1. München: C.H.Beck, S. 176-201.

Leinfelder, R. (2007): Der deutsche Kreationismus und seine

Rahmenbedingungen aus der Sicht eines Paläontologen. In: Kutschera, U. (Hrsg.): Kreationismus in Deutschland. Fakten und Analysen. (= Naturwissenschaft und Glaube, Bd. 1). Berlin: Lit, S. 277-326.

Leitung der Sektion Schulbiologie der Biologischen Gesellschaft in der DDR (1971): VIII. Schulbiologentage 1971. In: Biologie in der Schule, 20. Jg., H. 1, S. 40-41. 
Lemke, W. (1948): Zur Methodik und Praxis des Biologieunterrichts in der Grundschule. Berlin etc.: Volk und Wissen.

Lemke, W. (Hrsg.) (1951): Lehrbuch der Biologie für das 8. Schuljahr. Berlin etc.: Volk und Wissen.

Lemke, W. (Hrsg.) (1953): Lehrbuch der Biologie für das 8. Schuljahr. Berlin: Volk und Wissen.

Lengert, W. (1959): Zum Biologielehrplan für die sozialistische polytechnische Oberschule. In: Biologie in der Schule, 8. Jg., H. 3, S. 92-95.

Links, C. (2010): Das Schicksal der DDR-Verlage. Die Privatisierung und ihre Konsequenzen. 2. Aufl. Berlin: Ch.Links.

Löbel, F. \& Maschke, W. (Hrsg.) (1948): Lehrbuch der Biologie für das 7. und 8. Schuljahr. 3., durchg. Aufl. Berlin etc.: Volk und Wissen.

Löther, R. (2004): Kant und die biologische Evolutionstheorie. In: Sitzungsberichte der Leibniz-Sozietät, 69, S. 111-118.

Löther, R. (2010): Erinnerungen an meinen wissenschaftlichen Werdegang. In: Jahn, I. \& Wessel, A. (Hrsg.): Für eine Philosophie der Biologie. Festschrift to the 75th Birthday of Rolf Löther (= Berliner Studien zur Wissenschaftsphilosophie und Humanontogenetik, Bd. 26). München: Kleine, S. 81-96.

Löther, R. \& Schellhorn, M. (1964): Die Bedeutung der Abstammungslehre für die Herausbildung eines wissenschaftlichen Weltbildes. In: Böhme, H. (Hrsg.): Beiträge zur Abstammungslehre, Bd. 1. Berlin: Volk und Wissen, S. 7-24.

Lucretius Carus, T. (1973): De rerum natura. Welt aus Atomen. Stuttgart: Reclam.

Markert, M. (2006): Zwischen Wissenschaft und Politik: Der zweifache Rektor der Universität Jena Otto Schwarz (1900-1983). Online im Internet unter http://www.db-thueringen.de/servlets/DerivateServlet/Derivate29117/Markert,\%20M.\%20-\%20Otto\%20Schwarz\%20$\% 20$ Zwischen $\% 20$ Wissenschaft $\% 20$ und $\% 20$ Politik.pdf $<15.11 .2014>$.

Markus, U. (Hrsg.) (2009): Was war unsere Schule wert? Volksbildung in der DDR. Berlin: Das Neue Berlin.

Marquardt-Mau, B. \& Rojek, R. (2011): Kinder auf den Spuren Charles Darwins - Evolutionsbiologie im Sachunterricht. In: Dreesmann, D., Graf, D. \& Witte, K. (Hrsg.): Evolutionsbiologie. Moderne Themen für den Unterricht. Heidelberg: Spektrum, S. 43-64. 
Matthes, E. \& Schütze, S. (2014): Methodologie und Methoden der Schulbuch- und Lehrmittelforschung - Einleitung. In: Knecht, P., Matthes, E., Schütze, S. \& Aamotsbakken, B. (Hrsg.): Methodologie und Methoden der Schulbuch- und Lehrmittelforschung. (= Beiträge zur historischen und systematischen Schulbuchforschung). Bad Heilbrunn: Julius Klinkhardt, S. 9-18.

Matthes, E. \& Heinze, C. (Hrsg.) (2005): Das Schulbuch zwischen Lehrplan und Unterrichtspraxis. (= Beiträge zur historischen und systematischen Schulbuchforschung). Bad Heilbrunn/Obb.: Julius Klinkhardt.

Matthes, E. \& Schütze, S. (Hrsg.) (2016a): „1989“ und Bildungsmedien. (= Beiträge zur historischen und systematischen Schulbuchforschung). Bad Heilbrunn: Julius Klinkhardt.

Matthes, E. \& Schütze, S. (Hrsg.) (2016b): Schulbücher auf dem Prüfstand. (= Beiträge zur historischen und systematischen Schulbuchforschung). Bad Heilbrunn: Julius Klinkhardt.

Matthes, W. (1946): Landwirtschaftliche Biologie für die Oberschule. Berlin etc.: Volk und Wissen.

Mayr, E. (1995): ... und Darwin hat doch recht. Charles Darwin, seine Lehre und die moderne Evolutionstheorie. 2. Aufl. München: Piper.

Mayr, E. (2002): Die Entwicklung der biologischen Gedankenwelt. Vielfalt, Evolution und Vererbung. Nachdruck der Aufl. von 1984. Berlin etc.: Springer.

Mayr, E. (2005a): Das ist Evolution. 3. Aufl. München: Goldmann.

Mayr, E. (2005b): Konzepte der Biologie. Stuttgart: S. Hirzel.

Mayr, E. (2008): Charles Darwins Autobiographie. In: Barlow, N. (Hrsg.): Charles Darwin. Mein Leben. Frankfurt am Main etc.: Insel, S. 9-21.

Mayr, E. (2010): Das ist Biologie. Die Wissenschaft des Lebens. unveränderter Nachdruck. Heidelberg: Spektrum.

Mayring, P. (2010): Qualitative Inhaltsanalyse. Grundlagen und Techniken. 11. Aufl. Weinheim etc.: Beltz.

Meisert, A. (2008): Wie kann Biologieunterricht geplant werden? In: SpörhaseEichmann, U. \& Ruppert, W. (Hrsg.): Biologie-Didaktik. Praxishandbuch für die Sekundarstufe I und II. 5. Aufl. Berlin: Cornelsen Scriptor, S. 241-274.

Menck, P. (2014): Schulbuchforschung - Anmerkungen zu ihrer Methodik. In: Knecht, P., Matthes, E., Schütze, S. \& Aamotsbakken, B. (Hrsg.): Methodologie und Methoden der Schulbuch- und Lehrmittelforschung. (= Beiträge zur historischen und systematischen Schulbuchforschung). Bad Heilbrunn: Julius Klinkhardt, S. 333-343. 
Merbold, W.-E. (1964): Schulbiologentage 1964. In: Biologie in der Schule, 13. Jg., H. 10, S. 457-458.

Meyer, H. (2004): Was ist guter Unterricht? Sonderausgabe mit 65 Min.Vortrag (DVD). Berlin: Cornelsen Scriptor.

Meyers, P. (1976): Methoden zur Analyse historisch-politischer Schulbücher. In: Schallenberger, H. (Hrsg.): Studien zur Methodenproblematik wissenschaftlicher Schulbucharbeit. Kastellaun: Aloys Henn, S. 47-73.

Ministerium für Volksbildung der Deutschen Demokratischen Republik (Hrsg.) (1951a): Lehrplan für Grundschulen. Biologie. 5. bis 8. Schuljahr. Berlin: Volk und Wissen.

Ministerium für Volksbildung der Deutschen Demokratischen Republik (Hrsg.) (1951b): Lehrplan für Oberschulen. Biologie. 9. bis 12. Schuljahr. Berlin: Volk und Wissen.

Ministerium für Volksbildung der Deutschen Demokratischen Republik (Hrsg.) (1951c): Lehrplan für Zehnjahrschulen. Biologie. Berlin etc.: Volk und Wissen.

Ministerium für Volksbildung der Deutschen Demokratischen Republik (Hrsg.) (1952): Lehrplan für Grundschulen. Biologie. 5. bis 8. Schuljahr. Berlin: Volk und Wissen.

Ministerium für Volksbildung der Deutschen Demokratischen Republik (Hrsg.) (1953a): Lehrplan für Grundschulen. Biologie. 5. bis 8. Schuljahr. Berlin: Volk und Wissen.

Ministerium für Volksbildung der Deutschen Demokratischen Republik (Hrsg.) (1953b): Lehrplan für Oberschulen. Biologie. 9. bis 12. Schuljahr. Berlin: Volk und Wissen.

Ministerium für Volksbildung der Deutschen Demokratischen Republik (Hrsg.) (1954): Lehrplan für Oberschulen. Biologie. 9. bis 12. Klasse. Berlin: Volk und Wissen.

Ministerium für Volksbildung der Deutschen Demokratischen Republik (Hrsg.) (1955): Direktive zur Arbeit mit dem Lehrplan für das Fach Biologie. Schuljahr 1955/56. Berlin: Volk und Wissen.

Ministerium für Volksbildung der Deutschen Demokratischen Republik (Hrsg.) (1956): Lehrplan für Mittelschulen. Biologie 10. Klasse. Berlin: Volk und Wissen.

Ministerium für Volksbildung der Deutschen Demokratischen Republik (Hrsg.) (1958): Direktive (vorläufiger Lehrplan). Biologieunterricht Oberschule. Berlin: Volk und Wissen. 
Ministerium für Volksbildung der Deutschen Demokratischen Republik (Hrsg.) (1959a): Lehrplan der zehnklassigen allgemeinbildenden polytechnischen Oberschule. Berlin: Volk und Wissen.

Ministerium für Volksbildung der Deutschen Demokratischen Republik (Hrsg.) (1959b): Lehrplan der zwölfklassigen erweiterten Oberschule für das Schuljahr 1959/60. Berlin: Volk und Wissen.

Ministerium für Volksbildung der Deutschen Demokratischen Republik (Hrsg.) (1961): Lehrplan der zwölfklassigen erweiterten Oberschule. Berlin: Volk und Wissen.

Ministerium für Volksbildung der Deutschen Demokratischen Republik (Hrsg.) (1966): Lehrplan für das Fach Biologie. Klassen 7 bis 10.

Nachdruck des Lehrplans von 1959 unter Berücksichtigung aller seit 1959 durchgeführten verbindlichen Veränderungen. Berlin: Volk und Wissen.

Ministerium für Volksbildung der Deutschen Demokratischen Republik (Hrsg.) (1967a): Anweisung und Korrektur des Lehrplans Biologie der Erweiterten Oberschule. Berlin: Volk und Wissen.

Ministerium für Volksbildung der Deutschen Demokratischen Republik (Hrsg.) (1967b): Lehrplan für den Biologieunterricht der Vorbereitungsklassen 9 und 10 zum Besuch der Erweiterten Oberschule (Präzisierter Lehrplan). Berlin: Volk und Wissen.

Ministerium für Volksbildung der Deutschen Demokratischen Republik (Hrsg.) (1968): Präzisierter Lehrplan für Biologie Klasse 8. Berlin: Volk und Wissen.

Ministerium für Volksbildung der Deutschen Demokratischen Republik (Hrsg.) (1969): Lehrplan für Biologie. Klassen 9 und 10. Berlin: Volk und Wissen.

Ministerium für Volksbildung der Deutschen Demokratischen Republik (Hrsg.) (1971): Gesamtbedarfsplan für Unterrichtsmittel der zehnklassigen allgemeinbildenden polytechnischen Oberschule der DDR. Klassen 1 bis 10. Berlin: Volk und Wissen.

Ministerium für Volksbildung der Deutschen Demokratischen Republik (Hrsg.) (1972): Gesamtbedarfsplan für Unterrichtsmittel im obligatorischen Unterricht der erweiterten allgemeinbildenden polytechnischen Oberschule der DDR Klassen 11 bis 12. ergänzt durch die Bedarfspläne der zehnklassigen allgemeinbildenden polytechnischen Oberschule der DDR Klassen 9 und 10. Berlin: Volk und Wissen.

Ministerium für Volksbildung der Deutschen Demokratischen Republik (Hrsg.) (1980): Gesamtausstattungsplan für Unterrichtsmittel der zehnklassigen allgemeinbildenden polytechnischen Oberschule der DDR. Klassen 1 bis 10. Teil II. Berlin: Volk und Wissen. 
Ministerium für Volksbildung der Deutschen Demokratischen Republik (Hrsg.) (1988): Lehrplan Biologie. Klassen 5 und 10. Berlin: Volk und Wissen.

Ministerium für Volksbildung der Deutschen Demokratischen Republik (Hrsg.) (1989): Lehrplan der zehnklassigen allgemeinbildenden polytechnischen Oberschule. Biologie. Klassen 5 bis 10. Berlin: Volk und Wissen.

Miram, W. \& Scharf, K.-H. (1988): Biologie heute S II. Hannover: Schroedel.

Miram, W. \& Scharf, K.-H. (1997): Biologie heute S II. Ein Lehr- und Arbeitsbuch. Hannover: Schroedel.

Müntz, K. \& Wobus, U. (2013): Das Institut Gatersleben und seine Geschichte. Genetik und Kulturpflanzenforschung in drei politischen Systemen. Berlin etc.: Springer.

Nationale Akademie der Wissenschaften Leopoldina (2017): Evolutionsbiologische Bildung in Schule und Universität. Deutsche Akademie der Naturforscher Leopoldina e. V. Nationale Akademie der Wissenschaften. Halle (Saale).

Neffe, J. (2008): Darwin. Das Abenteuer des Lebens. München: C. Bertelsmann.

Neigenfind, Fritz (1974): Anschauung und Anschauungsmittel. (= Schriftenreihe Unterrichtsmittel, Heft 43). Berlin: Volk und Wissen.

Nestler, H. (1963): Lebensspuren im Stein. (= Bücher für Schüler). Berlin: Volk und Wissen.

Neumann, D. (2015): Bildungsmedien Online. Kostenloses Lehrmaterial aus dem Internet: Marksichtung und empirische Nutzungsanalyse. (= Beiträge zur historischen und systematischen Schulbuchforschung). Bad Heilbrunn: Julius Klinkhardt.

Neuner, G. (1969): Das Lehrplanwerk als Ganzes. In: Drefenstedt, E. \& Neuner, G. (Hrsg.): Lehrplanwerk und Unterrichtsgestaltung. Berlin: Volk und Wissen, S. 38-71.

Neuner, G. (1989): Allgemeinbildung. Berlin: Volk und Wissen.

Neuner, G. (1996): Zwischen Wissenschaft und Politik. Ein Rückblick aus lebensgeschichtlicher Perspektive. Köln etc.: Böhlau.

Neuner, G. (2009): Haben wir zu viel verlangt? In: Markus, U. (Hrsg.): Was war unserer Schule wert? Volksbildung in der DDR. Berlin: Das neue Berlin, S. $125-162$.

Neupert, D. (1987): XXII. Schulbiologentage in Neubrandenburg (10. bis 13. Mai). In: Biologie in der Schule, 36. Jg., H. 2/3, S. 87-89. 
Osche, G. (1979): Evolution. Grundlagen - Erkenntnisse - Entwicklungen der Abstammungslehre. 10. Aufl. Freiburg im Breisgau: Herder.

Pädagogisches Institut Mühlhausen und Abteilung Biologie des volkseigenen Verlages Volk und Wissen (1960): Zu den Lehrbüchern für die 10. Klasse unserer Oberschule. In: Biologie in der Schule, 9. Jg., H. 9, S. 428-430.

Pälchen, H. (1974): Das Schulbuch für den Biologieunterricht und die Entwicklung schöpferischer Tätigkeit der Schüler. In: Biologische Gesellschaft der DDR (Hrsg.): Mitteilungen der Sektion Schulbiologie der Biologischen Gesellschaft, H. 14.

Paul, A. (2009): Die „Sonderstellung“ des Menschen im Lichte neuerer Erkenntnisse der Evolutionsbiologie. In: Der mathematische und naturwissenschaftliche Unterricht, Sonderheft Evolution, S. 5-10.

Paul, A. (Hrsg.) (2004): Biologie heute entdecken SII. Ein Lehr- und Arbeitsbuch. Braunschweig: Schroedel.

Pietsch, A. (1959): Zehn Jahre Biologieunterricht. Gedanken zum 10. Jahrestag unserer Deutschen Demokratischen Republik. In: Biologie in der Schule, 8. Jg., H. 10, S. 440-452.

Pöggeler, F. (2003): Schulbuchforschung in der Bundesrepublik Deutschland nach 1945. In: Wiater, W. (Hrsg.): Schulbuchforschung in Europa Bestandsaufnahme und Zukunftsperspektive. Bad Heilbrunn/Obb.: Julius Klinkhardt, S. 33-53.

Porges, K. (2014): Evolutionsbiologie im Biologieunterricht der SBZ/DDR für die 8. Klasse - Methoden und Ergebnisse einer Dokumentenanalyse. In: Knecht, P., Matthes, E., Schütze, S. \& Aamotsbakken, B. (Hrsg.): Methodologie und Methoden der Schulbuch- und Lehrmittelforschung. (= Beiträge zur historischen und systematischen Schulbuchforschung). Bad Heilbrunn: Julius Klinkhardt, S. 162-174.

Porges, K. (2016a): Kritik eines "kritischen Lehrbuches". Der Disput um Legitimierung und Deligitimierung eines kreationistischen Schulbuches. In: Matthes, E. \& Schütze, S. (Hrsg.). Schulbücher auf dem Prüfstand. Bad Heilbrunn: Julius Klinkhardt, S. 220-230.

Porges, K. (2016b): Die deutsche Wiedervereinigung und ihr Einfluss auf die Darstellung der Evolutionsbiologie im Biologielehrbuch der Klasse 10 aus dem Verlag Volk und Wissen. In: Matthes, E. \& Schütze, S. (Hrsg.): "1989" und Bildungsmedien (= Beiträge zur historischen und systematischen Schulbuchforschung). Bad Heilbrunn: Julius Klinkhardt, S. 192-201. 
Porges (im Druck): Evolution und Schule. In: Kaasch, M., Kaasch, J. \& Himmel, T. K. D. (Hrsg.): Biologie in der DDR. (= Verhandlungen zur Geschichte und Theorie der Biologie, Bd. 20.). Berlin: Wissenschaft und Bildung.

Porges, K. \& Hoßfeld, U. (2017): Evolutionsbiologie im Biologieunterricht der SBZ und der DDR. In: MNU Journal, 70. Jg., H. 6, S. 369-375.

Porges, K., Hoßfeld, U. \& Levit, Georgy S. (2017): Genetik in Schulbüchern der Sowjetischen Besatzungszone und DDR. In: Biospektrum, 23. Jg., H. 5, S. 603-604.

Porges, K., Hoßfeld, U., Mielewczik, M. \& Simunek, M. V. (2016): Zur Fachdisziplin Genetik und Gregor Johann Mendel in den Lehr- und Lernmaterialien der SBZ/DDR. In: Folia Mendeliana, 52/1, S. 45-66.

Porges, K. \& Porges, A. (2017): Die Evokids-Boxen im Praxistest. Ein didaktisch-methodisches Konzept für einen inklusiven Unterricht. In: Aamotsbakken, B., Matthes, E., Schütze S. (Hrsg.): Heterogenität und Bildungsmedien. Bad Heilbrunn: Julius Klinkhardt, S. 252-265.

Prehn, K. G. (1962): Die Bildrolle - ein wertvolles Anschauungsmittel. In: Biologie in der Schule, 11. Jg., H. 5, S. 234-235.

Prehn, K. G. (1964): Internationale Schulbiologentagung in Berlin. In: Biologie in der Schule, 13. Jg., H. 3, S. 134-136.

Ralle, B. \& Graf, D. (Hrsg.) (2009): Mathematischer und Naturwissenschaftlicher Unterricht. Sonderausgabe Evolution. Neuss: Klaus Seeberger.

Räuber, H. (1973): Zur Bedeutung der Farbe im Biologieunterricht (II). In: Biologie in der Schule, 22. Jg., H. 4, S. 128-133.

Referat für Presse- und Öffentlichkeitsarbeit der Max-Planck-Gesellschaft zur Förderung der Wissenschaften e. V. (Hrsg.) (2007): MaxPlanckForschung. Fokus Evolution.

Reif, W.-E., Junker, T. \& Hoßfeld, U. (2000): The synthetic theory of evolution: general problems and the German contribution to the synthesis. In: Theory in Biosciences, 119. Jg., H. 1, S. 41-91.

Reischock, W. (2009): Polytechnik und Persönlichkeit. In: Markus, U. (Hrsg.): Was war unserer Schule wert? Volksbildung in der DDR. Berlin: Das neue Berlin, S. 32-37.

Reuter, L. R. (1998): Administrative Grundlagen und Rahmenbedingungen. In: Führ, C. \& Furck C.-L. (Hrsg.): Handbuch der deutschen Bildungsgeschichte, Bd. 6. 1945 bis zur Gegenwart, Teilbd. 2. Deutsche Demokratische Republik und neue Bundesländer. München: Beck, S. 37-53. 
Reuter, L. R. (1998): Rechtliche Grundlagen und Rahmenbedingungen. In: Führ, C. \& Furck C.-L. (Hrsg.): Handbuch der deutschen Bildungsgeschichte, Bd. 6. 1945 bis zur Gegenwart, Teilbd. 2. Deutsche Demokratische Republik und neue Bundesländer. München: Beck, S. 26-36.

Richter, W. (1967): Die Lehrerbildung in der DDR. Eine Sammlung der wichtigsten Dokumente und gesetzlichen Bestimmungen für die Ausbildung der Lehrer, Erzieher und Kindergärtnerinnen. Berlin: Volk und Wissen.

Richter, W. (1972). Die Lehrerbildung in der DDR. Eine Sammlung der wichtigsten Dokumente und gesetzlichen Bestimmungen für die Ausbildung der Lehrer, Erzieher und Kindergärtnerinnen. Berlin: Volk und Wissen.

Rieppel, O. (2001): Georges-Luis Leclerc, Comte de Buffon (1707-1788). In: Jahn, I. \& Schmitt, M. (Hrsg.): Darwin \& CO. Eine Geschichte der Biologie in Portraits, Bd. 1. München: C.H.Beck, S. 31-50.

Rockstuhl, H. (2006): Schulgesetz der DDR 1965-1991/92. Gesetz über das einheitliche sozialistische Bildungssystem der DDR vom 25. Februar 1965. Bad Langensalza: Rockstuhl.

Rohde, A. (o. J.): Auf Fossiliensuche an der Ostsee. Gesteine, Fossilien, Fundmöglichkeiten. Neumünster: Wachholtz.

Rohde, A. (2007): Fossilien sammeln an der Ostseeküste. Trilobiten, Seeigel, Donnerkeile und Co. fossilführende Gesteine des südwestlichen Ostseeraumes. Neumünster: Wachholtz.

Rojek, R., Marquardt-Mau, B. \& Werther, J. (2012): Evolution als Thema im Sachunterricht - zwischen Instruktion und Konstruktion. In: Hartmut Giest, H., Heran-Dörr, E. \& Archie, C. (Hrsg.): Lernen und Lehren im Sachunterricht. Zum Verhältnis von Konstruktion und Instruktion. (= Probleme und Perspektiven des Sachunterrichts, Bd. 22). Klinkhardt, S. 111-118.

Rommel, K. (2006): Evolutionsbiologie und Schule. Unveröffentlichte Examensarbeit, Jena.

Rose, S. (2000): Darwins gefährliche Erben. Biologie jenseits der egoistischen Gene. München: C.H.Beck.

Roth, L. (Hrsg.) (1994): Pädagogik. Handbuch für Studium und Praxis. Studienausgabe. München: Ehrenwirth.

Rothmaler, W. (1952): Homologie und Analogie. In: Biologie in der Schule, 1. Jg., H. 8, S. 341-345. 
Rothmaler, W. \& Uschmann, G. (1957): Lehrbuch der Biologie für das 12. Schuljahr. Die Entstehung des Lebens auf der Erde und seine Entwicklung bis zum Menschen. Zur Geschichte der Abstammungslehre. Berlin: Volk und Wissen.

Rupke, N. (2001): Richard Owen (1804-1892). In: Jahn, I. \& Schmitt, M. (Hrsg.): Darwin \& Co. Eine Geschichte der Biologie in Portraits, Bd. 1. München: C.H.Beck, S. 254-259.

Russell, B. (2009): Philosophie des Abendlandes. Ihr Zusammenhang mit der politischen und der sozialen Entwicklung. 2. Aufl. Zürich: Europa.

Sandfuchs, U. (2010): Schulbücher und Unterrichtsqualität - historische und aktuelle Reflexionen. In: Fuchs, E., Kahlert, J. \& Sandfuchs, U. (Hrsg.): Schulbuch konkret. Kontexte Produktion Unterricht. Bad Heilbrunn: Klinkhardt, S. 11-26.

Schäfers, A. (2008): Zur Geschichte der Evolutionstheorien. In: Praxis der Naturwissenschaften-Biologie in der Schule, Jg. 57, H. 1, S. 40-45.

Schallenberger, H. (Hrsg.) (1976): Studien zur Methodenproblematik wissenschaftlicher Schulbucharbeit. Kastellaun: Aloys Henn.

Schmidt-Salomon, M. (2006): Manifest des evolutionären Humanismus. Plädoyer für eine zeitgemäße Leitkultur. 2. Aufl. Aschaffenburg: Alibri.

Schmidt-Salomon, M. \& Held, E. (Red.) (2011): Aufklärung im 21. Jahrhundert.

Scholl, B. (2014): Die Darstellung der Evolutionsbiologie in schulischen Biologie-Lehrbüchern der Oberstufe in Deutschland. Unveröffentlichte Examensarbeit, Jena.

Schubert, F. (1989a): Hinweise zur Fernsehsendung „Die Alten aus Bilzingsleben“. In: Biologie in der Schule, 38. Jg., H. 4, S. 139-140.

Schubert, F. (1989b): Informationen und Empfehlungen zur Arbeit mit den Sendungen des Bildungsfernsehens der DDR für das Fach Biologie Klassenstufe 10. Potsdam: Wissenschaftlich-Technisches Zentrum der Pädagogischen Hochschule „Karl Liebknecht“ Potsdam, als Manuskript gedruckt.

Schulz, J. (2001): Julian Huxley (1887-1975) und seine Familie. In: Jahn, I. \& Schmitt, M. (Hrsg.): Darwin \& Co. Eine Geschichte der Biologie in Portraits, Bd. 2. München: C.H.Beck, S. 128-145.

Schulze-Marx, A. (1954): Bemerkungen zur Lehrmittelforschung. In: Biologie in der Schule, 3. Jg., H. 11, S. 488-494. 
Schwarz, O. (1960): Vorwort. In: Biologische Gesellschaft in der Deutschen Demokratischen Republik (Hrsg.): Arbeitstagung zu Fragen der Evolution. Zum Gedenken an Lamarck - Darwin - Haeckel (20. bis 24. Oktober 1959 in Jena). Jena: G. Fischer, S. V.

Sehmrau, W. (1976): Langfristige analytische Tätigkeit und Nutzung ihrer Ergebnisse für die konkrete Führung des Fachunterrichts. In: Biologie in der Schule, 25. Jg., H. 7/8, S. 333-339.

Sekretariat der Ständigen Konferenz der Kultusminister der Länder in der Bundesrepublik Deutschland (Hrsg.) (2004): Beschlüsse der Kultusministerkonferenz. Einheitliche Prüfungsanforderungen in der Abiturprüfung Biologie. München: Luchterhand.

Sellin, V. (2008): Einführung in die Geschichtswissenschaft. 2., erw. Aufl. Göttingen: Vandenhoeck \& Ruprecht.

Shubin, N. (2008): Der Fisch in uns. Eine Reise durch die 3,5 Milliarden Jahre alte Geschichte unseres Körpers. Frankfurt am Main: Fischer.

Simon, H. (1971): Hinweise und Erläuterungen zum UnterrichtsmittelBedarfsplan. In: Biologie in der Schule, 20. Jg., H. 8, S. 334-335.

Simpson, G. G. (1984): Fossilien. Mosaiksteine zur Geschichte des Lebens. Heidelberg: Spektrum der Wissenschaft.

SKUS Lehrmittel GmbH (о. J.): Über uns. Online im Internet unter http://web2.cylex.de/reviews/viewcompanywebsite.aspx?firmaName $=$ sk us-lehrmittel-gmbh\&companyId=1986660 (23.02.2013).

Sohn, W. (2001): Hugo de Vries (1848-1935). In: Jahn, I. \& Schmitt, M. (Hrsg.): Darwin \& Co. Eine Geschichte der Biologie in Portraits, Bd. 2. München: C.H.Beck, S. 9-27.

Sommer, R. J. (2011): Zum Wissenschaftsverständnis der modernen Evolutionsbiologie. In: Graf, D. (Hrsg.): Evolutionstheorie - Akzeptanz und Vermittlung im europäischen Vergleich. Berlin etc.: Springer, S. 91-98.

Spörhase-Eichmann, U. \& Ruppert, W. (Hrsg.) (2008): Biologie-Didaktik. Praxishandbuch für die Sekundarstufe I und II. Berlin: Cornelsen Scriptor.

Staatliches Kontor für Unterrichtsmittel und Schulmöbel (Hrsg.) (1974): Biologie. Unterrichtsmittel. Leipzig: Dewag Werbung.

Stefhan, B. (1975): Die Urvögel als Modell für Abstammung und Entwicklung. In: Biologie in der Schule, 24. Jg., H. 11, S. 454-459.

Stein (1994): Schulbücher in Lehrerbildung und pädagogischer Praxis. In: Roth, L. (Hrsg.): Pädagogik. Handbuch für Studium und Praxis. Studienausgabe. München: Ehrenwirth, S. 752-759. 
Steinhaus, M. (2014): Kein Kreationismus bzw. „Intelligent Design“ an der FSU Jena! Ein öffentlicher Appell an Universitätsrektor Klaus Dicke und OB Albrecht Schröter. Online im Internet unter http:/ /www.unijena.de/unijenamedia/Downloads/faculties/bio_pharm/ag_didaktik/GB S_Brief_HST.pdf $<15.06 .2014>$.

Steinheit, B. (1959): Zum Übergangslehrplan für den Biologieunterricht an der erweiterten Oberschule. In: Biologie in der Schule, 8. Jg., H. 8, S. 371-375.

Storch, V., Welsch, U. \& Wink, M. (2001): Evolutionsbiologie. Berlin etc.: Springer.

Storch, V., Welsch, U. \& Wink, M. (2007): Evolutionsbiologie. 2. Aufl. Berlin etc.: Springer.

Stritzel, H. (1989): Das Schulbuch im Unterricht. Berlin: Volk und Wissen.

Stürmer, V. (2014): Kindheitskonzepte in den Fibeln der SBZ/DDR 19451990. Bad Heilbrunn: Julius Klinkhardt.

Sujew, D. (1986): Das Schullehrbuch. Berlin: Volk und Wissen.

Taylor, M. P. (2009): A re-evaluation of Brachiosaurus altithorax Riggs 1903 (Dinosauria, Sauropoda) and its generic separation from Giraffatitan brancai (Janensch 1914). In: Journal of Vertebrate Paleontology, 29 (3), 787-806.

Thüringer Ministerium für Bildung Wissenschaft und Kultur (Hrsg.) (2012):

Lehrplan für den Erwerb der allgemeinen Hochschulreife. Biologie.

Online im Internet unter https://www.schulportal-

thueringen.de/web/guest/media/detail?tspi=2284 <25.04.2014>.

Thüringer Ministerium für Bildung Wissenschaft und Kultur (Hrsg.) (2012):

Lehrplan für den Erwerb des Hauptschul- und Realschulabschlusses.

Biologie. Online im Internet unter https:/ /www.schulportalthueringen.de/web/guest/media/detail?tspi=2283 <25.04.2014>.

Tille, R. (1992a): Lehrpläne und Biologieunterricht in der DDR: Erinnerungen und Erfahrungen (1). In: Biologie in der Schule, 41. Jg., H. 10, S. 321-324.

Tille, R. (1992b): Lehrpläne und Biologieunterricht in der DDR:

Erinnerungen und Erfahrungen (2). In: Biologie in der Schule, 41. Jg., H. 11, S. 382-386.

Tille, R. (1992c): Lehrpläne und Biologieunterricht in der DDR: Erinnerungen und Erfahrungen (3). In: Biologie in der Schule, 41. Jg., H. 12, S. 427-429.

Tille, R. (1993a): Lehrpläne und Biologieunterricht in der DDR: Erinnerungen und Erfahrungen (4). In: Biologie in der Schule, 42. Jg., H. 4, S. 134-140.

Tille, R. (1993b): Lehrpläne und Biologieunterricht in der DDR:

Erinnerungen und Erfahrungen (5). In: Biologie in der Schule, 42. Jg., H. 7/8, S. 268-270. 
Tille, R. (1993c): Lehrpläne und Biologieunterricht in der DDR: Erinnerungen und Erfahrungen (6). In: Biologie in der Schule, 42. Jg., H. 11, S. 385-388.

Tille, R. (1993d): Lehrpläne und Biologieunterricht in der DDR:

Erinnerungen und Erfahrungen (Schluß). In: Biologie in der Schule, 42. Jg., H. 12, S. 430-432.

Todes, D. B. (2009): Darwins malthusische Metapher und russische Evolutionsvorstellungen. In: Engels, E.-M. (Hrsg.): Charles Darwin und seine Wirkung. Frankfurt am Main: Suhrkamp, S. 203-230.

Tröhler, D. \& Oelkers, J. (2005): Historische Lehrmittelforschung und Steuerung des Schulsystems. In: Matthes, E. \& Heinze, C. (Hrsg.): Das Schulbuch zwischen Lehrplan und Unterrichtspraxis. (= Beiträge zur historischen und systematischen Schulbuchforschung). Bad Heilbrunn/Obb.: Julius Klinkhardt, S. 95-107.

Uhe, E. (1976): Quantitative Verfahren bei der Analyse von Schulbüchern. In: Schallenberger, H. (Hrsg.): Studien zur Methodenproblematik wissenschaftlicher Schulbucharbeit. Kastellaun: Aloys Henn, S. 74-93.

Uhlig, A., Baer, H.-W., Dietrich, G., Fischer, H., Günther, J., Hopf, P. \& Loschan, R. (1962) (Hrsg.): Didaktik des Biologieunterrichts. Berlin: Deutscher Verlag der Wissenschaften.

Uhlig, G. (1970): Monumenta Paedagogica, Bd. 6. Dokumente zur Geschichte des Schulwesens in der Deutschen Demokratischen Republik. Teil 1: 1945-1955. Berlin: Volk und Wissen.

Unterbrunner, U. (2006): Biologie-Schulbücher. In: Gropengießer, H. \& Kattmann, U. (Hrsg.): Fachdidaktik Biologie. 7. Aufl. Köln: Aulis Deubner, S. 370-376.

Urania-Verlag (Hrsg.) (1974): 50 Jahre Urania-Verlag: 1924-1974. Leipzig etc.: Urania.

Uschmann, G. (1960): Ernst Haeckel. In: Biologische Gesellschaft in der DDR (Hrsg.): Arbeitstagung zu Fragen der Evolution. Zum Gedenken an Lamarck - Darwin - Haeckel (20. bis 24. Oktober 1959 in Jena). Jena: G. Fischer, S. 1-13.

Virtuelle Fachbibliothek der Universitätsbibliothek Johann Christian Senckenberg in Frankfurt am Main (o. J.): Top Ten plus eins. Online im Internet unter http://www.vifabio.de/darwinjahr2009/TopTen+1.html $<13.04 .214>$.

Vogelbein, L. (1959): Zur Behandlung der Entwicklungslehre in der 10. Klasse. Hinweise zur Arbeit mit dem Übergangslehrplan für das Schuljahr 1959/60. In: Biologie in der Schule, 8. Jg., H. 10, S. 452-461. 
Voss, J. (Hrsg.) (2008): Charles Darwin. Das Lesebuch. Frankfurt am Main: S. Fischer.

Wagner, P. (2008): Naturkundemuseen im Wandel. Von der Raritätenshow zum Forschungsinstitut. In: Praxis der Naturwissenschaften-Biologie in der Schule, 57. Jg., H. 1, S. 6-8.

Wagner, P. (2016): Englischunterricht in der DDR im Spiegel der Lehrwerke. Bad Heilbrunn: Julius Klinkhardt.

Wallin, A. (2011): Zu einer inhaltsorientierten Theorie des Lernens und Lehrens der biologischen Evolution. In: Graf, D. (Hrsg.):

Evolutionstheorie - Akzeptanz und Vermittlung im europäischen Vergleich. Berlin etc.: Springer, S. 119-139.

Wandel, P. (о. J.): Die demokratische Einheitsschule - Rückblick und Ausblick. Rede gehalten auf dem Pädagogischen Kongress. Leipzig. September 1947. Berlin etc.: Volk und Wissen.

Waschke, T. \& Lammers, C. (2011): Evolutionstheorie im Biologieunterricht (k)ein Thema wie jedes andere? In: Dreesmann, D., Graf, D. \& Witte, K. (Hrsg.): Evolutionsbiologie. Moderne Themen für den Unterricht. Heidelberg: Spektrum, S. 505-534.

Weber, H. (2006): Die DDR 1945-1990. München: Oldenbourg.

Wehner, R. \& Gehring, W. (1995). Zoologie. 23. Aufl. Stuttgart etc.: Georg Thieme.

Weinbrenner, P. (1992): Grundlagen und Methodenprobleme sozialwissenschaftlicher Schulbuchforschung. In: Fritzsche, K. P. (Hrsg): Schulbücher auf dem Prüfstand. Perspektiven der Schulbuchforschung und Schulbuchbeurteilung in Europa. Frankfurt am Main: Diesterweg, S. 33-54.

Weismann, A. (1904): Vorträge über Deszendenstheorie. Gehalten an der Universität zu Freiburg im Breisgau. 2. Aufl. Jena: Gustav Fischer.

Wendt, E. (1957): Die Entwicklung der Lehrerbildung in der sowjetischen Besatzungszone seit 1945. Bonn: Deutscher Bundes-Verlag.

Wermke, M., Kunkel-Razum, K. \& Scholze-Stubenrecht, W. (Hrsg.) (2006): Duden. Die deutsche Rechtschreibung. 24. Aufl. Mannheim etc.: Duden.

Wernecke, A. (1967a): Zur Einführung der Lehrpläne für die

Vorbereitungsklassen 9 und 10 zum Besuch der Erweiterten Oberschule. In: Biologie in der Schule, 16. Jg., H. 1, S. 1-12.

Wernecke, A. (1967b): Zur staatsbürgerlichen Erziehung im

Biologieunterricht. In: Biologie in der Schule, 16. Jg., H. 11, S. 457-467.

Wernecke, A. (1971): Zur Einführung des neuen Lehrplans für Klasse 10. In: Biologie in der Schule, 20. Jg., H. 8, S. 305-316. 
Wessel, H. (1974): Übrigens ist der Darwin ganz famos. In: Neues

Deutschland, 13. Februar, S. 11.

Wessel, H. (1977): Marx, Darwin und Aveling. Neuer Fund klärt alte Frage. In:

Neues Deutschland, 26./27. März, S. 15.

Wiater, W. (2003): Das Schulbuch als Gegenstand pädagogischer Forschung.

In: Wiater, W. (Hrsg.): Schulbuchforschung in Europa -

Bestandsaufnahme und Zukunftsperspektive. Bad Heilbrunn/Obb.: Julius

Klinkhardt, S. 11-21.

Wiater, W. (2006): Lehrplan, Curriculum, Bildungsstandards. In: Arnold, K.H., Sandfuchs, U. \& Wiechmann, J. (Hrsg.): Handbuch Unterricht. Bad Heilbrunn: Klinkhardt, S. 169-178.

Wiater, W. (Hrsg.) (2003): Schulbuchforschung in Europa -

Bestandsaufnahme und Zukunftsperspektive. Bad Heilbrunn/Obb.: Julius Klinkhardt.

Wilms, G. (2009): „Also kommst du nach Berlin!“‘. In: Markus, U. (Hrsg.): Was war unsere Schule wert?. Volksbildung in der DDR. Berlin: Das neue Berlin, S. 21-22.

Windelband, A. (1966a): Abstammungslehre III: Vergleichende Morphologie und Anatomie. (= Beihefte zur Lichtbildreihe). Berlin: Volk und Wissen.

Windelband, A. (1966b): Abstammungslehre IV: Ontogenie. (= Beihefte zur Lichtbildreihe). Berlin: Volk und Wissen.

Windelband, A. (1966c): Abstammungslehre V: Biogeographie. (= Beihefte zur Lichtbildreihe). Berlin: Volk und Wissen.

Windelband, A. (1967): Abstammungslehre VI: Anthropogenese. (= Beihefte zur Lichtbildreihe). Berlin: Volk und Wissen.

Windelband, A., Kurze, M. \& Freydank, W. (1969): Komplettiertes GesamtInhaltsverzeichnis.

Zabel, E. (1974): Eröffnung. In: Biologische Gesellschaft der DDR (Hrsg.): Mitteilung der Sektion Schulbiologie, H. 14, S. 3-7.

Zabel, E. (1998): Beiträge zur Fachdidaktik Biologie aus drei Jahrzehnten (1965 bis 1997). Güstrow: Selbstverlag.

Zabel, E. (2002): Sektion Schulbiologie der Biologischen Gesellschaft der

DDR. Online im Internet unter http:/ / www.erwinzabel.homepage.tonline.de/sektion.htm $<12.05 .2013>$.

Zabel, E. (2007): Geschichte und Erinnerungen. Güstrow: Eigenverlag.

Zabel, N. (2009): Zur Geschichte des Deutschen Pädagogischen Zentralinstituts der DDR. Eine institutionsgeschichtliche Studie. Dissertation, Chemnitz. Online im Internet unter http://dnb.info/1002480337/34 (25.08.2014). 
Zacharias, M. (1964): Biologie IV. Genetik, Evolution und Züchtung. Lehrbuch für die erweiterte Oberschule. 12. Klasse (2. Teil). Berlin: Volk und Wissen.

Zentralinstitut für Weiterbildung der Lehrer und Erzieher beim Ministerium für Volksbildung der Deutschen Demokratischen Republik (1969):

Lehrprogramm zur Weiterbildung in Kursen für Lehrer im Fach Biologie. In: Biologie in der Schule, 18. Jg., H. 6, S. 245-274.

Zentralinstitut für Weiterbildung der Lehrer und Erzieher Ludwigsfelde (1971): Studienhilfen für ausgewählte fachwissenschaftliche und didaktisch-methodische Themen des Lehrprogramms zur Weiterbildung in Kursen für Lehrer im Fach Biologie. In: Biologie in der Schule, 20. Jg., H. 1, S. 41-44.

Zillmer, H.-J. (2009): Kontra Evolution. DVD. Rottenburg: Kopp.

Zirnstein, G. (2001): August Weismann (1834-1914). In: Jahn, I. \& Schmitt, M. (Hrsg.): Darwin \& Co. Eine Geschichte der Biologie in Portraits, Bd. 1. München: C.H.Beck, S. 411-433.

Zott, R. \& Zott H.-J. (2010): Klaus Kloss. Online im Internet unter http://www.banzianer.de/p/Klaus.Kloss.htm <19.11.2014>. 



\section{Personenregister}

Abel, O. 48

Agricola 48

Alt, R. 79

Ambrosius, H. 109, 214, 216, 217

Anaximandros 40

Aristoteles 40

Arnold, H. 106, 109, 183, 194

Arrhenius, S. 45

Aveling, E. 52

Bach, H. 11, 33, 106, 110, 111, 117, 214, 215, 217, 225, 226, 227, 239, 240, 242, 243, 244, 246, 247

Baer, H.-W. 53, 83, 84, 88, 91, 101, 125,129

Bauer, G. 48

Baumgartner, G. 52, 63

Bayrhuber, H. 31

Beese, W. 14, 59

Behrens, H. 84, 84

Beljajew, D. K. 110

Berck, K.-H. 13, 16, 20, 21, 26, 87, 90, 91, 99, 264

Bernhardt, D. 109, 117, 118

Blume, M. 59

Böhme, H. 90

Böhme, W. 53

Boje, L. 106, 143

Bonnet, C. 41

Borneleit, P. 68

Brasseur, A. 58, 59

Breidbach, O. 54, 55

Brömer, R. 14, 54

Bucharin, N. I. 53, 54

Buffon, G. 40, 181

Butzmann, G. 75

Campbell, N. A. 31

Carroll, S. B. 51

Cassube, M. 127
Chambers, R. 41

Choppin, A. 23, 26

Comenius, J. A. 19

Crome, W. 109, 117

Cuvier, G. 41, 48, 156, 181, 233

Darwin, C. 14, 37, 39, 40, 41, 42, 43, 45, 46, 47, 48, 50, 51, 52, 53, 55, 56, 57, 58, 59, 82, 123, 132, 138, 149, 151, 156, 157, 160, 161, 163, 169, 173, 177, 178, 181, 182, 183, 184, 187, 188, 195, 202, 204, 209, 212, 213, 214, 215, 217, 231, 233, 238, 239, 240, 241, 242, 246, 262, 263

Dawkins, R. 56, 57

Depaepe, M. 17, 20, 21

Deschner, K. 57

Detlev, F. 22, 52

Diamond, J. M. 44, 57

Diehl, E. 69

Dietrich, G. 52, 89, 90, 91, 95, 96, $97,98,103,105,113,153,154$, 256, 258

Dobzhansky, T. 44, 47

Dorber, H. 111, 112

Dornhof, C. 25

Drefenstedt, E. 78, 152

Emmerich, F. 109

Empedokles 40

Engel, D. 26

Engels, E.-M. 14, 46, 55

Engels, F. 51, 53, 145, 155

Epikur 40

Fees, K. 11

Fehling, G. 85

Feuer, J. 79

Fey, C.-C. 22, 24

Fischer, G. 82 
Fischer, H. 91, 109

Fischer, J. 12

Fischer, M. S. 42, 52, 55, 129

Freudig, D. 43, 44, 45, 46

Friedrich, G. 20, 30, 53, 55, 71, 74, 81, 84, 85, 87, 89, 106, 109

Fritsch, H. 110, 133

Fritsche, K. P. 20

Fritz-Roy, R. 41

Gehlhaar, K.-H. 85, 91

Gehring, W. 31

Geisenhainer, F. 120, 121

Geißler, G. 63, 64, 65, 66, 67, 68, $70,71,72,73,75,76,77,79,92$, 93, 104

Gensel, P. 48

Gerber, T. 116

Gesang, K. 5, 127

Gießmann, H.-G. 106

Goethe, J. W. von 41, 46, 156

Gottschalk, R. 91, 106

Gould, S. J. 43

Graef, H. 52, 111, 112, 113, 114, 129, 198

Graf, D. 13, 14, 16, 20, 21, 26, 58, $87,90,91,99,264$

Griffhorn, B. 14

Grönke, O. 87, 88, 90, 91, 98, 120, 125

Gropengießer, G. 13, 21, 22, 104

Grotewohl, O. 84

Grümme, T. 31

Grümmer, G. 90

Gruner, H.-E. 115, 240, 242, 244, 245, 246

Günther, J. 91, 123

Günther, K.-H. 63, 64, 65, 66, 67, $68,69,70,71,72,73,75,76,77$, $78,79,80,92,93,96,152$

Gürtler, A. 119, 120
Haeckel, E. 14, 42, 43, 45, 46, 51, 54, 55, 82, 109, 127, 156, 157 , 181, 182, 188, 209, 212, 220, 231, 233, 238, 242, 246, 247, 262

Hagemann, R. 90

Hagen, A. 88

Heberer, G. 55

Hebig, D. 52, 63

Hedewig, R. 84

Heichler, G. 152

Heinze, C. 22

Heinzel, I. 85, 90, 91

Helms, J. 111

Henke, W. 33, 57

Herbst, A. 70, 72, 73, 75

Herrmann, L. 74, 119

Hoffmann, J. 84, 84

Högermann, C. 31

Honecker, M. 76, 92, 98, 125

Horn, F. 91, 92, 98, 112, 113, 139

Hoßfeld, U. 5, 12, 14, 20, 26, 32, 40, 41, 42, 43, 44, 45, 46, 47, 51, 54, 55, 56, 57, 59, 81, 82, 106, 109, 260, 264, 268, 272, 273, 276, 277 , 278, 279, 280, 282, 289

Höxtermann, E. 81, 82

Höxtermann, J. 81

Humboldt, A. von 55, 71, 81, 82, 116

Hundt, R. 88, 90, 91, 103, 129

Huxley, T. H. 42, 47, 156, 182, 233

Jacobsen, H.-J. 56

Jahn, I. 40

Jockel, S. 26

Jordan, S. 25

Junker, T. 14, 32, 40, 41, 43, 44, 45, $46,47,48,58,59$

Jürgens, U. 11, 22, 23, 24

Kant, I. 40

Kattmann, U. 13, 43, 56, 84, 104 
Kiper, H. 21

Kirchhöfer, D. 70

Kirsch, W. 83, 87, 98, 122

Kirschke, S. 55

Klinkhardt, J. 20

Kloss, K. 109

Knecht, P. 20

Knorr, H. A. 128

Knorre, D. von 54

Koch, M. 71

Köhler, H. 61, 64, 66, 67

Kolchinsky, E. I. 53, 54

König, I. 112

Körnig, G. 113

Kowalewsky, A. O. 182, 233

Kowalewsky, W. O. 182, 233

Kress, H. 116

Kreße, E. 88, 129

Krombholz, H. 108

Krüger, D. 12, 122

Krüger, K. 88

Krull, H.-P. 31

Krull, W. 14

Kuckartz, U. 28

Kühn, O. 106, 116, 143, 144, 168

Kull, U. 31

Kummer, G. 5, 9, 10, 13, 33, 70, 76, 79, 80, 81, 82, 89, 90, 91, 95, 96, 103, 105, 109, 110, 111, 112, 113, 117, 195, 199, 200, 215, 217

Kupke, A. 81, 82, 83, 91

Kurze, M. 85, 91

Kutschera, U. 14, 31, 32, 40, 41, 42, 43, 44, 45, 47, 48, 49, 50, 51, 56, 57, 58, 59, 189, 190, 194, 221, 249

Küttner, R. 31

Laitko, H. 54, 115

Lamarck, J.-B. de 41, 45, 46, 53, 55, $82,156,160,161,163,181,202$, 204, 209, 212, 231, 233, 262

Lammers, C. 14, 58

Lang, J. 64, 71
Langlet, J. 14

Lapworth, C. 189

Lässig, S. 11, 21, 22

Lefèvre, W. 41

Leinfelder, R. 42, 59

Lemke, W. 87, 105, 133, 176, 183, 184, 187, 188, 190, 191

Lengert, W. 52, 108, 256

Lenin, W. I. 55

Lepel, W.-D. 91

Lerchner, W. 91, 103

Levit, G. 5

Linke, W. 109

Linné, C. von 40, 156, 181

Lobeck, K. 88

Löbel, F. 105, 169, 183, 185

Löther, R. 40, 52, 54, 90, 118, 239

Lukrez 40

Luthardt, H. 81

Lyell, C. 182, 213, 240

Lyssenko, T. D. 54, 55, 134, 140, 156, 158, 181, 184, 216, 231, 242, 264

Markert, M. 5, 81

Marquardt-Mau, B. 14

Marx, K. 52, 53, 71, 120, 145, 239

Maschke, W. 105, 169, 183, 185

Matthes, E. 5, 19, 20

Matthes, W. 115

Matzke, M. 91, 112

Mayr, E. 31, 32, 39, 42, 44, 46, 47, 48, 49, 50, 51, 56, 57, 59, 263

Mayring, P. 24, 28, 29

McDougall, W. 156

Meincke, I. 88, 111

Meisert, A. 13

Meißner, K. 31, 91

Mendel, G. J. 15, 47, 140

Merbold, W.-E. 83

Meyer, H. 22

Meyers, P. 27, 28, 30

Millat, U. 88 
Miller, S. 32

Miram, W. 31

Mitschurin, I. W. 134, 138, 140, 156, 158, 181, 184, 216, 231, 242

Morgan, T. H. 140

Müller, A. H. 229

Müller, H. 14, 111, 246

Muller, H. J. 55

Müller, J. 91

Müntz, K. 90, 118

Naudin, C. V. 41

Neffe, J. 57

Nestler, H. 106, 109, 118, 123

Neumann, D. 24

Neuner, G. 12, 51, 52, 64, 75, 76, $77,78,152,256,260$

Neupert, D. 85

Nitschmann, J. 109

Nolte, M. 31

Oelkers, J. 20

Oparin, A. I. 32, 162

Osche, G. 43, 45

Owen, R. 50

Padberg, W. 109

Pälchen, U. 91, 103

Paul, A. 31, 43

Paul, S. 58

Pews-Hocke, C. 91

Pietsch, A. 75, 81, 82, 106

Plato 40

Plesse, W. 90, 111

Ploog, H. 103

Pöggeler, F. 12, 19, 20, 22, 28

Porges, A. 14

Porges, K. 12, 14, 15, 26, 58, 140, 258, 260, 264, 288, 289

Prehn, K. G. 83, 122

Räuber, H. 259

Reif, W. 45, 47
Reif, W.-E. 263

Reinhard, H. 115

Reischock, W. 67

Renatus, K. 115

Rensch, B. 47

Reuter, L. R. 64, 65, 75

Richter, W. 74, 89

Rieppel, O. 40, 41

Rockstuhl, H. 69, 74, 78, 80, 92, 98, 290

Rohde, A. 49

Rojek, R. 14

Romanes, G. J. 46

Rommel, K. 15, 15, 30

Rosenow, G. 79

Rothacker, D. 111

Rothe, H. 33, 57

Rothmaler, W. 81, 87, 90, 109, 115, 116, 232, 240, 242, 244

Russell, B. 40, 52, 181, 238

Rux, D. 90

Saint-Hilaire, E. G. 41, 156, 233

Sandfuchs, U. 19

Sauermost, R. 43, 44, 45, 46

Schaaffhausen, H. 41

Schäfers, A. 40

Scharf, K.-H. 31

Scheiding, K. 123

Schellhorn, M. 52

Schleiden, U. 110

Schlüter, W. 88

Schmidt-Salomon, M. 56, 57

Schmitt, M. 40

Schneider, G. 54, 115

Scholl, B. 15

Schröder, H. 88

Schubert, A. 88

Schubert, F. 123

Schulz, J. 42, 47

Schütze, S. 5, 19, 20

Schwarz, O. 80, 81, 82, 87

Schwier, H.-J. 91, 112 
Sehmrau, W. 125

Sellin, V. 11

Shubin, N. 49

Simon, F. 17, 20, 21, 125

Simpson, G. G. 47, 48, 128

Smith, W. 226

Sohn, W. 47

Sommer, K. 90, 112

Sommer, R. J. 46

Spencer, H. 43

Stalin, J. 54

Stebbins, G. L. 47

Stein, G. 22, 24, 123

Steinhaus, M. 59

Steinheit, B. 116, 168

Stephan, B. 128

Storch, V. 31, 32, 33, 40, 41, 43, 49, 50,59

Stresemann, E. 90

Stritzel, H. 260

Stürmer, V. 12, 20

Sujew, D. 23, 26

Taubert, E. 81, 82, 83

Taylor, M. P. 128

Teile, M. 111

Theuerkauf, H. 112, 113

Tille, R. 12, 75, 88, 93, 94, 95, 96, 97, 98, 107, 108, 114, 125, 131, 140

Timirjasew, K. A. 156, 182, 231, 233

Todes, D. B. 182

Tröhler, D. 20

Tse-Tung, M. 55

Uhe, E. 30

Uhlig, A. 87

Uhlig, G. 63, 64, 65, 66, 67, 68, 69, $70,71,72,73,75,76,77,78,79$, $80,92,93,96$

Ulbricht, W. 55

Ullrich, H. 112
Unterbrunner, U. 20, 22, 23, 24

Uschmann, G. 82, 106, 109, 115, 116, 182, 232, 240, 242

Vaas, R. 59

Vogt, H. 12

Voss, J. 50

Vries, H. de 47, 108

Wagner, P. 12, 16, 20, 128

Wallace, A. R. 41, 43, 45, 46, 55, 156, 181, 182, 213, 238, 240

Wallin, A. 13, 44

Wandel, P. 61, 63, 65, 70, 71, 78, 79, 120

Waschke, T. 14

Weber, H. 63, 64, 65, 69, 70

Wehner, R. 31

Weinbrenner, P. 26, 27, 28

Weismann, A. 41, 44, 46, 47, 140 , 156, 184, 216, 217, 242

Welsch, U. 31

Wendt, E. 76

Wermke, M. 51

Wernecke, A. 69, 92, 110, 153, 154

Wessel, H. 52, 53, 55

Wessely, I. 109, 111

Wiater, W. 19, 20, 21, 22, 24, 25

Wiesner, E. 116

Wiljams, W. R. 231

Willbrandt, H. 103

Windelband, A. 121, 122, 127

Wink, M. 31

Wobus, U. 118

Wolff, C. F. 53

Zabel, E. 5, 76, 81, 84, 85, 86, 90, 91, 97

Zacharias, M. 118

Zillmer, H.-J. 59

Zirnstein, G. 47

Zott, H.-J. 109

Zott, R. 109 



\section{Abbildungsverzeichnis}

Abbildung 1: Inhaltsanalytisches Kommunikationsmodell .........................................25

Abbildung 2: Grundformen des Interpretierens ………………………………......29

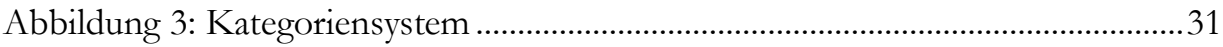

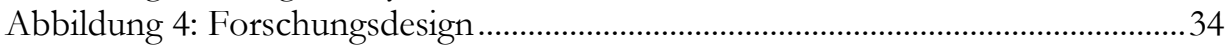

Abbildung 5: Strukturwandel des Schulsystems …………………………………....62

Abbildung 6: Struktur des Schulwesens bis 1950 .....................................................65

Abbildung 7: Struktur des Schulwesens seit dem Ende 1980er Jahre........................68

Abbildung 8: Lehrplan-Lehrbuch-Verschränkung für die 8. Klasse........................104

Abbildung 9: Lehrbuch Klasse 8 (1946) ..................................................................105

Abbildung 10: Lehrplan-Lehrbuch-Verschränkung für die 10. Klasse...................107

Abbildung 11: Lehrbuch Klasse 10 (1960) ...............................................................109

Abbildung 12: Vertreter der zweiten Hälfte des Paläozoikums ................................110

Abbildung 13: Lehrbuch Klasse $10(1968,1971)$....................................................111

Abbildung 14: Biologie in Übersichten (1975) ………………………………......112

Abbildung 15: Lehrbuch Klasse 10 (1988) .............................................................113

Abbildung 16: Tafelbild (1985) .............................................................................114

Abbildung 17: Unterrichtshilfen Klasse 10 (1985) ..................................................114

Abbildung 18: Lehrplan-Lehrbuch-Verschränkung für die 12. Klasse...................115

Abbildung 19: Lehrbuch Klasse 12 (1952)...........................................................115

Abbildung 20: Lehrbuch Klasse 12 (1965) .............................................................117

Abbildung 21: Verfasser und ihre Fachdisziplinen ................................................118

Abbildung 22: Skelettvergleich Gorilla-Mensch (SKUS 1974) ................................121

Abbildung 23: Literarische Mittel ............................................................................123

Abbildung 24: Lageplan für einen Biologiefachraum (1969)...................................126

Abbildung 25: Katalog Biologie, Unterrichtsmittel................................................126

Abbildung 26: Lehrbuch Klasse $10(1964,1987)$....................................................128

Abbildung 27: Stundenumfang, Lehrplan Klasse 8 (1951)....................................135

Abbildung 28: Stundenumfang, Lehrplan Klasse 8 (1952).....................................136

Abbildung 29: Stundenumfang, Lehrplan Klasse 8 (1953) ......................................137

Abbildung 30: Stundenumfang, Direktive Klasse 8 (1955) .....................................139

Abbildung 31: Lehrpläne Klasse 8, Grobstruktur......................................................141

Abbildung 32: Relative Häufigkeit der Kategorien, Lehrpläne Klasse 8 ................143

Abbildung 33: Stundenumfang, Lehrplan Klasse 10 (1951) .....................................145

Abbildung 34: Stundenumfang, Lehrplan Klasse 10 (1966) .....................................147

Abbildung 35: Stundenumfang, Lehrplan Klasse 10 (1967)....................................149

Abbildung 36: Stundenumfang, Lehrplan Klasse 10 (1969)...................................150

Abbildung 37: Stundenumfang, Lehrplan Klasse 10 (1988).....................................152

Abbildung 38: Lehrpläne Klasse 10, Grobstruktur................................................153

Abbildung 39: Stundenumfang, Lehrplan Klasse 12 Kurs B (1953).......................157

Abbildung 40: Stundenumfang, Lehrplan Klasse 12 Kurs A, C (1953)..................157 
Abbildung 41: Stundenumfang, Lehrplan Klasse 12 Kurs B (1954)......................158

Abbildung 42: Stundenumfang, Lehrplan Klasse 12 Kurs A, C (1954)..................159

Abbildung 43: Stundenumfang, Lehrplan Klasse 12 Kurs B (1956) .......................160

Abbildung 44: Stundenumfang, Lehrplan Klasse 12 Kurs A, C (1956).................160

Abbildung 45: Stundenumfang, Lehrplan Klasse 12 Kurs B (1959) .......................162

Abbildung 46: Stundenumfang, Lehrplan Klasse 12 Kurs A, C (1959).................162

Abbildung 47: Stundenumfang, Lehrplan Klasse 12 Kurs A, B (1961)..................164

Abbildung 48: Stundenumfang, Lehrplan Klasse 10/11 Kurs C (1961) ................164

Abbildung 49: Stundenumfang, Lehrplan Klasse 12 Kurs A, B (1967)..................165

Abbildung 50: Lehrpläne Klasse 12, Grobstruktur..................................................167

Abbildung 51: absoluter und relativer Umfang, Lehrbücher Klasse 8...................170

Abbildung 52: Abbildungen, Lehrbücher Klasse 8 ...............................................171

Abbildung 53: Biologische Begriffe, Lehrbuch Klasse 8 (1946) ..............................173

Abbildung 54: Kategorien im Lehrbuch Klasse 8 (1946).........................................174

Abbildung 55: Biologische Begriffe, Lehrbuch Klasse 8 (1951) .............................175

Abbildung 56: Kategorien im Lehrbuch Klasse 8 (1951) .........................................175

Abbildung 57: Biologische Begriffe, Lehrbuch Klasse 8 (1953) ..............................176

Abbildung 58: Kategorien im Lehrbuch Klasse 8 (1953).........................................177

Abbildung 59: Biologische Begriffe, Lehrbuch Klasse 8 (1957) ..............................178

Abbildung 60: Kategorien im Lehrbuch Klasse 8 (1957).........................................179

Abbildung 61: Umfang der Kategorien, Lehrbücher Klasse 8...............................180

Abbildung 62: Darwin, Wallace, Haeckel - Lehrbücher Klasse 8.............................181

Abbildung 63: Charles Darwin um 1880 .....................................................................181

Abbildung 64: Mitschurin, Lyssenko - Lehrbücher Klasse 8....................................184

Abbildung 65: Gehirne verschiedener Wirbeltiere, Handskelette..............................185

Abbildung 66: Knochengerüst des Grönlandwals ....................................................186

Abbildung 67: Die Scholle und ihre Entwicklung.......................................................188

Abbildung 68: Überblick über die Geschichte des Lebens.......................................189

Abbildung 69: Die Evolution der Lungenfische und Pferde.....................................191

Abbildung 70: absoluter und relativer Umfang, Lehrbücher Klasse 10 ................197

Abbildung 71: Abbildungen, Lehrbücher Klasse 10................................................197

Abbildung 72: Vordergliedmaßen der Wirbeltiere .....................................................199

Abbildung 73: Biologische Begriffe, Lehrbuch Klasse 10 (1960) ...........................202

Abbildung 74: Kategorien im Lehrbuch Klasse 10 (1960) ……………………......203

Abbildung 75: Biologische Begriffe, Lehrbuch Klasse 10 (1965) ............................204

Abbildung 76: Kategorien im Lehrbuch Klasse 10 (1965) …………………….....205

Abbildung 77: Biologische Begriffe, Lehrbuch Klasse 10 (1968) ...........................206

Abbildung 78: Kategorien im Lehrbuch Klasse 10 (1968) ……………………......206

Abbildung 79: Biologische Begriffe, Lehrbuch Klasse 10 (1971)............................208

Abbildung 80: Kategorien im Lehrbuch Klasse 10 (1971) .....................................208

Abbildung 81: Biologische Begriffe, Lehrbuch Klasse 10 (1988)...........................209

Abbildung 82: Kategorien im Lehrbuch Klasse 10 (1988) …………………….......210

Abbildung 83: Umfang der Kategorien, Lehrbücher Klasse 10...............................210 
Abbildung 84: Darwin, Wallace, Haeckel - Lehrbücher Klasse 10........................213

Abbildung 85: Mitschurin, Lyssenko, Weismann - Lehrbücher Klasse 10 ..........216

Abbildung 86: Die vier Hauptfarbmuster des Fliegenfängers.................................217

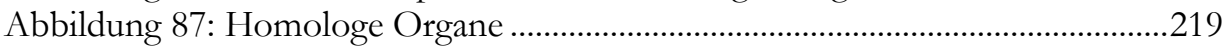

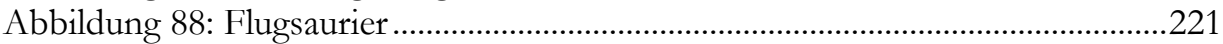

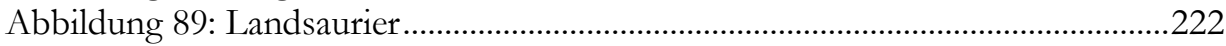

Abbildung 90: Der Urvogel und Merkmale von Reptilien und Vögeln..................224

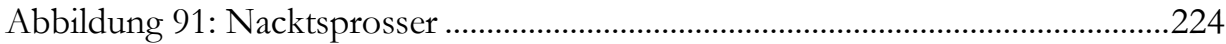

Abbildung 92: absoluter und relativer Umfang, Lehrbücher Klasse 12 ................228

Abbildung 93: Abbildungen, Lehrbücher Klasse 12...........................................228

Abbildung 94: Nautilus pompilius aus dem Indik................................................229

Abbildung 95: Biologische Begriffe, Lehrbuch Klasse 12 (1952) ...........................231

Abbildung 96: Kategorien im Lehrbuch Klasse 12 (1952) .....................................232

Abbildung 97: Biologische Begriffe, Lehrbuch Klasse 12 (1957)..........................233

Abbildung 98: Kategorien im Lehrbuch Klasse 12 (1957) .....................................234

Abbildung 99: Kategorien im Lehrbuch Klasse 12 (1965) .....................................234

Abbildung 100: Biologische Begriffe, Lehrbuch Klasse 12 (1965).........................236

Abbildung 101: Umfang der Kategorien, Lehrbücher Klasse 12 ..........................238

Abbildung 102: Ernst Haeckel ..............................................................................238

Abbildung 103: Darwin, Wallace, Haeckel - Lehrbücher Klasse 12.....................239

Abbildung 104: Das Forschungsschiff Beagle ........................................................241

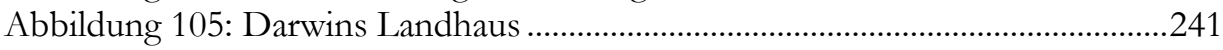

Abbildung 106: Mitschurin, Lyssenko, Weismann - Lehrbücher Klasse 12 ........243

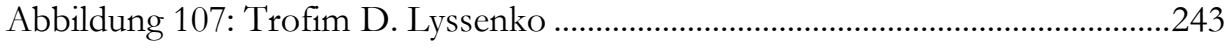

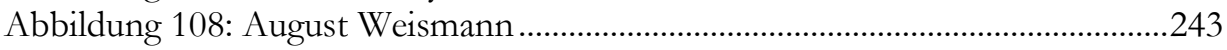

Abbildung 109: Ausbildung und Umwandlung der Hinterleibsgliedmaßen .........244

Abbildung 110: Niedere Krebse und sich ähnelnde Larven....................................247

Abbildung 111: Entwicklungsstadien verschiedener Wirbeltiere..............................248

Abbildung 112: Die Entwicklung des Lebens auf der Erde ....................................249

Abbildung 113: Die Entfaltung der Nautiloide im Alt-Paläozoikum .....................250

Abbildung 114: Rekonstruktion des Urvogels Archaeopteryx................................252

Abbildung 115: Evolutionsbiologie in den Jahrgängen 8, 10 und 12.....................257

Abbildung 116: Evolutionstheorien in den Lehrbüchern der SBZ/DDR............263 



\section{Tabellenverzeichnis}

Tabelle 1: Funktionen von Schulbüchern.....................................................................23

Tabelle 2: Anforderungen aus der Praxis an ein Lehrbuch ......................................24

Tabelle 3: Bezugssysteme und Aspekte des Forschungsansatzes ..............................27

Tabelle 4: Primärquellen und Referenzliteratur............................................................31

Tabelle 5: Typologie von Sach- und Ideologiebezug ...................................................35

Tabelle 6: Weiterentwicklung der Postulate des klassischen Darwinismus...............46

Tabelle 7: Geologische Zeitskala bzw. stratigrafische Tabelle ...................................4 49

Tabelle 8: Geschichte der Schule der DDR ……………………………………......62

Tabelle 9: Themen der Schulbiologentage.................................................................... 86

Tabelle 10: Ausbildungsmöglichkeiten für Fachlehrerinnen und -lehrer .................89

Tabelle 11: Lehrstühle für Biologiedidaktik der DDR ………………………….......91

Tabelle 12: Entwicklung der Stundentafel für das Fach Biologie..............................93

Tabelle 13: Lehrplankonzeption von 1947 .................................................................94

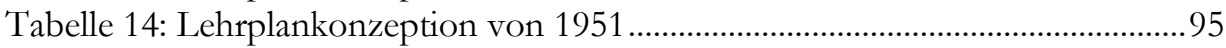

Tabelle 15: Lehrplankonzeption ab 1959 ..................................................................

Tabelle 16: Lehrplankonzeption ab 1965 ..............................................................98

Tabelle 17: Lehrplankonzeption ab 1988 ....................................................................99

Tabelle 18: Epochal bedeutsame Lehrpläne und Lehrbücher..................................102

Tabelle 19: Institutionen für Unterrichtsmedien.......................................................119

Tabelle 20: Unterrichtsmedien von 1962 und 1969 ................................................122

Tabelle 21: Unterrichtsmedien von 1971 und 1972 ...............................................124

Tabelle 22: Unterrichtsmedien von 1980 ...............................................................127

Tabelle 23: Sach- und Ideologiebezug, Lehrpläne Klasse 8......................................140

Tabelle 24: Lehrpläne Klasse 8, Kapitelüberschriften und Stundenumfang.........142

Tabelle 25: Lehrpläne Klasse 8, Verteilung der Kategorien .....................................142

Tabelle 26: Sach- und Ideologiebezug, Lehrpläne Klasse 10 ...................................153

Tabelle 27: Lehrpläne Klasse 10, Kapitelüberschriften und Stundenumfang.......154

Tabelle 28: Lehrpläne Klasse 10, Verteilung der Kategorien ...................................155

Tabelle 29: Inhalte, Lehrplan Klasse 12 .....................................................................156

Tabelle 30: Sach- und Ideologiebezug, Lehrpläne Klasse 12 .................................166

Tabelle 31: Lehrpläne Klasse 12, Kapitelüberschriften und Stundenumfang.......167

Tabelle 32: Lehrpläne Klasse 12, Verteilung der Kategorien .....................................168

Tabelle 33: äußeres und inneres Design, Lehrbücher Klasse 8...............................172

Tabelle 34: Strukturierung der Lehrbücher für die Klasse 8 ....................................180

Tabelle 35: Frequenzanalyse (Gesamt), Lehrbücher Klasse 8...................................181

Tabelle 36: Darwins Leben und Werk, Lehrbücher Klasse 8..................................182

Tabelle 37: Darwin'schen Theorien, Lehrbücher Klasse 8......................................183

Tabelle 38: Systemtabelle, Lehrbücher Klasse 8 ……………………………….......190

Tabelle 39: Entwicklungsreihen, Lehrbücher Klasse 8...............................................191

Tabelle 40: Zwischenformen und Entwicklungsreihen, Lehrbücher Klasse 8.....192 
Tabelle 41: Fossilisation, Lehrbücher Klasse 8.........................................................193

Tabelle 42: äußeres und inneres Design, Lehrbücher Klasse 10.............................201

Tabelle 43: Strukturierung der Lehrbücher für die Klasse 10 ..................................211

Tabelle 44: Frequenzanalyse (Gesamt), Lehrbücher Klasse 10................................212

Tabelle 45: Darwins Leben und Werk, Lehrbücher Klasse 10.................................213

Tabelle 46: Darwin'schen Theorien, Lehrbücher Klasse 10.......................................214

Tabelle 47: Systemtabelle, Lehrbücher Klasse 10.....................................................221

Tabelle 48: Entwicklungsreihen, Lehrbücher Klasse 10............................................223

Tabelle 49: Zwischenformen und Entwicklungsreihen, Lehrbücher Klasse 10...223

Tabelle 50: Fossilisation, Lehrbücher Klasse 10....................................................225

Tabelle 51: äußeres und inneres Design, Lehrbücher Klasse 12.............................230

Tabelle 52: Strukturierung, Lehrbücher Klasse 12 ………………………………...237

Tabelle 53: Frequenzanalyse (Personen), Lehrbücher Klasse 12 ..............................239

Tabelle 54: Darwins Leben und Werk, Lehrbücher Klasse 12................................240

Tabelle 55: Darwin'schen Theorien, Lehrbücher Klasse 12....................................240

Tabelle 56: Systemtabelle, Lehrbücher Klasse 12...................................................249

Tabelle 57: Entwicklungsreihen, Lehrbücher Klasse 12............................................251

Tabelle 58: Zwischenformen und Entwicklungsreihen, Lehrbücher Klasse 12...251

Tabelle 59: Fossilisation, Lehrbücher Klasse 12......................................................253

Tabelle 60: Lehrpläne mit evolutionsbiologischen Inhalten.....................................257

Tabelle 61: Sach- und Ideologiebezug der Klassen 8, 10 und 12............................258

Tabelle 62: Vergleich der Inhaltsstruktur der Lehrbücher und Lehrpläne.............261

Tabelle 63: Kategoriale Raumanalysen der Lehrbuchtexte ........................................262 
Annals of the History and Philosophy of Biology, Volume 18 (2013)

Karl Porges: Evolutionsbiologie im Biologieunterricht der SBZ/DDR

Inhalt

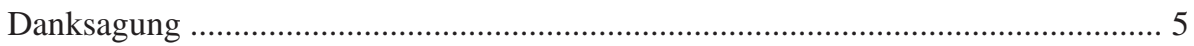

Geleitwort von Gertrud Kummer .......................................................................... 9

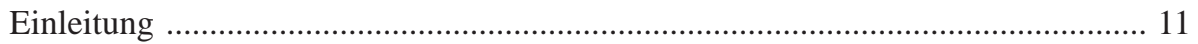

Zum Stellenwert der Schulbuchforschung ........................................................... 19

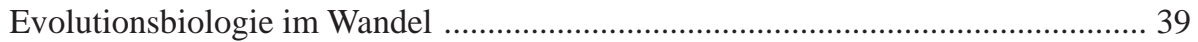

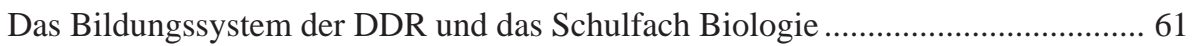

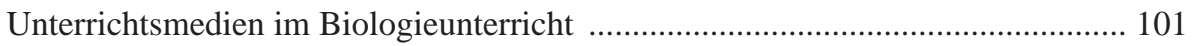

Der Lehrplan für Biologie ............................................................................. 131

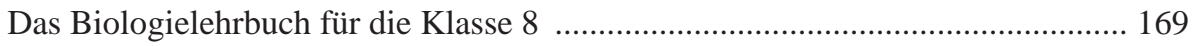

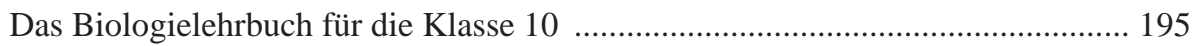

Das Biologielehrbuch für die Klasse 12 ........................................................ 227

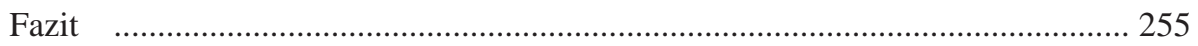

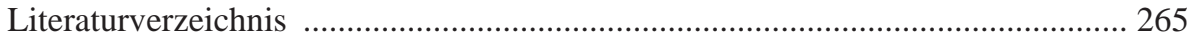

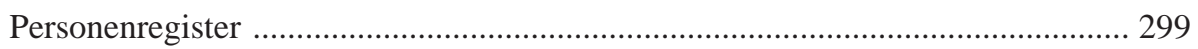

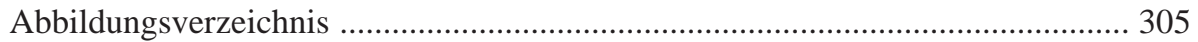

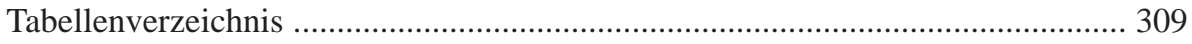


The name DGGTB (Deutsche Gesellschaft für Geschichte und Theorie der Biologie; German Society for the History and Philosophy of Biology) reflects recent history as well as German tradition. The Society is a relatively late addition to a series of German societies of science and medicine that began with the "Deutsche Gesellschaft für Geschichte der Medizin und der Naturwissenschaften", founded in 1910 by Leipzig University's Karl Sudhoff (1853-1938), who wrote: "We want to establish a 'German' society in order to gather German-speaking historians together in our special disciplines so that they form the core of an international society...". Yet Sudhoff, at this time of burgeoning academic internationalism, was "quite willing" to accommodate the wishes of a number of founding members and "drop the word German in the title of the Society and have it merge with an international society". The founding and naming of the Society at that time derived from a specific set of historical circumstances, and the same was true some 80 years later when in 1991, in the wake of German reunification, the "Deutsche Gesellschaft für Geschichte und Theorie der Biologie" was founded. From the start, the Society has been committed to bringing studies in the history and philosophy of biology to a wide audience, using for this purpose its Jahrbuch für Geschichte und Theorie der Biologie. Parallel to the Jahrbuch, the Verhandlungen zur Geschichte und Theorie der Biologie has become the by now traditional medium for the publication of papers delivered at the Society's annual meetings. In 2005 the Jahrbuch was renamed Annals of the History and Philosophy of Biology, reflecting the Society's internationalist aspirations in addressing comparative biology as a subject of historical and philosophical studies.

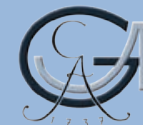

GEORG-AUGUST-UNIVERSITÄT GÖTTINGEN

ISBN: 978-3-86395-333-1

eISSN: $2512-5923$

\section{Universitätsverlag Göttingen}

